La montagne explorée, étudiée et représentée : évolution des pratiques culturelles depuis le $\mathrm{XV} \|^{\mathrm{e}}$ siècle

Louis Bergès (dir.)

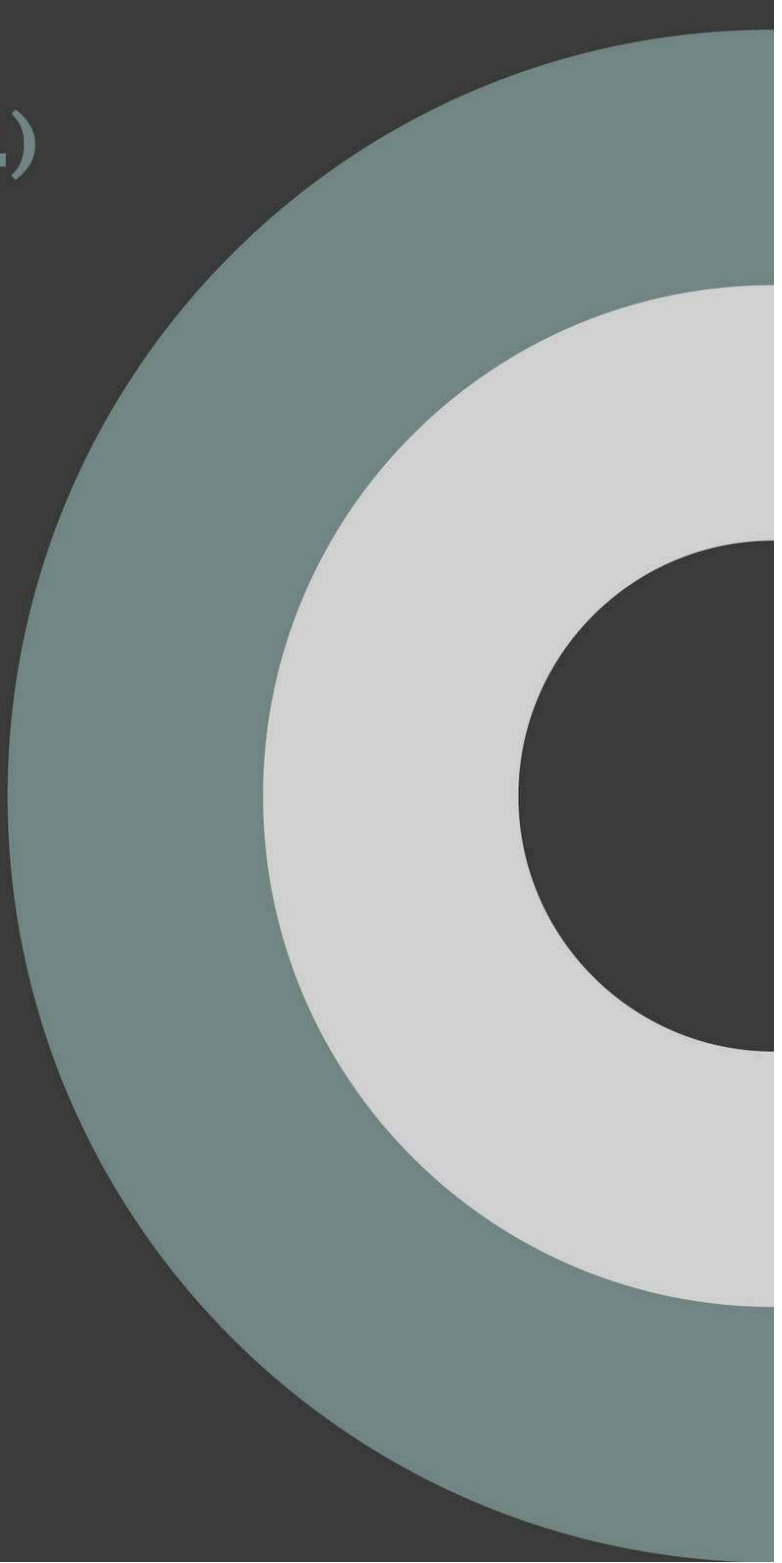




\title{
La montagne explorée, étudiée et représentée : évolution des pratiques culturelles depuis le $\mathrm{XVIII}^{\mathrm{e}}$ siècle
}

\author{
Louis Bergès (dir.)
}

DOI : $10.4000 /$ books.cths. 11252

Éditeur : Éditions du Comité des travaux historiques et scientifiques Année d'édition : 2020

Date de mise en ligne : 9 juin 2020

Collection: Actes des congrès nationaux des sociétés historiques et scientifiques

ISBN électronique : 9782735508877

\section{Sbooks}

http://books.openedition.org

\section{Référence électronique}

BERGĖS, Louis (dir.). La montagne explorée, étudiée et représentée : évolution des pratiques culturelles depuis le xvIII siècle. Nouvelle édition [en ligne]. Paris : Éditions du Comité des travaux historiques et scientifiques, 2020 (généré le 20 novembre 2020). Disponible sur Internet : <http://

books.openedition.org/cths/11252>. ISBN : 9782735508877. DOl : https://doi.org/10.4000/ books.cths. 11252 .

Ce document a été généré automatiquement le 20 novembre 2020.

(c) Éditions du Comité des travaux historiques et scientifiques, 2020

Conditions d'utilisation:

http://www.openedition.org/6540 
Le Siècle des lumières, qui consacre l'ouverture des élites européennes à la modernité scientifique, est aussi celui qui pousse les mêmes sociétés vers les sommets et les glaciers des montagnes. Objet de fascination et non plus de crainte, la montagne apparaît, à la suite de Rousseau et Senancour, dans toute sa majesté, à la fois vierge, mystérieuse, repliée sur elle-même et porteuse d'un message d'universalité. Après avoir longtemps suscité peur et préjugés depuis l'Antiquité, la montagne est devenue au $\mathrm{XVIII}^{\mathrm{e}}$ siècle un territoire de conquête et de découverte générant toute une mythologie et un imaginaire qui vont modifier le rapport des sociétés européennes avec le milieu des sommets.

Le Congrès national des sociétés historiques et scientifiques rassemble chaque année universitaires, membres de sociétés savantes et jeunes chercheurs. Ce recueil est issu de travaux présentés lors $\mathrm{du} 142^{\mathrm{e}}$ Congrès sur le thème «Circulations montagnardes, circulations européennes ».

\section{LOUIS BERGÈS}

Conservateur général du patrimoine, membre du CTHS, section Histoire du monde moderne, de la Révolution française et des révolutions 
SOMMAIRE

Introduction

Louis Bergès

\section{Naissance et développement d'une nouvelle sensibilité}

Des nouvelles des cimes : les échanges naturalistes sur la montagne au XVII' siècle (correspondance entre Villars, Picot de la Peyrouse et Ramond de Carbonnières) Émilie-Anne Pépy

D'une montagne à une autre : trois naturalistes étroitement liés

L'expérience des marges de la République des sciences

La montagne dans la communication scientifique

Jean-André Deluc (1727-1817) et la montagne comme objet d'étude et norme éthique Sabine Kraus

Le concept de Nature

Lettres physiques sur les montagnes, objet d'investigation scientifique

Lettres Morales sur les Montagnes, norme éthique

Le récit guerrier en montagne dans la fiction littéraire européenne (1780-1850)

Louis Bergès

À la recherche des origines: mythes fondateurs et courants de pensée

Le champ de bataille de montagne ou les champs de l'imaginaire

Le guerrier montagnard, héros romantique

Un nouvel l'horizon : l'Orient guerrier et ses montagnes

Figures romantiques de la mobilité et de l'immobilité montagnardes : les voyages aux Alpes et aux Pyrénées de Victor Hugo (1825-1843)

Odile Parsis-Barubé

Des mobilités étagées

Figures romantiques de l'immobilité montagnarde

Analyse du discours de la circulation en montagne : l'influence de John Ruskin sur la géographie alpine (1858)

Samia Ounoughi

Les modalités de la mise en discours de la géographie alpine ou la subversion du genre épistolaire

Contexte du voyage

De l'objet spatial à l'objet géographique

Voyages pittoresques dans les anciennes Pyrénées : déambulations romantiques et villégiature de montagne (1800-1860)

Viviane Delpech

Le triomphe de la nature promoteur de la villégiature

Récit de l'histoire et invention culturelle

L'homme et la société industrielle au cœur de l'univers

Apparat social et illusion de l'altérité

Épilogue : du roman romantique pyrénéen au mythe du thermalisme 
La montagne : lieu de résistance aux règles de l'Académie des beaux-arts et thème de prédilection des artistes de la modernité au tournant du siècle Véronique Richard-Brunet

La résistance aux règles académiques de l'Académie des beaux-arts

Paul Cézanne (1839-1906), face à la Sainte-Victoire, contre l'art des bourgeois

Henri Matisse (1869-1954), les fauves à Collioure : de la dynamite au pied des Albères

Pablo Picasso (1881-1973), la naissance d'un cubisme radical dans les Pyrénées

Les expressionnistes allemands et les Alpes de Bavière, comme un volcan bouillonnant

La montagne et l'art moderne

Le royaume dans le ciel : regard des pionniers béarnais de la Société des missionnaires évangéliques de Paris sur les montagnes du Sotho africain (1833-1880)

Laurence Espinosa

Une ligne sur l'horizon

L'Écoulement

Horizons

Circulations savantes et production des identités territoriales dans les Pyrénées centrales (1880-1930)

Arnauld Chandivert

Les territoires de l'érudition pyrénéenne : développement, recrutement social et univers d'action Centres d'intérêt, registres des singularités et modes de production

Savoirs érudits : organisation des échelles de la légitimité et formes de division du travail

La circulation d'une légende épique de fondation sur les chemins de Saint-Jacques : la légende de Dame Carcas et ses adaptations pyrénéennes, ibériques et occitanes Gauthier Langlois

La Légende de Dame Carcas : un récit épique

Des légendes similaires

Héroïnes : princesses et châtelaines

\section{Nouvelles appropriations contemporaines}

De la montagne comme adjuvant à la cure au site de loisirs urbains : le Revard et Aix-lesBains $\left(\mathrm{XIX}^{e}-\mathrm{XX}^{e}\right.$ siècle)

Elsa Belle et Philippe Gras

De la montagne agropastorale à la montagne climatique : de la propriété des marquis d'Aix à une invention aixoise

Relier la ville thermale et la station d'altitude

De la station climatique à la station mondaine de sports d'hiver : la proximité et la complémentarité de la ville thermale

De la station mondaine au site de loisirs urbains : un poumon urbain de proximité

Arts, culture, patrimoine du Queyras (XIX $-\mathrm{XX}^{e}$ siècles)

Jean-Gérard Lapacherie

Une population lettrée

Pratiques artistiques

Vitraux, estampes et lithographies

Meubles et objets sculptés 
Les Apennins comme espace d'expérimentation d'une nouvelle approche méthodologique de l'histoire de l'art : la naissance de la notion de bene culturale (1968-1971)

Sandra Costa et Anna-Lisa Carpi

Aux origines du rapport entre l'art et les lieux

La région des Apennins comme terrain d'expérimentation méthodologique pour le patrimoine culturel

L'institutionnalisation d'une expérience et l'actualité d'un héritage culturel

L'introduction des principes de la lecture de paysage et de l'art contemporain dans

l'interprétation de géosites sur le territoire de la réserve naturelle géologique de HauteProvence

Christel Venzal

La lecture de paysages, outil d'interprétation des géosites

La Nature, matière première de l'artiste : introduction au Land Art

Les montagnes andines et ses habitants : le Pérou sous l'œil de la caméra de l'alpiniste français Lionel Terray (1952-1964)

Chloé Tessier-Brusetti

Représentations françaises des Indiens du Pérou : antécédents des images de Terray Regard de Lionel Terray sur les Indiens : analyse des images rapportées du Pérou

Considération pour les Indiens au Pérou

La représentation de la montagne corse dans la littérature du XXI siècle Pierre Bertoncini

La représentation de la montagne chez Marie Susini

La représentation de la montagne chez Marcu Biancarelli

La représentation de la montagne dans Murtoriu

La représentation de la montagne chez Jérôme Ferrari

La montagne, un lieu tragique?

Un classique du roman de montagne : Joseph Peyré (1892-1968)

Pierre Peyre

La fresque romanesque de Joseph Peyré

Entre le réel et l'imaginaire, l'univers littéraire et montagnard de Peyré

Joseph Peyré (1892-1968) : radiographe pyrénéen

Christian Manso

Radiographie de son enfance

Réflexion au soir de la vie

Une ré-Vision du Pyrénéisme pour le $\mathrm{XXI}^{e}$ siècle

Manel Rocher Gonzalez et Claude Molinier

Le $\mathrm{xxI}^{\mathrm{e}}$ siècle

La vision

« Il faut poursuivre l'éducation avec les valeurs du Pyrénéisme »

Éducation et Pyrénéisme : la vision d'Henri Beraldi révisée 
NOTE DE L'ÉDITEUR

Les articles de cet ouvrage ont été validés par le comité de lecture des Éditions du Comité des travaux historiques et scientifiques dans le cadre de la publication des actes du $142^{\mathrm{e}}$ Congrès national des sociétés historiques et scientifiques tenu à Pau en 2017. 


\title{
Introduction
}

\author{
Louis Bergès
}

1 Le Siècle des lumières, qui consacre l'ouverture des élites européennes à la modernité scientifique, est aussi celui qui pousse les mêmes sociétés vers les sommets et les glaciers des montagnes. Objet de fascination et non plus de crainte, la montagne apparaît, à la suite de Rousseau et Senancour, dans toute sa majesté, à la fois vierge, mystérieuse, repliée sur elle-même et porteuse d'un message d'universalité. Après avoir longtemps suscité peur et préjugés depuis l'Antiquité, la montagne est devenue au $\mathrm{XVIIII}^{\mathrm{e}}$ siècle un territoire de conquête et de découverte générant toute une mythologie et un imaginaire qui vont modifier le rapport des sociétés européennes avec le milieu des sommets.

2 Avec l'émergence d'une culture spécifique et des pratiques culturelles nouvelles comme le tourisme et les sports d'hiver, la vie montagnarde, ses acteurs, ses promoteurs vont se transformer pour offrir un visage profondément renouvelé. L'analyse historique de ce mouvement qui se déploie depuis deux siècles jusqu'à aujourd'hui a fait l'objet de dix-neuf contributions, présentées au cours du Congrès national des sociétés historiques et scientifiques de Pau en 2017 puis rassemblées dans le présent volume.

3 Un premier groupe traite à la fois des premières études naturalistes et leurs auteurs, de l'observation géographique et anthropologique, de l'analyse littéraire et artistique des mythes et des nouvelles représentations montagnardes en couvrant une période qui va du Siècle des lumières à la fin des décennies flamboyantes du romantisme européen. Le second groupe de textes s'attache à développer des problématiques liées aux mutations de la période contemporaine: patrimonialisation des sites, nouvelles mobilités, circulation des idées et des arts, promotion de la civilisation des loisirs, alternatives environnementales.

4 Les approches de la montagne sont multiples: scientifique, militaire, artistique ou littéraire. De chacune d'entre elles ont émergé des discours divers qui interrogent le rapport au progrès, aux traditions ancestrales, à la conservation du milieu paysager, au monde extérieur en général et à ses mouvements d'exploration du territoire. À travers la naissance d'un monde nouveau, découvert par les sociétés occidentales, apparaît aussi une civilisation montagnarde traversée depuis longtemps par des influences 
nombreuses, fruit notamment d'un mouvement régulier d'émigration et de retour de sa population active. À partir des années 1750, les Alpes, les Pyrénées, puis des montagnes exotiques comme celles de Bernardin de Saint-Pierre à l'île Maurice sont l'objet de toutes les attentions des scientifiques, des voyageurs pleins de curiosité, des écrivains et artistes, dans un grand foisonnement d'idées et de projets où se mêlent l'analyse des variations glaciaires et la poésie des cimes.

5 Cette évolution du sentiment collectif vis-à-vis de la montagne ne s'est pas faite d'un seul élan. D'une fascination originelle de type métaphorique, celle des émotions littéraires du préromantisme ainsi que d'une phase d'appropriation des connaissances, on est progressivement passé à une véritable conquête du terrain, progressant du pittoresque au romanesque, du symbolique à la réalité montagnarde : la montagne s'est retrouvée enrichie à l'occasion de valeurs objectives et subjectives centrées autour de l'authenticité, de la pureté des mœurs, de l'enracinement fort dans la terre nourricière, de la fierté et du goût profond de liberté, toutes susceptibles d'admiration pour l'harmonie vivifiante qu'elle dégage.

6 Ainsi, à partir du moment où elle est l'objet de découverte et d'exploration passionnées, la montagne se voit attribuer des qualités qui vont d'une part être confinées dans l'ordre des représentations à travers l'art et la littérature, et d'autre part trouver une expression concrète grâce à des pratiques nouvelles, faisant parfois appel à des expériences alternatives, notamment dans les domaines touristique et culturel.

7 Après les lieux, les hommes. Les hommes vivant en montagne, les "montagnards ", sont eux aussi l'objet d'un regard nouveau de la part de l'extérieur : c'est l'apparition d'un type social qui, au même titre que l'ouvrier ou le bourgeois, va servir dans certains pays à construire une mythologie populaire et nationale. C'est aussi la définition de traits culturels spécifiques qui vont singulariser l'identité du montagnard liée à son mode de vie. Le montagnard suscite la même volonté encyclopédique, la même fréquentation visuelle que la roche ou la hauteur des sommets, il est, lui aussi, photographié comme le panorama d'un glacier.

8 Dès lors qu'elle a été conquise, au siècle suivant, la montagne est devenue familière, espace de développement, de circulation et d'échanges culturels avant de générer un sentiment collectif, comme pour la nature en général, d'affection protectrice face à de nouveaux dangers qui la menacent, ceux de la pollution et du dérèglement climatique.

L'analyse des différents textes présentés lors du congrès de Pau traduit la grande diversité des approches qui ont rythmé les travaux du congrès autour du thème de la montagne étudiée et représentée. Dans la première partie consacrée à la période de naissance et de développement de la nouvelle sensibilité vis-à-vis de la montagne, la communication d'Émilie-Anne Pépy met l'accent sur le premier vecteur de transformation des mentalités, l'observation du paysage montagnard, où se déploie l'activité pionnière de trois savants, à savoir Picot de Lapeyrouse, Villars et Ramond de Carbonnières, contemporains du grand explorateur des Alpes, Saussure, le futur père du pyrénéisme. Les difficultés de la diffusion du savoir accumulé témoignent du combat mené par les premiers explorateurs scientifiques pour faire reconnaitre leurs découvertes. Avec le naturaliste genevois Jean-André Deluc, Sabine Kraus ajoute à l'étude du naturalisme montagnard les résultats des travaux d'un autre pionnier, 
reconnu comme précurseur par Saussure et Cuvier : théologie, géologie et météorologie se mêlent à l'anthropologie pour faire de la montagne alpine un laboratoire pour l'étude du climat et de son influence sur la vie des habitants.

Les représentations de la montagne se développent dans l'art et la littérature à mesure que les élites européennes s'approprient le territoire comme objet familier d'étude et d'exploration: Louis Bergès s'intéresse au récit guerrier contenu dans des œuvres majeures de la littérature européenne, de Guillaume Tell de Schiller aux Carbonari de la montagne de Giovanni Verga en passant par Salammbô de Gustave Flaubert, qui posent à la fois le cadre de nouvelles intrigues romanesques et l'ébauche d'une sacralisation littéraire. Odile Parsis-Barubé scrute les récits de voyage de Victor Hugo dans les Alpes et les Pyrénées pour y dessiner les contours d'une approche romantique fondée sur la fascination pour les sommets, sur une orientation marquée à la fois pour l'étrangeté et la pureté de la nature montagnarde, auxquelles s'amalgame toute une palette de sensations liées à la verticalité. La période considérée (1825 à 1843) correspond au début du processus d'investissement touristique, ce qui fait de la montagne hugolienne une destination romantique par excellence.

11 John Ruskin, célèbre critique d'art, contemporain des grands écrivains romantiques, apporte le regard du voyageur britannique sur les Alpes, à partir duquel Samia Ounoughi dégage à la fois une analyse critique sur les représentations montagnardes de ses contemporains et un discours novateur sur l'étude de la géographie alpine. Les artistes vont emboîter le pas aux hommes de lettres dans la quête d'un nouveau paysage: Viviane Delpech évoque l'aventure exotique et dépaysante d'artistes voyageurs dans les Pyrénées, de Viollet-le-Duc et Dauzats à Delacroix et Devéria, qui produisent une iconographie pittoresque à l'origine du roman romantique pyrénéen et de ses stations thermales. L'observation de montagnes inconnues en Europe au milieu du XIX ${ }^{e}$ siècle fait l'objet de l'étude anthropologique de Laurence Espinosa qui nous transporte sur les pas de missionnaires protestants béarnais observant à partir de 1833 la chaîne du Drakensberg à la frontière nord-est du Lesotho dans le Sud-Est africain : décrites comme des lignes sur l'horizon qui racontent des histoires, les sommets des Maloti deviennent, pour ces hommes habitués à regarder les montagnes, les Pyrénées de l'Afrique australe.

12 L'esthétique finit par prendre une place importante dans cette quête générale d'une montagne idéale. La fin du siècle voit éclore une génération de grands peintres de la montagne, de Cézanne face à la montagne Sainte-Victoire à Klee dans les monts Zugspitze des Alpes bavaroises, à Henri Matisse au pied de la chaîne des Albères à Collioure, ou à Picasso dans le massif de Pedraforca en Catalogne : chacun à sa manière, selon Véronique Richard-Brunet, s'éloigne des règles académiques pour puiser de nouvelles sources d'inspiration à travers leur propre vision du monde.

13 Sur l'ensemble de la période, on peut se demander comment les élites locales des montagnes prennent en charge l'exploration savante et tout le travail de description et d'inventaire de leurs territoires. Arnauld Chandivert cherche à y répondre en examinant la circulation des nouveaux savoirs grâce au développement des sociétés savantes et de l'érudition dans les Pyrénées ariégeoises.

14 Ces transformations de la sensibilité européenne vis-à-vis de la montagne n'ont pas empêché les légendes traditionnelles des populations locales de prospérer: le cas de Dame Carcas, étudié par Gauthier Langlois, tend à prouver qu'une légende épique diffusée à travers les Pyrénées par les chemins de pèlerinage de Saint-Jacques a pu 
parvenir jusqu'à nous et fonder diverses identités de la péninsule Ibérique jusqu'en Provence.

La seconde partie des communications est tournée vers la période la plus contemporaine au cours de laquelle la montagne fait l'objet d'appropriations nouvelles. L'apparition, et le développement de sites de loisirs et de cures, est l'un des bouleversements majeurs dans l'économie et la société montagnarde au début du xx siècle. Le travail de monographie d'Elsa Belle et Philippe Gras sur l'une des premières stations de sports d'hiver créée en France, celle du Revard à Aix-les-Bains, permet de mettre en lumière le formidable mouvement touristique dont a bénéficié le massif alpin. Plus au sud, avec le massif du Queyras, c'est le défi de l'isolement géographique qui est posé par la situation d'un territoire qui a souffert au $\mathrm{xx}^{\mathrm{e}}$ siècle d'une réputation d'arriération et d'enfermement: Jean-Gérard Lapacherie s'emploie à mettre en valeur les richesses patrimoniales d'une vallée de haute montagne reposant sur une longue tradition spirituelle et immatérielle et une ancienne prospérité économique tout en dénonçant la vision tronquée qui a présidé au statut de zone témoin créée en 1952. À l'inverse, l'expérimentation développée entre 1968 et 1971 sur le massif des Apennins dans l'Émilie-Romagne italienne présentée par Sandra Costa montre que la nouvelle approche méthodologique autour de la notion de bien culturel appliqué à un espace géographique offre un nouveau sens à la sauvegarde d'un territoire montagneux.

Les géosites, comme espaces naturels montagnards d'un nouveau type où c'est notamment l'art contemporain qui contribue à la lecture du paysage, participent de l'émergence de nouvelles formes de mise en valeur territoriale comme le géotourisme que Christel Venzal décrit comme une démarche innovante susceptible de répondre aux questions essentielles de préservation de l'équilibre écologique. Le cas de la réserve géologique naturelle de Haute-Provence, créée en 1984, est symptomatique d'une volonté à la fois locale et nationale de valoriser une zone de montagne comme un patrimoine riche de son sous-sol, de ses espaces et de son habitat.

17 La vie des populations montagnardes est intéressante à observer dans les pays aussi éloignés de l'Europe que les territoires andins d'Amérique latine où Chloé TessierBrusetti nous invite à suivre l'un des plus grands alpinistes français, Lionel Terray, qui a posé son regard de cinéaste amateur sur le quotidien des Indiens quichuas des hauts plateaux, pris dans les bouleversements politiques du Pérou. De son côté, la littérature contemporaine reste tout aussi fascinée qu'à l'époque romantique par une montagne rêvée: en Corse, Pierre Bertoncini voit dans des romans de Jérôme Ferrari, Marc Biancarelli et Marie Susini les marques de l'authenticité de l'île-montagne. Dans les Pyrénées, c'est l'œuvre d'un grand écrivain de montagne, romancier de la solitude, le Béarnais Joseph Peyré (prix Goncourt 1935), qui est mise en perspective par Pierre Peyré et Christian Manso. Le regard de nos contemporains sur la montagne a évolué de façon radicale depuis un siècle: Manel Rocher Gonzalez, constatant le déclin des valeurs du pyrénéisme, prend acte de cette situation et propose diverses mesures visant à construire un nouvel avenir transpyrénéen. 
18 Un constat s'impose après l'examen d'une trajectoire historique aussi riche en évolution et innovation: les représentations de la montagne comme objet géographique continuent à interroger les historiens, les géographes, les géologues comme les artistes et les écrivains qui peuvent reprendre à leur compte l'idéal des pyrénéistes décliné par Beraldi, l'auteur des Cent ans aux Pyrénées (1902) :

« Savoir à la fois ascensionner, écrire et sentir. »

\section{AUTEUR}

\section{LOUIS BERGÈS}

Conservateur général du patrimoine, membre du CTHS, section Histoire du monde moderne, de la Révolution française et des révolutions 
Naissance et développement d'une nouvelle sensibilité 


\title{
Des nouvelles des cimes : les échanges naturalistes sur la montagne au XVIII ${ }^{\mathrm{e}}$ siècle (correspondance entre Villars, Picot de la Peyrouse et Ramond de Carbonnières)
}

\author{
Émilie-Anne Pépy
}

1 Dès le XVII ${ }^{\mathrm{e}}$ siècle, les Alpes puis les Pyrénées deviennent un véritable laboratoire pour les savants venus de l'Europe entière. La mode de l'exploration des montagnes se diffuse en Suisse dès la première moitié du siècle, exaltée par le poème Die Alpen d'Albrecht von Haller (1732). Dans la seconde moitié du xvIII ${ }^{e}$ siècle, ces montagnes sont parcourues, étudiées et cartographiées. Le contexte économique et culturel est alors favorable au développement des circulations internationales, engendrant une mise en contact sans précédent des voyageurs avec la montagne. Plusieurs phénomènes coexistent sans nécessairement se juxtaposer. Une transformation culturelle profonde conduit à dépasser les préjugés et appréhensions traditionnellement associés aux paysages de montagne. Cette nouvelle sensibilité amène les savants à s'intéresser aux mœurs des populations montagnardes. Ils enrichissent aussi les savoirs naturalistes sur la montagne, contribuant à constituer des champs d'étude spécifiques: minéralogie, glaciologie, botanique, ornithologie, etc. Dès le $\mathrm{XvI}^{\mathrm{e}}$ siècle, les botanistes ont investi les alpages. Les représentants des autres branches des sciences naturelles, comme les minéralogistes, sont généralement arrivés plus tard sur le terrain, au gré des enquêtes diligentées par le pouvoir monarchique pour recenser les ressources minières du royaume ${ }^{1}$. La montagne sert enfin de terrain d'expérimentation pour les sciences physiques, la météorologie et la cartographie.

2 Les recherches de Dominique Villars, Philippe-Isidore Picot de Lapeyrouse et Louis Ramond de Carbonnières portent sur les trois règnes de la nature (minéral, végétal, 
animal), non sans la tentation encyclopédique d'y inclure les sciences physiques, la météorologie et la topographie, qui entrent moins directement dans leurs préoccupations. Si leur production scientifique reste calibrée par les normes de l'inventaire naturaliste élaborées au $\mathrm{XVIII}^{\mathrm{e}}$ siècle $^{2}$, ils contribuent aussi, par leurs observations, à l'introduction de nouveaux questionnements sur le milieu naturel montagnard. Si ces trois savants, appartenant à la génération d'Horace Benedict de Saussure (1740-1799), ont été choisis comme point de départ de l'étude, c'est autant pour la richesse de leurs correspondances, que parce que leurs trajectoires suivent sensiblement la même course. L'objectif de cet article est de montrer quelles sont les difficultés auxquelles sont confrontés des savants travaillant dans et sur des espaces périphériques, qui doivent contourner les contraintes de la centralisation et tirer parti des modalités de la communication scientifique pour faire connaître les milieux naturels montagnards.

\section{D'une montagne à une autre : trois naturalistes étroitement liés}

3 Villars, Lapeyrouse et Ramond de Carbonnières gravitent dans une nébuleuse de naturalistes en transit entre le système académique d'Ancien Régime et le nouveau modèle institutionnel issu de la Révolution. Ils traversent sans trop d'encombres la période révolutionnaire et l'Empire, et profitent du contexte politique favorable aux savants, à partir du Directoire, pour recycler leurs compétences dans les nouvelles institutions, voire pour occuper des fonctions politiques et développer des stratégies d'ascension sociale inédites.

\section{Des hommes de terrain aux carrières parallèles}

Dominique Villars (1745-1814) est considéré comme l'un des principaux découvreurs des Alpes dauphinoises, et comme il l'écrit au couchant de sa vie :

« Il n'existera pas beaucoup de botanistes qui pendant cinquante ans ait fait plus de

cinquante courses parmi les montagnes. ${ }^{3}$ ”

5 La grande affaire de la vie de ce médecin reste la botanique de montagne, qui l'amène à prendre en charge la fondation du premier jardin botanique de Grenoble au début des années 1780. À partir de 1795, ayant perdu son poste à l'hôpital militaire, il devient professeur de sciences naturelles à l'École centrale, avant d'obtenir un bâton de maréchal comme professeur à la Faculté de médecine de Strasbourg, dont il finit doyen. Si l'Histoire des plantes du Dauphiné (publié de 1786 à 1789) constitue son œuvre maitresse, il est aussi l'auteur prolifique d'opuscules et mémoires dans lesquels il entend faire contribuer au progrès les connaissances sur la montagne. Il a également laissé des correspondances abondantes, partiellement éditées, et conservées au Muséum d'histoire naturelle de Grenoble. La relation épistolaire entre Villars et Lapeyrouse, engagée en 1786, se poursuit jusqu'en $1809^{4}$.

6 Philippe-Isidore Picot de Lapeyrouse (1744-1818), né dans une famille de négociants aisés, profite, dès le milieu de la décennie 1770, d'un riche mariage, puis d'un héritage généreux, pour quitter ses fonctions d'avocat à la chambre des Eaux et Forêts du parlement de Toulouse. Il se consacre exclusivement à l'animation de la vie académique toulousaine, et à l'histoire naturelle des Pyrénées. Entre 1763 et 1797, il effectue une 
dizaine de longs séjours dans les montagnes, qui lui fournissent matière à écrire une trentaine d'ouvrages et mémoires sur les fossiles, les minéraux, la faune et la flore, l'histoire géologique de la chaîne. Au début de la Révolution, il occupe un poste clé dans l'administration du district de Toulouse, puis renonce à toute fonction politique en 1792, ce qui ne l'empêche pas d'être emprisonné à partir d'octobre 1793. Après Thermidor, une double carrière scientifique s'offre à lui. Inspecteur des mines en 1794, il assure après 1796 des cours à l'École centrale de Toulouse et à l'École des mines de Paris. Il est nommé doyen de la Faculté des sciences de Toulouse en 1811 et contribue à la fondation d'un muséum d'histoire naturelle, alors qu'il est maire de la ville entre 1800 et 1806. Lapeyrouse entretient des relations amicales, puis concurrentielles, avec un nouveau venu dans les Pyrénées, Ramond de Carbonnières. C'est à propos de la découverte du Mont Perdu que leurs échanges sont de plus en plus intenses. En 1797, ils s'efforcent d'effectuer ensemble l'ascension de ce sommet, encore tenu pour le point culminant des Pyrénées. Ce projet fournit matière à une correspondance suivie pour la période 1795-17985.

7 De dix ans plus jeune que Villars et Lapeyrouse, Louis Ramond de Carbonnières (1753-1827), issu de la bourgeoisie strasbourgeoise confinant à la noblesse, est juriste de formation. Rien ne le destine à devenir le pyrénéiste que l'on sait. Un voyage en Suisse effectué en 1777 le sensibilise pourtant à la montagne. Il doit au cardinal de Rohan, dont il est le secrétaire, la découverte de l'Auvergne, puis des Pyrénées en 1787, lorsque le prélat exilé à la Chaise-Dieu se rend aux eaux de Barèges. Ayant quitté le service du cardinal en 1788, il suit les cours d'histoire naturelle dispensés au Jardin du roi. Membre du club des Feuillants, Ramond obtient une députation en 1791. En août 1792, devenu suspect de par sa prise de position en faveur du clergé réfractaire, il se réfugie à Barèges où il poursuit ses courses et ses travaux d'herborisation. Emprisonné à Tarbes en janvier 1794, il n'est libéré qu'après Thermidor. En 1796, il devient professeur d'histoire naturelle à l'École centrale de Tarbes, et reprend contact avec le monde scientifique. Après 1800 , ses activités politiques, qui lui vaudront un titre de baron d'Empire en 1809, l'éloignent des Pyrénées. Préfet puis député du Puy-de-Dôme, Ramond poursuit néanmoins ses recherches naturalistes dans les montagnes d'Auvergne.

\section{Les enjeux des rapports interpersonnels}

8 En tant que spécialistes des montagnes françaises, Villars, Lapeyrouse et Ramond entretiennent des relations étroites. Villars et Lapeyrouse se reconnaissent mutuellement comme des pairs, ce qui leur permet d'établir une relation de confiance. Chacun se considérant comme spécialiste de son terrain, qui le Dauphiné, qui les Pyrénées, le risque de concurrence est moindre. En 1786, Lapeyrouse, à l'occasion de ses recherches sur les saxifrages, sollicite l'expertise de Villars pour identifier des spécimens douteux; leurs échanges à propos des plantes se poursuivent des années durant (fig. 1). Une certaine défiance à l'égard des botanistes parisiens les rapproche, particulièrement après leur commune nomination à l'Institut. Les querelles entre botanistes éclatent souvent autour d'accusations de plagiat ou de pillage d'herbiers; le nom de Lamarck (1744-1829), accusé de s'approprier sans vergogne des spécimens montagnards, revient souvent dans leurs lettres. Ils échangent sans fard leurs impressions sur leurs collègues, tant sur leurs travaux que sur leurs caractères ${ }^{6}$. Villars comme Lapeyrouse reprochent aux savants de cabinet de se perdre dans l'élaboration 
de nouveaux systèmes au détriment de la connaissance de terrain. Eux restent intellectuellement proches des botanistes linnéens de Lyon et de Montpellier ${ }^{7}$. Alliances et antagonismes reflètent les lignes de failles du paysage éclaté de la systématique à la fin $\mathrm{du} \mathrm{XVIII}^{\mathrm{e}}$ siècle. Les botanistes parisiens de la nouvelle génération défendent la méthode dite naturelle face aux systèmes artificiels, visant particulièrement le système de Linné (1707-1788). Lapeyrouse, qui a choisi la classification linnéenne pour le premier volume de sa flore paru en 1795, se brouille successivement avec Lamarck, Cuvier (1769-1832) et Candolle (1778-1841), auxquels il reproche, en sus des questions de méthode, d'investir son terrain par le truchement de Ramond. Avant la Révolution, Lapeyrouse conservait un quasi-monopole sur les Pyrénées; sa position académique à Toulouse lui permettait d'écarter les concurrents potentiels. L'arrivée de Ramond, qui procure de nombreux spécimens aux concurrents parisiens, lui semble mettre en péril sa position mandarinale.

Fig. 1. - Extrait d'une lettre de Villars à Lapeyrouse du 17 mai 1796.

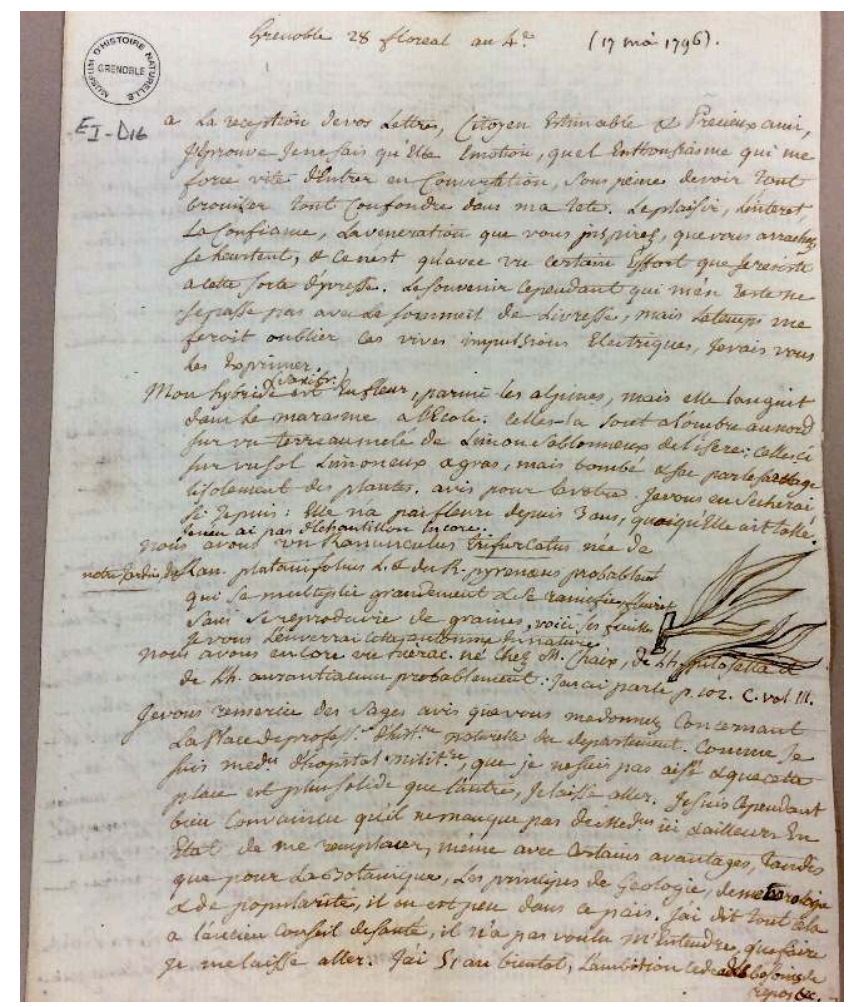

Bibliothèque du Muséum d'histoire naturelle de Grenoble, Villars El-D16.

9 Si le binôme constitué par Villars et Lapeyrouse fonctionne sur la longue durée de manière relativement harmonieuse, il n'en va pas de même de la relation entre Lapeyrouse et Ramond. Ramond se considère initialement comme le disciple de Lapeyrouse, mais la situation se tend lorsque l'élève dépasse le maître. Lapeyrouse se sent menacé par les premières publications de Ramond, alors que lui-même ne parvient pas à achever son chef-d'œuvre sur la flore pyrénéenne. Les lettres échangées entre 1795 et 1798 montrent ce retournement de situation: plus Ramond gagne en expérience, plus Lapeyrouse se crispe sur ses prérogatives, jusqu'au faux pas du Mont Perdu. En août 1797, leur tentative d'ascension a conduit leur collaboration dans une impasse. Quelques semaines après l'aventure, Ramond commence à publier ses 
observations ${ }^{8}$; Lapeyrouse, y voyant une tentative de le doubler, n'a alors de cesse que de lui mettre des bâtons dans les roues. Ramond lui reproche d'avoir fait détruire par ses étudiants, in situ, des échantillons de roche et des plantes sur lesquels il travaillait ${ }^{9}$. Il riposte par la publication des Voyages au Mont Perdu en 1801, où il se donne à voir comme un explorateur performant ayant guidé les pas d'un vieillard diminué. La brouille est consommée malgré les tentatives de médiation de Villars. Cet exemple montre que les correspondances privées en apprennent beaucoup sur les rapports de force entre savants. Les controverses constituent des moments révélateurs des forces en présence, qui pèsent dans la balance au moment de statuer sur la vérité scientifique. En botanique, les problèmes à résoudre portent sur l'identification des plantes, la découverte de nouvelles espèces, leur place dans des systèmes de classification dont on ne cesse d'éprouver les limites et que l'on veut réformer. Dans le domaine de l'étude des roches, les débats portent sur l'origine et les modalités de l'élévation des montagnes, la mesure de leur altitude, et leur composition géologique. Ramond, comme Palassou (1745-1830), s'oppose aux hypothèses formulées par Dolomieu (1750-1801) et Lapeyrouse sur la datation des couches calcaires observées dans les Pyrénées, et donc sur le scénario de l'histoire géologique de la chaîne.

\section{L'expérience des marges de la République des sciences}

10 Pour comprendre le positionnement des savants dans les controverses, il convient de rappeler l'importance de l'économie de l'information dans l'élaboration et dans la communication des savoirs. Lorsque l'on s'occupe de milieux naturels périphériques, dans un contexte de centralisation de la production savante et de l'expertise, il est par ailleurs impératif de s'insérer dans les réseaux académiques adéquats pour faire connaître ses travaux et gagner en réputation.

\section{Une culture matérielle fondée sur l'échange}

11 Les sciences d'observation exigent des lectures et des comparaisons de spécimens qui génèrent un commerce matériel important entre les savants. Avant la Révolution, le réseau français de Villars comprend davantage de naturalistes de terrain et de praticiens que de membres des institutions parisiennes. Font partie de ses correspondants réguliers les minéralogistes Guettard (1715-1786) et Faujas de SaintFond (1745-1819), les botanistes méridionaux Amoreux (1741-1824) et Gouan (1733-1821), les Lyonnais La Tourrette (1729-1793) et Gilibert (1741-1814). Son intérêt pour la flore à l'échelle de l'arc alpin le conduit à développer son réseau en direction des pays germaniques et des Balkans, en passant par le Piémont (Allioni) et la Suisse (Saussure). Lapeyrouse connaît également bien Amoreux ${ }^{10}$; dans les années 1780 , l'orientation de ses recherches le conduit à renforcer ses contacts avec des spécialistes des mines, comme Gillet de Laumont (1747-1834), qui devient en quelque sorte son représentant à Paris. Si les lettres peuvent emprunter les services de courrier habituels, il n'en va pas de même avec les herbiers, livres, graines et échantillons divers, souvent conditionnés dans des caisses. On recourt plutôt à des intermédiaires de confiance; les correspondances s'étendent longuement sur la logistique des échanges. Outre les aléas des services postaux, les collections des naturalistes ont eu à souffrir du contexte 
politique. Lors de son départ précipité pour Barèges en 1792, Ramond abandonne ainsi à Paris un petit cabinet de minéralogie, et trois herbiers totalisant plusieurs milliers de spécimens. Avant son arrestation, il a le temps de reconstituer un nouvel herbier, qui lui tient compagnie dans les geôles de Tarbes, mais dont il se plaint de la dégradation en $1795^{11}$. En 1796, sa nomination à l'École centrale de Tarbes l'oblige à reconstituer rapidement ses collections naturalistes, pour disposer de matériel pédagogique. Il doit alors faire face à des difficultés matérielles liées à l'éloignement des métropoles culturelles : en juin 1797, il demande par exemple à Lapeyrouse de lui procurer des yeux de verre destinés aux dépouilles d'oiseaux qu'il est contraint d'empailler luimême, faute d'avoir pu trouver un homme de l'art ${ }^{12}$. La stratégie de Ramond consiste alors à renforcer ses contacts avec les principaux représentants des institutions parisiennes, l'Académie des Sciences et l'Institut, dont se rapprochent dans une moindre mesure Villars et Lapeyrouse.

\section{Le savant de province face aux préjugés}

Des trois savants, seul Ramond acquiert une dimension véritablement nationale. C'est aussi celui dont la carrière scientifique ne décolle qu'après la Révolution, pendant le Directoire, au sein de cette République des savants qui encourage le recours systématique à leur expertise sur la société et sur l'économie. Villars et Lapeyrouse sont quant à eux représentatifs d'une autre catégorie, celle des savants des villes de province de la seconde moitié du XvIII ${ }^{\mathrm{e}}$ siècle. Ils contribuent au développement des équipements culturels à Grenoble et à Toulouse, en promouvant l'installation de jardins botaniques associés à des bibliothèques et cabinets de curiosité. Ils s'investissent dans la vie culturelle de la cité par le biais des académies. Tous deux ont mené des recherches initiées à des fins utilitaires par les agents de l'État monarchique. Villars a ainsi participé à un voyage d'exploration du Dauphiné commandité par Pajot de Marcheval, intendant de Grenoble en 1775-1776, en compagnie de Faujas de Saint-Fond et Guettard, pour repérer le potentiel minier de la région. Quant à Lapeyrouse, il publie en 1786 un Traité sur les mines de fer et les forges du comté de Foix, à la demande des États du Languedoc.

La revendication d'une expertise régionale constitue un premier pas vers une reconnaissance de la part des institutions centrales, qu'il convient de fréquenter régulièrement. Villars effectue une année de formation à Paris, est introduit au Jardin du roi, et y rencontre des personnalités scientifiques : les frères Bernard (1699-1777) et Joseph (1704-1779) Jussieu, Thouin (1747-1824), Le Monnier (1717-1799), Daubenton (1716-1800). Quant à Lapeyrouse, il entre en contact avec les réseaux parisiens grâce à des travaux d'ornithologie qui intéressent les encyclopédistes. Son élection à l'Académie royale des sciences de Stockholm en tant que membre étranger en 1785 contribue à asseoir sa stature. Il devient ensuite correspondant de la Société royale d'agriculture de Paris. Avant la Révolution, Villars est pour sa part membre de plusieurs sociétés savantes de province, correspondant de la Société royale de médecine de Paris et de l'Académie royale des sciences de Turin.

Pour Ramond, ces stratégies de rattachements académiques n'interviennent qu'après 1796. Comme Villars et Lapeyrouse, il est nommé à l'Institut, mais il est le seul à faire de cette position un tremplin. Alors qu'il est encore professeur à l'École centrale de Tarbes, les conclusions scientifiques qu'il tire de l'exploration du Mont Perdu lui 
assurent un succès d'estime parmi les savants de son temps, puis une place à l'Académie des sciences en 1802. Villars et Lapeyrouse exercent aussi des fonctions de professeurs dans les Écoles centrales. Lapeyrouse, en tant qu'inspecteur des mines, a en outre l'occasion de résider régulièrement à Paris après 1794, ce qui lui ouvre les portes des cabinets de travail des savants les plus réputés; il obtient même de Le Monnier le manuscrit original de Tournefort (1656-1708), botaniste officiel de Louis XIV, la Topographie botanique des Pyrénées, ambitionnant d'en être le continuateur. Sa position est pourtant affaiblie par ses incessantes querelles, et les savants de la nouvelle génération le considèrent comme un provincial mal dégrossi qui n'a eu le mérite que de profiter d'un terrain d'études quasiment inexploré ${ }^{13}$. Villars aurait pu également prétendre à une carrière plus élevée: il n'a dû sa nomination à Strasbourg qu'à l'intervention de Fourcroy (1755-1809) dont il a conservé l'estime.

Les trois savants ont bien conscience de la nécessité de fréquenter Paris, capitale scientifique dont ils reconnaissent la primauté en matière d'équipements culturels indispensables aux naturalistes. Les institutions savantes centrales constituent la clé de la validation des savoirs produits. À la fin du XVIII siècle, les jardins et serres du marchand collectionneur Cels (1740-1806), ainsi que le Jardin du roi devenu Jardin national en 1793, apparaissent comme des lieux de sociabilité incontournables pour les botanistes de quelque ambition, qui peuvent y consulter des herbiers et des collections vivantes de référence pour reconnaître précisément une plante. Dès le XVII ${ }^{\mathrm{e}}$ siècle, la flore des massifs de montagne est représentée dans les banquettes du Jardin du roi. Les collections s'étoffent par la suite grâce aux voyages de savants comme Tournefort, et à la mise en place de réseaux de collecteurs procédant à des envois réguliers de graines et de plantes vivantes. Nos trois naturalistes s'inscrivent dans une longue tradition. Leurs échanges épistolaires montrent la confiance dont ils honorent l'institution et ses principaux représentants. Après sa sortie de prison, Ramond reprend contact avec les botanistes parisiens, essentiellement Desfontaines (1750-1833) et Lamarck, auxquels il a coutume d'adresser les échantillons qu'il ne peut identifier avec certitude. Il justifie sa démarche auprès de Lapeyrouse :

« Vous avez raison. On ne fait point sans livres la critique botanique et l'on ne cite point de sinonimes sans auteurs. Aussi je consulte, et peut-être ne consulterais-je pas si j'étais au milieu des bibliothèques et des herbiers. ${ }^{14}$ »

16 Il faut dire que Ramond se définit avant tout comme un découvreur, à la différence de ses deux collègues qui se sont plongés bien avant lui dans les arcanes des classifications. La sollicitation fréquente de ses contacts parisiens, auxquels il apporte du matériau inédit sans se mêler de leurs constructions intellectuelles, lui permet de parachever sa réputation d'explorateur, et de devenir un maillon incontournable des réseaux naturalistes.

\section{La montagne dans la communication scientifique}

L'expérience des marges ne se résume pas à un déficit de visibilité. Pour les savants des périphéries, la publication de travaux spécialisés devient vite un défi, en raison de contraintes tant économiques que matérielles. En revanche, les récits de voyage et d'exploration en montagne conservent les faveurs d'un public sensibilisé aux savoirs naturalistes par le développement de cours publics, puis d'un enseignement spécifique pour la jeunesse. 


\section{Du rocher au papier, publier sur la montagne}

18 savante, dès la seconde moitié du XVIII ${ }^{e}$ siècle. Pour les savants, la voie de presse permet autant de faire valoir ses positions et de s'approprier symboliquement un terrain d'expertise, que de faire circuler de manière neutre et objective l'information scientifique. Sous l'Ancien Régime, les mécanismes de sélection institutionnelle favorisent les savants d'envergure européenne, dont les résultats sont publiés dans les Mémoires de l'Académie royale des sciences, le Journal de physique, etc. Quant aux académies locales, elles soutiennent des publications de moindre écho. Les spécialistes des espaces de montagne peuvent cependant communiquer dans des périodiques moins confidentiels, comme les Affiches du Dauphiné dans les années 1780, ou le Journal de Genève, qui relate les principaux voyages naturalistes après 1787. Après la Révolution, les revues des sociétés savantes locales reprennent le flambeau des publications des académies de province. À l'échelle nationale, la Décade philosophique, littéraire et politique, en activité entre 1794 et 1807, apparaît comme un des journaux de référence des savants dans un moment politique qui leur est favorable. Les domaines relevant des sciences naturelles y sont particulièrement mis en valeur ${ }^{15}$. Villars, qui ne connaît alors Ramond que de nom, peut y lire avec intérêt sa description de la cime du Pic du Midi ${ }^{16}$, publiée avec le soutien de Desfontaines ${ }^{17}$. Le Journal de physique, de chimie et d'histoire naturelle, et le Journal des mines (qui paraît entre 1794 et 1815), constituent également des organes majeurs de la communication scientifique. La présence de membres de l'Institut dans les comités de lecture favorise la publication par leurs pairs.

Dans les représentations des savants, la monographie reste au sommet de la hiérarchie symbolique des publications. À la fin de la décennie 1780, l'objectif scientifique premier de Villars et Lapeyrouse consiste à produire, sous forme de flores, un état des lieux du savoir sur la végétation montagnarde. Leurs auteurs s'appuient autant sur les écrits des botanistes anciens, que sur leurs propres recherches exploratoires pour découvrir et classer de nouvelles espèces. Il reste difficile de s'arroger la paternité d'une découverte, sachant qu'il est d'usage de consulter ses pairs avant de publier, donc de faire passer les spécimens entre plusieurs mains plus ou moins probes. La diffusion d'opuscules préparatoires, ou d'articles dans la presse savante, fait partie des stratégies pour baliser son terrain. En 1786, Villars a fait publier un prospectus pour annoncer son Histoire des plantes du Dauphiné, en partie pour se prémunir des menées peu délicates de Lamarck ${ }^{18}$.

Pour publier une flore, il faut aussi résoudre le problème de la mise en image des plantes, en sollicitant le concours d'artistes spécialisés, plus difficiles à trouver dans les villes de piémont qu'à Paris. On connaît l'importance de l'image dans l'administration de la preuve. Lorsque l'on entend décrire une espèce botanique nouvelle ou rare, la mise en image facilite la communication auprès des pairs ${ }^{19}$. Beaucoup de botanistes manient eux-mêmes la plume, avec plus ou moins de talent : si Villars reconnaît ses limites en la matière, Ramond est en revanche un dessinateur accompli. En 1795, il entend finaliser une flore des Hautes-Pyrénées par l'ajout de gravures réalisées à partir de ses propres dessins. Faute d'avoir trouvé à Tarbes un graveur compétent, il sollicite l'aide de Lapeyrouse, en juin 1798, pour se procurer des planches de cuivre et une presse $^{20}$. Recourir aux services de graveurs professionnels fait grimper le coût déjà élevé des monographies, ce qui ne facilite pas l'écoulement de ce type de production 
éditoriale, surtout dans le contexte économique de la Révolution. Les problèmes de financement donnent lieu à de récurrentes complaintes. En témoigne ce cri du cœur de Villars en décembre 1789 :

«J'ai déboursé 7000 livres. Je dois encore plus de 3 [000] à mon imprimeur et je n'en ai retiré que trois environ. Que faire ? Je suis dans l'eau, il a fallu nager. ${ }^{21}$ »

21 Avant la Révolution, Picot de Lapeyrouse entend puiser dans sa fortune personnelle pour produire un véritable chef-d'œuvre, tant scientifique qu'artistique. Sa magistrale flore des Pyrénées doit être publiée en plusieurs volumes, comportant chacun cent planches coloriées divisées en décades. Dès 1788, Lapeyrouse, par l'intermédiaire de son ami Gillet de Laumont, a l'ambition de faire travailler des graveurs parisiens renommés ; son choix se porte sur Jauinet ${ }^{22}$. Il souhaite un grand format, in $4^{\circ}$, pour un ouvrage destiné à faire date. Les évènements politiques, puis la perte de ses biens en 1793, le contraignent à mettre en sommeil ses ambitions. Après Thermidor, il tente de relancer le projet, avec la publication en 1795 d'un premier volume, essentiellement consacré aux saxifrages, illustré de 43 planches coloriées ${ }^{23}$. Faute de fonds, la série est interrompue sans que ne puisse paraître le second tome, conçu pour être illustré de 200 planches gravées. Renonçant à sa flore encyclopédique, Lapeyrouse, qui a compris que le récit de voyage contribuerait autant, si ce n'est davantage, à sa renommée, fait paraître en 1813 un ouvrage mixte, à la fois traité de botanique et compilation de ses itinéraires ${ }^{24}$.

Depuis le début du $\mathrm{xvIII}^{\mathrm{e}}$ siècle, les écrits sur la montagne sont protéiformes, mais le genre reste dominé par les récits d'exploration, composés à partir de la matière contenue dans les carnets de voyage tenus régulièrement sur le terrain. L'édition récente des Carnets pyrénéens de Ramond pour la période 1792-1795 permet de prendre la mesure de l'importance de ce type d'écrit dans la culture matérielle du naturaliste ${ }^{25}$. De même, une douzaine de carnets de voyage manuscrits de Villars sont conservés au Muséum de Grenoble; l'auteur en a fait imprimer quelques-uns, d'autres ont été publiés de manière posthume ${ }^{26}$. La rédaction des carnets s'effectue sur le terrain, souvent quelques jours après l'observation. Ces notes, parfois illustrées de croquis, sont ensuite si nécessaire retravaillées à la plume pour pouvoir faire passer un document intelligible à d'autres savants, ou préparer une publication. Les recherches actuelles tendent à réévaluer le poids de cette littérature grise manuscrite dans l'élaboration des hypothèses et dans la construction des savoirs.

Un des premiers succès éditoriaux de récit d'exploration en montagne est l'Ourésiphoïtès Helvéticus (1723) du médecin naturaliste Johann Jakob Scheuchzer (1672-1733), qui relate ses voyages dans les Alpes suisses. Ramond est sans doute celui qui s'inscrit le plus dans cette tradition ; avant la Révolution, il est autant, sinon davantage, homme de lettres que de sciences ${ }^{27}$. Sainte-Beuve le qualifie d'ailleurs de Saussure des Pyrénées, estimant que sa sensibilité poétique transparaît davantage que dans les textes de son confrère suisse. Parue en 1779, l'originalité de sa traduction des Lettres sur la Suisse de William Coxe est d'intégrer des commentaires tirés de son propre voyage en Suisse en 1777. Les Observations faites dans les Pyrénées (1789), et les Voyages au Mont Perdu (1801) reprennent les codes du récit d'exploration dont raffolent les lecteurs. La presse savante en fait également son miel, sachant que ce type de publication touche un public bien plus large que les cercles académiques. En 1797, le Journal des Mines publie les deux versions de l'expédition au Mont Perdu envoyées presque simultanément par les ascensionnistes $^{28}$. L'affaire tourne en défaveur de Lapeyrouse, qui a livré un récit 
ambigu dans lequel il semble s'arroger la primeur des découvertes. Son diagnostic sur des formes pétrifiées observées près du lac, sous le sommet, achève de le discréditer aux yeux de la communauté scientifique. Là où Ramond décrit de manière convaincante des coquilles et organismes marins, plaidant en faveur de l'existence d'une couche de calcaire " secondaire ", Lapeyrouse a vu avec enthousiasme des os de grands animaux, sans renoncer à la thèse du calcaire " primitif » que son collègue vient de mettre à mal. Entre les lignes de ces récits d'exploration se joue tout autant une controverse scientifique sur la datation des roches, qu'une bataille d'ego entre deux savants qui reste imperceptible pour le public non initié.

\section{La diffusion des savoirs naturalistes sur la montagne auprès du grand public}

Avant la Révolution, Villars et Lapeyrouse jouent le rôle de passeurs de sciences auprès d'un public large. Les villes de piémont s'équipent plus tardivement que les autres de jardins botaniques, grâce à l'engagement des élites éclairées et des agents de la monarchie ${ }^{29}$. La flore montagnarde se doit d'y être bien représentée. Les collections sont mises en valeur dans le cadre des cours publics de botanique. Villars et Lapeyrouse se conforment aux méthodes de la démonstration telles que pratiquées au Jardin du roi/Jardin national. Les cours théoriques, qui abordent l'histoire de la botanique et présentent les grands systèmes de classement (Tournefort, Linné, Jussieu), s'accompagnent de démonstrations relevant d'une démarche plus expérimentale. Lors de ces séances, publiques, très matinales, et estivales pour profiter de la floraison, le professeur décrit les grandes familles de plantes en s'attardant sur leur anatomie et sur leur utilité pour l'homme, notamment dans le domaine thérapeutique. Initialement conçus pour les étudiants en médecine, chirurgie, apothicairerie et obstétrique, les cours publics s'ouvrent à un auditoire plus large d'amateurs, et de professionnels autres que ceux de la santé. La connaissance de la flore peut intéresser les cultivateurs, les artistes, voire les artisans utilisant des teintures végétales. Les objectifs des amateurs et curieux sont moins utilitaires : certains considèrent la botanique comme un loisir scientifique, d'autres veulent aller plus loin dans la connaissance de la nature, encouragés par les ouvrages de vulgarisation assez largement diffusés par les éditeurs. Les cours sont prolongés par des herborisations hors les murs organisées par les professeurs de botanique, et qui contribuent à initier le public savant ou amateur aux déambulations dans le milieu naturel montagnard. Ces pratiques d'enseignement de la botanique par l'expérience sensible sont transposées dans Écoles centrales à partir de 1795.

Sous le Directoire, le chantier de l'enseignement secondaire débouche sur la création de ces établissements pensés pour offrir un enseignement scientifique solide en sus des classiques humanités ${ }^{30}$. Élus par un jury départemental sur présentation de leurs travaux, les professeurs des Écoles centrales ont toute latitude pour construire leur enseignement disciplinaire. Il leur faut inventer la méthode et les outils pédagogiques adaptés à un programme fort ambitieux, qui comporte l'étude des trois règnes de la nature, alors qu'il n'existe pas encore de synthèse dûment éditée. Dans leurs lettres, Ramond, Villars et Lapeyrouse partagent leurs expériences et leurs projets. Ils sont convaincus de la nécessité de combiner théorie et expérience, et entendent transmettre le goût des études de terrain. Le rythme des enseignements se calque sur la temporalité spécifique aux activités naturalistes: les dissections en zoologie se font l'hiver, 
l'observation botanique à l'époque de la floraison. La belle saison est aussi propice aux excursions pédagogiques, programmées toutes les semaines, parfois sur plusieurs jours $^{31}$. Pour les jours de classe, la question des supports pédagogiques est cruciale. Villars organise son cours en deux temps: la leçon magistrale que les élèves retranscrivent sur des cahiers, puis les observations pratiques lors d'un temps de dialogue $^{32}$. L'activité pédagogique des savants est étroitement liée à leurs propres pratiques scientifiques et à l'état de leurs recherches. Ramond envisage la publication de son cours, qu'il appelle son cahier de botanique, pour servir de manuel d'apprentissage, et pour présenter les nouvelles espèces qu'il a découvertes, même si leur identification n'est pas établie avec certitude ${ }^{33}$. Les étudiants les plus avancés ont l'opportunité de suivre leur maitre dans ses propres courses. Dans les Pyrénées, Ramond comme Lapeyrouse puisent dans le vivier des élèves des Écoles centrales pour repérer des disciples de talent.

Pour être en mesure de publier des travaux reconnus sur la montagne, les savants de province doivent faire leurs preuves au sein d'un système académique centralisé. Si leur production scientifique a pu être contrariée par des conditions matérielles défavorables à la réalisation de monographies spécialisées, notamment en raison du coût des illustrations, ils profitent en revanche de la fascination croissante du grand public pour ce type de milieu naturel, qui se traduit par un goût marqué pour les récits d'exploration. Villars, Lapeyrouse et Ramond ont joué le rôle de passeurs de science dans les villes de piémont, initiant à la montagne une nouvelle génération de citadins, qui contribuent à la généralisation de la promenade naturaliste.

\section{BIBLIOGRAPHIE}

DEMEULENAERE Christiane, « Une tentative d'inventaire des richesses minières de la France : l'enquête du Régent, 1716-1718 ", Documents pour l'histoire des techniques, $\mathrm{n}^{\circ} 16,2^{\mathrm{e}} \mathrm{sem} .2008$, p. 9-16.

DROUIN Jean-Marc, « L'histoire naturelle à travers un périodique, la Décade philosophique », dans CORVol Andrée (dir.), La Nature en révolution, 1750-1800, Paris, L’Harmattan, 1993, p. 175-181.

DURIS Pascal, Linné et la France, 1780-1850, Genève, Droz, 1993.

DURIS Pascal, «L'enseignement de l'histoire naturelle dans les écoles centrales (1795-1802) », Revue d'histoire des sciences, t. 49, n 1, 1996, p. 23-52.

LAPEYROUSE Philippe-Isidore Picot (de), Figures de la flore des Pyrénées, avec des descriptions, des notes critiques et des observations, t. 1, Paris, imprimerie de Du Pont, 1795.

LAPEYROUSE Philippe-Isidore Picot (de), Histoire abrégée des plantes des Pyrénées et itinéraire des botanistes dans ces montagnes, Toulouse, imprimerie de Bellegarrigue, 1813. 
LAPEYROUSE Philippe-Isidore Picot (de), « Voyage au Mont-Perdu, et observations sur la nature des crêtes les plus élevées des Pyrénées, 15 fructidor an V », Journal des Mines, premier trimestre, vendémiaire, brumaire, frimaire an VI, p. 39-66.

ORLANDO Francesco, «L'histoire des montagnes et l'harmonie du monde chez Ramond de Carbonnières », dans BERTRAND Gilles et GUYOT Alain (dir.), Des « passeurs » entre science, histoire et littérature, contribution à l'étude de la construction des savoirs (1750-1840), Grenoble, ELLUG, 2011, p. 167-220.

PÉPY Émilie-Anne, « Décrire, nommer, ordonner. Enjeux et pratiques de l'inventaire botanique au XVIII ${ }^{\mathrm{e}}$ siècle ", Études rurales $1 / 2015$ ( ${ }^{\circ}$ 195), p. 27-42.

PÉPY Émilie-Anne, «La connaissance de l'environnement au service du progrès. Le cas du médecin naturaliste Dominique Villars (1745-1814)», dans NJENHUIS-BESCHER Andreas, PÉPY Émilie-Anne, CHAMPELEY Jean-Yves (éd.), L'Honnête homme, l'or blanc et le duc d'Albe, Mélanges offerts à Alain Becchia, Chambéry, LLSETI, 2016, p. 499-528.

PINAULT-SøRENSEN Madeleine, Le livre de botanique (XVII et XVIII ${ }^{e}$ siècles), Paris, Imprimerie Nationale, 2008.

RAMOND DE CARBONNIÈRES Louis, Carnets pyrénéens, édition établie par Pierre Debofle, Pau, Monhélios, 2014.

RAMOND DE CARBONNIÈRES Louis, « Extrait d'une lettre du citoyen Ramond, associé de l'Institut national, et Professeur d'histoire naturelle à Tarbes, au citoyen Haüy, membre de l'Institut à Paris, sur deux voyages au Mont-Perdu, sommet le plus élevé des monts Pyrénées, 5 fructidor an V », Journal des Mines, premier trimestre, vendémiaire, brumaire, frimaire an VI.

RocHAS Joëlle, Muséum de Grenoble, une histoire naturelle, Grenoble, Éditions du Muséum de Grenoble, 2007.

RUSQUE Dorothée, «Faire circuler les objets naturalistes au XVIII ${ }^{\mathrm{e}}$ siècle, Jean Hermann comme intermédiaire dans les échanges entre la France méridionale et l'espace germanique », Liame, éd. numérique, 26 | 2016,

[URL : http://liame.revues.org/568]

SAIGEY Jacques-Frédéric, RASPAIL François-Vincent, Mémoires des sciences d'observation, t. 4, Paris, Rouen frères, 1830.

VILLARS Dominique, Mémoires sur la topographie et l'histoire naturelle, extraits du cours de l'École centrale du département de l'Isère, Lyon, Reymann et Cie, Paris, Bruno, an IX.

\section{NOTES}

1. C. Demeulenaere, "Une tentative d'inventaire des richesses minières de la France ", p. 9-16.

2. É.-A. Pépy, « Décrire, nommer, ordonner », p. 27-42.

3. Bibliothèque Municipale de Grenoble, N 3335, lettre de M. Villars à M. de la Salette, s.d.

4. Bibliothèque du Muséum d'Histoire Naturelle de Grenoble (désormais Bibl. MHN Grenoble), Villars EI-D1 à EI-D42, 42 lettres de Villars à Picot de Lapeyrouse, 1786-1809. 
5. Bibliothèque du Muséum national d'Histoire naturelle (désormais Bibl. MNHN), ms. 1994/699-714, 16 lettres de Ramond à Picot de Lapeyrouse.

6. Bibl. MHN Grenoble, Villars EI-D18, lettre de Villars à Lapeyrouse du 13 octobre 1796.

7. P. Duris, Linné et la France, 1780-1850, p. 151-152.

8. Bibl. MNHN, ms. 1994/710, lettre de Ramond à Lapeyrouse du 6 octobre 1797.

9. Bibl. MHN Grenoble, Villars EI-D33, lettre de Villars à Lapeyrouse du 7 août 1801.

10. D. Rusque, « Faire circuler les objets naturalistes au XviII ${ }^{\mathrm{e}}$ siècle ».

11. Bibl. MNHN, ms. 1994/700, lettre de Ramond à Lapeyrouse du 5 novembre 1795.

12. Bibl. MNHN, ms. 1994/708, lettre de Ramond à Lapeyrouse du $1^{\mathrm{er}}$ juin 1797.

13. J.-F. Saigey, F.-V. Raspail, Mémoires des sciences d'observation, p. 157.

14. Bibl. MNHN, ms. 1994/701, lettre de Ramond à Lapeyrouse du 16 décembre 1796.

15. J.-M. Drouin, «L'histoire naturelle à travers un périodique, la Décade philosophique », p. 175-181.

16. Bibl. MHN Grenoble, Villars EI-D14, lettre de Villars à Lapeyrouse du 9 janvier 1796.

17. Bibl. MNHN, ms. 1994/702, lettre de Ramond à Lapeyrouse du 9 mars 1796.

18. Bibl. MHN Grenoble, Villars EI-D1, lettre de Villars à Lapeyrouse du 24 mai 1786.

19. M. Pinault-Sørensen, Le livre de botanique (xvII et XVIII siècles).

20. Bibl. MNHN, ms. 1994/714, lettre de Ramond à Lapeyrouse du 17 juin 1798.

21. Bibl. MHN Grenoble, Villars EI-D5, lettre de Villars à Lapeyrouse du 31 décembre 1789.

22. Bibliothèque Municipale de Toulouse, ms. 2093, correspondance de Lapeyrouse avec Gillet de Laumont (26 lettres).

23. P.-I. Picot de Lapeyrouse, Figures de la flore des Pyrénées, avec des descriptions, des notes critiques et des observations.

24. P.-I. Picot de Lapeyrouse, Histoire abrégée des plantes des Pyrénées et itinéraire des botanistes dans ces montagnes.

25. L. Ramond de Carbonnières, Carnets pyrénéens, édition établie par Pierre Debofle.

26. E.-A. Pépy, « La connaissance de l'environnement au service du progrès. Le cas du médecin naturaliste Dominique Villars (1745-1814)», p. 499-528.

27. F. Orlando, «L'histoire des montagnes et l'harmonie du monde chez Ramond de Carbonnières ", p. 167-220.

28. P.-I. Picot de Lapeyrouse, « Voyage au Mont-Perdu, et observations sur la nature des crêtes les plus élevées des Pyrénées, 15 fructidor an V», p. 39-66. L. Ramond de Carbonnières, « Extrait d'une lettre du citoyen Ramond, associé de l'Institut national, et Professeur d'histoire naturelle à Tarbes, au citoyen Haüy, membre de l'Institut à Paris, sur deux voyages au Mont-Perdu, sommet le plus élevé des monts Pyrénées, 5 fructidor an $\mathrm{V} »$, p. 35-38.

29. J. Rochas, Muséum de Grenoble, une histoire naturelle, p. 46-54.

30. P. Duris, "L'enseignement de l'histoire naturelle dans les écoles centrales", p. 23-52.

31. D. Villars, Mémoires sur la topographie et l'histoire naturelle, extraits du cours de l'École centrale du département de l'isère. 
32. Bibl. MHN Grenoble, Villars J. I 10, Cours de botanique, programme de cours, s.d.

33. Bibl. MNHN, ms. 1994/710, lettre de Ramond à Lapeyrouse du 6 octobre 1797.

\section{RÉSUMÉS}

Naturalistes spécialisés dans l'étude des milieux montagnards, Villars, Lapeyrouse et Ramond partagent une même passion pour les études de terrain, revendiquant la pratique régulière de courses en montagne, dans les Alpes et les Pyrénées. À la croisée des XVIII ${ }^{\mathrm{e}}$ et XIX ${ }^{\mathrm{e}}$ siècles, leurs objets d'étude, ainsi que leurs carrières, les entraînent dans des relations épistolaires suivies. L'étude de ces correspondances révèle les difficultés que doivent surmonter ces savants de province pour faire connaître leur expertise de terrain, alors que la production de savoirs sur ces milieux naturels s'avère de plus en plus concurrentielle, dans un contexte de recomposition des institutions savantes.

\section{AUTEUR}

\section{ÉMILIE-ANNE PÉPY}

Maître de conférences en histoire moderne, Université Savoie - Mont-Blanc, laboratoire LLSETI 


\title{
Jean-André Deluc (1727-1817) et la montagne comme objet d'étude et norme éthique
}

\author{
Sabine Kraus
}

1 Issu d'une famille calviniste genevoise, Jean-André Deluc est l'un des premiers savants à explorer les Alpes, accompagné de son frère Guillaume-Antoine (1729-1812). Tous deux sont les fils de François Deluc (1698-1780), né à Genève d'une famille considérée, et dont les membres remplissent les premières charges de la République. Maître horloger de son métier, membre actif du parti populaire, auteur de nombreux ouvrages religieux et politiques, François Deluc est également un ami de longue date de Jean-Jacques Rousseau.

2 Jean-André et son frère Guillaume-Antoine montrent dès l'enfance un goût pour l'étude de l'histoire naturelle, et commencent de bonne heure à former un cabinet minéralogique, dont une partie des collections est aujourd'hui conservée au muséum d'histoire naturelle de Genève. Jean-André Deluc s'intéresse aux instruments et méthodes de la physique, et se consacre à perfectionner le baromètre et le thermomètre, dans le but de déterminer la hauteur des montagnes à l'aide d'observations barométriques. Jean-André n'a que dix-sept ans, et son frère quinze, lorsqu'ils font leur première excursion scientifique dans les Alpes en 1744. En 1765, ils sont les premiers à tenter l'ascension de la cime glacée du Buet ${ }^{1}$. Après deux nouvelles tentatives, ils atteignent le sommet le 23 septembre 1770 en passant du côté de SixtFer-à-Cheval, et y font de nombreuses observations. Après plus de vingt années d'explorations et d'expérimentations, leurs travaux sont réunis par Jean-André Deluc dans un ouvrage qui paraît en 1772 à Genève: Recherches sur les modifications de l'atmosphère: contenant l'histoire critique du baromètre et du thermomètre, un traité sur la construction de ces instrumens, des expériences relatives à leurs usages, et principalement à la mesure des hauteurs \& à la correction des réfractions moyennes.

3 Par ailleurs, les deux frères ayant hérité des principes politiques et religieux de leur père, Jean-André s'engage également dans la vie politique genevoise. Mais suite à un changement de régime, il est contraint à l'exil et s'établit en 1773 en Angleterre, où sa 
réputation scientifique lui vaut d'être nommé un an plus tard lecteur de la Reine Charlotte, épouse de Georges III, à Windsor. Bien qu'il ait été le premier à proposer le terme de géologie pour qualifier une cosmologie qu'il entend comme « connaissance de la Terre, et non de l'Univers ${ }^{2}$ ", Jean-André Deluc a été longtemps discrédité par les historiens. La raison en est qu'il cherchait à concilier ses observations scientifiques sur l'histoire de la Terre et de l'homme avec le livre de la Genèse, s'inscrivant à ce titre dans le courant de la théologie naturelle britannique. De même, si l'on reconnaît que la montagne devient à l'avènement des Lumières le lieu d'une nouvelle perception de la nature, dans laquelle les Suisses jouent un rôle prédominant et dont Rousseau se fait l'artisan, le rôle majeur de la théologie naturelle dans la transformation de cette perception reste largement méconnu.

\section{Le concept de Nature}

\section{Science et religion}

4 L'importance historique du travail de Jean-André Deluc a été oblitérée par la permanence de la thèse du conflit entre science et religion, dans laquelle l'Église est perçue comme contrecarrant chaque avancée scientifique. Beaucoup d'affirmations à propos de leur relation passée, supposée conflictuelle, sont dues à l'application inadéquate de nos catégories modernes à la lecture du passé. Les œuvres doivent être étudiées à l'intérieur de leur contexte historique, et en relation avec des sphères de pensée plus larges.

Beaucoup de chrétiens acceptaient la science de leurs temps, et recevaient ses avancées en harmonie avec la théologie traditionnelle sans grand conflit. Calvin, théologien fondateur de la Réforme, reconnaissait déjà que la Bible n'avait pas été écrite pour enseigner l'astronomie, mais pour adresser un message spirituel aux humains de façon à ce que celui-ci soit entendu et compris de tous ${ }^{3}$. Le principe d'accommodation du Réformateur genevois permit ainsi aux Protestants d'accepter les découvertes de la science. Durant la révolution scientifique, science et religion sont intimement liées, et la théologie naturelle est prédominante dans la communauté scientifique britannique $\mathrm{du} \mathrm{XvII}{ }^{\mathrm{e}}$ siècle. Ce sont des savants qui, lorsqu'ils étudient la Nature, sont convaincus qu'ils étudient les œuvres de Dieu. Partant, le compte rendu de la Création dans la Genèse fait l'objet d'un examen minutieux, et la Bible n'est plus considérée comme une forme d'autorité compétente pour expliquer de façon rationnelle comment la Création est advenue. Pour connaître le monde naturel et les lois qui le gouvernent, c'est la science, ou "philosophie naturelle ", qui fait alors autorité. À travers son alliance avec la théologie naturelle, l'image calviniste d'un dieu châtiant de manière arbitraire ses créatures est remplacée par une déité qui s'identifie avec la rationalité des processus naturels. Cette nouvelle conception place le pouvoir de création dans la nature ellemême, une création où tout ce qui est sur la Terre est bon, vivant et capable de vertu ${ }^{4}$. C'est donc une certaine théologie de la Création qui contribue à l'émergence d'une science à la fois rationnelle et expérimentale ${ }^{5}$.

6 Après la place du globe terrestre, c'est l'âge de la Terre qui occupe la cosmologie du $\mathrm{XVIII}^{\mathrm{e}}$ siècle, et les arguments théologiques en faveur d'une chronologie plus étendue ont précédé, plutôt que succédé, les découvertes géologiques. Vers 1750, un nombre croissant de savants est convaincu d'un temps géologique très vaste, et 
l'accommodation théologique est en phase avec ces idées, et non en réaction contre elles. De plus, la nature en tant que système est au cœur de la théologie naturelle qui, s'appuyant sur le récit mosaïque et la sagesse providentielle, a fourni les fondations des travaux de nombreux savants britanniques. Et peu de théologiens s'opposent à la géologie, science inaugurée par Deluc ${ }^{6}$.

7 Ce courant de pensée ouvre ainsi la voie au concept de Nature comme à la fois objet d'investigation scientifique et norme éthique. La notion de Nature en usage ici est celle empruntée aux Grecs. Pour Aristote, la nature est dans les êtres qu'elle crée le principe et la cause du mouvement et du repos. La Nature n'est donc pas entendue comme notre environnement, elle est considérée comme une force vive dans le monde. Son étude équivaut à apprendre à lire le Livre de la Nature, c'est-à-dire les Euvres de la Création, dans un émerveillement constant du fait de sa beauté.

\section{Le fait de la beauté}

8 La perception de la nature conçue comme un produit de la Chute a cependant prévalu dans la théologie chrétienne jusqu'à l'aube du xviII ${ }^{\mathrm{e}}$ siècle. Les immensités des montagnes, déserts, volcans ou océans apparaissent dans cette optique comme les monuments terribles du sacrilège humain ${ }^{7}$. Grâce à l'alliance de la science et de la religion dans le milieu intellectuel protestant de l'Angleterre du xvII siècle, une transformation de cette perception de la nature s'est imposée. La nature sauvage des montagnes n'y est plus considérée comme produit de la Chute et châtiment divin suscitant l'effroi. Par les richesses et diversités offertes au regard de celui qui les contemple et les étudie, la nature est perçue comme un ouvrage d'art dégageant un sentiment de beauté.

9 Une distinction va s'opérer alors dans la théorie de la perception, entre faits physiologiques et faits mentaux. La perception n'est plus le processus passif défini par la théorie des idées représentant les objets du monde extérieur. L'acte perceptuel est considéré comme la construction d'une connaissance permettant une action, car le premier stade sensoriel de la perception est porteur d'information et de signification. La perception nécessite donc à la fois l'activité physiologique des organes des sens et du système nerveux, et le rôle actif et vivant des opérations de l'intellect, c'est-à-dire là où le rôle de la matière finit. Ainsi, ce sont des médecins du xvIII ${ }^{e}$ siècle, comme le théologien et naturaliste William Derham (1657-1735), ou plus tard le philosophe et encyclopédiste Paul-Joseph Barthez (1734-1806), qui vont nous offrir une véritable éducation du regard pour contempler la beauté d'une nature vivante avant que d'être matérielle. Par ce regard sensible porté sur des paysages grandioses tels que les montagnes, celles-ci apparaissent alors comme les plus grandes beautés que la nature a données à la terre. Dans sa Théorie du beau dans la nature et les arts, Barthez traite « Du sentiment de la beauté des différentes parties du spectacle de la nature »:

« Il est surprenant que Buffon ait pu dire que sur la terre la nature brute est hideuse et mourante, et que le travail de l'homme seul peut la rendre agréable et vivante. Cependant il est certain que, dans plusieurs lieux de la terre, la nature produit d'elle-même, et sans être dirigée par les travaux de l'homme, des beautés semblables à celles des pays les plus cultivés. Mais de plus, combien la variété et la grandeur des objets agréables, que la nature répand dans ces paysages qu'elle crée sans le secours de l'homme, rendent leur beauté supérieure à celle des scènes de la plus riche culture, et même à celle des jardins les plus magnifiques, où l'art, dès 
qu'il se fait sentir, manifeste ses limites, et une sorte d'uniformité qui rapetisse tous ses effets! Dans les beaux paysages que produit la seule nature, elle travaille en grand, et la liberté de ses développements ajoute à la richesse de ses productions. ${ }^{8}$ " ouvrant ainsi le massif montagneux à la curiosité scientifique et à l'exploration. Dans l'optique de ce concept de Nature à valeur physique autant que morale, lors de ses explorations alpines, Deluc va donc associer observations et recherches scientifiques, observations anthropologiques sur le genre de vie des Montagnes, ou de l'état de l'âme sur les Montagne, ainsi que descriptions pittoresques de la vie rude des hautes cimes ou des vallées riantes de verdure et de population.

\section{Lettres physiques sur les montagnes, objet d'investigation scientifique}

\section{Météorologie et cosmologie}

11 En l'année 1754, Deluc fait avec son frère, « un voyage à la partie des Alpes la plus voisine de Genève, pour examiner de près ces masses énormes qu'il est si important de bien connaître pour établir une bonne théorie du globe ${ }^{9}$ ». En s'intéressant à la science de l'atmosphère terrestre, l'objectif de Jean-André Deluc est en premier lieu de perfectionner la physique de son temps, ainsi que les méthodes d'investigation et les instruments inventés pour découvrir les lois gouvernant les phénomènes observés et leurs rapports. Le compte rendu de vingt années d'explorations météorologiques en haute montagne est publié dans ses Recherches sur les modifications de l'atmosphère en 1772 .

C'est un extraordinaire recueil d'observations de terrain et d'expérimentations, qui lui donnent à chaque fois de nouveaux problèmes à résoudre car, comme il l'écrit dans ses Recherches, «il est fort ordinaire en Physique, que la théorie ne soit pas confirmée par l'expérience. Quelque cause imprévue survient, qui dérange les calculs ${ }^{10} »$. Les travaux pionniers de Deluc s'appuient sur une étude minutieuse et détaillée des écrits de ses prédécesseurs concernant l'invention du baromètre, les expériences décrites par les différents auteurs ainsi que les hypothèses qu'ils en ont déduites. Ce qui mène Deluc à nous offrir une foule de détails pratiques et de précautions à prendre, afin de pouvoir travailler dans de bonnes conditions d'observation visant à obtenir des mesures plus certaines. À un moment où toute l'Europe savante s'occupe de baromètre et de thermomètre pour connaître la nature de l'air et de l'atmosphère, les travaux de Deluc ont porté à une très grande exactitude les mesures barométriques des hauteurs, ouvrant un nouveau champ de recherche, tant pour la fabrication d'instruments plus fiables que pour la découverte des causes physiques de phénomènes observés. Par la poursuite des expériences de Blaise Pascal en 1648 (preuve de l'existence du vide au sommet du Puy de Dôme) et de Edmund Halley en 1686 (publication de la première carte météorologique) sur les relations entre l'altitude et la pression atmosphérique, Deluc est la première personne à avoir déterminé le rapport mathématique entre la pression de l'air et l'altitude. Il a montré que l'augmentation de l'altitude est proportionnelle à la diminution du logarithme de la pression de l'air, et inversement proportionnelle à la température de l'air ${ }^{11}$. En 1770, Deluc a calculé la hauteur du Mont- 
Blanc à 14346 pieds au-dessus du niveau de la mer. Horace-Bénédict de Saussure, après l'ascension et des observations au sommet en 1787, obtient la mesure de 15700 pieds $^{12}$.

\section{Météorologie et médecine}

13 Au cours de son travail d'amélioration des instruments à l'usage des physiciens, Deluc prend soin de ne pas négliger l'usage public du thermomètre commun. Aucun terme fixe n'existait dans les thermomètres, chacun les fabriquant et les graduant à sa guise. Les liquides utilisés dans leur fabrication étaient très divers : mercure, huile d'olive, huile de lin, huile essentielle de camomille ou de serpolet, eau saturée de sel marin, espritde-vin, ou encore eau commune. Réaumur avait fixé le terme inférieur zéro au point où l'eau gèle. Pour le terme fixe supérieur, Deluc rectifie le thermomètre de Réaumur en substituant le mercure à l'esprit-de-vin qui, à 80 degrés, est dissipé en vapeur. Il fixe ainsi le terme supérieur au point où l'eau bout. Pour le public, Deluc préférera revenir aux thermomètres d'esprit-de-vin moins coûteux, qui ont un avantage important pour l'usage ordinaire : on les observe plus aisément, car l'esprit-de-vin est coloré.

14 Le thermomètre est, en outre, devenu d'une grande utilité pour les médecins, qui faisaient des relevés météorologiques dans le cadre de leur pratique, afin d'étudier les variations saisonnières et leurs effets sur la santé des humains ainsi que des récoltes. En effet, dans le mouvement de vitalisation de la nature qui s'opère au XvIII ${ }^{\mathrm{e}}$ siècle, la médecine, qui reposait sur les connaissances anatomiques du corps humain, se tourne vers une approche plus globale qui étudie les organismes vivants en lien avec le monde extérieur ambiant. Les notions de milieu ou de terrain sont alors introduites, en référence au Traité des airs, des eaux et des lieux d'Hippocrate. L'étude des variations de l'atmosphère devient l'objet de nombreuses réflexions à propos de l'état de santé, tant sur les influences du poids de l'atmosphère sur notre corps, que sur l'effet que peut produire un air plus ou moins dense sur les mouvements du cœur et des poumons, ou encore sur certains états de l'atmosphère considérés comme cause de maladies.

Les recherches de Deluc ont fait l'objet d'une grande réception dans le monde médical et dans l'Europe des Lumières. Le renouveau de la médecine vitaliste est mené par PaulJoseph Barthez à Montpellier, ainsi que par William Cullen à Édimbourg. Ainsi, c'est en Écosse que se rendent les compatriotes de Deluc pour obtenir la meilleure éducation possible, le centre médical d'Édimbourg dominant alors le monde occidental. Par l'intermédiaire de ses amis médecins suisses, notamment Louis Odier qui sera le médecin d'Horace-Bénédict de Saussure, Jean-André entre en contact avec William Cullen. Ce dernier recevra les souscriptions pour 17 exemplaires de ses Recherches sur l'atmosphère, expédiés en 1773, année de l'exil de Deluc en Angleterre, ainsi que de la nomination de William Cullen comme président du Royal College of Physicians of Edinburgh ${ }^{13}$.

\section{Lettres Morales sur les Montagnes, norme éthique}

\section{Approche anthropologique}

16 Au cours de ses expéditions alpines, Deluc a l'occasion d'éprouver lui-même l'effet physiologique des variations de l'atmosphère, autant qu'il remarque leur influence sur les populations locales. En observant la vie des montagnards qui passent chaque jour 
des vallées au sommet de hautes montagnes, et qui ne ressentent aucune incommodité lors de grands changements dans la pression ou la densité de l'air, Deluc s'aperçoit alors de l'équilibre et de la régularité des mouvements du corps en haute montagne, et de l'adaptation des hommes et de leurs animaux au milieu. Conjointement à ses recherches sur le milieu physique alpin, Deluc s'intéresse extrêmement au mode de vie, ainsi qu'à l'histoire des gens qui habitent ces lieux. Aussi nous livre-t-il ses observations sur le genre de vie des habitants des Montagnes, puisque son frère et lui en font l'expérience quotidiennement au cours de la progression de leurs voyages successifs dans les hauteurs inexplorées. De plus, la route qu'il leur faut découvrir est connue seulement des gens qui y vivent, et dont ils dépendent, que ce soit pour le gîte grâce aux officieux chanoines des vallées, ou pour trouver des guides destinés à les conduire vers les sommets. Les différents types d'habitat selon les altitudes et les activités humaines sont observés de façon très minutieuse, de celui des paysans de riantes vallées à celui des chasseurs au chamois de belles solitudes enneigées. Le caractère pittoresque de ses récits les rend très vivants, il se dégage une grande cordialité dans les relations que les frères Deluc entretiennent avec les gens qui participent de la réussite de leur entreprise, à travers l'hospitalité accueillante qu'ils reçoivent dans les villages pour pourvoir à leurs besoins, ou bien encore par le savoir-faire des habiles artisans qui fabriquent les tubes de leurs précieux instruments. Partant, Deluc nous fait entrer dans les ateliers des ouvriers de verrerie, décrivant par le menu les opérations successives, ainsi que leurs conséquences sur l'état de l'atelier, allant jusqu'à faire circuler parmi eux des conseils afin de remédier aux défauts de fabrication des produits. C'est à une écologie de la vie que Deluc nous invite, toute séquence d'actions engagées par ses travaux devenant l'occasion d'une peinture de la vie. Et la réalité de la vie alpine se dessine au long de ces pages, ouvrant sur une esthétique de la nature ordinaire, animée tout à la fois par la variété des plantes, des sols et des aspects, les changements incessants de la route qui en est intéressante, ainsi que par la variété des occupations humaines qui s'y déroulent quotidiennement. Le sens pratique de Deluc lui fera noter la propriété des sapins de bien garantir de la pluie, en évoquant quelques montagnards qui étaient restés dans cette montagne pour y garder une vache blessée, aussi bien que le trait d'hospitalité des bergers dont les cabanes fournissent sans cesse l' exemple.

\section{Nature et Culture}

17 À la lecture de ses récits, Deluc nous fait découvrir « une Nature qui tend partout à donner les montagnes à l'homme, et dans les lieux même qui semblent encore les plus inutiles pour lui ${ }^{14}$ ». En observant les phénomènes des paysages alpins parcourus lors de ses expéditions, qui lui procurent une multitude de sensations agréables autant qu' inattendues, l'attention de Deluc est surtout portée à l'esprit d'entraide et d'obligeance des villageois habitant dans ces lieux reculés, bien peu connus, et dans lesquels il retrouve les beautés de la simple Nature, beautés pour les yeux, mais surtout pour l' esprit et le cœur. En 1778, ses Lettres sur les Montagnes sont autant d'exemples multipliés qu'il apporte de l'harmonie qui règne dans la Nature entre le moral et le physique. Il trouve dans ces montagnes l'hospitalité désintéressée de la Nature, il y apprend à quoi se réduisent les vrais besoins de l'homme; ce qu'il peut par la force de l'habitude ; mais surtout il y apprend, dans quel doux calme est son âme lorsqu'il reste entre les mains de la Nature, Deluc nous fait part de son admiration devant : 
«Le montagnard qui reste toujours l'homme simple et heureux de la Nature, l'homme commun, l'homme resté bon. Son bonheur reste attaché à la satisfaction des premiers besoins de la Nature, et il sent avec énergie celui d'obliger ses semblables comme l'un des premiers. Tout cela marche ensemble comme le premier penchant de la Nature... L'homme, comme tous les autres êtres sensibles, cherche avant tout son bonheur; c'est la plus générale et la plus belle des lois que nous présente l'étude de la Nature. L'homme aussi trouve une partie essentielle de son bonheur à obliger ses semblables; c'est la première et la plus admirable des lois naturelles ; c'est celle qui contribue le plus à former société. ${ }^{15}$ »

Nous retrouvons dans les réflexions de Jean-André Deluc le concept de Nature tel qu'il est mis en œuvre par l'alliance entre la science et la théologie naturelle. C'est cette nouvelle vision de la Création, où tout ce qui est sur terre est bon, vivant et capable de vertu, qui lui permet de déborder le hiatus entre nature et civilisation, un hiatus qui nous a fait oublier que l'homme est, sans aucun doute, une partie de la nature.

C'est l'expression d'une nature vitalisée, où tout est lié, qui s'impose au $\mathrm{xvIII}^{\mathrm{e}}$ siècle. Deluc nous fait partager ses connaissances sur une nature dont la conception ne correspond pas à ce que l'on entend aujourd'hui par ce terme. C'est une nature beaucoup plus vaste, puisqu'elle inclut également toutes les activités humaines en rapport avec des productions naturelles. C'est donc une vision dans laquelle il n'y a pas d'opposition entre nature et culture comme aujourd'hui, pas plus qu'il n'y a de séparation entre nature et esprit, la psychologie étant une partie de la science de la nature, telle que conçue par la philosophie grecque.

De plus, c'est une nature à qui il est toujours agréable de pourvoir aux besoins et aux plaisirs de ses habitants quels qu'ils soient. Bien qu'il soit un grand ami de Rousseau avec qui il partage son admiration pour l'homme simple et heureux de la nature, pour qui la vie est le premier des biens, Deluc nie l'idée de contrat social, car pour lui, la société est un fait naturel, primitif, nécessaire, permanent et universel :

«Sans doute cependant que les villes sont un bien à d'autres égards, puisqu'elles sont si généralement répandues sur la Terre. Car il paraît de là que les hommes tendent à s'entasser les uns auprès des autres comme les abeilles et les fourmis. C'est donc une sorte d'instinct qu'ils ont reçu de l'Auteur de la Nature: ainsi mettons la main sur la bouche. ${ }^{16}{ }^{\prime}$

\section{BIBLIOGRAPHIE}

ALlABY Michael, Atmosphere: A Scientific History of Air, Weather, and Climate, New York, Infobase Publishing, 2009.

BARTHEZ Paul-Joseph, Théorie du beau dans la nature et les arts : ouvrage posthume de P.-J. Barthez, Paris, Chez Léopold Colin, 1807.

DELUC Jean-André, Recherches sur les modifications de l'atmosphère, volumes I et II, Genève, 1772. 
DELUC Jean-André, Lettres physiques et morales, sur les montagnes et sur l'histoire de la terre et de l'homme, Suisse, les Libraires Associés, 1778.

DELUC Jean-André, Lettre de Jean-André à William Cullen, Glasgow University, Special Collections, Correspondence of William Cullen, MS Cullen 83, Londres, le 2 août 1773.

DURANT Will and Ariel, The Age of Voltaire: A History of Civlization in Western Europe from 1715 to 1756, The Story of Civilization, Volume IX, New York, Simon and Schuster, 2011.

EUVÉ François, « Bulletin théologie de la création et sciences », Recherches de Science Religieuse, t. $100, \mathrm{n}^{\circ} 2,2012$, p. 295-312.

GIPPER Andreas, « La nature entre utilitarisme et esthétisation. L'abbé Pluche et la physicothéologie européenne », dans GEVREY Françoise, BOCH Julie et HAQUETTE Jean-Louis (dir.), Écrire la nature au XVIII siècle : autour de l'abbé Pluche, Paris, Presses Paris Sorbonne, 2006.

GOSCHLER Isidore, abbé, Du panthéisme, Paris, Gaume frères et J. Duprey (éditeurs), 1862.

PERROT Alain, Le visage humain de Jean Calvin, Genève, Éditions Labor et Fides, 1986.

ROBERTS Michael B., « Genesis Chapter 1 and geological time from Hugo Grotius and Marin Mersenne to William Conybeare and Thomas Chalmers (1620-1825) », Geological Society of London, Special Publications, vol. 273, January 2007, p. 39-49.

SIGRIST René, La Nature à l'épreuve. Les débuts de l'expérimentation à Genève (1670-1790), Paris, Classiques Garnier, 2011, 704 p.

\section{NOTES}

1. Le Mont Buet, culminant à $3096 \mathrm{~m}$ d'altitude, autrement dénommé le Mont-Blanc des dames, l'un des sommets du massif du Giffre en Haute-Savoie.

2. J.-A. Deluc, Lettres physiques et morales sur les montagnes, p. VII.

3. A. Perrot, Le visage humain de Jean Calvin, p. 220-222.

4. I. Goschler, Du panthéisme, p. 36.

5. F. Euvé, « Bulletin théologie de la création et sciences », p. 301.

6. M. B. Roberts, « Genesis Chapter 1 and geological time from Hugo Grotius and Marin Mersenne to William Conybeare and Thomas Chalmers (1620-1825) », p. 40.

7. A. Gipper, La nature entre utilitarisme et esthétisation. L'abbé Pluche et la physico-théologie européenne, p. 34.

8. P.-J. Barthez, Théorie du beau dans la nature et les arts, p. 327-328.

9. J.-A. Deluc, Recherches sur les modifications de l'atmosphère, vol. 1, p. 185.

10. Ibid., p. 278.

11. M. Allaby, Atmosphere: A Scientific History of Air, Weather, and Climate (Discovering the Earth), p. 112.

12. W. et A. Durant, The Age of Voltaire: A History of Civlization in Western Europe from 1715 to 1756 , p. 91.

13. J.-A. Deluc, Lettre à William Cullen, MS Cullen 83.

14. J.-A. Deluc, Lettres physiques et morales sur les montagnes, p. 35.

15. Ibid., p. 159. 
16. Ibid., p. 169-170.

\section{RÉSUMÉS}

Les montagnes ont longtemps été considérées comme des monuments terribles du sacrilège humain. Une transformation de cette perception s'opère dans le milieu intellectuel protestant britannique au $\mathrm{XVII}^{\mathrm{e}}$ siècle. Les montagnes apparaissent alors comme un ouvrage d'art animant un sentiment de beauté. Issu d'une famille calviniste genevoise, Jean-André Deluc et son frère Guillaume-Antoine font partie des premiers savants à explorer les Alpes. Jouissant d'une grande renommée de son vivant, Deluc a été longtemps discrédité, car il cherchait à concilier ses travaux scientifiques avec la Genèse. Le résultat de vingt ans d'explorations météorologiques en haute montagne est publié dans Recherches sur les modifications de l'atmosphère. C'est un recueil d'observations de terrain et d'expériences empiriques, afin de perfectionner les instruments et les méthodes d'investigation dans les mesures altimétriques. Ce recueil nous révèle aussi tout un versant d'études anthropologiques sur le genre de vie des habitants des montagnes, sur le savoirfaire des artisans. Les travaux de Deluc exercèrent une grande influence sur Horace-Bénédict de Saussure et Georges Cuvier.

\section{AUTEUR}

\section{SABINE KRAUS}

Architecte de formation, doctorante en Histoire et civilisations, EHESS - Paris 


\title{
Le récit guerrier en montagne dans la fiction littéraire européenne (1780-1850)
}

\author{
Louis Bergès
}

1 Né en temps de guerre, le mouvement romantique est marqué par les conflits intérieurs et extérieurs, ceux des révolutions et des insurrections nationales. Sur la scène littéraire et artistique, la poésie et le roman ont puisé dans le paysage de montagne les bases d'un matériau romanesque considérable parmi lequel figurent le personnage du guerrier et le combat en altitude. Au début, l'inspiration de l'auteur romantique se nourrit des préjugés hérités des siècles passés, regardant la montagne comme un territoire mystérieux, proche du divin, où les parois rationnelles ont tendance à disparaître. Des représentations littéraires du Moyen Âge et de la Renaissance, ne ressort au début du $\mathrm{xvIII}^{\mathrm{e}}$ siècle qu'un milieu montagnard menaçant fait de clichés sombres et violents, souvent liés à la topographie, expression d'une Nature féroce, à la fois lieu de catastrophes et théatre d'opérations de peuples guerriers autochtones en guerre contre voyageurs et envahisseurs.

2 Ainsi, sera-t-il intéressant de voir comment une nouvelle approche romantique de la montagne, issue de l'imaginaire rousseauiste et des nombreux pèlerinages littéraires effectués depuis Bernardin de Saint Pierre, est parvenue à intégrer le récit guerrier dans la littérature européenne.

Pour cette recherche, il est proposé d'examiner un corpus se composant de plusieurs types de textes: en premier lieu, le roman, genre littéraire majeur de la période, qu'il soit français ou étranger, traduit et repris sous la forme de récits historiques ou de narrations fictives accompagnant une intrigue dramatique. La recherche s'est étendue ensuite aux textes poétiques portant sur des événements historiques qui se réfèrent soit à un passé mythique soit à un événement contemporain fondateur lié par exemple à une guerre d'indépendance. J'ai exclu d'examiner les nombreux récits de voyage d'écrivains en montagne dans la mesure où ils n'ont pas recours à la fiction littéraire. J'ai retenu enfin plusieurs pièces de théâtre contemporaines significatives de la période 
qui ont introduit dans un cadre comique ou dramatique un récit guerrier lié à ses habitants.

\section{À la recherche des origines: mythes fondateurs et courants de pensée}

4 D'où vient cette idée que la montagne serait un territoire de guerre peuplé de soldats nés pour combattre ? Le récit guerrier, tel qu'il apparaît dans les premiers écrits de la littérature préromantique, s'appuie sur des mythes fondateurs qui associent la montagne à la guerre et à ses développements, qu'il s'agisse de batailles légendaires ou réelles, de combattants célèbres ou du caractère guerrier des habitants. Mais un nouveau souffle face à l'appréhension des batailles commence à irriguer la fiction littéraire utilisant le cadre montagnard, comme si la présence de la guerre en montagne avait aussitôt fait partie de l'outillage mental de l'écrivain romantique.

Plusieurs indices existent au $\mathrm{xVIII}^{\mathrm{e}}$ siècle qui contribuent à construire cette représentation nouvelle des sites de montagne comme lieux de bataille. Vers 1750, Montesquieu se penche sur l'esprit guerrier qu'il considère comme lié à certaines caractéristiques physiques des territoires et de leurs habitants : les montagnards «se défendent aisément, ils sont attaqués difficilement; les munitions de guerre sont assemblées et portées contre eux avec beaucoup de dépenses; il est donc plus difficile de leur faire la guerre, plus dangereux de l'entreprendre... ${ }^{1} »$. Pour le philosophe, le relief est un obstacle à la guerre, il favorise en même temps l'esprit de liberté et la combativité du bon soldat, mais l'indiscipline du montagnard n'est pas évoquée. Buffon évoque plus précisément le cas des montagnards Kimos, qualifiés de nains blancs de Madagascar, vus comme des guerriers nés ${ }^{2}$. On peut relever aussi la définition donnée par l'Encyclopédie méthodique de Pancoucke :

«Les habitants des montagnes sont pour l'ordinaire plus guerriers que ceux du plat pays, qui est presque toujours plus fertile, qui, par cette abondance, rend ceux qui l'habitent plus délicats, plus vicieux et plus lâches. ${ }^{3}$ "

6 Les historiens militaires des Lumières renvoient eux aussi à des modèles façonnés par les récits de l'Antiquité : Folard évoque en 1754 à propos de la bataille de Sélasie entre Macédoniens et Spartiates en 222 avant notre ère les particularités des combats de montagne ${ }^{4}$. Charles Rollin évoque le cas des Miquelets dans les Pyrénées sous l'Antiquité: le général romain Sertorius, pour franchir les cols, fait face à "ces misérables montagnards », auxquels il doit payer « une espèce de tribut et de droit de passage $\mathrm{e}^{5}$.

7 Il se dessine une représentation de la montagne que l'on pourrait situer entre celle d'un paysage qui recèle de nombreux dangers et celle d'un refuge, havre de paix, entre celle d'un montagnard, bon sauvage, pacifique et celle du guerrier barbare dont l'aspect pittoresque apporte une touche exotique au récit. La grande proximité du montagnard avec l'état de nature est vue comme la cause principale de son caractère belliqueux : au sujet de cette passion guerrière censée habiter les populations de montagne, Rousseau n'évoque pourtant pas cet aspect pour les Corses et les Suisses au sujet desquels il se contente de parler simplement de "courage dans les combats", mais il souligne le mercenariat comme une dérive propre au "génie» de ces habitants ${ }^{6}$. Clausewitz enrichit les réflexions précédentes de considérations stratégiques en insistant sur la géographie au service de la défense d'un territoire avec le développement de la " petite 
guerre » ou guérilla propre aux zones de montagne et de bois ${ }^{7}$. Pour Jomini enfin, tout combat en montagne nécessite des camps retranchés ou des fortifications ${ }^{8}$.

8 À la fin $\mathrm{du}^{\mathrm{x}} \mathrm{xVIII}^{\mathrm{e}}$ siècle, paraissent des romans précurseurs qui se réfèrent à des traditions historiques nationales de combats libérateurs dans des pays de montagne: c'est le cas de la Suisse avec la renaissance du personnage mythique de Guillaume Tell. Florian montre un héros des montagnes d'Altorf qui se bat contre le tyran Gesler ${ }^{9}$; le thème est aussitôt popularisé par Schiller en 1804 sous la forme d'une pièce de théâtre ${ }^{10}$. De même, Andreas Höfer, le révolté du Tyrol, est célébré en 1809 par le poète anglais Wordsworth comme le guerrier descendant des montagnes pour lutter contre le "tyran Napoléon ${ }^{11}$ ». Mais ces héros semblent moins portés par le cadre de leurs montagnes natales que par la singularité de leur engagement.

9 Pendant la même période, dans ses premiers romans américains, Chateaubriand accorde au paysage des montagnes et à ses habitants une place mythique accompagnée d'un jeu de métaphores guerrières : dans les Natchez ${ }^{12}$, il imagine le berceau des tribus indiennes dans les Rocheuses, "montagnes bleues" situées près des rives du "Meschacebé » (Mississipi) entre l'Ohio et le Kentucky. Vastitude et étrangeté se mêlent pour faire des montagnes lointaines des lieux sauvages comme leurs habitants, où l'activité guerrière entre tribus rivales ou envahisseurs constitue une sorte de microcosme naturel.

10 Tous ces récits précurseurs représentent en ordre dispersé la part des premières influences que l'on peut résumer en trois modes littéraires qui vont irriguer de façon prédominante l'écriture poétique et romanesque en Europe au cours de la période.

11 En premier lieu, figure la celtomanie, née du succès littéraire des antiques légendes gaéliques recréées par le poète James Mac Pherson dans les montagnes d'Écosse vers 1760. Cette mode esthétique, née en France à partir de 1774 autour du personnage imaginaire du barde "calédonien » Ossian ${ }^{13}$, fait du paysage des Highlands un topos privilégié : l'auteur imagine autour de la bataille mythique de Temora, qui se serait déroulée vers la fin $\mathrm{du} \mathrm{III}^{\mathrm{e}}$ siècle avant notre ère, un modèle métaphorique qui va bercer tous les grands esprits militaires de la fin du XviII ${ }^{\mathrm{e}}$ siècle jusqu'à celui de Napoléon luimême ${ }^{14}$. Sophie Cottin avec son Malvina décline le modèle au cœur des montagnes "sauvages et escarpées" de Bread Alben ${ }^{15}$. À la même époque, Senancour fait une synthèse pleine de mélancolie entre la Suisse et l'Ecosse : les sommets écossais «qui tombent dans la mer du Nord " mettent en lumière l'image des "filles de guerriers " qui «sur l'Océan tranquille, chantent les combats et l'espérance de la patrie ${ }^{16}$ ». Les premières œuvres de Chateaubriand affectent aussi une sensibilité celtomane débridée : dans Les Martyrs, le sacrifice de Velléda la druidesse gauloise se déroule dans le décor montagnard d'une forteresse digne des Iles britanniques transposé en Armorique ${ }^{17}$.

12 Le second courant de pensée qui envahit la littérature européenne, c'est l'hispanisme qui prend racine dans le passé mauresque de la Reconquista au détriment de celui, cruel et ténébreux, de l'Inquisition décrié par les philosophes: puissance de la sensibilité, pathétique exacerbé, profondeur mystique, viennent remplacer les anciennes images d'une Espagne arriérée. Florian se fait en 1791 l'écho de la légende montagnarde de La Pena de los Enamorados à Antequera au nord de Malaga ${ }^{18}$, qui a abrité les amours tragiques d'une princesse musulmane et d'un chevalier chrétien. Chateaubriand explore lui aussi le thème avec Le dernier abencérage, ou le récit du retour 
mythique dans les montagnes d'Andalousie de Boabdil, "Ben Hamet», dernier souverain musulman d'Espagne ${ }^{19}$.

L'attrait exercé par l'Espagne revêt ensuite des aspects très divers : au départ, il s'agit d'un orientalisme fondé sur le passé historique musulman de la péninsule ibérique avec l'image du Maure victorieux à Roncevaux mais vaincu en 1492; puis, à partir des années 1820 , se manifestent des dérivés géographiques de type patriotique et national avec l'«euskarisme» qui s'est développé à la suite des travaux de l'ethnologue allemand Humboldt en 1817 et du Chant guerrier de Lelo ${ }^{20}$. Humbolt est à l'origine d'une littérature historico-légendaire basque qui émerge avec l'œuvre du poète Chaho et de son personnage légendaire Aïtor, le patriarche montagnard, fondateur de la race guerrière des "Euskariens $»^{21}$. D'autre part, il faut évoquer un autre avatar de l'hispanisme des «romanceros", l'« americanidad» des colons espagnols révoltés contre Madrid : il s'agit alors de la guerre d'indépendance menée par Bolivar à partir de zones montagneuses-refuges en Amérique latine dès 1813 et célébrées par la poésie en langue espagnole publiée et traduite en Europe ${ }^{22}$.

Le dernier courant de pensée qui transforme en profondeur les sources d'inspiration littéraire, c'est le philhellénisme, né de la guerre d'indépendance contre l'Empire ottoman et de la fondation de l'État grec à partir de 1821, qui surgit dans un terreau intellectuel largement favorable. Depuis Le voyage du jeune Anarcharsis en Grèce de l'abbé Barthélémy en 1788, la Grèce des montagnes est le lieu pittoresque par excellence pour l'écrivain français qui peut y lire une description de la bataille des Thermopyles au pied et au sommet du mont Kolonos, théâtre de l'ultime résistance spartiate ${ }^{23}$. La geste héroïque conduite par des chefs militaires insurgés sous la forme d'une guerre de montagne, représente un combat alliant révolte esthétique et désir de liberté qui réunit tous les intellectuels européens à partir de Byron : Adamantios Korais, poète et éditeur grec réfugié en France, suscite l'Appel aux Grecs et aux Français pour que tous se rejoignent dans un même élan de soutien ${ }^{24}$. Tandis que brillent des héros comme l'«armatole », Athanasios Diakos, fait prisonnier et mort empalé, ce sont les valeurs nationales de la Grèce antique qui galvanisent les insurgés contre l'ennemi turc dans le cadre montagnard du Péloponnèse. Après une première victoire, celle de Valtetsi du 12 mai 1821, ce sont des images à la fois souffrantes et victorieuses du « capetan » et du «klephte », guerriers montagnards, qui sont popularisées en France par le linguiste Fauriel à travers ses Chants populaires de la Grèce ${ }^{25}$ et le poète Kalvos, auteur de la « lira ${ }^{26}$ ».

\section{Le champ de bataille de montagne ou les champs de l'imaginaire}

15 Dans les récits guerriers, la montagne est mise en scène comme un haut lieu de conflictualité : zone-frontière, la montagne est un enjeu stratégique qui suscite la convoitise. L'action militaire en montagne signifie une expédition, c'est-à-dire une errance parfois dédaléenne, à la fois pour ceux qui descendent dans la plaine combattre leurs ennemis et pour ceux qui gagnent les hauteurs la poursuite d'un adversaire. Le théâtre de la bataille à son point d'arrivée peut se trouver au pied d'une montagne, dans une vallée ou sur les contreforts d'une colline à proximité des sommets. 
Plusieurs batailles, mises en scène dans des œuvres très diverses, retiennent l'attention: les combats des troupes révolutionnaires en 1793 au pied du Mont Cenis vus par Joseph Bonaparte ${ }^{27}$; le dernier affrontement des clans écossais "jacobites " contre les Anglais à Culloden au pied des Highlands (16 avril 1746) décrits par Campbell ${ }^{28}$; la victoire de Bolivar sur l'armée coloniale espagnole à Junin dans les Andes péruviennes ( 6 août 1824) célébrée dans le poème épique de Joaquin José Olmedo $^{29}$. Toutes ces batailles se rapportent à des scènes réelles qui sont parfois mythifiées : c'est le cas du champ de bataille de Roncevaux glorifié comme une victoire militaire des Basques dans le chant d'Altabiscar ${ }^{30}$ puis repris par Victor Hugo dans la Légende des siècles ${ }^{31}$. Georges Sand raconte elle aussi l'histoire d'une bataille médiévale mythique, la victoire de Jean Ziska et de ses Taborites contre les « Utraquistes » pragois à Malesko ${ }^{32}$.

La montagne, autrefois mystérieuse, désormais pittoresque, se transforme pour devenir guerrière dans sa forme: la géographie participe de la qualité du récit et de sa dramaturgie. Un type de décor s'impose le plus souvent. Si la plaine où descendent les héros est un lieu de prédilection pour affronter les ennemis, le cœur de la montagne, où se trouve la fortification militaire, repaire de tyran ou refuge contre l'envahisseur, est un lieu incontournable, théâtre de combats ou de sièges violents. On retiendra le château assiégé d'Alisberthe de Hapdé dans la guerre des sept montagnes ${ }^{33}$; celui de Nideck en Forêt noire vu par Erckman-Chatrian dans Hugues le loup ${ }^{34}$ ou bien celui de Windeck en ruines dans Othon l'archer d'Alexandre Dumas où vient s'installer pour la nuit un groupe d'archers en expédition ${ }^{35}$. Dans le cas du château de Duino situé dans le golfe de Trieste au pied des Alpes dinariques décrit par Nodier dans Jean Sbogar, il s'agit plutôt d'un antre, le " pied de ce promontoire » à partir duquel déferlent sur «tous les rivages du golfe où ils portent la désolation et la terreur " les «frères du bien commun», sorte «de troupe sanguinaire» composée de bandits montagnards illyriens ${ }^{36}$.

18 La maison familiale dans l'habitat montagnard est aussi un lieu de bataille: dans le village mythique de Pietranera en Corse décrit par Mérimée dans Colomba, il s'agit de l'affrontement d'une forteresse familiale contre l'autre. La tour du Nord s'oppose à celle du Sud comme symbole de deux puissances ancestrales qui s'affrontent à mort, et au milieu, se trouve la place du village; dans "les deux maisons ennemies», on "pratique des archères aux fenêtres donnant sur cette place $^{37}$ ". Alexandre Dumas verse dans un registre identique dans Les frères corses, notant qu'à Sullacaro près de Sartène, les maisons de ce village montagnard, "bâties en manière de forteresse ", "paraissent avoir soutenu des sièges... ${ }^{38}$ "

19 La gorge ou le ravin et leurs sinuosités représentent un espace privilégié pour le combat: dans Les Orientales d'Hugo, Le ravin est le lieu ultime où s'amassent les cadavres des combattants ottomans et grecs insurgés au cours d'une bataille de la guerre d'indépendance ${ }^{39}$. Une œuvre majeure du romantisme français fait aussi appel à ce topos pour en faire le point central d'un poème dramatique : sur le mont Erymanthe, Lamartine montre les femmes et enfants grecs se précipitant au fond d'un gouffre pour échapper à leurs poursuivants ${ }^{40}$.

La caverne est vue comme une sorte d'orifice des monts et des collines avec ses galeries labyrinthiques, ses mines et ses tunnels, creusés dans le ventre de la montagne, comme autant d'arènes pour des affrontements meurtriers. Xavier de Montepin situe le repaire du capitaine Lacuzon, rebelle franc-comtois au XVII ${ }^{e}$ siècle, dans les montagnes du Jura, 
à l'« extrême limite de la forêt de Morbier" dans un endroit escarpé où il est poursuivi ${ }^{41}$. Chez Lamartine, la grotte des Aigles près de Grenoble est le dernier refuge de deux proscrits poursuivis par des soldats de la Révolution (24 août 1793) ${ }^{42}$. La caverne où s'est réfugié avec sa famille l'heiduque monténégrin Christich Mladin, " couché sur des cailloux aigus ", cerné par ses ennemis, aurait été son tombeau si, comme le raconte Mérimée, il n'avait décidé d'en sortir pour mener l'ultime combat les armes à la main avec ses trois fils ${ }^{43}$.

21 Le champ de bataille romantique apparaît en fin de compte comme un espace de mélange conflictuel constitué en territoire hors norme, brouillant les repères identificateurs traditionnels, fruit d'une géographie indistincte: dans Han d'Islande d'Hugo, la montagne du Nord de la Norvège, près du cercle polaire, est faite de trous, de cavernes, de forêts, de ruines où se cache un monstre, le Han. Ce bout du monde est un repaire de brigands qui descendent vers la capitale de la province au sud (Drontheim). Il cache dans ses entrailles des lieux hostiles (grotte de Wesberhog, la mine d'ApsylCorh ou la clairière de Ralph le géant $)^{44}$. La montagne peut enfin servir de champ de bataille allégorique. Dans Les chants de Maldoror, Lautréamont fait d'une montagne imaginaire le lieu de toutes les violences et transgressions: accompagné de son bouledogue, Maldoror tue, viole, éventre, sous les yeux d'un berger ${ }^{45}$.

\section{Le guerrier montagnard, héros romantique}

Sur le champ de bataille de montagne, différents personnages se côtoient ou se succèdent : le lieu est imaginé pour fabriquer des héros guerriers typiques.

Le montagnard, conservateur des mœurs et de la pureté originelle, est belliqueux dans l'âme parce qu'attaché à défendre à tout prix son cadre de vie et celui de ses ancêtres : monstre protecteur imaginaire (le Han d'Islande, l'Aïtor des Basques), brigand de grands chemins qui pille et tue les habitants des villes (l'illyrien Jean Sbogar), bardesoldat des monts d'Armorique chez Petrus Borel ${ }^{46}$. Andreas Hofer, l'aubergiste tyrolien, homme du peuple, est vu comme l'un des premiers soldats de la liberté par Wordsworth, mais le dernier roi maure d'Espagne de Chateaubriand est un guerrier vaincu, réfugié dans les montagnes au-dessus de Grenade, victime de l'histoire.

Plusieurs ethnotypes figés par une identité nationale font appel à la réminiscence historique: le montagnard caucasien sur le modèle de Moullah Nour, «le fléau du Daghestan ", l'ennemi intime des Russes, dépeint par Dumas ${ }^{47}$; le rebelle bohémien, aveugle, Jean Ziska, rusé et cruel, qui sème la terreur à la tête de ses Taborites ${ }^{48}$. Le «klephte » issu des montagnes grecques tient sans doute une place à part : rebelle à toute autorité, icône guerrière de la lutte nationale, célébré par le poète Kalvos comme l'aigle des sommets, « immortel monument des rochers de Spezzia, Hydra et Psara où ne pénétra jamais la crainte du danger ${ }^{49} »$. Tous ces personnages de roman ont en commun d'être l'archétype du guerrier montagnard idéal, habité par la foi du charbonnier, ennemi redoutable pour tout combattant "terrestre», autonome, endurant, mobile et insaisissable.

D'autres traditions littéraires font appel à des figures contemporaines. Le guerrier basque, berger et contrebandier, dépeint par Humbolt se retrouve sous les traits d'un chef militaire navarrais célébré par le poète Camoussary : le général Zumalacarregui, mort au combat pendant la première guerre carliste, dont la réputation exceptionnelle 
a donné lieu à une importante postérité littéraire et artistique ${ }^{50}$. Une génération après, l'historien militaire Ducuing en a relevé tout l'intérêt :

«Ce qu'il y a de force native et de grandeur primitive dans la population basconavarraise fournirait d'innombrables thèmes à une époque héroïque digne du romancero. ${ }^{51}$ »

Le brigand calabrais s'est doté d'un profil pittoresque grâce à l'occupation française dans le royaume de Naples sous la Révolution et l'Empire: avec son profil de conspirateur révolutionnaire, Fra Diavolo (Michele Pezza), chef de la rébellion montagnarde contre les Français de 1798 à 1806, est un véritable personnage de théâtre mis en scène par Eugène Scribe. Mais le guérillero sanguinaire, devenu à la mode sur les planches des théâtres parisiens, s'est transformé en bandit mondain, fringant et charmeur, dans le cadre idyllique de l'hôtel de Terracine sur les bords de la mer Tyrrhénienne ${ }^{52}$. Chez Dumas, le conspirateur " carbonaro ", Placido Brandi père, cinq fois condamné à mort et rescapé, est victime d'injustice et réfugié en montagne par nécessité ${ }^{53}$. Avec Giovanni Verga, on revient au portrait classique du "carbonaro » italien destiné à accompagner l'expédition garibaldienne: Corrado en 1810 est un agitateur politique anti-français, retiré dans son château du Monte della Torretta, combattant secrètement l'occupant ${ }^{54}$.

Le montagnard monténégrin véhicule une image relativement positive. Évoquée par la voix d'un des héros de Nodier, Lothario, qui voit dans Jean Sbogar, un «homme formidable " protecteur du pays illyrien ${ }^{55}$, celle-ci se développe aussi chez Mérimée qui en fait le thème central de la mystification littéraire créée autour du personnage imaginaire de Hyacinthe Maglanovich et de ses ballades de la Guzla. Féroce mais excessivement courageux, le « morlaque » ou « heiduque » serbe, affronte l'ennemi turc sans espoir et pour l'honneur ${ }^{56}$. Un autre montagnard serbe, célébré par Nerval dans son opéra-comique et son Chant monténégrin, est assiégé par Napoléon qui ne parviendra pas à le vaincre ${ }^{57}$. Le poème serbe «Gorski Viyenatz » de Pietr. Njegos, publié en 1847 et traduit en français sous le titre Les lauriers de la montagne, exalte l'action de combattants comme l'évêque Danilo ou le voïvode Drachko dans une lutte générale du peuple serbe contre l'occupant ottoman ${ }^{58}$.

Le brigand solitaire corse peut être considéré comme une sorte de guerrier domestique, porteur d'une vendetta perpétuelle, constamment sur ses gardes, à l'image d'Orso della Rebbia chez Mérimée. En revanche, dans le poème La Corse de Petrus Borel où le peintre voyageur est assassiné par erreur dans la montagne près d'oletta, il est revêtu d'une image hostile sans rapport avec ses autres congénères européens ${ }^{59}$.

Les figures historiques de héros montagnards bénéficient généralement d'un portrait avantageux, celui de résistants, morts pour la défense de leur pays, mais la posture prise peut dépendre de la nationalité de son promoteur ; ainsi, Andreas Hofer, le héros du Tyrol en 1809 contre Napoléon, glorifié par Wordsworth, est réincarné dans une posture falote de guide de montagne dans le théâtre français ${ }^{60}$. Alfred de Musset, sans citer son héros historique, fait néanmoins l'éloge du peuple "tyrolien", "peuple héroïque et fier/montagnard comme l'aigle et libre comme l'air ${ }^{61}$ ".

Les figures féminines ne sont pas absentes, car la montagne a ses guerrières. Sur le modèle des amazones, magnifié par Théophile Gautier dans le Thermodon ${ }^{62}$, voici Alisberthe, personnage légendaire de la pièce de Hapdé, assiégée et finalement vaincue dans son château par Henri de Saxe ${ }^{63}$. Oum Djehane, la danseuse de Shamakha, est une guerrière accomplie comme ses ancêtres de la tribu caucasienne des Lesghis: son 
aventure, relatée par Gobineau, est un morceau de bravoure où la beauté se révèle à la hauteur de la férocité de l'héroïne ${ }^{64}$.

31 Tous ces êtres qui peuplent les montagnes sont-ils prédestinés au combat? La surcharge folklorique et la célébration des vertus guerrières dans le cadre de la mythologie nationale ont permis de construire une image de soldat-citoyen pour le Suisse des montagnes et de brigand héréditaire en Corse et en Calabre. Pour les Illyriens, les Monténégrins et les Grecs, il se dégage plutôt une image charnelle de race martiale qui affleure lorsque le combat de ces peuples est évoqué. Chaque peuple montagnard dispose d'attributs guerriers qui appartiennent à ses traditions ancestrales et ses habitudes de combat, même si comme le fait remarquer Dumas :

«Il y a dans la montagne du pain, de l'eau, de la poudre et des balles, c'est tout ce

qu'il faut à un montagnard..$^{65}$ ”

Chacun dispose d'une arme symbolique : l'arbalète et la dague suisses, le stylet corse, le «hanzar» monténégrin, la carabine tyrolienne, le couteau de Calabre («spada del popolo »), le fouet à longue mèche tcherkesse du Caucase, la sagaie des Kimos de Madagascar, la "claymore» (épée) et le «dirk» (sorte de lame) des Ecossais; la "framée " des anciens Germains (sorte de lance longue et légère), le bouclier des Taborites de Ziska, le sabre ou « kandjar » du Daghestan.

Le récit guerrier ne se réfère pas qu'à des objets, des lieux, des personnages historiques ou légendaires. Certains auteurs comme Alfred de Vigny ont une vision intemporelle du guerrier de montagne, multidimensionnelle, presque dégagée de tout repère géographique parce que modélisée à partir de l'épopée ossianique : avec le personnage du Trappiste au Monserrat en Aragon, Vigny distingue, sans leur conférer des attributs particuliers, "parmi ces montagnards, ces robustes bergers, aventuriers hardis, chasseurs aux pieds légers, qui rangent sous sa loi sa troupe volontaire... ${ }^{66}$ » Balzac à son tour, fait de Wilfrid, son héros allégorique de Seraphita, perché avec Minna sur le mont du Falberg en Norvège, un simple montagnard du Nord, lassé de porter l'épée dans les plaines ${ }^{67}$.

\section{Un nouvel l'horizon : l'Orient guerrier et ses montagnes}

Les montagnes d'Orient tiennent une place particulière dans les récits guerriers de l'époque romantique. Les peuples montagnards du Proche-Orient et d'Asie aux lois incertaines, considérés comme inaptes aux libertés modernes, sont au cœur du désir de scènes exotiques, de tableaux bigarrés, d'images fantastiques et somptueuses, de rêveries sensuelles, bourrées d'éléments étranges et séduisants qui constituent une théâtralisation éloignée de la grisaille européenne dans laquelle la violence guerrière est un trait particulier à la fois des lieux et des hommes.

Ces montagnes imaginées par le romantisme littéraire ont un périmètre très étendu puisqu'elles commencent en Espagne et trouvent leurs limites asiatiques dans l'Azerbaïdjan et le Caucase russe. C'est dans cette vaste aire géographique que l'on retrouve les sommets escarpés d'Andalousie, les monts du Monténégro, du Liban, de Grèce, d'Afrique du Nord, de Crimée et d'Asie centrale.

Un premier texte significatif de ce mouvement paraît à la fin de la Révolution en France : Dans Le Vieux de la Montagne, Delisle de Sales s'attache à mettre en valeur tous les caractères d'un récit oriental dans le cadre imaginaire de la montagne libanaise avec 
en ligne de mire les amours contrariés d'Ariel, le plus beau des anges et Bouton de rose, la sultane, sur fond de " grotte aux merveilles " et de septième croisade ${ }^{68}$.

À partir de Chateaubriand, grand initiateur du voyage littéraire en Orient, ce sont des «pèlerinages d'écrivains promeneurs et excursionnistes » qui permettent de cultiver cette source nouvelle d'inspiration: l'Orient mythique est ainsi fait de montagnes étranges peuplées de guerriers farouches en Kabylie, au Caucase et au Liban en passant par la Grèce et l'Anatolie que l'écrivain souhaite découvrir afin d'en faire connaître le sens et les secrets à tous ses lecteurs ${ }^{69}$.

Peut-être faut-il voir dans ces voyages la confirmation d'une inspiration préconçue plus que la découverte d'un nouvel horizon ; la méconnaissance du pays et de ses habitants n'empêchera d'ailleurs pas des écrivains comme Victor Hugo de s'inspirer du paysage oriental, quitte à utiliser comme Georges Sand des traductions d'œuvres littéraires existantes ${ }^{70}$.

La rêverie « orientale » prend parfois une dimension tragique. Chez l'orientaliste Ernest Fouinet, Hamid, le héros de La caravane des morts, va accomplir un crime presque rituel «en plongeant son poignard dans le sein d'un homme..., près d'une montagne carrée... » mythique, celle constituée par « les immenses débris de la cité détruite [de Babylone] et de la tour géante de Nemrod ${ }^{71}$ ». Dans Les anémones de Noman, c'est la montagne-désert qui sert de refuge au moine Arsenios face au danger situé dans la plaine, celui du Roi de Hira, le tyran Noman qui massacre son peuple avec force détails de cruauté. Les allées et venues de guerriers envoyés pour tirer l'ermite de son refuge font du sommet de la grotte où il se cache le point central du récit: l'arbitre de la montagne obtient finalement la conversion du Roi et triomphe de la violence guerrière qui se situait cette fois-ci dans la plaine ${ }^{72}$.

Théophile Gautier sur les rives du Thermodon au pied des monts d'Amazonie, au bord de la Mer Noire, raconte la terrible bataille qui voit la défaite légendaire des Amazones face aux héros grecs, Hercule et Thésée : la vision du carnage est d'autant plus singulière qu'il s'agit de corps féminins démembrés ${ }^{73}$. Au pied du défilé de la Hache près de Carthage se déroule une autre bataille célèbre, celle de la guerre des mercenaires de l'Antiquité cours de laquelle Hamilcar, le chef carthaginois, tend un piège aux révoltés ; Flaubert les imagine cernés sur la montagne dont ils descendent seulement pour mourir crucifiés par les vainqueurs ${ }^{74}$.

41 Edmond About, dont l'hostilité envers le peuple grec est avérée, considère le " paysage affreux » des montagnes de l'Attique comme un décor oriental : parlant du brigand Hadji-Stavros, il le voit comme un "héros en ruine", entouré de ses chiens qui dévorent vivants ceux que le maître désigne ${ }^{75}$. Dans le Caucase, Kouban, le terrible seigneur de la montagne, émir des Tcherkesses, manifeste sa barbarie, selon Ponson du Terrail, contre les Russes qui tentent de s'emparer de son «aoul»: aux prisonniers français et russes qui ont tenté de pénétrer dans la forteresse par les souterrains, il promet les yeux crevés, la langue et les mains coupées ${ }^{76}$.

42 À côté de la violence des combats, il y a aussi place pour la clémence : c'est ce que retient surtout Georges Sand dans son portrait de Kourroglou, le bandit-ménestrel turkmène de la vallée de Samas en Azerbaïdjan : poursuivi par Reyhan l'arabe, le héros écrase son ennemi, mais au lieu de lui ôter la vie, il le conduit dans son repaire pour l'accueillir dignement ${ }^{77}$. 
Les montagnes d'Orient ont une histoire multimillénaire où la guerre est partout présente, elles façonnent l'habitant comme cette jeune esclave kurde dépeinte par Fouinet : le khan de Perse fait défiler devant lui une prisonnière de guerre enlevée dans les gorges du Kurdistan, « son teint robuste et ses yeux pénétrants et la hardiesse de sa démarche annonçaient une jeune fille grandie dans l'air fortifiant et salubre des montagnes, habituée à les gravir, d'un pas ferme et sans crainte ${ }^{78}$ ».

La montagne orientale est aussi un lieu de sacrifice : dans l'épisode de la mort du poèteguerrier arabe Antar, Lamartine décrit une montagne mythique d'Arabie, où le héros, blessé à mort, se tient toute une nuit à l'entrée d'un défilé, " la vallée des gazelles » faisant face à ses ennemis pour permettre à sa famille et sa caravane de rejoindre les tentes d'une tribu amie ${ }^{79}$.

En Orient, plus qu'ailleurs, les valeurs de liberté et de résistance farouche à l'envahisseur attirent l'attention des écrivains : chez Ponson du Terrail, le chef guerrier libanais Mar Eddin rencontre le sultan de Constantinople pour proclamer le désir d'émancipation de son peuple :

«Les peuples du Liban sont des peuples guerriers; le vent de la liberté a toujours soufflé au sommet de nos montagnes et rafraîchi nos vallées profondes. Nous résisterons ${ }^{80}$ !»

Le récit guerrier « oriental ", à travers ses particularités, a puissamment contribué à populariser l'image d'une montagne, lieu de guerre et de souffrance.

4 La figure littéraire du guerrier montagnard et de son champ de bataille suscite plusieurs questions dans le cadre de cette étude : tout d'abord, la mythification de la guerre de montagne a-t-elle intéressé les stratèges militaires autant que les amateurs de fiction? Partant du point de vue de Jomini sur les guerres nationales en zone de montagne, on peut remarquer l'affirmation d'une force structurante du nationalisme au cours $d u$ xix ${ }^{e}$ siècle révélée à travers les combats menés par les peuples montagnards (Calabrais, Basques, Cosaques, Azeris, Libanais, Tyroliens, etc.). Alexandre Dumas insiste sur la géographie montagnarde au service de l'esprit d'indépendance mais pas forcément de celui de la liberté81.

$\mathrm{Au}$ terme de cette exploration où la réflexion esthétique tient une place importante, il convient de s'interroger aussi sur la place de la guerre dans la sacralisation littéraire de la montagne. Où sont passés les décors d'apothéose? Le récit guerrier n'est pas un discours élégiaque, il est inutile de rechercher de sublimes décors de spectacle dans la violence guerrière; peut-être pourra-t-on voir dans la redécouverte des langues perdues des guerriers des hauts sommets (le basque, le gaélique) une sorte de beau, idéal à contempler. Mais cette quête du beau fait ici sentir ses difficultés autour des contradictions assumées d'un modèle multiforme, traversé par le discours politique ou religieux. Quelques morceaux de bravoure existent pourtant sur le nombre de récits guerriers étudiés : la poursuite des Grecs au sommet du mont Erymanthe de Lamartine, l'extermination des mercenaires révoltés par Hamilcar vu par Flaubert. Ils témoignent de la vigueur de récits aux accents dramatiques qui se veulent une belle fenêtre ouverte sur la montagne, ses paysages et ses secrets. 


\section{BIBLIOGRAPHIE}

ABOUT Edmond, Le roi des montagnes, Paris, 1857.

ARTOLA CORNU Maria Guadalupe, « Jean-Baptiste Camoussary (1815-1842), una primera aproximacion a su vida e a su obra », Anuario del seminario de Filologia vasca Julo de Urquijo, San Sebastian, 22 février 1988, p. 391-443.

ATRIA Rosario Marco, «Confini aperti del romanzo storico : I carbonari della montagna », La letteratura degli Italiani : rotte, confini, passaggi, $\mathrm{XIV}^{\mathrm{e}}$ congresso nazionale degli Italianisti, Genova, 2010, DIRAAS, Università degli di Genova 2012, sessioni parallele, ed. electronica, 13 p.

BALZAC Honoré de, Louis Lambert suivi de Seraphita, Paris, 1842, 325 p.

BARTHÉLÉMY Jean-Jacques (abbé), Le voyage du jeune Anacharsis en Grèce, Paris, 1788, 5 tomes.

BLANCHARD RUBIO Laetitia, «Impressions de guerre : images et imaginaires de la première guerre carliste (1833-1840) », Cahiers de la Méditerranée, $\mathrm{n}^{\circ}$ 83, 2011, p. 147-162.

BONAPARTE Joseph, Moïna ou la villageoise du Mont-Cenis, Paris, 1799.

BOREL Petrus, Rhapsodies, Paris, 1832.

BUFFON, Histoire naturelle, générale et particulière, tome IV, supplément 1777.

CHATEAUBRIAND, Cuvres complètes, tome XVIII, Les martyrs, livre X, Paris, 1826.

CHATEAUBRIAND, Le dernier abencérage, Paris, 1807.

CLARIS DE FLORIAN Jean-Pierre, Guillaume Tell ou la Suisse libre, Paris, nouvelle éd., 1832.

CLARIS DE FLORIAN Jean-Pierre, Gonzalve de Cordoue ou Grenade reconquise, tome 3, Paris, 1791.

CLAUSEWITZ Carl Von, Schriften-Aufsätze-Studien-Brife, herausgegeben von Werner Hahlweg, vol. 1, Göttingen, 1966.

CATEL Olivier, «Les Natchez de Chateaubriand : histoire et destin d'un texte en sursis à travers la correspondance (1797-1827) ", La lettre et l'œuvre ; perspectives épistolaires sur la création littéraire et picturale au XIX siècle, Université Clermont-Ferrand II, 2009, p. 72-81.

CIROT Georges, « Le chant d'Altabiscar », Bulletin hispanique, 1936, vol. 38, p. 65-67.

COTTIN Sophie, Malvina, Paris, an IX, (3 vol.).

DELISLE DE SALES Jean-Baptiste Claude, Le vieux de la montagne : histoire orientale traduite de l'arabe, Paris, an VII, (4 vol.).

DUCUING François, La guerre de montagne (Navarre 1834-5 et Kabylie 1841-7), Paris, 1868.

DUMAS Alexandre, La comtesse de Charny, nouvelle éd. complète électronique, Paris, 2016.

DUMAS Alexandre, La boule de neige, Paris, 1879, 270 p.

DUMAS Alexandre, Maître Adam le calabrais, Paris, 1839, 150 p.

DUMAS Alexandre, Othon l'archer, Paris, 1853, éd. illustrée.

DUMAS Alexandre, Les frères corses, Paris, éd. illustrée, 1853.

DUMAS Alexandre, Ammalat Beg, Paris, A. Cadot, 1859, (2 vol.). 
Encyclopédie méthodique : art militaire, Paris, 1797, tome IV supplément, article guerre.

ERCKMANN-CHATRIAN, Contes de la montagne, Hugues le loup, Paris, 4e éd. 1873.

FAURIEL Claude, Chants populaires de la Grèce moderne. Chants historiques, Paris, 1824-1825, (2 vol.).

FLAUBERT Gustave, Salammbô, Paris, 1863, 475 p.

FOLARD Jean-Charles de (Chevalier), L'esprit du chevalier Folard, tiré de ses « Commentaires sur l'histoire de Polybe ", pour l'usage d'un officier, de main de maître, tome 1, Paris, 1754.

FOUCHER Paul, Guillaume Colmann ou les deux guides, Paris, 1837, 32 p.

FOUINET Ernest, la caravane des morts, Paris, 1836, tome 1.

FOUINET Ernest, Les anémones de Noman, Tours, 1855, 284 p.

FOUINET Ernest, La caravane des morts..., tome 2.

GAUTIER Théophile, La comédie de la mort, Paris, 1838.

GOBINEAU Arthur de, Nouvelles asiatiques, Paris, 1876.

HAPDÉ Jean-Baptiste Augustin, La guerrière des sept montagnes ou la laitière des bords du Rhin,

mélodrame en trois actes, Paris, 1805, 49 p.

HUGO Victor, Les Orientales, Paris, 1829, XVII.

HUGO Victor, Han d'Islande, Paris, 1823, (2 vol.).

HUGO Victor, La légende des siècles, cycle héroïque chrétien, V, le jour des rois, Paris, 1862.

нUмBоLт Wilhem von, Berichtigungen und Zusätze... Ueber die Cantabrische oder Vaskische Sprache, Berlin, 1817.

JOMINI Antoine de, Précis de l'art de la guerre, Paris, 1838, nouvelle éd., Perrin, 2001.

JOUTY Sylvain et ODIER Hubert, Dictionnaire de la montagne, Paris, 2009, 883 p.

KÁLVos Andréas, La Lyre patriotique de la Grèce, odes traduites du grec moderne de Kalvos, traduction Stanislas JULIEN, Paris, Peytieux, 1824, 95 p.

KORAY Adamance, Appel aux Grecs traduit du grec moderne d'Atromète, natif de Marathon, avec la proclamation d'Ypsilanti aux Français, Paris, 1821.

LAMARTINE Alphonse de, Le dernier chant du pèlerinage d'Harold..., XXVI-XXVII.

LAMARTINE Alphonse de, Jocelyn :épisode, journal trouvé chez un curé de campagne, tome $1,3^{\mathrm{e}}$ époque, Paris, 1836, (2 vol.).

LAMARTINE Alphonse de, Antar, Paris, 1864.

LAUTRÉAMONT, Les chants de Maldoror, Paris-Bruxelles, 1869-1874, chant III.

MÉRIMÉE Prosper, Colomba, Paris, 1841.

MÉRIMÉE Prosper, La Guzla ou choix de poésies illyriques recueillies dans la Dalmatie, la Bosnie, la Croatie et l'Herzégovine, Paris, 1827.

MONTEPIN Xavier de, Le médecin des pauvres, Paris, [1843], éd. illustrée.

MONTESQUIEU, CEuvres complètes, L'Esprit des Lois, livre XVIII, Paris, 1796. 
MOREL-BOROTRA Natalie, « Le chant et l'identification culturelle des Basques (1800-1950) », Lapurdum, revue d'études basques, V, 2000, p. 351-381.

MUSSET Alfred de, Un spectacle dans un fauteuil, La coupe et les lèvres, poème dramatique, Paris, 1833.

NERVAL Gérard de, Les Monténégrins, opéra-comique en trois actes, Paris, 1849, 54 p.

NODIER Charles, Jean Sbogar, Paris, 1832.

OLMEDO Joaquin José, Canto a Bolivar, Londres, 1826, 80 p.

PAVLOF Caroline, Les Préludes, Lochiel Warning, traduction du poème de Thomas Campbell, Paris, 1839.

PETROVITCH-NJEGOCH Pierre II, Les Lauriers de la montagne, Paris, 1917, 162 p.

PICAUD-MONNERAT Sandrine, « La réflexion sur la petite guerre à l'orée du XIX ${ }^{\mathrm{e}}$ siècle : l'exemple de Clausewitz (1810-1812) », Stratégique, n 97-98, 2005, p. 123-147.

PIVERT de SENANCOUR Étienne, Oberman, Paris, 1804, tome 2, lettre LXX.

PONSON DU TERRAIL Pierre-Alexis de, Les Fils de Judas. Un conte des mille et nuits, Paris, 1866.

PONSON DU TERRAIL Pierre-Alexis de, Le voleur du grand monde, III, Le seigneur de la montagne, Paris, 1870.

ROLLIN Charles, Histoire romaine, Paris, 1782, tome 10.

ROUSSEAU Jean-Jacques, Projet de constitution pour la Corse, édité par R. Chesnais, Paris, Nautilus, 2000.

SAND Georges, «Les aventures et les improvisations de Kourroglou... », La Revue indépendante, $3^{\mathrm{e}}$ rencontre, janvier 1843, p. 422-425.

SAND Georges, CEuvres de George Sand, Simon. Consuelo. Jean Ziska. Melchior. Mouny-Robin, Bruxelles, Hauman et Cie, vol. IV, 1843.

SCRIBE Eugène, Cuvres complètes, tome 10, Fra Diavolo, livret opéra comique, Paris, 1830.

SINARDET-SEEWALD Emmanuelle, « La victoria de Junin. Canto à Bolivar de Joaquin José Olmedo », America, cahiers du CRICCAL, $\mathrm{n}^{\circ}$ 41, 2012, p. 197-205.

SCHILLER Friedrich, Wilhelm Tell, schauspiel von Schiller, Zubingen, 1804.

VAN THIEGHEM Paul, Ossian en France, Paris, 1917, 2 tomes.

VERGA Giovanni, I carbonari della montagna, Catania, 1861, (4 vol.).

VIGNY Alfred de, Le trappiste : poème, Paris, 1823.

WILLIAMS John, Wordsworth translated: a case study in the reception of british romantic poetry in Germany, 1804-1914. Blombury Publ. 2009.

WORDSWORTH William, « Hofer, 20th october 1809 », The friend, 1815.

Yovanovitch Voyslav, La «Guzla » de Mérimée : étude d'histoire romantique, Paris, 1911, 602 p. 


\section{NOTES}

1. Montesquieu, CEuvres complètes, p. 5.

2. Buffon, Histoire naturelle, générale et particulière, p. 506.

3. Encyclopédie méthodique : art militaire, article guerre, p. 677.

4. Chevalier de Folard, Abrégé des commentaires sur Polybe, p. 390.

5. Ch. Rollin, Histoire des Romains, p. 315.

6. J.-J. Rousseau, Projet de constitution pour la Corse, p. 45.

7. C. Von Clausewitz, Shriften-Aufsätze-Studien-Brife, herausgegeben von Werner Hahlweg, Göttingen. Voir S. Picaud-Monnerat, «La réflexion sur la petite guerre à l'orée du XIX ${ }^{\mathrm{e}}$ siècle : l'exemple de Clausewitz (1810-1812)».

8. A. de Jomini, Précis de l'art de la guerre, p. 241-247.

9. J.-P. Claris de Florian, Guillaume Tell ou la Suisse libre.

10. F. Schiller, Wilhelm Tell, schauspiel von Schiller.

11. W. Wordsworth, «Hofer, 20th october 1809 ». Voir J. Williams, Wordsworth translated: a case study in the reception of british romantic poetry in Germany, 1804-1914.

12. Chateaubriand écrit Les Natchez entre 1797 et 1798, mais ne publiera que beaucoup plus tard un texte remanié (1826). Il se sert ensuite du matériau primitif pour René et Atala, respectivement publiés entre 1801 et 1802. Voir O.Catel, "Les Natchez de Chateaubriand: histoire et destin d'un texte en sursis à travers la correspondance (1797-1827) », p. 72-81.

13. La première traduction française est celle du marquis de Saint Simon, Temora: poème épique en VIII chants composé en langue erse ou gallique par Ossian fils de Fingal. La plus connue est celle de F-P-F. Le Tourneur, Poésies galliques traduites sur l'anglais de M. Mac Pherson.

14. La bataille est racontée au chant VII. Voir P. Van Thieghem, Ossian en France.

15. S. Cottin, Malvina.

16. É. Pivert de Senancour, Oberman, p. 196-197.

17. Chateaubriand, Euvres complètes, p. 90.

18. Voir J.-P. Claris de Florian, Gonzalve de Cordoue ou Grenade reconquise, p. 157-158.

19. Chateaubriand, Le dernier abencérage.

20. W. Von Humbolt, Berichtigungen und Zusätze...ueber die Cantabrische oder Vaskische Sprache. Le chant de Lelo qui parle du combat des Basques dans les Pyrénées au temps de César date du Xvi ${ }^{\mathrm{e}}$ siècle.

21. Joseph-Augustin Chaho (1811-1858), poète basque originaire de Tardets, élève de Nodier, fondateur du journal Ariel en 1844, y publie son texte «Aïtor, une légende cantabre » (numéros 36 à 40, juin-juillet 1845).

22. Il s'agit de l'œuvre du poète équatorien Joaquin José Olmedo, Canto a Bolivar, publié à Guayaquil en 1825, puis à Londres et à Paris en 1826. Voir E. Sinardet-Seewald, « La victoria de Junin. Canto à Bolivar de Joaquin José Olmedo », p. 197-205.

23. J.-J. Barthélémy, Le voyage du jeune Anacharsis en Grèce. L'épisode est narré au tome premier, introduction, p. 128 à 133. 
24. A. Koray, Appel aux Grecs traduit du grec moderne d'Atromète, natif de Marathon, avec la proclamation d'Ypsilanti aux Français.

25. C. Fauriel, Chants populaires de la Grèce moderne.

26. Voir La lyre patriotique de la Grèce, odes de Zante Kalvos traduites par Stanislas Julien.

27. J. Bonaparte, Moïna ou la villageoise du Mont-Cenis. La bataille évoquée est celle conduite par le général Dumas le 9 mai 1794, au cours de laquelle les troupes françaises reprennent le col aux Austro-sardes.

28. Voir C. Pavlof, Préludes, Lochiel Warning, p. 35-38.

29. J. J. Olmedo, Canto a Bolivar. Le poème de 909 vers, dans sa version de 1826, n'a pas fait l'objet à l'époque d'une traduction française.

30. Sur l'authenticité douteuse de l'œuvre, voir G. Cirot, "Le chant d'Altabiscar ", p. 65-67 et N. Morel-Borotra, "Le chant et l'identification culturelle des Basques (1800-1950)», p. 351-381.

31. V. Hugo, La légende des siècles, p. 113-127.

32. G. Sand reprend la version du récit de Jacques Lenfant dans sa Guerre des Hussites parue en 1731 : la bataille dite de "Maleschaux », datée du 6 juin 1424, se déroule dans les montagnes de Bohème au nord de Prague. Voir aussi G. Sand, Jean Ziska, p. 214.

33. J.-B.-A. Hapdé, La guerrière des sept montagnes ou la laitière des bords du Rhin, mélodrame en trois actes.

34. Erckmann-Chatrian, Contes de la montagne, p. 81-276.

35. A. Dumas, Othon l'archer, p. 18.

36. C. Nodier, Jean Sbogar, p. 55.

37. P. Mérimée, Colomba, p. 104-105.

38. A. Dumas, Les frères corses, p. 2-3.

39. V. Hugo, Les Orientales, p. 185-186.

40. A. de Lamartine, Le dernier chant du pèlerinage d'Harold..., p. 97-100. Le poète se réfère à un événement survenu pendant le soulèvement d'Épire réprimé par Ali Pacha, le 16 décembre 1803 à Zalongo, mis en scène en 1827 par Ary Scheffer dans un tableau célèbre, La ronde des femmes Souliotes au bord du précipice.

41. X. de Montepin, Le médecin des pauvres, p. 14.

42. A. de Lamartine, Jocelyn : épisode, journal trouvé chez un curé de campagne, p. 92-95.

43. P. Mérimée, La Guzla ou choix de poésies illyriques recueillies dans la Dalmatie, la Bosnie, la Croatie et l'Herzégovine, p. 67-69.

44. V. Hugo, Han d'Islande.

45. Lautréamont, Les chants de Maldoror, p. 141-182.

46. P. Borel, Rhapsodies, p. 83-85.

47. A. Dumas, La boule de neige.

48. L'aventure de Jean Ziska, aux côtés de l'« hérésie » de Jean Huss, est évoquée dans Consuelo (1842), La Comtesse de Rudolstadt (1843) et Jean Ziska (1844).

49. La lyre patriotique de la Grèce, odes de Zante Kalvos traduites par Stanislas Julien, p. 92-93.

50. Voir M. G. Artola Cornu, «Jean-Baptiste Camoussary (1815-1842), una primera aproximacion a su vida e a su obra », p. 391-443, et L. Blanchard Rubio, «Impressions de guerre : images et imaginaires de la première guerre carliste (1833-1840)», p. 147-162. 
Le poème sur Zumalacarregui fait partie des poèmes basques écrit par Camoussary en 1839 (manuscrit numérisé consultable : http://www.bilketa.eus/fr/).

51. Voir F. Ducuing, La guerre de montagne (Navarre 1834-5 et Kabylie 1841-7), p. 24.

52. E. Scribe, Euvres complètes, p. 85-180.

53. A. Dumas, Maître Adam le calabrais.

54. G. Verga, I carbonari della montagna. Voir R. Atria, «Confini aperti del romanzo storico : I carbonari della montagna ».

55. C. Nodier, Jean Sbogar..., p. 93-97.

56. P. Mérimée, La Guzla..., Les Monténégrins, p. 245-248. Voir V. Yovanovitch, La « Guzla » de Mérimée : étude d'histoire romantique.

57. G. de Nerval, Les Monténégrins; pour le chant monténégrin, voir Poèmes divers.

58. P. II Petrovitch-Njegoch, Les lauriers de la montagne.

59. P. Borel, Rhapsodies..., p. 33-37.

60. Voir la pièce de P. Foucher, Guillaume Colmann ou les deux guides.

61. A. de Musset, Un spectacle dans un fauteuil, p. 23.

62. T. Gautier, La comédie de la mort, p. 339-350.

63. J.-B.-A. Hapdé, La guerrière des sept montagnes..., p. 2-47.

64. A. de Gobineau, Nouvelles asiatiques, p. 11-98.

65. A. Dumas, Ammalat Beg..., p. 45.

66. A. de Vigny, Le trappiste : poème, p. 12-13.

67. H. de Balzac, Louis Lambert suivi de Seraphita.

68. J.-B. C. Delisle de Sales, Le vieux de la montagne : histoire orientale traduite de l'arabe.

69. Plusieurs écrivains français ont voyagé en Orient : Chateaubriand en Palestine et en Turquie en 1806-1807, Lamartine en Palestine, au Liban, en Syrie et en Turquie en 1832-1833, au Liban et en Turquie en 1849; Baudelaire sur les côtes d'Égypte et de Palestine en 1841-1842, Nerval en Égypte, en Syrie au Liban et en Turquie en 1843, Dumas en Algérie en 1846 et au Caucase en 1858-1859, Flaubert en Égypte, en Syrie et au Liban de 1849 à 1851, Théophile Gautier en Algérie en 1845, en Turquie en 1853 et en Égypte en 1869.

70. En 1843, dans la Revue indépendante, Georges Sand publie une traduction libre de l'épopée de Kourroglou, chanteur turkmène, recueillie oralement du persan par l'orientaliste et poète polonais Alexandre Chodzko (1804-1891) qui l'a ensuite mis en forme en anglais.

71. E. Fouinet, la caravane des morts.

72. E. Fouinet, Les anémones de Noman.

73. T. Gautier, La comédie de la mort..., p. 343-344.

74. G. Flaubert, Salammbô. Il s'agit de la bataille du défilé de la Scie (Djebel Ressas près de Zaghouan dans le Nord tunisien) en 238 av. J.-C.

75. E. About, Le roi des montagnes.

76. P.-A. Ponson du Terrail, Le voleur du grand monde, III, Le seigneur de la montagne.

77. G. Sand, « Les aventures et les improvisations de Kourroglou... ».

78. E. Fouinet, La caravane des morts... 
79. A. de Lamartine, Antar, p. 171-178.

80. P.-A. Ponson du Terrail, Les Fils de Juda, un conte des mille et nuits.

81. A. Dumas, La comtesse de Charny, p. 664.

\section{RÉSUMÉS}

La quête du sublime et de l'émotion artistique et littéraire dans le paysage de montagne fait partie de la formation aujourd'hui bien connue de l'écrivain romantique français de Rousseau à George Sand. De l'expression de l'infini et de l'harmonie, la perception romantique est aussi passée par celle des vestiges terribles de la violence de la Nature. De là peut découler une représentation guerrière intégrée du paysage et de ses habitants. Il est proposé d'analyser ici ce processus de construction littéraire à partir de l'écriture romantique française d'une montagne à la fois visitée et rêvée. Récits de voyage, poésies et romans de Rousseau à George Sand seront interrogés au regard de modèles et de schémas hérités ou cultivés ; la représentation du guerrier montagnard est au cœur de l'esthétique littéraire de cette période: Suisses, Bavarois, Monténégrins, Basques et Caucasiens, Druses et Maronites, sont mis en scène pour caractériser l'identité collective des peuples de montagne. Au-delà du sublime, la recherche de l'écrivain romantique est aussi celle du pittoresque : le guerrier est enraciné dans sa terre et ses traditions qui constituent un inépuisable décor.

\section{AUTEUR}

\section{LOUIS BERGÈS}

Conservateur général du patrimoine, membre du CTHS, section Histoire du monde moderne, de la Révolution française et des révolutions 


\title{
Figures romantiques de la mobilité et de l'immobilité montagnardes : les voyages aux Alpes et aux Pyrénées de Victor Hugo (1825-1843)
}

\author{
Odile Parsis-Barubé
}

1 L'emboîtement thématique des différentes sections composant ce colloque sur les circulations montagnardes invite à croiser trois problématiques : celle des conditions matérielles de la circulation au sein de l'espace montagnard européen, celle des modalités de la construction des systèmes de représentations de la montagne dans la culture occidentale, celle, enfin, des formes d'expression littéraire qui ont accompagné ce processus.

2 Sans doute avons-nous fort peu de mérite à avoir choisi, pour illustrer cette triple articulation, de nous inscrire dans ce temps du romantisme qui est à la fois celui où se parachève le processus, enclenché au milieu du $\mathrm{xvIII}^{\mathrm{e}}$ siècle, d'appropriation par les élites européennes d'espaces jusque-là considérés comme répulsifs - les littoraux et les montagnes - et celui où se forge un modèle de voyage traversé par des enjeux à la fois esthétiques, idéologiques et scientifiques - l'égotisme, le pittoresque, le sublime, la réinterprétation des traditions locales, l'interrogation sur les origines du monde -, enjeux qui, chacun à leur manière, nous ramènent une question littéraire majeure : celle de la spécificité de la poétique qui en accompagne le récit ${ }^{1}$.

Les Voyages de Victor Hugo constituent sans aucun doute l'une des illustrations les plus convaincantes de cette articulation entre ce qui relève de la culture matérielle du voyage, de l'imaginaire de l'espace et des contraintes formelles en fonction desquelles se constitue l'écriture viatique. Entre 1825 et 1843, l'écrivain accomplit deux voyages aux Alpes - le premier en 1825 en compagnie d'Adèle, le second en 1839 en celle de Juliette Drouet - et un voyage aux Pyrénées en 1843, qui est en réalité un voyage en 
Espagne demeuré dans l'historiographie hugolienne comme le voyage au retour duquel il apprend, au hasard d'une halte à Rochefort, la noyade de Léopoldine.

4 Les Voyages de Victor Hugo sont constitués de textes de nature très diverse - lettres, réelles ou fictives, fragments de journal ou de carnets, récits, parfois recomposés postérieurement sous la forme épistolaire, albums. Reconstituer les conditions de leur édition n'est pas chose aisée : certains de ces écrits ont été publiés du vivant de l'auteur - comme les Fragments d'un voyage aux Alpes, parus en deux étapes successives, en 1829 dans la Revue de Paris puis, en 1831, dans la Revue des Deux Mondes ${ }^{2}$ - ; d'autres l'ont été à titre posthume, comme l'ensemble des écrits relatant ses voyages de 1839 dans les Alpes, le midi de la France et la Bourgogne ainsi que celui de 1843 dans les Pyrénées. Les titres attribués à ces différents ensembles ont également pu varier au gré des éditions : ainsi les voyages de 1839 et 1843 ont-ils été regroupés dans une première publication par Paul Meurice chez Hetzel-Quentin en 1890 sous le titre En voyage - Alpes et Pyrénées, puis sous celui Alpes et Pyrénées en 1910 dans l'édition dite "de l'Imprimerie nationale » où on leur a adjoint de nouveaux textes tirés des Albums ainsi que la lettre écrite à l'intention de Louis Boulanger que nous évoquerons plus loin'.

5 Au fil des trois voyages accomplis par Hugo entre 1825 et 1843 aux Alpes et aux Pyrénées, se déploie toute une gamme de sensations liées aux modalités spécifiques de la pénétration de la montagne et de l'appropriation de sa verticalité ${ }^{4}$ : la montée, l'escalade, le dévalement des pentes. Les propriétés géométriques et topographiques de l'espace imposent ici, avec les contraintes matérielles qui leur sont liées, une nouvelle mécanique du regard où vient s'enrichir la quête du pittoresque et s'expérimenter l'abandon à la sublime horreur. Mais si la montagne hugolienne se donne à voir comme le territoire de mobilités multiples, elle n'en constitue pas moins, pour l'écrivain, un espace clos, refermé sur des populations préservées de tout contact avec l'extérieur. Un de ces isolats où la première génération romantique a cru pouvoir saisir les vestiges d'un âge d'or originel et pratiquer cette archéologie de l'étrangeté chère aux celtomanes du début du siècle. Un espace de méditation, surtout, laboratoire privilégié du voyage au-dedans de soi.

\section{Des mobilités étagées}

6 Hugo, comme nombre de ses contemporains, voit d'abord dans les massifs montagneux des barrières dont le franchissement relève du défi. Avant de comparer, en 1843, les Pyrénées à " une grande muraille dressée $e^{5}$ ", il convoque, dès son premier voyage aux Alpes de 1825, le souvenir historique des traversées mythiques des cols alpins, celles d'Hannibal et de Bonaparte, se demandant:

" Avec effroi, presque avec incrédulité, comment le lourd attirail d'une armée a pu voyager par des routes qui semblent souvent refuser de l'espace et de la solidité aux pieds aériens du chamois, et comment il a réussi à doubler deux fois ces hauts promontoires qui baignent dans les nuages et plongent si profondément dans le ciel. ${ }^{6}{ }^{\prime}$

7 Les massifs qu'il parcourt entre 1825 et 1843 n'en apparaissent pas moins dans ses notes de voyage comme des espaces relativement accessibles pour qui arrive de la plaine, au point de s'ouvrir de plus en plus largement, d'un voyage à l'autre, à la figure nouvelle $d u$ « touriste ». C'est en fait le passage du plan horizontal des circulations en plaine à celui vertical de la pénétration au cœur des massifs qui marque le point de rupture 
entre deux perceptions des mobilités montagnardes. Sous la plume de l'écrivain, 1 'enfoncement dans la montagne devient une expérience physique et sensorielle inédite, affrontement à des dangers spécifiques qu'accompagne et souligne l'adaptation des moyens de déplacement aux propriétés orographiques du terrain.

\section{Des territoires en voie d'appropriation}

8 À plusieurs reprises, l'écrivain souligne le bon état relatif des routes conduisant au pied des massifs. Il lui arrive même de constater, d'un voyage à l'autre, les améliorations dont certaines voies de communication ont pu bénéficier :

« Il y a quinze ans, note-t-il lors de son deuxième voyage aux Alpes en 1839, le chemin de Zug à Art était un sentier impraticable où trébuchait le meilleur cheval. C'est maintenant une grande route excellente laquelle ne cahote pas même l'espèce d'omnibus-charrette qui la parcourt avec des cargaisons de voyageurs le sac sur le dos. ${ }^{7}$ »

9 La même année, il écrit à Adèle que, sur la route de Thun à Berne, «son cabriolet à quatre roues trottait sur une route excellente ${ }^{8}$ ». C'est que les quelque dix-huit années séparant le premier voyage aux Alpes du voyage aux Pyrénées correspondent au début du processus d'investissement touristique de la montagne ${ }^{9}$, marqué par l'inscription du voyage aux Glacières dans l'itinéraire du Grand Tour, les séjours estivaux des riches anglais dans les hôtels de "Chamouny", le tout début de l'«ascensionnisme" romantique. Dès le premier voyage aux Alpes de 1825, Hugo perçoit les premiers frémissements du phénomène touristique et s'alarme de ce que cela risque de faire perdre à la montagne. Dans la vallée de Chamonix, là où depuis le $\mathrm{XI}^{\mathrm{e}}$ siècle, les chasseurs jouaient de ces échos qui avaient entretenu l'épouvante autour des Monts Maudits, il pointe, non sans nostalgie, la métamorphose de cette tradition en amusement touristique et sa mercantilisation :

\footnotetext{
"Aujourd'hui, dans ce même lieu, des voyageurs élégants, des femmes parées descendent de leur char à bancs sur une route assez bien nivelée. De petits garçons déguenillés accourent avec un long porte-voix. Ils en tirent des sons aigus qui ressemblent encore à l'ancienne adjuration du chasseur. Une voix des montagnes les répète distinctement sur un ton plus faible et plus lointain. Et puis, si vous demandez à ces enfants : qu'est cela? ils vous répondent : c'est l'écho, et tendent la main.

- Où est la poésie ${ }^{10} » ?$
}

Si le touriste n'est pas vraiment le bienvenu dans le paysage chez Hugo, c'est que ce moment, qui est celui de la quasi-invention du terme - Stendhal publie ses Mémoires d'un touriste en 1838, un an avant le second voyage aux Alpes - est aussi celui de sa tournure en dérision. "Contagion inévitable du monde élégant ${ }^{11}$ » selon Guichardat, le tourisme, que Littré ne va pas tarder à définir comme la pratique « des voyageurs qui ne parcourent des pays étrangers que par curiosité et désœuvrement ${ }^{12}$ ", trouble la quiétude du rêveur et compromet l'esprit des lieux. Lors de son second voyage aux Alpes, en 1839, Hugo s'agace de voir le Rigi-Kulm ${ }^{13}$ servir de succédané du Mont-Blanc à des hordes de voyageurs suréquipés et raille ces "espèces de don Quichotte des montagnes qui sont déterminés à faire une ascension et qui escaladent cette butte avec tout l'attirail de Cachat-le-Géant ${ }^{14} »$. Parvenu au sommet, où l'ampleur de la vue qui le saisit lui inspire une de ses méditations sur les merveilles de la nature dont sont émaillées tant de ses lettres de voyage, il prend soin de distinguer soigneusement sa propre attitude de celle des touristes : 
«Le touriste vient y chercher un point de vue; le penseur y trouve un livre immense où chaque rocher est une lettre, où chaque lac est une phrase, où chaque village est un accent et d'où sortent pêle-mêle comme une fumée deux mille ans de souvenirs. ${ }^{15} »$

\section{S'enfoncer dans la montagne}

11 Mais si le piémont des montagnes est, aux yeux de l'écrivain, de plus en plus facilement accessible, les propriétés topographiques de la montagne - la pente, le ravin, le précipice, l'altitude - dans ces années 1820 à 1840, font encore des hauteurs des lieux périlleux.

12 La pénétration des profondeurs des massifs impose des difficultés telles qu'elle constitue, pour le voyageur parisien, un sujet d'étonnement, d'effroi parfois, une expérience physique, toujours, dont il tient à relater les moindres détails. Terrain d'élection de la ligne serpentine, le milieu montagnard constitue, aux yeux de l'écrivain-voyageur, un espace de circulation particulier :

«Les montagnes produisent deux sortes de routes, écrit Hugo en 1843 : celles qui serpentent à plat sur le sol comme des vipères; celles qui serpentent en ondulant par soubresauts comme les boas. ${ }^{16}$ "

13 Les choses se compliquent lorsqu'à l'occasion des montées - Hugo met lui-même le terme en italiques - on passe de la route au "chemin ». Vieille distinction historique qui renvoie au caminus gaulois, lien de proximité qui, contrairement à la via rupta d'origine romaine tranchant dans le relief, s'adapte aux irrégularités du terrain. Première perception de cette pénétration dans l'inconnu lors du voyage de 1825 sur le chemin qui mène de Servoz à Chamonix où le voyageur expérimente des dangers inédits :

"C'est un chemin étroit et rapide, laborieusement tracé le long d'un escarpement effrayant, auquel rien ne peut se comparer, si ce n'est la pente de la montagne qui borde l'Arve de l'autre côté [...] On y glisse à chaque instant sur de larges dalles de granit qui font étinceler les fers des mulets. À droite on doit prendre sur sa tête la racine des grands mélèzes déchaussés par les pluies, à gauche, on peut pousser $d u$ pied la tête effilée comme l'aiguille d'un rocher. ${ }^{17}$ »

14 En 1843, lors du voyage aux Pyrénées où l'écrivain semble vivre une confrontation physique à la montagne plus brutale que dans les Alpes, il amplifie, par le recours à l'ironie, le caractère hasardeux du déplacement sur les chemins d'altitude. Empruntant à cheval celui qui mène au cirque de Gavarnie, il note :

«Profondeur effrayante du gave. Mon cheval aime le bord. Je le laisse faire. C'est son genre. ${ }^{18}$ »

15 C'est que, explique-t-il, les espèces autochtones sont adaptées aux conditions spécifiques des circulations montagnardes :

«Les chevaux de montagne sont admirables, patients, doux, obéissants, pleins d'instincts variés. Ils montent des escaliers et descendent des échelles. Ils vont sur le gazon, sur le granit, sur la glace. Ils côtoient le bord extrême du précipice. Ils marchent délicatement et avec esprit comme des chats. De vrais chevaux de gouttières..$^{19}$ ”

Les figures de l'affrontement de l'écrivain-penseur-voyageur aux périls des déplacements sur les chemins montagnards constituent ici l'hyperbole du topos romantique du face-à-face Paris/province. Sur son « cheval de gouttière » pyrénéen, 
Hugo symbolise cette inadaptabilité foncière du parisien aux périls des circulations montagnardes :

«Il avait l'air de se dire : ce monsieur est un artiste, un amateur. Il faut lui faire bien voir tout ! Ah! Tu veux des torrents, Parisien! Tu veux des gaves, des cascades, des gouffres, des précipices, des émotions ! Eh bien, en voilà. Tiens, regarde, penche-toi, ici, et ici, et ici. En as-tu assez ? Je trottais ainsi en surplomb sur des escarpements de huit cents pieds de profondeur avec un petit gave bleu et sombre sous les yeux $[\ldots]^{20} »$

\section{Poétique de l'effort et quête de la " sublime horreur »}

17 Dans le milieu montagnard, dont la pénétration s'ordonne selon les règles de la verticalité, l'adaptation aux contraintes de la déclivité répond à une double logique d'étagement: celle des moyens de transport utilisés mais aussi celle des efforts physiques fournis par le voyageur en quête active d'émotions visuelles. En écho au Rousseau des Confessions - «Il me faut des torrents, des rochers, des sapins, des bois noirs, des montagnes, des chemins raboteux à monter et à descendre, des précipices à mes côtés qui me fassent bien peur ${ }^{21} »-$, les pérégrinations alpines et pyrénéennes de Victor Hugo révèlent ainsi toute une gamme d'expériences sensibles engageant le corps dans son entièreté.

L'abandon de la voiture pour le char à bancs «attelés de mulets, et formés d'une seule banquette transversale où l'on est assis de côté sous une façon de petit dais en cuir " dont Hugo fait la première expérience en 1825 sur le chemin de Sallanches à Chamonix marque une rupture essentielle :

«Cette nouvelle manière de voyager vous avertit que vous passez, en quelque sorte, d'une nature à une autre. Voici que vous pénétrez dans la montagne. Le sabot rond et plat des chevaux ne convient plus à ces chemins âpres, escarpés et glissants. La roue des voitures ordinaires se briserait dans ces sentiers étroits, à tout moment déchirés par des pointes de rocs et rompus par les torrents. Il faut des chariots légers et solides qui puissent se démonter dans les passages difficiles, et les traverser avec vous sur les épaules des guides et des muletiers. ${ }^{22}$ »

Le passage du char à bancs à l'ascension à dos de mulets constitue une deuxième rupture qui confère à l'expérience une dimension sensorielle inédite : "Jusqu'ici, vous n'avez fait que voir les Alpes, maintenant vous commencez à les sentir », note Hugo. Expérience rugueuse du cahot à laquelle ne tarde pas à succéder celle de l'ascension pédestre qui engage plus encore toutes les fonctions physiques, de l'effort musculaire à la physiologie de l'équilibre :

«Vous avancerez encore et alors le vertige, ou quelque autre invincible obstacle, vous forcera de descendre de vos montures et de continuer à pied votre voyage hasardeux, jusqu'à ce qu'enfin vous ayez atteint ces lieux où l'homme lui-même est contraint de reculer, ces solitudes de glace, de granit et de brouillard, où le chamois, poursuivi par le chasseur, se réfugie audacieusement entre les précipices prêts à s'ouvrir et des avalanches prêtes à tomber. ${ }^{23}$ "

Sur les pentes des montagnes, s'expérimente ainsi cette culture de l'effort que consacreront à la fin du siècle les écrits des clubs alpins et que récompense, à l'arrivée au sommet, la sidération visuelle du panorama. Il y a dans cet engagement de tout le corps dont Hugo n'est jamais avare d'évocation, quelque chose qui renvoie aux conditions premières de la quête du sublime dans l'Angleterre de la fin du XVIII siècle et dont Baldine Saint-Girons a montré qu'elle supposait un affrontement physique aux 
éléments de la nature ${ }^{24}$. Hugo en fait l'expérience bouleversante lors de son ascension du Rigi en 1839 quand, au terme d'une progression de quatre heures, il se retourne, «[se] demandant à quel être supérieur et choisi la nature servait ce merveilleux festin de montagnes, de nuages et de soleil, et, cherchant un témoin sublime à ce sublime paysage ${ }^{25} »$.

21 Le long et périlleux parcours qui, depuis la plaine, hisse le voyageur jusqu'aux sommets illustre le besoin de confrontation de l'homme du XIX siècle à cette "énergie des vacuités dynamisées ${ }^{26}$ » évoquée par Alain Corbin à propos de l'apprivoisement romantique du littoral, lequel s'accomplit pratiquement au même moment que celui de la montagne. Gagnées de haute lutte physique, les hauteurs sont regardées par Hugo pour ce qu'elles recèlent d'éléments chaotiques, de traces d'accidents géologiques, de reliefs tourmentés, de violences visuelles et auditives; pour ces « vacarmes de l'œil » que, lors d'un autre voyage, il évoque devant la Fontaine de Vaucluse et dont se nourrit l'esthétique de la "sublime horreur ». Là encore, c'est au sommet du Rigi, où il va jusqu'à s'aplatir en surplomb du vide pour mieux capter la violente étrangeté du paysage, qu'il trouve à vibrer de tout son être au spectacle d'une nature déchaînée :

«Sur des sommets comme le Rigi-Kulm, il faut regarder, mais il ne faut plus peindre. Est-ce beau ou est-ce horrible ? Je ne sais vraiment. C'est horrible et c'est beau tout à la fois. Ce ne sont plus des paysages, ce sont des aspects monstrueux. ${ }^{27}$ »

L'horreur que les romantiques n'hésitent pas à associer à la beauté quand nous-mêmes en avons fait le superlatif de la laideur, doit être comprise comme une forme suprême d'effroi qui suscite une vibration de tout l'être au contact de la violence des éléments. En 1825, sur la route de Sallanches à Chamonix, Hugo salue ainsi «l'horrible beauté " du site sauvage du Nant noir ${ }^{28}$, «lugubre et désolé », et où, sous des crêtes nues et des rochers en surplomb « les échos ...se répètent les hurlements furieux du torrent ${ }^{29}$ ».

Beauté "horrible», aussi, que celle qui se dégage de la violente énergie du torrent pyrénéen qui surprend l'écrivain en août 1843 sur le chemin de Gavarnie et qui renvoie à cette esthétique du choc par laquelle l'âme sensible ne demande qu'à se laisser submerger :

« De larges entonnoirs forment de grandes cuves où l'eau saute et bout couverte d'écume comme dans une marmite énorme chauffée à un feu qui ne s'éteint jamais. Des souches d'arbres, monstrueuses, des racines hideuses, décharnées et difformes, roulent dans le torrent comme des carcasses d'hydres.

- L'horrible est partout..$^{30}$ ”

Mais pour être pleinement aboutie, la quête de la sublime horreur suppose, au terme de l'effort, des formes d'immobilité, des capacités à s'abstraire des fluidités ambiantes celles de la route, des foules et des circuits déjà trop convenus du pittoresque - pour s'abandonner, dans la solitude la plus absolue, aux transports intérieurs qui font de la montagne un espace de contemplation, un isolat propice à toutes les formes de dépassement du réel, une invitation à regarder "au-dedans de soi ». Un espace où s'expérimentent des formes spécifiques d'immobilité. 


\section{Figures romantiques de l'immobilité montagnarde}

\section{La montagne comme espace de contemplation}

25 C'est sur le chemin de Sallanches à Chamonix que, lors de son voyage de découverte des Alpes en 1825, Hugo fait une première fois l'expérience de ce passage, au sens métaphysique du terme, dans un monde différent :

"Après avoir gravi péniblement un chemin encombré de pierres roulantes, qui sonnent sous le pied des mulets, on traverse le village de Chède, et on laisse la belle cascade derrière soi, pour s'enfoncer dans la montagne. [...] Tout à coup, le taillis s'ouvre et s'écarte comme à plaisir, un spectacle rempli d'un charme inattendu est devant vos yeux [...] On se croirait magiquement transporté dans une autre contrée, sous un autre ciel $[. . .]^{31} »$

Cette expérience du "transport », au sens cette fois symbolique du terme - qui renvoie à l'idée, elle aussi duale, d'un « ravissement », c'est-à-dire d'un élan qui à la fois vous charme et vous enlève du réel - nous introduit à la problématique de l'immobilité en ce qu'elle suppose un détachement des contingences matérielles du voyage pour s'abîmer dans la contemplation, laquelle, chez Hugo, n'est jamais loin de la réflexion métaphysique.

Récompense des efforts de la montée, le panorama polarise la promenade et autorise le repos. Par lui, la montagne hugolienne peut devenir un espace privilégié de méditation. «Comment ai-je passé cette journée au sommet du Rigi ? , feint-il de se demander en 1839 après avoir détaillé les phases de son ascension. « Je ne sais. J'ai erré, j’ai regardé, j'ai songé $e^{32} »$. Vue des sommets, les lignes organisatrices du paysage sur lesquelles glisse le regard ne sont plus verticales mais horizontales. La vue circulaire permet d'en mesurer la variété des composantes, d'en ordonner, plan par plan, la saisie visuelle comme on le ferait avec un tableau et de laisser, dans la vacuité du temps de la contemplation, remonter les références culturelles à la surface.

Ce panoptisme, indissociablement géographique et historique, suppose l'immobilité du spectateur. Devant ses évocations paysagères, on pense au peintre Pierre-Henri de Valenciennes qui, apprenant à goûter au panorama, précisait, à la fin du XVIII ${ }^{\mathrm{e}}$ siècle, que la Nature devait être " envisagée d'un seul coup d'œil ; la tête immobile ${ }^{33}$ ". " Après une heure passée sur le Rigi-Kulm, écrit Hugo en 1839, on devient statue, on prend racine à un point quelconque du sommet ${ }^{34}$ ". Sommet où il faut, dit-il, « regarder mais [...] ne plus peindre », faire abstraction de toute activité, réaliser en quelque sorte le vœu que, devant le panorama de Riom, avait formulé au siècle précédent Le Grand d'Aussy qui souhaitait, " comme Argus [...] en ce moment être tout œil ${ }^{35}$ ».

Sur ces points privilégiés de dévoilement de la structure des massifs que sont les "points de vue », le regard de l'écrivain s'arrête sur les détails de la morphologie du paysage. Là encore, Hugo est bien l'enfant d'un siècle que taraudent les interrogations des géologues sur l'origine du monde et qui sonde du regard les falaises et les sommets des montagnes comme autant de conservatoires des " archives de la terre ${ }^{36}$ ». La vision de l'aspect fantasmagorique des blocs alpins et des gorges pyrénéennes lui inspire questionnements scientifiques et interrogations métaphysiques. Devant le massif du Mont-Blanc qui se révèle à lui en 1825 sur le chemin de Chède à Chamonix, il se pose des questions scientifiques :

"Je ne sais, et nul ne peut dire, comment se déplaça le centre où reposait l'équilibre

de ce grand corps; quelle cause mina la base sur laquelle posaient ses immenses 
terrasses, ses plateaux, ses dômes, ses pentes, ses aiguilles. Est-ce une convulsion intérieure du globe ? Est-ce une goutte d'eau lentement distillée depuis des siècles?

... Felix qui potuit... ${ }^{37}$ » démasquer, sous les formes qui s'offrent à la vue panoramique, «l'ostéologie colossale des Alpes ». Mais derrière ces reliefs bouleversés dont la vue panoramique lui révèle la sublime violence, Hugo cherche surtout la main du Créateur et ses méditations au sommet se confondent souvent avec la posture de l'action de grâce. Dernier relent du courant physico-théologique qui, dans l'Europe du XvIII siècle, a érigé la nature en spectacle, changé le promeneur en admirateur des bontés infinies d'un Dieu organisateur de l'Univers et l'a invité à l'inventaire insistant des merveilles de la Création $^{38}$ ? Il y a quelque chose de cela dans les émerveillements hugoliens devant les Alpes et les Pyrénées. Comme, par exemple, dans ceux qu'il éprouve lors de ce premier voyage de 1825 où la vision du massif du Mont-Blanc et de la Mer de glace lui fait considérer la vallée de Chamonix comme :

«Le cabinet de curiosité de la nature [...] une sorte de laboratoire divin où la providence tient en réserve un échantillon de tous les phénomènes de la création, ou plutôt [...] un mystérieux sanctuaire où reposent les éléments du monde visible. ${ }^{39}$ $»$

31 De même qu'il voit, en 1839, dans le panorama du Rigi, « un ensemble prodigieux de choses harmonieuses et magnifiques pleines de la grandeur de Dieu ${ }^{40} »$ ou, en 1843 , dans le cirque de Gavarnie, "l'édifice le plus mystérieux du plus mystérieux des $\operatorname{architectes}^{41} »$.

\section{La montagne comme isolat}

Taraudé, au fil de ses trois voyages aux Alpes et aux Pyrénées, par un incessant questionnement sur les mystères de l'ordonnancement des merveilles de la nature, Hugo postule l'étrangeté absolue du monde auquel introduit l'altitude. À la dynamique des successions paysagères toujours renouvelées, découvertes à la faveur des fameuses « montées ", succède, sous la plume du voyageur parvenu au cœur des massifs, la vision statique d'un milieu montagnard conçu comme un isolat, le conservatoire d'une primitivité préservée, un laboratoire de l'étrange.

Cela se lit d'abord dans la proximité qui continue de lier, dans une écologie élémentaire, les populations montagnardes à la nature qui les environne. Étrangeté des corps et des esprits autochtones, qui, dans un subtil jeu d'analogies mâtinées de déterminisme, se fondent dans celle du paysage. Au sommet du Rigi, face à la sidération visuelle du panorama, où tout n'est qu'un «chaos d'exagérations absurdes et d'amoindrissements effrayants", Hugo convoque la démesure des propriétés géométriques de l'espace alpin pour expliquer les troubles physiques et mentaux de populations quotidiennement confrontées à une expérience visuelle hors norme. Il écrit :

«Le paysage est fou. En présence de ce spectacle inexprimable, on comprend les crétins dont pullulent la Suisse et la Savoie..$^{42}$ ”

Le constat de la fréquence des anomalies physiques chez les populations montagnardes n'est pas fondamentalement nouveau à l'époque où Hugo parcourt les Alpes. Montaigne s'en était, en son temps, déjà fait l'écho et, depuis le début du XIX siècle, nombre de voyageurs - de Huchet de La Bédoyère ${ }^{43}$ à Albert Montémont en $1821^{44}$ - s'étaient 
effrayés de la "grosse gorge " des Valaisans et des Savoyards. La vieille idée hippocratique selon laquelle le corps de l'homme serait une composante de l'univers trouve matière à une vigoureuse réactualisation dans le contexte d'un romantisme attentif au genius loci, à la diversité infinie de la nature, au sens du relatif et du particulier. Ainsi, en 1839, Hugo peut-il affirmer que, si «les Alpes font beaucoup d'idiots ", c'est qu' « il n'est pas donné à toutes les intelligences de faire ménage avec de telles merveilles et de promener du matin au soir sans éblouissement et sans stupeur un rayon visuel terrestre de cinquante lieues sur une circonférence de trois cent ${ }^{45} »$.

Marque d'une primitivité préservée aussi, la pérennité des superstitions que Hugo rattache aux données esthétiques de l'environnement. Ainsi, peut-il, à l'occasion de sa découverte en 1825 du Torrent noir ${ }^{46}$ dans les environs de Chamonix, lier, dans la représentation qu'il construit de ce lieu, sa curiosité pour le merveilleux à la jouissance esthétique que lui a procurée la vision lugubre de l'endroit :

«J'avouerai cette infirmité de mon esprit, il aurait manqué pour moi quelque chose à l'horrible beauté de ce site sauvage, si quelque tradition populaire ne lui eût empreint un caractère merveilleux. Je me suis arrêté avec complaisance sur ces détails, parce que j'aime les superstitions : elles sont filles de la religion et mères de la poésie. ${ }^{47}$ "

\section{La montagne comme laboratoire des figures romantiques de la promenade intérieure}

Juxtaposition statique de «tableaux » invitant à la méditation, territoire de l'étrange, la montagne hugolienne se donne également à voir comme le laboratoire où s'expérimentent les figures de la promenade romantique. En 1843, Hugo écrit à Louis Boulanger depuis Cauterets :

«Figurez-vous, Louis, que je me lève tous les jours à quatre heures du matin, et qu'à cette heure sombre et claire tout à la fois je m'en vais dans la montagne. Je marche le long d'un torrent, je m'enfonce dans une gorge la plus sauvage qu'il y ait, et sous prétexte de me tremper dans de l'eau chaude et de boire du soufre, j'ai tous les jours un spectacle nouveau, inattendu, merveilleux [... ${ }^{48}$ »

Entre la plaine où l'écrivain est encore pris dans la cinétique de la route et contraint à subir l'inconfort d'un voisinage forcé avec des «touristes » toujours plus nombreux et la solitude absolue des sommets où il fait l'expérience de la sublime horreur, viennent ainsi s'intercaler les espaces intermédiaires de la promenade. Niveau accessible au "villégiaturiste » en quête de calme, les rives des torrents et les chemins pastoraux accrochés aux flancs des montagnes lui permettent de se laisser ravir par des spectacles d'un autre ordre et qui ramènent à la moyenne montagne rousseauiste.

L'équilibre pictural de la beauté classique prend ici le pas sur la violence des codes du sublime. Il y a dans cette poétique de l'adoucissement de toute chose, très clairement perceptible chez Hugo, quelque chose qui renvoie à L'art de se promener publié l'année même de la naissance de l'écrivain par Karl Gotllob Schelle, lequel définissait la promenade comme un art de vivre caractérisé par le sens de la mesure ${ }^{49}$. Hugo peut ainsi décrire à Louis Boulanger, la "vie obscure et charmante » qui animait le flanc ténébreux des montagnes en ce matin d'août 1843 :

«Pas un nuage, pas une vapeur [...] on y distinguait l'herbe, les fleurs, les pierres, les bruyères, dans une sorte de fourmillement doux et joyeux. Le bruit du gave n'avait plus rien d'horrible ; c'était un grand murmure mêlé à ce grand silence..$^{50}$ » 
À ce modèle de déambulation où le promeneur se fait accueillant à l'esprit du lieu, préside le principe de liberté51. Nous le voyons clairement, chez Hugo s'opposer à la dynamique de la "quête " qui guide ses ascensions vers les limites de la sublime horreur. En 1843, il évoque à l'intention d'Adèle cet abandon absolu du promeneur au hasard, à la digression, dans l'indécision totale d'un itinéraire que l'écrivain revendique de n'avoir pas choisi :

«Une route s'était présentée, je l'avais acceptée au hasard, et j'allais. Je marchais dans la montagne sans trop savoir où j'étais. ${ }^{52}$ "

Il y a, on l'aura compris, dans cette volontaire déstructuration de l'espace montagnard en une série aléatoire de chemins dont on ne sait où ils mènent, un procédé littéraire qui transforme la promenade en déambulation hors-sol. Hasard du choix de la direction qui, par une coquetterie rhétorique, fait croire au caractère fortuit de la découverte. Le paysage n'est plus ici, comme dans les codes du pittoresque ${ }^{53}$, «surprise au détour du chemin ", mais lente instillation dans l'âme du promeneur. "Le spectacle d'un paysage se dévoilant peu à peu est pour l'esprit un plaisir particulier » écrivait Schelle en $1802^{54}$. C'est que le promeneur romantique marche en quête des subtiles harmonies qui relient son âme à la nature et aux météores. Hugo narre à Louis Boulanger deux promenades effectuées dans les environs de Cauterets. L'une, effectuée au lendemain d'une nuit pluvieuse où le cri du torrent lui paraît «hideux et terrible» et où tout lui paraît " ténébreux et pensif » autour de lui ; l'autre, au lendemain d'une nuit sereine où il fait l'expérience de "cette fraîcheur, cette grâce, cette transparence mélancolique et inexprimable du matin ${ }^{55}$ ». « Il me semble, mon ami, que ces choses-là sont plus que des paysages $^{56}$ ", écrit-il. Elles sont l'expression de ce retournement du regard évoqué par l'écrivain à propos de la promenade effectuée en 1843 aux environs de Saint-Sébastien et dont il s'ouvre à Adèle en ces termes bien connus :

«Peu à peu le paysage extérieur que je regardais vaguement avait développé en moi cet autre paysage intérieur que nous nommons la rêverie; j'avais l'œil tourné et ouvert au-dedans de moi, et je ne voyais plus la nature, je voyais mon esprit. ${ }^{57}$ »

41 Forme suprême d'immobilité qui mène à cette abolition des réalités physiques déjà expérimentée par le Rousseau de la cinquième Promenade :

«De quoi jouit-on dans une pareille situation? De rien d'extérieur à soi, de rien sinon de soi-même et de sa propre existence, tant que cet état dure on se suffit à soi-même comme Dieu. ${ }^{58}$ "

Le principal mérite des passages des Voyages de Hugo consacrés aux Alpes et aux Pyrénées est sans doute de révéler cette variété des formes de mobilité inspirées par l'espace montagnard: le déplacement en voiture, la montée en char à bancs, la chevauchée, l'ascension pédestre. Il est aussi de décliner les formes d'immobilité dans lesquelles le penseur s'abîme - la méditation, la rêverie, la réflexion scientifique - et où la montagne s'enferme - la résistance à la marche de l'histoire, le repli des communautés pastorales, l'immuabilité des traditions, la fatalité de la transmission des tares.

Mais il est peut-être aussi de mettre en évidence, entre ces deux extrêmes, des coulées par lesquelles s'insinuent des mouvements intermédiaires, des formes subtiles de mobilité - la promenade, l'errance, la déambulation sans but - qui font de la montagne 
un de ces territoires d'élection des « sentiers vagabonds faits pour les pensées flâneuses et les causeries à arabesques » évoqués par Flaubert ${ }^{59}$.

Considérée en tant que source d'inspiration, la montagne - cela se voit très fort chez Hugo - pose la question de l'autonomie du récit de voyage en tant que genre littéraire et des interactions qu'il entretient avec d'autres formes artistiques, notamment picturales. La dynamique qui guide la quête, inscrite dans la verticalité, de la sublime horreur, la recherche de la surprise pittoresque au détour du chemin, l'ordonnancement du regard panoptique encadré dans les limites du panorama, témoignent que la montagne est aussi le lieu où le premier $\mathrm{xIX}^{\mathrm{e}}$ siècle fait l'apprentissage d'une nouvelle culture de la visualité, toute entière inscrite elle-même dans cette dialectique de la mobilité et de l'immobilité qui, de toute évidence, excède largement le champ historique et littéraire.

\section{BIBLIOGRAPHIE}

ANTOINE Philippe, «Une rhétorique de la spontanéité : le cas de la promenade », dans GUYOT Alain, MASSOL Chantal (Textes réunis et présentés par), Voyager en France au temps du romantisme. Poétique, esthétique, idéologie, Grenoble, Ellug, 2003, p. 131-146.

BOYER Marc, Histoire de l'invention du tourisme XVI ${ }^{e}$-XIX ${ }^{e}$ siècle. Origine et développement du tourisme dans le Sud-Est de la France, Paris, éditions de l'Aube, 2000.

CORBIN Alain, L'homme dans le paysage, Paris, Textuel, 2001.

CORBIN Alain, Le territoire du vide. L'Occident et le désir de rivage, Paris, Aubier, 1988.

GÉLY Claude, Euvres complètes, Voyages, Paris, Robert Laffont, « Bouquins », 1987.

GUYOT Alain, MASSOL Chantal (Textes réunis et présentés par), Voyager en France au temps du romantisme. Poétique, esthétique, idéologie, Grenoble, Ellug, 2003.

FLAUBERT Gustave, Par les champs et par les grèves (Voyages en Bretagne), Paris, Arléa, 1998.

LA BÉDOYÈRE Henri Huchet de, Voyage en Savoie et dans le Midi de la France en 1804 et 1805, Paris, Giguet et Michaud, $1807,439 \mathrm{p}$.

LE GRAND D'AUSSY, Voyage d'Auvergne, Paris, Chez Eugène Onfroy, 1788.

LETHUILLIER Jean-Pierre, PARSIS-BARUBÉ Odile (dir.), Le pittoresque. Métamorphoses d'une quête dans l'Europe moderne et contemporaine, Paris, Classiques Garnier, 2011.

MONTÉMONT Albert, Voyage aux Alpes et en Italie, ou Lettres en prose et en vers, Paris, Lelong, 1821. PARSIS-BARUBÉ Odile, « Un combat, deux géants. Dialogues de la mer et de la montagne dans les Voyages de Hugo ", dans Alain CABANTOUS, Jean-Luc CHAPPEY, Renaud MORIEUX, Nathalie RICHARD et François WALTER (dir.), Mer et montagne dans la culture européenne (XVI ${ }^{e}-\mathrm{XIX}{ }^{e}$ siècle), Rennes, PUR, 2011, p. 259-272.

SAINT-GIRONS Baldine, Le sublime de l'Antiquité à nos jours, Paris, Desjonquères, 2005. 
VALENCIENNES Pierre-Henri de, Elemens de perspective pratique à l'usage des artistes suivis de réflexions et conseils à un élève sur la peinture et particulièrement sur le genre du paysage, Paris, an VIII.

ROUSSEAU Jean-Jacques, Les rêveries d'un promeneur solitaire, Paris, H. Rodier éd., Classiques Garnier.

\section{NOTES}

1. A. Guyot, Ch. Massol, Voyager en France au temps du romantisme. Poétique, esthétique, idéologie, 2003, p. 11-16.

2. Et repris tels quels par Madame V. Hugo en 1863 dans le Victor Hugo raconté par un témoin de sa vie [chapitre XLII].

3. Pour plus de détails sur les avatars de la publication de ces textes, on se reportera aux notices de l'édition de C. Gély, dans Euvres complètes, notamment celle de Alpes et Pyrénées, p. 1262-1263. C'est à cette édition que sont empruntées toutes nos citations.

4. O. Parsis-Barubé, « Un combat, deux géants. Dialogues de la mer et de la montagne dans les Voyages de Hugo », p. 259-272.

5. V. Hugo, O.C. Voyages, p. 845.

6. Ibid., p. 508.

7. Ibid., p. 661.

8. Ibid., p. 671.

9. On se reportera sur ce sujet aux travaux de Marc Boyer, Histoire de l'invention du tourisme $\mathrm{XVI}^{e}-\mathrm{XIX}$ siècle. Origine et développement du tourisme dans le Sud-est de la France, p. 225-238.

10. V. Hugo, O.C., Voyages, p. 513-514.

11. Cité dans Marc Boyer, Histoire de l'invention du tourisme XVI ${ }^{e}-X I X^{e}$ siècle, p. 191.

12. Ibid., p. 191.

13. Sommet des Alpes suisses culminant à $1797 \mathrm{~m}$ au bord du lac de Lucerne.

14. V. Hugo, O.C., Voyages, p. 673.

15. Ibid., p. 677-678.

16. Ibid., p. 821.

17. Ibid., p. 512.

18. Ibid., p. 856.

19. Ibid., p. 856.

20. Ibid., p. 856.

21. J.-J. Rousseau, Les Confessions, livre IV.

22. V. Hugo, O.C., Voyages, p. 507.

23. Ibid., p. 507.

24. B. Saint-Girons, Le sublime de l'Antiquité à nos jours. Sur l'esthétique du sublime comme code d'appréciation du paysage à l'époque romantique, on relira aussi avec profit les pages pénétrantes d'Alain Corbin, L'homme dans le paysage, p. 86-90. Voir aussi, du même auteur, Le territoire du vide. L'Occident et le désir de rivage, p. 148.

25. V. Hugo, O.C., Voyages, p. 676. 
26. A. Corbin, Le territoire du vide. L'Occident et le désir de rivage 1750-1850, p. 190.

27. V. Hugo, O.C., Voyages, p. 662.

28. Il prend sa source aux Avanchers (Savoie) à $1500 \mathrm{~m}$ et forme dans sa partie haute une gorge étroite et roule à certaines périodes d'orage une forte lave qui lui vaut cette dénomination.

29. Ibid., p. 510.

30. Ibid., p. 881.

31. Ibid., p. 509.

32. Ibid., p. 679.

33. Cité dans A.Corbin, L'homme dans le paysage, p.164, d'après P. H. Valenciennes, p. 340 .

34. V. Hugo, O.C., Voyages, p. 677.

35. Le Grand d'Aussy, Voyage d'Auvergne, p. 10.

36. A. Corbin, L'homme dans le paysage, p. 115-127.

37. V. Hugo, O. C., Voyages, p. 511.

38. Sur ce sujet, on se reportera avec profit à la synthèse donnée par A.Corbin, Le territoire $\mathrm{du}$ vide, p. 34-44.

39. V. Hugo, O. C., Voyages, p. 516.

40. Ibid., p. 676.

41. Ibid., p. 860.

42. Ibid., p. 677.

43. La Bédoyère et Huchet, Voyage en Savoie et dans le Midi de la France en 1804 et 1805.

44. A. Montémont, Voyage aux Alpes et en Italie, ou Lettres en prose et en vers.

45. V. Hugo, O. C., Voyages, p. 677.

46. Autre appellation du Nant noir.

47. Ibid., p. 510.

48. Ibid., p. 851.

49. Ph. Antoine, "Une rhétorique de la spontanéité: le cas de la promenade", p. 131-146.

50. V. Hugo, O. C., Voyages, p. 851.

51. Ph. Antoine, « Une rhétorique de la spontanéité : le cas de la promenade », p. 132.

52. V. Hugo, O. C., Voyages, p. 791.

53. J.-P. Lethuillier, O. Parsis-Barubé (dir.), Le pittoresque. Métamorphoses d'une quête dans l'Europe moderne et contemporaine.

54. Cité par Ph. Antoine, p. 132.

55. V. Hugo, Voyages, p. 851.

56. Ibid., p. 851.

57. Ibid., p. 791.

58. J.-J. Rousseau, Les rêveries d'un promeneur solitaire, p. 71.

59. G. Flaubert, Par les champs et par les grèves, p. 219. 


\section{RÉSUMÉS}

Entre 1825 et 1843, Victor Hugo accomplit deux voyages aux Alpes et un voyage aux Pyrénées. Au fil de la correspondance qui les accompagne, se dessinent les grandes lignes de l'appropriation, par l'écrivain, de cet espace montagnard au moment où les élites européennes en font leur nouveau terrain de jeu. Destination romantique où continue de se construire le dialogue amorcé par Rousseau entre le paysage intérieur du poète et le paysage extérieur qui s'offre à sa vue, la montagne, en ce premier XIX ${ }^{\mathrm{e}}$ siècle, constitue aussi le lieu d'expériences sensorielles inédites où s'invente un nouveau rapport du corps à l'espace. Mais si la montagne hugolienne se donne à voir comme le territoire de mobilités multiples, elle n'en constitue pas moins, pour l'écrivain, un espace clos, refermé sur des populations préservées de tout contact avec l'extérieur. Un de ces isolats où la première génération romantique a cru pouvoir saisir les vestiges d'un âge d'or originel et pratiquer cette archéologie de l'étrangeté chère aux celtomanes du début du siècle.

\section{AUTEUR}

\section{ODILE PARSIS-BARUBÉ}

Maître de Conférences HDR en histoire contemporaine, Université de Lille, membre associé du laboratoire IRHiS (CNRS UMR 8529), membre du CTHS, section Histoire contemporaine et du temps présent 


\title{
Analyse du discours de la circulation en montagne : l'influence de John Ruskin sur la géographie alpine (1858)
}

\author{
Samia Ounoughi
}

$1 \quad \mathrm{Au}$ XIX ${ }^{\mathrm{e}}$ siècle en Grande Bretagne, le Grand Tour est le type de circuit le plus pratiqué et comprend nécessairement un itinéraire dans les Alpes. Les jeunes gens des middle classes Britanniques sont censés y parfaire leur éducation par la découverte des espaces géographiques-phares du continent. En parallèle, alpinistes, scientifiques et artistes viennent aussi séjourner régulièrement en moyenne et en haute montagne où ils conquièrent les sommets et contemplent les paysages alpins ${ }^{1}$. Dans tous les cas, le voyage en montagne implique systématiquement une production textuelle dont la vocation est à la fois de rendre compte d'un itinéraire et d'une expérience en montagne, et de préparer les futurs touristes ou alpinistes à s'y rendre. Aussi, l'écriture de récits d'ascension fait-elle partie du règlement de l'Alpine Club de Londres afin d'améliorer la sécurité des membres lors de leurs futurs périples². Pour le commun des gens aisés, les guides de voyages comme le Murray ou le Baedeker sont très répandus et favorisent le tourisme en montagne. S'ils permettent de rendre la découverte des Alpes plus accessible, les guides tendent aussi à uniformiser le circuit et la temporalité du voyage.

2 En marge de cette littérature, des figures influentes de la société britannique comme Samuel Butler (1835-1902) et John Ruskin (1819-1900), produisent des textes à partir d'une longue expérience de la montagne toute personnelle, empreinte d'anti-tourisme ${ }^{3}$. Ils s'intéressent à la montagne pour sa dimension universalisante ${ }^{4}$ et comptent ainsi donner à leurs récits et à leur expérience en montagne davantage de portée. Leur cheminement dans les Alpes s'accompagne d'une réflexion sur les plans social, philosophique et esthétique, sur le rapport de l'homme à la montagne et au-delà, sur le rapport de l'homme au monde. Ces approches originales de la montagne sont menées à l'appui d'une écriture qui déconstruit les canons des genres et qui fait ainsi émerger 
par le discours de nouvelles modalités de circulation et de connaissance de la montagne. Le cas de Samuel Butler a fait l'objet d'un travail de recherche ${ }^{5}$. Il est proposé de s'intéresser ici à la correspondance de John Ruskin Letters from the Continent (1858) : il s'agit d'une série de lettres qu'il adresse à son père pendant son séjour dans les Alpes suisses, françaises et italiennes de mai à septembre 1858.

3 À travers une analyse du discours menée à l'appui des outils de la pragmatique, nous souhaitons mettre au jour la manière dont un mode de voyage et d'écriture spécifiques à John Ruskin révèle de nouvelles formes d'accès à la connaissance de la montagne. Le genre épistolaire est justement celui du texte en circulation. Nous commencerons dès lors par analyser comment Ruskin subvertit les règles du genre pour conditionner son regard pendant le voyage tout en continuant à communiquer avec Londres. Nous verrons ensuite en quoi son circuit en montagne sur les traces de J.-M. William Turner lui permet de confronter la montagne perçue et la montagne représentée. Enfin, à partir d'un mode d'observation rigoureusement conditionné de l'espace montagneux, nous verrons comment Ruskin compose sa montagne.

\section{Les modalités de la mise en discours de la géographie alpine ou la subversion du genre épistolaire}

Ruskin part de Douvres le 13 mai 1858 et rentre à Londres le 14 septembre de la même année. À cette époque, il est en train de rédiger le dernier des cinq volumes de Modern Painters, mais il doit s'interrompre car il est physiquement et moralement épuisé. Son voyage est motivé par un besoin de se distraire de la réflexion sur l'esthétique par la pratique de l'art sur le terrain. Il choisit la représentation de paysages alpins et part sur les traces de William Turner qu'il considère comme l'un des peintres majeurs de son époque :

I thought I might rest myself by hunting down these Turner subjects, and sketching what I could of them, in order to illustrate his compositions. ${ }^{6}$

Pendant ces quatre mois, il rédige 134 lettres dont 121 sont adressées à son père et 13 seulement à d'autres correspondants. L'analyse du style de ces lettres et la modalité du discours qu'il y développe sont cruciales pour saisir le mode d'appréhension de la géographie par Ruskin. En effet, les conditions et les finalités du travail du géographe, sa formation, constituent son "horizon d'attente", infléchissent ses observations, ses analyses et le discours dans lequel il les transmet ${ }^{7}$.

6 Pour commencer, on rappellera cette contribution essentielle de la phénoménologie qui permet de comprendre le caractère intentionnel des représentations. La production de représentations et des systèmes d'objets dont elles sont faites a des finalités pratiques ; elle guide l'action et dans le même temps, est motivée par elle. Elles font que chacun de ces objets, une fois désigné et circonscrit, une fois rapporté à une catégorie particulière, constitue un " horizon d'attente ${ }^{8}$ ", « qui oriente les pratiques des usagers de l'objet en question ${ }^{9} »$.

7 Le point de vue du géographe rejoint ici l'analyse du discours, notamment la théorie de la réception ${ }^{10}$. La construction de toute représentation quelle qu'en soit la forme, en l'occurrence ici, de la montagne, prend racine chez le sujet qui la décrit et la raisonne et dont il convient de connaître la démarche énonciative. Cela se prolonge dans la lecture des lettres elles-mêmes, car Ruskin les écrit avant tout pour lui-même. Il s'agit 
donc, dans cette démarche épistolaire d'un circuit fermé de la lettre dont la composition et la réception par un même auteur-lecteur vise à construire une (auto) réflexion au sens littéral sur la montagne.

Ruskin semble tout à fait conscient de cet engagement total du sujet observateur et de son influence sur sa représentation de la montagne. Il commence donc par établir des conditions d'isolement et de rupture avec son entourage, ce qui lui donne d'autant plus de liberté dans l'observation et la représentation de l'espace alpin. Le discours de Ruskin se caractérise par la liberté qu'il prend pour reformuler ou corriger les écrits de spécialistes de la montagne :

Carrying on my mineralogical dictionary by the help of Jameson's three-volume Mineralogy,

[...] comparing his descriptions with the minerals in the British Museum, and writing my own more eloquent and exhaustive accounts. ${ }^{11}$

9 Ruskin s'instruit puis se détache de ses lectures pour construire sa propre connaissance de la montagne. Cette démarche s'inscrit jusque dans le métadiscours de son écriture dans lequel il redéfinit le genre épistolaire en amont, notamment en altérant les règles de la circulation des textes et les modalités de leur composition ${ }^{12}$. Il se réapproprie ainsi la correspondance, ce qui va conditionner ses observations et sa production du discours sur la montagne. Letters from the Continent se présente comme une analyse de terrain qui appréhende le géographe comme sujet, qui, au fil de son itinéraire et de sa plume, construit l'objet géographique, à savoir ici, la moyenne et la haute montagne. Ruskin ouvre sa première lettre sur un métadiscours par lequel il expose la fonction de toutes ses lettres :

I mean to write my diary as much as I can by letter. It will amuse mama \& you, and be just as useful to me as if in a book. (Calais, Thursday evening [13 May]) ${ }^{13}$

10 Les lettres ont la vocation secondaire d'informer et de divertir ses parents, mais son père a surtout le rôle de conservateur de ces lettres que Ruskin considère comme un ouvrage (book) dont il prévoit de se servir après son retour (useful). Alors qu'il s'engage sur le continent, première étape du périple qui doit le mener en montagne, ce projet d'écriture, énoncé sur le mode performatif, envisage le retour ${ }^{14}$ en même temps que le voyage et la réception en même temps que l'écriture. Sur le plan spatial, la construction de sa connaissance sur la montagne se fait dans un double mouvement du pied et de la plume. Elle s'inscrit également dans une temporalité complexe qui met en regard dans un même projet d'écriture et dans un même voyage une expérience passée et une expérience en cours qui prépare déjà une rétrospection. Au fil des mots et du voyage, Ruskin met en place un mode de circulation complexifié et individualisé du texte et du voyageur, mode de circulation qui lui permet de poser un regard renouvelé sur la montagne.

11 Ruskin expédie ses observations et ses pensées au fil du trajet. Chaque missive témoigne d'une expérience isolée qui n'est pas écrite à l'aune d'un récit déjà commencé. Chaque randonnée est une expérience unique et c'est du recueil final des lettres qu'émane la diversité en montagne, car Ruskin n'en fait jamais un objet générique. Avant d'en arriver au stade du recueil, il lui faut un récepteur intime, idéal. C'est son père qu'il désigne pour tenir ce rôle et ce dernier sera quasiment son seul correspondant pendant tout le voyage. Dans un premier temps, le père donne une légitimité au genre, car la démarche de Ruskin consiste à envoyer les lettres pour pouvoir les lire dans un second temps. Le père est choisi comme vecteur de transmission des lettres donc il est moins le destinataire que le simple récepteur et 
conservateur. Par ailleurs, Ruskin redéfinit la démarche d'écriture mutuelle inhérente à la correspondance :

I think you may send letters of this kind. I needn't answer unless I like. ([Rheinfelden] 20 th May, Evening [Thursday] $]^{15}$

12 Sa correspondance n'est clairement pas un échange. S'il tient à ce que son père lui écrive, il n'a pas l'intention de lui répondre. Il limite l'échange à sa convenance personnelle, signe d'une indépendance d'esprit bien consciente marquée à la fois par une volonté de prise en charge totale de l'énonciation de toute la correspondance qu'il envoie, mais aussi de celle qu'il reçoit et sur les conditions de ses observations qui seront la base de son voyage et donc de ce qu'il aura à dire sur son expérience de la montagne et de l'art.

Ruskin devient donc très restrictif sur ce qu'il accepte de lire. Son discours prend un tour très directif envers son père, à qui il donne des consignes détaillées sur l'agencement et le contenu de son discours épistolaire :

Have all right yours of 16 th with letters, yours of 18 th with slips of Athenaeum, yours of 20 th, too short and no enclosures. Don't be short. I don't want any letters but yours, but I want them long. Put the things requiring answers always at the top, or in any separate place you like; or underline them, and I won't miss them and then please chat away as you used to do. (Rheinfelden, Sunday [23 May]) ${ }^{16}$

14 Avec cinq occurrences de l'impératif, deux formes négatives, et l'exigence manifestée par la sémantique de want, Ruskin modélise sa réception jusque sous la plume de son seul correspondant à qui il dicte la teneur de son discours. Enfin, but yours et them sont en italiques dans le texte original; Ruskin insiste sur son refus de lire quiconque à l'exception de son père. Il ne le laisse écrire qu'à la seule condition qu'il applique à la lettre ses consignes d'écriture. Ruskin a décidé de n'engager de dialogue avec personne. Le genre épistolaire tel qu'il le redéfinit ici passe de la correspondance à la rupture nécessaire qui contribue à maîtriser les influences potentielles sur sa réception et éviter ainsi de la polluer.

Ruskin prolonge cette démarche jusque dans le conditionnement de ses idées, lisant des journaux que son père lui envoie et dans lesquels il lit et surtout relit seulement ce qu'il y a dans son horizon d'attente. C'est le cas des articles sur les accidents de la voie ferrée, à laquelle il est farouchement opposé :

I have been reading railroad accidents in the Times till I've frightened myself. ${ }^{17}$

Ruskin ne veut pas que sa réception soit infléchie par un contact avec le milieu dont il est parti. Son horizon d'attente est un tableau qu'il compose lui-même par le dirigisme envers son père et le silence envers le reste du monde. Si sa réception de la montagne ne peut être que soumise à son expérience passée et à ses connaissances, ses textes montrent avec force un auto-conditionnement et un isolement maximal grâce auxquels il sera libre d'être « un faiseur de montagne », pour emprunter le titre de Debarbieux et Rudaz.

\section{Contexte du voyage}

Suivant une démarche de géographe de terrain, Ruskin va dans le Jura et dans les Alpes, observer par lui-même les endroits dont Turner a peint des paysages. Ceci lui donne l'occasion de critiquer les tableaux de Turner et de livrer sa propre représentation des mêmes espaces dont il en fait des objets géographiques. Pendant tout le $\mathrm{XIX}^{\mathrm{e}}$ siècle, en 
Grande-Bretagne, en effet, la géographie de cabinet est encore en vogue et s'oppose à la géographie de terrain émergeante ${ }^{18}$, celle des voyageurs et des explorateurs comme David Livingstone (1813-1873), Richard Burton (1821-1890) ou John Hanning Speke (1827-1864). Ruskin se distingue des géographes qui se limitent aux archives et aux sources secondaires pour faire des déductions logiques. S'il se documente lui aussi, il finit toujours par aller sur le terrain pour y relever des données.

Le voyage de Ruskin dans les Alpes se situe donc au cœur d'une époque riche en bouleversements sur la représentation de l'espace, mais comme beaucoup, il est persuadé que la connaissance de la montagne est fondamentale pour notre connaissance et notre rapport à la terre. A priori, pourtant, l'intention de Ruskin n'est pas une expédition scientifique :

Despite his inclination for rest, Ruskin's tour was affected by various interests and intentions. There was, first of all, his concern with "Turnurian topography", a term he used to refer to the imaginative, rather than factual, depiction of a scene. ${ }^{19}$

19 Il faut cependant la voir à travers le prisme du personnage qui travaille tous les jours pendant ce voyage censé le reposer. Il occupe son temps aux déplacements, à l'écriture, à la peinture et au dessin jusqu'à épuisement. Pour Ruskin, l'esthétique préside à notre mode d'observation et à la construction de notre connaissance. Si l'enjeu de son voyage est esthétique, son œuvre, Modern Painters, vise à prouver qu'à travers leurs représentations de la nature, les peintres de son époque révèlent, bien mieux que leurs prédécesseurs, la vérité de la beauté de la création divine. Pour y parvenir, c'est la qualité de l'observation, donc la sensibilité et la formation du sujet, qui doit transparaître dans la représentation des montagnes afin de leur rendre à cet espace la beauté sublime que d'autres ne sauraient apprécier.

Une préparation et un engagement total du sujet sont donc nécessaires pour saisir la beauté et en ce sens, la vérité du paysage. C'est pourquoi Ruskin demeure très strict sur la représentation littérale des montagnes. Il n'accepte aucun libre remodelage de la réalité naturelle. Un peintre ne doit pas se permettre d'ajouter une cime pour équilibrer la symétrie de son tableau, par exemple. La peinture a une valeur documentaire et le peintre n'a donc pas plus de liberté que le cartographe. Pour illustrer la valeur cartographique des peintures, citons l'exemple de Rheinfelden. Ruskin demande à son père d'aller voir les peintures de Rheinfelden produites par Turner à la National Gallery pour visualiser l'endroit où il se trouve :

If you want to see where I am, just call at the National Gallery as soon as you go back to town; I fancy you will be back before I leave; and ask Wornum to let you look at the frames Nos. $86,87,88,89,90 .{ }^{20}$

21 Ainsi, le travail de l'artiste, l'écriture du voyageur et la carte géographique participent de la démarche du géographe. La rigueur scientifique de Ruskin face à la composition du paysage se retrouve dans sa formation. Critique d'art et voyageur passionné, Ruskin est avant tout minéralogiste et géologue. Depuis l'âge de douze ans, il s'intéresse à la minéralogie et à la géologie dont il parle comme la passion de sa vie :

But fate having ordered otherwise, I mourn the loss to engraving less than that before calculated, or rather incalculable, one, to geology !21

Grâce à son père, il connaît déjà les travaux d'Horace-Bénédict de Saussure (1740-1799) dont les Voyages dans les Alpes (1779-1796 en 4 volumes) donnent déjà l'orientation de Ruskin vers le terrain plutôt que dans un cabinet. Rappelons que Ruskin compose même son dictionnaire de minéralogie : 
And then, fourthly, there was the unabated, never to be abated, geological instinct, now fastened on the Alps. My fifteenth birthday gift being left to my choice, I asked for Saussure's "Voyages dans les Alpes" and thenceforward began progressive work, carrying on my mineralogical dictionary by the help of Jameson's three-volume Mineralogy, (an entirely clear and serviceable book) comparing his descriptions with the minerals in the British Museum, and writing my own more eloquent and exhaustive accounts in a shorthand of many ingeniously symbolic characters it took me much longer to write my descriptions in, than in common text, and which neither I nor anybody else could read a word of, afterwards. 22

Il aide le Dr William Buckland (1784-1856), ami de la famille et professeur de géologie à Oxford en produisant des croquis de géologie qui sont versés au fond documentaire de l'université alors qu'il n'a que 19 ans. Il suivra ensuite les cours de Buckland à Oxford. Enfin, Ruskin produit lui-même des articles très scientifiques dont un "Notice Respecting some Artificial Sections illustrating the Geology of Chamouni" publié dans les Proceedings of the Royal Society of Edinburgh, IV (1857-8), 82-4 et dans Works XXVI 545-7. Il y produit une description de la composition des Alpes et décrit la nature minérale des strates qui composent le Mont-Blanc et la région de Courmayeur. Il publie par ailleurs un nombre substantiel d'articles en géologie et en minéralogie ${ }^{23}$ parmi lesquels on peut citer :

On the Forms of the Stratified Alps of Savoy (1863).

À l'image de la globalité de son œuvre, Letters from the Continent montre combien l'engagement de Ruskin dans le champ de l'esthétique ne laisse jamais de côté les questions géographiques car pour lui, les deux ne font qu'un. La logique de ses lettres, comme de son œuvre conserve la méthode de la libre observation du terrain, ce qui infléchit le discours qu'il produit.

En amont, cette méthode consiste à se documenter tout en gardant un esprit libre et critique et à ne jamais reléguer sa propre perception à l'arrière-plan. Ce sont les premières conditions de sa réception et de sa créativité dans le mode d'observation et de représentation des montagnes.

\section{De l'objet spatial à l'objet géographique}

La pensée ruskinienne se caractérise par son ambition d'accéder à une connaissance générale de la création et de la nature qu'il entend livrer au plus grand nombre. On retrouve cette démarche dans son observation de la montagne qui est guidée par l'idée de domination. Il emploie les termes de command (dominer dans les deux sens du terme) et distance à plusieurs reprises ${ }^{24}$. On le retrouve dans son mode d'écriture de la lettre, empreinte de sa maîtrise du genre épistolaire adaptée à son voyage et à son mode d'observation. La montagne, comme repère de verticalité et d'histoire immémoriale, doit être saisie dans son ensemble pour servir l'ambition de sa perception et de sa réflexion. C'est ce qui explique son intérêt pour la haute montagne qui seule, est à la hauteur de son projet philosophique. Il ne s'agit pas là d'une posture d'esthète romantique, mais bien d'une condition de travail du géographe comme l'indique sa vue de La Tour (Torre Pellice) où il donne une description détaillée de la vallée avec des distances précises " 28 miles as the bird flies ${ }^{25}$ » et où il décrit les villes avoisinantes qu'il dit saisir dans leur ensemble comme s'il consultait une carte : «all as clear as on a pocket map ${ }^{26} \%$. La domination revient à la connaissance parfaite et l'on retrouve ainsi command dans ses recommandations sur l'éducation des jeunes : 
I believe the essence of education, as given by old people to young people, to consist in giving them command of their minds and bodies; when the command is obtained - the young man or woman - perfect in Bodily and Mental Form - must choose for themselves the kind of knowledge they wish to acquire in the world - and the direction in which they will apply they powers. ${ }^{27}$

Dans ce qu'elle donne du sens au savoir dans sa globalité, Ruskin choisit donc pour sa part la domination des vastes espaces montagneux qui sert à la fois ses critiques d'art et la géographie physique qu'il nourrit de ses travaux de recherche et de ses observations en montagne.

Au fil de son voyage, Ruskin va constater sur le terrain ce que Turner a représenté en peinture. Malgré sa grande admiration pour l'œuvre de Turner dont il essaie de saisir la technique, Ruskin émet des critiques sur le manque d'adéquation entre la toile et l'espace :

The near side of the port with the garden and trees, must from the first have been drawn out of Turner's head, as there are large houses on that side (of the towers) which clearly date from the beginning of last century. But the terrible roguery is in the hills. No such hills are or ever were, in sight from Arona. They are gathered together, hill by hill - partly from the battes of Oleggio - partly from above the town here - partly from half way up the way near Baveno - and then all thrown out together in one grand imaginary chain. I have roughly sketched the real view from here; with some boats in the port - and I have daguerreotyped the towers; but I cannot quite apologise for Mr. Turner this time. Usually, his work is only inaccurate in detail in order to give a more complete impression of the place; but the "Arona" is far more beautiful than anything that can anywhere be seen, or fancied by plain people, on all lago Maggiore. It has made all the real lake look mean and blank, \& its mountains low, to me, in spite of the sweet pensive character which I enjoy in them, as I told you, more than ever. ${ }^{28}$

Pour Ruskin, l'art doit dire la vérité : "Truth, of course is always precious in art ${ }^{29}$ ». Il emploie donc le champ lexical de l'imagination dont il accuse Turner: "drawn out of Turner's head»; «one grand imaginary chain ». En observateur rigoureux, Ruskin avait déjà relevé quelques inexactitudes dans les tableaux de Turner: "his work is only inaccurate in detail ». Néanmoins, son admiration pour le plus talentueux des peintres ne pourrait pas le faire renoncer à ses principes de l'art comme document géographique : «but I cannot quite apologise for Mr. Turner this time ». Pour Ruskin, le voyageur-artiste a la responsabilité morale et presque scientifique de représenter la montagne telle qu'elle se compose dans la nature et va jusqu'à accuser Turner de malhonnêteté (roguery). Le commentaire de Ruskin alterne la critique contre Turner et ses propres observations qui le conduisent à la représentation du lieu réel sur un simple croquis : « I have roughly sketched the real view ». S'il n'a pas le talent de Turner, Ruskin se crédite au moins le mérite de produire un document fiable et emploie d'ailleurs l'adjectif (real) à deux reprises. Ruskin ne pardonne pas à Turner d'avoir mis son immense talent au seul service d'un paysage librement inspiré par la montagne. Si la montagne retient l'œil de l'artiste au fil de son parcours, il doit tout faire pour en révéler la beauté, qui, pour Ruskin, est synonyme de vérité. Tout comme le voyage en montagne, la littérature, la peinture et toute forme d'art participent donc de la production du savoir.

Peindre la montagne doit faire accéder un public à la connaissance de la montagne. La fiabilité de la représentation de la géographie physique doit correspondre au travail du cartographe. Ruskin donne d'ailleurs des tableaux de la National Gallery comme documents de référence pour que son père puisse le localiser depuis Londres.

If you want to see where I am, just call at the National Gallery as soon as you go back to town; I fancy you will be back before I leave; and ask Wornum to let you look at the frames 
Nos. 86, 87, 88, 89, 90 ; they are all very like except only that the town, which Mr Turner has made about the size of Strasburg, consists of one street and a few lanes, and what he has drawn as mountains are only the wooded Jura, but pretty in shape. ${ }^{30}$ simple de vérité générale : cette perception in situ lui arroge la légitimité du géographe de terrain. Cependant, il corrige par avance les erreurs de Turner. L'accumulation de comparaisons marque la rhétorique de son discours par lequel il renforce l'idée que la composition esthétique est indissociable du raisonnement géographique. Entre les montagnes et les représentations qu'en donne le peintre, Ruskin procède à trois types de comparaisons : "they are all very like» et "what he has drawn as mountains ». La première procède d'une forte similitude avec like dont l'étymologie indique «de la même forme, littéralement, dont le corps correspond parfaitement ", " having the same form, literally with a corresponding body ", soit la duplication et le réalisme nécessaires de la représentation des montagnes sur la toile. Par la suite, il fait deux autres comparaisons quantitatives. Dans la première, il donne des indications de taille et de nombre :

The town, which Mr Turner has made about the size of Strasburg only consists of one street and a few lanes.

Ruskin compare ici des données quantifiables. Il ne passe pas par des termes grammaticaux de comparaison; il met directement face à face deux séries de données pour constater qu'il n'y a pas de reflet en miroir alors que c'est là le travail du voyageur-artiste. Enfin, il fait une troisième comparaison: "what he has drawn as mountains are only the wooded Jura ». Contrairement à like qui indique une similitude de forme, as indique une identification que l'on pourrait gloser ainsi : «ce que Turner a dessiné en lieu et place de ». Ruskin reproche à Turner d'avoir pris la liberté de donner des caractéristiques de montagne à un espace géographique qui n'en est pas une ; à tout le moins, que Ruskin ne définit pas comme une montagne.

Ruskin pose ici la question du respect des échelles. L'emploi de l'adverbe only marque une prise de position subjective par laquelle Ruskin affirme que le Jura est bien moins qu'une montagne sans pour autant lui attribuer d'autre hyperonyme géographique. Il se contente du toponyme Jura pour le désigner. Ruskin s'engage ici à donner une définition de ce que la montagne n'est pas et ici, elle n'est pas un relief boisé. Il précise en effet : «only the wooded Jura ». Du point de vue de la géographie physique, ce détail a son importance. Notons d'ailleurs que wooded comble le déficit définitoire, car le toponyme Jura vient du celte Jor et du latin romain juris, qui tous deux signifient «forêt ${ }^{31}$ ». Ainsi, pour Ruskin, la saillance d'un relief dans le paysage ne suffit pas à en faire une montagne. Pour lui, mountain n'est pas un hyperonyme, il désigne uniquement la haute montagne, un endroit hostile et désert. Parmi les bases anthropologiques universelles de l'imaginaire de la montagne telles que les rappelle Debarbieux ${ }^{32}$, elle est le plus puissant repère de verticalité sur la terre. Elle assure le lien entre le souterrain et le céleste, explique Debarbieux. Elle est un repère bien plus puissant qu'un arbre, qu'une forêt. On retrouve d'ailleurs cette opposition entre "forêt " et "montagne " ailleurs dans la littérature comme chez Ann Radcliffe (1764-1823) dans The Mysteries of Udolpho (1794) et surtout dans The Romance of the Forest (1791). On comprend alors la démarche de Turner mais aussi celle de Ruskin dans ce qu'ils définissent ou non comme une montagne. Pour ce dernier, la saillance ne suffit pas à reconnaître un élément géographique comme une montagne. Dans son aspiration à une prise de hauteur, de 
distance, pour accéder à une observation globale et à une réflexion globale, seule la haute montagne reste le repère adéquat.

Au-delà de l'attrait pour le paysage, on retrouve dans la correspondance de Ruskin des parallèles notoires avec une approche constructiviste de la géographie avec toujours un fondement sur la science dure qu'est la géographie physique.

Les pionniers de la géographie ne se désignaient pas eux-mêmes comme des géographes. C'est l'histoire, qui au regard de leurs travaux fondateurs, les reconnaîtra comme des géographes. Les travaux de Paul Claval en histoire de la géographie humaine créditent à Ruskin la paternité de la discipline :

«Le rôle que nous attribuons à Ruskin dans l'éclosion de besoins nouveaux en matière de géographie est un rôle indirect, comme on le verra. Mais sa pensée influença directement certains des grands géographes du siècle dernier. Il est curieux de remarquer qu'il s'agit surtout de ceux qui, rompant avec le déterminisme, attribuent un rôle important à l'homme dans la transformation de la nature..$^{33}$ "

Elle s'inscrit à la fin d'une longue démarche qui part de la créativité et de la liberté d'observation de la montagne et des gens de l'Alpe. Pour Ruskin, la montagne est une matière physique dont il fait un objet géographique et un thème esthétique suivant un mode de pensée aux fondements similaires. De l'apprentissage par les œuvres à la constatation de la réalité de terrain pour laquelle il se met dans des conditions de son libre choix.

Nourri par ses précédents voyages, par ses lectures et par les travaux de Turner, l'horizon d'attente de Ruskin confronté à sa perception lui permet de proposer une redéfinition de la montagne dans une modalité discursive, à la fois empreinte de subjectivité mais également d'assertions à valeur générale. Par là, Ruskin s'avance avec une légitimité qu'il semble s'arroger, mais dont on peut constater qu'elle est tout à fait justifiée dans différentes branches de la géographie. À ces fins, il redéfinit le genre épistolaire, s'implique fortement dans l'énonciation de son discours et souligne ses connaissances de terrain dans sa correspondance. Les textes de Ruskin révèlent en lui " un faiseur de montagne " selon l'expression de Debarbieux et Rudaz, mais aussi un écrivain qui défait les montagnes lorsqu'au bout du parcours, entre l'horizon d'attente et le perçu sensoriel, il ne constate pas la concordance escomptée. C'est dans l'espace de ce décalage que Ruskin, littéralement, déplace les montagnes.

BIBLIOGRAPHIE

BAEDEKER Karl, Handbook for Travellers: Switzerland and the Adjacent Portion of Italy, Savoy, and Tyrol, Leipzig, Karl Baedeker Publisher, 1897. 
CLAVAL Paul, Essai sur l'évolution de la géographie humaine, Annales Littéraires de l'université de Besançon, Paris, 1964.

CLAVAL Paul, Histoire de la géographie, Paris, Presses Universitaires de France « Que sais-je ? », 1995. DARLING W., The Complete Letter-Writer, Edinburgh, Printed by and for W. Darling, 1778.

DEBARBIEUX Bernard, Tourisme et montagne, Paris, Economica, 1995.

DEBARBIEUX Bernard, «Les montagnes : représentations et constructions culturelles », Y. VEYRET (dir.), Les montagnes : discours et enjeux géographiques, Paris, SEDES, 2001.

DEBARBIEUX Bernard, « Présentation générale. De l'objet spatial à l'objet géographique », dans DEBARBIEUX Bernard, FOURNY Marie-Christine, L'effet géographique, Publications de la MSH-Alpes, Grenoble, 2004, p. 11-33.

DEBARBIEUX Bernard, RUDAZ Gilles, Les faiseurs de montagne : imaginaires politiques et territorialités, XVIII ${ }^{e}$-XXI ${ }^{e}$ siècle, Paris, CNRS éd., 2010.

ENGEL Claire Éliane, La littérature alpestre en France et en Angleterre aux XVIII et XIX siècles, Chambéry, Dardel, 1930.

HÉLARD André, John Ruskin et les cathédrales de la terre, Chamonix, Guérin, 2006.

HOIBIAN Olivier (dir.), L'invention de l'alpinisme, La montagne et l'affirmation de la bourgeoisie cultivée, 1786-1914, Paris, Belin, 2008.

MURRAY John, (1852), A Handbook for Travellers in Northern Italy Embracing the Continental States, London, John Murray, 1858.

oUNOUGHI Samia, « La traversée des cols alpins : analyse du discours de la liminalité », E-rea, éd. numérique, 14.1 | 2016, [URL : https://journals.openedition.org/erea/5531]

oUNOUGHI Samia, « Analyse du discours de la liminalité : Butler, de l'autre côté du Saint-Gothard entre passage et ancrage », ILCEA, éd. numérique, 28 | 2017, [URL : http://ilcea.revues.org/4099] PÉAUD Laura, La géographie, émergence d'un champ scientifique : France, Prusse et Grande-Bretagne, Paris, Éditions de l'ENS, 2016.

PILLET Fabien, «Que reste-t-il de l'École de Constance ? ", Études Germaniques, éd. numérique, 2011/3, n² 263, p. 763-781, [URL :https://www.cairn.info/revue-etudes-germaniques-2011-3page-763.htm?contenu=resume]

RADCLIFFE Ann, The Romance of the Forest [1791], Oxford, Oxford University Press, 1992.

REGNAULD Hervé, L'espace, une vue de l'esprit ?, Rennes, Presses Universitaires de Rennes, 1998.

REICHLER Claude, SDREGNO Emma, John Ruskin : Écrits sur les Alpes, Paris, Presses Universitaires de la Sorbonne, 2013.

RICOEUR Paul, Temps et récit, tome 3, Paris, Éditions du Seuil, 1985.

RUSKIN John, Letters from the Continent, University of Toronto Press, 1982.

RUSKIN John, « Notice Respecting some Artificial Sections illustrating the Geology of Chamouni », dans Proceedings of the Royal Society of Edinburgh, IV (1857-8), 82-4 also in Works XXVI 545-7, Cambridge, Cambridge University Press, 1857-58.

RUSKIN John, Letters from the Continent [1858], Toronto, University of Toronto Press, 1982. 
RUSKIN John, « Notes on the Shapes and Structure of some Parts of the Alps, with Reference to Denudation », T. Rupert Jones (ed.), The Geologigal Magazine, London, Longmas, Green \& Co, 1865. RUSKIN John, « On Banded and Brecciated Concretions », wOODWARD Henry (ed.), The Geological Magazine, London, Trübner \& Co., 1870.

RUSKIN John, Deucalion: Collected Studies of the Lapse of Waves and Life of Stones, volume I, George Allen, Sunnyside, Orpington, Kent, 1879.

RUSKIN John, « Notes for the Intended Continuation of Deucalion», E.T. Cook, WEDDERBURN Alexander (eds.), The Works of John Ruskin, London, George Allen, New York, Longmans, Green, and Co., 1906, p. 363-370.

RUSKIN John, « On the Distinctions of Forms in Silica » [1884], E.T. Cook, WEDDERBURN Alexander (eds.), The Works of John Ruskin, London, George Allen, New York, Longmans, Green, and Co., 1906, p. 373-391.

RUSKIN, John, Praetaria, London, George Allen, 1907.

VIVIÈs Jean, Revenir/devenir. Gulliver ou l'autre voyage, Paris, Éditions de la rue d'Ulm, 2016.

ZDANSKI Clarice, « Samuel Butler, Local Identity, and the Periodizing of Northern Italian Art: The Travel Writer-Painter's View of Art History », J. PARADIS (dir.), Samuel Butler, Victorian against the Grain: a Critical Overview, Toronto, University of Toronto Press, 2007.

\section{NOTES}

1. O. Hoibian, (dir.), L'invention de l'alpinisme, La montagne et l'affirmation de la bourgeoisie cultivée, 1786-1914.

2. http://www.alpine-club.org.uk/ac2/about-the-ac/mission

3. C. Zdanski, «Samuel Butler, Local Identity, and the Periodizing of Northern Italian Art: The Travel Writer-Painter's View of Art History », J. Paradis (dir.), Samuel Butler, Victorian against the Grain: a Critical Overview.

4. B. Debarbieux, Tourisme et montagne, p. 9.

5. S. Ounoughi, «Analyse du discours de la liminalité : Butler, de l'autre côté du SaintGothard entre passage et ancrage », URL : http://ilcea.revues.org/4099

6. J. Ruskin, Works, VII, 5, p. XIII-XIV.

7. B. Debarbieux, L'effet géographique, p. 25.

8. Terminologie adoptée par Reinhart Koselleck, Vergangene Zukunft. Zür semantik geschichtlicher zeiten. 1979; reprise par Paul Ricoeur en 1985 dans Temps et récit, tome 3, p. 377.

9. B. Debarbieux, L'effet géographique, p. 25.

10. F. Pillet, «Que reste-t-il de l'École de Constance ?», p. 763-781.

11. J. Ruskin, Praeteria, p. 173-174.

12. La correspondance est un genre très normé depuis le XVIII ${ }^{\mathrm{e}}$ siècle. On trouve nombre de manuels à cet usage comme W. Darling, The Complete Letter-writer.

13. J. Ruskin, Letters from the Continent, p. 3.

14. J. Viviès, Revenir/devenir. Gulliver ou l'autre voyage. 
15. J. Ruskin, Letters from the Continent, p. 10.

16. Ibid., p. 12.

17. J. Ruskin, Letters from the Continent, 1858, p. 83.

18. L. Péaud, La géographie, émergence d'un champ scientifique: France, Prusse et GrandeBretagne, p. 35-36.

19. J. Hayman in J. Ruskin, Letters from the Continent, p. XIII.

20. J. Ruskin, Letters from the Continent, p. 9.

21. J. Ruskin, Praeteria, p. 173.

22. J. Ruskin, Praeteria, p. 173-174.

23. On citera encore "Notes on the Shapes and Structure of some Parts of the Alps, with Reference to Denudation (1865) "; « On Banded and Brecciated Concretions" (1867-1870) ; «Deucalion: Collected Studies of the Lapse of Waves and Life of Stones » (1875-1883); «Notes for the Intended Continuation of Deucalion»; «On the Distinctions of Form in Sicilia » (1884). Tous ces travaux se trouvent dans son œuvre complète, volume 26 .

24. J. Ruskin, Letters from the Continent, p. 109-110 ; 139-145.

25. Ibid. p. 145.

26. Idem.

27. Ibid., p. 152.

28. J. Ruskin, Letters from the Continent, p. 85-86.

29. Ibid., p. 156-157.

30. J. Ruskin, Letters from the Continent, p. 9.

31. Dictionnaire Larousse.

32. B. Debarbieux, Tourisme et montagne, p. 9.

33. P. Claval, Histoire de la géographie, p. 112.

\section{RÉSUMÉS}

Cet article est une analyse interdisciplinaire (analyse du discours, anglistique, géographie, histoire) de Letters from the Continent (1858) de John Ruskin (1819-1900) et vise à montrer la contribution de cette figure influente $d u$ xIX siècle sur notre mode d'appréhension de la géographie des Alpes. La déconstruction des canons du genre épistolaire et les conditions qu'il s'impose pour circuler dans les Alpes dans un isolement unilatéral lui permettent de conditionner son regard sur la montagne et de critiquer les représentations qui en sont faites, notamment celles de William Turner dont il suit les traces pendant cinq mois en 1858. Il met ainsi au jour le rôle de l'écriture de voyage autant que de la peinture de paysage dans la production du savoir sur la montagne. 
AUTEUR

SAMIA OUNOUGHI

Maître de conférences en langue et littérature anglo-saxonnes, Université Grenoble-Alpes, LIDILEM 


\title{
Voyages pittoresques dans les anciennes Pyrénées : déambulations romantiques et villégiature de montagne (1800-1860)
}

\author{
Viviane Delpech
}

Cet article est publié dans le cadre du programme de recherche FEDER TCV-PYR (Thermalisme Culture Villégiature dans les Pyrénées) (Fonds FEDER UE/Région Occitanie) mené entre 2017 et 2020 par l'Université de Toulouse - Jean-Jaurès, l'Université de Pau et des Pays de l'Adour, l'Université de Perpignan-Via Domitia ainsi que les services régionaux de l'Inventaire du patrimoine d'Occitanie et de Nouvelle-Aquitaine.

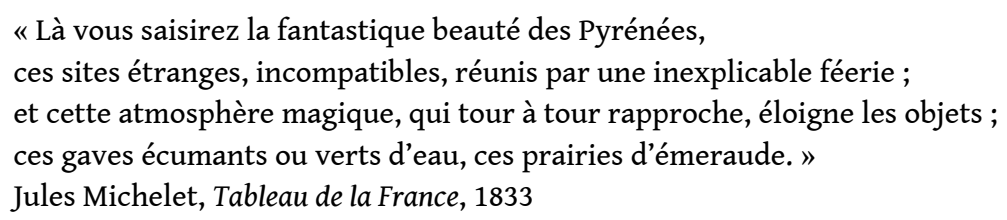

En 1795, dans sa Lettre sur l'art du dessin dans les paysages, François-René de Chateaubriand annonçait l'approche à la fois intuitive, réaliste et affranchie des normes qui inspira par la suite les artistes voyageurs romantiques. Cinq ans plus tard, Les Élémens de perspective pratique à l'usage des artistes de Pierre-Henri de Valenciennes (1750-1819) jetaient les jalons de la peinture paysagiste pyrénéenne. Par la suite, le paysage ne connote plus seulement la grandeur de la nature mais il magnifie aussi l'héritage des Anciens. Ainsi, en réaction au vandalisme révolutionnaire bafouant les souvenirs de l'Ancien Régime, un groupe d'intellectuels rassemblés autour du baron Isidore Taylor et de l'écrivain Charles Nodier lança une publication sérielle intitulée Les voyages pittoresques et romantiques dans l'ancienne France, qui donna son nom à une pratique excursionniste bientôt devenue phénomène de mode pour les élites intellectuelles et sociales ${ }^{1}$. Leur ambition visait à parcourir les territoires reculés de France afin d'en redécouvrir et de compiler le patrimoine matériel et immatériel mis en péril depuis la Révolution de 1789, et ce, dans l'optique de les préserver et de les sauver de la décrépitude. En parallèle, se développèrent les pratiques liées à la prise des eaux 
et au thermalisme moderne ${ }^{2}$ attirant graduellement la haute société et, dans son sillage, maints artistes, qui se plurent à représenter - et donc documenter - le patrimoine bâti et paysager et les populations de province. Dans les pas de grandes figures engagées comme l'Abbé Grégoire ou Prosper Mérimée, les Voyages pittoresques en tant que publication œuvrèrent à la définition de la notion de patrimoine durant la première moitié du XIXe siècle tandis que, comme pratique sociale, ils donnèrent lieu à une importante production artistique, particulièrement dans les Pyrénées où ils contribuèrent amplement à l'émergence du pyrénéisme formalisé en 1898 par Henri Beraldi ${ }^{3}$. Les villes d'eaux pyrénéennes furent en effet prisées dès le tournant du XVIII ${ }^{\mathrm{e}}$ siècle et à l'époque romantique grâce à l'aménagement routier, qui atteignit l'ensemble des principales stations thermales sous le premier Empire, ainsi qu'à une littérature médicale abondante et au diktat grandissant de la mode ${ }^{4}$. Cependant, l'étude du mouvement pyrénéiste met souvent en lumière l'approche cartésienne des voyageurs du second $\mathrm{XIX}^{\mathrm{e}}$ siècle qui, tour à tour géographes, géologues ou amateurs éclairés, ont fondamentalement enrichi la connaissance de ce massif montagneux ${ }^{5}$. La démarche intellectuelle des écrivains romantiques, transportés, à l'instar de George Sand, Victor Hugo et Hippolyte Taine, par le charme inspirant des hauteurs, s'avère également fort connue. Si l'on excepte le cas de Delacroix, les Pyrénées sont moins souvent évoquées pour leur dimension esthétique et symbolique, y compris chez des artistes complets comme Viollet-le-Duc, réduits dans ce contexte à leur rationalisme. Témoignant de la prise de conscience du patrimoine, dès la fin du XVIII ${ }^{e}$ siècle et jusqu'à la Monarchie de Juillet, le massif pyrénéen accueillit pourtant nombre d'artistes, albums à dessins sous le bras, peintres ou dessinateurs, travaillant l'aquarelle, la lithographie ou l'huile sur toile. Imprégnés du romantisme ambiant, ils offrent de ces lieux une vision équivoque, à la fois imaginaire et naturaliste, irrationnelle et scientifique, bien plus complexe qu'une étude purement morphologique du paysage. Ainsi Marguerite Gaston démontret-elle parfaitement l'ampleur et l'intérêt de la création picturale pyrénéenne durant la période romantique ${ }^{6}$. Dépassant les enjeux du renouveau de la peinture paysagiste et de l'histoire géographique, ces œuvres apportent un intéressant éclairage sur les déambulations physiques et intérieures de ces voyageurs esthètes, sur leur façon de vivre la montagne mais aussi sur leur perception fantasmée de la ruralité et du territoire pyrénéen. Dans la première moitié du XIX siècle, ces parcours initialement militants, dans la lignée des Voyages pittoresques, thérapeutiques - systématiquement motivés par la pratique de la cure ${ }^{7}$-, et de plaisance - liés à l'essor de la villégiature -, finissent par condenser les questionnements existentiels de la société industrielle naissante.

\section{Le triomphe de la nature promoteur de la villégiature}

\section{Des itinéraires balisés}

2 Pour les artistes romantiques, parcourir la montagne constitue un but en soi autant que l'agrément de leur voyage. Fréquentant les lieux déjà renommés de villégiature thermale, ils aiment à s'éloigner de ces points de convergence de la société mondaine pour s'imprégner de "l'authenticité » de la nature et de sa population afin d'éprouver le "sentiment de la montagne ${ }^{8}$ ». Aussi suivent-ils un itinéraire obligé qui les mène depuis les points névralgiques que sont ces stations vers des sites naturels de référence 
que, selon Valenciennes et les lois de la mode, tous doivent avoir vus et qui nourrissent leurs processus créatifs. Localisées principalement dans les Pyrénées centrales, les stations de villégiature irradient et exercent ainsi leur influence économique, sociale et artistique sur leur environnement naturel proche: des Eaux-Bonnes et des EauxChaudes, les artistes excursionnistes, loin d'être effrayés par la difficulté du terrain, se rendent au Pont d'Enfer, à la forêt de Gabas et au Pic du Midi d'Ossau ; depuis Argelès, ils rayonnent vers Saint-Savin, le Val d'Azun et les Gorges de Pierrefitte; de Cauterets vers le Pont d'Espagne, le lac de Gaube ou le Vignemale; de Bagnères-de-Bigorre, Campan et Barèges vers le Pic du Midi ou le Tourmalet; de Cambo vers la vallée de la Nive et le Pas de Roland; de Bagnères-de-Luchon vers les lacs d'Oô et d'Espingo ; ou encore, depuis Gèdre, ils visitent non sans mal, mais assidûment, le cirque de Gavarnie, le Marboré et la Brèche de Roland ${ }^{9}$; autant de sites grandioses que l'iconographie prépyrénéiste érige en motifs récurrents.

3 Par ailleurs, l'observation de ces parcours démontre que sous leurs apparences aléatoires, ces itinéraires sont en réalité bel et bien organisés, ne serait-ce que par la culture collective, et s'apparentent à un pèlerinage culturel élitiste où il faut avoir vu les chefs-d'œuvre de la nature et du patrimoine ${ }^{10}$. S'ils ont pour habitude de croquer et de dessiner sur le vif les paysages majestueux qu'ils contemplent, c'est souvent depuis les pensions rassérénantes de leurs points de chute, renouant avec le confort matériel auquel ils renoncent en montagne, qu'ils se posent pour finaliser leurs esquisses et rédiger leur journal de voyage; quoique, en excursion, ils sachent aussi se délecter de haltes pittoresques chez l'habitant. Malgré l'appel de la montagne et la transcendance romantique, ils bénéficient donc, de façon pragmatique, des infrastructures du tourisme naissant, en tête desquelles les hébergements et les établissements de loisirs et de thérapie ${ }^{11}$.

\section{La sublimation de la montagne}

Il n'en demeure pas moins que la nature constitue un thème iconographique central du romantisme et, partant, des voyages aux Pyrénées conduisant les artistes «dans les lieux les plus inconnus, les plus reculés ${ }^{12}$ » qui impliquent de dompter les sommets. Le regard porté sur la montagne, personnifiée et iconique à l'égal du ciel et de l'océan, oscille entre l'idéalisation et la célébration de ce qu'elle est intrinsèquement, deux modes de perception distincts que réunissent le principe du pittoresque - en son sens étymologique - et une démarche de sublimation et de sacralisation des éléments naturels. Compte tenu du contexte intellectuel, on réserve en outre une large place à l'émotion, ce que manifeste matériellement le recours fréquent aux outils de l'instantané et de la spontanéité que sont l'aquarelle et la mine de plomb ${ }^{13}$.

Dès lors, dans la continuité du classicisme, certains artistes n'hésitent pas à accentuer les formes, les hauteurs, l'élancement des montagnes, afin de les rendre plus majestueuses encore qu'elles ne le sont, à l'instar de Louis-François Lejeune (1775-1848), qui séjourne à Barèges, Ussat, aux Eaux-Bonnes et dont les travaux sont fortement empreints de sa formation académique ${ }^{14}$. Le lac d'Oô dans sa Chasse à l'Ours (fig. 1) s'apparente alors à un paradis perdu né de l'idéalisation de l'exotisme émergeant durant le siècle des Lumières. Il aurait pu illustrer la théorie du «bon sauvage » avec ses arbres bien plus fournis que dans la réalité, ses sommets arrondis, sa végétation luxuriante tandis que les animaux et les personnages proviennent, quant à 
eux, d'études académiques et de modèles standards. Lejeune crée à partir du paysage pyrénéen un monde idéalisé, une utopie où, tels les ours capturés au premier plan, la nature devenue inoffensive est maîtrisée par l'homme. Cette approche académique, qui vaut surtout dans la transition entre classicisme et romantisme, n'est cependant pas généralisée. Aussi un même motif peut-il faire l'objet d'interprétations variées et plus réalistes, qui, de par leur degré de précision graphique, renseignent sur l'histoire du paysage pyrénéen. Si Lejeune innove par le sujet, ses méthodes picturales, qui se doivent de l'enjoliver, demeurent traditionnelles. En revanche, d'autres comme Eugène Viollet-le-Duc (1814-1879), qui vit un véritable voyage initiatique en $1833^{15}$, ou Eugène Cicéri (1813-1890) ${ }^{16}$, tous deux ébahis par le caractère spectaculaire des paysages, la nature se suffit à elle-même et ne nécessite aucun artifice pour manifester sa beauté (fig. 2). CEuvrant à la célébration de la montagne, avec sa magnificence mais aussi ses failles et son essence chaotique, faisant émerger le genius locii, connectant l'homme à sa spiritualité profonde en cette cathédrale de la création, tout un ensemble de procédés picturaux contribue à la glorification des monuments naturels, à commencer par les cadrages qui évincent les plans intermédiaires et renforcent la sensation de hauteur et de vertige, ainsi que les couleurs et l'aquarelle, qui favorisent l'exactitude et l'instantané. Répondant à cette exaltation du monde propre à la pensée romantique et magnifiant sa quintessence prodigieuse, certains sites très inspirants ou d'autres détails générant l'enchantement de la nature, comme les torrents, les grottes ou la grandeur des espaces, ont les faveurs des artistes, à l'instar du lac d'Oô, du Mont-Perdu et du cirque de Gavarnie, lequel ressemble bien souvent au jardin d'Éden qu'il n'est pas. D'ailleurs, l'eau, avec la cascade ou le lac bienfaisant, constitue un leitmotiv symbolique assimilable à la source de vie. Le ciel, cristallisant les états d'âme et le discours de l'artiste, le fascine par ses effets de lumière et de variations atmosphériques, de même que ses tourmentes et ses nuages, le point du jour et le clair de lune, si bien que les hauteurs célestes, indissociables de la montagne infranchissable, contribuent à la stimulation de l'imaginaire. Par exemple, La route de Saint-Just-Ibarre à Mauléon par Viollet-le-Duc évoque le cadre dans lequel l'artiste et son compagnon de route vivent une situation ubuesque, interrogés par un suspicieux contrebandier dans une petite guérite au cœur d'une tempête mémorable ${ }^{17}$. Sublimant à son tour les Pyrénées, Adrien Dauzats (1804-1868), dont les dessins illustrent plusieurs volumes des Voyages pittoresques, hésite entre l'idéal académique et le réalisme émergent ${ }^{18}$. Ses représentations de paysages pyrénéens, avec leurs jeux de lumière, leur ciel chargé et en mouvement, confinent parfois au mysticisme, telle sa Vallée supérieure du Gripp, sorte de vision miraculeuse où les rayons du soleil surgissent prodigieusement de l'arrière de la montagne en perçant les nuages comme par magie (fig. 3). Ses réalisations, notamment L'une des sources de l'Adour (fig. 4), se rapprochent des compositions apocalyptiques de John Martin (1789-1854), comme La destruction de Sodome et Gomorrhe (1852), ou des cieux tourmentés telle l'âme profonde de William Turner (1775-1851) alors que, rappelant ceux de Lejeune, son dessin des arbres trahit le trait académique. Parachevant le portrait de cette nature vivante, les œuvres de Dauzats et de ses confrères recèlent fréquemment des animaux endémiques, tels les isards, les biches et autres rapaces (fig.4), qui confèrent davantage encore de poésie à la nature et en extraient savamment la « fantaisie sauvage ${ }^{19} »$. 
Fig. 1. - Louis-François Lejeune, La chasse à l'ours. Huile sur toile, 152 × 181 cm, 1834.

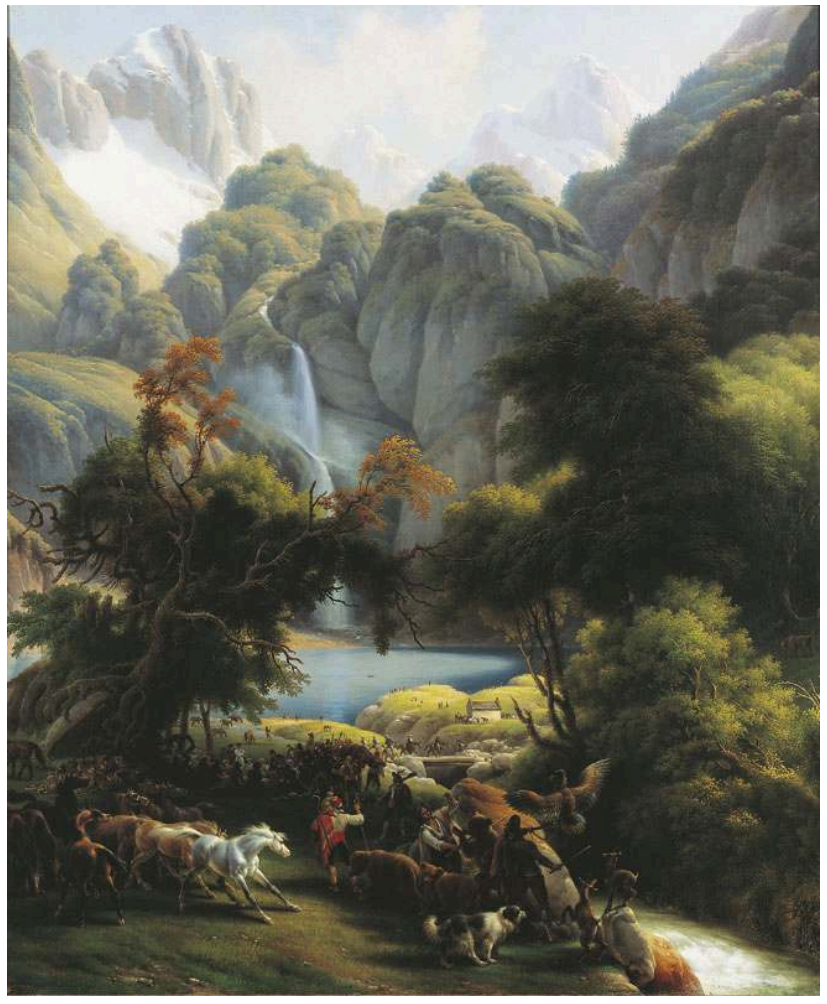

Inv. 2000-7-7, Musée des Augustins ( Daniel Martin

Fig. 2. - Eugène Viollet-le-Duc, Le lac d'Oô (ou Séculéjo). Aquarelle, 1833.

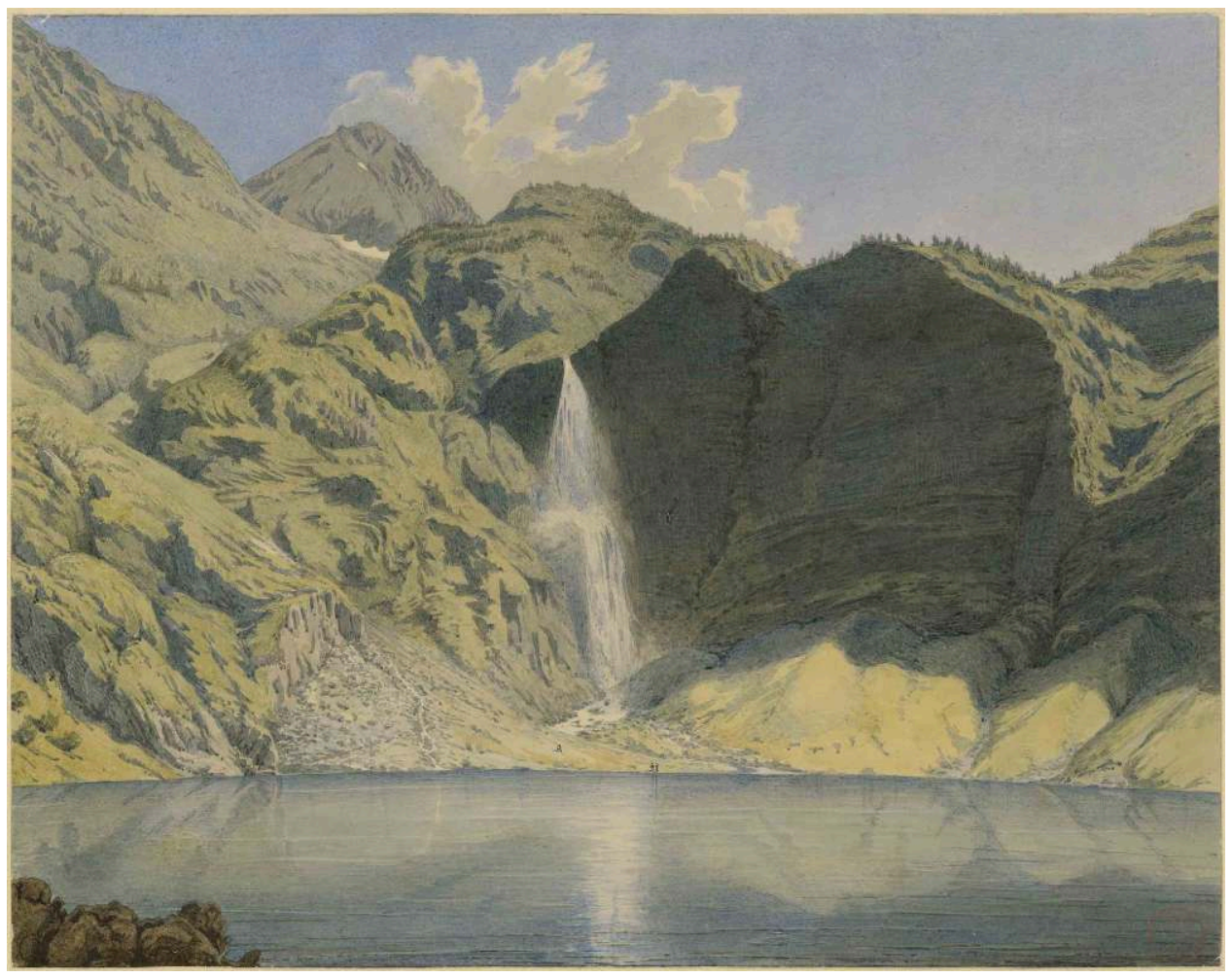

(c) Ministère de la culture - Médiathèque de l'architecture et du patrimoine 
Fig. 3. - Adrien Dauzats, Vallée supérieure du Gripp. Lithographie.

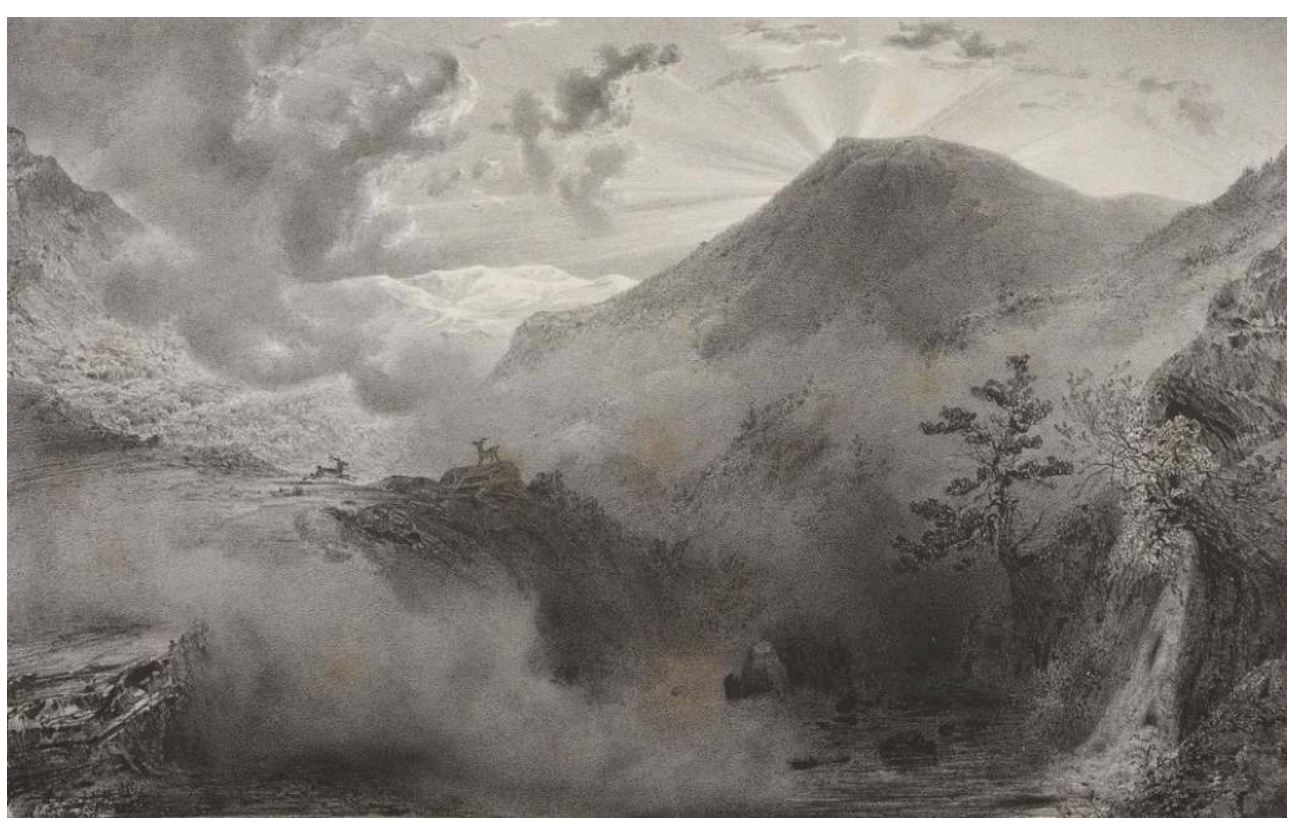

Extrait de Nodier C., Taylor I., Cailleux A. (de), Voyages pittoresques et romantiques dans l'ancienne France, vol. Languedoc, t. 2, pl. 224 (c) Libre de droits

Fig. 4. - Adrien Dauzats, L'une des sources de l'Adour. Lithographie.

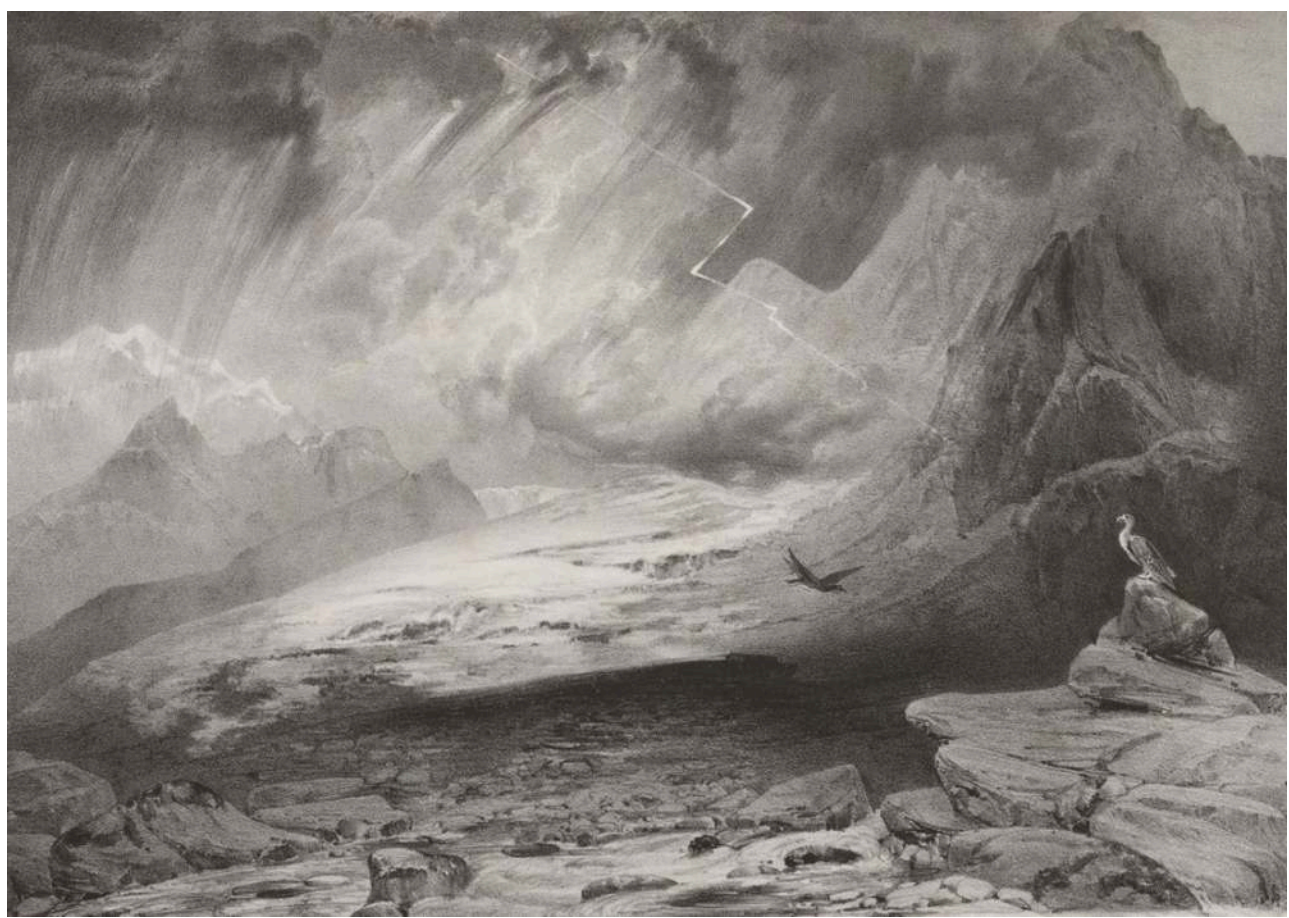

Extrait de Nodier C., Taylor I., Cailleux A. (de), Voyages pittoresques et romantiques dans l'ancienne France, vol. Languedoc, t. 2, pl. 220 (c) Libre de droits

6 En tout état de cause, au-delà de célébrer le patrimoine paysager de leurs destinations, les artistes diffusent une image méliorative et fantasque du territoire pyrénéen. À l'aube du XIXe siècle, aux côtés des médecins, ils participent dès lors, délibérément ou 
indirectement, à la promotion des stations thermales et à l'essor des séjours de plaisance, et ce, d'autant plus que nombre de leurs œuvres sont présentées au Salon annuel, lors d'expositions ou éditées dans les guides d'excursion, les récits de voyage illustrés et autres publications « pittoresques » en vogue ${ }^{20}$.

\section{Récit de l'histoire et invention culturelle}

Dans les œuvres romantiques, le paysage, témoignant du lien indivisible entre nature et culture, fait l'objet de représentations pour lui-même mais il favorise aussi l'exégèse du passé et de la mémoire locale et universelle. Ses interprétations iconographiques traduisent l'évolution du regard porté sur le déroulement de l'histoire, désormais perçue comme une longue continuité et non plus comme une juxtaposition d'évènements. La fuite du temps, qui préoccupe fondamentalement le romantisme, se matérialise en des solutions picturales proclamant l'insignifiance de l'homme face à la nature insaisissable.

Dans cette perspective, le culte du passé et de l'identité régionale et nationale s'exprime par la récurrence des vestiges ${ }^{21}$, habiles conteurs des temps révolus, de constructions et de traces du présent. L'emploi fréquent de l'aquarelle et de la mine de plomb et le geste instantané inscrivent l'œuvre dans un processus de mémorisation immédiate et de fabrique du souvenir. Les allusions à l'histoire de la civilisation se confondent alors avec le moment personnel et intime, présent et ressenti, de l'artiste. Les ruines et l'architecture de l'Ancien Régime, évoquant la puissance seigneuriale et ecclésiastique d'antan, constituent une motivation primordiale de la publication des Voyages pittoresques et des itinéraires de découverte qui en découlent afin de raconter ce que la société révolutionnaire, héroïque, a paradoxalement mis en péril, voire détruit ${ }^{22}$. Ainsi les artistes pré-pyrénéistes contribuent-ils à cette grande entreprise de réconciliation de l'homme post-révolutionnaire avec lui-même tout en offrant, malgré eux, à la société de loisir émergente la substance culturelle vouée à son épanouissement et son divertissement ${ }^{23}$.

Mû par l'exaltation romantique, surtout durant sa jeunesse, Viollet-le-Duc compose, dans cet esprit, des œuvres profondes, mettant en scène des ruines vers Argelès, l'église de Bossost ou les cabanes de Tramezaïgues ${ }^{24}$. Ces fameux refuges de montagne inspirent quantité d'artistes comme Émilien Frossard (1802-1881) (fig. 5) parce qu'elles incarnent le motif pittoresque par excellence, sublimant le passé, la tradition et une prétendue authenticité. Lejeune croque, pour sa part, l'église de Luz-Saint-Sauveur, «bâtie par l'ordre des Templiers ${ }^{25}$ », dont la vue en contre-plongée accentue le caractère magistral et glorifie ses mythiques et mystiques pères fondateurs. De façon générale, et plus spécifiquement concernant le patrimoine bâti, les cadrages revêtent une essentielle fonction discursive, car ils relèvent d'une complexe réflexion scénique, esthétique et philosophique. Les ruines vers Argelès par Viollet-le-Duc, sises au premier plan, et le couvent de Saint-Savin par Louise-Joséphine Sarazin de Belmont (1790-1871) (fig. 6) laissent admirer la montagne, à la fois protectrice et menaçante, et des siècles d'histoire humaine. Ainsi, sont légion les vues d'ensemble de villages comme Barèges (fig. 7), amas infimes nichés au cœur de la montagne, nourris par elle et livrés à sa merci, qui laissent entrevoir la tension ressentie par le visiteur profane en proie à la fascination aussi bien qu'à la terreur qu'elle inspire, en une "sorte d'équilibre des contraires $^{26} »$. 
Fig. 5. - Émilien Frossard, Pic du Midi.

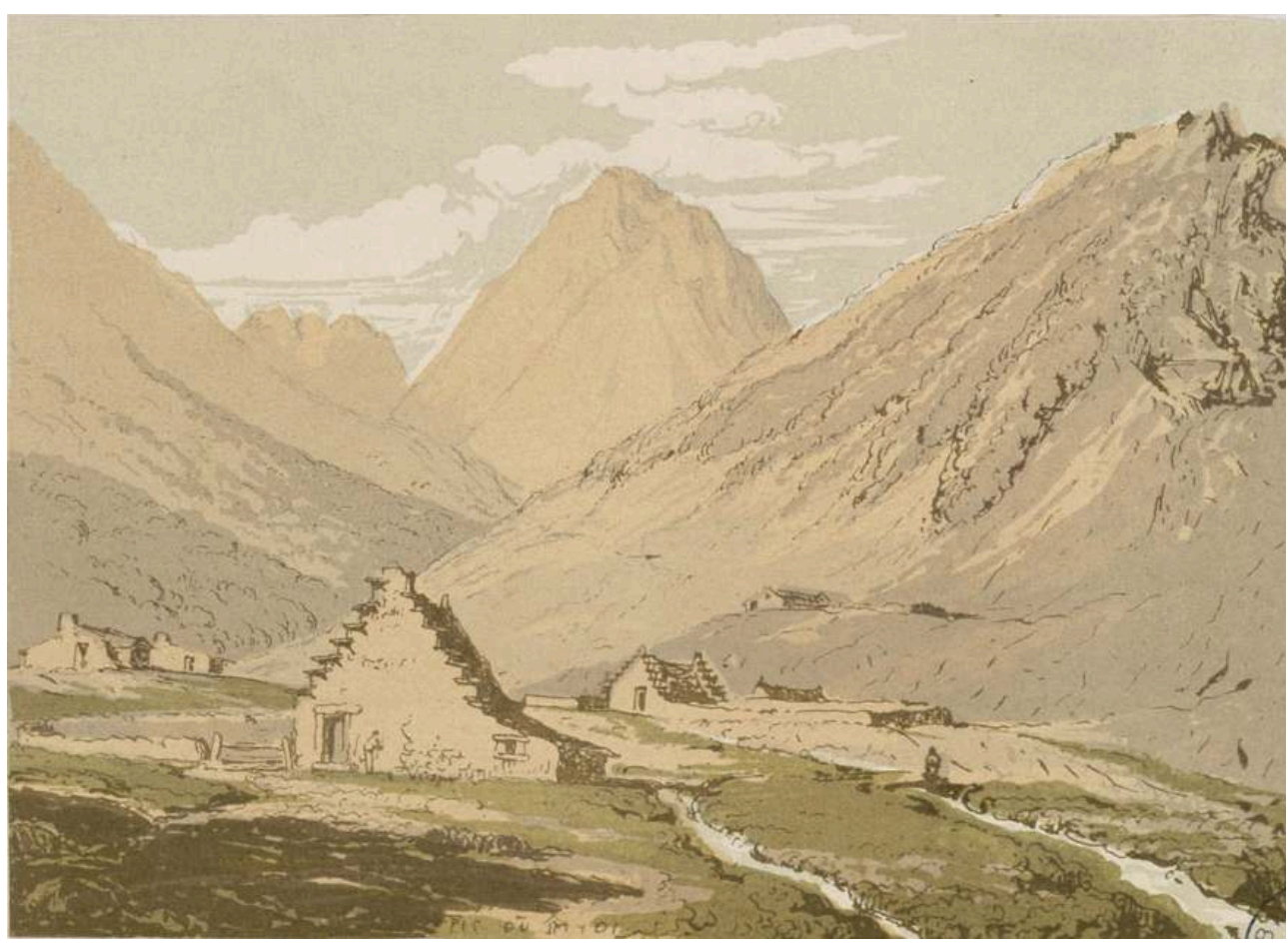

Reproduction imprimée en couleurs d'aquarelle, A-Frossard (2-9) ( Bibliothèque municipale de Toulouse-Domaine public

Fig. 6. - Louise-Joséphine Sarazin de Belmont, Vue du couvent de Saint-Savin et de la vallée d'Argelès. Huile sur toile, $104 \times 65 \mathrm{~cm}, 1831$.

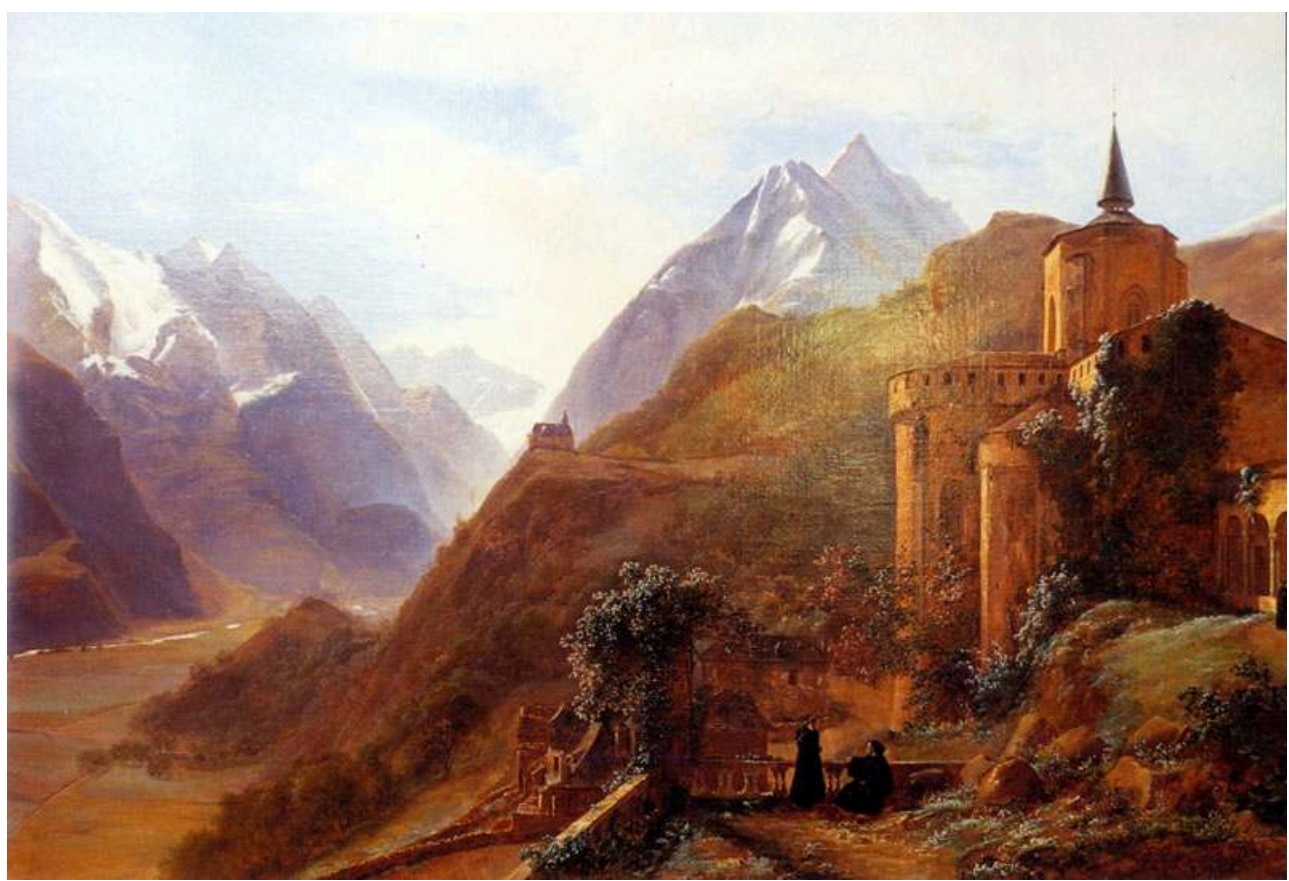

Inv. RO 257, Musée Salies, dépôt Musée des Augustins (c) Ville de Toulouse - Musée des Augustins 


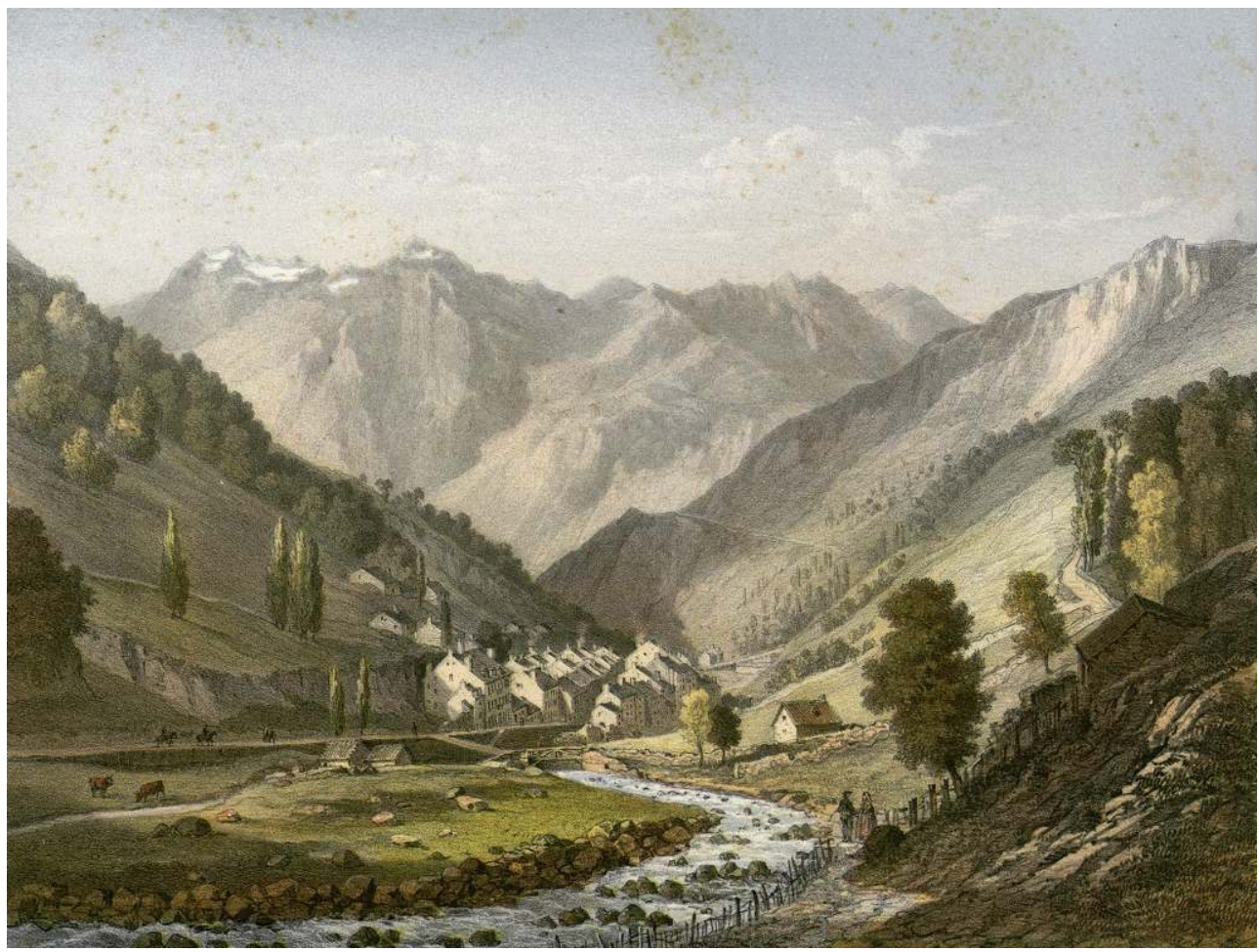

Extrait de La France de nos jours, pl. 336 (c) Bibliothèque municipale de Toulouse-Domaine public

Comme les sites naturels, le patrimoine bâti, et à travers lui l'histoire universelle, est donc vivement célébré par les romantiques à l'échelle nationale et internationale. Toutefois, là encore, et eu égard à la forte diffusion de leurs œuvres par le biais de la lithographie et des expositions, ils en livrent la matière profonde à la promotion touristique, suivant et enrichissant les itinéraires de visites d'agrément destinés à la clientèle bourgeoise en villégiature, qui profite des mêmes destinations qu'eux. Au séjour thérapeutique, conditionné par la prise des eaux et les promenades de santé dans les parcs et aménagements prévus à cet effet, s'adjoignent ainsi les visites culturelles évoquant un passé lointain ravivé par les romantiques, un récit national et régional que s'approprie l'économie thermale en plein essor. C'est bien, en ce sens, la méditation exaltée sur le passé, la contemplation opposée au tumulte de la société urbaine, que cultivent les guides touristiques jusqu'à la Première Guerre mondiale ${ }^{27}$, à l'image de l'Itinéraire des Hautes-Pyrénées (1818) par Arnaud Abbadie (1797-1870), qui, aux côtés des grottes, lacs et autres monastères, compile quelques contes historiques, et, surtout, du Guide Richard (1834), dont les développements romanesques - souvent plagiés - sont connus de la plupart des voyageurs aux Pyrénées ${ }^{28}$.

\section{L'homme et la société industrielle au cœur de l'univers}

Le patrimoine naturel et architectural compose un cadre idyllique où évolue la figure humaine, omniprésente dans l'iconographie des Pyrénées et au cœur du romantisme, qu'elle soit traitée en simple élément du paysage ou comme sujet en soi. Cette démarche relève $d u$ "romantisme social ", qui voit émerger divers stéréotypes humains, réducteurs par définition, captivant l'imaginaire des artistes ${ }^{29}$ et donnant à 
voir la nature animée, voire incarnée par ses habitants qu'elle modèle. Si l'homme croit posséder la montagne, les effets de proportions démontrent la domination et la toutepuissance divine de celle-ci. La population pyrénéenne, rurale et donc pittoresque, est généralement représentée en pleine activité par les archétypes de l'agriculteur, du bouvier, du berger, de la lavandière ou du contrebandier, car, plus que l'illustrer, il s'agit de définir, d'après les canons nationaux, le peuple autochtone, ce à quoi s'attelle scrupuleusement Lejeune en $1829^{30}$. De fait, les romantiques mettent l'actualité en images à leur façon en idéalisant les usages exogènes qu'ils amalgament au passé. Malgré l'intérêt documentaire de leurs œuvres, les autochtones sont ainsi les ambassadeurs bien involontaires d'une vision archaïque de leur mode de vie et de l'idéologie nationaliste de rigueur. Cette perception ethnocentrée et, pour partie, illusoirement philanthropique repose sur une dualité schématique opposant deux mondes, la rusticité et l'urbanité, le dur labeur et l'oisiveté, la sincérité et la superficialité, le salut et la damnation. Plaçant l'individu au centre de l'univers, certains voyageurs n'hésitent pas à brosser leur autoportrait, ou du moins à dépeindre la figure de l'artiste en situation, comme Viollet-le-Duc chutant dans un torrent ${ }^{31} \mathrm{ou}$, plus tard, Henry de Triqueti (1803-1874) illustrant un dessinateur au travail ${ }^{32}$, faisant ainsi converger l'expérience personnelle et la réalité de l'exotisme. Comme le suggère Viollet-le-Duc dans sa correspondance et son dessin du Val d'Azun ${ }^{33}$, ces représentations matérialisent et se font l'écho des réflexions existentielles caractéristiques du romantisme, s'interrogeant sur le sens de la vie, sur l'histoire, sur une forme de transcendance irrationnelle ${ }^{34}$. Ainsi les albums de dessins, les journaux de voyage et les écrits épistolaires témoignent-ils du cheminement physique et intellectuel de l'artiste, de son parcours initiatique. Intégrée dans le paysage, la figure humaine cristallise ses propres atermoiements, d'autant plus que peindre la montagne signifie nécessairement de l'avoir vaincue dans la douleur ${ }^{35}$.

Ceci étant, lorsqu'ils s'intéressent aux individualités, c'est pour ce qu'elles représentent d'exotisme et de dépaysant. Les études ethnographiques sont systématiques chez ces voyageurs de bonne famille, en attestent les travaux d'Eugène Delacroix (1798-1863), Eugène Devéria (1805-1865), Pierre Gorse ${ }^{36}$ (1816-1875) et des sœurs Blanche Hennebutte Feillet (1815-1886) et Hélène Feillet (1812-1889), qui comptent parmi les exemples les plus emblématiques alors que les albums de Gavarni (1804-1866), James Duffield Harding (1798-1863) et Édouard Pingret (1785-1869) en constituent la documentation graphique de référence à l'époque. Tous subliment la vaillante femme pyrénéenne et s'accordent sur le "héros montagnard» incarné par le berger ou le contrebandier ${ }^{37}$, ce qu'illustrent les portraits idéalisateurs d'Alfred Dartiguenave (1821-1885) (fig. 8). Relevant de la scène de genre, tant appréciée du romantisme et qui fera les beaux jours du réalisme, les thèmes bucoliques et collectifs, tels les fêtes traditionnelles, les moments de vie populaire et les transhumances, favorisent la scénographie et la diffusion des mœurs tout en s'adonnant à une fondamentale réflexion esthétique sur le mouvement, les matières et la lumière. Bien connu des pyrénéistes, Guillaume-Sulpice Chevalier, dit Gavarni, compose ainsi des vues enchanteresses, dont le Cirque de Gavarnie (fig. 9), avec son refuge pittoresque et ses montagnards en habits traditionnels, bâton de marche en main, ménageant une pause bien méritée sur les rochers, ou le Paysage des Pyrénées (1827) et sa foule paisible ${ }^{38}$. Les drapés et les couleurs des costumes stimulent fortement Delacroix, qui rend hommage aux femmes avec ses Deux Ossaloises, vêtues de costumes chamarrés, foulards noués sur la tête ${ }^{39}$, ou encore Devéria avec ses paysannes des Eaux-Bonnes (fig. 10). Les visages 
révélant les ethnotypes interpellent les artistes, qui, tels Delacroix, Devéria ou Violletle-Duc, en brossent des portraits naturalistes louant leur noblesse naturelle et, au grand dam des partisans de l'académisme, élevant ces sujets ordinaires aux rangs les plus prestigieux de la hiérarchie des genres en peinture. Comme Léon Soulié (1804-1862) avec son Poste de frontière dans les Pyrénées ${ }^{40}$ ou dans les dessins de Dauzats ${ }^{41}$, les autochtones endossent souvent une double fonction discursive et didactique dans la composition, en illustrant d'une part le caractère typique de la région et en s'insérant d'autre part en tant que points de repère rendant lisibles les proportions extraordinaires des paysages.

Fig. 8. - Alfred Dartiguenave, Contrebandiers aragonais. Lithographie, 1855.

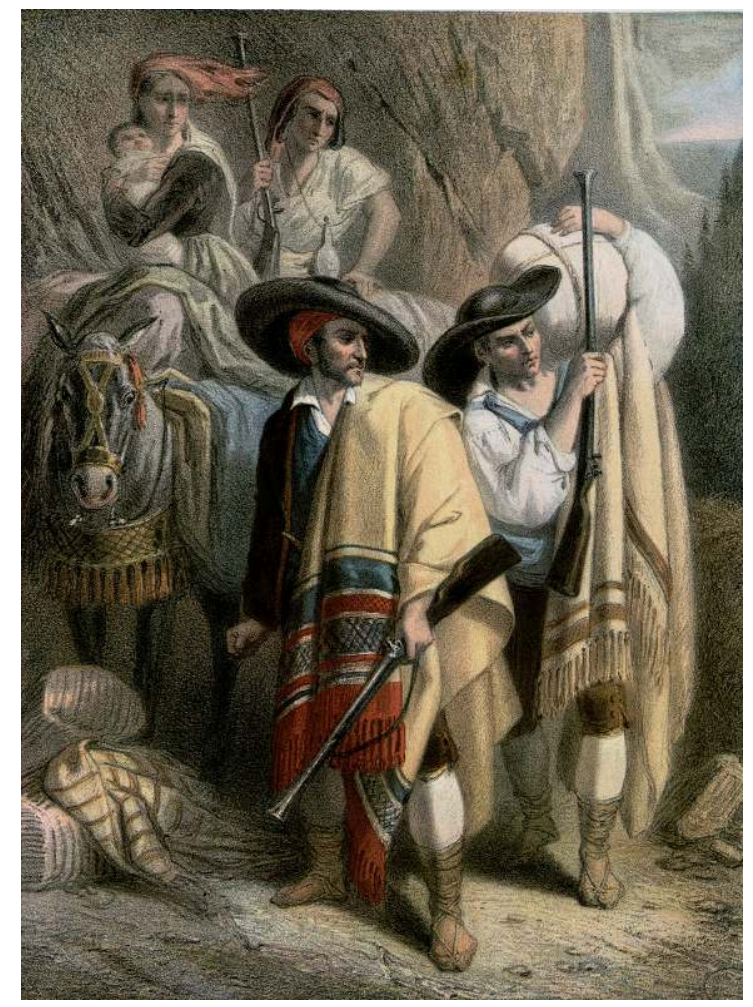

Inv. 55-7-1 (c) Bibliothèque municipale de Toulouse-Domaine public 
Fig. 9. - Guillaume-Sulpice Chevalier, dit Gavarni, Cirque de Gavarnie, 1829.

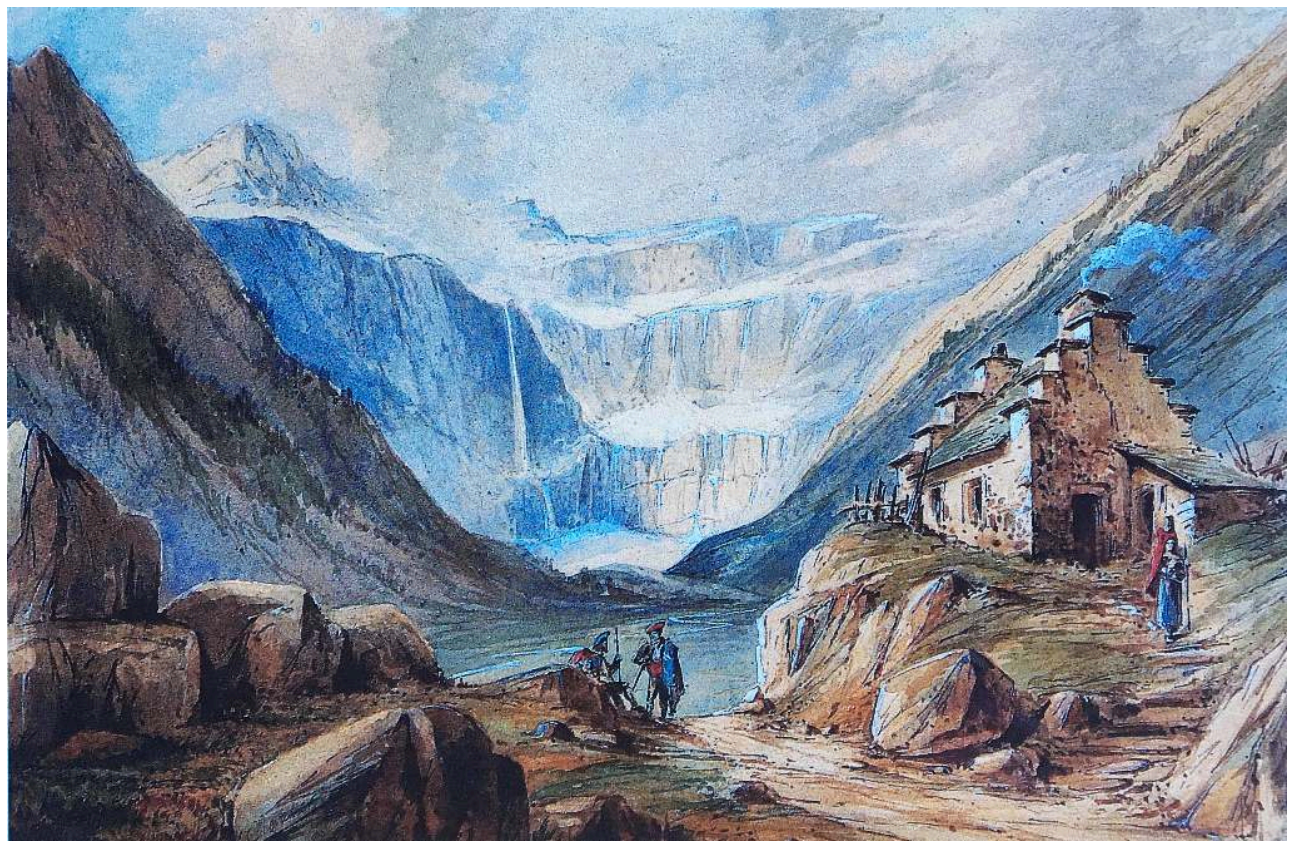

(c) Musée Pyrénéen, Lourdes

Fig. 10. - Eugène Devéria, Costume des Eaux-Bonnes (femmes portant un grand panier sur la tête). Lithographie, 1844.

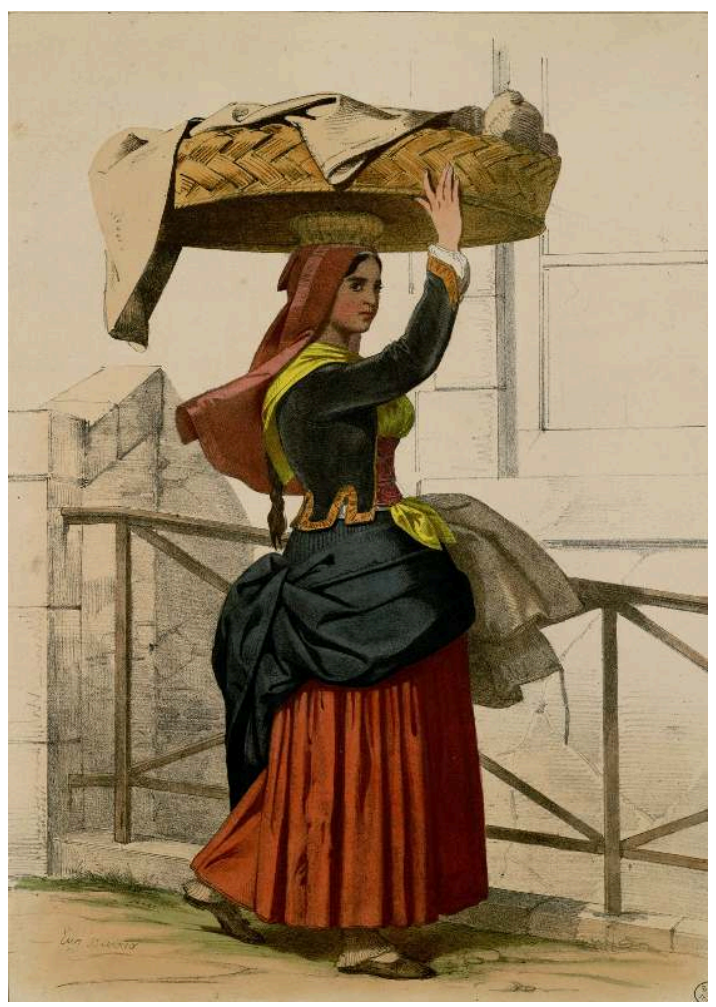

(C) Bibliothèque municipale de Toulouse-Domaine public

13 Au demeurant, la découverte de cet environnement et de sa population s'apparente aux explorations des terrae incognitae plus lointaines dans le contexte impérialiste. Les 
observateurs aguerris, quoique amateurs que sont les voyageurs aux Pyrénées, exploitent des méthodes similaires d'étude ethnographique inspirées de la démarche géographique multidisciplinaire théorisée par Alexander von Humboldt (1769-1859) qui, du reste, s'intéressa aussi aux peuples pyrénéens -, où l'on cherche à décrypter tous les mécanismes du monde en puisant dans l'ensemble des sciences ${ }^{42}$. Les pléthoriques études de costumes et de types physiques produites par les romantiques impliquent un regard extérieur et potentiellement biaisé, parfois involontairement condescendant, en tout cas ethnocentriste et ne résultant jamais, ou rarement, des autochtones eux-mêmes. Fondée sur la raison et l'onirisme, nourrissant l'imaginaire romantique et sa quête de progrès et de savoir, l'observation de l'homme pyrénéen parachève le processus de patrimonialisation énoncé dans les Voyages pittoresques en honorant le folklore et les traditions menacés d'oubli suite à l'action révolutionnaire et son empreinte jacobine. Mais, paradoxalement, loin d'annihiler la modernité, cette nostalgie du passé vise au contraire à la construire ${ }^{43}$. En plein engouement de la découverte de l'altérité, les artistes romantiques aux Pyrénées enrichissent en effet la connaissance du territoire national dans les pas du Tableau de la France dressé par Jules Michelet en $1833^{44}$.

\section{Apparat social et illusion de l'altérité}

14 À trop rechercher l'authenticité dans le dépaysement, ce qui relève d'une quête de connaissance mais aussi de distraction et d'épanouissement personnel, les artistes se risquent à recomposer des images "préconstruites » émanant de leur imaginaire collectif et d'un conditionnement intellectuel. Non sans émerveillement, ils viennent in situ confirmer des représentations artificielles qui résultent des réflexions de l'élite nationale, d'autant que leur nomadisme d'agrément témoigne et découle d'un statut privilégié, dont la possibilité de voyager est un marqueur social. D'ailleurs, la haute société en villégiature reproduit son organisation hiérarchique urbaine le temps d'une cure et ne cultive avec la population locale qu'un rapport mercantile ou logistique, réduit à l'achat de souvenirs ou aux prestations de guides d'excursion. Certaines stations privilégiées, en particulier Bagnères-de-Bigorre devenue «l'Athènes des Pyrénées ", reconstituent même, sous l'affluence, un microcosme culturel effervescent réunissant la société artistique et littéraire selon les usages de la capitale ${ }^{45}$. Au final, les artistes peuvent être captivés moins par la réalité d'une culture vivante que par la réitération des émotions suscitées par l'exotisme ${ }^{46}$ ou par la nécessité mondaine. En leur attribuant une valeur patrimoniale sacrée, ils instaurent une sorte de muséification des communautés pyrénéennes jugées en voie de disparition. Comme ils dressent les inventaires archéologiques visant à la restauration de la France féodale, ils procèdent au recensement des costumes et des coutumes, les albums de paysages côtoyant ceux de l'habillement, l'indigène devenant une curiosité et un patrimoine à protéger autant qu'un agrément supplémentaire de voyage destiné à la société industrielle avide de distraction. De fait, le Guide de Madame Joudou ${ }^{47}$ (1818) et le Guide Richard dépeignent avec force détails les mœurs et les vêtements éclatants pyrénéens ${ }^{48}$ au moment où Michelet incite à visiter la foire de Tarbes qui donne à voir un concentré de «toutes les races et tous les costumes des Pyrénées ${ }^{49}$ ». Par la force des choses, ces observations conditionnées par la culture collective, impliquant un regard distancié sur une société perçue comme "moins évoluée ", se destinent à une élite autoproclamée et réduisent les peuples pyrénéens à des attractions culturelles au même titre que les 
ruines ou les sites naturels d'exception, tous - pierres, lacs, arbres, hommes, femmes étant promus par la lithographie, support publicitaire et objet souvenir par excellence jusqu'au second Empire ${ }^{50}$. De la même façon que l'on admire alors les chefs-d'œuvre de l'art à Rome ou Florence, on s'émerveille de ceux de la tradition au sein du musée vivant à ciel ouvert que deviennent les contrées pyrénéennes. Sans remettre en cause la sincérité des romantiques, car certains, tels Viollet-le-Duc et Hippolyte Taine, exècrent et démystifient l'ambiance mondaine des stations, cet intérêt envers l'humain et l'ailleurs relève du jeu des apparences sociales parce qu'il a valeur de témoignage et prouve la véracité du voyage ${ }^{51}$. L'idée de la représentation sociale se lit aisément dans les mœurs urbaines transposées vers le «microcosme thermal " autant que dans son architecture formatée, si bien que «l'espace fictif de la station fut souvent dénoncé ${ }^{52}$ ». Selon l'exigence implicite de leurs usages, les artistes romantiques clament ainsi "qu'ils y étaient » et ont vécu la rencontre de bon ton de l'altérité, tout comme leurs confrères - ou eux-mêmes - en Orient et en Afrique. Cette équivoque quête d'évasion et de transcendance intellectuelle et spirituelle, qu'il faut replacer dans son contexte sociopolitique, reflète en définitive les hésitations d'une époque charnière mue entre progrès techniques fulgurants, avancées sociales inédites sur le plan des libertés individuelles, résistance au changement et prise de conscience des excès que ces enjeux supposent.

\section{Épilogue : du roman romantique pyrénéen au mythe du thermalisme}

15 Voyager dans les Pyrénées selon la démarche pittoresque implique en somme des degrés de lecture dépassant les apparences premières. L'artiste romantique contribue d'une part à l'inventaire monumental entrepris sous la Restauration et renseigne sur le patrimoine de l'Ancien Régime de même que sur l'histoire des paysages pyrénéens. Il propose des œuvres instantanées et documentaires, qui se présentent avec plus ou moins d'exactitude à un stade donné de leur évolution. Un second niveau d'analyse fait surgir, d'autre part, les sentiments animant cet esthète contemplatif vis-à-vis du monde qui l'entoure. Après avoir glorifié l'histoire de ses pères, il fait l'éloge de la nature, création transcendantale digne de perfection. En gravissant les contreforts indomptables d'autrefois, il atteint les sommets de sa propre quête intérieure et assoit la montagne en objet sacré, mythique et hiératique, dont la redescente équivaudrait, dans son ressenti existentialiste, à une sorte de chute du paradis originel. L'artiste est alors d'autant plus submergé et envahi par le sentiment de la montagne que celle-ci lui offre grâce et plénitude, fascination et effroi, avant de lui permettre de se révéler à luimême et de mettre à nu ses croyances profondes.

Cependant, les voyages pittoresques ne concernent qu'une infime partie de la société du premier xix esiècle, en l'occurrence la notabilité parisienne, dont les artistes sont les émissaires délibérés ou non et produisent des témoignages matériels pérennes trahissant une vision ethnocentrée valable tant aux confins du monde que de l'imaginaire. Cet état de fait se révèle par l'iconographie humaine qui, en illustrant les us et coutumes de cet ailleurs à la fois si proche et si lointain, aboutit souvent à une image stigmatisante de la population locale. Généralement issue de la haute société, cette communauté artistique en plaisance est d'autant plus subjuguée par les mœurs et les costumes traditionnels indigènes qu'elle évolue dans un microcosme confortable et 
illusoire se désintéressant des autres sphères sociales de son propre environnement quotidien.

Ainsi, ces voyageurs en révèlent beaucoup sur leurs intentions personnelles mais aussi sur la culture collective d'une certaine élite sociale et intellectuelle à un moment clé de l'histoire française, partagée entre l'héritage encyclopédiste des Lumières et le tumulte de l'industrialisation. Bien plus qu'ils ne le laissent entendre, ils contribuent à la promotion du massif pyrénéen et de ses villégiatures thermales à l'échelle nationale et internationale, ce qui répond aussi de la quête plus pragmatique et géopolitique de la maîtrise des espaces à l'heure de l'expansion impérialiste. Qu'ils le veuillent ou non, leurs itinéraires et leurs destinations sont calqués sur ceux de la bourgeoisie urbaine et révèlent l'influence et le pouvoir de la culture collective et de la " comédie humaine ", à laquelle ils participent à leur corps défendant, sur leur appréhension du voyage et des autochtones. Ces pratiques interrogent somme toute sur les motivations paradoxales de ces nomades à la fois oisifs et studieux, en pleine ascèse et à la rencontre de leur prochain, dont les qualités de voyageur et de touriste se confondent et s'entrecroisent. Témoignant d'une nouvelle perception du monde et d'un mouvement de pensée inédit centré sur l'humain, l'identité et l'histoire, l'iconographie des voyages pittoresques concourt de façon essentielle à l'élaboration de l'image onirique des stations thermales pyrénéennes, lieux d'évasion du corps et de l'esprit, à l'écriture du « roman romantique des Pyrénées » et à la genèse du mythe moderne de la montagne et du culte des eaux.

\section{BIBLIOGRAPHIE}

BERALDI Henri, Cent ans aux Pyrénées, 1898-1904, (7 vol.).

CLARAC Marie, « Henry de Triqueti, gentleman voyageur et paysagiste ", dans 1848-1849. Une saison aux Pyrénées. Dessins de voyage d'Henry de Triqueti, Pau, Musée des Beaux-arts de Pau, 2010, p. 52-69. DALZIN Claire, PENENT Jean, Gouffres, chaos, torrents et cimes, les Pyrénées de peintres, Toulouse, Musée Paul Dupuy/Éditions Privat, 2007.

DELPECH Viviane, «Viollet-le-Duc à la découverte des Pyrénées en 1833. Une science romantique », dans DEBOFLE Pierre, SANCHEZ Jean-Christophe (dir.), Pays pyrénéens et environnement, Société Ramond/Fédération Historique des Pyrénées, 2016, p. 481-496.

DUVIOLS Jean-Paul, MINGUET Charles, Humboldt, savant citoyen du monde, Paris, Gallimard, 1994. FOUCART Bruno, « Isidore Séverin Justin Taylor, découvreur et inventeur culturel », dans La Fabrique du romantisme. Charles Nodier et les Voyages pittoresques, Paris, Musée de la Vie romantique/Flammarion, 2014, p. 69-77.

FOUCART Bruno, "La montagne dans la peinture française au XIX siècle », dans Le sentiment de la montagne, Grenoble/Paris, Glénat/Musée de Grenoble/Éditions de la RMN, 1998, p. 29-35.

FOUCART Bruno, « La Mission Taylor-Dauzats dans l'Ancienne France », dans FOUCART Bruno (dir.), Adrien Dauzats et Les Voyages pittoresques et romantiques dans l'ancienne France du baron Taylor, Paris, Fondation Taylor, 1990, p. 3-47. 
FOURCASSIÉ Jean, Le romantisme et les Pyrénées, Paris, Gallimard, 1940.

GASTON Marguerite, Images romantiques des Pyrénées, Lourdes, Les Amis du Musée Pyrénéen, 1975.

JARRASSÉ Dominique, Les thermes romantiques. Bains et villégiatures en France de 1800 à 1850, ClermontFerrand, Publications de l'Institut d'Études du Massif Central, 1992.

JAZÉ-CHARVOLIN Marie-Reine, «Les stations thermales : de l'abandon à la renaissance. Une brève histoire du thermalisme en France depuis l'Antiquité », In Situ. Revue des patrimoines, éd.

numérique, $\mathrm{n}^{\circ} 24,2014$,

[URL : https://insitu.revues.org/11123]

MARQUEZ Christophe, «Le général baron Lejeune (1775-1848), peintre pyrénéiste », dans DEBOFLE Pierre, SANCHEZ Jean-Christophe (dir.), Pays pyrénéens et environnement, Société Ramond/

Fédération Historique des Pyrénées, 2016, p. 497-508.

MICHELET Jules, Tableau de la France. Géographie physique, politique et morale, Paris, Lacroix, 1875 (1 1 ère édition, 1833).

NODIER Charles, TAYLOR Isidore, CAILLEUX Alphonse (de), Voyages pittoresques et romantiques dans l'ancienne France, Paris, Firmin Didot, 1820-1878, 24 volumes (Languedoc : tome I, 1833-1834 ; tome II, 1835 ; tome III, 1837).

RAUCH André, « Le voyageur et le touriste », In Situ. Revue des patrimoines, éd. numérique, $\mathrm{n}^{\circ} 15$, 2011, [URL : https://insitu.revues.org/533]

SAULE-SORBÉ Hélène, « Les Pyrénées. Marches et démarches de peintres », dans BERDOULAY Vincent (dir.), Les Pyrénées. Lieux d'interaction des savoirs (XIX'-XX ${ }^{e}$ siècles), Paris, Éditions du CTHS, 1993, p. 98-113.

VAILLANT Alain (dir.), Dictionnaire du romantisme, Paris, CNRS Éditions, 2012.

VANDER GUCHT Daniel, Ecce homo touristicus. Identité, mémoire et patrimoine à l'ère de la muséalisation du monde, Paris, Quartier libre, 2006.

\section{NOTES}

1. C. Nodier, I. Taylor, A. de Cailleux, Voyages pittoresques et romantiques dans l'ancienne France.

2. M.R. Jazé-Charvolin, «Les stations thermales : de l'abandon à la renaissance. Une brève histoire du thermalisme en France depuis l'Antiquité ", [https:// insitu.revues.org/11123].

3. H. Beraldi, Cent ans aux Pyrénées.

4. J. Fourcassié, Le Romantisme et les Pyrénées, p. 51-53.

5. H. Saule-Sorbé, « Les Pyrénées. Marches et démarches de peintres », p. 98-113.

6. M. Gaston, Images romantiques des Pyrénées.

7. D. Jarrassé, Les thermes romantiques, p. 251.

8. J. Fourcassié, Le romantisme et les Pyrénées, p. 308-312.

9. M. Gaston, Images romantiques des Pyrénées, p. 87-101 et plan annexe.

10. D. Vander Gucht, Ecce homo touristicus, p. 103-104.

11. Ibid., p. 103. 
12. J. Fourcassié, Le romantisme et les Pyrénées.

13. M. Gaston, Images romantiques des Pyrénées, p. 147-163.

14. C. Marquez, «Le général baron Lejeune (1775-1848), peintre pyrénéiste», p. 497-508.

15. V. Delpech, «Viollet-le-Duc à la découverte des Pyrénées en 1833. Une science romantique », p. 481-496.

16. Médiathèque Pau-Pyrénées, cote Ee3211, Les Pyrénées: dessinées d'après nature et lithographiées par E. Cicéri, album de 36 lithographies.

17. V. Delpech, «Viollet-le-Duc à la découverte des Pyrénées en 1833. Une science romantique », p. 481-496.

18. B. Foucart, « La Mission Taylor-Dauzats dans l'Ancienne France », p. 3-47.

19. B. Foucart, « La montagne dans la peinture française au XIX $X^{\mathrm{e}}$ siècle », p. 29-35.

20. M. Gaston, Images romantiques des Pyrénées, p.60-80. D. Jarrassé, Les thermes romantiques, p. 246.

21. Ibid., p. 103-121.

22. B. Foucart, «Isidore Séverin Justin Taylor, découvreur et inventeur culturel », p. 69-77.

23. D. Vander Gucht, Ecce homo touristicus, p. 111.

24. Médiathèque de l'architecture et du patrimoine, Fonds Viollet-le-Duc, cotes 41076 à 41125, vues des Pyrénées, 1833.

25. Musée Paul Dupuy, Inv. 008-12-1, L.-F. Lejeune, carnet de croquis, 1829.

26. B. Foucart, « La montagne dans la peinture française au XIX siècle », p. 29-35.

27. A. Rauch, "Le voyageur et le touriste», In Situ, revue des patrimoines [https:// insitu.revues.org/533]

28. J. Fourcassié, Le Romantisme et les Pyrénées, p. 68-72.

29. Ibid., p. 325-347.

30. Musée Paul Dupuy, Inv. 008-12-1, L.-F. Lejeune, carnet de croquis, 1829.

31. Médiathèque de l'architecture et du patrimoine, Fonds Viollet-le-Duc, cote 2014/024/105, E. Viollet-le-Duc, lettre du 28 juin 1833.

32. M. Clarac, « Henry de Triqueti, gentleman voyageur et paysagiste », p. 52-69.

33. V. Delpech, «Viollet-le-Duc à la découverte des Pyrénées en 1833. Une science romantique », p. 485-489.

34. B. Foucart, « La montagne dans la peinture française au XIX siècle », p. 29-35.

35. H. Saule-Sorbé, « Les Pyrénées. Marches et démarches de peintres », p. 98-113.

36. Pierre Gorse, originaire de Gironde, installé à Pau, a fixé, comme spécialiste de la lithographie en camaïeu, de nombreux panoramas des principales stations pyrénéennes, notamment dans ses Pyrénées monumentales et pittoresques publiées vers 1860.

37. M. Gaston, Images romantiques de Pyrénées, p. 123-140.

38. C. Dalzin, « Guillaume-Sulpice Chevalier dit Gavarni », dans Gouffres, chaos, torrents et cimes. Les Pyrénées des peintres, p. 42-43. 
39. E. Delacroix, Deux Ossaloises, aquarelle, gouache, mine de plomb, $34 \times 26.2 \mathrm{~cm}$, Chicago Art Institute.

40. C. Dalzin "Léon Soulié », dans Gouffres, chaos, torrents et cimes. Les Pyrénées des peintres, p. 38-39.

41. Par exemple, Vue générale de Saint-Savin, dans Voyages pittoresques..., vol. Languedoc, t. 2, pl. 192 et Cabanes de Tramezaïgues, vol. Languedoc, t. 2, pl. 223.

42. J.-P. Duviols, C. Minguet, Humboldt, savant citoyen du monde.

43. A. Vaillant, « Du romantisme à la modernité », p. LXXVIII-XCI.

44. J. Michelet, Tableau de la France. Géographie physique, politique et morale.

45. D. Jarrassé, Les thermes romantiques, p. 238-239.

46. D. Vander Gucht, Ecce homo touristicus, p. 107-112.

47. Le guide du voyageur aux bains de Bagnères, Barèges, Saint Sauveur et Cauteretz, attribué par Beraldi à une Madame Joudou, est l'œuvre de Jean-Baptiste-Marie Joudou (1778-1859), journaliste et historien tarbais.

48. J. Fourcassié, Le Romantisme et les Pyrénées, p. 68-73.

49. J. Michelet, Tableau de la France. Géographie physique, politique et morale, p. 31.

50. D. Jarrassé, Les thermes romantiques, p. 254.

51. D. Vander Gucht, Ecce homo touristicus, p. 114-115.

52. D. Jarrassé, Les thermes romantiques, p. 241.

\section{RÉSUMÉS}

Dans la première moitié $d u \mathrm{XIX}^{\mathrm{e}}$ siècle, les Pyrénées deviennent un but de voyage et de réflexion esthétique à la mode pour les artistes romantiques. Dépassant les enjeux du renouveau de la peinture paysagiste et de l'histoire géographique, leurs œuvres apportent un intéressant éclairage sur leurs déambulations physiques et intérieures, sur leur façon de vivre la montagne mais aussi sur leur perception fantasmée de la ruralité et du territoire pyrénéen. Ces parcours initialement militants, dans la lignée des Voyages pittoresques, thérapeutiques - systématiquement motivés par la pratique de la cure -, et de plaisance - liés à l'essor de la villégiature -, finissent par condenser les questionnements existentiels de la société industrielle naissante. La célébration de la montagne et de ses habitants contribue cependant à la promotion touristique des villégiatures thermales et cultive l'art de la représentation sociale de leurs cercles d'origine.

\section{AUTEUR}

\section{VIVIANE DELPECH}

Chercheur, projet FEDER TCV-PYR, Université de Pau et des Pays de l'Adour 
La montagne : lieu de résistance aux règles de l'Académie des beaux-arts et thème de prédilection des artistes de la modernité au tournant du siècle

\author{
Véronique Richard-Brunet
}

\title{
La résistance aux règles académiques de l'Académie des beaux-arts
}

1 Les artistes de la fin du XIX ${ }^{\mathrm{e}}$ siècle les plus avancés, précurseurs d'un art qui s'interroge sur lui-même, sur sa nature, son rôle et sa destination, sont liés au concept de modernité qui émerge, dès 1850 , pour désigner les grands changements survenus après les révolutions technique et industrielle. La modernité est alors perçue, par les théoriciens d'une avant-garde balbutiante, comme la manifestation d'un mode de pensée, de vie et de création, basé sur le changement, et non plus sur les traditions anciennes d'une institution. L'Académie des beaux-arts, membre de l'Institut de France, créée le 21 mars 1816, entend, en effet, perpétuer les principes esthétiques des Académies royales de peinture et de sculpture fondées à Paris en 1648. Sous son égide, les écoles d'art dispensent aux élèves une formation scientifique (géométrie, anatomie et perspective) et humaine (histoire et philosophie), et leur enseignent les techniques et les savoir-faire nécessaires à l'élaboration d'une œuvre. Elles transmettent les diktats d'une culture classique, attachée à la recherche de l'idéal du beau et de l'essence éternelle des choses, que les artistes suivent pour produire l'art des salons officiels, obtenir des commandes publiques, et s'attirer les faveurs d'une clientèle fortunée ${ }^{1}$.

2 En 1863, toutefois, un vent de révolte souffle chez les artistes parisiens, car le jury du Salon de peinture et de sculpture, désigné par les membres de l'Académie, refuse plus 
de 3000 œuvres sur les 5000 envoyées à l'institution. Les postulants exclus, dont Antoine Chintreuil (1814-1873) ou Édouard Manet (1832-1883), critiquent vigoureusement l'intransigeance des membres du jury du Salon et réclament un lieu d'exposition pour montrer leurs œuvres au public parisien. Informé du conflit, l'Empereur Napoléon III décide de financer une exposition « des Refusés », qui doit se tenir au Palais de l'Industrie à Paris. Cet évènement obtient un certain succès, malgré la polémique née de la présentation d'un tableau provocateur, Le Déjeuner sur l'herbe de Manet, qui met sur le devant de la scène la question de l'émancipation des peintres.

De ce fait, trop conscients des limites qui leur sont imposées, nombre d'artistes décident de se former dans des ateliers privés, dont l'enseignement est moins conventionnel que celui des Beaux-Arts, dans le but d'affirmer leur talent. C'est le cas du jeune Paul Cézanne, qui a échoué au concours d'entrée de l'École des beaux-arts de Paris, en 1861, en raison d'un tempérament coloriste jugé excessif, et qui va suivre les cours de l'Académie de Charles Suisse, en 1862, où il rencontre Alfred Sisley (1839-1899), Camille Pissarro (1830-1903), Claude Monet (1840-1926) et Auguste Renoir (1841-1919). Ces derniers refusent les règles académiques et veulent baser les principes de leur création sur leur sensibilité, s'emparer de sujets déclarés jusqu'alors triviaux. Enfin, ils s'intéressent à la nature d'une façon plus libre que leurs aînés, cherchant à transcrire les variations de la lumière, la fluidité des formes, pour animer leurs tableaux, suivant les postulats des peintres installés à Barbizon, à partir de 1850 .

\section{Paul Cézanne (1839-1906), face à la Sainte-Victoire, contre l'art des bourgeois}

4 Pour donner plus de force à son travail, Cézanne écarte les teintes sombres et les nuances trop lisses, façonne un modelé qui lui est propre, constitué de touches divisées, susceptibles de traduire la richesse de sa perception, comme de révéler les aspects les plus secrets de la nature. Une peinture jaillie de l'intérieur, stimulante, celle «des tripes ", totalement désavouée par l'Académie des beaux-arts, voit ainsi le jour. Paul Cézanne cherche, à tâtons, un art solide, équilibré, structuré, porteurs de valeurs stables, universelles. Des années s'écoulent avant que le style cézanien ne s'affirme. Certes, l'artiste a toujours recours aux lois plastiques qui sous-tendent toute œuvre d'art, comme la loi de contraste des formes et des couleurs, la loi de composition et de représentation du sujet, mais il veut les utiliser autrement pour découvrir la vraie nature de la peinture et lui conférer une structure durable. Cézanne côtoie Pissarro et Armand Guillaumin (1841-1927) et participe, le 27 décembre 1873, à la fondation de la Société anonyme coopérative des artistes-peintres avec Edgar Degas (1834-1917), Monet et Renoir. Lors de la première exposition impressionniste de 1874, chez le photographe Nadar (1829-1910), le public réserve un accueil peu encourageant, voire scandalisé, aux toiles de Cézanne qui, dès lors, déserte de plus en plus souvent la capitale.

5 À partir de 1876, il se réfugie dans le Midi, et séjourne à L'Estaque, petit port environné d'une nature encore préservée, où il peint des tableaux pour son ami Victor Chocquet (1821-1891). Ainsi, la plupart des œuvres qui sont montrées à la troisième manifestation du groupe impressionniste, en 1877, ont été exécutées à L'Estaque. Le public est toujours hostile à l'art de Cézanne, jugé malhabile, brutal, mais l'artiste s'obstine dans ses recherches. À Paris, il peint un portrait, qui, plus tard, sera considéré comme l'un de ses chefs-d'œuvre, Madame Cézanne à la robe bleue, qui détonne par une gamme de tons 
bleus très poussée, mais aussi par une déclinaison de verts remarquables. Cézanne se voit comme l'artisan d'un art nouveau qui rejette toute convention bourgeoise, toute concession aux «effets " à la mode, et c'est dans une tenue d'ouvrier, cotte bleue et veste de toile blanche couverte de taches de peinture, qu'il travaille. En 1881 et 1882, l'artiste s'installe avec sa famille, à Pontoise, près de Pissarro, avec lequel il découvre les nouvelles théories de la couleur, celles du chimiste français Eugène-Michel Chevreul (1786-1889) et du physicien américain Ogden Rood (1831-1902), qui nourrissent sa réflexion. Cette même année, il est admis au Salon, se déclarant l'élève d'Antoine Guillemet (1841-1918) de l'école de Barbizon, mais il poursuit sa quête, de plus en plus solitaire. Il peint, désormais, en appliquant des touches juxtaposées, et accentue la technique du clair-obscur pour obtenir des effets descriptifs forts sur ses toiles. Les paysages sont construits par plans successifs, suivant une perspective aérienne, déjà utilisée par les impressionnistes, et constitués d'une succession de traits et de lignes disjointes, qui décrivent de façon de plus en plus synthétique les objets ou les figures. En 1883, Cézanne en sait assez pour suivre, seul, son chemin, et il décide de rentrer chez lui, en Provence, pour pousser plus loin ses investigations, dans l'atelier de la demeure familiale. L'artiste développe sa technique en travaillant essentiellement sur le motif pour saisir la beauté des paysages nimbés de soleil et transcrire le sens profond de leur nature. Viscéralement attaché à ses racines, il aime étudier sans relâche ses sujets, en pleine campagne, dans la solitude des paysages méditerranéens d'une austère beauté, près des carrières de Bibémus, du château de Vauvenargues ou dans le village du Tholonet.

6 En 1886, l'artiste s'installe pour un an, à Gardanne, avec sa famille, où il commence une série de peintures sur la Sainte-Victoire qu'il représente comme sujet à part entière, et de façon récurrente, dans plus de quatre-vingts œuvres, développant un style de plus en plus épuré. La montagne provençale, rattachée dans son histoire géologique, aussi bien à l'ancienne chaîne pyrénéenne, qu'à celle des Alpes occidentales, devient le cadre d'un laboratoire de recherche. Il ne s'agit pas, pour Cézanne, de s'épancher sur une "nature complice», mais de capter ses qualités intrinsèques, son pouvoir à exprimer une énergie, à stimuler l'imaginaire, pour la transcender. La tradition académique considère alors l'observation personnelle et sensible de la nature comme inférieure à l'expérience intellectuelle, alors qu'elle permet aux premiers artistes de l'art moderne de trouver un support d'expression solide et varié .

7 La peinture de Cézanne suscite jusqu'en 1887, à Paris, les railleries de détracteurs qui parlent de « visions cauchemardesques » et autres « atrocités à l'huile ». Puis, grâce aux collectionneurs éclairés et à des critiques indépendants, grâce au soutien de marchands d'art comme Ambroise Vollard (1866-1939) et Durand-Ruel (1831-1922), elle finit par obtenir un vrai succès. En 1888, une série d'articles mentionnent son œuvre en termes flatteurs et il est admis à l'exposition de l'Art français pour l'Exposition universelle de Paris de 1889. Cézanne commence à être reconnu et apprécié pour son audace picturale, la solidité de ses compositions, enfin, sa touche incomparable. Il peut, dès lors, vivre de son art, mais il est déjà malade, et effectue des cures pour se soigner. En 1906, alors qu'il est installé sur le motif, l'artiste prend froid et contracte une pneumonie, dont il ne se remet pas. Il meurt le 22 octobre, chez lui, en Provence.

8 À Paris, le monde de l'art est en deuil et lui rend hommage en organisant, au Grand Palais, pour le Salon d'automne de 1907, une grande rétrospective de ses œuvres. Le public découvre ses baigneuses, ses natures mortes, ses portraits, ainsi que les paysages 
de la Sainte-Victoire, et salue le talent et la ténacité du peintre. Pour tous, il est clair, qu'après Cézanne, l'art ne sera plus le même car l'héritage pictural que le maître d'Aix transmet, met à rude épreuve ses anciens fondements. La montagne Sainte-Victoire devient, dès lors, l'emblème de la volonté de l'artiste, tout debout contre l'ordre établi, de rénover l'art, car elle a été le refuge de celui qui, en marge d'une société étriquée et des dogmes de l'Académie des beaux-arts, a voulu se modifier lui-même, en profondeur, pour découvrir la vérité de la peinture.

\section{Henri Matisse (1869-1954), les fauves à Collioure : de la dynamite au pied des Albères}

9 En 1905, Henri Matisse (1869-1954), lui aussi, cherche la tranquillité, près de la montagne, pour entamer une longue méditation sur la couleur et s'affranchir des postulats de la peinture académique, encore pesants, malgré les défis lancés par les peintres postimpressionnistes, dans des toiles-manifeste. Matisse éprouve bien des difficultés à exprimer son propre tempérament de peintre et à se libérer du cadre, encore rigide, des enseignements qu'il a reçus. Tout d'abord, à Bohain, dans le Nord, puis à l'École des Arts Décoratifs de Paris, enfin, en 1895, à l'École des beaux-arts, dans l'atelier de Gustave Moreau (1826-1898). Le maître symboliste encourage ses élèves à penser leur peinture, à la rêver, au-delà d'une virtuosité technique, à dépasser leurs propres limites. Toutefois, après son apprentissage, Matisse décide de suivre les cours de l'Académie de la Grande Chaumière, dans l'atelier d'Eugène Carrière (1849-1906), où il rencontre André Derain (1880-1954), qui lui présente Maurice de Vlaminck (1876-1958). Tous entretiennent une passion pour la peinture cézanienne.

10 L'artiste est déjà un peintre reconnu lorsqu'il arrive à Collioure. En effet, en 1896, ses toiles ont été exposées au Salon de la Société Nationale des Beaux-Arts, dont il est devenu membre associé, sur proposition de Pierre Puvis de Chavannes (1824-1898). Cette fonction lui a permis de montrer, sans passer par un jury, un art expressif, qui s'inscrit dans l'air du temps. Matisse s'intéresse, en effet, à la peinture de son époque : les impressionnistes, qu'il découvre au musée du Luxembourg en 1897, et les pointillistes qui exercent une grande influence sur son travail, grâce au traité du peintre Paul Signac (1863-1935) de 1899, D’Eugène Delacroix au néo-impressionnisme. Les œuvres qu'il présente au Salon des indépendants de 1901, puis à la première édition du Salon d'automne de 1903, en témoignent, tout comme celles qui figurent chez Vollard, lors de la première exposition que le marchand consacre à l'artiste en 1904. L'été suivant, à Collioure, Matisse trouve le cadre idéal pour mener ses recherches : la mer, face à lui, juste à quelques mètres de la maison de pêcheur qu'il a louée, et la montagne, la chaîne majestueuse des Albères, enserrant le petit port, offrent un spectacle stimulant. Les rochers, les collines, les bateaux, les petites chapelles environnantes, constituent des sujets de choix, qu'il traite avec talent.

11 Rejoint par le jeune André Derain durant l'été, qui arrive avec de nouvelles idées, il s'attelle à l'élaboration d'un langage original, qui va peu à peu se démarquer de la peinture pointilliste qu'il a pratiquée, l'été précédent, aux côtés de Paul Signac, à SaintTropez. Les artistes exécutent ainsi, grâce à une collaboration quotidienne, des œuvres emplies de soleil, aux forts contrastes, dynamisant des plans de couleur pure, qui feront sensation au Salon d'automne de Paris, en 1905, dans la cage aux Fauves. Le travail novateur de Matisse, développé dans des toiles telles que La femme au chapeau, ou 
Intérieur à Collioure, aboutissant à la disparition des références au réel, par la déformation des lignes et l'élaboration de plans de plus en plus autonomes, rompt avec les conventions classiques de représentation. Matisse propose une peinture qui entame le concept de l'art comme esthétique plaisante, loin d'un idéal de beauté préétabli, dans des compositions régies par l'émotion. Près de la montagne, il parvient à redéfinir l'acte pictural, en harmonisant ses sentiments à d'autres systèmes de représentation. Aux côtés de Derain, véritable théoricien de la couleur, il est plus réceptif au fait plastique pur et s'engage dans une nouvelle voie chromatique.

Grâce à une réflexion poussée, les artistes ordonnent et équilibrent des zones de couleurs franches, parfois explosives, comme de la dynamite, dans leurs toiles, renonçant aux lois classiques de l'optique. Ils parviennent à accroître la conscience de la réalité matérielle du tableau pour demeurer au plus près de la vie immédiate d'un monde lumineux, fort et dense. Les peintres parisiens abandonnent le ton local pour passer aux tons non réalistes, en ayant recours aux aplats, et mettent fin à la peinture illusionniste. Le sujet est traité comme un instantané, et s'impose dans une vision lyrique du monde avec une sensibilité qui n'exclut pas la dissonance. Tout un système d'équivalence triomphe du chaos lumineux et permet de restituer l'essence de chaque chose. L'acte pictural se charge d'une spiritualité nourrie par la matière et la couleur, fruit d'une méditation éclairée sur le monde. L'artiste effectue plusieurs séjours fructueux à Collioure jusqu'à la guerre, où il rencontre le sculpteur Maillol travaillant non loin, à Banuyls-sur-Mer ${ }^{3}$. Cézanne recommandait aux artistes d'aiguiser leur vision face à la nature et d'observer les moindres détails avec leur propre sensibilité, afin que la peinture demeure un art vivant, qu'elle ne décline pas en se perdant dans la peinture des générations précédentes. Tel fut le but de Matisse, mais aussi celui de Picasso, tout au long des divers séjours qu'il effectua près de la montagne, milieu naturel riche et poétique, qui allait inspirer le peintre et l'amener vers le cubisme.

\section{Pablo Picasso (1881-1973), la naissance d'un cubisme radical dans les Pyrénées}

Pour les artistes en quête d'authenticité, comme Cézanne, puis Matisse, la montagne constitua en effet, un ancrage moral et esthétique face aux préceptes dominants des anciennes académies, et devint le symbole de leur résistance aux institutions. Ce fut le cas, aussi, au tout début du siècle, pour le peintre Pablo Picasso, qui remit en cause l'enseignement des écoles, dès qu'il eut conscience de leurs limites et effectua plusieurs séjours près des montagnes. Fils d'un professeur d'art, Picasso reçut une formation artistique classique dès son enfance, avant de suivre, en 1896, les cours de l'école des beaux-arts de Barcelone, puis ceux de la Llonja, où son père enseignait. L'année suivante, à seize ans, il réussit le concours d'entrée de l'Académie royale de San Fernando, et fut admis à l'école des beaux-arts de Madrid, la plus prestigieuse d'Espagne, où bien des artistes renommés avaient séjourné. Toutefois, le jeune homme ne poursuivit pas son enseignement à San Fernando, qu'il trouvait trop contraignant, et qui ne lui permettait pas de suivre son propre tempérament.

14 Aussi, retourna-t-il à Barcelone, en 1898, pour travailler seul, mais il tomba malade. Il effectua sa convalescence à Horta de Sant Joan, le village de son ami Manuel Pallarès (1876-1974), situé près de la ville de Tarragone, où il partagea la vie des paysans. Ce séjour de quelques mois fut, pour l'artiste, une véritable révélation. La présence de la 
montagne, la Santa Barbara, le contact quotidien avec la nature, et un mode de vie campagnard, rustique, l'enthousiasma. Des visions puissantes, capables de nourrir son imaginaire, stimulèrent sa création, pendant de longues années. Plus tard, l'artiste se plaira à répéter :

"Tout ce que je sais, je l'ai appris dans le village de Pallarès. ${ }^{4}$ "

En avril 1899, de retour à Barcelone, Picasso, fréquente le cabaret Els Quatre Gats, lieu de convivialité bohème, où les artistes peuvent exposer leurs œuvres, échanger leurs points de vue sur l'art. Là, il retrouve Miguel Utrillo (1883-1955), Carlos Casagemas (1880-1901), Ricardo Opisso (1880-1966), Julio Gonzalez (1876-1942), se lie d'amitié avec le poète Jaime Sabartès (1881-1968) qui deviendra son secrétaire particulier et expose quelques œuvres en 1900.

Picasso, aimait le pays de sa jeunesse, la Catalogne, mais il savait que ce serait une chance de le quitter pour parfaire sa formation et étudier les grands maîtres de la peinture à Paris, destination obligatoire pour tout peintre qui avait de l'ambition. Plusieurs séjours dans la capitale l'aidèrent à préciser son orientation et ses choix esthétiques. À la période bleue, triste et dure, succéda la période rose, nostalgique et déliquescente, qui trouva un public de connaisseurs et d'amateurs, le succès fut vite au rendez-vous. Cependant, pour faire partie des maîtres les plus doués de sa génération, l'artiste devait élaborer un nouveau langage et la tâche était ardue. En 1905, il fut chargé de faire le portrait de Gertrude Stein (1874-1946), une poétesse américaine qui soutenait le jeune Catalan et il lui fallait se démarquer de son principal rival, Matisse, pour satisfaire son mécène. Pour se lancer dans de nouvelles expérimentations, il chercha à regagner son pays afin de s'immerger dans un monde qui l'avait fortement inspiré, quelques années auparavant, celui de la montagne, et aller au-delà de ce que les écoles lui avaient appris.

Picasso partageait avec Matisse, Derain, Georges Braque (1882-1963) et d'autres artistes de sa génération, un vif sentiment d'admiration pour Cézanne et son œuvre. Il voulait, comme lui, rester au contact de son pays natal, pour se fortifier et progresser. À l'instar du maître d'Aix, il éprouvait ce sentiment qu'ont les Méditerranéens que leur terre est celle des dieux, où sont nées les grandes mythologies, et qu'elle constitue pour les créateurs une source d'inspiration inépuisable. Comme Cézanne, il pensait qu'il fallait s'éloigner du monde artistique parisien pour s'imprégner d'une nature non domestiquée par l'homme, afin de révéler la vérité de la peinture.

Pour rénover son art, Picasso effectue alors, durant l'été 1906, un séjour en Catalogne, dans un village de montagne très retiré, accessible uniquement à dos de mulet, nommé Gósol. Le massif de Pedraforca, qui ferme de façon imposante la perspective de la petite vallée, offre un spectacle grandiose, comme celui de la Sainte-Victoire, et l'artiste peut aller sur le motif pour capturer des images. La volonté du peintre de produire un art différent est autant soutenue par la quête d'un retour à la nature et aux racines, prôné par Cézanne, que par la recherche de nouvelles références puisées dans l'art de Gauguin (1848-1903), le Greco ou encore empruntées à l'art roman et ibérique local. Le travail effectué à Gósol a un impact majeur sur l'œuvre de Picasso puisqu'il débouche sur l'achèvement du Portrait de Gertrude Stein, qui présente des inventions sans précédent, comme la réduction du visage humain à son masque, marquant un jalon dans l'histoire du portrait. À la suite de ce séjour fécond, naît un chef-d'œuvre, Les Demoiselles d'Avignon dont l'angulosité des volumes annonce le premier cubisme. 

Horta de Sant Joan, où il avait séjourné dans sa jeunesse, à la suite d'une maladie, chez son ami Pallarès. Tout près de la montagne Santa Barbara, il réalise des œuvres remarquables comme Usines à Horta, le Réservoir de Horta de Sant Joan, ou encore des portraits de Fernande ${ }^{5}$, au "cou-montagne", qui poussent toujours plus loin les postulats cézanniens. L'artiste élabore une nouvelle syntaxe, bouleversant les principes classiques de perspective et de modelé, basée sur un système rythmé de formes et de couleurs, définissant le cubisme analytique. Picasso est alors reconnu comme un artiste et il veut approfondir ses recherches. Il effectue alors un troisième séjour près de la montagne, durant l'été 1910, dans un petit port du Cap de Creus, Cadaquès, où il s'établit avec sa compagne Fernande. Il veut aller jusqu'au bout de sa démarche, ne faire aucune concession au naturalisme pour rompre définitivement avec les règles de représentation illusionniste académique. Il exécute alors des œuvres frôlant l'abstraction, telles que Guitariste, ou encore Port de Cadaquès, qui mènent à un cubisme conceptualisé, mental. Bien qu'entouré de montagnes, de sujets marins fortement évocateurs, le réel disparaît. La présence d'André Derain, l'ancien collaborateur de Matisse à Collioure, le conforte dans sa démarche.

De 1911 à 1914, Picasso revient à la montagne, effectuant plusieurs séjours à Céret, en Catalogne du nord. Au pied du Pic de Fontfrède, montagne marquant la frontière avec l'Espagne, l'artiste connaît une période de grande effervescence, aux côtés des peintres cubistes Braque, Auguste Herbin (1882-1960) et Juan Gris (1887-1927). Des œuvres novatrices, marquées par une composition pyramidale, rappelant la montagne, comme L'Indépendant, de plus en plus élaborées, voient alors le jour. En 1912, à la suite d'aléas sentimentaux, il effectue, enfin, un séjour en Provence avec sa nouvelle compagne Eva ${ }^{6}$. À Sorgues, près du Mont Ventoux, il agence des formes géométriques pures, et multiplie les expérimentations techniques, dans des compositions inventives, qui aboutissent aux collages et engendrent, comme le voulait Cézanne, une nouvelle réflexion sur la nature de l'art. Les séjours près de la montagne ont marqué la production de Picasso de façon éloquente.

\section{Les expressionnistes allemands et les Alpes de Bavière, comme un volcan bouillonnant}

21 En Allemagne aussi, les artistes les plus avancés sont convaincus que les œuvres d'après l'antique ne tiennent plus, et que les arts doivent être natifs de la terre même où leur inspiration se développe, car seule la terre peut les vivifier, apporter des réponses proches de la réalité et des nouvelles considérations esthétiques, philosophiques, sociologiques, culturelles. La montagne, repère majestueux d'un espace et véritable concentré d'une nature originelle, symbolise ce postulat émergeant en Allemagne, valorisant le sentiment d'appartenance à un territoire, à une histoire personnelle et collective. En tant que trait fort du paysage, elle incarne l'identité d'un pays, établie depuis des temps anciens, mais aussi la relation sentimentale de l'homme avec un lieu. Les Alpes de Bavière deviennent ainsi le théâtre d'un renouveau artistique sans précédent.

22 Après la mort de Cézanne, le 22 octobre 1906, les peintres installés en Allemagne commencent à s'intéresser aussi au rôle du lieu de création, à la façon dont on peut expérimenter les formes et les couleurs, loin de la ville. Remettant en cause la société 
industrielle, la pression néfaste de la culture dominante sur l'art, ils veulent réformer leur attitude, leur fonctionnement personnel. Comme le maître d'Aix, ils décident de travailler sur le motif, dans une nature préservée, de côtoyer une population encore liée à la terre, évoluant parmi des formes simples, voire primitives, pour rénover le langage plastique. Outre-Rhin, la montagne, symbole de métamorphoses de grande envergure, assimilée à un volcan bouillonnant, incarnant un désir de liberté et de retour vers les forces et les lois fondamentales de la nature, joue ainsi un rôle important dans le parcours de grands maîtres de la modernité tels que Vassily Kandinsky (1866-1944), Paul Klee (1879-1940), Alexej von Jawlensky (1864-1941) ou encore Frantisek Kupka. Ainsi, à partir de 1908, des artistes quittent la ville de Munich pour passer l'été à Murnau, un village pittoresque de Haute-Bavière, où la lumière est appréciée pour sa subtilité, et où la montagne créée un espace majestueux. Le lieu, exaltant, inspire à plusieurs peintres, dont Kandinsky, Jawlensky, Gabriele Münter (1877-1962) et Marianne von Werefkin (1860-1938), une palette expressive, qui donne vie à de flamboyants paysages. L'image de la montagne, et sa silhouette imposante, est omniprésente dans les œuvres créées à Murnau, et la palette des fauves, qui ont travaillé à Collioure, est reprise avec un sens chromatique éblouissant. De ce contexte particulier naît, en 1911, un mouvement de rénovation tourné vers l'expressionnisme, dirigé par Kandinsky, consolidé par August Macke (1887-1914) et Franz Marc (1880-1916), le «Blaue Reiter» (le cavalier bleu), qui :

«Vise à montrer, par la diversité des formes représentées, comment le désir intérieur des artistes peut prendre des formes multiples. ${ }^{7}$ "

En effet, Vassily Kandinsky (1866-1944) cherche à rénover l'art pour en faire une arme contre la société industrielle, capitaliste, jugée décadente. En 1908, il s'éloigne de Munich, et d'une culture convenue, pour effectuer des séjours à Murnau, avec sa compagne Gabriele Münter. Il s'immerge dans une nature de haute montagne, baignée de lumière, renouant avec un monde simple, et son œuvre commence à se transformer. Une peinture de 1909 intitulée La Montagne bleue résume les recherches de cette période et annonce le tournant pris par l'artiste vers un art plus libre. Le large emploi de la couleur, dans un style expressionniste, et la simplification des formes aboutissent à un traitement non-figuratif du sujet. Ce que Kandinsky appelle le " chœur des couleurs ", est un vocabulaire coloré, issu de la peinture cubiste et fauve, qui peut se charger d'un fort pouvoir émotionnel et d'une dimension cosmique dynamique.

L'année suivante, Kandinsky peint sa première œuvre abstraite intitulée, Sans titre, une création spirituelle qui ne procède que de la seule nécessité intérieure de l'artiste, dans le sillage des Improvisations. En 1911, il écrit un traité d'esthétique, Du spirituel dans l'art et dans la peinture en particulier, où il invite le créateur à substituer, à l'apparence visible, la réalité pathétique et invisible de la vie. Il veut ainsi redéfinir l'objectif de l'œuvre d'art :

«Une œuvre d'art n'est pas belle, plaisante, agréable. Elle n'est pas là en raison de son apparence ou de sa forme qui réjouit nos sens. La valeur n'est pas esthétique. Une œuvre est bonne lorsqu'elle est apte à provoquer des vibrations de l'âme, puisque l'art est le langage de l'âme et que c'est le seul. [...] L'art peut atteindre son plus haut niveau s'il se dégage de sa situation de subordination vis-à-vis de la nature, s'il peut devenir absolue création et non plus imitation des formes du modèle nature. ${ }^{8}$ " abstrait qu'il veut révolutionnaire, pour exprimer l'intériorité spirituelle abstraite, en 
perpétuelle évolution. Dans sa production des années vingt, il combine les formes géométriques et les couleurs pour révéler un monde mystérieux, issu de la musique, ou du cosmos, parfois résumé à une épure. Des tableaux comme Schwarzer Raster (1922), ou Einige Kreise (1926), où son trait s'est solidifié, montrent un lyrisme cérébral. Dans Steroscopic exhibition, les deux triangles aigus bleutés, dynamisant la composition, sont, pour cet artiste cultivé, une sorte de « private joke».

À partir de 1910, Paul Klee (1879-1940) se rapproche des peintres « de la montagne » qui posent de nouvelles problématiques, liées à la nature et à l'objectif de l'art. Comme eux, il est convaincu que la notion d'idéal et de beauté est tout à fait désuète, dépassée. Certes, l'artiste, dont l'œuvre est intuitive, a une personnalité bien affirmée. Refusé à l'Académie des beaux-arts de Munich en 1898, il est dirigé vers l'atelier d'Heinrich Knirr où il étudie le dessin figuratif. En 1900, il est finalement admis aux Beaux-Arts, dans la même classe que Kandinsky, mais à l'instar du jeune artiste russe, il a déjà défini son objectif artistique et il entend peindre selon sa propre conception du monde. Il se rend à Paris en 1912 et découvre chez les marchands l'art "des cubes». Il s'intéresse aux œuvres de Robert Delaunay (1885-1941), Braque, Picasso, et Derain, dépositaires de l'héritage artistique de Paul Cézanne, qui fait l'objet de nombreuses études. Les inventions des pionniers du cubisme et des collages, Picasso et Braque, ont alors atteint tous les cercles d'art et suscité un vif enthousiasme. Le traité rédigé par Jean Metzinger (1883-1956) et Albert Gleizes (1881-1953) en 1912, Du cubisme, a permis de diffuser à l'étranger les principes d'une esthétique qui fait scandale, car elle incite les artistes à refuser toute convention de représentation. L'année suivante, Paul Klee traduit un texte de Robert Delaunay qui l'intéresse particulièrement, De la lumière, et a recours, pour rénover son art, aux principes esthétiques « orphistes » développés par les Delaunay, Sonia et Robert, qu'il invite en Allemagne, pour y exposer leurs œuvres.

En 1914, Klee rejoint le «Blaue Reiter », et fonde avec Jawlensky, Kandinsky, Münter et Alexander Kanoldt (1881-1939), un mouvement artistique rénovateur, «La Nouvelle Sécession » de Munich. Puis il entreprend un voyage en Tunisie avec ses amis Macke et Louis Moilliet (1880-1962). En avril, avant d'embarquer à Marseille, i1 séjourne à L'Estaque, dans l'intention de photographier le viaduc peint par Cézanne et Braque, qu'il admire. Lorsque la guerre éclate, les artistes doivent trouver refuge dans les pays neutres pour continuer de peindre ou s'engager. Jawlensky et Kandinsky, de nationalité russe, doivent s'exiler. August Macke est tué sur le front de Champagne, le 26 septembre 1914, tout comme Franz Marc, l'ami de toujours, le sera deux ans plus tard à Verdun. Mobilisé, Klee obtient, grâce à son père, d'être affecté dans un régiment de réserve à Munich où il peut encore exercer son art. En cette période, éprouvé par les événements, ses sensations sont intenses et près des montagnes, notamment la Zugspitze, dans le massif du Wetterstein, la production de l'artiste se transforme, marquant un réel tournant stylistique. En 1917, il expose à la galerie Der Sturm de Berlin, et son travail remporte un grand succès. On note que Klee a intégré l'idée de révolution dans son art, ayant pris conscience que l'art moderne est un défi jeté à la culture bourgeoise, avec, pour emblème, le triangle, une forme géométrique universelle, qui figure dans une aquarelle devenue célèbre, Le Niesen, datée de 1915.

Dans cette œuvre, l'imposante masse pyramidale du Niesen, culminant à 2563 mètres, est représentée selon les préceptes du maître d'Aix, dans un style qui oscille entre le fauvisme et le cubisme triomphant. La montagne est traitée avec un lavis bleu azur, lui 
conférant une légèreté métaphysique, résonnant avec les aplats bigarrés, lumineux des arbres, de forme orthogonale.

Paul Klee, qui avait dit « je suis Dieu" », a rempli le ciel d'étoiles scintillantes, côtoyant la lune et le soleil, pour créer une ambiance onirique. L'héritage cubiste est bien présent dans cette belle composition qui est un prélude aux chefs-d'œuvre qui toucheront le public par leur sincérité expressive, comme Senecio, de 1922, ou Château et soleil, de 1928.

De son côté, le peintre tchèque Frantisek Kupka (1871-1957) tisse aussi un lien particulier avec la montagne. Dans une œuvre emblématique, Méditation, (1897) il s'est représenté nu, agenouillé devant un imposant paysage de montagne. L'artiste se pose des questions cruciales pour l'évolution de son art, quant à la réalité des choses et leur représentation. La montagne lui révèle l'articulation entre physique et métaphysique, entre phénomène et noumène, l'interroge sur le sens de la vie. Kupka s'installe à Paris en 1896 et devient l'auteur d'une création picturale très originale. Il est le premier à affronter le public avec des œuvres non figuratives lors du salon d'automne de 1912. Il crée une autre réalité, rejetant toute référence au monde sensible ${ }^{10}$.

31 La rénovation voulue par le maître d'Aix permet aussi de donner un statut moderne à l'art des cubo-futuristes russes, théorisé, en 1912, par le peintre du suprématisme, Kasimir Malevitch (1879-1935), qui conçoit le Carré blanc sur fond blanc, jugé scandaleux, en 1918, puis, à celui des constructivistes révolutionnaires, tout debout contre «l'ordre ancien » :

«Malevitch avait bien vu que le principe dynamique était déjà présent, à l'état d'embryon, chez Paul Cézanne et, à sa suite, dans les toiles cézannistes géométriques de Georges Braque ou de Pablo Picasso. ${ }^{11}$ "

Dans son manifeste Une gifle au goût du public, David Burliouk (1882-1967) avait réaffirmé que Paul Cézanne, le gardien de la Sainte-Victoire, était le père de toute l'avant-garde picturale, un mouvement de contestation international qui mena, après la Grande Guerre, à l'anti-art. Dada, fruit de la rébellion systématique contre toute esthétique établie, vit, lui aussi le jour près de pics majestueux, à Zurich, la capitale de la Suisse alémanique. L'art de l'absurde, d'Hugo Ball (1886-1927), Tristan Tzara (1896-1963), Richard Huelsenbeck (1892-1974) et Francis Picabia (1879-1953), déboucha sur le surréalisme, qui évoluera sur les hautes terres de l'inconscient et de la folie, avant d'être stoppé net par le chaos de la Seconde Guerre mondiale.

\section{La montagne et l'art moderne}

La montagne, repère-témoin de parcours créatifs exemplaires, peut être vue comme le fil reliant les œuvres d'artistes modernes, d'origine et de cultures variées, à celles de Paul Cézanne. Ceux-ci ont cherché à porter un nouveau regard sur la nature, l'opposant de façon aiguë au nouveau monde industriel, asservissant et dégradant l'homme. La montagne, véritable concentré de nature, monde préservé et intact, leur a fourni un refuge pour s'interroger, en se donnant pour mission, à l'instar du maître d'Aix, d'investir le paysage, afin que celui-ci cesse d'être un document géographique, ou scientifique, pour devenir un exercice purement plastique et psychologique. Ce dernier considérait l'œuvre comme un témoignage particulier, d'un moment d'introspection et de réflexion du peintre face à la nature. L'homme, tout entier, devait se transformer pour apporter au tableau sa vraie substance, par un regard plus perçant et plus 
conscient, tant sur le monde, que sur lui-même. Pour Cézanne, la Sainte-Victoire était l'un des moyens d'y parvenir. Cette dernière devint l'emblème de la volonté de l'artiste, debout contre l'ordre établi, de rénover l'art en profondeur. La montagne peut ainsi être considérée comme le symbole de la révolte contre les diktats des académies qui a rassemblé les plasticiens voulant puiser tant dans une idéologie vivante que dans un environnement à forte identité.

Picasso et Matisse ont aussi cherché dans la nature des réponses à des questions cruciales. Matisse, bien qu'installé près de la mer, à Collioure, s'est imprégné de la dimension mystique et sauvage des Albères, enserrant le port, pour élaborer son langage fauve. De même, la montagne, véritable laboratoire de recherche à ciel ouvert, a incarné pour Picasso autant une volonté créative sans borne, qu'une farouche opposition aux règles des Beaux-Arts. L'immersion de Picasso dans le milieu montagnard, de 1906 à 1914, de Gosol à Céret, le propulsa sur le devant de la scène. Les séjours près de la montagne ont marqué, de façon éloquente, l'évolution du style des deux artistes, rendant hommage, par une démarche forte, «au bon dieu de tous les peintres». En effet, plusieurs œuvres significatives de la modernité attestent que la montagne, entité puissante, à la fois physique et métaphysique, propice à une réflexion sur le sens et la nature du réel et de l'art, a constitué le lieu privilégié de la résistance aux anciens préceptes, et nourri une expression plus dynamique. Les artistes allemands et ceux d'Europe du Nord, trouvèrent aussi dans le milieu montagnard les conditions propices à la formulation d'un art qui ne s'attache plus à la réalité physique mais aux états d'âme du créateur.

Selon Kirchner, les peintres ne devaient plus s'imposer de règles et l'inspiration devait couler librement afin de donner une expression immédiate à leurs pressions psychologiques. La production de Kandinsky, réalisée à Murnau dès 1909, affirmait un art dénué de sa fonction de reproduction du réel pour renforcer sa composante subjective jusqu'à formuler une abstraction lyrique, issue d'un profond désir spirituel qu'il appelait la "nécessité intérieure ", et qu'il tenait pour un principe essentiel de l'art. Paul Klee, près des monts Zugspitze dans les Alpes, après des mois d'une longue maturation et d'une intense réflexion théorique au contact de la montagne, fondée sur son expérience et sur une démarche esthétique proche de celle des Delaunay, émit lui aussi de nouveaux principes sur la forme et la couleur, et exposa la première théorie systématique des moyens picturaux purs, qui conduisit à une clarification exceptionnelle des possibilités contenues dans les procédés abstraits. De tels procédés seront également explorés par les cubo-futuristes russes et par le maître du suprématisme, Malévitch, enfin, par Kupka, dont la réflexion se calquait sur l'image de la montagne, comme le montre l'œuvre Méditation.

Ainsi, tout comme on ne peut pas regarder la production de Cézanne sans penser à sa relation avec la Provence et la Sainte-Victoire, on ne peut ignorer le rôle joué par la montagne, selon un principe de filiation spirituel inédit, dans l'évolution du sentiment esthétique de plusieurs figures majeures de l'art $d u x^{e}$ siècle, qui mena à l'élaboration de nouvelles théories, et ouvrit la voie aux avant-gardes les plus audacieuses. 


\section{BIBLIOGRAPHIE}

BRULLÉ Pierre, Exposition Frantisek Kupka, Galerie Antoine Laurentin, Paris, 8

septembre-29 octobre 2016.

NAKOv Andrei, Malévitch : Aux avant-gardes de l'art moderne, Paris, Découvertes Gallimard, 2003.

KANDINSKI Vassily, Du spirituel dans l'art et dans la peinture en particulier, Paris, Éd. de Beaune, 1911.

KLEE Paul, Théorie de l'art moderne, Paris, Gallimard (Folio Essais), 1998.

LHOTE André (préface), Exposition L'influence de Cézanne, 1908-1911, Galerie de France, Paris, 14

janvier-15 février 1947.

MAILLARD Robert et ELGAR Frank, Picasso, étude de l'œuvre par Frank Elgar et étude biographique par Robert Maillard, Paris, Éd. Fernand Hazan, 1955.

MARCADE Jean-Pierre, Exposition Le futurisme, 2008-2009, Centre Georges-Pompidou, Paris, 15 octobre 2008-26 janvier 2009.

MONNIER Gérard, L'Art et ses institutions en France, De la révolution à nos jours, Paris, Gallimard (Folio histoire, $\mathrm{n}^{\circ}$ 66), 1995.

SCHNEIDER Pierre, Matisse, Paris, Flammarion, 2002

VEZIN Annette et VEZIN Luc, Kandinsky et le Cavalier bleu, Paris, Éd. P. Terrail, 1991.

\section{NOTES}

1. G. Monnier, L'Art et ses institutions en France, De la révolution à nos jours, p. 61.

2. A. Lhote, Catalogue de l'exposition L'influence de Cézanne, 1908-1911, 1947, p. 5.

3. P. Schneider, Matisse, p. 45.

4. R. Maillard et F. Elgar, Picasso, étude de l'œuvre et étude biographique, p. 3.

5. Fernande Olivier (1881-1966), de son vrai nom, Amélie Lang, compagne de l'artiste de 1904 à 1912. Modèle de plusieurs peintres montmartrois, elle rencontre Picasso au bateau-lavoir de la place Émile Godeau.

6. Eva Gouel (1885-1915), née Marcelle Humbert, chorégraphe et modèle de Picasso de 1911 à 1915.

7. A. Vezin, L. Vezin, Kandinsky et le Cavalier bleu, p. 223.

8. V. Kandinski, Du spirituel dans l'art et dans la peinture en particulier, p. 21.

9. P. Klee, Théorie de l'art moderne, p. 11.

10. P. Brullé, Catalogue de l'exposition Frantisek Kupka, 2016.

11. J.-P. Marcade, Catalogue de l'exposition Le futurisme, 2008-2009, p. 59. 


\section{RÉSUMÉS}

Les artistes de la fin du XIX ${ }^{\mathrm{e}}$ siècle sont liés au concept de modernité qui émerge, dès 1850, pour désigner les changements survenus après la révolution industrielle. La modernité est perçue comme la manifestation d'un mode de pensée, de vie et de création, basé sur le changement, et non plus sur les traditions esthétiques de l'Académie des beaux-arts. Bien des artistes, refusant d'adapter les œuvres des anciens au goût contemporain, vont refonder les principes de la création sur leur propre vision du monde, liée à leur terre d'origine. La montagne, et plus particulièrement, la Sainte Victoire, peinte par Cézanne, va symboliser ce postulat émergeant, en devenant le lieu de résistance aux institutions. Matisse, puis Picasso, reprennent cette posture en quittant Paris pour peindre dans le sud, en induisant un autre rapport à la nature. À leur suite, les artistes d'Allemagne, Kandinsky, Klee, face aux Alpes, vont modifier leur perception du monde, pour « re-créer » les formes selon un processus mental qui évoluera jusqu'à l'abstraction.

\section{AUTEUR}

\section{VÉRONIQUE RICHARD-BRUNET}

Historienne de l'art, membre associé de l'Académie des lettres et arts de Mâcon, membre associé de la Société d'histoire de l'art français - Paris 
Le royaume dans le ciel : regard des pionniers béarnais de la Société des missionnaires évangéliques de Paris sur les montagnes du Sotho africain (1833-1880)

\author{
Laurence Espinosa
}

\title{
Une ligne sur I'horizon
}

1 Alors que je passais par le jardin du château de Pau pour rejoindre le centre de la ville, j'ai aperçu les montagnes couchées sur l'horizon. Plusieurs images ont alors défilé à la surface de mon esprit, comme ces mots d'élisée Reclus: "Sans trop savoir où me conduisaient mes pas, j'étais sorti de la ville bruyante, et je me dirigeais vers les grandes montagnes dont je voyais le profil denteler le bout de l'horizon ${ }^{1}$ ", des pages du livre de l'anthropologue écossais Tim Ingold sur les lignes ${ }^{2}$ ou de mes recherches consacrées aux relations entre les Sotho d'Afrique australe et les pionniers de la Société des missions évangéliques de Paris (SMEP) ${ }^{3}$. Ces images étaient-elles les éléments d'une succession? Comment pouvaient-elles se conjuguer? N'étaient-elles que les éclats éparpillés d'une brève pensée?

\section{Méthode et pistes théoriques - Les terrains : le terreau ou/et} I'humus

Dans le cadre de ma thèse, ouverte dans un premier temps au domaine historique, j'ai arpenté le terrain des archives. J'ai parcouru les textes pour observer les humains et le non humain dans leurs interrelations. En d'autres termes, j'ai accompli mon service ethnographique sur le papier. J'ai plus explicitement exploré les récits des missionnaires de la SMEP publiés dans les Journaux des missions évangéliques (JME) 
entre 1834 et 1880. De cette situation, j'ai retenu une appréciation lâche du terrain. J'ai abordé le texte comme un tissu (textus), un ensemble de fibres enchevêtrées ou/et une surface organique. Au moment de cette rédaction, je n'avais pas encore accédé aux travaux de Tim Ingold. Leur découverte a aiguisé ma curiosité. Le texte est la trame de fils enchevêtrés qui est en train de se faire. À ce propos, Tim Ingold préfère la notion de meshwork (maillage) à celle de network (réseau). J'avoue être également peu réceptive au réseau trop rigide. Étymologiquement, il s'agit d'un filet fabriqué pour capturer les animaux. Par analogie, il représente tout ce qui entrave. Ce sont aussi des lignes entrecroisées ou des lieux et personnes qui communiquent entre eux, reliés eux-mêmes à un point central ${ }^{4}$. Le réseau ignore la surface. Il l'enjambe. La trame est imparfaite. Elle suppose des vides (ajours du textus) inexplorés ou inexplorables, des ruptures et des impensés ou impensables. Pour rendre compte, de ce contexte, j'hésite entre les notions quasi synonymiques de terreau (vers le terrain ou la terre) et d'humus (vers l'humain).

3 Parce que l'un de leurs principaux champs de recherche a été les échanges entre les Tswana et des missionnaires méthodistes britanniques au $\mathrm{xIX}^{\mathrm{e}}$ siècle, dans une perspective d'anthropologie historique, les travaux et les itinéraires intellectuels de Jean et John Comaroff m'ont interpellée. D'abord sensibilisés au Cap, en Afrique du Sud, puis à Manchester, en Grande Bretagne, aux études de l'anthropologie sociale britannique, les deux anthropologues ont rejoint l'université de Chicago en 1979. Dans ce contexte, ils ont été amenés à participer activement aux débats interdisciplinaires portés par la revue Public Culture ou le Chicago Center for Contemporary Theory. Sensibles aux idées développées par les promoteurs des subaltern studies, ils ont toutefois essayé de ne pas s'y laisser enfermer. Leur approche est composite. Leurs recherches partent de faits, d'un terrain (les territoires septentrionaux de l'Afrique du Sud principalement) qu'ils ont choisi d'explorer sur le long terme. Aussi, se revendiquent-ils plus volontiers d'une "néomodernité ", modernité non englobante, "plurifocale», que d'un postmodernisme jugé trop souvent hors sol ${ }^{5}$. Dans la contribution qui suit, j'essaie donc de jongler avec ces approches théoriques. La montagne se meut en ligne transformable à saisir au fil des sens.

\section{Itinéraires}

4 Le récit que je propose est celui d'une superposition imprécise de lignes, celles formées à l'horizon par les Pyrénées et les Maloti' ${ }^{6}$ J'ai eu envie de parcourir leurs angles et leurs volutes. L'horizontalité géologique pourrait servir de terrasse à cette incrustation.

Dans un premier point, je parlerai d'écoulement. J'évoquerai d'abord le remplissage du lieu (la montagne) qu'accomplit le chef Sotho Moshoeshoe ${ }^{7}$ au début du XIx ${ }^{e}$ siècle. Puis, je m'attarderai sur la situation contemporaine. Le départ des hommes vers les mines et la captation de l'eau pour abreuver la province du Gauteng dont l'agglomération de Johannesburg, s'apparentent à des dessiccations.

6 Dans un second point, j'envisagerai les lignes en tant qu'horizon «romantique » des missionnaires ou errance générationnelle. La ligne, écriture ou voix, tente de justifier un canevas qui se noue et s'étiole. Les religieux ont écrit et parlé de poésie. 


\section{L'Écoulement}

\section{Remplissage}

7 Le royaume des Sotho est né dans la tourmente du lifacane ou difacane pour les SothoTswana et mfecane, pour les Ngunis (Zulu ou Xhosa). Ce processus dont la traduction littérale est «mouvement tumultueux de populations » est un phénomène complexe. Il serait imputable à une pression démographique accrue, à des changements climatiques et également aux perspectives commerciales qui commencent à se développer avec la région européanisée du Cap ${ }^{8}$. Cette situation favorise la concentration du pouvoir et l'émergence de chefferies concurrentielles qui veulent contrôler les ressources. Certaines d'entre elles acquièrent une suprématie en incorporant d'autres clans ou individus désorientés par les mutations en cours. Les royaumes constitués par le chef Zulu Shaka (1787-1828), qui s'engage dans une politique résolument expansionniste ou le Sotho Moshoeshoe qui parie sur une stratégie défensive, illustrent cette évolution. Pour éviter l'affrontement armé, les hommes de Moshoeshoe, à l'instar d'autres peuples, remettent un tribut aux Zulu sous forme de têtes de bétail. Malgré ces arrangements, dans ce contexte troublé, des conflits éclatent fréquemment. Ainsi, les Tlookwa qui appartiennent également au sous-groupe des Sotho-Tswana attaquent à plusieurs reprises les Sotho. Les affrontements, qui se succèdent entre 1822 et 1823 , aboutissent en 1824 au siège de Botha-Bothe. Moshoeshoe se met alors en quête d'un refuge un peu plus sûr. Il le trouve à quatre-vingts kilomètres au sud. En 1828, cette place défensive, Thaba-Bosiu, permet aux Sotho de résister aux Ngwane, un groupe de Nguni mené par le chef Sobhuza $1^{\text {er }}$ (1795-1836), qui seront connus ultérieurement sous le nom de Swazi. En 1829, ils chassent de nouveau les Tlookwa. Enfin en 1831, ils sont victorieux des Ndebele, un groupe d'hommes qui a suivi Mzilikazi (1790-1868), un lieutenant rebelle de Shaka. Cette date marque la fin du lifacane pour les Sotho. Les missionnaires de la SMEP Thomas Arbousset (1810-1877), Eugène Casalis (1812-1891) et Constant Gosselin (1800-1875) arrivent chez les hommes de Moshoeshoe en juillet 1833. Jusqu'à la fin des années 1840, la cohabitation semble productive pour les uns et les autres 9 . Pour les jeunes missionnaires, les Sotho sont les brebis égarées aptes à être mises sur le bon chemin. Cette sympathie pour les réfugiés dans les Maloti m'autoriset-elle à esquisser un lien avec l'histoire des protestants dont la résistance dans les Cévennes est l'un des faits constitutifs ${ }^{10}$ ? Dans l'instant, je me contenterai de singulariser cet épisode comme l'initiale d'un phrasé à venir. La construction politique des Sotho, qui émerge dans ce contexte particulier, a pu préserver ses frontières et son intégrité politique pendant la période d'apartheid en Afrique du Sud (1948-1991). Pour autant, le royaume n'a cessé de nourrir son puissant voisin - le Lesotho est littéralement englouti par l'Afrique du sud - d'abord en hommes pour les mines et actuellement en eau pour alimenter Johannesburg et son territoire (Gauteng). Associés à l'érosion des sols qui ampute le Lesotho de ses terres les plus fertiles, le départ des hommes et l'écoulement de l'eau s'apparentent à des dessiccations. 


\section{Dessiccations}

\section{Dessiccation 1 : les hommes}

8 Le Lesotho est un pays complètement enclavé dans la République sud-africaine. Région montagneuse (basses terres: $1500 \mathrm{~m}$, hautes terres : 2500-3 000 m), au nord du Drakensberg, elle couvre $30355 \mathrm{~km}^{2}$ et abritait en 2015 environ 2 millions d'habitants qui vivent concentrés dans les basses terres. Il pleut en moyenne 800 millimètres d'eau par an (300 mm dans les basses terres et 1600 dans les hautes terres) principalement entre octobre et avril. Janvier est le mois le plus chaud $\left(30^{\circ}\right)$. Il peut faire jusqu'à - $20^{\circ}$ en montagne. Le pays a été surnommé le royaume dans le ciel. Le relief est très accidenté. Les vallées encaissées forment parfois des canyons. Les pentes rudes sont nues. L'érosion est très active et condamne les rares terres fertiles à la disparition ${ }^{11}$.

L'économie est fragile. La moitié de la population vit en dessous du seuil de pauvreté. Le produit intérieur brut (PIB) par habitant est de 1330 \$. L'agriculture est la base de l'économie, mais elle est structurellement déficitaire. Les Sotho produisent du maïs, du sorgho et des fruits. Les gains sont peu importants parce que seulement $10 \%$ des terres sont arables. Ce secteur en crise occupe $85 \%$ de la population. L'industrie, principalement de transformation (laine, peaux, cuirs, alimentation), est majoritairement aux mains d'entrepreneurs étrangers. Des entreprises chinoises se sont progressivement installées au Lesotho, mais les exigences d'un accord commercial passé avec les États-Unis ont mis fin à leurs investissements ${ }^{12}$. Par ailleurs, le Lesotho exploite des mines de diamant et possède des dépôts d'uranium.

Depuis l'indépendance, en 1966, jusqu'au début des années 2000, le rapatriement des salaires des travailleurs migrants et la redistribution des recettes douanières dans le cadre de la Southern Africa Customs Union (SACU), l'union douanière sud-africaine, ont été les principales entrées financières. Elles sont en diminution actuellement. En effet, la crise minière et agricole en Afrique du Sud a entraîné une baisse de la demande de main-d'œuvre (127000 en 1991 et 51000 en 2006). De plus, la libéralisation de l'économie sud-africaine, avec l'ouverture des frontières, a eu un effet négatif sur les recettes douanières. En conséquence, le Lesotho s'appauvrit et devient encore plus dépendant économiquement de son puissant voisin.

\section{Dessiccation 2 : l'eau}

11 Les pionniers de la SMEP décrivent un pays riche en eau («foule de petits lacs», « abondance d'eau »). Parmi les lacs et sources dénombrés, dénommés ou non, règne le Caledon, l'un des principaux affluents de l'Orange, respectivement Mohokare et Sinqu pour les Sotho. Les missionnaires sont les premiers à renseigner leurs contemporains européens sur les caractéristiques de ce fleuve. Ils les informent de la situation géographique de la source notamment. Ainsi, ils écrivent:

«Le Calédon mérite de compter parmi les principales rivières du sud de l'Afrique. D'après les rapports des Bassoutos, il prend sa source dans les Monts Witteberg, près du pays des Mantaetis. Il coule Ouest-Sud-Ouest et se jette dans l'Orange, à deux ou trois heures de la station des Bushmen, c'est-à-dire à seize ou dix-huit lieues Sud-Est de Philippolis. ${ }^{13}$ "

En août 2016, la presse internationale reprenait cette annonce :

«Les habitants de Sephareng, au Lesotho, ont la vue sur l'immense barrage de Katse, mais ils n'ont pas accès à l'eau : elle part en Afrique du Sud. Une ironie 
d'autant plus cruelle que tous souffrent de la sécheresse provoquée par le

phénomène climatique El Niño en Afrique australe. ${ }^{14}$ "

13 rôle de château d'eau. Le point culminant est le Thabana Ntlenyana. Le Lesotho Highlands Water Project (LHWP) est un projet de transfert hydraulique transfrontalier entre l'Afrique du Sud et le Lesotho destiné à alimenter Johannesburg et son agglomération ( 12 millions d'habitants sur les 52 millions de Sud-Africains) à des fins d'irrigation agricole et d'approvisionnement urbain. Le projet a démarré après la signature d'un accord entre le Lesotho et l'Afrique du Sud en 1986. Les deux plus grands barrages sont le Katse Dam et le Mohale Dam. L'eau est transférée de barrage en barrage, et passe par un tunnel long de $80 \mathrm{~km}$ creusé au travers du massif des Maloti pour atteindre la région de Johannesburg après un parcours de près de $500 \mathrm{~km}$. Lorsque la construction des infrastructures du LHWP sera à son terme, prévue autour de 2020-2030, des barrages seront construits sur tous les principaux cours d'eau du Lesotho et près de la moitié du volume hydrologique du Lesotho sera détournée vers l'Afrique du Sud ${ }^{15}$. Le projet est contesté par la majeure partie de la population :

«En deux décennies, seulement la moitié des villages aux abords de l'ouvrage d'art ont bénéficié d'un accès à l'eau, avec un robinet pour six à sept foyers. ${ }^{16}$ "

Il reçoit cependant le soutien des élites politiques du Lesotho, notamment comme moyen de renouveler les sources extérieures de mannes financières. En 2016, le ministre des affaires étrangères Lekheto Rakuoane, soulignait que l'apport de l'Afrique du Sud représentait $6 \%$ du budget du pays ${ }^{17}$. Graphiquement, le Lesotho s'écoule vers l'Afrique du Sud. Ce mouvement donne à ce petit pays l'aspect d'une peau de chagrin, un dessin incrusté dans un plus grand destin.

\section{Horizons}

«Vue de la plaine, la montagne est de forme bien simple: c'est un petit cône dentelé s'élevant, parmi d'autres saillies d'inégale hauteur, sur une muraille bleue, rayée de blanc et de rose, qui borne tout un côté de l'horizon. Il me semblait voir de loin une scie monstrueuse aux dents bizarrement taillées; une de ces dents est la montagne où se sont égarés mes pas. ${ }^{18}$ "

Les lignes ne sont pas toujours visibles. Elles s'entendent parfois, se devinent ou bien se perdent. Tim Ingold induit une parenté entre «marcher, tisser, observer, chanter, raconter une histoire, dessiner et écrire ${ }^{19}$ ». Quand Eugène Casalis se retrouve face aux échancrures des Maloti, son regard ravive la ligne des Pyrénées qu'il a souvent vue. Il compare les massifs, mais le brouillage des lignes n'avalise pas pour autant la confusion. Le paysage familier rend la trame de Tim Ingold palpable. La ligne sur l'horizon invite le jeune pionnier à retrouver ses traces sur un parcours à baliser.

Thaba-Bosiu, le refuge de Moshoeshoe, est un plateau sablonneux situé entre l'Orange et le Caledon. Le mot thaba signifie montagne et bosiu, nocturne. Cette montagne est magique parce qu'elle grandit la nuit et s'amenuise le jour. Ceux qui osent s'y aventurer sont irrémédiablement absorbés par ses profondeurs ${ }^{20}$. 


\section{Génération}

17 Indéniablement, ces jeunes protestants sont des fils des Lumières comme le fait constater Alain Ricard. Pour autant, ils ont une vingtaine d'années en $1830^{21}$. Ils ont l'âge d'être romantiques :

« En 1830, chacun se sentait gros de parler, comme le disait le véhément ami de Job.

En politique, en philosophie, en religion, c'était pour tout la même chose : nous

vivions dans une atmosphère d'enthousiasme. ${ }^{22}$ "

Le romantisme n'est pas qu'un mouvement littéraire et artistique. Il s'accompagne d'une pensée politique et sociale ${ }^{23}$. Dans leur choix pour la mission, les jeunes missionnaires expriment leur désir d'aller au-delà des frontières physiques et idéologiques. Ils sont sourds aux injonctions de leurs détracteurs qui les accusent de négliger ce qu'il reste à accomplir en France. Leur nationalisme est idéal. Ils ont besoin d'ailleurs, d'horizons nouveaux pour naître autrement.

19 La montagne est romantique. Le 31 août 1843, Victor Hugo s'émerveilla devant le cirque de Gavarnie. Eugène Casalis, originaire d'Orthez dans les Basses-Pyrénées, qualifie les Maloti de Pyrénées de l'Afrique australe. La ligne imperturbable qui hachure le ciel conserve son passé et prépare son devenir.

20 Les récits de convertis ponctuent régulièrement les pages des JME dans lesquels les missionnaires racontent leur quotidien. La montagne est présente dans ces témoignages. Elle clignote. Protectrice, elle empêche Entouta de se faire piétiner par un hippopotame :

«Le jeune chasseur, exténué de fatigue, allait inévitablement être déchiré, mais Dieu, qui veillait sur ses jours dirigea sa fuite vers un profond ravin que l'animal n'osa pas franchir ${ }^{24} »$.

21 Assassine, elle provoque la chute de Mara :

«Au temps des malheurs de sa patrie, elle avait vu tous ses parents périr par le fer des Zoulas: précipitée elle-même du haut d'une montagne par ces ennemis impitoyables, Dieu seul peut savoir comment elle échappa à la mort. ${ }^{25}$ "

Elle est également riante comme les collines de Corocoro ${ }^{26}$. La montagne est écrite au gré des sentiments des hommes.

\section{Un fil à la langue}

Pour Tim Ingold, l'écriture à l'encre sur du papier est une trace "additive». Les missionnaires ont écrit à la main les textes avant qu'ils ne soient publiés dans les JME. Des mots ont d'ailleurs été parfois mal retranscrits ${ }^{27}$. Les traces s'inscrivent sur des surfaces. Les fils s'entrelacent ou sont suspendus, mais «à chaque fois que les fils se transforment en traces, des surfaces se forment, tandis que lorsque les traces deviennent des fils, les surfaces disparaissent ${ }^{28}{ }^{2}$.

Les missionnaires se profilent dans les paysages qu'ils traversent et les hommes qu'ils côtoient. Quand ils croisent les Sotho pour la première fois, des échanges se font en hollandais. Pour être plus proches de leurs interlocuteurs, ils doivent apprendre leur langue ou trouver une langue à partager. La première publication autonome (hors des $J M E)$ est une étude linguistique : Eugène Casalis, Études sur la langue sechuana ${ }^{29}$. Alain Ricard considère le pionnier comme l'un des premiers « bantouistes », un précurseur de Wilhelm Bleek (1827-1875) ${ }^{30}$. Dans son ouvrage, Eugène Casalis complète les données 
grammaticales et lexicales par des informations historiques (constitution du royaume) et ethnographiques (collecte de louanges, proverbes, énigmes et contes populaires). Il nomme la langue qu'il étudie "sechuana " autrement dit Setswana, le parler des Bantu des plateaux centraux, mais les mots auxquels il se réfère majoritairement sont ceux des Sotho et plus précisément des Kwena, les hommes du clan de Moshoeshoe.

Pour Alain Ricard, l'opération de traduction est essentielle puisqu'elle "constitue le socle sur lequel toutes les autres entreprises s'édifieront ${ }^{31} »$. Les textes religieux sont traduits afin que leurs auditeurs se les attribuent ${ }^{32}$ :

«Lorsqu'ils entendent chanter les louanges de Dieu dans leur propre langue, les païens, malgré toute leur opposition, sont attirés à venir se joindre à leurs frères convertis et confondus avec eux, ils écoutent le message de miséricorde qui leur est annoncé. ${ }^{33}$ "

Le linguiste fait du pionnier Thomas Arbousset, l'un des premiers écrivains de la version écrite de la langue des Sotho. Le Sesotho écrit est une langue que j'ai qualifiée d'« emparée ». Elle est extraite de la bouche du locuteur originel pour être poursuivie sur le territoire instable qu'est la page. Comme l'explique Xavier Garnier à propos des langues des territoires évangélisés, les missions ont apporté les imprimeries et ont choisi les langues ${ }^{34}$. Les fils se sont alors embrouillés. Dans Moeti oa bochabela, (L'homme qui marchait vers le soleil levant), imprimé à Morija ${ }^{35}$, le catéchiste Thomas Mofolo puise son inspiration à la fois dans la Bible et les mythes fondateurs des Sotho ${ }^{36}$. Dans Chaka, une épopée bantoue ${ }^{37}$, le roman qui l'a fait connaître internationalement, Thomas Mofolo se réfère cette fois à l'ennemi zulu ou au contexte d'émergence de la construction politique de Moshoeshoe. Il semble ainsi superposer des origines pour défier une langue qui n'a jamais été «écrite ou parlée ainsi ${ }^{38}$ ". Le poète zimbabwéen Dambudzo Marechera (1952-1987) qui, dans House of Hunger ${ }^{39}$, décrit l'univers colonial rhodésien, fait, lui, le choix de l'anglais contre le shona pour éviter toute ghettoïsation. Il refuse, dans une attitude désemparée, de recourir à une langue « emparée».

Les missionnaires transmettent les Évangiles et leur civilisation. Alors qu'ils ont rêvé d'un monde loin des excès de l'industrialisation tel qu'il se profile en Europe, leur foi en Dieu et dans le progrès les conduit à favoriser l'entrée des Sotho dans le monde « moderne ». Ils les incitent ainsi à la culture du blé pour développer le commerce avec Le Cap et multiplier les échanges avec les Européens qui s'y sont établis.

Les poèmes héroïques, les dithoko, présentés par Eugène Casalis sont une parole de l'ordinaire, un «va-et-vient entre la communauté et le récitant ». Ils sont l'expression des éleveurs, des chasseurs, des guerriers, des hommes qui font alors la société sotho. Les poètes racontent à la communauté rassemblée leur quotidien comme pour rappeler ce qui les lie entre eux, aux ancêtres et à leurs fils.

En 1867, des gisements de diamant sont découverts à la frontière de l'État Libre d'Orange. De nombreux Africains, dont les territoires ne cessent d'être amputés et dont le mode de vie est définitivement bouleversé, rejoignent la ville minière en construction. En 1878, par la promulgation du Cape Peace Preservation Act (loi pour la préservation de la paix), les autorités du Cap ordonnent aux Africains de rendre leurs armes. La guerre dite des fusils éclate. Elle dure sept mois du 13 septembre 1880 au 29 avril 1881. L'issue du conflit se solde par un compromis. Les Sotho peuvent conserver leurs armes, mais doivent payer un droit de licence au Cap. Le chef Letsie ${ }^{40}$ qui était partisan d'une solution négociée, pacifique, n'a pas su imposer son autorité face aux chefs Lerotholi, Masopha et Joel. Les armes sont progressivement achetées avec 
l'argent de la mine qui devient un lieu de référence pour les Sotho qui sont contraints d'y travailler. La mine devenue lieu de souffrance, d'épreuves et en ce sens initiatique, s'immisce dans l'expression poétique des Sotho. Les dithoko deviennent lifela.

J'ai imaginé le Sotho, l'Africain du premier contact, en tant que " projeté » romantique du missionnaire parti «s'utopier» hors d'Europe. Le Sotho de Kimberley échappe à ce projet parce qu'il est incorporé au monde réel du Blanc, tel qu'il est en train de se faire. Cependant, si le pari romantique, poétique du pionnier est un chemin emprunté pour fuir l'horizon dévoyé qui se dessine en Europe, c'est également l'alibi poétique qui permet au Sotho de survivre, de ne se fondre qu'en partie dans le paysage minier naissant :

«Les lifela sont créés à partir de ressources traditionnelles dans le creuset du travail migrant. Ils montrent comment des gens qui luttent pour faire face à un pouvoir social et économique coercitif se taillent une sphère d'autonomie personnelle au sein de laquelle ils peuvent agir réellement. La réinterprétation de nouvelles épreuves à travers le remodelage de modèles culturels existants est essentielle à la survie sociale. L'autonomie est aussi en partie psychologique et culturelle. Elle exige que l'individu réaffirme de manière positive sa propre valeur humaine, à travers des formes comme le sefela, en opposition à une identité vécue en tant que simple unité de travail dans l'économie politique de l'Afrique du Sud. ${ }^{41}$ "

Tim Ingold exprime ainsi ces mutations :

«Une fois encore, je m'aperçus que les fils se transformaient en trace quand ils constituaient des surfaces : les surfaces de la règle, à quoi tout peut être relié. Mais à mesure que les certitudes de la modernité laissent place au doute et à la confusion, les lignes qui jadis allaient droit au but ont commencé à se fragmenter; aussi l'existence doit-elle une fois de plus se frayer un chemin à travers les fissures. ${ }^{42}$ »

Georges Mabille, arrière-petit-fils d'Eugène Casalis, est devenu missionnaire et a œuvré auprès des Sotho travaillant dans les mines. Un fil s'est mis en place par la généalogie et les mutations socio-économiques. Patrick Harries s'est intéressé au quotidien, aux fragilités et aux troubles partagés par ces hommes reliés ${ }^{43}$. Tim Ingold parle parfois de pointillé pour évoquer des lignes discontinues ou qui se perdent par endroits. Je perçois des points clignotants, des ombres ou des fantômes qui ont peut-être à voir avec les «zombies», errants déconnectés, produits du néolibéralisme, identifiés par Jean et John Comaroff ${ }^{44}$ ?

33 Le 20 décembre 1882, un chant d'Eugène Casalis écrit en béarnais est publié dans Le Protestant Béarnais $\left(\mathrm{n}^{\circ} 16\right)$. Cette même année, le missionnaire a quitté la Maison des missions dont il était directeur depuis 1856. En écrivant ce chant dans une langue de l'enfance, ne se saisit-il pas d'un fil enfoui pour laisser la trace d'une trame momentanément écartée, affirmant un lien ombilical à la langue?

Les lignes que les montagnes dessinent sur l'horizon peuvent raconter des histoires. Elles constituent un morceau de la trame faite et en train de se faire et un gisement d'imaginaires. Le Lesotho est un territoire qui s'épuise dans l'Afrique du Sud. Il est des hommes ordinaires qui ont croisé d'autres hommes ordinaires venus d'horizons différents et qui ont emmêlé leur quotidien. Je ne connais cette surface qu'à partir des traces qu'ont laissées ceux que l'on appelle missionnaires et j'ai tissé des fils pour simuler la trame d'un récit plausible. Je retiens, dans ce contexte, la montagne comme 
fond d'écran. Les Sotho se sont installés dans les Maloti aux XIX siècle. Avant, ils vivaient dans les plaines. Le crocodile, totem des Kwena, le clan de Moshoeshoe, laisse à penser que ces hommes sont venus de régions fluviales plus septentrionales (Limpopo). Tim Ingold invite à penser le monde à partir des lignes pour indiquer que celui-ci n'est pas une extériorité, mais que les hommes sont faits et traversés par les fils tissés de l'environnement. En ce sens, la ligne sur l'horizon se déroule et entrâne l'eau et les hommes, les sépare, les oppose, les réconcilie et murmure ou grave la fable de l'humus et du terreau.

\section{BIBLIOGRAPHIE}

AMBROSE David, The French and the kingdom of Lesotho: a bibliographical study, Bordeaux, Biographies du CEAN, 1989, $24 \mathrm{p}$.

ANKOMAH Baffour \& BAZID Khalid, « Lesotho: Africa's Best Kept Secret », New African, mai 2003, https://www.questia.com/magazine/1G1-101527743/lesotho-africa-s-best-kept-secret-an-icspecial

BLEEK Wilhelm Heinrich Immanuel, A comparative grammar of South African languages, London, Trübner, 1862, (2 vol.).

BÉNICHOU Paul, Le temps des prophètes : doctrines de l'âge romantique, Paris, Gallimard, 1977, 589 p.

BÉNICHOU Paul, Romantismes français II. Les mages romantiques. L'école du désenchantement, Paris, Gallimard, 2004, 989 p.

CASALIS Eugène, Études sur la langue séchuana, Paris, Imprimerie royale, 1841, 103 p.

CASALIS Eugène, Mes souvenirs, Paris, Fischbacher, 1884, 344 p.

Colombani Philippe, «Le Lesotho Highlands Water Project (LHWP) : une coopération réussie dans l'attente d'une politique régionale de l'eau. ", Afrique contemporaine, $\mathrm{n}^{\circ}$ 205, 2003, p. 91-102.

COMAROFF Jean \& John, Of revelation and revolution. Volume one, Christianity, colonialism, and consciousness in South Africa, Chicago, University Chicago Press, 1991, 414 p.

COMAROFF Jean \& John, Of revelation and revolution. Volume two, the dialectics of modernity on a South African frontier, Chicago, University Chicago Press, 1997, 588 p.

COMAROFF Jean \& John, Zombies et frontières à l'ère néolibérale. Le cas de l'Afrique du Sud post-apartheid, traduction Jérôme DAVID, Paris, Les Prairies ordinaires, 2010, 188 p.

COPLAN David Bellin, In township tonight ! : musique et théâtre dans les villes noires d'Afrique du Sud, traduction Catherine BELVAUDE, Paris, Karthala, Nairobi, CREDU, 1992, 468 p.

DEBARBIEUX Bernard, BART François, MORIN Serge \& SALOMON Jean-Noël, « Les montagnes tropicales : identités, mutations, développement », Revue de géographie alpine, tome 90, n 3, 2002, p. 114-115.

DELUMEAU Jean, Naissance et affirmation de la Réforme, Paris, Presses Universitaires de France, 1965, $417 \mathrm{p}$. 
ESPINOSA Laurence, «Anthropologie d'une rencontre - les Sotho dans les écrits des pionniers de la Société des Missions Évangéliques de Paris au XIX ${ }^{\mathrm{e}}$ siècle (1830-1880) », thèse de doctorat en ethnologie, Pau, Université de Pau et des Pays de l'Adour, 2014, 375 p.

GARNIER Xavier, « Nguni wa Thiong'o et la décolonisation par la langue », Les actes du colloque en ligne du musée du quai Branly, Musée du quai Branly (département de la recherche et de l'enseignement), 2011, p. 37-72.

HARRIES Patrick, Butterflies \& barbarians: Swiss missionaries \& systems of knowledge in South-East Africa, Oxford, James Currey, Harare, Weaver Press, Johannesburg, Wits University Press, Athens, Ohio University Press, 2007, 286 p.

HERVIEU Sébastien, «Durement touché par El Niño, le Lesotho craint la famine », Le Monde, le 24 février 2016, http://www.lemonde.fr/planete/article/2016/02/24/en-afrique-australe-lasecheresse-verte-et-l-angoisse-de-la-faim_4870581_3244.html

INGOLD Tim, Une brève histoire des lignes, traduction Sophie RENAUT, Bruxelles, Zones sensibles, Le Kremlin-Bicêtre, Les Belles lettres (4 ${ }^{\text {ème }}$ éd.), 2013, 269 p.

KUNENE Daniel, Heroic Poetry of the Basotho, Oxford, Clarendon Press, 1971, 203 p.

KUNENE Daniel, Thomas Mofolo and the Emergence of Written Sesotho Prose, Johannesburg, Ravan Press, 1989, $251 \mathrm{p}$.

LÖWY Michaël \& SAYRE Robert, Esprits de feu, figures du romantisme anti-capitalistes, Paris, Éditions du Sandre, $2010,288 \mathrm{p}$.

MARECHERA Dambudzo, House of Hunger, London, Ibadan, Nairobi, Heinemann, 1978, 154 p.

MOFolo Thomas, Chaka : une épopée bantoue, Paris, Gallimard, 1940, 271 p. (traduit du sesotho par Victor ELLENBERGER).

RECLUS Elysée, Histoire d'une montagne, Paris, Bibliothèque d'éducation et de récréation, J. Hetzel et $\mathrm{C}^{\mathrm{ie}}, 1875-1876,152 \mathrm{p}$.

RICARD Alain, «Casalis, les Bassoutos, la poésie », Cahiers d'ethnographie, Bordeaux 1, 1995, p. 95-106.

RICARD Alain, « "Un ouvrage d'imagination absolument original...” Moeti oa bochabela de Thomas Mofolo : premier roman africain » dans RICARD Alain \& VEIT-WILD Flora (éditeurs scientifiques), Interfaces between the oral and the written, Amsterdam, New-York, Rodopi, 2005, p. 87-97.

ZORM Jean-François, Le grand siècle d'une mission protestante : la mission de Paris de 1822 à 1914, Paris, Karthala Éditions, 2012, 791 p.

\section{NOTES}

1. E. Reclus, Histoire d'une montagne, p. 9.

2. T. Ingold, Une brève histoire des lignes.

3. L. Espinosa, «Anthropologie d'une rencontre - les Sotho dans les écrits des pionniers de la Société des Missions Évangéliques de Paris au XIX ${ }^{e}$ siècle (1830-1880)».

4. Entrée « Réseau », Trésor de la Langue Française, (en ligne).

5. J.\&J. Comaroff, of revelation and revolution.

6. La partie du Drakensberg au sein de laquelle est né le Lesotho. 
7. Moshoeshoe $1^{\text {er }}(1786-1870)$, chef de clan, maître du Basutoland, protectorat signé avec les Britanniques en 1843.

8. L. Espinosa, «Anthropologie d'une rencontre - les Sotho dans les écrits des pionniers de la Société des Missions Évangéliques de Paris au XIX siècle (1830-1880)», p. 129-133. Les Hollandais, puis les Britanniques sont présents dans la région du Cap depuis la première moitié $d u \mathrm{XVII}^{\mathrm{e}}$ siècle pour les premiers et la fin $\mathrm{du} \mathrm{XVIII}^{\mathrm{e}}$ siècle pour les seconds.

9. L. Espinosa, «Anthropologie d'une rencontre - les Sotho dans les écrits des pionniers de la Société des Missions Évangéliques de Paris au XIXe siècle (1830-1880) », p. 25-28.

10. Thomas Arbousset, l'un des pionniers, est né à Pignan, dans l'Hérault, à l'orée des Cévennes. Sur le protestantisme :J. Delumeau, Naissance et affirmation de la Réforme.

11. L. Espinosa, "Anthropologie d'une rencontre - les Sotho dans les écrits des pionniers de la Société des Missions Évangéliques de Paris au XIX ${ }^{e}$ siècle (1830-1880) », p. 14-16.

12. Les accords AGOA (African Growth Opportunities Act) prévoyaient que les confections exportées soient en tissus du pays exportateurs. Or, les Chinois avaient prévu d'écouler leur propre coton. Ils ont fermé les entreprises. (AGOA.info, https://agoa.info/profiles/ lesotho.html.)

13. JME 1834, p. 14.

14. La Libre Belgique, 11 août 2016, http://www.lalibre.be/actu

15. B. Debarbieux, F. Bart, S. Morin et J.-N. Salomon, "Les montagnes tropicales»: Identités, mutations, développement; P. Colombani, Le Lesotho Highlands Water Project (LHWP) : une coopération réussie dans l'attente d'une politique régionale de l'eau.

16. S. Hervieu, «Durement touché par El Nino, le Lesotho craint la famine », Le Monde, le 24 février 2016, http://www.lemonde.fr/planete/article/2016/02/24/en-afriqueaustrale-la-secheresse-verte-et-l-angoisse-de-la-faim_4870581_3244.html.

17. Ibid.

18. E. Reclus, Histoire d'une montagne, p. 10.

19. T. Ingold, Une brève histoire des lignes, p. 10.

20. B. Ankomah \& K. Bazid, « Lesotho: Africa's Best Kept Secret », New African, mai 2003, https://www.questia.com/magazine/1G1-101527743/lesotho-africa-s-best-kept-secretan-ic-special.

21. A. Ricard, «Casalis, les Bassoutos, la poésie ».

22. E. Casalis, Mes souvenirs, p. 51.

23. Je me réfère en particulier à : P. Bénichou, Le temps des prophètes: doctrines de l'âge romantique; Les mages romantiques. L'école du désenchantement; M. Löwy, R. Sayre, Esprits de feu, figures du romantisme anticapitalistes.

24. Lettre d'Eugène Casalis du 18 juillet 1842, dans L. Espinosa, "Anthropologie d'une rencontre - les Sotho dans les écrits des pionniers de la Société des Missions Évangéliques de Paris au XIX siècle (1830-1880)», p. 181.

25. Lettre d'Eugène Casalis du 10 novembre 1847, ibid.p. 181.

26. Lettre d'Eugène Casalis du 8 janvier 1847, ibid. p. 181.

27. D. Ambrose, The French and the kingdom of Lesotho: a bibliographical study, p. 1. 
28. T. Ingold, Une brève histoire des lignes, p. 8.

29. E. Casalis, Études sur la langue sechuana. Pour Alain Ricard, cet ouvrage est un précis linguistique mais aussi une ouverture sur une forme poétique de Bantu d'Afrique australe.

30. W.H.I. Bleek, A comparative grammar of South African languages.

31. A. Ricard, « Casalis, les Bassoutos, la poésie », p. 72.

32. Dans cette tâche, ils suivent les traces de Robert Moffat (1795-1883) de la mission de Londres, installé à Kuruman depuis 1817 parmi des peuples de langue tswana, qui a traduit la Bible entre 1830 et 1857. C'est la première version du livre saint dans une langue bantu d'Afrique australe.

33. JME 1848, p. 18, dans L. Espinosa, «Anthropologie d'une rencontre - les Sotho dans les écrits des pionniers de la Société des Missions Évangéliques de Paris au XIX siècle (1830-1880)», p. 229.

34. X. Garnier, « Ngugi wa Thiong'o et la décolonisation par la langue ».

35. Première station créée par les missionnaires de la SMEP où fut installée une imprimerie qui existe encore aujourd'hui.

36. A. Ricard, " "Un ouvrage d'imagination absolument original..." : Moeti oa bochabela de Thomas Mofolo : premier roman africain ».

37. T. Mofolo, Chaka: une épopée bantoue.

38. D. Kunene Daniel, Heroic Poetry of the Basotho ; Thomas Mofolo and the Emergence of Written Sesotho Prose.

39. D. Marechera, House of Hunger.

40. Letsie $1^{\text {er }}$ (1811-1891), règne de 1870 à 1891 sur le protectorat.

41. D. B. Coplan, In township tonight !: musique et théâtre dans les villes noires d'Afrique du Sud, p. 32-33.

42. T. Ingold, Une brève histoire des lignes, p. 11.

43. P. Harries, Butterflies \& barbarians : Swiss missionaries \& systems of knowledge in SouthEast Africa.

44. J.\&J. Comaroff, Zombies et frontières à l'ère néolibérale. Le cas de l'Afrique du Sud postapartheid.

\section{RÉSUMÉS}

La montagne est une ligne brisée sur l'horizon ou une horizontalité. Quand Eugène Casalis est arrivé en 1833 chez les Sotho, dans le Drakensberg, en Afrique australe, il a reconnu un dessin familier. Cette empreinte paysagère a-t-elle suffi à créer une proximité, voire une solidarité entre les ainsi nommés "Cavaliers de la montagne », le religieux et ses coreligionnaires? Sur ce sujet, les récits insérés dans les Journaux des Missions Évangéliques offrent des pistes en pointillé. Aujourd'hui le Lesotho s'épuise dans l'Afrique du Sud. Les derniers mineurs continuent sans doute de composer des lifela, héritages des dithoko, poèmes qu'Eugène Casalis fit connaître à ses 
lecteurs européens. M'inspirant des travaux de l'anthropologue Tim Ingold, je tente de nouer la ligne des montagnes, en l'occurrence les Maloti et les Pyrénées, couchée sur l'horizon aux mots laissés par le missionnaire pour suggérer un récit.

\section{AUTEUR}

\section{LAURENCE ESPINOSA}

Docteure en anthropologie, chercheure associée laboratoire Territoires, Expressions, Mobilités (ITEM - EA 3002), Université de Pau et des Pays de l'Adour 


\title{
Circulations savantes et production des identités territoriales dans les Pyrénées centrales (1880-1930)
}

\author{
Arnauld Chandivert
}

1 De nombreux travaux ont été consacrés en France à ce que l'on peut appeler la formation des images territoriales, l'institution des spécificités culturelles ou encore la construction des emblématiques, que ce soit à un niveau régional ou local. Ces travaux ont notamment concerné la question de la géographie administrative et de l'élaboration de représentations savantes des territoires ${ }^{1}$, le développement d'une fierté localiste ${ }^{2}$ ou le déploiement de l'érudition provinciale ${ }^{3}$. Ils ont aussi traité du développement d'une histoire elle aussi provinciale, autour de la figure de l'antiquaire par exemple $e^{4}$, de la construction des images régionales associées à certaines productions $s^{5}$ ou de leurs liens avec le développement du tourisme ${ }^{6}$, etc.

2 Ce type de perspective a bien sûr été appliqué aux espaces montagnards, depuis longtemps conçus comme spécifiques et supposés conservatoires de mœurs anciennes. Ce fut notamment le cas pour les Alpes, mais aussi pour les Pyrénées, quoique dans une moindre mesure et à partir de thématiques parfois quelque peu différentes de celles mentionnées ci-dessus - la frontière, le paysage ${ }^{7}$. En ce qui concerne ce massif pyrénéen, l'examen des mécanismes de fixation des caractères locaux semble avant tout lié à des moments (sa " découverte " à la fin du XvIII e siècle, le romantisme), des lieux (les villes d'eau) et des producteurs spécifiques, voyageurs ou "découvreurs" illustres, rédacteurs de diverses descriptions des territoires et de leurs habitants. Si Serge Briffaud a pu signaler l'existence d'une proportion non-négligeable de récits produits par des auteurs locaux ${ }^{8}$, il n'empêche que ces textes et ceux qui les ont rédigés n'ont que peu été analysés au titre de leur rôle dans l'élaboration d'une typicité pyrénéenne et dans la fixation du répertoire la concernant.

3 L'objectif de cet article est de traiter d'une période, de lieux et d'acteurs qui n'ont pas réellement retenu l'attention lorsqu'il s'agissait d'examiner ce travail d'établissement des représentations des spécificités territoriales et culturelles pyrénéennes. La période en question est celle qui court entre les années 1880 et les années 1930. La zone 
géographique concernée est celle d'une portion des Pyrénées centrales (Ariège et Haute-Garonne) qui, hormis le cas de Bagnères-de-Luchon, ne fut pas un des lieux centraux du thermalisme et qui, pour cela, a quelque peu échappé aux regards des voyageurs et découvreurs des $\mathrm{XVIII}^{\mathrm{e}}$ et $\mathrm{xIX}^{\mathrm{e}}$ siècles. Les acteurs sont ceux qui s'investissaient alors dans le développement des études locales, des sociétés d'érudition, et participaient ainsi aux formes de sociabilité et aux activités de la notabilité savante provinciale. Bien qu'exerçant à partir de positions localisées, ces acteurs n'opéraient certes pas en toute autonomie, et leur travail d'établissement des singularités territoriales et culturelles obéissait bien sûr à un ensemble de codes diffusés de manière plus générale au sein de la société. Mais les modalités de mise en œuvre de ce travail et les objectifs visés s'inscrivaient bel et bien, eux, dans un horizon de proximité marqué par cet ancrage localisé.

4 Pour autant, il serait inexact de considérer que les activités de ces érudits, aujourd'hui quelque peu ou totalement oubliés pour nombre d'entre eux, n'opéraient qu'à l'échelle du localisme dont ils faisaient montre dans leurs travaux. Car l'on ne peut au contraire qu'observer combien ce localisme était finalement multi-positionné. Cela concernait notamment les centres d'intérêt extrêmement variés de ces acteurs, auxquels répondait le recours à des genres narratifs et à des supports de publication eux-mêmes très divers. Cette multiplicité se manifestait aussi par la pluralité des affiliations à divers organismes savants ou artistiques et par une mobilité géographique assez marquée entre les scènes locales, départementales ou régionales. L'ensemble dessinait ainsi des réseaux d'érudition fortement entrecroisés, de réels «territoires circulatoires" (selon l'expression qu'Alain Tarrius a proposée dans le cadre de son analyse des phénomènes migratoires ${ }^{9}$ ), du localisme érudit, au travers desquels opérait la fixation des caractères des lieux et de leurs habitants.

5 L'analyse de ce travail de caractérisation n'entend donc pas se limiter aux espaces dont il sera question ici, soit en l'occurrence l'Ariège, avec ses divisions internes, et le Comminges voisin, région issue de l'ancien comté éponyme située au sud de la HauteGaronne et correspondant à l'actuel arrondissement de Saint-Gaudens, sous-préfecture de ce département. Il ne se limitera pas plus aux entreprises savantes et culturelles qui y étaient implantées - Société ariégeoise des sciences, lettres et arts ; Société des études du Couserans (ouest de l'Ariège); Société des études du Comminges, etc. Il s'agira au contraire de signaler tout ce que la définition des spécificités de ces espaces devait à l'insertion dans ces réseaux d'érudition. Cette insertion s'imposait de par certaines caractéristiques des territoires considérés. Adossés aux Pyrénées, majoritairement ruraux et à l'infrastructure urbaine peu développée, ceux-ci n'étaient pas prédisposés à jouer un rôle notable dans la production des savoirs, si ce n'est au titre de terrains d'enquête et d'exploration pour des travaux menés depuis des lieux plus centraux, à l'image de Toulouse, capitale régionale possédant une université ${ }^{10}$ et regroupant diverses sociétés et académies prestigieuses.

6 Cette position périphérique ne pouvait qu'avoir des conséquences dans la définition du cadre des entreprises érudites locales et dans la mobilisation des ressources qui leur étaient nécessaires. L'objectif était en effet, non pas nécessairement de contribuer aux avancées de la science, mais plutôt d'exhumer et de réunir tous les matériaux concernant les territoires d'action de ces sociétés savantes, montrant ainsi qu'ils étaient porteurs de certaines particularités, ceci en prenant appui sur les bonnes âmes souhaitant participer à cet effort et possédant quelques capacités et titres de légitimité 
pour ce faire. Le nombre de personnes disposant de ces ressources étant quelque peu limité in situ de par les caractéristiques des zones concernées, ce n'est finalement qu'au travers de l'insertion dans des réseaux plus larges que les objectifs de reconnaissance des singularités locales pouvaient être atteints.

7 Ce n'est donc qu'en examinant les modalités d'organisation des activités érudites, le recrutement social de ces sociétés et les formes de sociabilité qui y avaient cours que l'on peut comprendre les mécanismes de singularisation des territoires mis en œuvre au sein de ces entreprises scientifiques et culturelles et leur dimension " circulatoire ". Ce faisant, il devient possible de mettre à jour l'organisation des échelles de la légitimité savante entre différentes scènes sociales (locales, régionales ou nationales) ainsi que les formes de division du travail qui y étaient associées, tout en signalant en quoi elles ont été affectées par la spécialisation et la professionnalisation des savoirs qui opèrent sur la période.

\section{Les territoires de l'érudition pyrénéenne: développement, recrutement social et univers d'action}

Comme l'a montré Jean-Pierre Chaline, le mouvement de création des sociétés savantes se déploie sur l'ensemble du xix siècle et au cours du premier tiers du suivant, les années 1850 constituant un moment marquant dans l'amplification et la généralisation des implantations ${ }^{11}$. La situation est légèrement différente dans le Midi toulousain et pyrénéen, puisque le développement des activités académiques, érudites et culturelles y connaît une accélération un peu plus tardive, dans le dernier quart du XIX ${ }^{e}$ siècle. Ce mouvement, néanmoins comparable à celui que l'on perçoit à l'échelle nationale, permet d'appréhender une des logiques qui le guident. En effet, les institutions les plus anciennes et aussi les plus prestigieuses ont tout d'abord été créées à Toulouse, ville la plus importante de la région: le Consistoire du gai savoir au XIV siècle, devenu académie des jeux floraux à la fin du XVII ${ }^{e}$ siècle ; l'Académie des sciences, inscriptions et belles-lettres de Toulouse au milieu du XVII siècle ; la Société archéologique du Midi de la France en 1831. Ce n'est qu'autour du milieu du XIX siècle que ces créations ont concerné les grandes villes des départements voisins, soit par exemple l'Aude avec la naissance de l'Académie des arts et sciences de Carcassonne en 1846; Tarbes avec la création de la Société académique des Hautes-Pyrénées en 1856; les PyrénéesAtlantiques avec celle de la Société des sciences, lettres et arts de Pau en 1841, disparue en 1843 et refondée en 1871. Enfin, l'on a assisté dans le dernier quart du XIX siècle à une multiplication de ces sociétés, qui se sont implantées bien sûr dans les grandes villes de la région, mais aussi dans des centres urbains de moindre importance : la Société des arts, sciences et lettres de l'Ariège à Foix en 1882 et la Société des études du Couserans à Saint-Girons, sous-préfecture de l'ouest de ce même département, en 1899 ; la Société des études du Comminges à Saint-Gaudens, sous-préfecture de la HauteGaronne, en 1884, etc. Ce mouvement général permet ainsi d'apercevoir une des logiques qui guide ce champ du savoir tel qu'il est constitué à la fin du XIX siècle et au début du suivant, soit la corrélation entre distribution chronologique des créations de sociétés, échelle de distribution des implantations géographiques et distribution des hiérarchies du prestige savant - les plus anciennes sociétés les plus renommées ayant tout d'abord été créées dans les plus grandes villes et les sociétés de moindre renommée l'ayant été dans de petites villes et plus tardivement. L'objectif, au niveau 
local, est donc bien souvent de combler le retard, de participer à un mouvement déjà engagé, de porter l'attention sur des territoires n'ayant pas bénéficié jusque-là d'une attention érudite. Il s'agit de territoires que les membres de ces sociétés considèrent insuffisamment reconnus et appréciés par les savants, les touristes ou les industriels, et ce malgré ce qu'ils estiment être leur richesse.

9 Le recrutement social de ces sociétés correspond assez aux caractéristiques déjà signalées par J.-P. Chaline ${ }^{12}$. Elles regroupent une élite montante avant tout formée de professions libérales, de fonctionnaires ayant quelque rang, notamment dans l'enseignement, élite masculine, diplômée, finalement peu âgée, où les milieux d'affaires (commerçants, industriels) sont peu présents et où les nobles et les abbés côtoient les médecins et les avocats, quelques propriétaires terriens ainsi que des archivistes où des conservateurs de bibliothèque (tabl. 1).

Tabl. 1. - Répartition par domaines d'activité des effectifs des deux principales sociétés savantes de l'Ariège (Société ariégeoise des sciences, lettres et arts, 91 membres) et du sud de la HauteGaronne (Société des études du Comminges, 51 membres) à leur création (1882 et 1884).

\begin{tabular}{|c|c|c|c|}
\hline $\begin{array}{l}\text { Domaine } \\
\text { d'activité }\end{array}$ & Effectifs & Pourcentages & Précisions \\
\hline $\begin{array}{l}\text { Professions } \\
\text { libérales }\end{array}$ & 51 & 36 & $\begin{array}{l}\text { Dont médecins, avocats, notaires, avoués (en ordre } \\
\text { décroissant des effectifs). }\end{array}$ \\
\hline Enseignement & 21 & 15 & $\begin{array}{l}\text { Dont enseignants et fonctions administratives } \\
\text { (instituteurs jusqu'à directeur d'école } \\
\text { polytechnique). }\end{array}$ \\
\hline Administration & 29 & 20.5 & $\begin{array}{l}\text { Fonctions préfectorales, justice, armée, ponts et } \\
\text { chaussées, métiers de la culture (bibliothèque, } \\
\text { archives). }\end{array}$ \\
\hline $\begin{array}{l}\text { Hommes } \\
\text { politiques, élus }\end{array}$ & 12 & 8.5 & Prépondérance des républicains radicaux en Ariège. \\
\hline Industrie & 7 & 5 & Imprimeurs locaux. \\
\hline Clergé & 7 & 5 & Abbés locaux. \\
\hline $\begin{array}{l}\text { Propriétaires } \\
\text { terriens }\end{array}$ & 11 & 8 & \\
\hline Autres & 4 & 2.8 & Dont deux publicistes et deux libraires. \\
\hline Total & 142 & 100 & Dont une dizaine de membres communs. \\
\hline
\end{tabular}

Bulletin de la Société ariégeoise des sciences, lettres et arts, Foix, Imprimerie Barthe, 1882 ; Revue de Comminges, Saint-Gaudens, Imprimerie Abadie, 1885.

10 Bien qu'éclectique, cette composition sociale garantit un certain entregent, l'existence de ressources intellectuelles et temporelles pouvant être mises au service de l'activité érudite et le partage de formes de sociabilité spécifiques, liées à la tenue de séances où sont exposés divers travaux, à l'organisation de congrès, mais aussi de repas, avec leur lot de toasts ou de brindes. L'ensemble était associé à un ethos de bonne tenue correspondant à la figure de ce que l'on peut appeler l'honnête homme de science, où l'élégance du comportement s'exprime notamment dans la recherche de l'éloquence, le 
goût de la mesure et l'adoption de valeurs de politesse et de civilité, par-delà les clivages politiques, en étant portée par une ambition érudite commune.

Cette référence à l'honnête homme, idéal social du xvII siècle, se retrouve aussi au niveau des centres d'intérêt. Elle s'oppose finalement à la figure du savant comme spécialiste, mobilisant un savoir choisi et adéquat pour traiter de problèmes spécifiques, et promeut au contraire l'appui sur les ressources de la culture générale et sur la polyvalence pluridisciplinaire ${ }^{13}$. Certes, nombre des acteurs de ces réseaux d'érudition participent à l'essor de disciplines naissantes ou en plein développement (archéologie, préhistoire), mais leurs profils ne sont pas encore totalement ceux du savant spécialiste: leurs centres d'intérêt demeurent pluriels, de même que leurs supports éditoriaux, et ils prennent tous part aux activités de diverses sociétés et académies départementales ou régionales, notamment celles qui conservent une dimension généraliste, comme l'Académie des sciences, inscriptions et belles-lettres de Toulouse.

\section{Centres d'intérêt, registres des singularités et modes de production}

Les travaux effectués par ces sociétés frappent d'abord par leur éclectisme : histoire, archéologie, préhistoire, notes sur des courses en montagne, botanique, archéologie, description de monuments, travaux de géographie historique, notes sur les patois, collecte de proverbes et de contes, notes sur la vie de divers personnages locaux, etc. Cet éclectisme est avant tout le produit du recrutement social et de l'idéal intellectuel qui ont cours dans ces sociétés. En effet, leurs membres, bien qu'appartenant à une élite de capacité, viennent de divers horizons comme cela a été signalé et n'ont pas toujours de compétences pointues en tel ou tel domaine. Il convient dès lors de s'accommoder de la variété des centres d'intérêt résultant pour partie de la composition de ces sociétés. De plus, l'objectif est avant tout de participer aux séances avec des communications de bonne tenue donnant ensuite lieu à des articles sur tel ou tel sujet, sans présupposer chez les uns ou les autres des connaissances précises et avancées. La poursuite de cet objectif s'effectue en mobilisant les ressources d'une élocution et d'une écriture distinguées comme celles d'un bagage de culture classique. Mais il est aussi possible de réduire cet éclectisme à un principe directeur: tous ces travaux concernent les territoires d'action de ces sociétés. Les personnages comme les monuments sont locaux; les grottes préhistoriques récemment découvertes et visitées sont locales; l'histoire, souvent anecdotique, liée à la découverte de documents ou rappelant des événements passés des territoires concernés, perd ce caractère car elle est spécifiquement locale.

13 Ainsi, cette variété de centres d'intérêt et de travaux participe d'un mouvement visant à établir les spécificités des territoires d'action de ces sociétés, à en poser les fondations : rappeler les grandeurs du comté de Foix ou de celui du Comminges voisin, établir l'existence d'une histoire propre à ces espaces, une histoire à soi ${ }^{14}$, qui ne serait pas uniquement référée à l'histoire nationale; montrer qu'existent dans le Comminges et en Ariège des grottes préhistoriques marquant l'ancienneté du peuplement de ces zones géographiques; pointer la physionomie propre à ces vallées pyrénéennes en y consacrant une livraison de la revue; signaler l'existence de parlers locaux, qu'ils soient languedociens ou gascons, etc. 

perspective d'attachement savant à la "petite patrie», notion centrale au sein de la mouvance régionaliste à cette époque et que la Troisième République a fortement mobilisée au service du renforcement du sentiment d'appartenance nationale ${ }^{15}$. Mais ces opérations étaient aussi déterminantes pour assurer une certaine reconnaissance, non pas simplement aux territoires en question, mais aussi aux sociétés qui prenaient en charge leur étude. Créées tardivement, dans des espaces quelque peu périphériques au sein du champ du savoir et qui ne bénéficiaient pas d'une renommée aussi importante que celle d'autres lieux des Pyrénées, ces sociétés entraient dans le grand mouvement de l'érudition savante avec un handicap. Grandir le territoire en montrant les richesses, considérées jusque-là trop ignorées, qu'il possède, c'était en même temps grandir la société qui effectuait cette divulgation. Mais ces «manœuvres pour se grandir ${ }^{16} »$ ne pouvaient atteindre leur but qu'en bénéficiant d'une audience minimale. Il s'agissait alors d'attirer l'attention, de réussir par exemple à faire se dérouler sur le territoire le congrès annuel d'une société savante nationale; à faire participer à ses travaux un savant d'envergure régionale ou plus encore nationale; d'effectuer une découverte donnant lieu à une forte publicité; de s'inscrire dans des réseaux d'échanges avec d'autres sociétés et de prendre part à la structuration régionale de ces réseaux. Captation des ressources externes et externalisation des ressources endogènes étaient donc les deux mécanismes socio-symboliques et les principales formes de circulation qui permettaient d'assurer, dans le même mouvement, la reconnaissance des qualités et des spécificités du territoire et celle des sociétés qui se chargeaient de son étude.

Dans cette perspective visant à fonder l'intérêt et la singularité des zones concernées, de très nombreuses ressources pouvaient être mobilisées, jusqu'à la pierre, à l'image de la lherzolite, roche magmatique issue du manteau terrestre que l'on trouve rarement à la surface et cependant fortement présente dans un site ariégeois, qui pouvait dès lors servir d'appui à la mention de la spécificité du lieu. Néanmoins, certaines ressources offraient un intérêt particulier. Il s'agissait notamment des textes médiévaux comme les traités de lies et passeries et les chartes de coutumes. Ces dernières tout particulièrement démontraient l'existence de spécificités dans le régime du droit féodal, en révélant les libertés dont jouissaient les populations locales, pour ce qui est $\mathrm{du}$ droit d'exploitation des ressources par exemple (bois, parcours du bétail). Les publications de ces chartes furent nombreuses. Ce sont elles qui sont montrées aux participants au congrès de la Société française d'archéologie qui se déroulait en Ariège en 1884. C'est toujours un membre de cette société qui présente un mémoire sur les libertés et privilèges du comté de Foix à la fin du XIV et au commencement du Xve siècles au congrès des sociétés savantes de la Sorbonne en 1895. Ces chartes sont enfin l'objet d'un vœu en faveur de leur travaux à l'échelle du Midi de la France, formulé lors du congrès bordelais de l'Association pyrénéenne en 1891, association qui entendait réunir les sociétés savantes du grand Sud-Ouest et avait été créée à l'initiative de Julien Sacaze, fondateur de la Société des études du Comminges, et Félix Garrigou, acteur majeur de sa consœur ariégeoise.

16 Ces travaux, dont on comprend qu'ils s'inscrivaient dans les formes de circulation mentionnées précédemment, ouvraient la voie au développement du mythe des petites " républiques montagnardes ", utilement ramené à une plus juste raison par Christian 
Desplat notamment ${ }^{17}$. Ils permettaient d'introduire la référence à la fierté, à l'esprit d'indépendance, voire au caractère " rebelle » prêtés au montagnard ${ }^{18}$.

De manière plus générale, les travaux sur la préhistoire, l'archéologie, l'épigraphie et l'histoire de l'antiquité servaient eux aussi de ressources pour marquer la singularité de tel ou tel espace. Ils signalaient la profondeur historique des peuplements locaux, notamment grâce à l'archéologie préhistorique et à la fouille de nombreuses grottes, fortement présentes dans les massifs karstiques de l'Ariège et du sud de la HauteGaronne. Bénéficiant d'une certaine notoriété, ces grottes attiraient des savants renommés ou en passe de le devenir comme Édouard Piette (1827-1906), Émile Carthaillac (1845-1921) et Félix Régnault (1847-1908), mais aussi des amateurs érudits locaux participant à ce travail d'exhumation des traces du passé le plus lointain, à l'exemple de l'abbé Cau-Durban (1844-1908), membre de toutes les sociétés savantes locales de la région. La position de ces scientifiques déjà reconnus, qui faisaient des grottes locales leurs terrains d'exploration, concourrait à l'activation des mécanismes de reconnaissance conjointe des qualités du territoire et des sociétés qui se chargeaient de son étude, mécanismes dont j'ai signalé précédemment l'existence. En effet, ces scientifiques servaient de médiateurs entre plusieurs lieux emboîtés d'organisation des renommées savantes et territoriales. Ils publiaient les résultats de leurs travaux dans les revues des sociétés savantes locales, mais aussi régionales et nationales. Non seulement les revues locales étaient rehaussées grâce la publication du texte d'un auteur reconnu mais, par son intermédiaire, l'on connaissait aussi jusqu'à Paris l'existence de contrées pyrénéennes recelant de réels trésors. Par cet intermédiaire encore et suite à de belles découvertes, des amateurs locaux pouvaient voir leur notoriété s'accroître, leur permettant de communiquer à propos de leurs trouvailles dans des sociétés prestigieuses d'envergure régionale, le plus souvent à Toulouse, voire à Paris dans certains cas. De nouveau, la reconnaissance de la valeur des territoires et celle des érudits qui se consacraient à leur étude étaient liées, et cette liaison fonctionnait en prenant appui sur des dispositifs de circulation assez spécifiques.

L'archéologie de l'Antiquité et l'épigraphie fournissaient elles aussi un registre de singularisation des plus pertinents. Ces deux domaines, indissociables, en traitant de la période de la présence romaine, permettaient d'éclairer chacun quant à une histoire où, avant la constitution des territoires féodaux (comté de Comminges, vicomté du Couserans et comté de Foix d'ouest en est pour ce qui est de la zone ici étudiée), certains de ces espaces étaient déjà assez fortement individualisés. En effet, les travaux menés par les sociétés ariégeoise et commingeoise portaient leur attention sur l'existence de deux cités romaines (Lugdunum Convenarum et Lugdunum consoranorum, actuelles Saint-Bertrand-de-Comminges en Haute-Garonne et Saint-Lizier en Ariège), centres relativement importants de ce qui deviendra la province de Novempopulanie. Gisements archéologiques très riches, donnant lieu à une longue campagne de fouille soutenue par des membres de la Société des études du Comminges à partir de 1913 en ce qui concerne Saint Bertrand, la référence à ces cités servait la fierté localiste d'un passé glorieux tout comme elle offrait, par le jeu de la succession des périodes historiques (préhistoire, peuplement préromain, antiquité romaine), la possibilité d'évoquer l'idée d'une permanence quasi trans-historique des relations entre les lieux et leurs habitants. Ainsi, en 1899 lors de la création de la Société des études du Couserans, territoire de l'Ouest de l'Ariège, ancien pagus gallo-romain, une communication sur «Les premiers habitants du Couserans " permet à son auteur de noter que « avant d'être des Ariégeois, titre à peine séculaire, nous étions depuis 2000 
ans des Couserannais, Consorani, disent les géographes romains ${ }^{19} »$. Par ailleurs, l'on sait aussi combien sa contribution au développement de l'épigraphie, science naissante à la fin du XIX ${ }^{e}$ siècle, a permis à Julien Sacaze, fondateur de la Société du Comminges et initialement avocat à Saint-Gaudens, d'acquérir une renommée lui permettant d'obtenir un cours libre d'«Épigraphie et de géographie historique des Pyrénées à l'époque romaine " à la Faculté des lettres de Toulouse en 1887 - autre forme de circulation, qui est celle d'une notoriété grandissante donnant accès aux lieux centraux du savoir régional.

19 Sans pouvoir continuer la démonstration en envisageant d'autres domaines du savoir (géographie, linguistique et même la science naissante du folklore), notons cependant que, combinant différents mécanismes circulatoires, les contributions à ces différents domaines, puisqu'elles étaient régies par le principe d'une unité de lieu (le territoire d'action de chaque société), étaient porteuses d'un effet cumulatif quant à la singularisation de ces territoires. En effet, le marquage de leur spécificité était multiplié par les recoupements entre ces contributions. Ainsi, toujours au sujet de cette micro-région de l'Ouest de l'Ariège, le Couserans, pour lequel une société d'études ad hoc a été créée en 1899 , le président de cette société pouvait présenter une communication lors du Congrès national des sociétés savantes de 1925 sur «le Couserans, considéré comme une véritable région orographique, hydrographique, ethnique, linguistique, historique, économique, administrative et intellectuelle». À cette occasion, c'est finalement l'ensemble des travaux, pourtant éclectiques, de cette société qui était mobilisé au service d'une ambition érudite de singularisation territoriale. Remarquons aussi que cette ambition était atteinte en envoyant à Paris lors d'un congrès national le représentant d'une modeste société locale, qui accédait à une certaine reconnaissance tout en faisant exister aux yeux du monde savant une micro-région inconnue ou presque une décennie auparavant.

\section{Savoirs érudits : organisation des échelles de la légitimité et formes de division du travail}

Outre leur rôle jusque-là peu analysé dans l'établissement des spécificités territoriales et culturelles, ces sociétés, comme tant d'autres, offrent aussi un intéressant objet d'analyse des modes de production des sciences et des richesses locales, mais aussi nécessairement régionales et nationales, lorsque ces savoirs et ces richesses étaient identifiés à ces niveaux.

21 Ainsi, les cas de certains de ces acteurs permettent de relever ce que notait Stéphane Gerson, à savoir l'entrecroisement entre la production locale de l'histoire et de ses monuments et la participation au mouvement «central» d'inventaire ou de recensement des richesses, servant la constitution d'un corpus national ${ }^{20}$. Le fait que certains érudits soient nommés correspondants du ministère de l'Instruction publique, de l'administration supérieure des beaux-arts et soient en relation avec la Commission des monuments historiques, conduisait à un double renforcement de leurs positions : légitimation par et vers Paris de la valeur d'historiens et d'érudits de ces acteurs, et renforcement corollaire de leur emprise locale, en cette période de professionnalisation de la gestion du patrimoine ${ }^{21}$. Les relations local/national restaient pourtant tendues dans cette ambivalence que note Gerson ${ }^{22}$. Les correspondants locaux étaient régulièrement soupçonnés d'incompétence et de parti 
pris, alors que de leur côté, les érudits occupant ces fonctions ne cessaient de se plaindre de la difficulté à faire reconnaître les richesses locales et, quand c'était le cas, de ce qu'ils considéraient être leur pillage par la capitale. Mais l'existence des réseaux de notabilité savante permet aussi de remarquer la place grandissante de la scène régionale à cette période de la fin $\mathrm{du} \mathrm{XIX}^{\mathrm{e}}$ siècle et du début du siècle suivant.

En effet, comme cela a été signalé précédemment, les savants d'envergure régionale, principalement regroupés dans la Ville rose pour ce qui est des cas ici étudiés, jouaient un rôle déterminant de médiateurs entre les différents niveaux de la hiérarchie scientifique (local, départemental, régional, national). Ils faisaient des espaces alentour de réels terrains d'enquête, mais aussi d'observation et de sélection des travaux d'autres érudits susceptibles de retenir l'attention. Ils prenaient part aux activités des sociétés départementales ou locales, qu'ils faisaient briller par leur présence et où ils étaient susceptibles de distinguer des recherches ou découvertes d'importance, assurant en même temps la reconnaissance de leurs auteurs. En retour, ces «savants périphériques " pouvaient accéder à des positions au sein des sociétés et académies régionales les plus prestigieuses, voire à une certaine renommée parisienne, obtenue bien souvent par l'entremise des "savants régionaux». Cette spécificité des positions régionales, associée à la multiplication des sociétés savantes dans le dernier quart du $\mathrm{xIX}^{\mathrm{e}}$ siècle, a finalement donné lieu à ce que l'on peut appeler une première régionalisation des pratiques savantes et érudites, avec par exemple la création à Toulouse de l'Association pyrénéenne en 1888, dont l'organe, la Revue des Pyrénées et de la France méridionale, est paru dès l'année suivante.

L'importance de ce niveau régional s'est ensuite renforcée, grâce notamment au développement de l'université après la réforme de 1896 et plus encore après la Première Guerre mondiale. La place grandissante prise par les universitaires, producteurs les plus légitimes du savoir scientifique, conduisait aussi à un affaiblissement corrélatif des positions des érudits, renvoyés en quelque sorte au statut d'amateurs. Et ce n'est sans doute pas un hasard si la Revue des Pyrénées et de la France méridionale, issue de la régionalisation des activités érudites mentionnée ci-dessus, est publiée sous les auspices du Conseil de l'université à partir de 1905, avec l'ambition que les facultés "achèvent, précisent et systématisent, en s'offrant à guider méthodiquement leurs recherches, l'œuvre pieuse des sociétés locales » selon Célestin Bouglé (1870-1940), sociologue de renom rattaché à l'école durkheimienne alors en poste à Toulouse ${ }^{23}$.

Plus généralement, on assiste aussi durant le premier tiers $d u x^{e}$ siècle et à l'échelle nationale, au développement d'écoles scientifiques novatrices, qui se fondent sur un travail de spécialisation disciplinaire marqué. Que l'on songe à la géographie moderne post-vidalienne, à l'école des Annales autour de March Bloch et de Lucien Febvre, mais aussi au folklore qui se professionnalise et se spécialise fortement dans les années 1920-1930, l'ensemble de ces développements scientifiques conduisent à une déqualification de la figure de l'honnête homme de science, adepte de l'éclectisme, multi-positionné localement ou régionalement, et à une remise en cause des légitimités érudites. Les sociétés savantes sont loin de disparaître, mais leur composition sociale évolue cependant (entrée des instituteurs dans les années 1920-1930, initialement peu 
présents) et l'on assiste sans doute à un recul des conditions de réception et de validité de leurs travaux, sans pour autant que les répertoires des singularités à l'institution desquels elles ont participé se trouvent, eux, remis en cause. Ces répertoires trouveront en effet toutes les conditions d'une certaine pérennité dans le domaine du tourisme et des intérêts patrimoniaux, donnant lieu à la sédimentation, si l'on peut dire, des images territoriales et culturelles produites dans le cadre de l'engouement érudit du dernier quart du XIX $x^{e}$ siècle.

\section{BIBLIOGRAPHIE}

BENSA Alban et FABRE Daniel (dir.), Une histoire à soi. Figurations du passé et localités, Paris, Éditions de la Maison des sciences de l'homme, 2001.

BERTHO-LAVENIR Catherine, La roue et le stylo. Comment nous sommes devenus touristes, Paris, Odile Jacob, 1999.

BOLTANSKI Luc, «La dénonciation », actes de la recherche en sciences sociales, vol. 51, n 1, p. 3-40. BOUGLÉ Célestin, « La vie des facultés des lettres en province », Revue des Pyrénées, vol. 17, n 1 , 1905, p. 5-27.

BOURGUET Marie-Noëlle, Déchiffrer la France. La statistique départementale à l'époque napoléonienne, Paris, Éditions des archives contemporaines, 1988.

BRIFFAUD Serge, Naissance d'un paysage, la montagne pyrénéenne à la croisée des regards ( $\mathrm{XVI}^{e}$-XIXe siècle), Tarbes/Toulouse, Archives des Hautes Pyrénées et CIMA-CNRS-Université Toulouse le Mirail, 1994.

CHALINE Jean-Pierre, Sociabilité et érudition. Les sociétés savantes en France, Paris, Éditions du CTHS, 1995.

CHANET Jean-François, L'École républicaine et les petites patries, Paris, Aubier, 1996.

CAU-DURBAN Abbé, « Les premiers habitants du Couserans », Bulletin de la société ariégeoise des sciences, lettres et arts, vol. 16, 1899-1900, p. 164-167.

DESPLAT Christian, « Les “républiques” montagnardes des Pyrénées occidentales françaises à l'époque moderne : mythe et réalité ", actes du $108^{e}$ Congrès national des sociétés savantes, vol. 1, Grenoble, 1983, p. 47-65.

GENÊT-DELACROIX Marie-Claude, «État et patrimoine sous la III ${ }^{\mathrm{e}}$ République. De l'amateur au professionnel dans la gestion du patrimoine national », dans GRANGE Jean-Daniel et POULOT Dominique (dir.), L'esprit des lieux. Le patrimoine et la cité, Grenoble, Presses universitaires de Grenoble, novembre 2013, p. 147-159.

GERSON Stéphane, The Pride of Place. Local Memories and Political Culture in Nineteenth-Century France, Ithaca et Londres, Cornell University Press, 2003.

LAFERTÉ Gilles, La Bourgogne et ses vins. Image d'origine contrôlée, Paris, Belin, 2006. 
OZOUF-MARIGNIER Marie-Vic, La formation des départements. La représentation du territoire français à la fin $d u$ XVIII ${ }^{e}$ siècle, Paris, Éditions de l'EHESS, 1989.

PARSIS-BARUBÉ Odile, La province antiquaire. L'invention de l'histoire locale en France (1800-1870), Paris, Éditions du CTHS, 2011.

SAHLINS Peter, Frontières et identités nationales. La France et l'Espagne dans les Pyrénées depuis le XVII siècle, Paris, Belin, 1996.

SAPIRO Gisèle, " Défense et illustration de "l'honnête homme" ", actes de la recherche en sciences

sociales, $\mathrm{n}^{\circ} 153$, p. 11-27.

SOURRIEU Abert, « La révolte de la Barousse en 1848 », Revue de Comminges, vol. 2, $3^{\mathrm{e}}$ trimestre, 1886, p. 196-207.

TARRIUS Alain, « Territoires circulatoires et espaces urbains ", Annales de la Recherche Urbaine, vol. 59, n 1, 1993, p. 50-60.

THIESSE Anne-Marie, Écrire la France. Le mouvement littéraire régionaliste de langue française entre la Belle Époque et la Libération, Paris, PUF, 1991.

THIESSE Anne-Marie, Ils apprenaient la France. L'exaltation des régions dans le discours patriotique, Paris, Éditions de la Maison des sciences de l'homme, 1997.

\section{NOTES}

1. M.-V. Ozouf-Marignier, La formation des départements. La représentation du territoire français à la fin du XVIII siècle; M.-N. Bourguet, Déchiffrer la France. La statistique départementale à l'époque napoléonienne.

2. S. Gerson, The Pride of Place. Local Memories and Political Culture in Nineteenth-Century France.

3. J.-P. Chaline, Sociabilité et érudition. Les sociétés savantes en France.

4. O. Parsis-Barubé, La province antiquaire. L'invention de l'histoire locale en France (1800-1870).

5. G. Laferté, La Bourgogne et ses vins. Image d'origine contrôlée.

6. C. Bertho-Lavenir, La roue et le stylo. Comment nous sommes devenus touristes.

7. P. Sahlins, Frontières et identités nationales. La France et l'Espagne dans les Pyrénées depuis le XVII siècle.

8. S. Briffaud, Naissance d'un paysage, la montagne pyrénéenne à la croisée des regards (XVI XIX ${ }^{e}$ siècle), p. 232.

9. A. Tarrius, « Territoires circulatoires et espaces urbains».

10. Les diverses facultés toulousaines furent réunies en un corps des facultés en 1893, qui devint lui même une université suite à la réforme de 1896.

11. J.-P. Chaline, Sociabilité et érudition. Les sociétés savantes en France, p. 49.

12. Ibid.

13. G. Sapiro, « Défense et illustration de "l'honnête homme” ".

14. A. Bensa et D. Fabre (dir.), Une histoire à soi. Figurations du passé et localités. 
15. A.-M. Thiesse, Écrire la France. Le mouvement littéraire régionaliste de langue française entre la Belle Époque et la Libération; A.-M. Thiesse, Ils apprenaient la France. L'exaltation des régions dans le discours patriotique ; J.-F. Chanet, L'École républicaine et les petites patries.

16. Selon l'expression qu'utilise Luc Boltanski dans « La dénonciation », p. 19-23.

17. Ch. Desplat, «Les "républiques montagnardes" des Pyrénées occidentales françaises à l'époque moderne : mythe et réalité ».

18. Cette référence à l'esprit de liberté des habitants de ces vallées des Pyrénées centrales se retrouvait dans la narration d'épisodes postérieurs de l'histoire locale, comme les révoltes contre l'imposition du Code forestier au milieu du xix ${ }^{e}$ siècle - voir par exemple A. Sourrieu, « La révolte de la Barousse en 1848 ».

19. A. Cau-Durban, «Les premiers habitants du Couserans ».

20. S. Gerson, The Pride of Place. Local Memories and Political Culture in Nineteenth-Century France.

21. M.-C. Genêt-Delacroix, « État et patrimoine sous la III ${ }^{e}$ République. De l'amateur au professionnel dans la gestion du patrimoine national ».

22. S. Gerson, The Pride of Place. Local Memories and Political Culture in Nineteenth-Century France, particulièrement chapitre 8.

23. C. Bouglé, La vie des facultés des lettres en province.

\section{RÉSUMÉS}

Cet article est consacré à l'examen de l'élaboration des images territoriales et des représentations de l'identité culturelle dans les Pyrénées centrales, et ce dans le dernier quart du $\mathrm{XIX}^{\mathrm{e}}$ siècle et le premier quart du siècle suivant. Il se concentre sur la contribution de plusieurs sociétés d'érudition à cette élaboration, en portant l'attention sur les différents registres de singularisation alors utilisés. Il analyse notamment l'importance prise par des «circulations savantes » entre différents lieux et échelles d'action, qui seules permettaient d'assurer ce que visaient ces sociétés : montrer que leur territoire de référence était porteur d'un certain nombre de spécificités, qui en faisaient un objet d'études des plus intéressants. Cependant, il est aussi possible de comprendre l'importance grandissante prise sur la période par l'échelon régional dans l'organisation des activités scientifiques et d'examiner les conditions ayant conduit à une déqualification progressive de ces savoirs érudits.

\section{AUTEUR}

\section{ARNAULD CHANDIVERT}

Maître de conférences, Université Paul-Valéry - Montpellier III, LERSEM 


\title{
La circulation d'une légende épique de fondation sur les chemins de Saint-Jacques : la légende de Dame Carcas et ses adaptations pyrénéennes, ibériques et occitanes
}

\author{
Gauthier Langlois
}

1 Cette étude s'inscrit dans une recherche pluridisciplinaire menée depuis 2007 sur la Légende de Dame Carcas, ses origines et son utilisation jusqu'à nos jours ${ }^{1}$. Dans une démarche comparative, j'ai recensé et étudié les légendes similaires pour comprendre les origines de la légende carcassonnaise, pour contribuer à la dater et caractériser son influence sur le légendaire européen. Dans une perspective plus large, je me suis interrogé sur la circulation des légendes de ce groupe. Pour cela, j'ai combiné des approches géographiques, chronologiques et thématiques.

\section{La Légende de Dame Carcas : un récit épique}

2 La Légende de dame Carcas dérive d'une ou plusieurs chansons de geste centrées sur le siège de Carcassonne par Charlemagne contre les Sarrasins. Ces chansons de gestes sont perdues, mais la légende est connue par la tradition orale recueillie à l'époque moderne (fig. 1), des allusions dans la littérature épique et une fresque du xII ${ }^{e}$ siècle au château comtal de Carcassonne. Voici le résumé de la version la plus commune :

«Au début du siège, Charlemagne capture puis exécute le défenseur de la ville, le roi sarrasin Balahac. Sa veuve, Dame Carcas, se retrouve alors à la tête de la ville et s'efforce de la défendre par divers stratagèmes: le «stratagème de l'armée augmentée " pour faire face au manque de défenseurs; le "stratagème de la nourriture augmentée » pour faire face à la famine. C'est ainsi qu'elle remplace les soldats morts par des mannequins de paille placés sur les remparts pour donner l'illusion d'une importante garnison. Puis, quand la nourriture vient à manquer, elle sacrifie le dernier animal, une truie nourrie avec le dernier sac de blé, qu'elle 
précipite par-dessus les remparts. Écrasée au pied des remparts, la truie laisse échapper du blé de son ventre, donnant l'illusion que la ville est encore pourvue de vivres pour longtemps. Découragé, Charlemagne lève le siège. Mais Dame Carcas sonne du cor pour le rappeler. Au moment où elle ouvre les portes à l'empereur, une tour s'abaisse par miracle pour le saluer. En hommage à la résistance de la dame, Charlemagne baptise la ville Carcassonne, jeu de mots évoquant le geste de l'héroïne. Il baptise également Dame Carcas, puis la marie avec un comte franc auquel il confie la ville. Et l'on dit que c'est de ce couple que descendent les seigneurs de Carcassonne. »

Fig. 1. - Carte de localisation des livres, manuscrits et auteurs rapportant la Légende de Dame Carcas aux $\mathrm{XVI}^{\mathrm{e}}$ et XVII ${ }^{\mathrm{e}}$ siècle. Une diffusion internationale.

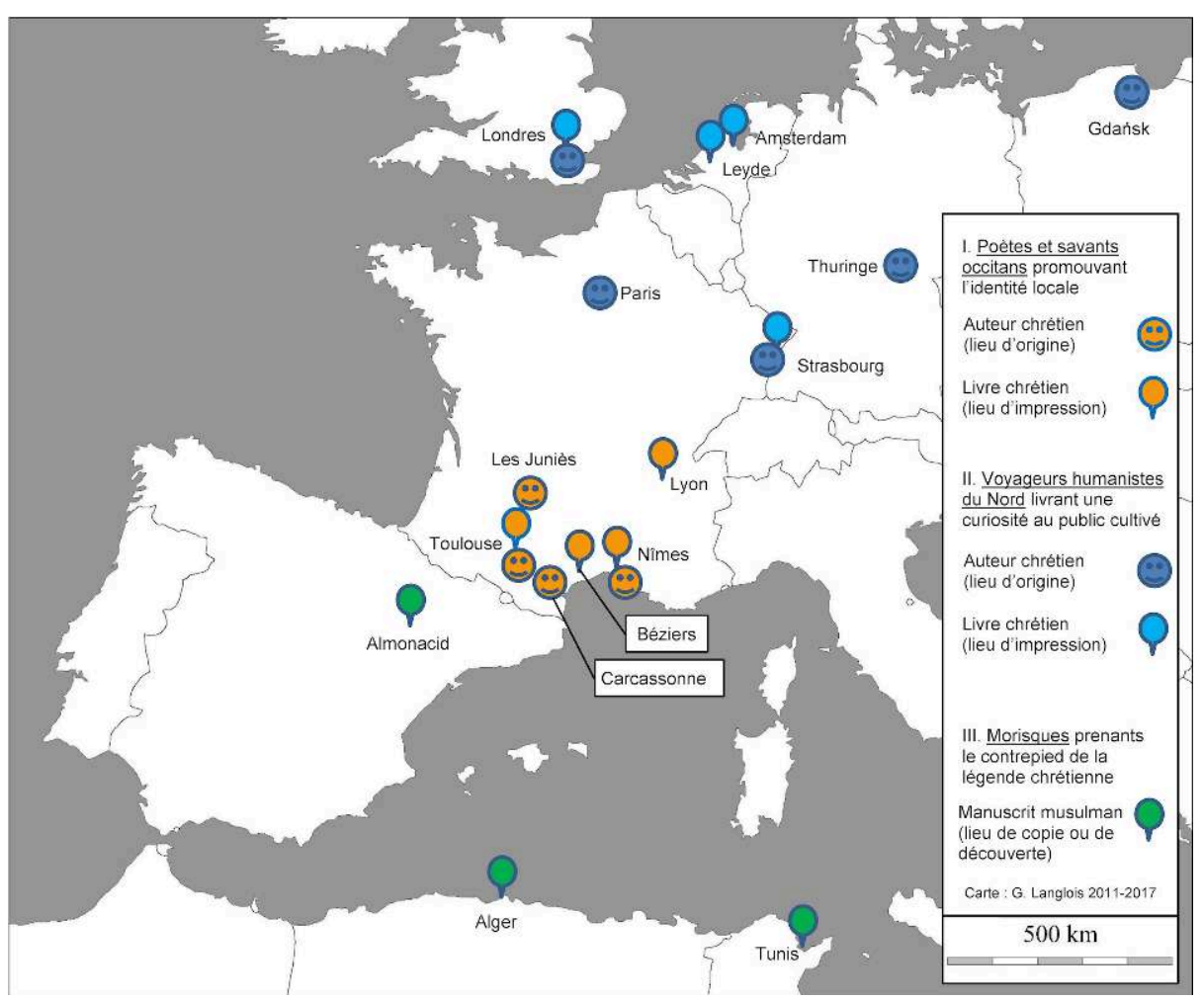

(c) G. Langlois

3 À travers ses multiples représentations et usages (littéraires, théâtraux, iconographiques...) la Légende de Dame Carcas répond depuis le Moyen Âge à des fonctions multiples qui expliquent sa popularité et sa célébrité. C'est d'abord une légende étiologique : elle explique les origines et le nom de la ville. Au Moyen Âge, elle remplit une fonction politique en renforçant la légitimité des seigneurs de Carcassonne et en glorifiant leur dynastie, ainsi qu'une fonction sanctifiante et chrétienne à travers les miracles de saint Charlemagne et la conversion de Dame Carcas. Elle possède aussi une fonction identitaire en renforçant la cohésion des habitants, notamment par l'usage de Dame Carcas comme allégorie (fig. 2). Elle transmet des valeurs morales ou courtoises telles que la résistance, la liberté, l'amour. Il s'agit enfin d'une histoire divertissante. 
Fig. 2. - Buste de Dame Carcas.

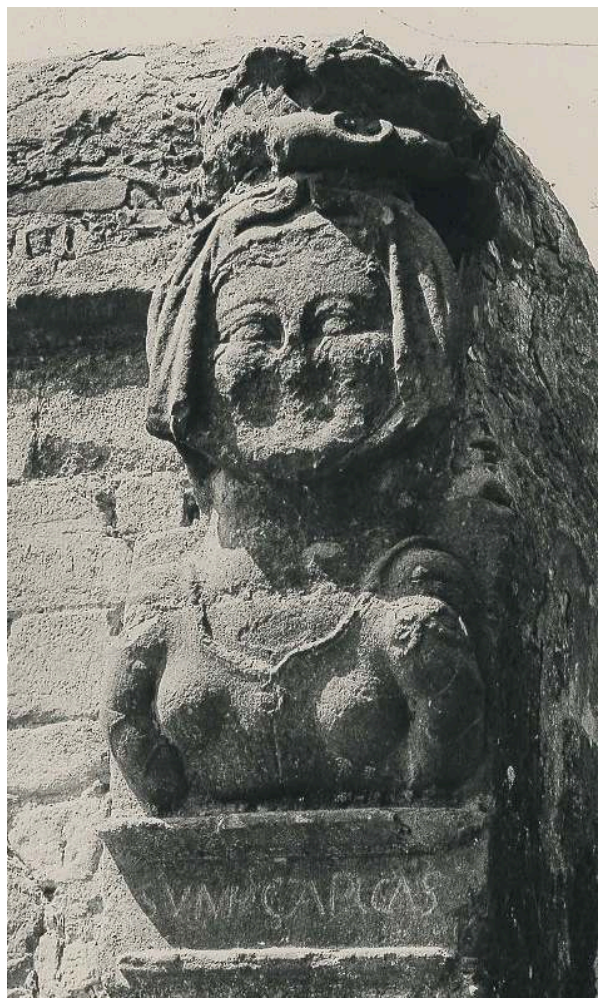

En érigeant cette sculpture à l'entrée de la cité vers 1538, les édiles carcassonnais affirment le caractère allégorique et identitaire de l'héroïne.

Photographie de l'abbé Verguet, 1899. A. D. Aude 7 Fi 16 et 2 Fi 1532.

\section{Des légendes similaires}

4 Les stratagèmes et fonctions de la Légende de Dame Carcas se retrouvent dans les légendes similaires. Dans une perspective comparative, j'ai tenté de repérer tous les récits et légendes dans lesquels un belligérant trompe l'ennemi sur ses forces réelles en vivres ou en soldats. Cette tâche n'a pas été facile car il n'existe pas pour les légendes de classification ou de catalogue comparable à ce qui a été fait pour les contes et les mythes. Le repérage s'est donc fait essentiellement par mots-clés sur internet dans les langues romanes, en anglais et en allemand.

5 La moisson de cette recherche est abondante puisque le stratagème de la nourriture augmentée apparaît dans cinquante-six localités (fig. 3) et celui de l'armée augmentée dans vingt-deux (fig. 4), le tout réparti sur quinze pays. Je les ai regroupés par tranches chronologiques; d'abord les sources littéraires antiques, puis, pour les autres, en tenant compte non pas de l'époque de rédaction qui est rarement connue, mais de celle à laquelle se situe l'action de ces légendes. Ce classement a permis de constituer plusieurs ensembles possédant également une cohérence thématique et géographique. Le premier ensemble que j'ai intitulé Le légendaire barbare de l'Antiquité tardive regroupe des légendes, principalement italiennes, se situant dans la période de transition entre l'Antiquité et le Haut Moyen Âge. Le second ensemble intitulé Le légendaire sarrasin du haut Moyen Âge, même s'il déborde un peu sur le Bas Moyen Âge, regroupe des légendes se situant pendant la conquête arabe et la reconquête chrétienne entre le $\mathrm{VIII}^{\mathrm{e}}$ et le $\mathrm{XII}^{\mathrm{e}}$ 
siècle en France et dans la péninsule ibérique. Le dernier ensemble Le légendaire européen du Bas Moyen Âge regroupe des légendes européennes et nord-africaines se situant à la fin du Moyen Âge. Il déborde un peu sur l'époque moderne puisque j'y ai ajouté deux légendes situant leur action $\mathrm{au} \mathrm{XVI}$ siècle.

Fig. 3. - Carte de répartition du stratagème de la nourriture augmentée dans les récits et légendes. Les numéros sur la carte renvoient au catalogue des légendes en annexe.

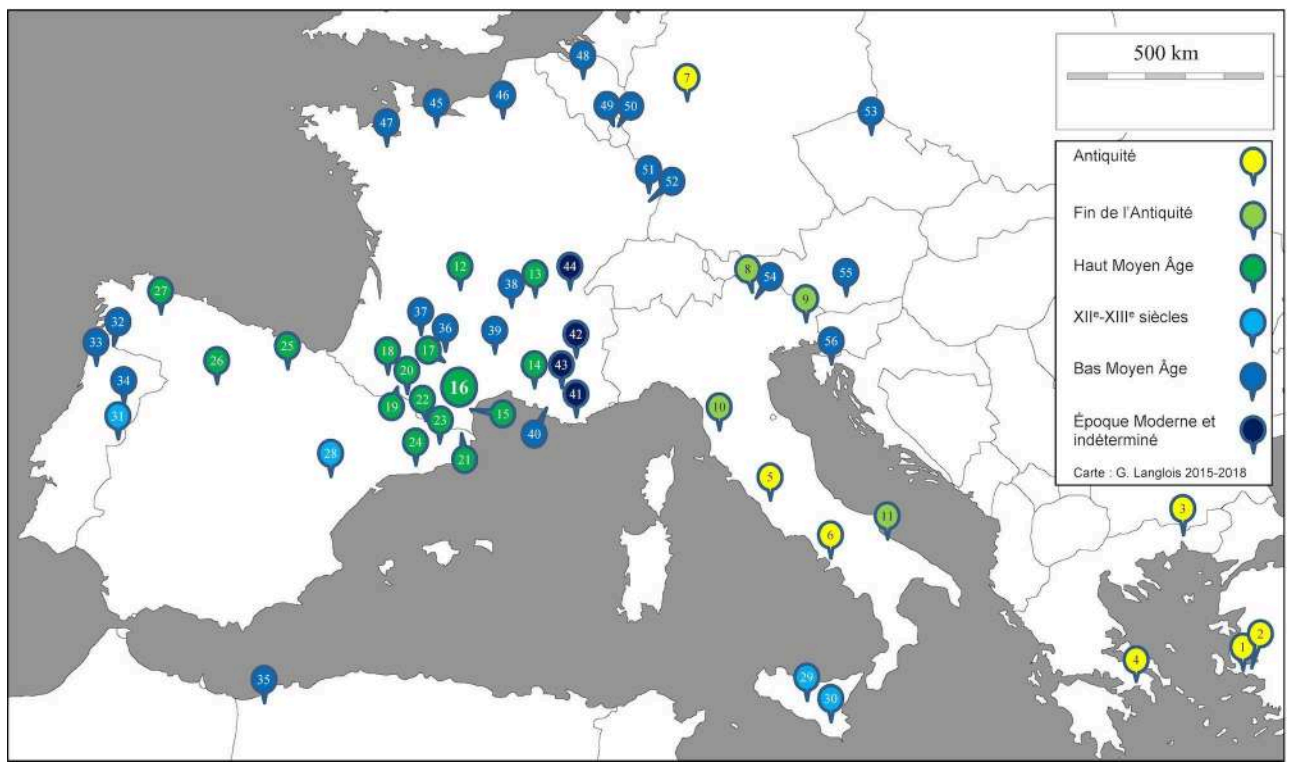

(c) G. Langlois

Fig. 4. - Carte de répartition du stratagème de l'armée augmentée dans les récits et légendes. Les numéros sur la carte renvoient au catalogue des légendes en annexe.

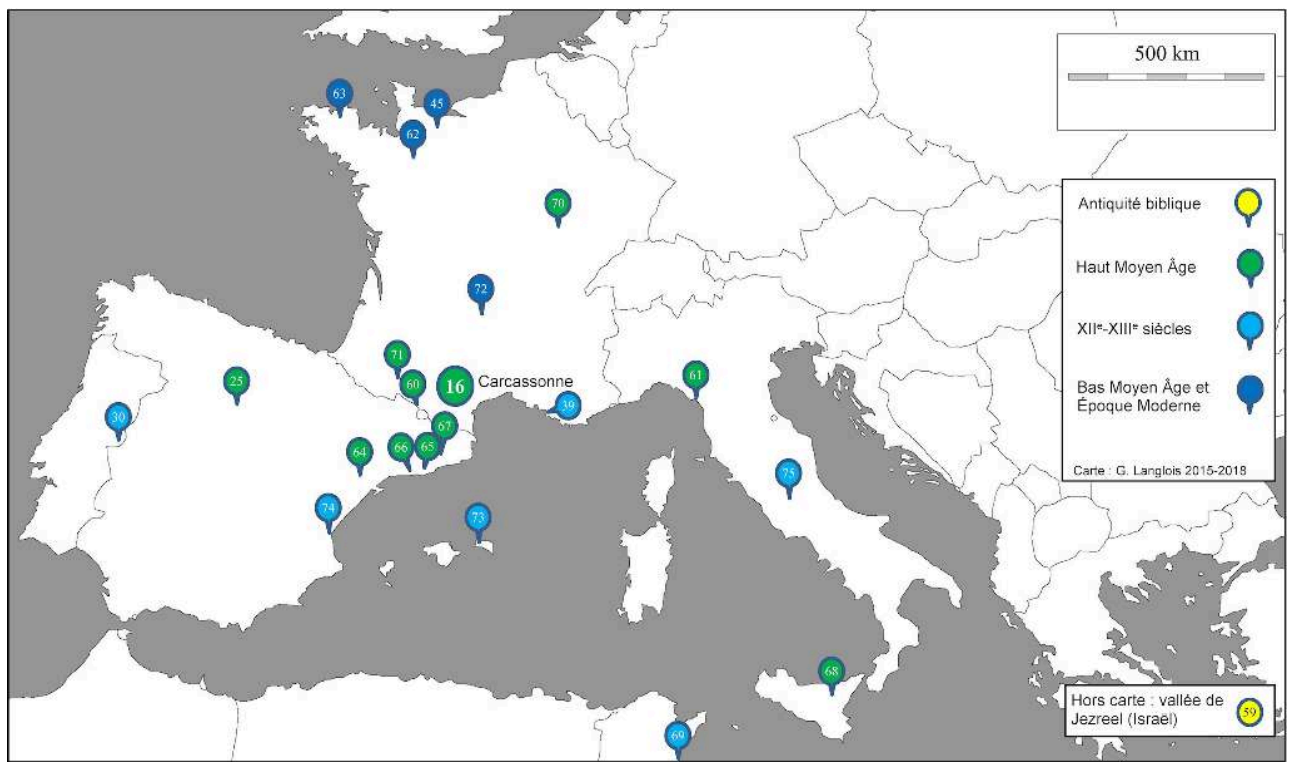

(c) G. Langlois

6 La répartition topographique et chronologique des stratagèmes (fig. 3 et 4) permet déjà de faire quelques constatations. Les légendes étudiées ne dépassent pas le monde gréco-romain et l'Europe occidentale. Elles dérivent donc d'un ou plusieurs modèles 
européens. La forte densité de légendes autour de Carcassonne est un indice d'une diffusion aréale ou de proche en proche depuis cette ville. Carcassonne apparait également comme le centre de gravité de l'ensemble de l'espace étudié et semble donc constituer le principal foyer de diffusion de ces stratagèmes. Pour valider cette hypothèse, étudions maintenant la répartition chronologique des légendes en nous appuyant sur quelques exemples.

\section{Les sources littéraires antiques gréco-romaines}

7 Dès l'Antiquité, faire croire à l'assiégeant que la ville est suffisamment pourvue en vivres pour résister encore longtemps semble un lieu commun des récits de sièges. Les écrivains grecs et romains en donnent plusieurs exemples. La plupart de ces ruses ont été relevées par Frontin, général et homme politique romain du $\mathrm{I}^{\mathrm{er}}$ siècle après J.-C., dans son traité d'art militaire appelé Stratagèmes. Mais la ruse la plus intéressante est rapportée par Diogène Laërce, un écrivain grec du $\mathrm{III}^{\mathrm{e}}$ siècle après J.-C. Celui-ci fait état de la ruse, employée par le philosophe Bias assiégé dans la ville de Priène par le roi de Lydie. Bias fit engraisser de blé deux mulets qu'il envoya à l'ennemi, ce qui engagea ce dernier à renoncer au siège.

Ces récits littéraires sont, aux côtés de mythes antiques disparus ou ayant survécu par la tradition orale, les sources des légendes médiévales que nous allons étudier. Mais, qu'ils se rapportent à des faits réels ou imaginaires, ils ne sont pas à proprement parler des légendes. Ils n'ont pas le caractère merveilleux ni édifiant des légendes postérieures.

\section{Le légendaire barbare de l'Antiquité tardive et du Haut Moyen Âge}

9 Sous l'effet de la christianisation de l'Empire romain, les guerres contre les barbares prennent une dimension religieuse que l'on retrouve dans trois légendes italiennes. Le héros y est une reine qui fait figure de sainte ou le saint patron d'une ville. Ces récits sont donc des légendes au premier sens du terme, c'est-à-dire des vies de saints. Ils témoignent de l'effort de christianisation des cultes et récits antiques où le saint chrétien se substitue au héros de la mythologie. Les récits hagiographiques ont certainement servi de source aux légendes du groupe suivant parmi lesquelles se situe celle de Carcassonne.

\section{Le légendaire sarrasin du Haut Moyen Âge}

10 Parmi les récits et légendes repérés, plus du tiers situe leur action au Moyen Âge dans le cadre des conflits entre les chrétiens et musulmans, dont une vingtaine pendant la reconquête carolingienne. L'action de ces légendes est localisée dans une zone dont Carcassonne constitue le centre de gravité et qui a effectivement été concernée par ces conflits au viII ${ }^{\mathrm{e}}$ siècle et au début du $\mathrm{IX}^{\mathrm{e}}$ siècle : le sud de la France et le nord de la péninsule ibérique. Cette localisation montre que ces légendes s'appuient sur des faits réels, même si ces faits ont pu être transposés d'un lieu à un autre, déformés et adaptés.

11 Si la plupart de ces légendes ne sont connues, parfois de manière très succincte, que par des sources modernes ou contemporaines, celles connues par des sources médiévales sont riches et développées. C'est le cas des légendes d'Uzerche et de Lourdes 
servant chacune d'introduction à un cartulaire, le premier du milieu du XII siècle et le second du xiII ${ }^{e}$ siècle. Ces deux légendes, manifestement inspirées de celle de Carcassonne, partagent avec cette dernière des personnages, le stratagème de la nourriture augmentée et les mêmes fonctions. Sauf que, rédigées pour un public de religieux - les moines d'Uzerche et pour Lourdes, les chanoines du Puy-en-Velay - elles sont adaptées à la mentalité cléricale. C'est ainsi que l'héroïne laisse place à un héros et que la peu chrétienne truie laisse place à un bœuf gavé de blé à Uzerche, ou à une truite miraculeusement apportée par un aigle à Lourdes. Le motif de la pêche miraculeuse se retrouve ensuite dans huit autres légendes, six dans les Pyrénées et deux au Portugal.

12 L'influence de la Légende de Dame Carcas semble directement s'exercer dans une autre légende médiévale, celle de San Vitorez de Cerezo, un saint particulièrement honoré en Castille. Sa vita, datée du $\mathrm{xv}^{\mathrm{e}}$ siècle, mêle des motifs empruntés aux vies de saints céphalophores et des motifs empruntés aux légendes de siège. On y retrouve en particulier le motif de la princesse sarrasine qui se convertit, le stratagème de l'animal gavé et le miracle de la fontaine (accompli à Carcassonne par Charlemagne).

\section{Le légendaire européen du Bas Moyen Âge}

13 Autant qu'on puisse s'en assurer, ces légendes reposent sur des faits réels, des sièges dont l'existence est rapportée par des sources contemporaines. Des sièges menés par les Anglais pendant la Guerre de Cent ans en France, par les Castillans au Portugal, par les Vénitiens en Croatie, les Marocains au Maghreb... La moitié de ces sièges se situent dans le cadre de guerres opposant deux nations. Les légendes qui s'y rapportent ont donc un caractère nationaliste plus ou moins marqué. Les autres se situent dans le cadre de conflits entre seigneurs locaux. Le caractère religieux présent dans le légendaire du Haut Moyen Âge a disparu. À cela il faut ajouter trois exceptions se situant pendant les guerres de religion: entre catholiques et hussites au $\mathrm{xv}^{\mathrm{e}}$ et entre catholiques et protestants au XvI ${ }^{\mathrm{e}}$ siècle. Il est possible que certaines de ces légendes aient été actualisées, un ennemi s'étant substitué à un autre. Le cas est attesté à Carcassonne où une version substitue les Anglais aux Sarrasins.

\section{Les aliments de la ruse}

14 Une autre façon de comparer les légendes est d'étudier les aliments mis en œuvre dans le stratagème de la nourriture augmentée, quand ils sont précisés (fig.5). Des regroupements significatifs apparaissent. Les trois-quarts des suidés (truies, cochons, sangliers) sont localisés en Occitanie, au nord de Carcassonne. Ils attestent de l'influence du modèle carcassonnais dans cette zone. Les trois-quarts des poissons sont localisés dans les Pyrénées occitanes et catalanes, au sud de Carcassonne. Ils attestent de l'influence du modèle de Lourdes vers l'Est, mais aussi vers le Portugal. Hors de l'espace catalano-occitan domine le bovin, plus compatible que le cochon avec les religions monothéistes.

Les animaux gavés de céréales sont présents dans près de la moitié des légendes et se concentrent pour plus de la moitié autour de Carcassonne (fig. 6). D'autres groupements apparaissent, notamment du pain dans le Nord-Ouest de la péninsule ibérique, du fromage en Sicile. Ces groupements témoignent de l'influence exercée par des modèles secondaires sur des légendes voisines. 
Fig. 5. - Carte des animaux utilisés dans les récits et légendes du stratagème de la nourriture augmentée.

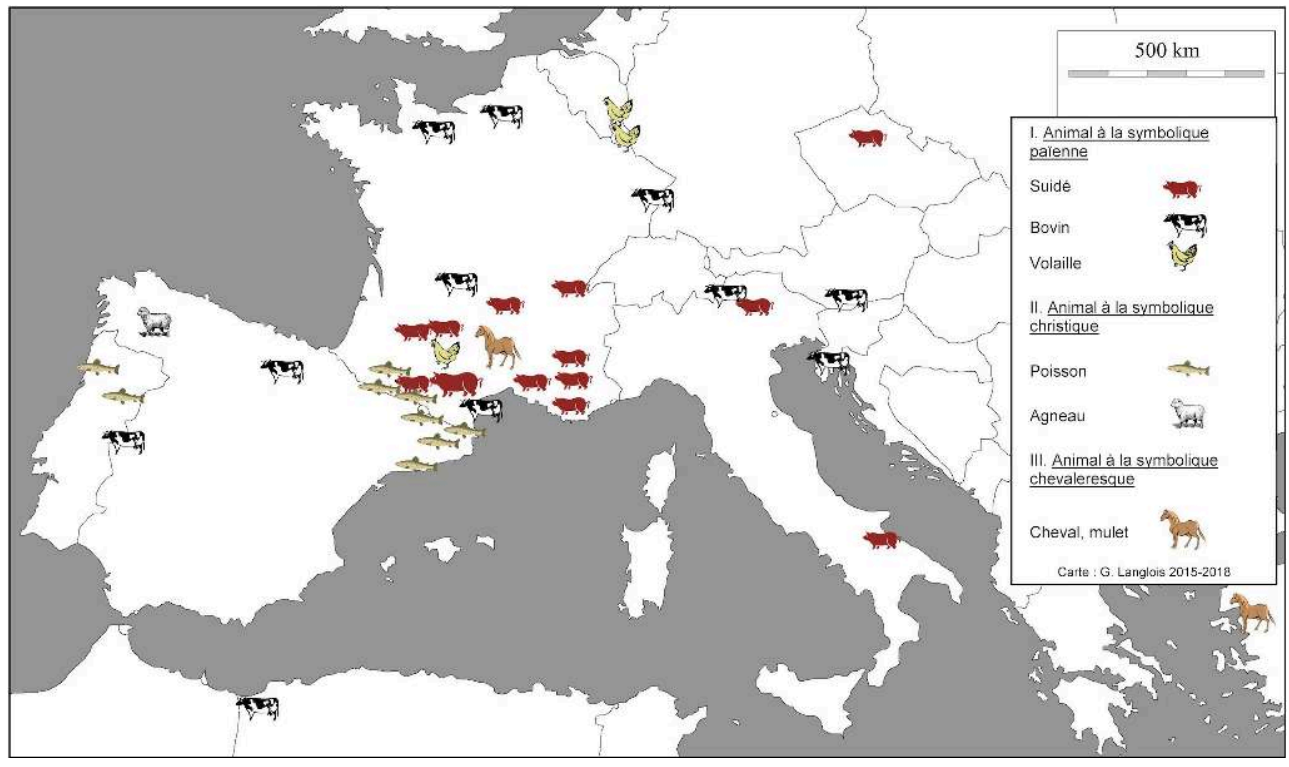

(c) G. Langlois

Fig. 6. - Carte de la nourriture non carnée utilisée dans les récits et légendes du stratagème de la nourriture augmentée.

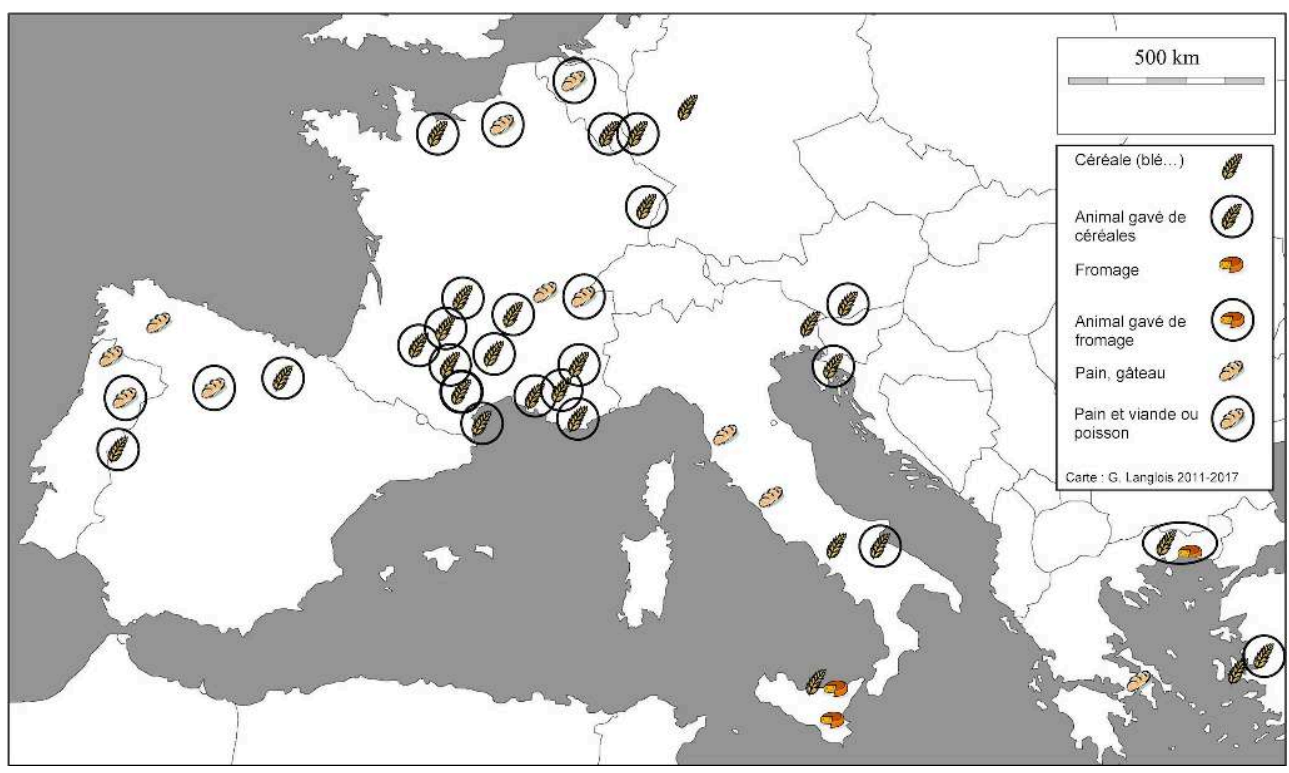

(c) G. Langlois

\section{Les variantes du stratagème de l'armée augmentée}

Ce stratagème comporte deux variantes, l'une diurne, l'autre nocturne (fig. 7). Le jour, l'assiégé doit montrer un maximum de soldats en armes. Pour cela, on emploie des soldats morts à Urueña, des femmes déguisées à Marseille, ailleurs des mannequins de paille, de bois ou de chiffon affublés en soldats. Cette variante diurne est associée, dans 
cinq légendes sur neuf à la ruse de la nourriture augmentée. C'est un argument supplémentaire en faveur de l'hypothèse de la Légende de Carcassonne comme archétype.

Fig. 7. - Carte de localisation des variantes du stratagème de l'armée augmentée dans les récits et légendes.

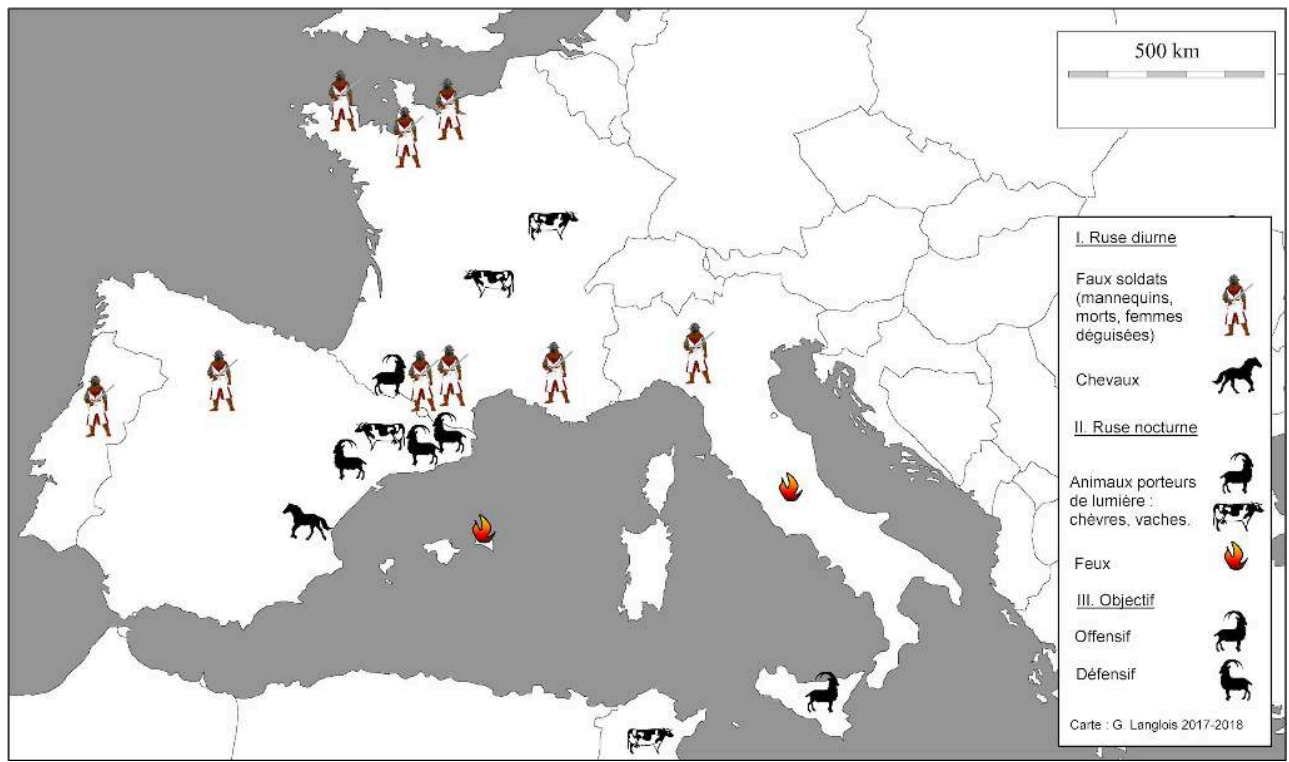

(c) G. Langlois

La nuit, l'illusion d'une armée importante à l'intérieur de la ville assiégée est réalisée grâce à des lumières, le plus souvent des lanternes, des cierges ou des torches attachées aux cornes d'un troupeau de chèvres ou de vaches. Cette variante nocturne peut être utilisée à titre défensif comme à titre offensif.

Ces ruses, nocturnes ou diurnes, ne sont pas seulement imaginaires. Dans son autobiographie, le roi Jaume $\mathrm{I}^{\mathrm{er}}$ d'Aragon présente les ruses qu'il a déployées pour cacher son infériorité numérique face aux Sarrasins. La première fois, à Minorque, il multiplie les feux de camps. La seconde fois, près de Valence, il place des chevaux derrière une ligne serrée de cavaliers.

\section{Héroïnes : princesses et châtelaines}

Même si les hommes dominent, notre corpus de légende possède une proportion notable d'histoires comportant des héroïnes qui ne sont pas seulement, contrairement à celles de la plupart des contes, des actrices passives (fig. 8). Ces héroïnes constituent une particularité méridionale et appartiennent, pour les trois-quarts, aux classes dominantes de la société. Ce sont des reines ou des princesses, comme Dame Carcas, des châtelaines, ou des bourgeoises à Marseille. Les héroïnes positives issues du peuple, telle la bergère d'Auzon ou Aïcha de Tlemcen, sont rares et ne se rencontrent que dans des histoires dont la tradition est très tardive. Trois légendes font également intervenir une héroïne négative. Comme dans les contes, elle est parfois le faire-valoir d'une hérö̈ne ou d'un héros positif. Ainsi aux Baux-de-Provence comme à Cerezo, c'est une femme égoïste qui cache l'animal et le blé nécessaires au stratagème. 
Fig. 8. - Carte des héroïnes dans les récits et légendes du stratagème de la nourriture augmentée : une répartition méridionale.

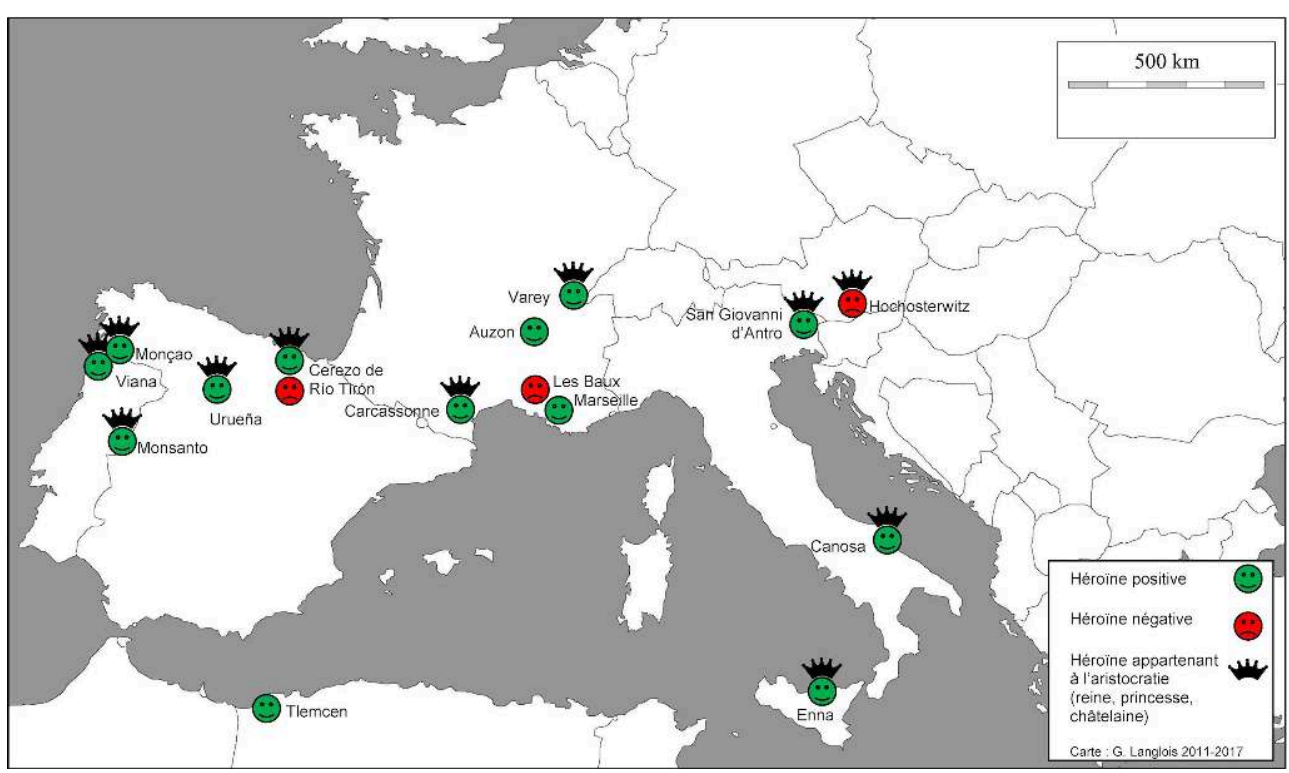

(c) G. Langlois

La présence d'une héroïne positive, croisée avec d'autres critères, est un indice sérieux de filiation avec la légende de Dame Carcas. C'est le cas notamment des légendes de Monçao (fig. 9), Monsanto et Tlemcen.

Fig. 9. - Deu la Deu, une dame Carcas portugaise.

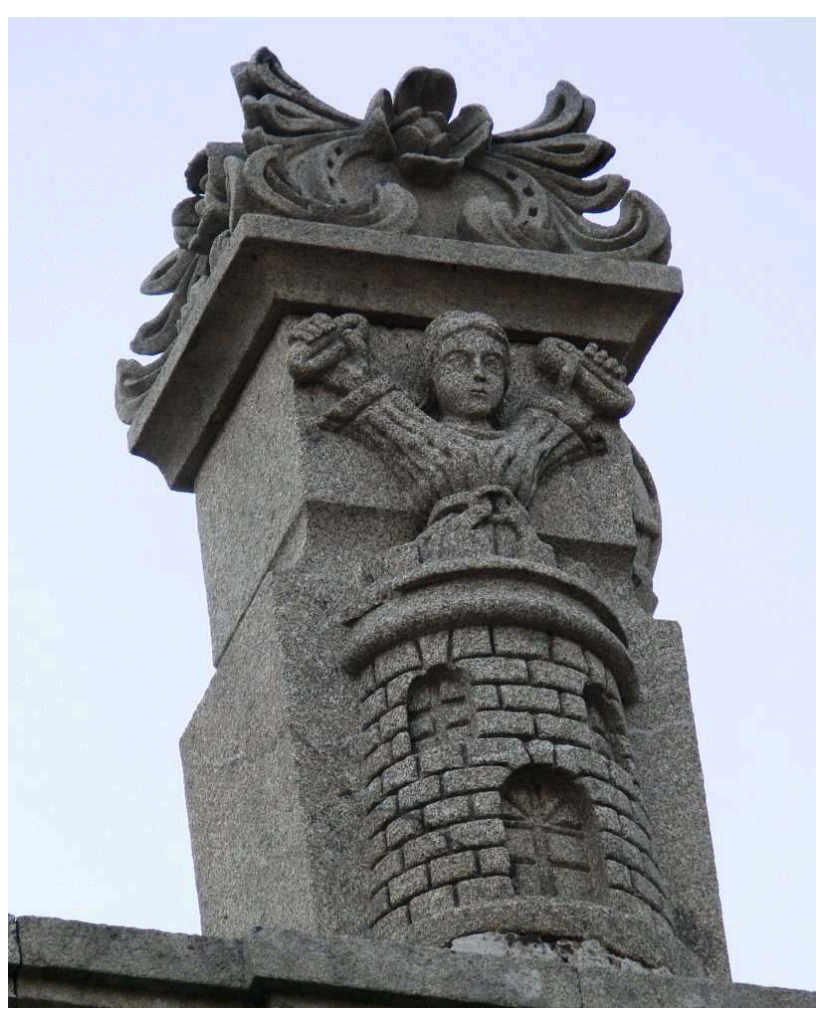

Au xıxe siècle, en faisant sculpter l'héroïne sur les cheminées de l'hôtel de ville, les édiles de Monçao se placent symboliquement sous la protection de l'allégorie de la ville.

(c) G. Langlois 

légendes ${ }^{2}$. Il convient maintenant de comprendre comment ces légendes ont circulé.

\section{La circulation des légendes}

Sur un champ chronologique et géographique aussi vaste, les modes de diffusion ont été nécessairement diversifiés et ont beaucoup évolué. Je m'en tiendrai donc à quelques remarques centrées sur le Moyen Âge, déduites du corpus ou inspirées par les travaux sur la diffusion des contes ou des chansons de geste.

Les cartes de répartition (fig. 3 et 4) ont fait apparaître, pour le Moyen Âge et l'époque moderne, une progression géographique et chronologique rayonnante centrée sur Carcassonne et délimitant trois types d'espace : un espace central très dense puis un espace vide et enfin un espace périphérique peu dense. L'absence, le plus souvent, de continuité entre centre et périphérie implique des modes et des rythmes de diffusion différents.

\section{Au centre, une diffusion de proche en proche}

Par imitation, des localités voisines de Carcassonne ont adapté la légende, puis ont servi à leur tour de modèle à d'autres localités. C'est ainsi que l'on peut expliquer la diffusion du motif de la pêche miraculeuse à travers les Pyrénées à partir de la légende de Lourdes, elle-même imitation de celle de Carcassonne. Cette diffusion s'est sans doute faite grâce à des vecteurs de diffusion variés et sur une longue période puisque certaines légendes, les plus éloignées du centre, se déroulent $\mathrm{au} \mathrm{XvI}{ }^{\mathrm{e}}$ siècle. Elle a été facilitée par l'unité linguistique : l'usage dans cet espace central de deux langues très proches, l'occitan et le catalan qui, au XI ${ }^{e}$ siècle, ne se sont pas encore différenciées.

\section{En périphérie : une diffusion via les chemins de pèlerinage et de reconquête}

La diffusion vers la périphérie, notamment hispanique, semble avoir privilégié les chemins de pèlerinage et particulièrement ceux de Saint-Jacques-de-Compostelle. Uzerche est située sur une variante de la "via Lemovicensis» entre Limoges et Toulouse; Couzan sur la "via Podensis» entre le Puy et Lyon. Le groupe le plus important constitué par Les Baux, Narbonne, Carcassonne, Saint-Julia de Gras Capou et Lourdes, se situe sur le « chemin d'Arles » ou l'une de ses variantes. Toutes ces voies se rejoignent à Puente-la-Reina en Navarre pour former le « Camino francès » sur lequel se trouvent Cerezo, entre Burgos et Logroño, et Lugo, non loin de Compostelle. Requesens, Quer Foradat, Balsareny et òlerdola en Catalogne se trouvent à proximité d'autres voies ibériques de Saint-Jacques. Enfin, des légendes dont l'action se situe à la fin du Moyen Âge peuvent également être associées à ces chemins de Saint-Jacques : quatre dans le Nord du Portugal, une en Aragon et quatre dans le Massif Central. Au total, près des deux tiers des légendes médiévales repérées en Europe sont localisées dans cet espace formé par le nord de la péninsule ibérique et l'espace occitan. Difficile d'y voir le simple effet du hasard. Alors quels liens peuvent entretenir ces légendes avec le pèlerinage de Saint-Jacques? 
26 Le pèlerinage prend de l'ampleur au $\mathrm{xI}^{\mathrm{e}}$ siècle grâce au rapprochement des rois espagnols avec l'Église romaine, notamment sous l'influence de moines venus de Cluny. Désormais, des pèlerins affluent de toute l'Europe. C'est peu après que se développe la représentation de saint Jacques matamoros, littéralement tueur de Maures, dont l'une des premières représentations orne le tympan d'une porte de la cathédrale de Compostelle. L'apôtre est figuré en chevalier, monté sur un destrier, brandissant son épée contre les musulmans. On lui attribue désormais de nombreuses victoires miraculeuses contre les ennemis de la foi. Or, à la même époque, se développe l'épopée de Charlemagne à qui l'on attribue le début de la reconquête chrétienne. Dans l'une des versions de cette épopée, l'Historia Karoli magni et Rothlandi, conservée à Santiago et datée de la première moitié $\mathrm{du} \mathrm{XII}^{\mathrm{e}}$ siècle, les deux légendes se rejoignent. Le chapitre liminaire fait de Charlemagne l'acteur principal de la découverte du tombeau de Jacques le Majeur. Le légendaire de Charlemagne et celui de saint Jacques se diffusent par les chemins de pèlerinage à travers toute l'Europe. Les héros de cette épopée servent de modèle aux chevaliers participant à la Reconquête, aussi bien dans la péninsule hispanique qu'en Italie ${ }^{3}$. Rien d'étonnant donc à ce que les légendes étudiées ici, qui s'inscrivent pour les plus anciennes dans le légendaire de Charlemagne, se retrouvent majoritairement localisées sur les chemins de Saint-Jacques.

\section{Les vecteurs de diffusion}

Les chansons de geste ont sans doute constitué le principal vecteur de diffusion de la Légende, soit directement à travers les chansons de geste disparues, centrées sur Carcassonne, soit à travers la reprise de certains motifs ou héros dans d'autres chansons. En témoignent les allusions plus ou moins longues au siège de Carcassonne dans la Chanson de Roland, la Chanson de la croisade et le Roman de Notre Dame de Lagrasse. Mais aussi la reprise de certains personnages ou motifs dans Les Narbonnais, Ogier le Danois, Fierabras... À côté de la littérature épique, essentiellement laïque, les traits de la légende se sont également propagés grâce à la littérature religieuse: légendes hagiographiques telles celle de San Vitorez de Cerezo, légendes de fondations monastiques telle celle d'Uzerche. Quant à la question de la langue, de l'auteur et du caractère populaire ou savant de ces œuvres, tentons de l'éclairer par un récit du Livre des miracles de Sainte Foy de Conques de Bernard d'Angers. En voici le résumé :

\footnotetext{
"L'histoire se situe en Rouergue dans la seconde moitié du $\mathrm{x}^{\mathrm{e}}$ siècle. Guibert est l'intendant d'un prêtre qui est aussi son parent et son parrain de confirmation. Il se rend en pèlerinage à Conques pour la fête de sainte Foy. En chemin, il est capturé par son parrain. Pour une histoire de femmes, le prêtre, jaloux, fait arracher les yeux de son filleul. Le pauvre Guibert, désormais aveugle, devient jongleur, c'est-àdire chanteur ou conteur d'histoires. Pendant un an, il gagne ainsi sa vie, jusqu'au jour où il a la vision de sainte Foy. La sainte lui promet de lui rendre ses yeux s'il achève son pèlerinage. Après une nuit passée dans le sanctuaire de Conques, il recouvre la vue. Il finit sa vie comme moine, en contant des histoires aux autres moines et aux pèlerins. »
}

Ne peut-on pas penser qu'au répertoire de Guibert figuraient, sous forme de récit ou de chanson rimée ${ }^{4}$, des légendes telle que celle de Carcassonne ? En tout cas, le répertoire de ce jongleur est nécessairement oral, puisqu'il est aveugle, et en occitan, puisque Bernard d'Angers qualifie Guibert d'illettré - c'est-à-dire n'ayant pas étudié le latin. Mais Guibert n'est pas seulement un interprète, c'est aussi un " trouveur " (en occitan troubadour): il invente ses propres histoires en agrémentant le récit de sa vie de 
détails miraculeux. Et grâce à Bernard d'Angers, certains récits du jongleur, mis par écrit en latin, vont pouvoir voyager sous forme de livres à destination d'un public lettré. Cette histoire montre que les sanctuaires situés sur les chemins de saint Jacques tels que Conques constituent des lieux privilégiés pour la transmission des récits et légendes. Laïcs porteurs de culture populaire tels que jongleurs, chevaliers ou pèlerins et religieux porteurs de culture savante s'y échangent des histoires.

Reste à comprendre pourquoi nos légendes sont absentes de grandes régions d'Europe alors que les chemins de pèlerinage irriguent toute l'Europe et y ont véhiculé partout certaines œuvres épiques telles que la Chanson de Roland.

\section{Une réception différenciée}

Si nos légendes sont généralement absentes des zones de plaine, comme le Bordelais ou le Bassin parisien, c'est peut-être parce que le cadre montagnard convient mieux au récit. Si elles ne dépassent guère les marges de l'Europe romane c'est sans doute une question de barrière linguistique. Pour autant ces explications sont insuffisantes, car bien des contes et des chansons de geste ne connaissent pas ces limites. C'est donc plutôt dans la culture et la mentalité qu'il faut chercher cette réception différenciée.

31 Les stratagèmes étudiés semblent inspirés de la littérature épique et appartiennent très majoritairement à des légendes de l'Europe méridionale. Comment expliquer alors leur faible présence dans les légendes du nord de l'Europe et leur quasi-absence dans les chansons de gestes françaises? La réponse semble liée à une différence de mentalité entre les Méridionaux et les Européens du Nord. Robert Lafont a établi cette différence en étudiant le couple Roland/Olivier dans les chansons de geste : "Roland est preux mais Olivier est sage » affirme la Chanson de Roland. Il en a conclu à l'existence de deux ethnotypes: Roland, le preux, le fanfaron, le matamore correspond à l'archétype du chevalier franc; Olivier courageux mais sage au sens de réfléchi et de savant, correspond à l'archétype du chevalier occitan ${ }^{5}$.

Cette différence n'est pas seulement littéraire, c'est une réalité sociale que j'ai pu observer à travers une étude relative à l'un des plus célèbres chevaliers occitans du XIII ${ }^{\mathrm{e}}$ siècle, Olivier de Termes ${ }^{6}$.

Les Méditerranéens, qu'ils soient occitans, italiens, grecs ou arabes, sont davantage imprégnés de culture antique où la ruse est nettement plus positive que dans la culture chrétienne. Ils voient dans les ruses une preuve d'intelligence qui valorise celui qui les emploie, tel Ulysse dans l'Iliade et l'Odyssée ou Sinbad dans les Mille et une nuits qui font preuve de ce que les Grecs appellent la « mètis».

Est-ce à dire que la ruse ou le stratagème sont perçus de la même manière chez les Occitans que chez les Grecs ou les Arabes? Le stratagème est-il systématiquement positif? Non. En matière de fidélité vassalique, le stratagème, désigné par le mot latin ingenium, signifiant littéralement machination, est associé à la trahison ${ }^{7}$. Mais le code féodal comme le code courtois souffrent quelques exceptions. Quand le seigneur ne remplit pas ses devoirs féodaux envers son vassal, quand le mari ne remplit pas ses devoirs envers son épouse ou se montre d'une jalousie excessive, le contrat vassalique ou marital est implicitement rompu. Et la machination, arme de celui, vassal ou épouse, qui se trouve en position d'infériorité, semble pleinement justifiée. Dans la littérature épique, la révolte d'Ogier le Danois contre Charlemagne et les stratagèmes qu'il emploie se justifient parce que l'empereur ne lui a pas rendu justice. Plusieurs nouvelles en 
langue occitane comme le Castia Gilos (le jaloux puni) de Raimond Vidal de Besalú, Las novas del papagay d'Arnaut de Carcassés et le Roman de Flamenca mettent en valeur les ruses ourdies par la dame, son amant ou leur complice pour tromper le mari ${ }^{8}$. Quel que soit le motif de rupture de fidélité, cette rupture n'est pas considérée par les Occitans comme quelque chose de très grave. Pendant la croisade albigeoise, nombre de seigneurs occitans vont se soumettre et prêter un serment de fidélité à Simon de Montfort ou, plus tard au roi de France, quand ils se sentent en position d'infériorité. Mais ce serment n'est pour eux qu'une ruse, une manière de faire le dos rond en attendant la fin de la tempête. Dès que la situation leur semble plus favorable, ils se révoltent. Olivier de Termes, chevalier dont la sagesse était louée, a agi ainsi plusieurs fois envers le roi de France. Pour un homme du Nord imprégné de morale cistercienne tel que Simon de Montfort, un serment est sacré. Rompre un serment est un crime très grave. Le parjure tout comme le relaps, celui qui est revenu sur ses aveux, est passible de mort.

Enfin, pour les Occitans, quand il n'existe pas de contrat de fidélité aucun code moral ne semble limiter l'usage du stratagème. C'est particulièrement vrai contre l'ennemi, dans le légendaire sarrasin comme dans la réalité. Dans son autobiographie, le roi d'Aragon Jaume $\mathrm{I}^{\text {er }}$ ne cache pas ses stratagèmes guerriers ou diplomatiques, au contraire. "En bon narrateur ", affirment Agnès et Robert Vinas, "Jaume raconte toujours avec délectation ses petites ruses de guerre [...]. Il se présente ainsi comme un guerrier astucieux et économe de ses forces ${ }^{9} »$. La vie d'Olivier de Termes, ami et compagnon de Jaume $\mathrm{I}^{\mathrm{er}}$, est ponctuée de ruses et stratagèmes.

En résumé, la ruse est généralement étrangère à la mentalité du chevalier français et plus généralement nord-européen. Il l'ignore ou la considère avec la traîtrise comme l'apanage de l'ennemi, notamment du sarrasin. C'est pourquoi les stratagèmes sont rares dans les légendes de siège du Nord de l'Europe, voire inexistants dans certaines régions comme les îles Britanniques ou l'Ile-de-France. Et quand le stratagème est présent, il n'est pas toujours valorisant. Il est parfois associé à la figure du traître comme dans les légendes normandes de Ganne, seigneur de la Lande-Patry. Dans l'Europe méridionale, tant qu'il ne rompt pas le code de fidélité, le stratagème est valorisé. C'est un signe de sagesse.

La méthode pluridisciplinaire et comparative s'est avérée pertinente. Elle a permis de comprendre comment et pourquoi ces légendes ont circulé en les replaçant dans leur contexte politique, religieux, social et littéraire. Les acquis en sont synthétisés dans la figure 10. Cette étude ouvre de nouvelles perspectives de recherche non seulement pour l'étude d'autres légendes, mais aussi pour l'étude de la littérature épique. 
Fig. 10. - Hypothèse de diffusion de la Légende de Dame Carcas et des légendes apparentées, d'après les stratagèmes de la nourriture augmentée et de l'armée augmentée.

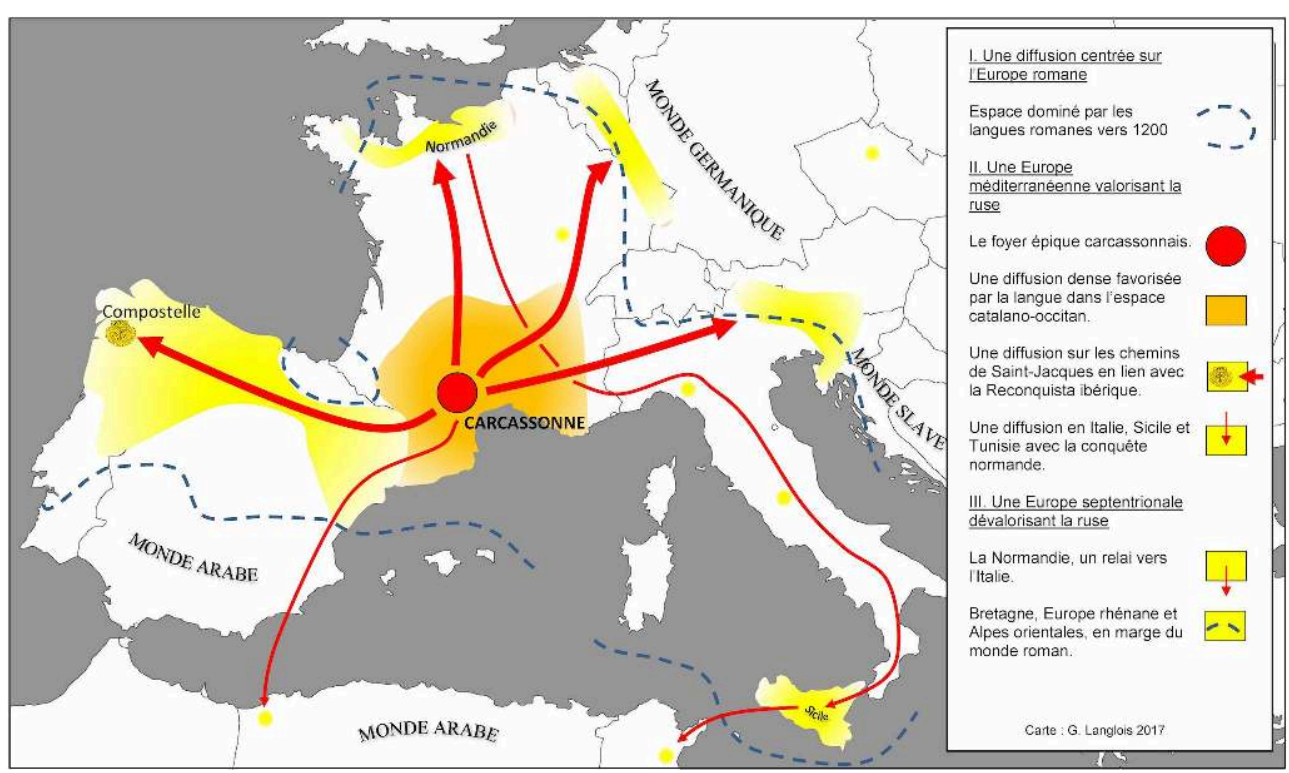

(C) G. Langlois

\section{BIBLIOGRAPHIE}

CAUCANAS Sylvie, «Dame Carcas, une héroïne sarrasine », Perpignan, l'histoire des musulmans dans la ville (du Moyen Âge à nos jours). Recueil des communications du colloque du 7 et 8 avril 2005, Perpignan: Archives communales, 2005, p. 33-44.

DÉBAX Hélène, La féodalité languedocienne $\mathrm{XI}^{e}$-XII ${ }^{e}$ siècles. Serments, hommages et fiefs dans le Languedoc des Trencavel, Toulouse, Presses universitaires du Mirail, 2003.

GICQUEL Bernard, La légende de Compostelle. Le livre de Saint-Jacques, Paris, Tallandier, 2003.

HUY Julien (d'), « Peut-on tester scientifiquement la phylogénétique des mythes ? À propos du motif de la chasse cosmique », Mythologie française, $n^{\circ} 263$, juin 2016, p. 20-24.

LAFONT Robert, La source sur le chemin : aux origines occitanes de l'Europe littéraire, Paris, L'Harmattan, 2002.

LANGLOIS Gauthier, « La légende de Dame Carcas. Les origines épiques », Bulletin de la Société d'études scientifique de l'Aude, tome CIX, 2009, p. 77-87 et tome CX, 2010, p. 45-58.

LANGLOIS Gauthier, « Note sur les origines du troubadour Arnaut de Carcassés ", Revue des langues romanes, tome CXII-1, 2008, p. 89-99.

LANGLoIs Gauthier, Dame Carcas, une légende épique occitane, Carcassonne, Société d'études scientifique de l'Aude, à paraître.

LANGLOIS Gauthier, Olivier de Termes, le cathare et le croisé (vers 1200-1274), Toulouse, Privat, 2001. 
LUCE-DUDEMAINE Dominique, Flamenca et les « novas » à triangle amoureux : contestation et renouveau de la « fin'amor », Montpellier, Presses universitaires de la Méditerranée, 2007.

MACÉ Laurent, (dir.), Entre histoire et épopée. Les Guillaume d'Orange (IXe-XIII siècles), Toulouse, CNRS, Université de Toulouse - le Mirail, 2005.

VINAS Agnès et Robert, Le Livre des Faits de Jaume le Conquérant, Perpignan, Société agricole, scientifique et littéraire des Pyrénées-Orientales, $114^{\mathrm{e}}$ volume, 2007.

\section{ANNEXES}

\section{Le stratagème de la nourriture augmentée}

\begin{tabular}{|c|c|c|c|c|c|}
\hline $\mathbf{N}^{\circ}$ & Lieu & $\begin{array}{l}\text { Période } \\
\text { historique }\end{array}$ & Assiégé & Ennemi & Nourriture \\
\hline 1 & Milet* & Antiquité & Grecs & Lydiens & Blé \\
\hline 2 & Priène* & Antiquité & Grecs & Lydiens & Blé, mulet gavé de blé \\
\hline 3 & Thrace* & Antiquité & Thraces & Grecs & $\begin{array}{l}\text { Bête gavée de blé et } \\
\text { fromage }\end{array}$ \\
\hline 4 & Athènes* & Antiquité & Grecs & Grecs & Pain \\
\hline 5 & Rome* & Antiquité & Romains & Gaulois & Pain \\
\hline 6 & Casilinum* & Antiquité & Carthaginois & Romains & Blé \\
\hline 7 & Teutoburg* & Antiquité & Romains & Gaulois & Blé \\
\hline 8 & Castel Regino & $\begin{array}{l}\text { Antiquité } \\
\text { tardive }\end{array}$ & Romains & Barbares & Bœuf rôti \\
\hline 9 & $\begin{array}{l}\text { San-Giovanni- } \\
\text { d'Antro }\end{array}$ & $\begin{array}{l}\text { Antiquité } \\
\text { tardive }\end{array}$ & Reine & Barbares & Blé \\
\hline 10 & Volterra & $\begin{array}{l}\text { Antiquité } \\
\text { tardive }\end{array}$ & Romains & Barbares & Pain \\
\hline 11 & Canosa-di-Puglia & $\begin{array}{l}\text { Haut Moyen } \\
\text { Âge }\end{array}$ & Reine d'Italie & Lombards & Porc gavé de blé \\
\hline 12 & Uzerche & $\begin{array}{l}\text { Haut Moyen } \\
\text { Âge }\end{array}$ & Francs & Sarrasins & Bœuf gavé de blé \\
\hline 13 & Couzan & $\begin{array}{l}\text { Haut Moyen } \\
\text { Âge }\end{array}$ & Francs & Sarrasins & Pain \\
\hline 14 & $\begin{array}{l}\text { Les Baux-de- } \\
\text { Provence }\end{array}$ & $\begin{array}{l}\text { Haut Moyen } \\
\text { Âge }\end{array}$ & Seigneur local & Sarrasins & Cochon gavé d'orge \\
\hline 15 & Narbonne & $\begin{array}{l}\text { Haut Moyen } \\
\text { Âge }\end{array}$ & Francs & Sarrasins & Vache gavée de blé \\
\hline 16 & Carcassonne & $\begin{array}{l}\text { Haut Moyen } \\
\text { Âge }\end{array}$ & $\begin{array}{l}\text { Princesse } \\
\text { sarrasine }\end{array}$ & Francs & Cochon gavé de blé \\
\hline
\end{tabular}




\begin{tabular}{|c|c|c|c|c|c|}
\hline 17 & $\begin{array}{l}\text { Saint-Julia de Gras } \\
\text { Capou }\end{array}$ & $\begin{array}{l}\text { Haut Moyen } \\
\text { Âge }\end{array}$ & Francs & Sarrasins & Chapon gras \\
\hline 18 & Lourdes & $\begin{array}{l}\text { Haut Moyen } \\
\text { Âge }\end{array}$ & Sarrasins & Francs & $\begin{array}{l}\text { Truite amenée par un } \\
\text { aigle }\end{array}$ \\
\hline 19 & $\begin{array}{l}\text { Seix, château de } \\
\text { Mirabat }\end{array}$ & $\begin{array}{l}\text { Haut Moyen } \\
\text { Âge }\end{array}$ & Sarrasins & Francs & Truites et marcassins \\
\hline 20 & Montréal-de-Sos & $\begin{array}{l}\text { Haut Moyen } \\
\text { Âge }\end{array}$ & Sarrasins & Francs & Truite \\
\hline 21 & Requesens & $\begin{array}{l}\text { Haut Moyen } \\
\text { Âge }\end{array}$ & Francs? & Sarrasins? & Poisson \\
\hline 22 & el Quer Foradat & $\begin{array}{l}\text { Haut Moyen } \\
\text { Âge }\end{array}$ & Francs & Sarrasins & Truite \\
\hline 23 & Balsareny & $\begin{array}{l}\text { Haut Moyen } \\
\text { Âge }\end{array}$ & Francs & Sarrasins & $\begin{array}{l}\text { Truite amenée par un } \\
\text { aigle }\end{array}$ \\
\hline 24 & òlerdola & $\begin{array}{l}\text { Haut Moyen } \\
\text { Âge }\end{array}$ & Francs & Sarrasins & Truite \\
\hline 25 & Cerezo de Río Tirón & $\begin{array}{l}\text { Haut Moyen } \\
\text { Âge }\end{array}$ & Francs & Sarrasins & Vache gavée de blé \\
\hline 26 & Urueña & $\begin{array}{l}\text { Haut Moyen } \\
\text { Âge }\end{array}$ & $\begin{array}{l}\text { Seigneur } \\
\text { castillan }\end{array}$ & Sarrasins & Pain \\
\hline 27 & Lugo & $\begin{array}{l}\text { Haut Moyen } \\
\text { Âge }\end{array}$ & Un comte & Sarrasins & Pain et agneau \\
\hline 28 & Mora de Rubiellos & $\begin{array}{l}\text { Haut Moyen } \\
\text { Âge }\end{array}$ & Sarrasins & Francs & Nourriture \\
\hline 29 & Enna & $\begin{array}{l}\text { Haut Moyen } \\
\text { Âge }\end{array}$ & Sarrasins & Francs & $\begin{array}{l}\text { Blé, fromage fait avec } \\
\text { du lait de femme }\end{array}$ \\
\hline 30 & Comiso & $\begin{array}{l}\text { Haut Moyen } \\
\text { Âge }\end{array}$ & Francs & Sarrasins & Fromage (ricotta) \\
\hline 31 & Monsanto & $\begin{array}{l}\text { Haut Moyen } \\
\text { Âge }\end{array}$ & Femme & Sarrasins & Génisse gavée de blé \\
\hline 32 & Monçao & $\begin{array}{l}\text { Bas Moyen } \\
\text { Âge }\end{array}$ & $\begin{array}{l}\text { Femme } \\
\text { portugaise }\end{array}$ & Castillans & Pain \\
\hline 33 & Viana-do-Castelo & $\begin{array}{l}\text { Bas Moyen } \\
\text { Âge }\end{array}$ & Reine d'Aragon & Roi d'Aragon & $\begin{array}{l}\text { Truite amenée par un } \\
\text { aigle }\end{array}$ \\
\hline 34 & Celorico-da-Beira & $\begin{array}{l}\text { Bas Moyen } \\
\text { Âge }\end{array}$ & Seigneur local & Seigneur local & $\begin{array}{l}\text { Truite amenée par un } \\
\text { aigle }\end{array}$ \\
\hline 35 & Tlemcen & $\begin{array}{l}\text { Bas Moyen } \\
\text { Âge }\end{array}$ & Vieille femme & Marocains & Veau gavé de blé \\
\hline 36 & Puycelsi & $\begin{array}{l}\text { Bas Moyen } \\
\text { Âge }\end{array}$ & Français & Anglais & Cochon gavé de blé \\
\hline 37 & Puymirol & $\begin{array}{l}\text { Bas Moyen } \\
\text { Âge }\end{array}$ & Français & Anglais & Cochon gavé de blé \\
\hline
\end{tabular}




\begin{tabular}{|c|c|c|c|c|c|}
\hline 38 & Auzon & $\begin{array}{l}\text { Bas Moyen } \\
\text { Âge }\end{array}$ & $\begin{array}{l}\text { Bergère } \\
\text { française }\end{array}$ & Anglais & Cochon gavé de blé \\
\hline 39 & Capluc & $\begin{array}{l}\text { Bas Moyen } \\
\text { Âge }\end{array}$ & Seigneur local & Seigneur local & Cheval gavé d'avoine \\
\hline 40 & Marseille & $\begin{array}{l}\text { Bas Moyen } \\
\text { Âge }\end{array}$ & Femmes & Seigneur local & Nourriture \\
\hline 41 & La Garde & $?$ & Seigneur local & $?$ & Porc gavé de blé \\
\hline 42 & Mévouillon & $\begin{array}{l}\text { Époque } \\
\text { moderne }\end{array}$ & Protestants & Catholiques & Cochon gavé de blé \\
\hline 43 & Fort-de-Buoux & $?$ & $?$ & $?$ & Cochon gavé de blé \\
\hline 44 & Varey & $\begin{array}{l}\text { Époque } \\
\text { moderne }\end{array}$ & $\begin{array}{l}\text { Comtesse } \\
\text { savoyarde }\end{array}$ & Français & Pain, truie \\
\hline 45 & La Lande Patry & $\begin{array}{l}\text { Bas Moyen } \\
\text { Âge }\end{array}$ & Seigneur local & Seigneur local & Vache gavée de blé \\
\hline 46 & Le Thuit & $\begin{array}{l}\text { Bas Moyen } \\
\text { Âge }\end{array}$ & Seigneur local & Seigneur local & $\begin{array}{l}\text { Pain et quartiers de } \\
\text { bœuf }\end{array}$ \\
\hline 47 & Rennes & $\begin{array}{l}\text { Bas Moyen } \\
\text { Âge }\end{array}$ & Français & Anglais & Nourriture \\
\hline 48 & $\begin{array}{l}\text { Grammont/ } \\
\text { Geraardsbergen }\end{array}$ & $\begin{array}{l}\text { Bas Moyen } \\
\text { Âge }\end{array}$ & Habitants & $?$ & Gâteaux \\
\hline 49 & Bourscheid & $\begin{array}{l}\text { Bas Moyen } \\
\text { Âge }\end{array}$ & Seigneur local & Seigneur local & Oie gavée de blé \\
\hline 50 & Vianden & $\begin{array}{l}\text { Bas Moyen } \\
\text { Âge }\end{array}$ & Seigneur local & Seigneur local & Oie gavée de blé \\
\hline 51 & Geroldseck & $\begin{array}{l}\text { Bas Moyen } \\
\text { Âge }\end{array}$ & Seigneur local & ? & Nourriture \\
\hline 52 & Guirbaden & $\begin{array}{l}\text { Bas Moyen } \\
\text { Âge }\end{array}$ & Seigneur local & $?$ & Vache gavée de blé \\
\hline 53 & Kost & $\begin{array}{l}\text { Bas Moyen } \\
\text { Âge }\end{array}$ & Hussites & Catholiques & Sanglier rôti \\
\hline 54 & Sauschloss & $\begin{array}{l}\text { Bas Moyen } \\
\text { Âge }\end{array}$ & Seigneur local & $\begin{array}{l}\text { Duc } \\
\text { d'Autriche }\end{array}$ & Porc rôti \\
\hline 55 & Hochosterwitz & $\begin{array}{l}\text { Bas Moyen } \\
\text { Âge }\end{array}$ & Seigneur local & $\begin{array}{l}\text { Duchesse de } \\
\text { Tyrol }\end{array}$ & Bœuf gavé d'orge \\
\hline 56 & Kožljak & $\begin{array}{l}\text { Bas Moyen } \\
\text { Âge }\end{array}$ & Seigneur local & Vénitiens & Vache gavée de blé \\
\hline
\end{tabular}

\section{Le stratagème de l'armée augmentée}

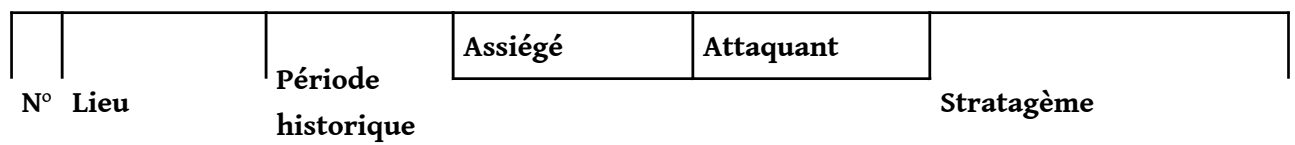




\begin{tabular}{|c|c|c|c|c|c|}
\hline \multirow[b]{2}{*}{59} & \multirow[b]{2}{*}{$\begin{array}{l}\text { Vallée de } \\
\text { Jezreel }\end{array}$} & \multirow[b]{2}{*}{$\begin{array}{l}\text { Antiquité } \\
\text { biblique }\end{array}$} & \multicolumn{2}{|c|}{$\begin{array}{l}\text { (L'astérisque indique l'utilisateur } \\
\text { du stratagème) }\end{array}$} & \\
\hline & & & Madianites & Hébreux* & $\begin{array}{l}\text { lanternes et sonnant de la } \\
\text { trompe }\end{array}$ \\
\hline 16 & Carcassonne & $\begin{array}{l}\text { Haut Moyen } \\
\text { Âge }\end{array}$ & $\begin{array}{l}\text { Princesse } \\
\text { sarrasine* }\end{array}$ & Francs & $\begin{array}{l}\text { Mannequins de paille } \\
\text { habillés en soldats }\end{array}$ \\
\hline 60 & Seix & $\begin{array}{l}\text { Bas Moyen } \\
\text { Âge? }\end{array}$ & Français* & Espagnols & $\begin{array}{l}\text { Mannequins de bois } \\
\text { habillés en soldats }\end{array}$ \\
\hline 61 & Castelforte & $\begin{array}{l}\text { Haut Moyen } \\
\text { Âge }\end{array}$ & Comte franc* & Charlemagne & $\begin{array}{l}\text { Mannequins de bois } \\
\text { habillés en soldats }\end{array}$ \\
\hline 62 & Châtenay & $\begin{array}{l}\text { Bas Moyen } \\
\text { Âge }\end{array}$ & Seigneur local* & Seigneur local & Arbres habillés en soldats \\
\hline 45 & Le Thuit & $\begin{array}{l}\text { Bas Moyen } \\
\text { Âge }\end{array}$ & Seigneur local* & Seigneur local & Arbres habillés en soldats \\
\hline 63 & Moncontou & $\begin{array}{l}\text { Bas Moyen } \\
\text { Âge }\end{array}$ & Seigneur local & Seigneur local* & $\begin{array}{l}\text { Mannequins de paille } \\
\text { habillés en soldats }\end{array}$ \\
\hline 30 & Monsanto & $\begin{array}{l}\text { Haut Moyen } \\
\text { Âge }\end{array}$ & Femme* & Sarrasins & Poupées de chiffons? \\
\hline 39 & Marseille & $\begin{array}{ll}\text { Bas } & \text { Moyen } \\
\text { Âge } & \end{array}$ & Femmes* & Seigneur local & $\begin{array}{l}\text { Femmes déguisées en } \\
\text { soldats }\end{array}$ \\
\hline 25 & Urueña & $\begin{array}{l}\text { Haut Moyen } \\
\text { Âge }\end{array}$ & $\begin{array}{l}\text { Seigneur } \\
\text { castillan* }\end{array}$ & Sarrasins & Soldats morts \\
\hline 64 & $\begin{array}{l}\text { Castell de } \\
\text { Cabres }\end{array}$ & $\begin{array}{l}\text { Haut Moyen } \\
\text { Âge }\end{array}$ & Chrétiens* & Sarrasins? & $\begin{array}{l}\text { Chèvres avec lanternes } \\
\text { attachées aux cornes }\end{array}$ \\
\hline 65 & La Barquera & $\begin{array}{l}\text { Haut Moyen } \\
\text { Âge }\end{array}$ & Chrétiens* & Sarrasins & $\begin{array}{l}\text { Moutons avec lanternes } \\
\text { attachées aux cornes }\end{array}$ \\
\hline 66 & Valldossera & $\begin{array}{l}\text { Haut Moyen } \\
\text { Âge }\end{array}$ & Chrétiens* & Sarrasins & $\begin{array}{l}\text { Bœufs avec lanternes } \\
\text { attachées aux cornes }\end{array}$ \\
\hline 67 & Gelida & $\begin{array}{l}\text { Haut Moyen } \\
\text { Âge }\end{array}$ & Chrétiens* & Sarrasins & $\begin{array}{l}\text { Bêtes avec lanternes } \\
\text { attachées aux cornes }\end{array}$ \\
\hline 68 & Troina & $\mathrm{XI}^{\mathrm{e}}$ & Sarrasins & Normands* & $\begin{array}{l}\text { Chèvres avec lanternes } \\
\text { attachées aux cornes }\end{array}$ \\
\hline 69 & Sfax & $\mathrm{XII}^{\mathrm{e}}$ & Normands & Sarrasins* & $\begin{array}{l}\text { Vaches avec bougies } \\
\text { attachées aux cornes }\end{array}$ \\
\hline 70 & $\begin{array}{l}\text { Alise-Sainte- } \\
\text { Reine }\end{array}$ & Antiquité & Gaulois & Romains* & $\begin{array}{l}\text { Bœufs avec cierges } \\
\text { attachés aux cornes }\end{array}$ \\
\hline 71 & Valcabrère & $\begin{array}{l}\text { Antiquité } \\
\text { tardive? }\end{array}$ & Gallo-romains? & Barbares ?* & $\begin{array}{l}\text { Chèvres avec flambeaux } \\
\text { attachés aux cornes }\end{array}$ \\
\hline 72 & Miremont & $\begin{array}{l}\text { Époque- } \\
\text { Moderne }\end{array}$ & Protestants* & Catholiques & $\begin{array}{l}\text { Vaches avec flambeaux } \\
\text { attachés aux cornes }\end{array}$ \\
\hline 73 & Minorque & $\mathrm{XIII}^{\mathrm{e}}$ & Sarrasins & Le roi Jaume $\mathrm{I}^{\mathrm{er} *}$ & Feux de camp \\
\hline
\end{tabular}




\begin{tabular}{|l|l|l|l|l|l|}
\hline 74 & $\begin{array}{l}\text { Puig de } \\
\text { Cebola }\end{array}$ & XIII $^{\mathrm{e}}$ & Sarrasins & Le roi Jaume $\mathrm{I}^{\text {er* }}$ & Chevaux \\
\hline 75 & Fiuggi & XIII $^{\mathrm{e}}$ & Seigneur local* & Troupes papales & Feux \\
\hline
\end{tabular}

\section{NOTES}

1. G. Langlois, «La légende de Dame Carcas. Les origines épiques ». L'ensemble de ces travaux vont être publiés sous le titre G. Langlois, Dame Carcas, une légende épique occitane. On y trouvera la totalité des références qui n'ont pu prendre place dans cet article. Voir aussi S. Caucanas, "Dame Carcas, une héroïne sarrasine", Perpignan, l'histoire des musulmans dans la ville (du Moyen Âge à nos jours). Recueil des communications du colloque du 7 et 8 avril 2005, Perpignan : Archives communales, 2005, p. 33-44.

2. Pour réaliser un arbre phylogénétique des différentes légendes, il aurait fallu compléter cette approche thématique par une approche statistique, comme l'a fait avec d'excellents résultats J.d'Huy sur des contes et des mythes: "Peut-on tester scientifiquement la phylogénétique des mythes? À propos du motif de la chasse cosmique ». Mais le temps nous a manqué.

3. B. Gicquel, La légende de Compostelle. Le livre de Saint-Jacques.

4. L'origine des premières chansons de geste est un sujet très polémique depuis le XIX ${ }^{\mathrm{e}}$ siècle, notamment sur la date ( $\mathrm{xl}^{\mathrm{e}}$ ou avant ?), la forme (chanson ou simple récit ?) et la langue (occitan ou français). Signalons cependant que les indices en faveur d'une origine occitane précoce s'accumulent. Voir en particulier les travaux de R. Lafont et plus récemment L. Macé, (dir.), Entre histoire et épopée. Les Guillaume d'Orange (IX ${ }^{e}$-XIII siècles).

5. R. Lafont, "Roland matamore, ou l'ethnotype du Franc fanfaron", La source sur le chemin : aux origines occitanes de l'Europe littéraire, p. 223 et suiv.

6. G. Langlois, Olivier de Termes, p. 141 et suiv.

7. Sur la fidélité méridionale voir H. Débax, La féodalité languedocienne XI ${ }^{e}$-XII ${ }^{e}$ siècles.

8. D. Luce-Dudemaine, Flamenca et les «novas » à triangle amoureux et G. Langlois, « Note sur les origines du troubadour Arnaut de Carcassés", Revue des langues romanes, p. 93-103.

9. A. et R. Vinas, Le Livre des Faits de Jaume le Conquérant, p. 187.

\section{RÉSUMÉS}

La Légende de dame Carcas dérive de chansons de geste perdues, centrées sur le siège de Carcassonne mené par Charlemagne face aux Sarrasins. Elle relate la défense de la ville par une femme, usant de stratagèmes pour tromper l'ennemi sur ses forces réelles. L'étude des légendes et récits comportant les mêmes stratagèmes permet d'arriver aux conclusions suivantes: la Légende de Dame Carcas s'est diffusée à travers les Pyrénées et l'Europe par les chemins du 
pèlerinage de Saint-Jacques-de-Compostelle, empruntés notamment par des chevaliers participant à la Reconquista, des troubadours et des clercs. Elle a alors inspiré la composition d'autres légendes fondatrices d'une identité locale, principalement en France méridionale et dans le Nord de la péninsule ibérique. L'absence de légendes similaires dans une grande partie de l'Europe du Nord s'explique par des différences de mentalité : pour la société aristocratique du Nord la ruse est infamante, pour celle du Sud elle est valorisante.

La leyenda de doña Carcas proviene de cantares de gesta perdidos, centrados en el sitio de Carcasona conducido por Carlomagno contra los Moros. Conta la defensa de la ciudad por una mujer, usando estratagemas para engañar al enemigo sobre sus fuerzas reales. El estudio de leyendas y narraciones que contienen las mismas estratagemas conduce a las siguientes conclusiones : la Leyenda de doña Carcas se extendió por los Pirineos y Europa a través de los caminos de peregrinación de Santiago de Compostela, tomados por los caballeros que participan en la Reconquista, trovadores y clérigos. A continuación, inspiró la composición de otras leyendas que fundaron una identidad local, principalmente en el sur de Francia y en la parte norte de la península Ibérica. La ausencia de leyendas similares en gran parte del norte de Europa puede explicarse por las diferencias de mentalidad: para la sociedad aristocrática del Norte, la astucia es infame; para la del Sur es gratificante.

\section{AUTEUR}

\section{GAUTHIER LANGLOIS}

Société d'études scientifiques de l'Aude, Académie des arts, sciences et lettres de Carcassonne 
Nouvelles appropriations contemporaines 


\title{
De la montagne comme adjuvant à la cure au site de loisirs urbains : le Revard et Aix-les-Bains (XIX ${ }^{\mathrm{e}}-\mathrm{XX}^{\mathrm{e}}$ siècle)
}

\author{
Elsa Belle et Philippe Gras
}

Dominant la ville d'Aix-les-Bains, la station du Revard se situe à 1545 mètres d'altitude dans le massif des Bauges en Savoie. Elle s'étend sur le territoire de cinq communes (Mouxy, Les Déserts, Le Montcel, Pugny-Chatenod et Saint-François de Salles) et se développe sur un plateau vallonné, marqué par de faibles dénivellations et en grande partie couvert de forêt. Les pentes du Revard, modestement équipées de quelques remontées mécaniques, sont parcourues par une clientèle familiale de skieurs tandis que le domaine de ski nordique, Savoie Grand Revard, est fréquenté par de nombreux fondeurs. Durant la saison estivale, le sommet et le site pâturés par les vaches attirent également des visiteurs contemplatifs, des randonneurs et des promeneurs ainsi que des cyclistes. Le cadre bâti, peu étendu et peu dense, comprend majoritairement des chalets individuels, quelques immeubles et quelques commerces.

La montagne du Revard entretient des liens intimes avec Aix-les-Bains depuis la fin du Moyen Âge. La station, fondée à la fin du XIX ${ }^{\mathrm{e}}$ siècle, s'est construite et développée dans une relation de complémentarité avec la ville thermale aixoise, ses besoins, ses ambitions. 


\section{De la montagne agropastorale à la montagne climatique : de la propriété des marquis d'Aix à une invention aixoise}

3 Les textes promouvant la fondation d'une station au sommet du Revard à la fin du XIX siècle, insistent sur la nécessité de mettre en valeur la montagne, perçue comme une ressource inutilisée malgré la présence ancienne d'une activité agropastorale.

En effet, depuis la fin du Moyen Âge et jusqu'à la Révolution, une grande partie de la montagne du Revard appartient à la famille des marquis d'Aix-les-Bains. On connaît une inféodation du comte de Savoie à Humbert de Seyssel, en 1344, pour les terres jusqu'au sommet de la montagne de Mouxy ${ }^{1}$. Chalets et pâturages, appelés Fermes du Revard, sont affermés à des fermiers prenant à l'estive les vaches de différents propriétaires issus des communes voisines. Intégrées dans le domaine foncier constitutif de la station dès sa création à la fin du xix siècle, les Fermes du Revard suivent les changements de propriétaires successifs et sont acquises par la Compagnie du Revard en 1923, puis par la Société hôtelière et touristique de la compagnie ParisLyon-Méditerranée (PLM) en 1930 et enfin par la Société Immobilière (STIR) en 1953. Depuis 2003, elles appartiennent à la Communauté d'agglomération Grand Lac, dont Aix-les-Bains constitue la ville centre, et sont exploitées par un groupement agricole d'exploitation en commun (GAEC).

5 Comme de nombreux sommets et sites d'altitude, le Mont-Revard fait l'objet d'une évolution des sensibilités et d'un changement de regard sur la montagne amorcés au $\mathrm{XVIII}^{\mathrm{e}}$ siècle $^{2}$. Jusqu'ici brièvement cité comme cadre de la station thermale aixoise, il est décrit à partir du milieu du $\mathrm{XIX}^{\mathrm{e}}$ siècle comme un site particulièrement remarquable, susceptible d'attirer des curieux et d'accueillir des malades. Après avoir décrit son ascension et son sommet en $1860^{3}$, l'auteur et éditeur de guide Adolphe Joanne propose d'y établir un chalet-hôtel qui :

«Serait certainement visité par tous les étrangers qui séjournent à Aix [...] et un grand nombre d'habitants de Lyon, de Grenoble et de toutes les vallées voisines viendraient aussi, soit pour jouir de la vue, soit pour y prendre des bains d'air si utiles dans un grand nombre de maladies. ${ }^{4}$ "

Plus qu'un simple écrin brièvement décrit comme cadre de la station thermale, le Mont-Revard est ici promu au rang de site à part entière. À partir de 1875, le MontRevard fait l'objet de l'attention du Club alpin français (CAF), créé l'année précédente à Paris. La présence d'Adolphe Joanne, fidèle client de la station aixoise, parmi les fondateurs, n'est probablement pas étrangère à cet intérêt qui se concrétise en 1876 par la création de la Société du Grand Revard. L'objectif de celle-ci, associant les efforts de la ville d'Aix-les-Bains, de la section aixoise du CAF fondée en 1876 et des médecins, réside dans la création d'une extension de la ville thermale au Revard ${ }^{5}$. Le Mont-Revard est en effet envisagé comme un lieu d'excursions et sa situation vue comme propice à l'établissement de cures d'air venant compléter les cures thermales.

7 Créer une telle station au Revard s'inscrit dans une démarche générale de la ville thermale, soucieuse d'augmenter son attractivité et de tenir son rang dans le contexte concurrentiel entre stations de villégiature. Depuis les années 1860-1870, cette concurrence doit compter avec le succès grandissant des stations d'altitude suisses, telles que Davos, ou celles des hautes vallées de l'Engadine comme Saint-Moritz: la 
clientèle anglaise, chère à Aix-les-Bains, porte, en effet, un intérêt croissant au charme et à l'air pur de la montagne et a tendance à abréger son séjour aixois pour se rendre en Suisse $^{6}$. L'établissement d'une cure d'air, envisagée comme complémentaire de la cure thermale, indispensable pour retenir baigneurs et étrangers, semble de plus offrir la possibilité d'attirer une nouvelle clientèle ${ }^{7}$. La campagne promotionnelle s'attache donc à montrer que le Revard constitue un site propice à l'établissement de cures d'air.

La promotion d'une station climatique au Revard trouve ses ressorts dans les principes de la climatologie médicale et sa justification scientifique dans les travaux du médecin aixois Jean Monard (1850-1923). Ce dernier mène une campagne très active, au travers de nombreuses publications spécialisées et de vulgarisation, pour créer une "vallée climatérique Aix-Revard ». Il défend en effet l'idée de caractériser les différents climats existants depuis le lac du Bourget jusqu'au sommet du Revard en passant par la station thermale, afin de traiter différentes pathologies ${ }^{8}$. À partir d'une série d'observations et de relevés météorologiques effectués en 1889-1890 au cours d'expéditions, Jean Monard affirme la supériorité du climat du Revard en particulier et des Alpes françaises en général sur les montagnes suisses ${ }^{9}$. Au Revard, le médecin propose l'installation d'un sanatorium pour tuberculeux et se heurte à une vive opposition qui considère la présence de contagieux comme préjudiciable à la ville thermale ainsi qu'à la future station d'altitude ${ }^{10}$.

Malgré l'ambiguïté entretenue par les documents promotionnels et l'imprécision du vocabulaire employé pour désigner la station, à son ouverture en 1892 et après, celle-ci n'abrite finalement aucun établissement dédié aux cures d'air et ne fonctionne que durant la saison estivale, à l'inverse des sites suisses ${ }^{11}$. Aussi, la saison du Revard suitelle celle de la ville thermale, confirmant la complémentarité et l'interaction souhaitées entre les deux stations sur lesquelles l'initiative originale reposait.

\section{Relier la ville thermale et la station d'altitude}

10 Lors de sa création en 1876, l'une des premières initiatives de la Société du Grand Revard consiste à rénover et à entretenir le chemin muletier du Pertuiset, décrit en 1872 par Adolphe Joanne comme en «très mauvais état, envahi par les broussailles et difficile à trouver sans guide $»^{12}$. Inauguré le 5 octobre $1876^{13}$, ce chemin facilite l'accès au Revard et permet d'acheminer les premiers excursionnistes à pied ou à dos de mulets.

11 La création d'autres moyens d'accès, plus rapides et plus aisés, est rapidement envisagée. Au cours des années 1880, l'idée d'établir des cures d'air au Revard permet de justifier la construction d'une route ou d'un chemin de fer. C'est d'ailleurs dans les rapports de présentation de ces projets qu'est développé l'argumentaire le plus éloquent. L'établissement de cures d'air et la réalisation de moyens d'accès sont désormais liés et se légitiment réciproquement.

12 Le projet de réalisation d'une route, envisagé dès 1880 et bien engagé en $1888^{14}$, est finalement abandonné au profit de la construction d'un chemin de fer à crémaillère, s'appuyant sur l'expérience suisse du Righi ${ }^{15}$ inauguré en 1871. Rapidité du trajet et des délais de réalisation, intérêt touristique et technique suscité par un tel équipement, expliquent le choix d'une voie ferrée aux dépens d'une desserte carrossable. En effet : 
«Si une route existait jusqu'au Revard, il faudrait six heures en voiture hippomobile contre une heure trente en train à vapeur pour atteindre le sommet. ${ }^{16}$

》) (CAF), finance la réalisation d'une étude préparatoire de construction d'un chemin de fer entre Aix-les-Bains et le Revard ${ }^{17}$. Après une recherche d'investisseurs, la concession pour la construction et l'exploitation du chemin de fer à crémaillère est confiée en 1890 à quatre personnalités genevoises, par ailleurs actionnaires de la Société genevoise des chemins de fer à voie étroite qui exploite des tramways ${ }^{18}$.

Ceux-ci se portent également acquéreurs d'un ensemble d'alpages et de forêts constituant un vaste domaine privé au sommet du Revard ${ }^{19}$. Dès 1892, ils y font édifier une gare d'arrivée, un hôtel (formé de deux bâtiments jumeaux) et un restaurant. La ligne ouvre aux voyageurs le 15 août 1892 et son inauguration par Jules Roche (1841-1923), ministre du Commerce et sénateur de la Savoie, a lieu le 5 septembre à l'occasion des Fêtes du Centenaire. La station d'altitude est désormais accessible en $1 \mathrm{~h} 15$ depuis le centre-ville de la station thermale (fig. 1).

Fig. 1. - La ville, son lac et le Mont-Revard.

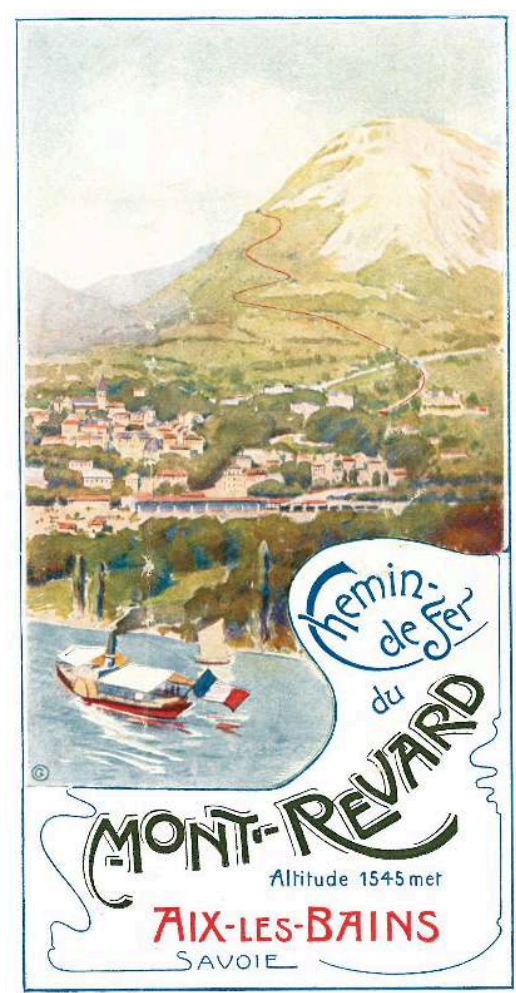

Couverture de l'un des premiers dépliants du chemin de fer à crémaillère, vers 1897.

Collection privée François Fouger (c)

Au tournant des années 1930, le chemin de fer fait l'objet de nombreuses plaintes liées à l'inconfort des voitures et aux délais d'attente lors des périodes de pointe (le dimanche en hiver) en raison de sa faible capacité de débit. La Société Hôtelière et Touristique de la Compagnie de chemin de fer Paris-Lyon-Méditerranée (SHT-PLM), propriétaire de la station et du chemin de fer depuis 1923, décide alors de substituer un transport aérien par câbles à la desserte ferroviaire ${ }^{20}$. 
Le téléphérique du Revard, mis en service le 21 décembre $1935^{21}$, permet d'accéder à la station en 18 minutes. La gare inférieure, implantée à quelques kilomètres d'Aix-lesBains au lieu-dit Les Mentens, sur la commune de Mouxy, est reliée à la gare ferroviaire aixoise par un service automobile. La convention passée entre le Département de la Savoie et la SHT-PLM pour la concession du téléphérique en 1935, prévoit l'obligation d'établissement d'un second tronçon reliant Aix-les-Bains aux Mentens au-delà d'un seuil de recettes qui ne fut jamais atteint ${ }^{22}$. En facilitant l'accès au Revard, la SHT-PLM agit dans les intérêts de la Compagnie de chemin de fer et cherche à augmenter le trafic sur les lignes ferroviaires à destination d'Aix-les-Bains pendant la période hivernale. Pour faire face aux faibles capacités d'accueil des hôtels, la société n'envisage pas d'investissements supplémentaires dans la station mais compte s'appuyer sur la rapidité et sur l'importante capacité de débit du téléphérique ainsi que sur les infrastructures d'hébergement aixoises ${ }^{23}$. Le fonctionnement de cet équipement cesse en 1969, face à la concurrence routière, faute d'entretien et de recettes satisfaisantes. Les déficits d'exploitation rencontrés depuis les années 1950, structurels puisque le téléphérique n'avait pas été pensé comme un équipement rentable, sont malgré tout imputés à l'éloignement de la gare inférieure du centre-ville aixois. Au cours des années 1970, les différents projets de rénovation envisagés, non réalisés faute de moyens financiers, proposent systématiquement une liaison, directe ou non, par voie ferrée ou par câbles, avec Aix-les-Bains ${ }^{24}$. La concurrence de la liaison routière, réalisée tardivement, écarte par ailleurs toutes possibilités de maintien d'un moyen de desserte alternatif.

17 Le projet de construction d'une route desservant le Revard renaît dans les années 1910 Celle-ci emprunte cependant un itinéraire partant de Chambéry et passant par le col de Plainpalais. Praticable mais peu commode, cette voie fait l'objet de travaux d'amélioration au cours des années 1920. Mobilisée dès 1910, la ville d'Aix-les-Bains participe financièrement à sa construction dans l'intention de la faire prolonger jusqu'à Aix. Les travaux, organisés en quatre sections selon le profil établi dans les années 1880, ne débutent qu'en 1932 grâce à l'action du sénateur-maire Maurice Mollard (1863-1947) et grâce à la participation financière du Département et de l'État. Le 23 novembre 1936, une caravane de trois voitures - celle de l'ingénieur principal Lehanneur et celles des journaux du Petit Dauphinois et de l'Écho de Savoie - parcourt pour la première fois et dans d'excellentes conditions « la boucle du prestigieux circuit touristique » qui permet de relier Chambéry à Aix-les-Bains en passant par le Revard.

18 Aujourd'hui, la route constitue le moyen d'accès principal au Revard depuis Aix-lesBains. L'itinéraire de la voie ferrée, dont les superstructures ont été démantelées dès 1937, a été transformé en sentier de randonnée et permet de rejoindre le sommet et la station à pied. L'existence du téléphérique s'incarne quant à elle dans la conservation de sa gare supérieure occupée par un restaurant.

\section{De la station climatique à la station mondaine de sports d'hiver : la proximité et la complémentarité de la ville thermale}

19 Présentée comme une extension de la ville thermale, la station mondaine du Revard appuie sa promotion sur la proximité d'Aix-les-Bains, à une heure quinze via le chemin 
de fer, et des ressources dont la ville dispose. L'existence d'une gare, d'une vie festive et mondaine par l'intermédiaire des théâtres et casinos, sont autant d'éléments présentés comme des points forts pour une station d'altitude. L'ingénieur grenoblois Édouard Lullin (1832-1912), dans une brochure de présentation du chemin de fer du Revard, construit son argumentaire en ce sens :

«Si pour Aix c'est une bonne fortune d'avoir, par cette station d'altitude, agrandi de la sorte son domaine thérapeutique, c'est également une bonne fortune pour le Revard d'être à une heure de cette station thermale et de sa gare PLM $[. .].]^{25}$ »

L'argument de proximité est mis en avant afin de réaffirmer la supériorité du Revard sur les stations suisses dont l'isolement et par conséquent le manque de distractions, ainsi que les difficultés d'accès sont régulièrement rappelés. Le fait que le Revard soit à la portée «de toutes ces distractions, qui manquent trop parfois à certaines stations d'altitude, dans les solitudes montagneuses de la Suisse ou de l'Allemagne ${ }^{26}$ se veut attractif. Aussi, des trains spéciaux sont-ils mis en place le matin et le soir pour faciliter les mouvements pendulaires avec la ville thermale ${ }^{27}$.

Dès son ouverture, la station d'été offre des possibilités sportives et contemplatives. La situation incomparable, en balcon sur Aix-les-Bains et le lac du Bourget, a inspiré, en 1893, la construction d'un observatoire en rondins de bois doté d'une table d'orientation et l'installation d'un magasin de souvenirs, remplacés avant la Première Guerre mondiale par un bâtiment en moellons de pierre ${ }^{28}$. À partir de 1893, la station s'équipe d'un tir aux pigeons, installé à proximité de la gare de chemin de fer. Il est créé et dirigé par M. Bertrand, fondateur d'un établissement similaire à Cannes, qui organise annuellement un grand concours international baptisé le « Prix du Revard ». Un court de tennis complète ces premières installations.

L'histoire du Revard se singularise cependant par la naissance et le développement précoce des sports d'hiver pratiqués dès 1906. Des pionniers, originaires d'Aix-les-Bains et de Chambéry, fondent le Club des Sports Aix-Chambéry-Revard (ARC) en 1908. L'année suivante marque le lancement de la saison et de la station hivernale du Revard désormais accessible par un service régulier de train pendant l'hiver. Appuyée par la Ville, l'organisation d'un grand concours international en présence du champion de ski norvégien, Durban- Hansen ${ }^{29}$, rencontre un immense succès médiatique et assure le lancement promotionnel de la station.

En 1924-1925, la SHT-PLM entreprend des travaux de modernisation destinés à améliorer le confort et à adapter les locaux aux conditions hivernales ${ }^{30}$. Avec la construction d'une patinoire, cette campagne confirme la vocation hivernale du site. En parallèle, le Club des sports d'hiver, grâce au docteur Hallberg ${ }^{31}$, introduit une nouvelle méthode de ski, dite autrichienne, ancêtre de notre pratique actuelle. L'école de ski qu'il crée au Revard en 1931, est à l'origine de l'École de ski français (ESF). Premier diplômé, l'alpiniste Roger Frison-Roche (1906-1999) anime ce centre de formation pendant l'hiver 1931-1932. Dans la foulée de la construction du téléphérique, la SHTPLM met en service en février 1936 le premier remonte-pente à câble de la station permettant désormais de pratiquer le ski sur des pentes plus raides.

24 La station, pionnière des sports d'hiver, dotée de nombreux équipements (patinoire, piste de luge, tremplin de ski, remontée mécanique) et d'établissements d'hébergement luxueux, connaît une renommée internationale jusque dans l'entre-deux-guerres. 


\section{De la station mondaine au site de loisirs urbains : un poumon urbain de proximité} radicalement différentes de celles préconisées dans les années 1950. Les nombreuses critiques du plan neige et des stations de troisième génération, villes hivernales créées pour la pratique exclusive du ski alpin, aboutissent à une nouvelle approche de l'aménagement de la montagne marquée par la directive montagne de 1977. Plusieurs études sur les potentialités de développement du Revard préconisent, en s'appuyant sur le constat de l'échec du projet des années 1950 et sur celui de la faiblesse des moyens des autorités publiques, un développement autour du "patrimoine existant». Ainsi, l'aménagement du Revard, désormais considéré comme un site de loisirs urbains pour les bassins aixois, chambérien et annécien, doit privilégier la préservation de son cadre dit « naturel » et favoriser le développement des sports d'hiver, notamment le ski de fond. Les infrastructures et l'encadrement de cette discipline, pratiquée par des amateurs de plus en plus nombreux depuis la fin des années 1960, apparaissent au Revard grâce à la forte implication du maire d'Aix-les-Bains et des services de la Ville. d'aménagement retenus il y a trente ans ${ }^{36}$, découlent un cadre bâti et des équipements 
très modestes. La station du Revard est désormais perçue d'abord comme un site et comme un poumon à disposition d'une clientèle aixoise et plus largement urbaine de proximité. Le bulletin municipal d'Aix-les-Bains le résume d'ailleurs en ces termes :

«Avec le lac du Bourget, [le Revard] c'est l'autre espace de jeux et de nature d'Aix-

les-Bains. »

\section{BIBLIOGRAPHIE}

BELLE Elsa, GRAS Philippe, LAGRANGE Joël, « Aix-les-Bains côté montagne », Arts et mémoire, $\mathrm{n}^{\circ}$ 90, août, numéro spécial, 2016.

JOANNE Adolphe, Itinéraire descriptif et historique de la Savoie. Réimpression de l'édition de 1860, Paris, Les Éditions du Bastion, 1992, p. 38-39.

JOANNE Adolphe, Dauphiné et Savoie. Paris, Hachette, 1872, p. 427-428.

MOUXY De LocHE Jules, Histoire d'Aix-les-Bains. Chambéry, Imprimerie Savoisienne, 1898.

\section{NOTES}

1. J. Mouxy de Loche, Histoire d'Aix-les-Bains.

2. Horace Benedict de Saussure, célèbre pour son ascension du Mont-Blanc en 1787, effectue celle du Mont-Revard en 1790.

3. A. Joanne, Itinéraire descriptif et historique de la Savoie, p. 38-39.

4. A. Joanne, Dauphiné et Savoie, p. 427-428.

5. Archives communales d'Aix-les-Bains (AC), A 762. «Trois jours en Savoie. Congrès des clubs alpins à Annecy. (août 1876)», 1877.

6. AC Aix-les-Bains. A 879. « Chemin de fer du Revard : Rapport présenté au Comité des Études ", 1888, p. 2.

7. AC Aix-les-Bains. LDA 343. "Aix-les-Bains. Grand-Revard. Rapport présenté au conseil municipal le 12 avril $1887 », 1887$, p. 18.

8. AC Aix-les-Bains. «L'Avenir d'Aix-les-Bains », 7 et 14 décembre 1890.

9. AC Aix-les-Bains. « L'Avenir d'Aix-les-Bains », 23 février 1890.

10. AC Aix-les-Bains. «L'Avenir d'Aix-les-Bains », 15 février 1891.

11. AC Aix-les-Bains. «L'Avenir d'Aix-les-Bains », 8 septembre 1892.

12. A. Joanne, Dauphiné et Savoie, p. 427-428.

13. AC Aix-les-Bains. A 879. «Chemin de fer du Revard: Rapport présenté au Comité des Études », 1888, p. 12-13.

14. AC Aix-les-Bains. LDA 343. «Aix-les-Bains. Grand-Revard. Rapport présenté au conseil municipal le 12 avril $1887 », 1887$. 
15. Le trajet part de Vitznau, au bord du lac des Quatre-Cantons, pour atteindre le sommet du Righi à $1810 \mathrm{~m}$, sur une distance de $8300 \mathrm{~m}$. C. Boissay, «Le chemin de fer du Righi ", La nature-revue des sciences, $n^{\circ} 1,1873$, p. 7-10.

16. AC Aix-les-Bains. A 879. «Chemin de fer du Revard: Rapport présenté au Comité des Études », 1888.

17. AC Aix-les-Bains. "L'Avenir d'Aix-les-Bains », 27 mai, 15 juillet et 27 septembre 1888.

18. Archives Départementales (AD) Savoie. 68 S 1. "Convention signée entre le Département et MM. Dupont-Buëche, Petit, Tronchet et Annevelle pour la concession de la crémaillère ", 1890.

19. AD Savoie. 70 SPC 3. «Chemin de fer à crémaillère d'Aix-les-Bains au Revard. Exposé général. Rapport sur la construction et le rendement ", 1891.

20. AD Savoie. 1210 W 127. "Société Hôtelière et Touristique, Note sur le projet de téléphérique », 9 mai 1935.

21. AD Savoie. 1164 W 92. "Arrêté préfectoral autorisant l'exploitation du téléphérique à titre provisoire ", 21 décembre 1935.

22. AC Aix-les-Bains. $160 \mathrm{~W}$ 36. "Projet de convention entre la Société Hôtelière et Touristique et le Département pour la concession du téléphérique », 1935.

23. AD Savoie. $1210 \mathrm{~W}$ 127. "Société Hôtelière et Touristique, Note sur le projet de téléphérique », 9 mai 1935.

24. AC Aix-les-Bains. 2 O 25. «Consultation d'entreprises pour la modernisation du téléphérique ».

25. AC Aix-les-Bains. 20 3. «Le chemin de fer à crémaillère d'Aix-les-Bains au MontRevard ", 1893, p. 38-39.

26. AC Aix-les-Bains. 20 3. «Le chemin de fer à crémaillère d'Aix-les-Bains au MontRevard ", 1893, p. 39.

27. AC Aix-les-Bains. «L'Avenir d'Aix-les-Bains », 24 août 1893.

28. D'après l'iconographie ancienne.

29. Harald Durban-Hansen (1876-1930), conseiller technique du Club alpin en 1905, champion du saut à ski à Montgenèvre (lors du premier concours international organisé du 9 au12 février 1907) avec un bond de $26 \mathrm{~m}$.

30. Archives privées Entreprise Léon Grosse. «Ensemble de dossiers de chantiers de construction au Revard».

31. F. Hallberg, auteur du Ski actuel publié en 1936 où l'auteur décrit la position «telemark».

32. AC Aix-les-Bains. BR 639. «Rapport sur l'aménagement du plateau du Revard», 22 avril 1952.

33. AD Savoie. $30 \mathrm{~J}$ 340. « Notice rédigée par Laurent Chappis en 1985 lors du versement de ses archives aux Archives départementales de la Savoie ».

34. AC Les Déserts. «Permis de construire accordé à la Ville de Pantin », 11 septembre 1956.

35. AC Aix-les-Bains. 160 W 21. "SIVOM du Revard, Urbanisme et lotissements", 1953-1986. 
36. Ces principes sont repris dans les documents d'urbanisme postérieurs et renforcés par l'intégration de la station dans le Parc naturel régional du massif des Bauges créé en 1995.

\section{RÉSUMÉS}

La station savoyarde du Revard, située à $1545 \mathrm{~m}$ d'altitude dans le massif des Bauges au-dessus d'Aix-les-Bains, voit le jour à la fin du $\mathrm{XIX}^{\mathrm{e}}$ siècle suite à une importante campagne promotionnelle. Des membres du Club alpin français, des médecins et des hommes politiques locaux portent le projet d'une station de cure d'air destinée à compléter la ville thermale. À partir de 1892, des investisseurs suisses réorientent le projet vers une station de villégiature estivale d'altitude. Par une sorte de renversement, le Revard appuie dorénavant sa promotion sur la proximité d'Aix-les-Bains et des ressources dont la ville dispose. Pionnière des sports d'hiver, la station connaît la renommée internationale avant que l'évolution du ski n'entraîne sa clientèle, après 1945, vers les pentes des nouvelles stations d'altitude. C'est enfin, depuis Aix, que sont impulsées de nouvelles orientations sportives attribuant au Revard, à partir des années 1970, le rôle de poumon urbain de proximité.

\section{AUTEURS}

\section{ELSA BELLE}

Chercheur, service Patrimoines et Inventaire général, région Auvergne-Rhône-Alpes

\section{PHILIPPE GRAS}

Chercheur, Archives municipales et Inventaire du patrimoine de la ville d'Aix-les-Bains et administrateur de la Société d'art et d'histoire d'Aix-les-Bains (SAHA) 


\title{
Arts, culture, patrimoine du Queyras ( $\mathrm{XIX}^{\mathrm{e}}-\mathrm{XX}$ siècles)
}

\author{
Jean-Gérard Lapacherie
}

1 "Une cellule fortement clôturée, difficilement accessible dans toutes les directions ", voilà dans quels termes Raoul Blanchard définit le Queyras ${ }^{1}$. Les métaphores de la clôture, de la chartreuse ou de la cellule de monastère, de la forteresse sont éloquentes. Si tant est que la métaphore de la cellule soit pertinente, dans la réalité, il n'y a pas une cellule, mais plusieurs, autant que de communautés ou communes, à savoir au nombre de sept, ou de villages et hameaux (une trentaine), entre lesquels n'est érigée aucune clôture. Ce ne sont pas les seules métaphores. Les nombreuses études que le géographe Raoul Blanchard (1877-1965) a publiées sur cette vallée de haute montagne entre 1908 et 1950 commencent par une description des conditions qu'il qualifie tantôt de naturelles, tantôt de physiques, tantôt de matérielles, à savoir le relief, l'altitude, la pluviométrie, l'hydrographie, l'exposition des versants, la nature et la composition des sols, l'érosion glaciaire, etc. La métaphore des "conditions" est empruntée à la grammaire logique : une action, à partir du moment où elle se réalise, entraîne une autre action. Là est le cœur du déterminisme. À ce déterminisme physique, dans les années 1940, Raoul Blanchard a essayé de substituer l'hypothèse d'un déterminisme culturel, l'état sinistré du Queyras s'expliquant par l'existence de routines ou de structures mentales archaïques. Au sujet de l'habitat et de la cohabitation avec les animaux dans une même salle commune, il écrit pour expliquer le phénomène :

« Nous soupçonnerions plutôt la persistance de vieilles habitudes héritées d'un régime social ou politique évanoui ; on sait à quel point la force d'inertie paralyse les transformations de l'habitat rural, particulièrement dans un milieu aussi fermé que le Queyras. ${ }^{2}$ "

2 Ces conditions physiques expliqueraient les techniques archaïques (araire, jachère, pas ou peu d'engrais, pas de chemins agricoles, pas de route carrossable jusqu'à la fin du $\mathrm{XIX}^{\mathrm{e}}$ siècle, transport des récoltes à dos d'homme ou par bêtes de somme, etc.) et des modes de vie héritées d'un passé séculaire (la cohabitation avec les animaux). Or, alors que ces conditions n'ont pas changé au cours des deux derniers siècles, ce qu'elles sont censées déterminer, à savoir l'agriculture de montagne, a disparu. 
3 La thèse d'une vallée de haute altitude close sur elle-même, reprise dans la seconde moitié du xx ${ }^{e}$ siècle, entre autres auteurs, par Robert Burns ${ }^{3}$ et Augustin Guillaume ${ }^{4}$, est en partie infirmée, entre autres faits, par deux réalités avérées, l'une matérielle, l'autre culturelle, à savoir l'irrigation de près de la moitié des terres cultivables grâce à un réseau très dense de canaux dont certains ont été creusés aux XIII ${ }^{\mathrm{e}}$ et $\mathrm{XIV}^{\mathrm{e}}$ siècles $^{5}$ et l'alphabétisation massive et précoce, dès le $\mathrm{xv}^{\mathrm{e}}$ siècle, de la population. De plus, les recherches menées à partir des années 1970 infirment, non pas les descriptions admirables de Raoul Blanchard, mais les conclusions qu'il en a tirées. À celles-ci, il est opposé la modernité démocratique des communautés d'Ancien Régime et le niveau élevé de développement économique et social que ces communautés ont atteint sans bourgeoisie et sans aide de l'État ${ }^{6}$; l'adaptation des Queyrassins aux conditions économiques changeantes ${ }^{7}$; les stratégies commerciales et de mobilité auxquelles ont eu recours au $\mathrm{xIX}^{\mathrm{e}}$ siècle les familles pour faire face aux crises économiques, et la maitrise de la culture écrite par des populations tenues souvent pour arriérées ${ }^{8}$. Ce sont surtout les études menées dans le cadre de l'Inventaire général des Monuments historiques et des Richesses artistiques de la France, en particulier celles de MariePascale Mallé9 qui ont substitué au Queyras réduit à un vestige archaïque du Moyen Âge un Queyras relativement heureux, prospère, inventif. Soit la maison-type, singulière et primitive, adaptée au climat et au relief, qui se serait maintenue, telle quelle, au fil des siècles ${ }^{10}$. Marie-Pascale Mallé montre qu'il n'y a pas une maison queyrassine, mais des maisons, que celles-ci ne se soumettent pas au seul même modèle, mais que la disposition des pièces et leur destination varient d'une maison à l'autre, que la quasi-totalité d'entre elles sont récentes, datant de la seconde moitié du XVIII ${ }^{\mathrm{e}}$ siècle et du tout début $\mathrm{du} \mathrm{xIX}^{\mathrm{e}}$ siècle, et que, modifiées, agrandies, rénovées, reconstruites, elles sont, en un mot, modernes, c'est-à-dire adaptées au mode de vie et de production de leur époque.

4 L'étude qui suit a pour objectif d'amplifier les hypothèses en germe dans l'Inventaire général et cela à partir des réalités suivantes : la haute culture d'une population lettrée, la pratique du théâtre et du plain-chant, les vitraux et le programme décoratif des églises, l'ornementation et les décors intérieurs.

\section{Une population lettrée}

$5 \quad$ Les études portant sur l'alphabétisation des habitants du Briançonnais et du Queyras écartent communément ce que l'on est contraint de nommer haute culture, puisque le nom culture désigne désormais à peu près tout et n'importe quoi. Cette haute culture s'exprime dans les innombrables écrits du XIX ${ }^{e}$ siècle et du début du $\mathrm{Xx}^{\mathrm{e}}$ siècle. Leurs auteurs se répartissent, suivant la profession qu'ils exerçaient, en curés, pasteurs, professeurs, médecins, instituteurs, fonctionnaires, ingénieurs, négociants ou industriels. Les domaines qu'ils abordent sont l'histoire locale, les mœurs, traditions et coutumes, l'analyse d'archives, le patois, les contes, la vie des saints ou des hommes morts en réputation de sainteté, la rhétorique religieuse, l'observation de cas médicaux communs, le développement économique, la pédagogie et les matières d'enseignement, la morale publique et la formation de soi. Ces écrits se présentent sous des formes diverses : manuscrits inédits, dont quelques-uns, signalés dans des notes d'ouvrages publiés, sont introuvables (par exemple le registre de la communauté de ChâteauQueyras, une histoire du Queyras par l'abbé Berge, une histoire de la communauté 
d'Abriès par l'abbé Richard (1834-1907), etc.); journaux familiaux oubliés dans des armoires; articles ou études publiés dans des revues savantes (Revue du Dauphiné, bulletins annuels ou semestriels divers (ceux de l'Académie delphinale, de la Société d'études des Hautes-Alpes, de la Société dauphinoise d'ethnologie et d'anthropologie, de la Société des statistiques, des sciences naturelles et des arts industriels du département de l'Isère), Annales des Alpes, Annuaire du Club Alpin Français, Annuaire de la Société des Touristes du Dauphiné); brochures imprimées d'une dizaine de pages et publiées soit par une institution, telle que le Syndicat d'Initiative du Queyras ou la Chambre de commerce de Marseille, soit à compte d'auteur; livres imprimés, pour beaucoup d'entre eux, à compte d'auteur. Les patronymes de ces auteurs sont ceux de longues lignées queyrassines: les abbés Jacques Gondret (1820-1893) et Pierre Berge (1868-1949) $)^{11}$, l'abbé Chaffrey-Martin (1813-1872) ${ }^{12}$, l'abbé Victor Gignoux (1810-1860), les abbés Jean-Laurent Merle (1808-1877) et Bourcier (1749-1816) ${ }^{13}$, l'instituteur Joseph Maritan (1820-1891), le docteur Richard-Calve (1799-1848), l'ingénieur Pierre Isnel (1864-1938), le docteur Chabrand (1812-1898), Claude Arnaud ${ }^{14}$, ou alliés à une lignée queyrassine : Jean Tivollier (1859-1938) ${ }^{15}$, le docteur Rozan de Château-Ville-Vieille ${ }^{16}$. Pour rendre compte de ces écrits, il faut distinguer ce qui est nommé histoire littéraire ou histoire de la littérature, enseignée dans les lycées et les universités de 1820 ou 1830 à la fin $\mathrm{du} \mathrm{xx}^{\mathrm{e}}$ siècle, de ce que les bénédictins de la Congrégation de Saint-Maur, entre autres auteurs Dom Vallet, Dom Rivet et Dom Clément, ont appelé l'Histoire littéraire de la France, qui a abouti à une entreprise éditoriale, souvent méconnue, de douze volumes publiés entre 1733 et 1763 et de trente et un autres publiés entre 1814 et 2015 par l'Académie des Inscriptions et Belles Lettres. L'adjectif littéraire a deux ou plus de deux sens. Pour les mauristes, il se rapporte aux connaissances que l'on peut acquérir dans les écrits de jurisconsultes, théologiens, Pères de l'Église, évêques, historiens, rabbins, hérésiarques, etc. que ces écrits soient des lettres, des cours d'université, des essais, des livres manuscrits ou imprimés. À la fin du XviII ${ }^{\mathrm{e}}$ siècle, l'adjectif littéraire s'est enrichi de sens nouveaux, la littérature devenant l'expression singulière du génie d'un peuple ou d'une nation ou d'un individu ou une œuvre de haut langage ou d'art du langage. Les catégories, telles que le néo-classicisme, le préromantisme, le romantisme, l'art pour art, le réalisme, le naturalisme, le symbolisme, avec lesquelles il est rendu compte de la littérature au $\mathrm{XIX}^{\mathrm{e}}$ siècle, sont inadéquates pour étudier les écrits queyrassins. En revanche, si l'on entend littéraire dans le sens donné par les mauristes, il est possible d'analyser les connaissances, souvent érudites, qui sous-tendent ces écrits et les sujets qui y sont développés. Pourtant, dans cette littérature savante, froide ou impartiale, dont l'érudition est historiquement datée, parfois caduque, il arrive que perce l'expression d'états d'âme ou d'une vie intérieure, faits qui relèvent de la littérature au sens moderne de ce terme, quand elle est l'expression d'une singularité individuelle. Ainsi, l'abbé Gondret exprime, dans un style désinvolte et volontiers ironique, une critique de Montaigne, dont il moque les préventions sur l'éducation des filles, réduite à la seule science du ménage, alors que, dans le Queyras, celles-ci reçoivent la même instruction que les garçons ${ }^{17}$. Citons l'ingénieur Isnel qui, effrayé par la brutalité de la guerre à laquelle il a participé, adopte dans les années 1920-1930 les positions d'historiens antimodernes, dont Funck-Brentano et Boulainvilliers, pour opposer aux horreurs du monde moderne la relative humanité de la féodalité et de l'Ancien Régime $^{18}$. On peut citer aussi l'instituteur Joseph Maritan, qui a écrit une histoire et une géographie de France, ainsi qu'une histoire sainte en alexandrins, quelques poèmes d'assez bonne qualité et un essai de morale publique sur la perfection de soi. Il est 
l'auteur d'un exercice tenu par les sémiologues pour l'essence même de la littérature ${ }^{19}$, à savoir la parodie, transformant les Lamentations de Jérémie, qui ont été mises en musique dans les Leçons de Ténèbres, en un texte pittoresque en patois provençal dans lequel il énumère les sobriquets des habitants des villages et hameaux du Queyras (pèlechiens, cure-toupins, tripes longues...) et les défauts qui leur étaient attribués.

Il est des faits qui confirment cette haute culture. Jean-Laurent Merle, curé de la très petite paroisse de l'Echalp, réunissant alors à peine deux cents fidèles en 1840, a constitué pendant quelques années une bibliothèque personnelle de près de deux cents livres, dont un tiers seulement a été sauvegardé, et qui comprend des ouvrages de théologie publiés entre le $\mathrm{xVI}^{\mathrm{e}}$ et le début $\mathrm{du} \mathrm{xIX}^{\mathrm{e}}$ siècle, et dont les auteurs sont protestants, jansénistes ou jésuites, mais aussi des ouvrages traitant d'histoire sainte, de philosophie morale, de bonnes mœurs, d'histoire profane, de grammaire, de langue française, de latin, de poésie. Dans un manuscrit intitulé Mes Souvenirs, Joseph Maritan, alors inspecteur des écoles primaires, écrit :

«En 1862, j'ai acheté, pour 97 francs, du libraire Rispaud, d'Embrun, plusieurs ouvrages de ma bibliothèque, entre autres, le Dictionnaire géographique en 20 volumes brochés, l'Encyclopédie Courtin 19 volumes cartonnés, L'Encyclopédie Diderot et d'Alembert, 38 volumes solidement reliés, etc., etc., etc. ${ }^{20}$ »

Maritan a compilé ces ouvrages savants pour écrire une trentaine de biographies, traitant l'une des écrivains illustres, l'autre des ecclésiastiques illustres, une troisième des médecins illustres, une quatrième des Dauphinois illustres, etc. déclinant les professions et les origines jusqu'à épuisement des données qu'il lisait dans les encyclopédies qu'il avait acquises.

Dans le Queyras, la haute culture excède dans d'importantes proportions ce qui est nommé literacy. Il semble qu'une partie de la population, et pas seulement les membres du clergé, connaissait le latin : on peut lire dans les églises, sur les cadrans solaires, sur les murs de quelques maisons, dans les archives des anciennes communautés, des textes, plus ou moins longs, en latin. Les anecdotes sans cesse répétées dans les ouvrages de vulgarisation (réunions d'un conseil municipal se faisant en latin $^{21}$ ou ingénieurs et minéralogistes, en mission à Saint-Véran au début du XIX siècle, interpellés en latin par les notables du village), même si elles embellissent sans doute la réalité des choses, acquièrent un vernis de vraisemblance parce qu'elles convergent avec des faits avérés. Ce que montrent les archives, en latin ou en français, de 1343 à 1789, c'est la parfaite connaissance que les consuls, notaires, secrétaires des communautés du Queyras avaient des arcanes de la justice d'alors, des procédures et des recours, des ordonnances et des arrêts, pour défendre leurs droits sur les alpages, les forêts, les pâturages, les cours d'eau, certaines terres, qui étaient, pour les communautés, des sources de revenus élevés, multipliant les procès à l'élection de Gap, au bailliage de Briançon, aux parlements du Dauphiné et de Bourgogne, au Conseil du Roi pour défendre leurs droits. En 1343, le Dauphin leur a cédé aussi les tailles et les offices municipaux: il incombait aux communautés d'établir l'impôt, de le répartir entre les divers caput domus ou chefs de maison suivant des péréquations subtiles et en tenant compte des revenus réels que chaque famille tirait de l'exploitation de ses terres, de le recouvrer; et pour cela, consuls et notaires ont rempli des livres de compte, dressé de nombreux registres (des propriétés, dit cadastre, des mutations, des cotes non liquidées, des recouvrements, des dépenses engagées par les consuls, etc.) et imaginé des procédures, dont la distinction entre l'ordonnateur (des dépenses) et le 
payeur et surtout l'audit, un auditeur vérifiant les comptes des consuls, avant que ceuxci ne les présentent aux propriétaires assemblés qui les approuvaient ou non par un vote. A donc été mise en place sur les ruines de la féodalité et à partir de rien une administration à la fois efficace et équitable. Les Queyrassins étaient rompus aux comptes, au négoce, aux transactions, comme le souligne assez justement le titre de la thèse d'Harriet Rosenberg, A Negociated World ${ }^{22}$, ce qui explique, entre autres raisons, qu'ils aient brillamment réussi, à compter de 1830, dans leurs entreprises commerciales à l'étranger ${ }^{23}$. Aussi les exemples de l'araire ou de la jachère sont-ils peu pertinents pour illustrer la supposée routine queyrassine ou le prétendu retard de l'économie de la vallée.

\section{Pratiques artistiques}

En 1909, quand Raoul Blanchard réside à Saint-Véran pour y étudier les singularités de ce village de haute montagne, il y apprend que, depuis plusieurs années, des habitants jouent chaque année une pièce de théâtre. Il cite quelques titres : Les deux orphelines, La porteuse de pain, Marceau ou les enfants de la République, une passion, des comédies de Molière. Sans étudier ces réalités, il va immédiatement aux causes. La cause (car il y en a une seule), ce sont les conditions physiques ou naturelles: la neige et le froid qui empêchent de travailler pendant six ou sept mois dans les champs ou dans les prés. Alors, on se divertit: jeux de cartes, discussions, repos, artisanat, meubles et objets sculptés, théâtre. Des essayistes ont avancé l'hypothèse d'une survivance des mystères médiévau $\mathrm{x}^{24}$, dont des érudits haut-alpins ont retrouvé des manuscrits dans les archives de communautés de la vallée de la Durance. Pourtant, dans aucun document, il n'est fait état de ces mystères. Tous les auteurs, qu'ils soient ou non queyrassins, confirment que, pendant un siècle (et peut-être davantage), de 1850 à 1950 environ, dans quasiment tous les villages de la vallée et pas seulement à Saint-Véran, des habitants ont fait du théâtre et les pièces préparées ont été jouées en public: à Fontgillarde et Pierre Grosse (Molines en Queyras), à Aiguilles, à La Monta (Ristolas), au Roux d'Abriès (Abriès). Il est possible que dans les deux communes qui ne sont pas citées ci-dessus, Arvieux et Château-Queyras, des habitants se soient essayés à cette activité, mais nous ne disposons pas de témoignage qui l'attesterait. En 1902, il existait à Aiguilles une société théâtrale, qui émettait, pour deux francs, une carte d'adhérent donnant accès aux spectacles à prix réduit. Parmi les pièces jouées dans ce village, on compte L'anglais tel qu'on le parle de Tristan Bernard (1866-1947), vaudeville en un acte créé en 1899. Le répertoire comprend, outre des comédies de Molière et des mélodrames ayant connu de vifs succès à la fin du XIXe siècle, des Passions jouées au moins à Saint-Véran et à Abriès, et, dans les années 1930, une adaptation pour le théâtre d'un roman réaliste, Nêne d'Ernest Perochon (1885-1942), qui a obtenu le prix Goncourt en 1920. Certes, des spectacles de comique troupier ont été montrés à Fontgillarde ou au Roux d'Abriès, des sketches comiques du genre de ceux qui étaient joués dans les music-halls de Marseille. Mais la plupart de ces pièces, exigeantes et ambitieuses, atteste que ceux qui les ont choisies et qui les ont jouées avaient un goût sûr et étaient informés de ce qui avait ou avait eu du succès dans les grandes villes de France; c'est donc qu'ils ne vivaient pas dans un univers clos et refermé sur lui-même.

La question du théâtre populaire, un théâtre émancipateur par le peuple et pour le peuple, permettant au plus grand nombre d'accéder à la culture, a hanté les 
théoriciens, historiens et responsables de théâtre pendant presque tout le $\mathrm{xx}^{\mathrm{e}}$ siècle. Or, le thêâtre queyrassin s'inscrit dans cette perspective. Certes, les acteurs ou les responsables n'ont pas senti la nécessité de mettre en récit leur expérience pour y donner un sens ou pour l'inscrire dans une histoire, comme cela a été fait à Bussang, dans les Vosges, où le Théâtre du Peuple, fondé en 1895, soit un demi-siècle après les premières expériences dans le Queyras, est tenu pour l'exemple parfait d'un théâtre populaire, qui renouerait, dans des formes différentes, avec les mystères de la fin du Moyen Âge. De fait, ces expériences queyrassines échappent à l'histoire de la culture et des formes, les traces qui restent étant trop ténues pour qu'il soit possible de reconstituer avec rigueur ce qui a été : pas ou plus de documents, pas d'indication sur le lieu où étaient jouées ces pièces, sinon à La Monta (Ristolas), pendant deux ou trois ans, le préau de la nouvelle école, détruite, comme tout le village en juin 1940, lors de l'attaque italienne, pas de nom d'acteur, sauf pour L'anglais tel qu'on le parle joué à Aiguilles, pas de nom de metteur en scène ou de directeur de troupe, pas d'éditorial ou de profession de foi exposant les intentions des acteurs et les buts qu'ils ont poursuivis.

11 La pratique du plain-chant dans la liturgie catholique avec deux chœurs de chantres, hommes et femmes, est également attestée à la fois par divers témoignages et par les manuscrits de messes chantées conservées dans les archives de la paroisse du Queyras. L'empan historique est plus large encore que pour le théatre : de la fin du XVII siècle aux années 1950, et les témoignages sont plus précis, puisqu'ils attestent l'existence à Saint-Véran d'une école de chantres, dans laquelle, une fois ou plus par semaine, enfants et jeunes gens étaient initiés, soit par le curé, soit par le maître d'école, à la technique subtile du plain-chant, c'est-à-dire à une activité artistique qui s'enracine dans le lointain passé de l'Europe chrétienne.

\section{Vitraux, estampes et lithographies}

Le cas des vitraux est encore plus éloquent. L'art du vitrail, qui a été délaissé pendant trois siècles, $\mathrm{du} \mathrm{XVI}^{\mathrm{e}}$ au début $\mathrm{du} \mathrm{xIX}^{\mathrm{e}}$ siècle, a connu un vif renouveau lors de la redécouverte de l'art ogival, dit gothique au $\mathrm{XVI}^{\mathrm{e}}$ siècle, par les auteurs romantiques. Cet art qui a touché les villes et surtout les régions du centre, du nord et de l'est de la France, dans le Sud-Est, s'est manifesté dans quelques grandes villes, mais n'a pas touché les campagnes :

«Les régions méridionales paraissent, dans l'ensemble, moins bien dotées, en quantité et en qualité, que les autres. ${ }^{25}$ »

Or, dans le Queyras, on dénombre dans huit églises paroissiales 46 vitraux (grisailles, vitraux à personnages, vitraux historiés d'une scène biblique), dont 14 dans la seule église du village d'Abriès. Ces vitraux, payés par de générateurs donateurs, ont été posés entre 1842 et les années 1930, la plupart d'entre eux datant des années 1860-1890. Les maîtres verriers auxquels ils ont été commandés et qui les ont livrés étaient établis à Lyon (Louis Payet), dans l'Ain (Jean-Augustin Bessac, 1858-1917), en Isère (Louis Balmet, 1876-1957 et Jean-Augustin Bessac), en Haute-Garonne (Amédée Bergès, LouisVictor Gesta, 1828-1894), dans la Loire (Félix Gaudin, 1851-1930), à Montreuil (Julien Vosch, 1885-1964 ${ }^{26}$. Le Queyras n'est pas resté à l'écart d'un phénomène culturel et artistique qui a touché pendant un demi-siècle les grandes villes de France, alors qu'il n'existe, dans cette vallée de haute altitude, aucune tradition architecturale qui puisse être qualifiée de gothique. 

que les Queyrassins étaient sensibles aux formes artistiques de la France centralisée. Il n'est pas de chapelles ou d'églises dont les murs ne soient pas décorés d'un chemin de croix, soit sous la forme de tableaux ou de tableautins, soit sous la forme de panneaux de bois sculptés en relief, soit sous la forme d'estampes, soit sous la forme de lithographies, de panneaux métalliques imprimés ou de céramiques. Les estampes qui datent du début du XIX ${ }^{e}$ siècle viennent de la maison Basset, établie rue Saint-Jacques à Paris, qui a produit des milliers d'estampes à sujet religieux ou profane, lesquelles sont conservées avec soin au titre d'objets patrimoniaux. Les lithographies ont été acquises auprès de maisons établies dans le quartier Saint Sulpice et qui ont diffusé des images de grande qualité graphique : la dynastie des Bouasse-Lebel, Letaille père et fils, Louis Turgis jeune et Turgis veuve, etc. ${ }^{27}$

\section{Meubles et objets sculptés}

est un dernier fait d'ordre culturel qui dément la thèse de la «cellule fortement clôturée » : ce sont les meubles (armoires, tables, buffets, lits clos, fauteuils, chaises, etc.) et autres objets (coffres, coffrets, coffins, boîtes, lampes, lanternes, coiffes, fuseaux, quenouilles, rouets, etc.) sculptés ou gravés ou peints (les cadrans solaires), en bois, en pierre ollaire, en cuir, en dentelle ou en métal, avec lesquels les Queyrassins pendant plusieurs siècles ont orné ou décoré leur maison ou procédé à leurs activités quotidiennes. Ces objets et meubles sont recensés pour une partie d'entre eux dans l'Inventaire général ou conservés dans des musées : le musée départemental de Gap, le musée dauphinois de Grenoble, le musée ethnographique de Genève. Ils sont considérés comme des objets patrimoniaux ou des œuvres d'art, relevant d'un art dit tantôt rustique, tantôt populaire, tantôt régional, tantôt alpin. Cet art, florissant du $\mathrm{XVI}^{\mathrm{e}}$ siècle à la seconde moitié du XIX ${ }^{\mathrm{e}}$ siècle, a été découvert par des collectionneurs cultivés au début du XXe siècle et les premières études ont été publiées dans les années 1920.

Ce qui caractérise ce patrimoine, c'est le changement de point de vue qui a été porté sur lui. Dans le musée cantonal d'Aiguilles, dit musée des Vallées du Queyras, où une partie de ces objets a été exposée de 1920 à 1980 (aujourd'hui, les collections ont été transférées au musée de Gap), les meubles, coffres, coffrets, ustensiles divers n'étaient pas au sens propre du terme exposés, mais déposés dans une salle voûtée, sombre, mal éclairée ; aujourd'hui, au musée départemental de Gap, les deux salles, parfaitement éclairées, qui y sont consacrées sont organisées suivant un principe scénographique qui met en valeur ces objets comme des objets qui se suffisent à eux-mêmes ou qui sont beaux en eux-mêmes. Ce changement de point de vue apparaît aussi dans les photographies. Le premier catalogue est celui d'un Petit Guide du Visiteur du musée dauphinois, publié en $1933^{28}$. Le papier n'est pas de grande qualité. Une page est consacrée au Queyras, illustrée de photos imprimées en noir et blanc sur du papier grisâtre qui n'ont d'intérêt que documentaire; il en va de même des photos qui illustrent dans la Gazette des Alpes ${ }^{29}$ (revue régionaliste : du Léman à la Côte d'Azur : les arts, les sports, le tourisme) et dans L'Art populaire en France ${ }^{30}$ une recension par Hippolyte Muller de ces objets. Aujourd'hui, il en va tout autrement: dans les quatre catalogues qui ont été publiés depuis une vingtaine d'années, à l'intérêt documentaire s'est ajouté l'intérêt artistique ; les photos imprimées sur du papier glacé sont l'œuvre d'excellents photographes d'art; les objets sont parfaitement éclairés, comme s'ils 
étaient devenus dans notre conscience des œuvres d'art en elles-mêmes leur propre $\mathrm{fin}^{31}$.

17 Les premières analyses, celles de l'archiviste Pinet de Manteyer (1867-1948) et de l'ethnologue Hippolyte Müller (1865-1933), sont fondées sur la thèse de la vallée refermée sur elle-même, à l'écart du monde et qui, de ce fait, est devenue un conservatoire de traditions disparues partout ailleurs en France, etc. Ce ne peut être qu'un art endogène. Pour en rendre compte est avancée l'hypothèse illyrienne ${ }^{32}$ ou celle du vestige d'un culte solaire disparu. Un passé très ancien aurait survécu dans le Queyras grâce à l'isolement. Or, les années 1920-1930 pendant lesquelles ces hypothèses sont avancées sont celles, en histoire de l'art, des arts décoratifs. Évidemment, cet art queyrassin n'a aucun lien avec les arts décoratifs des années 1920-1930, ni par les formes, ni par la périodisation, puisqu'il fleurit entre le $\mathrm{XVI}^{\mathrm{e}}$ et le $\mathrm{XIX}^{\mathrm{e}}$ siècle, mais le goût de l'ornement dans les meubles et les objets sculptés est aussi une réalité de l'histoire de l'art en France, certains artisans du Queyras se révélant dignes des ornemanistes du XVIII ${ }^{e}$ siècle. L'existence de ces arts d'ornementation peut être corrélée à la pose de vitraux dans les églises ou à l'acquisition d'estampes et de lithographies, ainsi qu'à l'importante littérature d'érudition, à la pratique du théâtre et à celle du plain-chant. Ces réalités convergentes façonnent une tout autre représentation que celle que produisent les seules conditions matérielles ou physiques ou naturelles.

Dans les Archives du Service historique de la Défense à Vincennes, sont conservés les mémoires d'officiers et ingénieurs aux ordres de Vauban qui avaient pour mission de transformer un château delphinal du xIII ${ }^{\mathrm{e}}$ siècle en forteresse militaire incluse dans le réseau des fortifications de la frontière. Dans un mémoire de cinq pages daté de 1694 et signé Ricord, sont énumérées les mesures à prendre pour assurer la défense de la vallée et surtout est analysé le mode de vie des Queyrassins. Ce qui est noté, c'est la prospérité : la seule communauté d'Abriès :

« Entretenait, auparavant la guerre [de la Ligue d'Augsbourg, 1691-1697], tous les ans, plus de quarante mille brebis, ce qui paraîtrait incroyable, si la chose n'était pas toute récente et de la connaissance de tout le Briançonnais. »

Et :

«Une brebis qui leur coûtait pendant la paix trois livres leur en rendait trois et quatre de revenus par an. ${ }^{33}$ "

Autrement dit, la rentabilité du capital investi dans l'élevage était très élevée. Cette prospérité était favorisée par des institutions d'un type inédit et sans exemple en France :

«Ces peuples se gouvernent en républicains, ne reconnaissant aucun chef parmi eux, n'ayant ni ne souffrant aucun noble [...] Dans les règlements de leurs affaires, ils convoquent des assemblées générales où tous ont droit d'assister et d'y dire leurs sentiments $[. . .]^{34}$ »

21 L'officier Ricord rappelle que les Queyrassins ont acquis, à la suite de transactions passées en 1343, «tous les droits seigneuriaux» (et féodaux) du Dauphin, et il en conclut ceci :

"Ce qui les a depuis toujours entretenus dans un degré de vanité insupportable..$^{35}$ "

Dans la quatrième édition de leur dictionnaire, les académiciens définissent la vanité comme " amour-propre qui vient de choses frivoles ou étrangères à la personne qui s'en prévaut ${ }^{36}$. Les notables queyrassins voyageaient et étaient en contact avec les 
autorités de la province et du royaume : par vanité, ils ont tenu à vivre eux aussi dans un décor soigné qui fît de l'effet ou qui donnât d'eux la plus belle image possible.

Toutes ces réalités spirituelles ou immatérielles forment le patrimoine du Queyras; elles existent partiellement à l'état de vestiges et elles étaient encore vivaces au début $\mathrm{du} \mathrm{Xx}^{\mathrm{e}}$ siècle quand Raoul Blanchard a fait du Queyras un objet d'étude. Certes, il est malaisé et sans doute vain d'en rechercher la cause, l'essentiel étant ce qu'elles dévoilent des réalités de cette vallée de haute montagne. En 1910, dans son étude consacrée à la commune de Saint-Véran, Raoul Blanchard souhaite que cette commune, entièrement vouée à l'agriculture de montagne, la seule activité adaptée aux conditions physiques, persiste dans son être et reste ce qu'elle est ${ }^{37}$. Si cela s'était réalisé et que la commune n'eût pas délaissé l'agriculture pour le tourisme, un siècle plus tard, SaintVéran serait désert. Avec deux autres confrères, Paul Veyret (1912-1988) et Pierre Chauvet, Raoul Blanchard a publié en octobre 1947 un rapport sur le Queyras ${ }^{38}$ et destiné à informer la sous-commission "Vie rurale en montagne» du Plan de modernisation de l'agriculture. Les propositions suggérées ont été en partie mises en œuvre avec d'importants moyens humains et financiers de 1952 à 1960, lorsque le Queyras est devenu une zone témoin ${ }^{39}$. Or, à peine cette expérience terminée, l'agriculture de montagne a quasiment disparu. Dans ce rapport de 1947, Raoul Blanchard et ses collègues ne tiennent pas compte de cette réalité qu'est le marché. Ils raisonnent comme si l'économie de la France était de type public. L'oubli est d'importance, car le Queyras a bénéficié d'une niche (un étroit segment de marché, avec une offre restreinte et une demande assez large, sans concurrence véritable), son économie étant fondée, non pas sur l'agriculture (de sorte que les questions de l'araire, des jachères, de l'absence d'engrais, etc. ont peu de pertinence), mais sur l'élevage et la vente, dans les villes du Piémont proche (à 5 ou 6 heures de marche), puis en Provence, des produits de l'élevage (agneaux, laine, fromages, beurre, lait), ainsi que du commerce de tout autre produit (colportage, parapluies, articles de ménage, textiles). Or, au XIXe siècle, avec une offre de produits laitiers, fromages, agneaux, qui a crû dans d'importantes proportions et avec une concurrence attisée par les chemins de fer et le développement de la circulation routière, cette niche a peu à peu disparu.

Comment des savants admirables ont-ils pu s'abuser sur cette vallée de haute montagne? Il faut, pour le comprendre, examiner leur épistémè, c'est-à-dire ce qu'ils présupposent avant toute analyse et les concepts qui organisent leur pensée, à savoir le positivisme et le déterminisme : les faits, mais uniquement les faits matériels, et ce que Blanchard appelle les conditions naturelles, physiques ou géographiques. Les réalités matérielles (le relief, l'altitude, les eaux, la pluviométrie, l'exposition au soleil, la déclivité des pentes, les températures, etc.) ne conditionnent, ni ne déterminent, l'habitat, les modes de production, le style de vie, les mœurs. L'exemple du Queyras le prouve. De plus, ce que Raoul Blanchard présuppose, c'est la convergence entre l'histoire de cette haute vallée alpine et l'histoire de France. La France a connu de 1820 à 1910, puis jusqu'à aujourd'hui, une prospérité sans précédent, conséquence d'une croissance économique régulière et parfois très forte. Le Queyras n'a rien connu de tel pendant la même période. La prospérité queyrassine est antérieure de plusieurs siècles et elle cesse à la Révolution, quand le Queyras perd ses libertés publiques, et pendant le 
XIX ${ }^{e}$ siècle, quand le Queyras perd ses marchés proches. L'histoire du Queyras et l'histoire de la France ne sont pas synchrones. Or, cette dimension historique est totalement absente des analyses de Raoul Blanchard, qui tient les réalités physiques pour immuables, et présente la vallée comme rétive à tout changement, alors que le changement est en train de se produire sous ses yeux.

Les études menées dans le cadre de l'Inventaire corrigent la vision d'une vallée isolée, refermée sur elle-même, à l'écart du monde. En 1964, mettant en place la Commission de l'Inventaire, André Malraux, alors ministre de la Culture, avait prévu que cette "nouvelle dimension [...] du savoir» entraînerait "une mise en question sans précédent des valeurs sur lesquelles ces connaissances se fondent $»^{40}$. En 1970, dans l'éditorial du numéro 9 de La Revue de l'Art, André Chastel a tiré un premier enseignement de cet Inventaire :

«Le recensement [...] est surtout générateur d'une nouvelle dimension intellectuelle du savoir. ${ }^{41}$ "

Michel Melot a montré que cette intuition était réalisée ${ }^{42}$. Mais il n'y a pas que le champ de l'art qui en ait été affecté : la connaissance que l'on pouvait se faire de toute une « civilisation » en était renouvelée :

«Une entreprise d'inventaire, avec son ambition énorme, [...], c'est, en somme, un effort passionnant et désespéré pour doter de mémoire, c'est-à-dire pour rendre intelligible à elle-même dans son développement, une civilisation qui tend, par son accélération propre, à perdre la dimension historique. $»^{43}$

C'est ce que montre l'étude du patrimoine du Queyras.

\section{BIBLIOGRAPHIE}

ALBERA Dionigi, Au fil des générations : terre, pouvoir et parenté dans l'Europe alpine (XIVe-XXe siècles), Presses Universitaires de Grenoble, coll. « La Pierre \& L'Écrit », 2011.

ALBERT Aristide, « Le pays briançonnais. Les Queyrassins négociants », Bulletin de la Société d'Études des Hautes-Alpes, huitième année, Gap, 1889.

ARNAUD Claude, « Une mémoire de Saint-Véran : vie traditionnelle et patois queyrassins », Le monde alpin et rhodanien, revue d'anthropologie régionale, 11-3, 1983, p. 5-103.

BERGE Pierre (abbé), Monographie de Saint-Véran, Gap, 1928.

BERGE Pierre (abbé), Monographie d'Abriès, Val-des-Prés, Éditions Transhumances, 2015.

BLANCHARD Raoul, «Le village de Saint-Véran. Monographie d'une commune de haute montagne », La Montagne, Revue du Club Alpin Français, tome VI, Paris, 1910.

BLANCHARD Raoul, VEYRET Paul, CHAUVET Pierre, « Les possibilités de modernisation d'une haute vallée alpestre (le Queyras) », Revue de géographie alpine, tome XXXVI, nº 4, Grenoble, 1948.

BLANCHARD Raoul, Les Alpes Occidentales, tome V : Les Grandes Alpes françaises du Sud, volume 2, Paris et Grenoble, B. Arthaud, 1950. 
BURNS Robert, « Saint-Véran : France's Highest Village », The National Geographic Magazine, volume $\mathrm{CXV}, \mathrm{n}^{\circ} 4$, National Geographic Society, Washington, avril 1959.

Catalogue d'exposition (en français et en italien), De l'habitation au musée : mobilier du Queyras, L'Arcière, Cuneo, 1989.

CHABRAND Armand, Vieilles coutumes et traditions briançonnaises, Grenoble, Drevet, 1901.

CHASTEL André, « Éditorial », Revue de l’Art, Éditions du CNRS, n 9, Paris, 1970.

CHEVALLIER Stéphane, PUTELAT Gaëlle et Pierre, Meubles et objets anciens du Queyras et des vallées voisines, Éditions du Queyras, Saint-Véran, 2012.

DELAFONT Polydore, « Un village des Hautes-Alpes », Revue du Dauphiné, tome troisième, Valence, 1838.

GENETTE Gérard, Palimpsestes. La littérature au second degré, Paris, Le Seuil, 1982.

GLÜCK Denise, Le Queyras I, les collections ethnographiques, Musée départemental, Gap, 1991.

GONDRET Jacques, Mœurs et coutumes des habitants du Queyras au XIX ${ }^{e}$ siècle, $2^{\mathrm{e}}$ éd., (chapitre III des Mémoires historiques sur le Queyras, années 1850, manuscrit non publié), Centre Alpin et Rhodanien d'Ethnologie, Grenoble, 1981.

GRANET-ABISSET Anne-Marie, La Route réinventée : les migrations des Queyrassins aux XIXe et XXe siècles, Presses Universitaires de Grenoble, 1994.

GRANET-ABISSET Anne-Marie, «Les Alpes “cultivées”. Le goût du livre et la maîtrise des savoirs écrits dans les sociétés alpines traditionnelles. L'exemple du territoire des Escartons », Anticléricalisme, minorités religieuses et échanges culturels entre la France et l'Italie, FORLIN Olivier (dir.), L'Harmattan, Paris, 2006.

Guillaume Augustin (général), Le Queyras, $1^{\mathrm{er}}$ éd., Société d'Études des Hautes-Alpes, Gap, 1968. ISNEL Pierre, « Avant-propos », Le Queyras, de Jean TIVOLLIER et Pierre ISNEL, Gap, 1938, (2 vol.). LAPACHERIE Jean-Gérard, « Les Canaux d'irrigation du Queyras », Quey'racines, n 19, Arvieux, février 2016.

LAPACHERIE Jean-Gérard, «Vie et œuvre de l'abbé Chaffrey Martin, écrivain queyrassin » et «Journal de l'abbé Chaffrey Martin », Bulletin de la Société d'Études des Hautes-Alpes, Gap, année 2013.

LAPACHERIE Jean-Gérard, « Bourcier, prêtre queyrassin réfractaire », Annales du Midi (revue de la France méridionale), tome 127, $\mathrm{n}^{\circ}$ 290, Toulouse, Privat, avril-juin 2015.

LAPACHERIE Jean-Gérard, « Les chemins de la Croix dans le Queyras », Bulletin de la Société d'Études des Hautes-Alpes, Gap, année 2015.

LAPACHERIE Jean-Gérard, « Les vitraux des églises du Queyras », Bulletin de la Société d'Études des Hautes-Alpes, Gap, année 2016.

MALLÉ Marie-Pascale, Queyras, Hautes-Alpes, coll. Images du Patrimoine, Association pour le Patrimoine de Provence, 1994.

MALLÉ Marie-Pascale, L'habitat du nord des Hautes-Alpes, patrimoine architectural et mobilier, coll. Cahiers du Patrimoine, Association pour le Patrimoine de Provence et Société d'Études des Hautes-Alpes, Aix-en-Provence et Gap, 1999.

MALRAUX André, Écrits sur l'Art, Gallimard, Paris, coll. Bibliothèque de la Pléiade, tome II, 2004. 
MARITAN Joseph, Mes Souvenirs, Bibliothèque de l'Association de Généalogie des Hautes-Alpes, Gap. MELOT Michel, Mirabilia. Essai sur l'Inventaire général du patrimoine culturel, Gallimard, Paris, coll. Bibliothèque des idées, 2012.

MULLER Hippolyte, «Quelques vieilles sculptures sur bois dans le Queyras », La Gazette des Alpes (Les Arts, Les Sports, Le Tourisme, Revue régionaliste paraissant le samedi), Grenoble, cinquième année, $\mathrm{n}^{\circ} 180,1925$.

MULLER Hippolyte, « Études d'art populaire dans le Queyras (Hautes-Alpes) », L'Art populaire en France, troisième année, Strasbourg et Paris, Istra, 1931.

RICORD, « Description et situation de la vallée du Queyras, des avantages que l'on peut retirer et de l'intérêt que l'on a d'y occuper deux postes de la dernière importance ", Archives du Service historique de la Défense, Fort de Vincennes, 1 VH 1512, carton 1, nº 7, 1694.

RIOU Yves-Jean, «Iconographie et attitudes religieuses. Pour une iconologie du vitrail du XIX siècle », Revue de l'Art, Paris, Éditions du CNRS, 1986.

ROSENBERG Harriet, A Negociated World, Three Centuries of Change in a French Alpine Community, University of Toronto Press, 1988. Version française : Un monde négocié, Grenoble, Le Musée Dauphinois, coll. Le Monde alpin et rhodanien, 2014.

SOULIÉ DE BRU, « Les maisons-types dans la région des Hautes-Alpes ", Enquête sur les conditions de l'habitation en France. Les maisons-types (éd. Alfred de Foville), Comité des Travaux historiques et scientifiques, Paris, Leroux, 1894.

TIVOLLIER Jean, Monographie de la vallée du Queyras, Gap, 1897.

TIVOLLIER Jean, Molines-en-Queyras, $1^{\mathrm{er}}$ éd., Lyon, 1913 ; $3^{\mathrm{e}}$ éd., Éditions Transhumances, Val-desPrés, 2016.

TIVOLLIER Jean et ISNEL Pierre, Le Queyras, Gap, 1938, (2 vol.).

\section{NOTES}

1. R. Blanchard, Les Alpes Occidentales (V, 2), p. 894.

2. Ibid., p. 935.

3. R. Burns, « Saint-Véran : France’s Highest Village », p. 570-588.

4. A. Guillaume, Le Queyras.

5. J-G. Lapacherie, « Les canaux d'irrigation du Queyras », p. 15-17.

6. H. Rosenberg, A Negociated World.

7. D. Albera, Au fil des générations, p. 405-409 et p. 447-458.

8. A-M. Granet-Abisset, La Route réinventée, p. 281, et «Les Alpes “cultivées”. Le goût du livre et la maîtrise des savoirs écrits dans les sociétés alpines traditionnelles. L'exemple du territoire des Escartons », p. 305-324.

9. M-P. Mallé, Queyras, p. 3-78; et L'habitat du nord des Hautes-Alpes.

10. Soulié de Bru, « Les maisons-type dans la région des Hautes-Alpes », p. 169-184.

11. P. Berge, Monographie de Saint-Véran et Monographie d'Abriès.

12. J-G. Lapacherie, « Vie et œuvre de l'abbé Chaffrey-Martin », p. 171-206. 
13. J-G. Lapacherie, « Bourcier, prêtre réfractaire queyrassin », p. 235-251.

14. Claude Arnaud (1892-1973), natif de Saint Véran, auteur d'une histoire de sa commune, publiée en 1983 par les soins de Jean-Claude Duclos, conservateur du musée dauphinois de Grenoble dans le Monde Alpin et Rhodanien.

15. J. Tivollier, Monographie de la vallée du Queyras ; Molines-en-Queyras; Le Queyras.

16. Le docteur François Rozan (1823-1891), médecin militaire, auteur d'une Physiologie du mariage (1866), dirige un service médical pendant les campagnes d'Italie du Second Empire.

17. J. Gondret, Mœurs et coutumes des habitants du Queyras au XIX siècle, p. 44.

18. P. Isnel, « Avant-propos », p. XV-XIX.

19. G. Genette, Palimpsestes.

20. J. Maritan, Mes Souvenirs, p. 54.

21. P. Delafont, « Un village des Hautes-Alpes », p. 302-314.

22. H. Rosenberg, A Negociated World, p. IX-XV.

23. A. Albert, « Le Pays briançonnais. Les Queyrassins négociants », p. 313-331.

24. A. Chabrand, Vieilles coutumes et traditions briançonnaises, p. 16-18.

25. Y-J. Riou, «Iconographie et attitudes religieuses. Pour une iconologie du vitrail du XIX ${ }^{\mathrm{e}}$ siècle », p. 41.

26. J-G. Lapacherie, « Les vitraux des églises du Queyras », p. 77-100.

27. J-G. Lapacherie, « Les chemins de la Croix dans le Queyras », p. 87-104.

28. H. Muller, Petit Guide du Visiteur, p. 12-15.

29. H. Muller, « Quelques vieilles sculptures sur bois dans le Queyras », p. 1-3.

30. H. Muller, «Études d'art populaire dans le Queyras (Hautes-Alpes) », p. 7-20.

31. D. Glück, Le Queyras I, Les collections ethnographiques, p. 21-80 ; M-P. Mallé, Queyras, p. 3-78; Catalogue d'exposition, De l'habitation au musée: mobilier du Queyras, p. 55-137;

S. Chevallier, G. et P. Putelat, Meubles et objets anciens du Queyras et des vallées voisines.

32. Les lointains ancêtres des Queyrassins viendraient d'Illyrie où existe une tradition du meuble sculpté.

33. Ricord, « Description et situation ... », p. 1-5.

34. Ibid.

35. Ibid.

36. Ibid.

37. R. Blanchard, «Le village de Saint-Véran (monographie d'une commune de haute montagne) », p. 680-691.

38. R. Blanchard, P. Veyret et P. Chauvet, «Les possibilités de modernisation d'une haute vallée alpestre (le Queyras) », p. 577-591.

39. L. Roche, « Les zones-témoins », Économie rurale, $\mathrm{n}^{\circ} 15,1953$, p. 11-19.

40. A. Malraux, Écrits sur l'Art, p. 1193-1194.

41. A. Chastel, «Éditorial », p. 4-5.

42. M. Melot, Mirabilia. Essai sur l'Inventaire général du patrimoine culturel, p. 21-28.

43. A. Chastel, «Éditorial », p. 4-5. 


\section{RÉSUMÉS}

Au sujet du Queyras (Hautes-Alpes), la thèse d'une vallée de haute montagne fermée, sans lien véritable avec l'extérieur et rétive au progrès a été exprimée par Raoul Blanchard et reprise par des historiens des Alpes et du Queyras (Robert Burns, général Guillaume, etc.). Or, elle est démentie par des études récentes ou des faits, naguère ignorés, matériels (irrigation de $50 \%$ des terres et «niche » de marché) ou immatériels, qui relèvent de la culture : tableaux, vitraux, statues, goût pour l'érudition, pratique du théâtre et du plain-chant, haut niveau d'instruction. Pour comprendre ce qui a donné du crédit aux thèses sur la fermeture, l'archaïsme, l'extériorité à l'histoire, il faut analyser l'épistémè de ceux qui ont formulé ces thèses, c'est-à-dire ce qu'ils ont présupposé avant toute analyse : importance accordée aux conditions (naturelles, physiques ou géographiques), positivisme, déterminisme, primauté donnée à l'agriculture sur le négoce.

\section{AUTEUR}

\section{JEAN-GÉRARD LAPACHERIE}

Professeur émérite, Langue et littérature française 
Les Apennins comme espace d'expérimentation d'une nouvelle approche méthodologique de l'histoire de l'art : la naissance de la notion de bene culturale (1968-1971)

\author{
Sandra Costa et Anna-Lisa Carpi
}

Entre 1968 et 1971, la Soprintendenza alle Gallerie di Bologna, alors dirigée par l'historien d'art Cesare Gnudi (1910-1981), organisa chaque année une campagna di rilevamento ${ }^{1}$ dans l'Apennin émilien, soit quatre campagnes d'inventaire du patrimoine artistique et culturel de cette région. L'initiative, tout à fait inédite pour l'époque, était menée par Andrea Emiliani (1931-2019), historien d'art et muséologue aujourd'hui internationalement reconnu ${ }^{2}$ : c'est à partir de l'expérience de ces campagnes qu'il mit à l'épreuve ses premières hypothèses méthodologiques ${ }^{3}$ grâce auxquelles, tout en partant d'une connaissance rigoureuse de la tradition historienne et critique italienne, il allait renouveler fondamentalement le champ des possibilités offertes à la discipline. Cette initiative menée dans les montagnes des Apennins se caractérisa par une interdisciplinarité d'une rare ampleur, et par l'implication de l'université, des communes, des pouvoirs politiques, des associations, et même des simples habitants de ces lieux souvent isolés, qui se trouvaient en grande difficulté économique, sociale et démographique dans les années soixante.

Novatrice sur le plan méthodologique, constituant un modèle exemplaire de révision des objectifs et des instruments de la connaissance de l'art, l'expérience réalisée dans les montagnes des Apennins émiliens a renouvelé en profondeur la façon de concevoir l'histoire de l'art et le rapport entre la culture matérielle et les lieux, avec des conséquences non seulement méthodologiques, mais également juridiques et institutionnelles, qui allaient avoir une grande importance en Italie durant les décennies suivantes. 
Cette contribution entend présenter - près de cinquante ans plus tard, grâce au témoignage direct de son acteur principal, Andrea Emiliani - les racines historiques, les expérimentations et le contexte de mise en œuvre de ces campagne di rilevamento. Il s'agit, en outre, de rechercher aujourd'hui l'héritage d'une proposition critique et méthodologique qui, à partir de l'analyse spécifique d'un territoire de montagne, est largement à l'origine d'une conception différente de l'histoire de l'art, et surtout d'une autre façon de penser les liens entre l'art et son territoire ${ }^{4}$.

\section{Aux origines du rapport entre l'art et les lieux}

En Italie, la réflexion sur les liens entre l'artiste et ses conditions de travail et de vie bénéficie de racines historiques profondes ; il suffit de songer à tout ce que l'abbé Luigi Lanzi (1732-1810) avait écrit dès la fin du $\mathrm{XVIII}^{\mathrm{e}}$ siècle dans sa Storia pittorica d'Italia sur la recherche d'une relation entre les lieux, les pratiques et les formes de l'art, selon une démarche déjà anthropologique.

La conscience de la nécessité de tisser des liens entre recherche scientifique et actions de protection, a été développée en Italie par Giovanni Battista Cavalcaselle (1819-1897), l'un des acteurs pionniers de la réflexion post-unitaire sur le patrimoine national et sur la nécessité de sa protection. Ce rôle fondateur lui a été reconnu par un grand nombre de critiques d'art italiens de la première moitié du $\mathrm{xx}^{\mathrm{e}}$ siècle. Andrea Emiliani lui attribue également le mérite d'avoir, le premier, imaginé une géographie de la culture. C'est avec Cavalcaselle que les questions de l'inventaire, de la conservation et de l'adoption d'une méthode scientifique se sont répandues dans le pays.

6 Au niveau international, au début du $\mathrm{xx}^{\mathrm{e}}$ siècle, un rôle significatif fut joué par l'École de Vienne, à laquelle Cavalcaselle faisait déjà référence quelques décennies plus tôt. $\mathrm{Au}$ sein de cette tradition, retenons l'essai d'Aloïs Riegl (1858-1905) sur la théorie des monuments, Der moderne Denkmalkultus ${ }^{5}$ : cette réflexion majeure s'avère être en mesure de réunir un renouvellement méthodologique des études et une action politique administrative, union réalisée par la synergie de forces diverses, académiques et politiques. Selon Sandro Scarrochia, c'est essentiellement grâce à Riegl que l'idée d'une valeur artistique distincte d'une valeur historique commença à être remise en cause.

7 Mais ce fut surtout dans les années 1960-70 que se développèrent diverses recherches tendant à inscrire l'art au sein d'une réflexion plus vaste sur la culture matérielle. En 1962, aux États-Unis, était publié The shape of time de George Kubler, tandis que quelques années plus tard, en 1971, la conférence de l'ICOM à Grenoble - là aussi un territoire de montagne - affirmait au niveau international et institutionnel la relation du patrimoine artistique et historique avec la communauté et l'environnement qui l'entourent ${ }^{6}$.

8 En Italie, la redéfinition plus large du patrimoine découla aussi d'une volonté de collaboration interdisciplinaire nouvelle pour une discipline académique encore jeune, l'histoire de l'art, désireuse de revendiquer son autonomie, ses prérogatives et ses spécificités. Pour comprendre comment se développèrent ces nouvelles hypothèses de travail, il convient de considérer les fondements intellectuels du travail d'Andrea Emiliani, qui correspondent à la synergie existante entre des réflexions liées à l'élargissement de la notion de patrimoine et des actions visant à unir réflexion méthodologique, connaissance disciplinaire et recherche sur le terrain. Pour Emiliani, il 
s'agissait du suivi d'un long fil théorique qui, partant du siècle des Lumières et des idées de Quatremère de Quincy (1755-1849), passait par Cavalcaselle pour parvenir jusqu'à son propre mentor, Cesare Gnudi.

9 Le concept proposé de museo diffuso rassemble le patrimoine historique, artistique et paysager, tout en associant recherche historique et recherche anthropologique. Dans ce cadre théorique, l'étude du paysage - à partir du paysage de montagne, objet des campagnes d'inventaire qui inaugurèrent ce type de recherche - participe d'un système visant à unir de façon organique nature et culture. Ainsi, ces années ont vu le développement d'une branche "anthropologique » de l'histoire de l'art, au sein de laquelle l'histoire, telle qu'elle est conçue dans les Annales de Marc Bloch et Lucien $\mathrm{Febvre}^{7}$, rejoint la géographie culturelle portée par Lucio Gambi ${ }^{8}$, s'appropriant des outils et des méthodes des sciences sociales ${ }^{9}$. Une approche de l'histoire qui aboutit à l'aventure éditoriale de la Storia d'Italia éditée chez Einaudi, à travers laquelle s'affirme l'avant-garde intellectuelle du pays dans les années 1960. Il s'agit d'une Italie encore imprégnée de l'idéalisme du philosophe Benedetto Croce (1866-1952) et des exploits de l'ekphrasis de Roberto Longhi ${ }^{10}$. Mais on peut parler aussi d'un pays dans lequel, depuis quelques années déjà, s'enracinaient les germes du pragmatisme anthropologique d'origine anglo-saxonne ${ }^{11}$.

10 Ce parcours méthodologique, ainsi conçu à partir d'un échantillon de régions de montagne, allait réunir la conscience civique de l'engagement pour le patrimoine historique aux actions résultant de programmes de planification administrative sur le territoire, et aux opérations de mobilisation sociale engageant la participation active de la collectivité. Par le biais de la réhabilitation du territoire, l'objectif serait celui d'une réappropriation culturelle de la dignité même de l'homme ${ }^{12}$.

11 Une différence significative cependant, entre tout ce qui avait été fait du XIXe siècle jusqu'au début $d u x^{e}$ siècle, et la réflexion issue des campagnes d'inventaire, concernait le rapport entre État et Régions, à savoir entre le centre et les périphéries. Elle était l'objet d'un débat historique de grande actualité, mais surtout au cœur de la tentative de dépasser l'opposition entre zones toujours plus urbanisées d'une part et campagnes ou montagnes d'autre part, laissées à l'abandon du fait d'une industrialisation tardive et quelquefois inaboutie.

Instrument de participation active et collective des citoyens, les campagnes d'inventaire eurent le mérite de déplacer la conception contemporaine de l'histoire de l'art vers des réflexions d'ordre «environnemental» et social, sans se contenter des seules considérations théoriques.

\section{La région des Apennins comme terrain d'expérimentation méthodologique pour le patrimoine culturel}

13 Dans les années 1960, la région des Apennins était considérée comme un espace qui se dégradait sur les plans économique, environnemental et social, affecté par un exode des populations qui laissaient derrière elles des villages entiers à l'abandon. C'est dans la région de Porretta $\mathrm{Terme}^{13}$, lieu de la première campagne d'inventaire, que furent mis au point les méthodes et les objectifs ensuite adoptés pour l'ensemble du projet. "Guidé », symboliquement au moins, par un historien de l'art, un groupe de 
spécialistes en géographie, linguistique, archéologie, architecture, anthropologie, mit en place une méthodologie suivant un plan d'action articulé en deux temps: tout d'abord l'analyse géographique et humaine du territoire, puis celle de sa culture matérielle, dont les résultats étaient communiqués à l'occasion de débats impliquant la participation de la population locale ainsi que des acteurs, politiques et administratifs ${ }^{14}$. Le fil conducteur de la méthode adoptée était l'étude directe, sur le terrain, des œuvres dans leur contexte d'origine, de la matérialité des objets du quotidien et des phénomènes, qui se substituaient à la centralité jusqu'alors accordée aux chefsd'œuvre.

15 À cette occasion, fut défini le concept de comprensorio, renvoyant à un espace homogène du point de vue territorial, économique et culturel, caractérisé par une cohérence paysagère et par des liens "anthropo-géographiques». Les espaces ainsi définis ne coïncidaient certes pas avec les frontières administratives et les délimitations institutionnelles, mais s'avéraient finalement bien plus adaptés dans la perspective d'une planification d'actions de protection du patrimoine ${ }^{15}$.

L'ensemble de la culture matérielle, dans ses témoignages artistiques, mais aussi archéologiques et historiques, se trouvait ainsi corrélé à la morphologie du territoire.

Le recensement du patrimoine et son inventaire ne portaient plus seulement sur des critères qualitatifs, mais tendaient à une prise en compte globale du site et des relations entre les éléments. La fiche d'inventaire, la photographie et la cartographie s'imposèrent comme les instruments fondamentaux d'analyse du patrimoine de ces régions de montagne, et leur complémentarité renforça la qualité du travail réalisé : la photographie s'avéra être une précieuse alliée pour la fiche d'inventaire traditionnelle, offrant un moyen efficace pour unir par l'image, l'objet et son contexte. Les clichés réalisés par le célèbre photographe Paolo Monti (1908-1992)16 étaient exemplaires et ils furent pris pour modèles dans les projets similaires qui suivirent. Monti partageait avec Emiliani une vision anthropologique du paysage :

«À partir d'une maison de montagne, avec seulement quelques pierres, il est intéressant d'observer comment l'homme a traité les matériaux, comment il a construit le toit, comment il a agencé les poutres les unes sur les autres afin qu'elles ne bougent pas. ${ }^{17}$ »

18 Si la photographie permettait de recueillir les données de façon «scientifique » et en même temps "dynamique ", la méthode de l'inventaire s'appuyait toujours sur les fondements de la grande tradition philologique italienne en histoire de l'art, autrement dit sur l'élucidation formelle, historique, attributive et bibliographique de chaque objet singulier. De cette façon, la tradition devenait la base du renouvellement méthodologique.

19 C'est ainsi qu'il fut possible de redécouvrir et de valoriser un patrimoine historique et artistique oublié, et pourtant d'une importance fondamentale pour le sentiment d'appartenance et l'identité des populations locales. L'exposition qui fit suite à la publication de l'opuscule Per un rilevamento dei beni artistici e culturali di Porretta Terme, présentant les résultats de la campagne de 1968, fut le premier aboutissement de la volonté de diffusion et de partage du travail mené ${ }^{18}$.

20 La deuxième campagne, qui adopta l'année suivante les mêmes méthodes et instruments que la première, se concentra sur la zone comprise entre la rivière Setta et le Reno ; la troisième, en 1970, eut comme objet un ensemble situé à l'ouest du Reno ; la quatrième, en 1971, fut consacrée aux Apennins de la région d'Imola. Ce fut 
précisément grâce à ces campagnes, menées durant quatre ans, que l'idée culturelle et civique de museo diffuso se développa en Italie, pour désigner une méthode non seulement d'étude mais aussi de gestion globale, culturelle et civique du patrimoine historique et artistique. Née d'un travail de terrain dans les montagnes des Apennins, cette conception allait se diffuser ensuite et prendre un sens plus large, s'appliquant à la valorisation du territoire national.

Peu après la fin des quatre campagnes furent publiés plusieurs textes fondateurs de cette nouvelle façon de concevoir le rapport entre objets et mémoire, entre mémoire et paysage. Citons en particulier, en 1974, Dal museo al territorio, ouvrage d'Andrea Emiliani dont le titre même rendait explicite le "glissement» fondamental qui s'opérait du patrimoine culturel conservé au sein du musée jusqu'à un panorama plus vaste et complexe associant culture et nature. De simple lieu de conservation du patrimoine artistique, le musée se transformait ainsi en levier pour des actions culturelles et surtout pour prendre en charge la connaissance historique du passé. Passant de l'intérêt pour le chef-d'œuvre à l'intérêt pour le territoire, l'étude de l'art commençait donc à offrir une importance plus grande aux aspects dynamiques et évolutifs qui caractérisent le rapport entre la société et la culture. Émergeait l'idée d'un réseau d'œuvres d'art directement lié au paysage et aux lieux. Ce réseau ne prenait véritablement sens qu'au sein d'une valorisation et d'une mise à disposition du public s'inscrivant dans le contexte d'origine, où le cadre géographique, la tradition historique et la création humaine étaient inextricablement liés ${ }^{19}$.

Le territoire montagneux des Apennins devenait un tissu historico-artistique multiforme, auquel devaient être étendues les fonctions de conservation et de valorisation jusqu'alors traditionnellement réservées aux objets conservés dans les musées. Le parti pris idéologique des campagnes d'inventaire prévoyait pour l'État un rôle de coordination, tandis que les administrations locales - avec leur compétence spécifique adaptée à chaque territoire - devaient se charger d'une protection du patrimoine ancrée dans le contexte d'origine ${ }^{20}$. La planification politique et territoriale en matière de conservation et valorisation du patrimoine artistique naquit ainsi de la synthèse entre recherche théorique et recherche sur le terrain. L'objectif était le suivant: en exportant hors du musée les exigences de conservation du patrimoine artistique, il s'agissait de proposer à la culture matérielle des cadres de référence, et aux diverses communautés de montagne des instruments concrets capables d'activer une participation collective à même de cimenter une identité locale. Les plans d'intervention se fondèrent donc sur la connaissance des réalités régionales locales : la finalité en était à la fois didactique, sociale et civique.

23 C'est ainsi que se construisit une connaissance pragmatique et interdisciplinaire, fondée sur des projets collectifs et partagés, et forte d'une étroite collaboration entre la Sovrintendenza et les institutions universitaires :

«Il apparaît aujourd'hui clairement que, de la sauvegarde de la nature et du paysage à l'intégrité des centres historiques, de la protection des objets d'art et des monuments à l'enregistrement des traditions locales, tout coïncide en un nouveau concept de conservation qui dépasse les seules et étroites frontières traditionnelles [...] En effet, ce que nous appelons œuvre d'art [...] devra continuellement se nourrir de toute une série de rapports, d'interrelations, d'échanges et de références, et seule une protection absolue et globale aujourd'hui peut en garantir l'avenir. ${ }^{21}$ ” apennin, permirent aussi une observation de l'état de dégradation dans lequel sombrait 
une grande part de ce patrimoine après des décennies de défaillances d'abord législatives et ensuite politiques ${ }^{22}$. En partant de la matrice identitaire afin de stimuler et promouvoir une conscience collective et sociale: l'idée de base restait celle d'attribuer une valeur didactique à l'information donnée sur le patrimoine historique et artistique, vue comme un moyen d'éducation et de progrès de la collectivité.

Par ailleurs, le sentiment profond qui unissait la nation aux citoyens s'enracinait dans la pensée des Lumières. Il suffit de songer à ce qu'avait déjà écrit Diderot :

«Or, vous savez mieux que moi quelle est l'influence du goût national sur les progrès de l'art. L'art reste misérable chez un peuple imbécile. Il marche avec rapidité chez un peuple instruit. ${ }^{23}$ "

Et peu après, aux considérations de l'abbé Luigi Lanzi, qui en outre rapprochait l'intérêt pour l'art du progrès social :

«Avec des subventions ainsi augmentées, la culture s'est étendue à toutes les classes sociales, elle qui était autrefois réservée à quelques-uns seulement; l'art prend de fait un tournant nouveau, également animé par l'honneur et par l'intérêt. ${ }^{24}$ »

Habituellement, les politiques culturelles ont pour caractéristique de naître au cœur des centres politiques, économiques ou administratifs, avant de se déployer, avec un certain décalage temporel et selon des modalités parfois diverses, aux périphéries. Dans ce cas, certes singulier, il se passa exactement le contraire : le modèle utilisé pour explorer et répertorier le patrimoine du territoire montagneux des Apennins fut en effet réinvesti par la suite, selon une méthodologie similaire, pour l'étude et l'action patrimoniale qui concerna le centre historique de Bologne, également dans un état de dégradation avancé ${ }^{25}$. Les acteurs en furent pour beaucoup les mêmes: il suffit de rappeler le rôle de l'architecte Pier Luigi Cervellati, compagnon d'Andrea Emiliani dans ses pérégrinations à travers les Apennins, mais également adjoint à l'urbanisme de la municipalité de Bologne ${ }^{26}$.

L'impératif politique né des campagnes d'inventaire se trouve résumé par les préconisations d'Emiliani :

«Connaître pour planifier; planifier pour conserver; conserver pour instruire et survivre. ${ }^{27} »$

29 La culture et l'art sont donc désormais considérés comme des sujets à aborder également dans le débat politique et civique, s'inscrivant dans une politique territoriale plus générale ${ }^{28}$; le recensement du patrimoine et son inventaire deviennent l'étape première de toute planification d'actions de restauration et de protection.

30 La conoscenza circolare est le modèle auquel fait référence aujourd'hui encore Emiliani, lorsqu'il relate ces campagnes d'inventaire : un modèle capable de mettre en œuvre un savoir appliqué et une recherche sur le terrain, en lien avec des territoires dotés d'un patrimoine historique et artistique, et avec les collectivités à qui en incombent la gestion et la protection ${ }^{29}$. Les notions de museo diffuso et de conoscenza circolare sont ainsi à l'origine d'une proposition organisationnelle concrète, en mesure d'assurer la conservation institutionnelle et de promouvoir la valorisation, également sociale, $\mathrm{du}$ patrimoine historique et artistique. 


\section{L'institutionnalisation d'une expérience et l'actualité d'un héritage culturel}

31 Le travail de recherche mené dans les montagnes des Apennins entre 1968 et 1971 devint en Italie un modèle de référence, repris en Ombrie par Bruno Toscano ${ }^{30}$ et dans le Piémont par Giovanni Romano, Sovrintendente de Turin à partir des années $1970^{31}$. Cependant seule l'Émilie-Romagne, à partir des résultats des campagnes, chercha à se doter de moyens institutionnels pour mettre en place une politique de développement culturel et de conservation des œuvres d'art attentive aux spécificités du territoire, et dans laquelle se retrouvèrent, organisés et systématisés, les méthodes et les instruments expérimentés lors des campagnes dans les Apennins.

32 C'est ainsi qu'est née cette « politique du patrimoine » qui a donné naissance en 1974 à l'Istituto per $i$ beni artistici culturali e naturali (IBC) : s'ancrant dans une institution, il s'agit certainement du résultat le plus pérenne produit par ce regard nouveau porté sur le territoire et le patrimoine historique et artistique, et par une conception élargie de la protection, face à une sélection reposant exclusivement sur des critères qualitatifs et typologiques.

33 L'IBC devint l'instrument de la planification culturelle, animée par la conviction que la conservation du patrimoine et la protection du paysage constituaient un service public et un acte politique capables de susciter la participation collective de la communauté et de la sensibiliser aux activités relevant de la compétence des instances locales ${ }^{32}$.

À la fin des années 1990, la montagne fit l'objet d'une nouvelle attention "muséologique », proche de l'esprit des campagnes d'inventaire et sous-tendue par une conscience civique aiguë, avec la recherche réalisée sur la dorsale apennine à partir du projet Linea gotica, lancé en 1997 par la région Émilie-Romagne. L'objectif était de réaliser l'inventaire du patrimoine historique de la Seconde Guerre mondiale, afin de créer un musée "intégré » croisant histoire, art et nature, et ce, grâce à la collaboration d'un grand nombre d'institutions diverses ${ }^{33}$. Comme dans les premières campagnes, l'inventaire du patrimoine historique fut effectué grâce à des acteurs d'horizons divers : historiens, architectes, photographes, archivistes ${ }^{34}$. Concernant plus directement le patrimoine naturel, citons la recherche dédiée dans les années 1990 par l'IBC à l'inventaire des arbres remarquables au sein des zones naturelles.

35 En 2004, a été publié le nouveau Code du patrimoine et du paysage (Codice dei beni culturali e del paesaggio), mis à jour en 2016, dans lequel une attention nouvelle est accordée au caractère dynamique du territoire et de la formation du paysage ${ }^{35}$, ainsi qu'au rôle actif qui doit être joué par les citoyens. Le Code propose également la

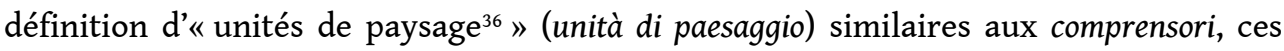
zones présentant une homogénéité historico-géographique, socio-culturelle, environnementale et économique, qui était née des recherches des campagnes d'inventaire. En effet, ces " unités de paysage » désignent des secteurs non seulement géo-morphologiques mais également historiques et artistiques, confirmant à nouveau l'actualité de la méthode transversale définie par les campagnes d'inventaire.

36 S'il y a bien eu une évolution méthodologique, celle-ci est perceptible dans l'intérêt croissant pour la notion de museo diffuso et son adoption avec l'invention récente du sistema museale. La volonté de créer des systèmes muséaux répond à l'exigence de «favoriser les liens entre les musées et l'intégration des collections existantes sur le 
territoire ${ }^{37}$ ", mais elle est aussi liée à l'orientation de plus en plus explicite vers une uniformisation des modèles d'organisation et de gestion, notamment financière. Toujours sur l'Apennin modénais, la collaboration entre le Museo storico de Montese et le Museo memorie d'Italia de Iola illustre cette nouvelle tendance: la coopération entre ces deux institutions a en effet favorisé la valorisation des deux collections, mais également celle de leur environnement.

L'appropriation de l'héritage méthodologique de l'expérience des campagnes d'inventaire conduites par Andrea Emiliani ainsi que la réflexion historique relative à son influence sur la recherche en histoire de l'art, ont été approfondies en 2014 avec la réédition d'Una politica dei beni culturali ${ }^{38}$, qui constituait à la fois la recherche fondatrice de l'IBC et la synthèse des résultats des campagnes. Véritable manifeste programmatique du projet de réforme initié par les campagnes d'inventaire, le texte présente à la fois les fondements théoriques et méthodologiques, résumés par la trilogie "connaître-conserver-valoriser ", et l'appareil organisationnel et législatif nécessaire à la traduction opérationnelle d'un projet qui considère la protection du patrimoine comme un service public ${ }^{39}$.

Mais l'attention au paysage, en tant que partie intégrante de la culture d'une communauté, a bénéficié au XxI siècle d'une reconnaissance d'envergure plus large encore, avec la Convention Européenne du Paysage, ouverte à la signature des États membres le 20 octobre 2000 et ratifiée par l'Italie en 2006 : son article premier précise que :

«Le paysage désigne une partie de territoire telle que perçue par les populations, dont le caractère résulte de l'action de facteurs naturels et/ou humains et de leurs interrelations. "

Musées et paysages culturels était également le thème de la conférence de l'ICOM qui s'est tenue à Milan en juillet 2016 : l'actualité de la recherche s'est exprimée à travers une exigence d'élaboration de bonnes pratiques de conservation et de valorisation du patrimoine historique et artistique ; dotées d'une forte dimension pédagogique, cellesci peuvent être portées à la fois par le territoire et la communauté de ses habitants, afin d'en valoriser la mémoire et d'enraciner la citoyenneté dans la conscience d'une appartenance historique ${ }^{40}$.

\section{BIBLIOGRAPHIE}

ALESSANDRONE PERONA Ersilia, « Dai luoghi della memoria alla memoria dei luoghi », dans IBC. Informazioni commenti inchieste sui beni culturali XII, éd. numérique, 2004, p. 62-67. [URL : http://rivista.ibc.regione.emilia-romagna.it/xw-200402/xw-200402-d0001/xw-200402-a0018] BLOCH Marc, Apologie pour l'histoire, ou Métier d'historien, Paris, Librairie Armand Colin, 1949. 
CARPI Anna-Lisa, « Un metodo antropologico. Andrea Emiliani : storia arte cultura », tesi di Laurea Magistrale in Arti Visive, Bologne, Alma Mater Studiorum-Università di Bologna, 2016.

CARPI Anna-Lisa, « Tra architettura, paesaggio e grandi capolavori : la "via” bolognese per un metodo interdisciplinare ", dans Histories of Postwar Architecture, vol. 1, n 0, 2017, p. 135-138.

CAVALCASELLE Giovanni Battista, Sulla conservazione di monumenti ed oggetti di Belle Arti e sulla riforma dell'insegnamento accademico, Turin, Tipografia Subalpina di Zoppis e Marino, 1863.

CECCHINI Folco, « Un sistema ecomuseale nel Parco del Delta del Po », dans NEGRI Massimo, PINI Laura (dir.), Verso l'ecomuseo del futuro, Bologne, Nuova Alfa Editoriale, 1994, p. 25-27.

CERvellati Pier Luigi, «Bologna Centro Storico », dans emiliani Andrea e scolaro Michela (dir.), L'Arte : un universo di relazioni : le mostre di Bologna, 1950-2001, Milan, Skira, 2002, p. 204-221.

CHASTEL André, « Roberto Longhi : il genio dell “ ekphrasis ” ", dans PREVITALI Giovanni (dir.), L'Arte di scrivere sull'arte. Roberto Longhi nella cultura del nostro tempo, Roma, Editori Riuniti, 1982, p. 56-65. costa Sandra (dir.), De l'art au Patrimoine, France et Italie : le transfert de modèles culturels et esthétiques à l'époque moderne, Grenoble, CRHIPA, 2003.

DIDEROT Denis, « Salon de 1769 », dans CEuvres complètes, Paris, Club Français du Livre, [1769] 1969-1973 vol. VIII.

EMILIANI Andrea, Per un rilevamento dei beni artistici e culturali di Porretta Terme : mostra documentaria 1969, Bologne, Tipografie Labanti, 1969.

EMILIANI Andrea, La Conservazione come pubblico servizio, Bologne, Edizioni Alfa, 1971.

EMILIANI Andrea, Dal museo al territorio, 1967-1974, Bologne, Edizioni Alfa, 1974.

EMILIANI Andrea, «I materiali e le Istituzioni », dans PREVITALI Giovanni (dir.), Materiali e problemi. Questioni e metodi. Storia dell'Arte Italiana, Turin, Einaudi, 1979, p. 97-162.

EMILIANI Andrea, «L'esperienza sul campo », dans AGOSTINI Grazia, MARI Cesare e ORLANDI Piero (dir.), L'esperienza sul campo. Per un'analisi del paesaggio appenninico : le Campagne di rilevamento dei beni culturali della provincia di Bologna (1968-1971), Bologne, Edizioni Alfa, 1981, p. XV-XXIV.

EMILIANI Andrea, L'elogio della mano, Faence, Carta Bianca Editore, 2015.

EMILIANI Vittorio, Lo sfascio del Belpaese. Beni culturali e paesaggio da Berlusconi a Renzi, Chieti, Edizioni Solfanelli, 2017.

FANTI Guido, «La legge per i centri storici », dans EMILIANI Andrea, Una politica dei beni culturali, Turin, Einaudi, 1974, p. 253-270.

FEBVRE Lucien, Pour une histoire à part entière, Paris, Éditions de l'École des hautes études en sciences sociales, 1962.

GAMBI Lucio, La cognizione del paesaggio, Bologne, Bononia University Press, 2008.

JALLA Daniele, « Un museo responsabile del territorio », dans DELL'ORso Silvia (dir.), Musei e territorio. Una scommessa italiana, Milan, Electa, 2009, p. 144-167.

JALLA Daniele, «Introduzione », dans GALLONI Valentina, SANI Margherita (dir.), Musei e paesaggi culturali in Emilia-Romagna, Bologne, Istituto per i beni artistici culturali e naturali, 2016, p. 4-5. LANZI Luigi, Storia pittorica della Italia, Bassano (Vi), Remondini, 1795-1796, vol. 2. 
MEYER Susanne Adina, « Il giudizio del pubblico e il ruolo dei critici. Il panorama europeo e il caso romano », dans RAO Anna Maria, POSTIGLIOLA Alberto (dir.), Il Settecento negli studi italiani. Problemi e prospettive, Rome, Edizioni di Storia e Letteratura, 2010, p. 323-340.

MONTI Paolo, Scritti e appunti sulla fotografia, Acerra (Na), ACM, 2008.

POULOT Dominique, « Greuze et l'intelligence publique de la peinture chez Thomas Crow », dans MC WILLIAM Neil, MORÉTEAU Constance, LAMOUREUX Johanne (dir.), Histoires sociales de l'art. Une anthologie critique, Paris, Les presses du réel, 2016, vol. 2, p. 413-427.

ROMANo Giovanni, Studi sul paesaggio, Turin, Einaudi, 1978.

SCARROCCHIA Sandro (dir.), RIEGL Alois, Il culto moderno dei monumenti : il suo carattere e $i$ suoi inizi, Bologne, Nuova Alfa, 1985.

SOGARI Chiara, «La proposta critica di museo diffuso in Emilia Romagna », tesi di Laurea in Discipline delle arti, della musica e dello spettacolo, Bologne, Alma Mater Studiorum-Università di Bologna, 2016.

TOSCANO Bruno, "Cattedrale e città : studio di un esempio ", dans Topografia urbana e vita cittadina nell'alto Medioevo in Occidente, Spoleto, Panetto \& Petrelli, 1974, (2 vol.), p. 711-747.

VERDE Simone, Cultura senza Capitale : storia e tradimento di un'idea italiana, Venise, Marsilio Editori, 2014.

\section{NOTES}

1. Ces campagne di rilevamento peuvent être traduites par campagnes d'inventaire.

2. Andrea Emiliani : lauréat du prix ICOM en 2012, cofondateur de l'Istituto per $i$ Beni Culturali dell'Emilia-Romagna, il a été durant de nombreuses années sovrintendente et directeur de la Pinacothèque de Bologne. Considéré comme le père de la notion de museo diffuso, il est l'auteur de textes devenus de véritables manifestes programmatiques d'une conception élargie du patrimoine.

3. Cette synthèse sur les aspects méthodologiques des campagnes fait partie d'une entreprise de valorisation de l'enseignement historique d'Andrea Emiliani, menée par Sandra Costa, qui a dirigé plusieurs recherches universitaires sur ce sujet, et par AnnaLisa Carpi à partir de sa Tesi di Laurea Magistrale (mémoire de Master) intitulée Un metodo antropologico. Andrea Emiliani: storia arte cultura. La traduction a été confiée à Maillys Liautard, élève à l'École Normale Supérieure de Paris et étudiante en master «Patrimoine et Musées» à l'université de Paris I - Panthéon-Sorbonne, qui s'est intéressée au sujet à l'occasion d'un séjour d'études à Bologne en juin-juillet 2017.

4. Cf. S. Verde, Cultura senza Capitale: storia e tradimento di un'idea italiana et C. Sogari, «La proposta critica di museo diffuso in Emilia Romagna ».

5. A. Riegl, Le culte moderne des monuments : son essence, sa genèse. Publié à Vienne en 1903, il est traduit en Italie seulement en 1985 (S. Scarocchia, Il culto moderno dei monumenti. Il suo carattere i suoi inizi).

6. La IX ${ }^{\mathrm{e}}$ Conférence générale de l'ICOM (International Council of Museum) a consacré le concept d'“écomusée ", précisément pour souligner les liens indéniables entre patrimoine, communauté et environnement. 
7. Cf. M. Bloch, Apologie pour l'histoire, ou Métier d'historien, et cf. L. Febvre, Pour une histoire à part entière. En Italie, l'École des Annales s'incarne essentiellement dans l'entreprise éditoriale majeure de la Storia d'Italia d'Einaudi, un projet pluridisciplinaire qui éveilla un grand intérêt précisément sur le plan méthodologique; cf. O. Rossi Pinelli (dir.), La storia delle storie dell'arte, p. 473.

8. Le géographe Lucio Gambi (1920-2006) a enseigné dans les universités de Milan et de Bologne, et a été membre de l'Accademia dei Lincei. Ses recherches, suivant une perspective historiciste et proches de la géographie humaine, annonçaient la vision interdisciplinaire qui serait à la base de la conception de la protection et de la conservation du patrimoine culturel élaborée par Andrea Emiliani.

9. En français : la méthodologie de la connaissance pour l'action sociale. L. Gambi, La cognizione del paesaggio, p. 75.

10. Sur la théorie de la description (ekphrasis) voir A. Chastel, « Roberto Longhi : il genio dell “ekphrasis " ", p. 56-65.

11. Cf. A.-L. Carpi, « Un metodo antropologico. Andrea Emiliani : storia, arte, cultura ».

12. Cf. A. Emiliani, L'elogio della mano.

13. Cf. A. Emiliani, Per un rilevamento dei beni artistici e culturali di Porretta Terme : mostra documentaria, 1969.

14. A.-L. Carpi, « Un metodo antropologico. Andrea Emiliani : storia, arte, cultura », p. 78.

15. Le concept de comprensorio (terme d'origine militaire qui correspond au "quartier " dans une zone quadrillée et qui pourrait être traduit ici par " unité ») avait également été employé par le groupe de chercheurs et de professionnels qui avait accompagné Adriano Olivetti dans son projet de planification territoriale de la Vallée d'Aoste.

16. Voir A. Emiliani, «L'esperienza sul campo », p. XV.

17. P. Monti, Scritti e appunti sulla fotografia, p.174; cf. A.-L.Carpi, "Un metodo antropologico. Andrea Emiliani : storia, arte, cultura », p. 191.

18. Cf. A. Emiliani, Per un rilevamento dei beni artistici e culturali di Porretta Terme: mostra documentaria, 1969.

19. Parmi les premiers partisans d'une conception du patrimoine émanant intrinsèquement de la société où il se trouve et avec laquelle l'œuvre d'art ne cesse de dialoguer, il faut citer le philosophe, homme politique et critique d'art, Quatremère de Quincy.

20. Bien que l'organisation régionale ait été prévue dès la Constitution de la République afin d'instituer un équilibre entre centre et périphérie, il fallut attendre vingt ans pour en voir la concrétisation, avec la création des régions en 1970. Durant ce laps de temps, la politique culturelle du pays fut particulièrement centralisatrice, ce qui ne changea guère même après l'établissement des régions, car les multiples lois alors promulguées pour définir les rapports entre régions et ministères donnèrent bien souvent lieu à une application confuse, quand elles ne restèrent pas simplement lettre morte; cf. V. Emiliani, Lo sfascio del Belpaese. Beni culturali e paesaggio da Berlusconi a Renzi, p. 16-20. L'échec de la dynamique centre-périphérie a contribué à mettre en lumière l'inadéquation des régions, définies selon des critères davantage administratifs que culturels.

21. A. Emiliani, Dal museo al territorio, 1967-1974, p. 258. 
22. Il faut en effet attendre 1985 pour que soit promulguée l'une des rares lois - la $n^{\circ}$ 431, dite aussi loi Galasso - qui témoigne d'une conscience de l'inadéquation de la réglementation en vigueur, exigeant des Régions la rédaction de plans " paysagistiques » ou " urbanistiques-territoriaux », avec pour objectif une protection qui dépasse la seule préoccupation esthétique, et reconnus comme des outils adaptés à la sauvegarde du territoire; cf. C. Sogari, «La proposta critica di museo diffuso in Emilia Romagna », p. 5.

23. Sur cette question, cf. D. Poulot, «Greuze et l'intelligence publique de la peinture chez Thomas Crow », p. 413, et cf. D. Diderot, « Salon de 1769 ».

24. L. Lanzi, Storia pittorica della Italia, p. 196 ; S. A. Meyer, «Il giudizio del pubblico e il ruolo dei critici. Il panorama europeo e il caso romano ", p. 328.

25. Les objectifs en furent identiques, mais également les instruments et les stratégies afin d'atteindre ces objectifs: pour la première fois, un centre historique était considéré dans sa globalité, comme s'il s'agissait d'un seul et unique monument, dont la structure complexe s'harmonisait tout naturellement avec la campagne qui l'entourait.

26. P. L. Cervellati, « Bologna centro storico », p. 204-221.

27. A. Emiliani, La conservazione come pubblico servizio, p. 10.

28. Point de vue soutenu par Guido Fanti (1925-2012), maire de Bologne (1966-1970) et premier président de la Région Émilie-Romagne de 1970 à 1976. Il fut, parmi les acteurs politiques, l'un des plus farouches partisans d'une vision réformiste et attentive aux exigences culturelles du territoire. Cf. G. Fanti, « La legge per i centri storici », p. 253-270.

29. En Émilie-Romagne, il existe plus de 500 musées et institutions connexes qui appartiennent aux collectivités locales.

30. Cf. B. Toscano, «Cattedrale e città : studio di un esempio ».

31. Cf. G. Romano, Studi sul paesaggio.

32. Le projet vit le jour grâce à la loi régionale $\mathrm{n}^{\circ} 46$ du 26 août 1974, se référant aux compétences transmises aux Régions en vertu des articles 117 et 118 de la Constitution de la République. Pour réussir à analyser, à plusieurs décennies de distance, les raisons qui ont empêché ce modèle de se traduire en actions concrètes, il faut revenir aux éléments qui en ont permis la naissance. De fait, celle-ci doit beaucoup aux circonstances heureuses qui virent la rencontre de personnalités hors du commun, dotées d'une sensibilité aux mêmes questions politiques, sociales et culturelles, dont elles partageaient une vision civique similaire.

33. Cf. E. Alessandrone Perona, "Dai luoghi della memoria alla memoria dei luoghi»; D. Jalla, «Un museo responsabile del territorio ».

34. L'objectif du projet "Linea gotica " était de créer une structure territoriale regroupant des musées, des fonds d'archives, des collections publiques et privées, constituant un véritable museo diffuso à ciel ouvert. Ce musée avait pour rôle d'intégrer le patrimoine local au sein du paysage environnant, la protection de l'art et celle du paysage représentant les deux composantes complémentaires d'une même action de valorisation dont le musée était le garant ; cf. D. Jalla, «Introduzione », p. 5.

35. Le Code du patrimoine et du paysage (Codice dei beni culturali e del paesaggio, DL 22 gennaio $2004, \mathrm{n}^{\circ} 42$ ) a finalement pris en compte une vision inclusive du paysage, représentatif de l'ensemble du territoire en tant que patrimoine à part entière, et, surtout, il en a mis en évidence le caractère très fortement identitaire. L'art. 131 qui 
ouvre quant à lui la Parte III, spécialement dédiée au patrimoine paysager (Beni Paesaggistici), affirme que «l'on entend par paysage un territoire qui exprime une identité, dont les caractéristiques résultent de l'action de facteurs naturels et humains, et de leurs interrelations ».

36. Le concept désigne les zones culturellement et historiquement homogènes, auparavant appelées comprensori et désormais identifiées grâce à une cartographie qui en définit les frontières et sert de cadre de référence pour l'application des dispositions programmatiques prévues par la réglementation régionale. Se concrétise ainsi un modèle de valorisation et de gestion du territoire fondé sur la reconnaissance des identités locales, et sur leur rôle central pour la protection de l'environnement.

37. Cette exigence trouve un exemple éloquent dans l'Ecomuseo delle Valli di Argenta. Créé entre 1991 et 2002, le musée est en fait un « réseau » qui rassemble les patrimoines de musées et d'instances locales du territoire et qui assure une valorisation globale de tout le tissu culturel local, à travers la coordination de visites guidées et de diverses actions de médiation; cf. F. Cecchini, «Un sistema ecomuseale nel Parco del Delta del Po », p. 25-27.

38. Cf. A.-L. Carpi, «Tra architettura, paesaggio e grandi capolavori : la "via" bolognese per un metodo interdisciplinare ».

39. Cf. A. Emiliani, «I materiali e le Istituzioni », p. 100, où A. Emiliani retrace l'évolution du terme "conserver", d'un emploi naturaliste à une fonction historique et anthropologique.

40. Le thème de la $24^{\mathrm{e}}$ conférence générale de l'ICOM (Milan, 3-9 juillet 2016), a été choisi à partir de l'idée selon laquelle le paysage italien avait toujours représenté la meilleure synthèse entre histoire et beauté naturelle, illustrée par exemple par la pratique du Grand Tour au XvIII ${ }^{e}$ siècle. Mais le choix a aussi trouvé son origine dans le constat de la quantité et de la variété des musées italiens, et dans la prise de conscience de la nécessité de créer des liens entre les musées et les contextes culturels dans lesquels ils s'inscrivent.

\section{RÉSUMÉS}

Entre 1968 et 1971, la Soprintendenza alle Gallerie di Bologna organisa quatre Campagne di rilevamento du patrimoine artistique et culturel dans l'Apennin émilien. L'initiative était menée par Andrea Emiliani, historien de l'art et muséologue internationalement reconnu, et se caractérisa par une interdisciplinarité d'une rare ampleur. Novatrice sur le plan méthodologique, constituant un modèle exemplaire de révision des objectifs et des instruments de la connaissance de l'art, l'expérience réalisée dans les montagnes des Apennins émiliens a renouvelé en profondeur la façon de concevoir l'histoire de l'art et le rapport entre la culture matérielle et les lieux. Près de cinquante ans plus tard, grâce au témoignage direct de son acteur principal, Andrea Emiliani, on présente les racines historiques, les expérimentations et le contexte de mise en œuvre des Campagne en même temps que l'héritage d'une proposition critique et méthodologique qui, à 
partir de l'analyse spécifique d'un territoire de montagne, est largement à l'origine d'une autre façon de penser les liens entre l'art et son territoire.

\section{AUTEURS}

\section{SANDRA COSTA}

Professeur des universités en muséologie, critique artistique et restauration à l'université Alma Mater Studiorum de Bologne, Italie, directrice du master Arts visuels

\section{ANNA-LISA CARPI}

Coordinatrice de la Collection collegArti de l'université Alma Mater Studiorum de Bologne, Italie 


\title{
L'introduction des principes de la lecture de paysage et de l'art contemporain dans l'interprétation de géosites sur le territoire de la réserve naturelle géologique de Haute-Provence
}

\author{
Christel Venzal
}

1 La notion de géotourisme, a été initiée en France par les gestionnaires de la réserve naturelle géologique de Haute-Provence (RNGHP) (fig.1). Cette aire géographique, implantée sur 52 communes, entre la nappe de charriage de Digne-les-Bains (Alpes de Haute-Provence), l'arc plissé de Castellane, le bassin de Digne-Valensole et les chaînons provençaux varois, s'est organisée en deux étapes. Dans un premier temps (1984-1997), une offre de découverte ludique et didactique est orchestrée à partir d'un ensemble de sites géologiques et/ou historiques, se traduisant à travers des aménagements pour la visite du public, des sentiers, des panneaux, des brochures, des expositions... Toutes ces réalisations se basent sur l'inventaire d'un ou plusieurs sites, identifiés à partir d'éléments de base (roches, fossiles, pierres, eau...), et induisent ainsi une construction spatiale de géosites. Dans un deuxième temps (1997-2017), on note une implication de cette notion par les gestionnaires de ces géosites, dans des programmes transnationaux dont l'objectif premier envisage le développement durable de territoires géotouristiques, dénommés géoparcs et labellisés par l'UNESCO depuis le début des années 2000. Afin de comprendre et analyser les fondements de la terminologie de géosite, nous avons étudié les réalisations entreprises sur le territoire de la RNGHP. Nous avons constaté une proximité avec les principes énoncés par Freeman Tilden sur le concept $d^{\prime}$ « interprétation du patrimoine ${ }^{1} »$. L'analyse de ces principes a guidé notre étude et notre réflexion sur les géosites. Dans un deuxième temps, nous avons étudié les orientations stratégiques du Centre d'Art Informel de Recherche sur la Nature (CAIRN), 
initié sur le territoire de la RNGHP. L'art représente dans cette approche un médiateur du géosite permettant de mettre en évidence des points particuliers du territoire. Les «sentinelles» réalisées par l'artiste britannique Andy Goldsworthy, sont installées le long de trois parcours d'interprétation du territoire et notamment afin de matérialiser les portes d'entrée de la RNGHP en soulignant l'angle de vue idéal pour apprécier un panorama ${ }^{2}$. L'artiste se positionne en médiateur de la protection de l'environnement en installant son œuvre dans l'éphémère naturel. Ces œuvres contemporaines s'intègrent dans la Nature en utilisant les éléments minéraux mais aussi végétaux ou encore liquides comme l'eau (ou solide selon les saisons, la glace, la neige). Ce courant de pensée artistique dénommé le Land Art est consacré par le travail d'artistes du naturel.

Fig. 1. - Présentation cartographique du territoire de la RNGHP.

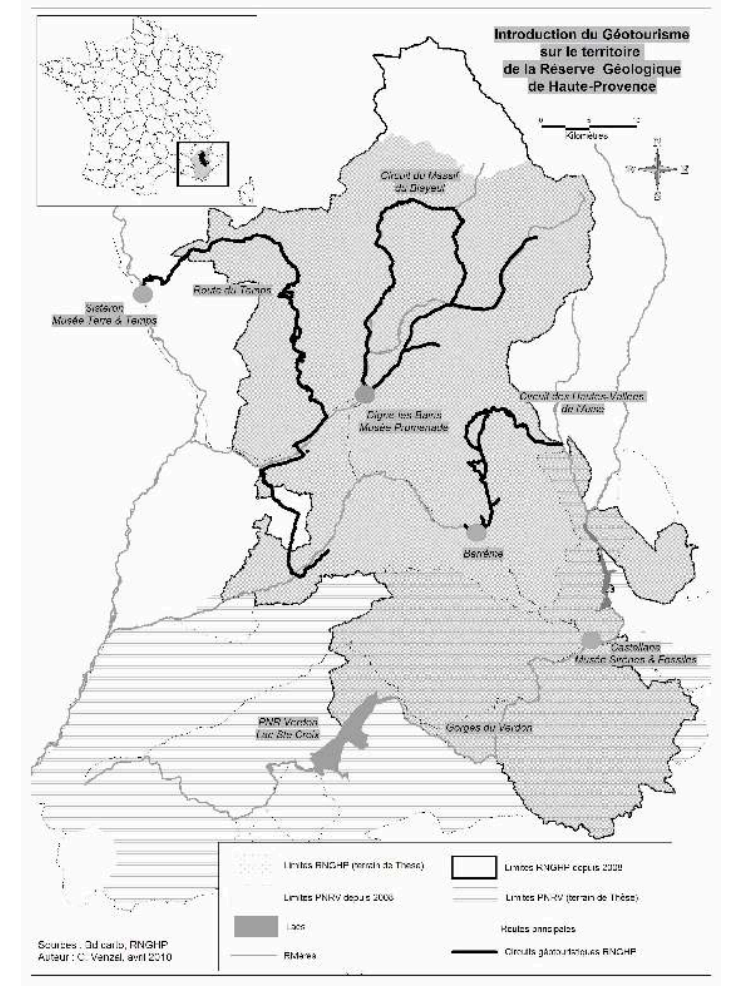

(C) Christel Venzal.

\section{La lecture de paysages, outil d'interprétation des géosites}

2 L'Interprétation du patrimoine se définit comme l'art de communiquer la signification et la valeur d'un lieu à ceux qui le visitent. Ce concept désigne une philosophie, des méthodologies et des moyens d'expression. Son développement sur le continent américain est fortement lié à l'histoire et aux principes des parcs nationaux. Dans les textes de création des parcs nationaux, le territoire est envisagé comme un espace de récréation et de connaissances pour les citadins.

«Le territoire protégé sera mis en réserve pour le plaisir, la détente et

l'enrichissement culturel de tous les citoyens. ${ }^{3}$ " 
3 L'interprétation permet d'expliquer, de rendre clair ce qui est obscur, de donner un sens, de commenter, de comprendre. Dans le cadre de l'association interprétation à patrimoine, il s'agit de donner à comprendre le territoire à travers toutes ses composantes naturelles mais également culturelles pour sensibiliser le visiteur. Plusieurs termes sont associés à ce concept : information, communication, animation, vulgarisation, éducation, pédagogie. Dans les parcs américains, les interprètes sont les employés chargés de guider les visites de groupes ou d'animer des veillées autour des feux de camp qui sont un moyen de communiquer avec le public, en raison de l'ambiance très particulière qui s'en dégage (flammes, nuits étoilées...). Comme un interprète permet de comprendre les paroles prononcées dans une langue étrangère, les guides des parcs traduisent le message du territoire. L'interprétation du patrimoine développe une ambivalence ressentie par l'ensemble des gestionnaires de parcs, de réserves naturelles ouvertes au public : comment mettre le visiteur en contact avec la richesse des ressources d'un site tout en assurant la protection et la conservation de ces ressources? L'interprétation recouvre toutes les activités liées à l'information des visiteurs: expositions, centres d'information, livres, brochures, cartes, panneaux disposés le long des sentiers, photographies, cartes postales... Les principes de Freeman Tilden sont basés sur l'expérience accumulée auprès des personnels des parcs nationaux américains et par l'abondance de ses observations de terrain. Il présente avant tout une philosophie de l'art du métier d'interprète. Tilden définit l'interprétation comme :

«Une activité qui veut dévoiler la signification des choses et leurs relations par l'utilisation des objets d'origine, l'expérience personnelle ou divers moyens d'illustration plutôt que par la communication d'une simple information sur des faits. ${ }^{4}$ "

4 Il insiste sur le fait qu'il ne s'agit pas uniquement de transmettre des données scientifiquement validées ou des coutumes ancestrales, mais de s'impliquer personnellement dans son discours afin d'associer le visiteur à cet exercice. À la différence de l'enseignant, l'interprète garde à l'esprit que le visiteur est en situation de loisir et non d'instruction académique. L'interprète rend son exposé attractif en se gardant de tout excès mais en souhaitant intéresser le visiteur. Dans le cas des sites géologiques très spectaculaires comme le Canyon du Colorado ou les Chutes du Niagara, l'interprétation doit se confondre avec le site. L'interprète sait se placer en retrait et se taire.

«La beauté pour elle-même n'a pas besoin d'être interprétée. Plus tard des questions peuvent venir à l'esprit. Quelles grandes forces naturelles sont à l'œuvre derrière tout ça ? C'est alors que l'heure de l'interprète est arrivée. ${ }^{5}$ "

5 L'interprète appréhende le visiteur dans son ensemble et il ne doit pas le sous-estimer ni surestimer son intelligence ou sa capacité à comprendre. Il garde à l'esprit que l'important n'est pas ce qu'il veut dire mais ce que souhaite connaître le visiteur. Toute représentation approchée de la réalité est en principe contraire à l'esprit scientifique, mais l'interprétation ne fait pas appel aux mêmes qualités que la recherche. Darwin a notamment utilisé les ressources de l'imagination pour interpréter les phénomènes qu'il étudiait $^{6}$. L'objectif de l'interprétation est donc de satisfaire le visiteur en lui révélant une réalité qu'il ignorait ou connaissait mal. Le sentiment d'avoir appris quelque chose est source de satisfaction, contrairement à la frustration que produit toute information indigeste qui infériorise le visiteur et excite parfois son agressivité, sa capacité de vandalisme. Pour présenter ses idées, l'interprète maîtrise l'art du 
suspense et de la rhétorique sans tronquer les bases scientifiques. Lorsqu'il n'y a pas de certitudes sur un sujet, l'interprète rend compte simplement au visiteur et fait de cette incertitude un motif d'intérêt et de curiosité. Tilden estime que la provocation est une des clés de l'interprétation ${ }^{7}$. Pour Tilden, provoquer signifie qu'il faut dépasser le niveau cognitif, qui est celui de l'instruction, pour toucher plus profondément le visiteur et agir au niveau de l'appréciation qui est celui des valeurs. Il est préférable que le visiteur ait retenu une portion de l'information, plus ou moins variable en fonction des individus, plutôt qu'il ait l'impression de n'avoir rien compris ou d'avoir reçu trop d'informations et trop peu de temps pour les assimiler. Il doutera ainsi de l'intérêt de protéger le site et l'interprétation aura échoué. D'autres auteurs ont travaillé sur ce concept notamment en l'adaptant à la situation du continent européen et à son patrimoine, principalement architectural.

6 Pour Don Alridge ${ }^{8}$, l'interprétation est un art qui ne peut être réduit à une technique traitée dans un manuel. Les qualités essentielles à une bonne interprétation font appel à l'émotion et à la sensibilité du visiteur. Avec l'interprétation de la nature, l'interprète cherche à montrer le caractère du site sur la base de l'interrelation entre les roches, les sols, la flore, la faune et l'homme. Il s'agit d'une prise de conscience sur l'interrelation entre l'Homme et son environnement naturel. La méthode qu'il préconise se base sur une approche thématique des sites naturels avec comme fil conducteur la place de l'Homme dans son environnement. Il estime qu'il est important de sensibiliser au préalable la majorité des visiteurs dans des centres d'accueil. La visite des sites naturels s'adresse à un nombre restreint de visiteurs conscients des ressources naturelles et culturelles du territoire. Cette activité rend le visiteur plus sensible à un lieu et l'incite à adapter son comportement. Deux acteurs aux rôles distincts : l'interprète (ensemble des interventions et moyens) transmet la connaissance au visiteur qui la reçoit et peut ainsi modifier son comportement envers le patrimoine.

7 Une des bases du principe du géotourisme se focalise sur la compréhension des paysages afin d'impliquer le guide et le visiteur dans l'interprétation des géosites. Le paysage est porteur de significations qui se diffusent d'autant plus que les paysages sont montrés et commentés'. Le géotourisme ne se limite pas à l'admiration d'un beau paysage ou d'un site rassemblant par exemple une grande variété de très beaux fossiles. L'interprétation des éléments géologiques, économiques et historiques accompagne l'introduction, sur un territoire géologiquement référencé, du géotourisme. Le paysage en tant qu'élément de base participe à la diffusion des intérêts du géosite. La traduction des paysages par les géologues et géomorphologues est porteuse de sens pour le visiteur qui ne subit plus un panorama mais qui peut en saisir toutes les évolutions. Le géotourisme envisage de dépasser l'aspect esthétique des paysages géologiques et géomorphologiques en donnant à comprendre: comment s'est formé ce pli, cette faille ou cette gorge. L'étendue du paysage se détermine en fonction de son observateur, de ses capacités visuelles et par le cadre virtuel qu'il s'impose. La lecture de paysage s'organise à une échelle significative pour l'observateur.

«Un paysage se définit par la vision de l'observateur et l'étendue de ce qu'il embrasse. ${ }^{10} »$

8 Pour le géologue, la première échelle d'analyse du paysage est temporelle afin de définir la durée et l'âge des processus qui le composent.

"C'est en regardant autour de lui que le géologue organise son cheminement d'un affleurement à un autre, et c'est par l'observation du paysage qu'il parvient peu à peu à reconstituer les structures en trois dimensions qui en donnent la clef. ${ }^{11}$ " 
9 L'analyse et la compréhension de la formation des plis et des failles permettent d'établir, dans le cadre de l'ascension d'une montagne, un rapport entre un déplacement géographique et un déplacement dans le temps géologique. Jean-Pierre Pralong pense également que :

«La randonnée pédestre offre la possibilité de parcourir un espace (vallon), et de découvrir les paléo-espaces (bord de mer, fond océanique) qui le constituent, ainsi que les paléotemps (Trias, Jurassique) qu'il représente; le but ultime étant de faire comprendre aux non-initiés que notre monde n'est pas immuable !12 "

10 Le choix de l'échelle d'observation se détermine en fonction des objectifs de la lecture (analyse scientifique ou simple observation du terrain) mais aussi de ses connaissances personnelles, de ses capacités à traduire différents phénomènes.

11 La lecture géologique du paysage constitue la base de la découverte d'un espace. Cette approche prend en compte les éléments et processus naturels mais également des données humaines et culturelles. La diversité géologique entraîne une diversité biologique favorisant ainsi l'approche pluridisciplinaire, principe essentiel de l'interprétation.

«Dans leur pratique quotidienne, l'archéologue et le naturaliste poursuivent chacun une quête qui dépasse le cadre de la coupe stratigraphique à étudier, et l'objectif est fondamentalement le même : comprendre l'espace, qu'il soit territoire des sociétés ou milieu naturel. ${ }^{13}$ "

12 Les activités humaines sont intégrées au géosite et constituent une partie de son intérêt (patrimonial, culturel et social). L'implication de la géologie dans la découverte du patrimoine bâti, en associant les matériaux de construction à leurs sites d'extraction, illustre par exemple cette orientation et toute la pertinence de rendre accessible les géosites au plus grand nombre.

«Arriver à valoriser un géosite ou une émergence paysagère est un défi car il faut les voir non pas comme des éléments isolés mais comme des témoins privilégiés d'un ensemble important constitué par des lieux généralement perçus comme anonymes ou de valeur limitée. ${ }^{14}$ »

Le géosite ne se résume pas seulement à un site isolé racontant une histoire géologique, il reflète l'histoire naturelle et humaine d'un espace qui ne peut être limité aux frontières administratives d'un parc ou d'une réserve. Le géosite permet de comprendre une globalité naturelle et culturelle sans compromettre la pérennité d'un point isolé. L'interprétation du géosite procède ainsi par étapes correspondant à des objectifs de compréhension. Ces objectifs doivent tenir compte des observateurs (visiteurs et interprète) c'est-à-dire de leur niveau, de leurs compétences, de leur expérience, de leurs capacités... Si l'interprète ne se fixe pas des objectifs adaptés à ses auditeurs, la visite se résume alors à une simple consommation touristique de paysages et de sites. L'approche géotouristique de l'espace correspond à la demande touristique, grande consommatrice de paysages, mais souhaite compléter cette option en procurant une vision éclairée et éclairante aux touristes. L'interprétation ayant éveillé la curiosité du visiteur l'incitera à rechercher d'autres formes structurales dans la composition de nouveaux paysages. Le paysage interprété n'est plus simplement consommé mais véritablement fréquenté puisque porteur de sens. Décodé, le paysage devient lisible donc appropriable comme familier et non plus rejeté ou ignoré comme étranger.

« Notre société, qui est par ailleurs grosse consommatrice d'images, ne connaît plus la différence entre le voir et le regarder, et une éducation à la lecture du paysage est à suivre par ceux qui veulent comprendre les logiques de son organisation pour 
mieux le fréquenter: le paysage ne se consomme pas comme un produit, il

s'apprend. ${ }^{15}$ »

14 L'introduction du géotourisme dans la découverte d'un territoire associe les données géologiques, économiques et historiques dans l'interprétation de géosites afin de former un ensemble commun, vecteur de sens. Il ne s'agit donc plus de réserver une portion d'un territoire protégé ayant été qualifié de remarquable et devant être sauvegardé, mais de dépasser les limites administratives des territoires par la compréhension des phénomènes géologiques dans leur globalité : donner à comprendre et rendre " lisible » afin de ne plus ignorer les paysages qui entourent les touristes et les pousser à les traverser.

\section{La Nature, matière première de l'artiste : introduction au Land Art}

15 Pour développer cette complémentarité du naturel et du culturel, l'approche artistique de la Nature par l'art représente la base du courant du Land Art. Qu'est-ce que l'art contemporain peut apporter à l'interprétation du géosite? Au-delà d'un courant de pensée, est-ce que l'approche artistique du géosite participe de son interprétation? Pour répondre à ces interrogations, nous avons analysé le travail de deux artistes dans le cadre du CAIRN (Centre d'Art Informel de Recherche sur la Nature) et complété notre étude par une prise en compte des réalisations de Benoît de Souza, «artiste-artisan $»^{16}$. Ces rencontres nous ont permis de saisir toute la richesse artistique inspirée par le géosite et comment l'artiste traduit ces phénomènes dans la matière.

16 L'objectif du Centre d'Art CAIRN, projet issu d'une collaboration entre la RNGHP et le musée départemental Gassendi de Digne-les-Bains, ambitionne d'inviter des artistes en résidence à Digne-les-Bains. Les artistes explorent le territoire et proposent des œuvres "visuelles et plastiques dans l'esprit des lieux ». Ces interventions peuvent se traduire sous forme d'expositions temporaires, d'installations permanentes ou éphémères, d'éditions, de productions d'œuvres ou encore de commandes publiques. L'ambition du CAIRN est de :

«Favoriser les rencontres entre les créateurs et le site de Digne-les-Bains vécu dans ses différentes composantes : rurale, écologique, sociale, historique et de faciliter la mise en œuvre des projets afin de constituer un patrimoine contemporain au cœur du pays dignois et de lier création contemporaine et développement durable. ${ }^{17}$ »

17 Il s'agit d'une mise en valeur du territoire par l'axe de l'art contemporain. L'introduction de l'art contemporain est envisagée comme un facteur permettant, à plus ou moins long terme, d'attirer de nouvelles clientèles ${ }^{18}$. Il s'agit d'une mise en scène des sites et des lieux favorisant une accessibilité aux thématiques de la géologie. L'art est le vecteur de communication des sites et du territoire. Toute la difficulté de cette médiation artistique réside dans l'adaptabilité des artistes choisis. Leur art doit être suffisamment accessible et ludique pour attirer et retenir l'attention des visiteurs. Ce programme est un outil de communication permettant au travers des différentes expositions, éditions de catalogues, de créer des événementiels culturels. Pour comprendre les orientations du programme CAIRN, nous avons étudié la démarche d'Andy Goldsworthy et le travail de Joan Fontcuberta.

18 L'analyse du travail d'Andy Goldsworthy sur le territoire de la RNGHP, nous permet d'aborder la problématique du Land Art. Installé en Écosse, le sculpteur contemporain 
Andy Goldsworthy a exploré pendant des années différents lieux dans le monde, en laissant derrière lui des sculptures, qu'il souhaite en harmonie avec l'environnement. Ses matériaux sont extraits de la nature et ses œuvres, comme toutes choses naturelles, sont essentiellement éphémères. Pour Goldsworthy, regarder et comprendre la nature est un moyen de renouveler nos liens avec la terre. Dans ses premières approches du territoire de la RNGHP, il a travaillé sur la thématique de la mémoire de la Terre (Earth Memory, en 1995 et 1997) en coopération directe avec le musée départemental de Digneles-Bains. Dans le cadre du programme CAIRN, il a élaboré plusieurs réalisations pérennes à Barles, Authon et Tartonne intitulées les « sentinelles »" ${ }^{19}$. Dans le parc boisé $\mathrm{du}$ « musée promenade $»^{20}$, il a installé des water-cairns tout au long d'un sentier qui emmène les visiteurs jusqu'aux salles d'exposition. Il a également imaginé, à la suite de plusieurs randonnées pédestres, un sentier traversant le territoire appelé le «sentier des arts " et jalonné de cinq Houses of Art. Ces maisons sont à la fois des lieux d'hébergement et des sites pouvant accueillir des expositions temporaires d'artistes. Le Land Art se définit comme une rencontre entre un artiste et la nature dans une optique de protection de l'environnement et de respect de la nature. L'artiste choisit un espace contenant les matériaux naturels qu'il utilisera dans son œuvre (arbre, végétal, roche, pierre...). Pour construire son œuvre, il utilise des outils, mais les matériaux de consolidation doivent être biodégradables. L'œuvre peut être totalement éphémère ou s'assurer, par sa structure, une certaine pérennité. Cette œuvre s'intègre dans le paysage en mettant en lumière la créativité de l'artiste. Cette tendance de l'art contemporain est apparue dans les paysages grandioses de l'Ouest américain ${ }^{21}$. Les artistes se réclamant du Land Art ne représentent pas seulement la nature, leur créativité se matérialise à travers les éléments qui la composent. Leurs lieux d'exposition sont souvent éloignés et cachés, la photographie joue alors un rôle essentiel pour montrer, témoigner et également financer leurs projets par la vente des ouvrages regroupant leurs travaux par thèmes (temps, spirales, roche...). Dans une interview accordée à la chaîne télévisée Arte (2005), Andy Goldsworthy déclare :

"Je veux aller sous la surface. Quand je travaille avec une feuille, une pierre, un bout de bois, ce n'est pas que le matériau en lui-même qui m'intéresse, c'est une ouverture aux processus de la vie, à l'intérieur et autour. Quand j'ai terminé, ces processus continuent. ${ }^{22}$ "

On note, dans ce propos, l'intérêt de l'artiste à travailler dans un territoire consacré à la protection et la mise en valeur du patrimoine géologique qui utilise des phénomènes passés pour expliquer les évolutions actuelles et futures de la nature. Les responsables du programme CAIRN lui ont commandé des œuvres pérennes qui participent à la découverte pédagogique et touristique des sites géologiques. Il a notamment réalisé des cairns $^{23}$. Dans le cadre de ce projet, la fourniture en pierres plates de qualité suffisante pour structurer l'œuvre s'est posée aux gestionnaires de la RNGHP. Les pierres qui composent les œuvres de Goldsworthy ont été importées. Le talent et l'expérience de l'artiste ont permis d'intégrer ces œuvres dans le milieu naturel. Ces cairns composent une nouvelle lecture du paysage. Ils constituent un ancrage visuel et les sentinelles marquent, à présent, l'entrée dans la RNGHP.

Nous notons par ailleurs que la symbolique du cairn, marqueur de passage, se réfère aux tas de pierres construits le long des sentiers par les randonneurs. La tradition établit que le randonneur passant devant un cairn réalisé pour marquer le chemin, devra apporter sa pierre à l'édifice et au moins ne pas casser cet ouvrage spontané. Le principe de ces cairns spontanés s'établit à l'inverse des cairns réalisés par Andy 
Golsworthy sur le territoire de la RNGHP, à partir des éléments minéraux disponibles sur place. Dans le cas du programme CAIRN, les gestionnaires de la RNGHP n'ont pas souhaité favoriser le ramassage d'éléments minéraux à proximité de sites référencés par leur intérêt géologique, ni l'ouverture de carrières autrefois développées sur la région. Ces projets de réalisations artistiques, dans et à partir de la nature, associent l'artiste et son milieu et constituent ainsi un vecteur d'éducation à l'environnement. Il ne s'agit pas de prendre des fossiles et de les mettre en scène pour réaliser une œuvre unique. Mais ces œuvres naturelles minérales ou végétales mettent en valeur un paysage géologique qui se trouve en arrière-plan ou qui entoure le projet. L'œuvre est un repère permettant l'interprétation et la lecture du milieu naturel et culturel. Les théories du Land Art correspondent aux problématiques de l'éducation à l'environnement.

Joan Fontcuberta a réalisé une exposition temporaire sur le thème des sirènes au " musée promenade» de Digne-les-Bains. Cette exposition et son catalogue de présentation s'intitulent « Volte-face à l'envers de la science, les leçons de l'histoire ». Joan Fontcuberta est artiste (photographe, plasticien) et enseigne à l'Université de Barcelone. Son travail de photographe s'intéresse :

"À une certaine culture de la vision qui se base sur la notion d'empreinte et de vestige, ainsi que sur la question du vrai-faux. ${ }^{24}$ "

Le monde des sirènes est assez obscur et spéculatif. Phineas T. Barnum a fait fortune en exhibant toutes sortes de monstres dans ses foires et notamment la fameuse Fejee mermaid ${ }^{25}$. Ces vraies fausses sirènes rencontraient un grand succès et fascinaient les visiteurs. Ces monstres de foire faisaient renaître le mythe des sirènes. C'est à partir de ce constat que Joan Fontcuberta a proposé un travail à deux phases :

«D'un côté, mon intervention dans la réserve géologique, introduisant de façon permanente de faux fossiles de sirènes, de l'autre une pseudo-documentation sur ces fossiles, tout un dispositif narratif dans lequel la photographie est utilisée comme élément authentificateur. »

Ce projet s'est réparti en deux lieux : la mise en œuvre dans la vallée du Bès d'un site renfermant un fossile d'hydropithecus (dite sirène fossile du Tanaron) et la réalisation, dans le premier espace d'accueil du "musée promenade ", d'une exposition contenant des photographies des sites, des documents scientifiques, des comptes-rendus de recherches, des objets fossiles... Dans un parcours idéal de découverte, les visiteurs se devaient d'appréhender les deux lieux, sinon ils n'obtenaient qu'une vision tronquée du projet. Joan Fontcuberta a souhaité que ces fossiles de sirènes « restent dans la nature le plus longtemps possible» et soient conservés. Tout ce travail reposait sur le paradoxe du faux vrai et de la relation étroite entre un site naturel et sa reconstitution, son artefact. Joan Fontcuberta a estimé que « dans la Réserve, on trouve des situations paradoxales ». Il a donné l'exemple du site d'empreintes de pas d'oiseaux, implanté en bord de route. Il s'agit en fait d'un moulage, le vrai site étant situé dans une zone dangereuse non accessible au public. L'objectif de ce projet était une prise de conscience face aux flux continus d'informations qui nous parviennent par les médias et face aux données annoncées et certifiées par les scientifiques. Le visiteur devait conserver son esprit critique face aux événements. Joan Fontcuberta a précisé que son travail est :

«Une confrontation entre vrai et faux, ou entre une logique scientifique et une autre, spéculative. » 
«Connaissant les mécanismes de la communication de la science officielle, celui-ci les utilise afin de nous convaincre comme Vallisnieri, qu'il convient toujours d'être un curieux sceptique.»

Nous avons pu rencontrer des visiteurs de cette exposition (une vingtaine de personnes), une majorité a apprécié ce travail mais certains n'ont pas compris la démarche de l'artiste qui n'est réellement explicitée que dans le catalogue de l'exposition. Une institutrice a transmis, par voie électronique, aux services de la RNGHP son indignation et son désarroi en faisant la remarque suivante :

«Traversant la réserve de géologie en juillet pour la première fois, je suis tombée, après plusieurs sites passionnants, sur la sirène de Tanaron. Après une seconde de stupéfaction, j'ai analysé le spécimen avec mes connaissances (j'ai une maîtrise de biologie) et j'ai conclu que c'était un faux. J'étais très choquée. Je ne comprenais pas d'où venait cette supercherie ni comment des scientifiques pouvaient la cautionner. "

Elle ajoute plus loin dans son message :

«Je m'adresse aux scientifiques, aux gens qui travaillent au service de la vérité : comment pouvez-vous cautionner ça ? On ne peut pas sacrifier la vérité au nom de l'art. »

Cette enseignante ne serait peut-être pas choquée par la visite d'un site comme la reconstitution de la Grotte de Lascaux ou bien encore par l'exposition de Vulcania en Auvergne, par contre elle n'a pas admis que les scientifiques de la RNGHP aient cautionné ce type de démarche. L'artiste, interrogé sur ce point, n’a pas souhaité indiquer ni sur le site ni à la fin de l'exposition les orientations de son travail. L'art contemporain apporte une autre vision sur les sciences et dans une certaine mesure, il peut permettre un autre accès aux sciences.

Dans le cadre de leur développement commercial et l'introduction des principes du géotourisme, les gestionnaires de la RNGHP (labellisé Géoparc de Haute-Provence par l'UNESCO) ont également imaginé un partenariat avec des artisans locaux qui travaillaient depuis des années sur la thématique des fossiles, notamment des ammonites. Des bijoux, utilisant au départ de véritables fossiles comme l'étoile de Saint Vincent puis des moulages, ont toujours été commercialisés. Plusieurs corps de métiers utilisent les fossiles comme modèles, par exemple dans les métiers de la céramique, de la faïence, du textile, mais également dans les métiers de bouche comme la boulangerie, la pâtisserie ou la chocolaterie. Ces artisans sont autorisés à utiliser le nom du Géoparc de Haute-Provence et les symboles de ses principaux sites comme la dalle à ammonites. Nous avons interrogé Benoît de Souza, artiste plasticien céramiste, originaire du Bénin, implanté à Digne-les-Bains. Depuis les débuts de son atelier, il a souhaité utiliser le symbole des fossiles présents sur le territoire comme les ammonites et l'étoile de Saint Vincent. Son travail s'articule sur un rapport esthétique et symbolique des fossiles. Il nous a indiqué que ses œuvres se vendent très bien à une clientèle de connaisseurs mais aussi à des touristes de passage dans le secteur. Benoît de Souza a également réalisé des pièces exposées dans l'exposition permanente du musée Sirènes \& Fossiles de Castellane qui a accueilli en 1999 un grand nombre de ses réalisations sur le thème des sirènes et siréniens. On remarque notamment la "sirène ammonite " qui se compose d'un buste sculpté associé à un moulage d'ammonites découvertes sur la commune de Barrême. Le vrai et le faux se mélangent dans cette pièce d'art et elle finalise l'approche artistique du géosite en associant un moulage d'ammonite réalisé à la suite de fouilles et à partir de données scientifiquement validées, à une œuvre sculptée, 
fruit du travail d'un artiste localement implanté et fortement inspiré par les sites géologiques référencés. Elle évoque l'anthropomorphisme ou bien encore le syncrétisme dans un mélange des genres en favorisant l'effet esthétique ${ }^{26}$. L'œuvre réalisée par Benoît de Souza nous a semblé pouvoir illustrer l'association du minéral, de l'art et du scientifique au service de l'interprétation du géosite. La recherche artistique participe ainsi à l'interprétation du géosite et représente un élément didactique de la protection de l'environnement naturel. Les œuvres réalisées par des artistes, sensibles et impliqués dans la préservation de la Nature, s'intègrent au site en utilisant les éléments naturels principalement de l'ordre du minéral mais également végétal, constituant ainsi un point d'ancrage artistique et culturel du géosite. Nous avons envisagé que l'approche artistique et culturelle, commencée sur des bases géologiques, participait à l'élargissement des thèmes traités par les sciences de la terre en introduisant un facteur supplémentaire d'interprétation. L'artiste est positionné comme un médium de l'interprétation au même titre que le guide-interprète ou bien encore la signalétique des circuits. Il utilise le facteur émotionnel engendré par son œuvre afin de sensibiliser comme le guide-interprète, le visiteur à une prise de conscience de l'intérêt de préserver l'environnement naturel.

29 L'introduction des principes de la lecture du paysage dans le développement du géotourisme, sur le territoire de la RNGHP (Géoparc de Haute-Provence), ne correspond pas uniquement à une stratégie de transfert des flux de touristes des zones périphériques vers le centre du territoire. L'interprétation par la lecture de paysage introduit la dimension spatiale et culturelle du géosite. Le site géologique ne se limite pas à un point fixe mais intègre un ensemble d'informations nécessaires à sa compréhension. L'approche du géosite par la lecture de paysage permet d'intéresser le touriste à des secteurs du territoire dont il n'aurait pas perçu seul les richesses géologiques, mais également historiques et économiques. Par ailleurs l'introduction de l'art contemporain dans l'interprétation de géosites démontre les limites d'une approche uniquement basée sur la géologie. Associer l'esthétique, le symbolique, l'artistique dans l'interprétation du géosite peut permettre d'intéresser un public plus large. L'association de l'art contemporain et de la lecture de paysage interroge le visiteur et l'incite à découvrir la variété des formes et structures visibles sur un territoire géologiquement référencé. Pour être complète l'interprétation de géosites dépasse les limites des territoires protégés et peut montrer l'intérêt d'une mise en réseau des sites, des territoires et de la culture scientifique au service du développement du géotourisme.

\section{BIBLIOGRAPHIE}

ALDRIDJE Don, Principe of country interpretation, Edimbourg: Her Majesty's stationary office, 1975. 
BELGRANO Elena, RIMONDI Daniela, Société et territoire : une approche qualitative, Laboratoire CRAFTS du département POLIS de la faculté d'architecture de Gênes, 2003.

BRUNET Roger, FERRAS Robert et THERY Hervé, Les Mots de la Géographie, Paris, RECLUS - La Documentation Française, $3^{\mathrm{e}}$ édition, 2001, p. 373-376.

COURTOT Roland, Agriculture, paysages et patrimoine, actes des Assises pour l'Agriculture des Alpes de Haute-Provence, décembre 2004, p. 115-119.

FÉRÉROL Marie-Ève, « Le Massif du Sancy et Horizons - Arts Nature : quand Land Art rime avec attractivité ", Journal of Alpine Research / Revue de géographie alpine, éd. numérique, 105-2 | 2017, [ URL : http://rga.revues.org/3750]

FONTCUBERTA Joan, Volte-face à l'envers de la science, les leçons d'histoire, Digne-les-Bains, Les Petites Affiches, 2000, $118 \mathrm{p}$.

GUYOT Sylvain et SAUMON Gabrielle, « La mise en art de la Blackfoot Valley (Montana, USA) ou comment (ré)concilier le front minier et le front écologique ? ", Journal of Alpine Research, Revue de géographie alpine, éd. numérique, 105-2, 2017, [URL : http://rga.revues.org/3690]

MATTAUER Maurice, Palimpsestes géologiques, Pour la Science, novembre 2004, nº 325, p. 56-61. PINCHEMEL Philippe, Lire les paysages ? - Lire les Paysages, La Documentation Photographique, avril 1987, $\mathrm{n}^{\circ} 6088$, p. 1-8.

PRALONG Jean-Pierre, « Valorisation et vulgarisation des sciences de la Terre : les concepts de temps et d'espace et leur application à la randonnée pédestre », dans REYNARD E. et al. (eds), Géomorphologie et Tourisme, actes de la Réunion annuelle de la Société Suisse de Géomorphologie (SSGm) Finhaut, 21-23 septembre 2001, Lausanne, Institut de Géographie, coll. « Travaux et recherches ", $n^{\circ} 24$, p. 115-127.

TILDEN Freeman, Interpreting Our Heritage, The University of North Carolina Press, 1957, 1967, 1977, $119 \mathrm{p}$.

\section{NOTES}

1. F. Tilden, Interpreting Our Heritage.

2. Panorama du Défens, commune de Tartonne sur le circuit des «Hautes Vallées de l'Asse » mais également le panorama de Lèbre près de la commune d'Authon.

3. Extrait du texte de création du parc national de Yellowstone en 1872.

4. F. Tilden, Interpreting Our Heritage, p. 8-9.

5. F. Tilden, ibid, définition et principes en page 114 .

6. Ibid., définition et principes en page 20.

7. Ibid., définition et principes en page 41.

8. D. Aldridje. Principe of country interpretation.

9. R. Brunet, R. Ferras, H. Thery, Les Mots de la Géographie, p. 373-376.

10. Ph. Pinchemel, Lire les Paysages, p. 1-8.

11. M. Mattauer, Palimpsestes géologiques, p. 56-61.

12. J-P. Pralong, Valorisation et vulgarisation des sciences de la Terre : les concepts de temps et d'espace et leur application à la randonnée pédestre, p. 125. 
13. M. Mattauer, Palimpsestes géologiques, Pour la Science, p. 56-61.

14. E. Belgrano, D. Rimondi, Société et territoire : une approche qualitative.

15. R. Courtot, Agriculture, paysages et patrimoine, p. 115-119.

16. Comme il nous l'a souvent répété et aime lui-même à le dire.

17. Nadine Gomez en introduction de l'ouvrage de J. Fontcuberta, Volte face à l'envers de la science, les leçons d'histoire.

18. M-E. Férérol, « Le Massif du Sancy et Horizons - Arts Nature : quand Land Art rime avec attractivité », http://rga.revues.org/3750

19. Élévations de pierres plates en forme d'œuf.

20. Centre administratif et muséographique de la RNGHP, installé dans le Parc Saint Benoît à Digne-les-Bains, et autrefois dénommé « Centre de géologie Saint Benoît ».

21. Sylvain Guyot et Gabrielle Saumon, «La mise en art de la Blackfoot Valley (Montana, USA) ou comment (ré)concilier le front minier et le front écologique ?» : http://rga.revues.org/3690

22. «I want to get under the surface. When I work with a leaf, rock, stick, it is not just that material in itself, it is an opening into the processes of life within and around it. When I leave it, these processes continue ».

23. Construction en pierres rappelant les bories provençales.

24. Tous ces propos sont extraits du catalogue de cette exposition: J. Fontcuberta, Volte-face à l'envers de la science, les leçons d'histoire, $118 \mathrm{p}$.

25. Phineas Taylor Barnum (1810-1891), entrepreneur de spectacles nord-américain, créateur en 1871 du cirque du même nom et de la fameuse sirène des îles Fidji, une sirène factice mi-singe mi-poisson.

26. Anthropomorphisme: tendance à concevoir la divinité à l'image de l'homme. Syncrétisme : fusion de deux éléments culturels, religieux différents. (Le Petit Robert, 2001).

\section{RÉSUMÉS}

Un géosite correspond à un espace naturel complexe, ne se résumant ni à un site géologique ni à un paysage. Il est référencé scientifiquement par ses intérêts géologiques et par ses valeurs historiques, économiques et culturelles. La lecture de paysage introduit la dimension spatiale et culturelle du géosite en intégrant un ensemble d'informations nécessaires à son interprétation (Freeman Tilden, 1956). Cette approche permet d'intéresser le touriste à des secteurs d'un territoire dont il n'aurait pas perçu seul les richesses géologiques, historiques voire économiques. L'introduction de l'art contemporain dans l'interprétation de géosites associe l'esthétique, le symbolique, l'artistique en permettant d'intéresser un public plus large. L'association de l'art contemporain et de la lecture de paysage interroge le visiteur et l'incite à découvrir la variété des formes et structures visibles sur un territoire montagnard, géologiquement référencé. 
AUTEUR

CHRISTEL VENZAL

Maître de Conférences Aménagement - Géographie, Université de Pau et des Pays de l'Adour, Membre du laboratoire Passages (UMR 5319, CNRS) 


\title{
Les montagnes andines et ses
} habitants : le Pérou sous l'œil de la caméra de l'alpiniste français Lionel Terray (1952-1964)

\author{
Chloé Tessier-Brusetti
}

1 Lionel Terray naît à Grenoble en 1921 dans une famille de «bourgeois aisés », selon ses propres termes. Son père, après des études d'ingénieur chimiste, part fonder une industrie au Brésil ; mais la Seconde Guerre mondiale l'obligera à rentrer rapidement en France, avec sa femme, pour s'acquitter de son devoir de soldat. Dans son autobiographie ${ }^{1}$, Terray se confie sur l'originalité de sa famille : un père passionné de compétitions automobiles, qui reprend des études en médecine après quarante ans de vie d'affaires, une mère audacieuse qui skie en pantalon et conduit sa propre voiture à une époque où les femmes commencent tout juste à s'émanciper, des aïeux « grands voyageurs en quête de fortune et d'aventure ». Terray, lui, se considère avant tout comme un montagnard, tel que la littérature a figé ce personnage dans les esprits : « un rude paysan aux manières frustes" confie-t-il; il se décrit d'ailleurs de nature « réservée et timide $»^{2}$.

2 Pendant longtemps, Terray a imaginé que la photographie était «une cuisine magique $»^{3}$ difficile à maîtriser, et que les photographes-cinéastes qui accompagnent les expéditions ne servent pas à autre chose qu'à ternir son plaisir d'alpiniste. Mais en 1952, pendant sa première expédition dans les Andes, à l'occasion de l'ascension de l'Aconcagua, il découvre le plaisir de fixer des images sur la pellicule lorsque son ami Georges Strouvé 4 lui prête un appareil photo et une caméra. Emballé par les résultats de ses clichés et rushes qu'il jugera plutôt convenables, il voit dans ces images de formidables souvenirs des courses auxquelles il a participé, mais également des documents qu'il pourrait utiliser pour collecter des fonds et financer de nouvelles expéditions. Il se lance donc, dès 1952, dans la réalisation d'un premier film sur la descente à ski de la face nord du Mont-Blanc, dont il est le protagoniste, puis tourne quelques images sur la vie et les mœurs des populations de l'Himalaya lors de son 
ascension de l'Annapurna en 1955. L'exploitation de ces deux premiers films lui permet de financer sa seconde expédition dans les Andes en 1956, longue de six mois, mais qu'il décidera de prolonger, estimant qu'il est « stupide de se rendre si loin pour une période si courte ${ }^{5} »$. Deux mois de plus qu'il met à profit pour gravir deux cimes de plus de 8000 mètres et tourner des images sur la vie des Indiens Quechuas. Il rapporte de nouvelles bobines sur les Indiens des montagnes péruviennes en 1962, lors de la première de la face est du Chacraraju. Ces dernières bandes viennent compléter les images des premiers rushes pour constituer le film C'est le Pérou, monté par ses amis cinéastes Marcel Ichac et Jean-Jacques Languepin ${ }^{6}$. Trois ans plus tard, Terray meurt après une terrible chute lors d'une ascension dans le Vercors.

\section{Représentations françaises des Indiens du Pérou : antécédents des images de Terray}

Concernant les images ayant précédé celles tournées par Terray, l'historique des portraits des Indiens du Pérou proposés par des Français fait remonter les premières descriptions au XVII e siècle, après que la couronne espagnole a autorisé l'Académie des sciences de France à organiser des expéditions scientifiques dans les colonies américaines qu'elle contrôle jalousement. Mais c'est avant tout la nature latinoaméricaine qui intéresse les naturalistes (faune, flore, climat, minéraux, etc.). Il faut attendre le $\mathrm{XIX}^{\mathrm{e}}$ siècle, notamment sa seconde moitié avec le développement de l'archéologie, pour voir fleurir dans les rapports et carnets de voyage des explorateurs français des passages entiers consacrés aux Indiens des Andes. Dans le même temps, la naissance de l'égyptologie a fait naître un véritable engouement pour les " antiquités ", poussant les collectionneurs-archéologues amateurs à prendre le chemin du Pérou en quête d'objets anciens. Dans leurs notes, ils détaillent la progression de leur voyage, la découverte des sites pré-hispaniques et livrent leurs impressions sur la vie dans les Andes. Ainsi, Laurent Saint-Cricq (1815-1887), voyageur bordelais, membre de l'expédition menée par le comte de Castelnau ${ }^{7}$ dans les années 1840 décrit les différences qu'il observe entre les Indiens de la côte et ceux des montagnes. Le premier a :

«Le masque rond, le nez aplati, les lèvres lippues, les yeux étroits à sclérotique jaune, obliques et bridés par les coins comme ceux des Chinois et des races mongoles. "

Tandis que l'autre possède un :

«Faciès ovale, des pommettes saillantes, un nez en bec d'aigle, des yeux obliques mais bien fendus, une chevelure noire, abondante et lisse, [et] semble rattaché à la grande famille indoue [...]»

5 Il termine son observation en indiquant que :

«Du mélange des deux races de la côte et de la sierra, il est résulté avec le temps bon nombre d'hybrides, dont une laideur hébétée est le trait distinctif. ${ }^{8}$ "

Ces observations raciologiques, récurrentes dans les journaux des voyageurs qui découvrent le Pérou et ses habitants à cette période, s'accompagnent parfois même de théories originales qui affirment qu'en grandissant, il arrive aux enfants indiens :

«Comme aux races inférieures telles que le chimpanzé, l'orang-outan et le gorille, que la partie inférieure du crâne et les mâchoires s'avancent à la recherche de la pâtée.» 
7 Comme l'indique l'archéologue Théodore Ber dans les années $1870^{9}$. En considérant les Indiens comme une catégorie inférieure d'humains, sortes d'infra-hommes, les voyageurs européens ne font en réalité que reprendre les théories darwinistes d'évolution de l'homme et les études anthropologiques de l'époque qui s'emploient à distinguer et hiérarchiser les races. Mais le discours sur les Indiens évolue aussi : on les décrit toujours comme des hommes sales et primitifs, mais on considère aussi leur paresse et leur apathie comme le résultat d'une colonisation mal menée qui a conduit le Pérou à sa perte :

"Courbé sous la lame d'acier, l'Indien apprenait à se prosterner à genoux devant la croix espagnole. Tremblant de peur, ahuri, indifférent, répétant sans les comprendre les prières adressées à un Être qu'il ne connaissait point, l'Indien semble avoir désappris le travail en écoutant la messe. Les Indiens avaient été d'excellents agriculteurs, on en a fait des mauvais mineurs; aller à la mine c'était aller à la mort. C'est ainsi que l'activité s'est éteinte, et que la nature des Andes au caractère inhospitalier a reparu pendant que les vastes cultures dont l'Indien l'avait recouverte se rétrécissaient de plus en plus. ${ }^{10}$ ”

Vers la fin du XIX ${ }^{e}$ siècle, au moment où l'ethnologie se développe, on craint désormais de voir disparaître les «races pures» et leurs mœurs sous l'effet du processus de métissage. L'invention de la photographie permet de figer les traits de ces gens ; ainsi, en 1889 , la société d'Anthropologie de Paris invite les explorateurs à réaliser des clichés des populations qu'ils rencontrent :

«Il faut réunir les portraits des hommes, des femmes, des enfants, des vieillards, des adultes surtout, avec les costumes, [...] Il faut se hâter car l'unification marche à grands pas. ${ }^{11}$ "

Ces portraits "ethniques", bien souvent, se rapprochent davantage des "natures mortes ", les Indiens posant devant une toile unie dans des positions parfois peu naturelles. Cette chosification en est d'autant plus accentuée lorsque les titres des photos réduisent les modèles à des catégories de métiers ou à des lieux, leur refusant ainsi toute identité individuelle, comme les photos que fit Charles Wiener en $1875^{12}$. Une trentaine d'années plus tard, les époux d'Harcourt (Raoul et Marguerite) sillonnent le Pérou pour enregistrer la musique traditionnelle des Indiens Quechuas. Ils craignent "l'effacement de l'élément indigène en faveur d'une musique affreusement mélangée ${ }^{13}$ ", abâtardie sous l'influence de la musique occidentale apportée par l'arrivée du phonographe, de la radio et du flot des touristes. Les photos que ramèneront les d'Harcourt de leurs explorations de terrain de 1913 et 1919 contrastent avec celles de la fin du siècle passé. Ce ne sont plus des portraits, mais des vues d'ensemble d'une place ou d'une rue, où se trouvent des gens; les photos sont prises sur le vif, sans préparation, capturant un moment de vie ${ }^{14}$. Les travaux des d'Harcourt et d'autres ethnologues s'intéressent désormais non plus aux Indiens, mais à leur vie quotidienne et à leurs pratiques culturelles: musique, savoirs techniques, langues, etc. Ce déplacement des objets d'étude illustre l'évolution du regard scientifique qui reconnaît à présent la diversité culturelle chez les autochtones des Andes. Mais si on étudie les caractéristiques des langues ou des vêtements de telle ou telle communauté, c'est pour consigner leurs spécificités avant que ce patrimoine immatériel ne disparaisse. Car c'est dans l'ordre des choses, l'unification de la population est la prochaine étape de l'histoire latino-américaine et il faut encourager l'assimilation «des races autochtones ", comme le dit Paul Rivet. Ce spécialiste des langues sub-amérindiennes, directeur du Musée de l'Homme de 1937 à 1949, écrit en 1948 un article intitulé « Sur 
l'Amérique latine : propos d'un ami ", dans lequel il livre ses espoirs pour la résolution $\mathrm{du}$ " problème indien » en Amérique latine. Il indiquera que pour ce faire :

«Il s'agit de chercher les meilleures méthodes pour éduquer ces masses allogènes, leur donner, peu à peu, une culture d'inspiration blanche et les incorporer à la nation. ${ }^{15}$ "

$10 \mathrm{Au}$ début des années 1950 naît le Programme Indien Andin (1953-1974), un plan d'assistance technique créé par l'agence des Nations Unies, qui a pour but d'accompagner les Indiens dans leur « développement » économique, social et culturel (programmes linguistiques, professionnels, éducatifs, etc.). Des anthropologues sont intégrés au programme pour apporter leur regard d'experts sur la faisabilité et la mise en place de ces projets. L'anthropologue suisse Alfred Métraux participe à une mission en 1954 au Pérou, puis en Bolivie deux ans plus tard. Dans un article publié dans la revue Le Courrier de l'UNESCO, il explique que « l'amélioration de leur niveau de vie est inconcevable sans un renouvellement de presque tous les aspects de leur culture hybride ", n'hésitant pas à reprendre des images stéréotypées qui les dépeignent comme des êtres arriérés végétant dans leur saleté :

«Ce n'est pas sans raison qu'ils ont la réputation d'être parmi les peuples les plus sales de la terre! L'inventaire des maladies dont ils sont atteints a de quoi faire frémir. ${ }^{16} "$

11 Les documents audiovisuels français consacrés au Pérou sont plutôt récents, et les films qui précèdent ceux tournés par Lionel Terray sont particulièrement rares. Une recherche dans les archives du Centre National du Cinéma et de l'Image Animée et dans celles de l'Institut National de l'Audiovisuel montre qu'avant 1952, seuls un film et un documentaire ont été diffusés en France : un document muet de 1931 au sujet de la récolte du guano au Pérou, commandé par le Ministère de l'Agriculture, et la version française d'un reportage allemand sur l'histoire préhispanique du Pérou, diffusé en $1950^{17}$. Le premier n'offre que de rares plans sur les travailleurs péruviens ramassant l'engrais naturel. Le second s'attarde largement sur l'héritage inca dans les pratiques culturelles indiennes; on note dans les commentaires un manque d'aisance dans la manipulation des termes autochtones («Titicac»), et quelques mélanges et approximations (notamment l'amalgame avec les cultures préhispaniques maya et aztèque qui n'appartiennent ni à la même aire géographique ni à la même période). Ceci montre à quel point le Pérou est loin de l'hexagone et méconnu. La démocratisation de la télévision dans les foyers français dans les années 1950 et le développement des expéditions anthropologiques vont favoriser l'émergence d'émissions dédiées à la découverte de la vie dans contrées lointaines, telles que "Voyage sans passeport ", qui diffuse 156 reportages entre 1956 et 1969 ; parmi eux, 5 numéros sont consacrés au Pérou entre 1959 et 1960 . Trois d'entre eux sont des images tournées par Lionel Terray : il s'agit en réalité de son film muet «Hommes et cimes du Pérou » qui a été divisé en trois parties et adapté au format de la télévision ${ }^{18}$. Les commentaires de la voix off sont ceux des réalisateurs de l'émission qui apportent un éclairage sur le regard que la société française portait sur les Indiens péruviens à cette époque. Les Indiens y sont présentés comme des gens « honnêtes, travailleurs et hospitaliers "; on décrit la splendeur des tissus qu'ils réalisent grâce aux techniques héritées de leurs ancêtres incas et on applaudit leur agilité pour attraper le bétail. Mais les remarques positives sont assez rares et le discours est presque unilatéral; il insiste avant tout sur leur caractère primitif et la vie de débauche qu'ils mènent. Ce sont des 
«misérables » vêtus de "loques » qui vivent dans des « huttes plus que sordides » ou des « maisons en pisé parfois délabrées »; la forte mortalité infantile est due à :

«L'usage excessif d'alcool, [...] l'effet toxique de la coca et aussi un total manque d'hygiène. "

12 La conclusion est sans appel, pour remédier à la :

«Décadence de la race indienne [...] il suffirait de peu de chose [...], probablement un léger travail d'éducation. »

13 L'image qui est donnée des autochtones est encore fortement influencée par le sentiment de supériorité européen qui a fait du progrès le critère fondamental d'évaluation des sociétés, ce techno-centrisme qui a servi les desseins coloniaux et plus tard les politiques interventionnistes comme le Programme Indien Andin par exemple.

\section{Regard de Lionel Terray sur les Indiens : analyse des images rapportées du Pérou}

14 Une partie des images tournées au Pérou par Lionel Terray et conservées par la famille a été numérisée, archivée et mise en ligne par la Cinémathèque d'Images de Montagne de Gap (CIMALPES) : plusieurs heures de bobine, à la fois des rushes, des films montés et des chutes d'images. Les lieux fixés sur la pellicule sont ceux que Terray a visités : la région cusquénienne, l'Altiplano et le littoral.

Dans l'ensemble des images qu'il rapporte du Pérou, on peut identifier trois périodes de tournage, qui correspondent à ses trois expéditions dans les Andes (1952, 1956 et 1962), et huit films d'une durée variant entre 15 et 25 minutes approximativement. La moitié traite des expéditions et des ascensions, l'autre moitié dépeint les autochtones dans leur vie quotidienne ${ }^{19}$. Le dernier film C'est le Pérou est un montage réalisé à partir de ses différents documents sur la vie des Indiens.

16 Trois grandes thématiques se détachent de ce corpus d'images: d'abord, la nature andine, avec ses vastes paysages et la démesure de la Cordillère; ensuite, les occupations quotidiennes des habitants, comme les tâches agricoles (l'élevage de camélidés, les récoltes) ou les travaux d'artisanat (la confection des vêtements traditionnels); enfin, les fêtes et les cérémonies rituelles (danses costumées, processions catholiques, célébration de l'Inti Raymi ${ }^{20}$ ). Il s'attarde d'ailleurs largement sur cette dernière thématique, et filme notamment la fin des fêtes où l'ébriété des gens est manifeste. On note que Terray cherche à filmer la vie de ces gens telle qu'elle s'offre à lui, dans sa différence et son authenticité : il propose un portrait pittoresque de la vie dans les Andes. Mais on ne peut pas nier, non plus, la poésie qui se dégage des images lorsqu'il filme les paysages de la Cordillère ou la côte Pacifique, qui rappelle sans aucun doute les descriptions romantiques de certains explorateurs européens du XIX $x^{e}$ siècle. Ainsi, Terray offre au spectateur des images qui correspondent avant tout à ce qu'il est et ce qu'il est venu chercher: un explorateur curieux en quête de rencontres et d'aventures.

17 Les plans d'ensemble l'emportent largement dans la construction de ces images. Terray opte très rarement pour des plans rapprochés ou serrés, préférant les plans larges ou moyens qui lui permettent d'offrir une vision globale d'un groupe ou d'un lieu animé, qu'il balaye parfois de sa caméra dans un panoramique horizontal. Cette manière de filmer montre à quel point il veut «embrasser » largement les scènes auxquelles il 
assiste, comme s'il voulait ne rien rater et rapporter le plus de choses. D'un autre côté, on voit clairement que ce qui l'intéresse n'est pas l'homme indien en tant qu'individu, mais les hommes indiens ou plutôt la culture indienne. Ceci montre que Terray conçoit les Péruviens autochtones comme appartenant à une catégorie ethnique homogène, ne distinguant ni les identités individuelles, ni la diversité des communautés. Il filme sans distinction les Quechuas de Ollantaytambo près de Cuzco, et les Aymaras de Puno dans l'Altiplano, les englobant dans une seule et même catégorie: les Indiens Quechuas. Ils ne partagent pourtant ni la même langue ni la même origine ethnique.

En prenant soin de ne jamais apparaître sur la pellicule lorsqu'il filme les Péruviens, Terray s'inscrit ouvertement dans une démarche testimoniale. Il semble tenir à ce que ses vidéos se rapprochent le plus possible d'une observation, d'une collecte d'images, comme le proposent les documents ethnographiques des chercheurs auxquels il se réfère parfois (Alfred Métraux, par exemple) ou les documentaires qui font leur apparition à la télévision française dans les années 1950, placés sous l'égide d'explorateurs bien connus, tels que Paul Rivet ${ }^{21}$. Les images de Terray, bien qu'enregistrées en $16 \mathrm{~mm}$ sans son, présentent parfois un accompagnement sonore lorsqu'elles sont montées : musique ou commentaires, voire les deux. Dans le premier cas, Terray choisit d'apposer systématiquement sur la bande de la musique traditionnelle andine (guitare, flûte de pan, percussions), ce qui a probablement pour objet de donner davantage d'authenticité encore, même si on perçoit parfois, dans les séquences de danses et de défilés rituels, que le rythme de la musique, captée lors d'une autre fête, ne s'accorde pas avec les images. On ne sait d'ailleurs pas exactement quelle est cette musique ni d'où elle est tirée, Terray se contentant d'indiquer, dans le bandeau d'introduction, "Musique du folklore péruvien ». Les commentaires, quant à eux, sont destinés à expliciter le contenu des images pourtant déjà très explicites. En dehors de cette redondance assez caractéristique des documentaires de l'époque, les explications, proposées par le vidéaste lui-même, décrivent ici les costumes traditionnels, là les techniques de battage de l'orge, ou encore les particularités des corridas andines. Dans son exposé, il livre également au spectateur ses sentiments sur la vie des Indiens Quechuas et, tout comme les propos qu'il tient dans son autobiographie, ses remarques laissent transparaître une représentation des Indiens assez ambivalente.

La manière dont il parle de la vie des gens qu'il observe laisse percevoir, d'emblée, sa curiosité pour l'autre (sans doute un héritage familial que ses expéditions autour du monde n'ont fait que renforcer). Il montre qu'il a conscience de la diversité culturelle en s'appliquant à décrire :

«[...] la couleur et l'originalité des vêtements, la bizarrerie des coutumes [qui] provoquent parfois un dépaysement plus grand encore que celui qu'on éprouve au cœur de l'Asie. ${ }^{22}$ »

Il reconnaît l'autre dans sa différence et c'est précisément ce qui le pousse dans sa démarche cinématographique : il se plaît à faire découvrir une autre façon de vivre. Les termes qu'il emploie traduisent un engouement pour ces terres et ses habitants; il se dit «[...] littéralement envoûté par le charme sauvage qui se dégage de ce peuple d'Indiens", qu'il décrit comme "magnifiques et colorés " ou encore " hospitaliers et aimables ${ }^{23}$ \#. Il ne tarit pas d'éloges, non plus, sur les savoirs transmis depuis l'époque incaïque, «la précision admirable » avec laquelle les blocs de pierre du Machu Picchu ont été taillés et encastrés, et "les siècles de patience et d'expérience ", dit-il, qui ont permis de domestiquer la vigogne ${ }^{24}$. On repère néanmoins dans le discours de Terray 
quelques adjectifs qui contrastent avec cette vision exaltée : « les pauvres indiens », «le misérable peuple des Andes », «les misérables indiens ${ }^{25}$ ». S'ils traduisent une forme d'apitoiement sur le sort de ces gens lorsqu'il décrit la situation d'asservissement que connaissent les Indiens des haciendas ou le dénuement dans lequel ils vivent, ces termes évoquent également un certain mépris, notamment lorsqu'ils sont assortis d'autres formules infériorisantes :

« [...] nous étions partis à trois dans un massif sauvage à peine peuplé par quelques misérables indiens que quatre siècles de conquête ont ramenés à l'état de bêtes

farouches..$^{26}$ "

D'autres descriptions font écho à ces considérations typiquement ethnocentrées, certaines juste suggérées, d'autres non dissimulées : ainsi apprend-on que les Indiens sont "malpropres et grossiers", "voleurs et brutaux", "sournois et ivrognes ${ }^{27}$ ", "qu'ils ont renouvelé l'exploit de la préhistoire» en poursuivant l'élevage des camélidés, ou encore que " c'est au treizième siècle - [alors que] chez nous, Notre Dame de Paris était déjà bâtie - que leur petite tribu montagnarde fonda [...] $\mathrm{Cuzco}^{28} »$. Terray dépeint donc les Indiens également comme un peuple de sauvages, primitifs, arriérés, ce qui n'est pas sans rappeler certains discours chez les chroniqueurs de l'époque coloniale. Ainsi, derrière cette caméra qui se veut objective et qui incarne la mutation de la pensée qui est en train de s'opérer dans les années 1950, qui commence à considérer l'Autre et essayer de le comprendre, se cache en réalité un regard qui ne parvient pas encore à s'affranchir de l'héritage de la pensée impérialiste européenne.

\section{Considération pour les Indiens au Pérou}

À cette époque, au Pérou, les populations des montagnes andines constituent une masse encore fortement marginalisée, malgré les réformes, les décrets et les lois promulgués pour gommer la fracture sociale entre Indiens et non-Indiens et " améliorer " leurs conditions de vie. Au Xvi siècle, déjà, des voix se sont élevées pour dénoncer l'usurpation des droits des Indiens et leur exploitation, comme le fit Bartolomé de las Casas, entraînant la création des Nouvelles Lois qui n'ont cependant pas fait que profiter aux Indiens (la suppression de la perpétuité des encomiendas, puis la suppression complète des encomiendas, en 1542, a permis à la Couronne espagnole de récupérer la pleine possession de ces terres et des Indiens qui y vivaient). Mais par la suite, et tout au long de la période coloniale, les différentes révoltes indiennes se sont systématiquement soldées par de violentes répressions et des exécutions magistrales (décapitation, écartèlement sur la place publique, dont l'exemple le plus connu est certainement celui de Túpac Amaru ${ }^{29}$ exécuté publiquement en 1572). Plus tard, au moment des indépendances latino-américaines, on a proclamé l'égalité des Blancs et des Indiens et construit les jeunes républiques à partir de ce critère. En 1815, Simón Bolívar, protagoniste clé de la guerre des indépendances latino-américaines, posait pourtant un regard générique, réducteur et méprisant sur "l'Indien», le décrivant comme un bon sauvage doux, pacifique, indolent et sans ambition :

«L'Indien est de caractère paisible; il ne souhaite que le repos et la solitude : il n'aspire pas à commander sa tribu, encore moins celle des autres [...]. Il ne réclame aucune autorité, car il n'en a pas l'ambition ni ne pense être apte à l'exercer, se contentant de sa tranquillité, sa terre et sa famille..$^{30}$ " bipolarisation de la société, a cherché à intégrer officiellement les Indiens et a déployé 
un arsenal de mesures et de lois en ce sens. Inscrit dans une démarche de construction nationale, le nouvel État place ces mesures dans un projet d'homogénéisation de la nation - projet clairement assimilationniste. En 1825, plusieurs décrets en faveur des Indiens sont promulgués par Bolívar à Cuzco, dont l'interdiction du service personnel aux Créoles (réduisant l'exploitation tacite des Indiens) et la répartition des biens des communautés indiennes en lots (sensée développer la production agraire sous l'effet de la propriété privée). Paradoxalement, Bolívar s'oppose aussi farouchement à l'intégration raciale des Indiens dans la société, c'est-à-dire au métissage : cela signifie, pour lui, la mise en marche d'une abâtardisation des races et la dégénérescence de la population américaine ${ }^{31}$. De son côté, le général José de San Martín (1778-1850), protecteur de la nouvelle république du Pérou, dans un décret qu'il publie au lendemain de la déclaration d'indépendance, annonce que :

«Désormais les autochtones ne seront plus appelés Indiens ou naturels; en tant qu'enfants et citoyens du Pérou, ils doivent être connus sous le nom de Péruviens. ${ }^{32}$

Cet ajustement terminologique est hautement symbolique et illustre à quel point on cherche à faire disparaître la composante ethnique indienne de la réalité sociale péruvienne en «l'absorbant». Si les Indiens sont bien des citoyens péruviens, leur condition sociale influera cependant directement sur certains de leurs droits : au fil des Constitutions, les Péruviens autorisés à voter devront, par exemple et selon les époques, être propriétaires, ou bien savoir lire et écrire, écartant de ce fait une grande majorité d'Indiens ${ }^{33}$.

Le début $\mathrm{du} \mathrm{xx}^{\mathrm{e}}$ siècle constitue une période charnière; il marque le début de "l'indigénisme", ce mouvement social, politique et artistique qui plaide la cause des masses indiennes opprimées et réfléchit à des mesures en leur faveur. L'essayiste, philosophe et théoricien radical Manuel González Prada (1844-1918) publie dès 1904 Nuestros Indios : il condamne le darwinisme social qui soutient que les races ont des capacités inégales et que « l'Indien » est « réfractaire à la civilisation ${ }^{34}$ ». Dans son essai, il juge que seuls les Indiens ont le pouvoir de mettre fin à l'oppression qui les maintient asservis depuis la conquête; il les appelle à se soulever sans attendre, leur suggérant notamment d'investir dans des armes et des balles plutôt que de gaspiller leur argent dans l'alcool et les fêtes et d'attendre que le cœur des Blancs s'attendrisse sur leur sort $^{35}$. En plus de l'image générique et stéréotypée - «l'Indien » ivrogne et apathique -, on remarque également dans son discours l'expression « le problème indien » (qui sera reprise par d'autres plus tard ${ }^{36}$ ), formulation qui laisse envisager que «l'Indien » est le problème et non que la société $\mathrm{a}$ un problème avec les Indiens.

Avec la nouvelle constitution de 1920, le gouvernement péruvien, qui s'inscrit dans une dynamique de modernisation du pays, propose des mesures qui octroient des droits spéciaux aux communautés indiennes: reconnaissance de la diversité ethnique et culturelle, reconnaissance de l'existence juridique des communautés indiennes et imprescriptibilité de ce statut ${ }^{37}$. Cependant, la politique indigéniste du président Augusto Bernardino Leguía (1863-1932) répond avant tout au projet national ${ }^{38}$ de modernisation du pays. S'il se considère comme un défenseur de " la race indienne » et qu'il soutient les actions des organisations pro-Indiens indépendantes (engagées dans la défense des Indiens au tribunal ou dans des campagnes d'alphabétisation), il cherche rapidement à les contrôler pour orienter les programmes ${ }^{39}$. L'objectif prioritaire est de favoriser le « développement » des communautés pour faciliter leur intégration dans la 
société péruvienne. L'État promulgue par ailleurs, en 1924, un nouveau code pénal (connu comme Código Penal Maúrtua) qui propose des peines spéciales pour les Indiens " sauvages [...] semi-civilisés ou abrutis par l'esclavage et l'alcoolisme ${ }^{40}$ ». Ce code pénal restera en vigueur jusqu'en 1991. La constitution de 1933 apporte quelques changements notables, particulièrement concernant les biens fonciers des Indiens (l'État s'engagera par exemple à fournir des terres aux plus démunis et à garantir leur inaliénabilité), mais l'alphabétisation reste une condition à l'exercice du droit de vote.

En 1946, sous l'impulsion de l'Institut Indigéniste Interaméricain (créé en 1940, ayant son siège à Mexico) où sont discutées les politiques indigénistes de chaque pays sudaméricain, naît l'Institut Indigéniste Péruvien. Organe du ministère du Travail qui se maintiendra jusqu'en 1981, il a pour but de réfléchir à la création de nouvelles mesures, de faire appliquer les lois et de coordonner les projets. Certains lui reprocheront, plus tard, d'avoir élaboré des programmes destinés à lisser l'image de l'Indien pour qu'il s'insère dans l'idée de nation qui était en train de mûrir: ce sont les programmes éducatifs qui reposaient sur la castillanisation, les programmes de santé réduits à une sensibilisation à l'hygiène et parfois, la protection des traditions anciennes, pour que soit exposé dans les musées ce qui fait l'orgueil du pays ${ }^{41}$.

Pendant el Ochenio du général Odría ${ }^{42}$, on continue à réfléchir aux manières de résoudre «le problème indien» dans une démarche assimilationniste. Les programmes s'inscrivent désormais dans une perspective scientifique et les Indiens deviennent objet d'étude. L'État péruvien signe, par exemple, un accord avec l'Université nordaméricaine de Cornell qui mène un projet anthropologique expérimental dans la région de Ancash, destiné à moderniser les Indiens qui vivent à Vicos. Là-bas, une hacienda est transformée en terrain d'étude et d'application: on observe les résultats d'apprentissage de quelque 2000 Indiens à qui on enseigne les modèles de la vie moderne (notamment la technologie agraire). Dans un long article intitulé "L'anthropologie culturelle et notre problème indien: Vicos, un cas d'anthropologie appliquée ", l'un des anthropologues en charge du projet, le Péruvien Mario Vázquez Várela, indiquait à l'époque que la société péruvienne reposait sur un socle culturel binaire avec, d'un côté, des modèles de culture occidentale et, de l'autre, des modèles de peuples arriérés ${ }^{43}$. Ceci laisse entrevoir le regard que le gouvernement péruvien pose sur la population indienne au moment où Lionel Terray tourne ses images.

Les représentations des Indiens proposées par la société péruvienne de cette première moitié du $\mathrm{xx}^{\mathrm{e}}$ siècle, notamment dans la photographie et les films, illustrent, quant à elles, l'autre versant du mouvement indigéniste. Car, si avant les années 50, les Indiens ne sont pas représentés dans le cinéma péruvien ${ }^{44}$ - illustrant bien la négation de leur existence -, le Club de Cuzco, à l'inverse, fera de l'identité indienne son sujet de prédilection dès 1955. Bien que l'industrie du cinéma au Pérou ne parvienne pas à se développer, écrasée sous le poids des productions nord-américaines ou mexicaines et abandonnée par le gouvernement péruvien, un groupe de cinéastes indiens s'engage dans la célébration de la culture indienne et la dénonciation de sa marginalisation, comme d'autres le feront en littérature ${ }^{45}$. Ainsi, plusieurs courts ou longs métrages autofinancés (et donc éloignés des modèles que veut imposer le gouvernement) sortent dans les années 1950 : Corpus en la ciudad de Cusco (1955), Corrida de toros y cóndores (1956) de Manuel Chambi (en coréalisation avec Eulogio Nishiyama) ou encore Policromía andina (1958) de Luis Figueroa Yábar. Tournés comme des documentaires, ils s'inscrivent dans une volonté d'intégrer l'identité indienne dans le cinéma péruvien. Ils 
se situent par ailleurs dans la lignée des premiers photographes péruviens indigénistes, Martín Chambí (originaire de l'Altiplano) et Daniel Cisneros Cáceres (natif de la région de Cuzco) qui ont accordé une large place aux Indiens dans leurs travaux. Pendant les premières décennies $\mathrm{du} \mathrm{xx}^{\mathrm{e}}$ siècle, les clichés saisis au Pérou ont fait la part belle aux portraits de studios, pensés comme des tableaux figés, commandés par des clients argentés (en grande majorité créoles ou métisses); et lorsque les photographes sortaient de leur atelier, ils choisissaient d'immortaliser des sujets statiques qui ne risquaient pas de compromettre la netteté de la photo (vues panoramiques de grandes villes, monuments, montagnes) ${ }^{46}$. La photographie de "plein air ", qui se développe en même temps que l'évolution des techniques, a ouvert de nouvelles perspectives: les photographes se sont alors intéressés aux scènes de vie et aux différentes classes socioprofessionnelles dans leurs activités (militaires, mineurs, religieux, écoliers, ouvriers $)^{47}$. La diversification des thèmes photographiés, à un moment où fleurissent les arts indigénistes qui mettent en valeur l'identité autochtone, permet progressivement aux Indiens de figurer parmi les sujets d'intérêt pour certains photographes, surtout ceux d'origine indienne. C'est ainsi que Martín Chambi (1901-1973) et Daniel Cisneros Cáceres (1901-1994), après avoir appris la photographie à Arequipa, retournent dans les hautes terres pour documenter la vie des hommes et des femmes des Andes, leurs fêtes et leurs coutumes. Martín Chambi deviendra l'un des photographes péruviens les plus reconnus internationalement. Ses photos, qui oscillent entre témoignage documentaire et esthétisme, sont des fenêtres qui s'ouvrent sur le monde andin autant que des miroirs qui reflètent l'identité du photographe. À sa mort, il laisse plus de 30000 négatifs; on lui consacrera des expositions dans différents pays d'Amérique latine, en Europe et aux États-Unis.

Les films-portraits des Indiens du Pérou que propose Lionel Terray correspondent sans doute au regard que la France et l'Occident portent sur les populations des montagnes andines à la même époque. Ils donnent à voir plusieurs images de «l'Indien", parfois très réductrices et stéréotypées (comme l'illustre l'emploi de l'article défini singulier et générique, qui rejette les identités plurielles indiennes), d'autres fois romantiques ou encore pittoresques. Elles reflètent probablement la mutation du regard de la société française, à cheval entre ethnocentrisme hérité de la pensée coloniale, curiosité de touriste et reconnaissance de l'Autre. Elles traduisent également la personnalité polyfacétique de Terray : un explorateur conquérant, mais aussi un aventurier curieux. On repère toutefois une évolution dans les images qu'il ramène du Pérou : les premières insistent davantage sur l'aspect rudimentaire de la vie des Indiens, la misère et leurs déviances, offrant ainsi l'image d'une population arriérée; les dernières montrent plutôt les savoirs et traditions hérités de la période pré-hispanique, montrant la vivacité des traditions et la force de l'identité indienne. L'une comme l'autre concorde avec l'image que le gouvernement péruvien de l'époque utilise pour assimiler cette population exclue: des Indiens qu'il faut moderniser, mais dont le folklore doit être préservé. 


\section{BIBLIOGRAPHIE}

BOLÍVAR Simón, Reflexiones políticas, Linkgua Digital, 2004.

FAVRE Henri, « Bolívar y los indios », Historica, vol. X, n 1, 1986, p. 1-18.

GONZÁLES Osmar, « El Instituto Indigenista Peruano : una historia por conocer », dans GIRAUDO Laura et MARTÍN-SÁNCHEZ Juan (dir.), La ambivalencia del Indigenismo. Campo Interamericano y trayectorias nacionales 1940-1970, Lima, IEP, 2011, p. 133-158.

GONZÁLEZ PRADA Manuel, « Nuestros indios », Latinoamerica, Cuadernos de cultura latinoamericana, 29, Mexico, Universidad Nacional Autónoma de Mexico, 1905.

HARCOURT Raoul \& Marguerite (d'), « La musique des Aymaras sur les hauts plateaux boliviens », Journal de la Société des Américanistes, 48, 1959, p. 5-133.

MARCOY Paul, Voyage à travers l'Amérique du Sud, de l'Océan Pacifique à l'Océan Atlantique par Paul Marcoy, Paris, Hachette, 1869, volume I.

MARIÁTEGUI José Carlos, Siete Ensayos de Interpretación de la Realidad Peruana, chapitre II, Lima, Editorial Minerva, 1928.

MÉTRAUX Alfred, «Les Indiens des Andes souffrent... de "la faim de la terre" " , Le Courrier de l'UNESCO, année 8, 2, 1955, p. 4-9.

Photographie ethnique. Participation à l'Exposition universelle de 1889 de la Société d'Anthropologie de Paris, Société d'Anthropologie de Paris, Paris, Imprimerie nationale, 1889. RIVET Paul, «Sur l'Amérique latine : propos d'un ami », Annales, Économies, Sociétés, Civilisations, $3^{\mathrm{e}}$ année, 4, 1948, p. 393-400.

RIVIALE Pascal, GALINon Christophe, Une vie dans les Andes : le journal de Théodore Ber (1864-1896), Paris, Ginkgo, 2014.

TERRAY Lionel, Les conquérants de l'inutile, Paris, Gallimard, 1961.

VARELA VÁZQUEZ Mario, « La antropología cultural y nuestro problema indio : un caso de antropología aplicada », Perú Indígena 2, n 5-6, 7-157.

WIENER Charles, Voyage au Pérou et en Bolivie (1875-1877), Paris, Ginkgo, 2010 [1880].

\section{NOTES}

1. L. Terray, Les conquérants de l'inutile, 1961.

2. Ibid., p. 3-4.

3. Ibid., p. 425

4. Georges Strouvé (1927-1988), photographe, fondateur des Cahiers de l'AFC (cahiers de l'association des directeurs de la photographie cinématographique).

5. Ibid., p. 442.

6. Marcel Ichac (1906-1994) cinéaste, explorateur et alpiniste, considéré comme l'un des grands spécialistes du film de montagne ; Jean-Jacques Languepin (1924-1994) cinéaste de montagne, président du Groupe de recherches et d'essais cinématographiques. 
7. François-Louis Nompar de Caumont La Force de Castelnau (1802-1880), fils illégitime de Joséphine de Caumont La Force et, selon certains auteurs, du roi d'Angleterre Georges IV. Explorateur, consul des États-Unis au Pérou en 1841-1842, puis de France, à Bahia, au Cap, Bangkok, Honolulu et Melbourne, membre de la société de géographie en 1851.

8. P. Marcoy, Voyage à travers l'Amérique du Sud, de l'Océan Pacifique à l'océan Atlantique par Paul Marcoy, p. 60. (Paul Marcoy est le pseudonyme sous lequel Laurent de Saint-Cricq a écrit).

9. Théodore Ber (1820-1900), secrétaire de Delescluze, ancien communard, s'établit à Lima en 1871. Il mène une mission archéologique dans les Andes, concurrente de celle de Wiener en 1875. Voir P. Riviale \& C. Galinon, Une vie dans les Andes: le journal de Théodore Ber (1864-1896), p. 408.

10. C. Wiener, Voyage au Pérou et en Bolivie (1875-1877), p. 303.

11. Photographie ethnique. Participation à l'Exposition universelle de 1889 de la Société d'Anthropologie de Paris, p. 303.

12. Charles Wiener (1851-1913), archéologue et diplomate, parti en mission archéologique officielle au Pérou entre 1875 et 1877. Musée du Quai Branly, Fond photographique, PP0024397 à PP0024412.

13. Raoul d'Harcourt (1879-1971), arrivé en 1912 comme directeur du port de Callao, ne cessera pas ensuite de parcourir le pays, accompagné de sa femme Marguerite, devenant notamment un grand spécialiste du textile préhispanique. Marguerite, musicienne de formation, s'est passionnée pour le folklore musical indien. Voir R. et M. d'Harcourt, «La musique des Aymaras sur les hauts plateaux boliviens », p. 6.

14. Musée du Quai Branly, Fond photographique PP0010186, PP0010189, PV0010189, PV0036341.

15. Paul Rivet (1876-1958), médecin anthropologue, homme politique, américaniste, arrive pour la première fois en Amérique latine en 1901 pour une mission géodésique en Équateur jusqu'en 1907. Il se fait connaître par la suite pour ses travaux sur les langues aymara et quechua. Voir P. Rivet, «Sur l'Amérique latine : propos d'un ami », p. 395.

16. Alfred Metraux (1902-1963), découvre l'Amérique latine en Argentine avec ses parents dans la région de Mendoza. Il suit la formation de l'École des chartes et des Langues orientales au début des années 1920, puis soutient une thèse de doctorat à la Sorbonne en 1928 sur les Tupinambas. Jusqu'en 1938, il parcourt les hauts plateaux boliviens et conduit une expédition à l'Ile de Pâque en 1934.Voir son article "Les Indiens des Andes souffrent... de "la faim de la terre" ", p. 6.

17. «Le guano naturel du Pérou» (1931). «Au pays des Incas» (1950). Archives de l'INA.

18. "Pérou: Cuzco et Lima", "Pérou: les Andes» (1959). "Pérou», "Pérou: l'Altiplano », et «Pérou: le lac Titicaca » (1960). Numéros de l'émission Voyage sans passeport. Archives de l'INA.

19. Victoire sur le Huantsán (1952). Le monde du vertige; Fête quechua; Hommes et cimes du Pérou : Soray; Hommes et cimes du Pérou : Cusco, Arequipa, Lima ; Hommes et cimes du Pérou : Deuxième partie, Cusco; De Ande expeditie (1956). C'est le Pérou (1964). Archives du CIMALPES. 
20. Fêtes du soleil en langue Quechua marquant le solstice d'hiver dans l'hémisphère Sud, à la date du 24 juin (Wawa inti raymi, celle du soleil naissant) et le solstice d'été à la date du 21 décembre (Capaq inti raymi, celle du grand soleil).

21. Le premier numéro du Magazine des explorateurs a été présenté par Paul Rivet le 23 février 1956.

22. L. Terray, Les conquérants de l'inutile, p. 446.

23. Ibid., p. 424.

24. C'est le Pérou.

25. C'est le Pérou et L. Terray, Les conquérants de l'inutile, p. 423.

26. Ibid., p. 423.

27. Ibid., p. 424.

28. C'est le Pérou.

29. Tupac Amaru (1535-1572), dernier Sapa Inca, ou dernier empereur cuzquénien, fils de Manco Capac II et demi-frère d'Athahualpa, le dernier empereur inca régnant à Cuzco, exécuté par les Espagnols en 1531.

30. S. Bolívar, lettre du 28 septembre 1815 faisant partie de la série d'articles qu'il publia comme des lettres dans The Royal Gazette of Kingston.

31. H. Favre, «Bolivar y los Indios", p. 14. L'auteur prend appui sur plusieurs lettres. Bolívar est convaincu que la population refusera, de toute façon, de se mélanger aux Blancs qui les ont dominés pendant des centaines d'années.

32. Décret du 27 août 1821.

33. Constitution de 1823 (article 17), constitution de 1826 (article 14). La loi électorale de 1890 écarte les analphabètes des élections.

34. M. González Prada, Nuestros indios, p. 14.

35. Ibid. p. 19.

36. C. Mariátegui, Siete Ensayos de Interpretación de la Realidad Peruana.

37. Article 58 de la Constitution de 1920.

38. Président du Pérou de 1908 à 1912 et de 1919 à 1930 ; deux fois réélu en 1924 et 1929, renversé par le général Sanchez Cerro le 25 août ; arrêté, il meurt au pénitencier de Lima.

39. Il remplacera par exemple en 1924 le Comité Pro-Derecho Indígena Tawantinsuyu, indépendant, par le Patronato de la Raza Indígena, dépendant de l'État.

40. Article 44 et 45 du Code pénal de 1924.

41. O. Gonzáles, El Instituto Indigenista Peruano : una historia por conocer, p. 133-158.

42. Général Manuel Odria (1897-1974), ministre de l'Intérieur de 1946 à 1948, président du Pérou de 1948 à 1956, en tant que chef d'une junte militaire.

43. M. Varela Vázquez, «La antropología cultural y nuestro problema indio : un caso de antropología aplicada », 7-157.

44. Les rares films de l'époque sont, soit des documentaires sur la vie galante de Lima, soit des fictions montrant des problématiques typiquement créoles ou encore des documents utilisés comme instrument par la Escuela Cinemática Ambulante pour l'éducation massive des Indiens et leur intégration au processus national. 
45. José María Arguedas, Yawar Fiesta (Fiesta de Sangre) 1941, ou Uku Mayu (Ríos profundos) 1958.

46. Maximiliano T. Vargas (1874-1959), principal animateur du «Centro artístico fotográfico » de Arequipa à partir de 1896.

47. Les frères Carlos (1885-1979) et Miguel (1886-1976) Vargas, par exemple.

\section{RÉSUMÉS}

Grande figure de l'alpinisme français de l'après-guerre, membre de huit expéditions, notamment dans les Andes et l'Himalaya, spécialiste des premières ascensions, Lionel Terray était passionné par la montagne, le cinéma et « poussé par la curiosité des hommes » [C'est le Pérou, film de Lionel Terray, en collaboration avec Marcel Ichac, Jean-Jacques Languepin (1964)]. Lors des ascensions de plusieurs sommets enneigés au Pérou en 1956 et à l'occasion de séjours postérieurs, il réalise des images sur la vie et les traditions des Indiens du Pérou; il rapporte en France quelques bobines de film à partir desquelles il réalisera différents documentaires, dont l'un gagnera le Grand Prix du Film de Montagne et de l'Exploration au Festival International de Trente. Les filmsportraits de Terray, bien qu'ils soient empreints d'exotisme et de stéréotypes, s'inscrivent avant tout dans la curiosité et la reconnaissance de l'autre, à une époque où la nation péruvienne peine à admettre sa pluralité culturelle.

\section{AUTEUR}

\section{CHLOÉ TESSIER-BRUSETTI}

Docteure en Études hispaniques, membre du Centre de recherche sur les identités nationales et l'interculturalité (CRINI, EA 1162, université de Nantes) 


\title{
La représentation de la montagne corse dans la littérature du XXI ${ }^{\mathrm{e}}$ siècle
}

\author{
Pierre Bertoncini
}

1 Si on considère la notice « Montagne » du Dictionnaire de la Méditerranée $e^{1}$, on saisit qu'il s'agit d'un univers complexe marqué par l'héritage antique, aujourd'hui caractérisé par le déclin. Dans Non lieux, Marc Augéz affirme :

«Le monde de la sur-modernité n'est pas aux mesures exactes de celui dans lequel nous croyons vivre, car nous vivons dans un monde que nous n'avons pas encore appris à regarder. "

La Corse vient d'obtenir le statut officiel d'« île montagne ». Cela apparaît comme la traduction politique de la formule classique de Friedrich Ratzel ${ }^{3}$ qualifiant cet espace de «montagne dans la mer». La société corse a néanmoins connu des transformations profondes entre la rédaction de ces deux textes. Aussi, il n'est pas question ici d'interroger la montagne comme nous invitait à le faire Élisée Reclus ${ }^{4}$ avec les outils dont il disposait en son temps. Le présent texte est à comprendre comme une contribution à l'élucidation de la question plus globale: comment appréhender la montagne corse d'aujourd'hui?

3 C'est avec des observations faites en Vallée d'Aspe que Guy di Meo a récemment investigué l'espace vécu montagnard pour comprendre les «territoires du quotidien ». Il se place à la suite d'Armand Frémont qui, en montrant comment les artistes ont évoqué «l'espace vécu », expliquait qu'il voulait "piéger la territorialité » que les « littérateurs » avaient déjà comprise ${ }^{6}$. Plus précisément, son ambition était d'étudier :

«Le rapport spatial, c'est-à-dire la manière dont l'individu vivant en société pratique l'espace au quotidien, se le représente, établit des relations tantôt rationnelles, tantôt affectives avec ses lieux de vie. La question soulevée porte aussi sur le rôle que jouent de tels espaces, différemment parcourus, représentés et vécus, en matière d'auto-identification des groupes sociaux localisés. ${ }^{7}$ "

Afin de mesurer comment le rapport à la topographie est vécu aujourd'hui en Corse, nous nous proposons d'analyser la représentation de la montagne en faisant un détour 
par la littérature, celle du Xxi ${ }^{\mathrm{e}}$ siècle. En effet, une étude sur la relation entre mémoire et tourisme en Corse $^{8}$ a amené à réaliser une lecture du roman Le Sermon sur la chute de $R o m e^{9}$. Est alors apparue la manière dont l'écrivain utilise un vocabulaire emprunté à la géographie dans sa forme la plus conventionnelle afin de peindre le décor où jouent ses personnages. La ville, posée sur le littoral, y est présentée comme l'opposé du village situé quant à lui dans la montagne. Cela semble reprendre le stéréotype comprenant le village comme lieu de l'authenticité dénoncé par José Martinetti ${ }^{10}$ alors qu'il observait comment les lieux de résidence et d'emploi de la population corse sont devenus depuis des décennies majoritairement citadins et littoraux. On relira enfin l'œuvre de Jérôme Ferrari que l'on comparera à celles de Marie Susini qui l'a précédée, puis à celle de Marcu Biancarelli, son contemporain, afin de montrer comment les auteurs de notre temps comprennent les mutations récentes des territoires du quotidien liés à la montagne corse.

\section{La représentation de la montagne chez Marie Susini}

\section{Présentation de La renfermée. La Corse}

En 1981, Marie Susini signe La renfermée. La Corse ${ }^{11}$. Il s'agit d'un texte divisé en douze chapitres. Il est accompagné par des photographies de Chris Marker ${ }^{12}$. Quand elle écrit ce livre, elle a déjà 65 ans. C'est à Paris qu'elle a principalement vécu et écrit les huit livres précédents parmi lesquels le roman $A$ fiera ${ }^{13}$. Dans ce récit, toute une communauté villageoise se retrouve au mois d'août pour la fête votive. Si le terme «village » est omniprésent dans A fiera, celui de « montagne » n'apparaît qu'une seule fois. Il faut peut-être comprendre que village est automatiquement à comprendre comme « village de montagne ».

6 La renfermée, la Corse pourrait être classé dans la catégorie "essai ». Son propos est d'une ambition démesurée : décrire en trente-huit pages ce qu'est la culture corse. On présentera ici dans quelle mesure et pour répondre à quelle volonté le fait montagnard apparaît. Notre analyse sera aidée par des cairns posés par Marie Susini. En effet, faisant un choix lexical complètement différent de celui d'A fiera, onze fois, elle a utilisé le mot «montagne». On va les présenter afin de comprendre le sens de la marche proposée au lecteur.

\section{Le terme « montagne » dans La renfermée}

$7 \quad$ La montagne participe au temps des légendes, elle correspond aux villages habités par des familles dont la culture tourne le dos à la mer, aux envahisseurs, à leurs villes. La représentation de la montagne corse que propose Marie Susini est comparable à celle que se faisait A. Frémont de la montagne algérienne du temps de la colonisation :

"Séculairement la montagne maghrébine est un refuge face aux hommes des

plaines. La colonisation l'a à peine touchée..$^{14}$ »

Dans cette représentation, cette montagne à partir des années 1960 est agressée par la télévision, par les touristes et les promoteurs immobiliers qui la voient comme un accessoire complémentaire de la mer. Quand Marie Susini évoque "Francesi fora " (Les Français dehors), elle qui avait donné une place d'honneur à la Pinzuta en fin d'A Fiera, c'est pour montrer la réaction des Corses à « l'invasion » présente. Précisons qu'avant 
même la constitution du FLNC, dès 1973 c'était le cri de ralliement de ses partisans ${ }^{15}$. Le texte est écrit en 1981, avant le printemps, alors que des dizaines de militants nationalistes sont emprisonnés. Marie Susini explique de Paris comment elle perçoit une société marquée par l'enfermement.

\section{Comparaison entre la représentation de la montagne dans La renfermée et dans Granit island}

9 On peut comparer la vision de la Corse de Marie Susini à celle de Dorothy Carrington. Cette dernière a vécu son rapport à l'île à l'inverse de l'auteur de Rennu. Britannique, elle débarque en Corse après guerre alors qu'elle a trente-huit ans. Installée à Ajaccio, elle y restera, jusqu'à son décès ${ }^{16}$. On évoquera ici son grand livre Granit island ${ }^{17}$. Écrit sous la forme d'un récit de voyage, ce texte est difficilement classable puisqu'il est à la fois un traité d'ethnologie et une œuvre littéraire. Afin de comprendre sa vision de la Corse, reprenons ce qu'a écrit Marie Susini :

« L'histoire commence le 15 août $1769 .{ }^{18}$ »

10 Dorothy Carrington prend cette tendance de l'historiographie corse à contre-pied puisque l'idée centrale de son essai est que l'acmé de la civilisation corse se retrouve à deux moments : à l'époque de la Préhistoire, avec les statues de Filitosa, qui venaient d'être analysées par l'archéologue Roger Grosjean après qu'elle les ait découvertes ${ }^{19}$ lors de ses premiers séjours dans l'île et au moment de l'indépendance au milieu du xvIII ${ }^{e}$ siècle. Ce que Dorothy Carrington a l'audace d'écrire, c'est que lorsque l'indépendance de l'île prend fin, le 15 août 1769, l'histoire n'est plus intéressante. Cette seule opinion sur l'histoire est révolutionnaire en soi dans le contexte historiographique du temps. Au sujet de la culture corse, Dorothy Carrington a décrit la manière dont elle a été victime de la trahison des élites corses. Elle met en lumière les actions de quelques rares professionnels du patrimoine. Selon l'auteur, à l'exception des couches les plus populaires de la population, la culture corse transmise depuis la préhistoire est alors méprisée et abandonnée.

11 Afin de saisir comment Dorothy Carrington se figure la montagne, reprenons un des parcours auxquels elle invite le lecteur. Elle part du littoral de la région de Balagne. Elle quitte Calvi en train. Quand elle passe devant l'Ile-Rousse elle décrit une baie dont le paysage est saccagé ${ }^{20}$. Elle condamne sans appel ces nouvelles constructions. Elle poursuit son chemin pour aller dans le Niolu. Là, avant que ne soient rédigées les thèses d'ethnologie de Georges Ravis-Giordani ${ }^{21}$ ou de Gérard Lenclud ${ }^{22}$ sur les communautés villageoises qui y résident, elle décrit comment, selon un avis qu'elle partage avec R. Grosjean ${ }^{23}$, les bergers qu'elles rencontrent sont liés directement au Néolithique ${ }^{24}$.

On peut établir une correspondance entre la vision de la montagne présentée dans Corsica, Granit island et dans La renfermée, la Corse. Les « envahisseurs ", parmi lesquels il faut compter les touristes de l'époque moderne, "saccagent» des sites littoraux. La culture corse se retrouve en altitude, chez les montagnards. Tandis qu'une transmission culturelle de la préhistoire à l'époque contemporaine a existé, depuis les années 1960, par la trahison des élites, par le poids idéologique de l'État français dont la télévision, dont il a le monopole des chaînes de diffusion, est le vecteur alors le plus moderne, par le développement du tourisme, cette culture est en péril. Les deux auteures sont témoins du développement du mouvement nationaliste né en réaction 
contre ce qui est perçu par ses partisans comme le processus de destruction du peuple corse.

\section{La représentation de la montagne chez Marcu Biancarelli}

\section{La représentation de la Montagne dans Prisonnier}

En 2000 est publié Prighiuneru ${ }^{25}$. Il est signé par Marcu Biancarelli et traduit en français (Prisonnier) par Jérôme Ferrari. L'auteur, professeur de corse travaillant en Corse et écrivant en langue corse, est présenté par la critique d'alors comme novateur dans le paysage culturel insulaire. Remarquons cependant, que dix-neuf ans après la publication de La renfermée, la Corse, un jeune auteur de trente-deux ans choisit pour titre de son premier grand texte un terme presque synonyme. Alors que l'enfermement était une sensation que Marie Susini décrit dans A Fiera, qu'elle fuyait en vivant à Paris, il s'agit maintenant d'examiner l'œuvre, dans une certaine continuité, au début du XxI ${ }^{\mathrm{e}}$ siècle, d'un narrateur qui se pense comme prisonnier de son village. Quelle lecture géographique peut-on faire des quatorze nouvelles que comprend ce recueil ? Dans la première nouvelle, Prisonnier, le personnage est statique. Comme dans les douze autres nouvelles, il vit à l'époque où le livre est écrit. Ce qui le caractérise, c'est « je suis assis à la terrasse d'un bar de mon village ». À partir de ce point de vue, il explique son dégoût des mois de juillet et d'août ainsi que l'ennui qui règne les dix autres mois de l'année. Le terme de «montagne » n'apparaît pas. Il est précisé que le narrateur est professeur de langue et culture corses dans un lycée de la ville voisine. Dans les autres textes, la dimension spatiale des récits va progressivement s'enrichir de nouvelles observations.

La géographie des nouvelles de Prisonnier est basée sur des éléments stables. Le centre en est le village. Il a son centre historique, où se trouvent la place et le bar. Plus loin, des maisons isolées sont situées sur d'anciennes exploitations agricoles. Quand on s'éloigne de ce noyau, on s'approche de la mer. On atteint alors des maisons en location dans le maquis, un village de vacances ou une propriété acquise par un " opportuniste ». Avant d'arriver dans la ville voisine où il est loisible de travailler, de suivre des études secondaires, il y a la côte. Là, s'y trouvent des restaurants et des boîtes de nuit. L'activité illégale de la côte modifie les rapports sociaux sur l'ensemble du territoire. L'empire colonial étant achevé depuis 1962, l'horizon où l'on s'exile pour longtemps, où l'on part un week-end, demeure Paris. Dans cet ensemble, la montagne est un espace qui a un statut ambigu, lieu de refuge, mais aussi lieu d'exécution pour des malfrats. C'est un lieu structurellement lié au reste mais où les phénomènes qui s'y observent sont hors normes.

Dès 2002, Marcu Biancarelli participe à une manifestation commémorative sur les trente ans du riacquistu ${ }^{26}$. Il y a présenté un texte qui peut nous apporter des éléments de compréhension de la géographie de Prisonnier. Dans ces pages autobiographiques, il indique comment "À cette époque, mon village de montagne était encore un vrai village ». Il précise :

«Avec des enfants partout qui couraient et se faisaient la guerre, des jardins où nous allions chaparder presque tous les jours, et des bandes de chèvres et de cochons que nous chassions des journées entières à coups de pierres. ${ }^{27}$ » 
ce court paragraphe, on prend connaissance d'un certain nombre d'éléments significatifs. Si l'auteur est né en 1968, les souvenirs évoqués doivent correspondre aux années 1972-1978, les années fortes du mouvement du riacquistu. Ce contexte est fondamental pour la compréhension du terme. Un village au cours de cette période est alors selon Philippe Pesteil un espace investi par des processus de recomposition d'identité, c'est « le lieu incontournable de la corsitude et de la formation identitaire ${ }^{28}$ ». On peut ainsi lire que pour le romancier existe son «village de montagne ». Village et montagne sont indissociablement liés. Aussi, une relecture de Prisonnier s'impose. Comme dans A fiera, les parties du récit qui concernent le village semblent pouvoir en fait être à comprendre comme "village de montagne». Aussi, quand il écrit "montagne », faut-il sans doute comprendre, zone d'alpage au-dessus du village. Le village de Prisonnier est, semble-t-il, à comprendre comme un espace en piémont qui se trouve comme la plupart des cœurs historiques des villages corses, à une altitude comprise entre 600 et 800 mètres d'altitude. Quand il évoque son village, Marcu Biancarelli utilise le passé. L'explication de cet usage ne se trouve pas réduite au fait que le texte corresponde à son enfance révolue. Il précise bien :

«Mon village de montagne était encore un vrai village. ${ }^{29}$ »

sous-entendu est qu'aujourd'hui, le même espace n'est plus un " vrai village ». Ce qui est en cause, ce n'est pas l'usage de la télévision que décrivait Marie Susini, ce ne sont pas les quelques verrues destructrices d'harmonie dénoncées depuis le début des années 1990 par l'architecte Jacques Poncin ${ }^{30}$. Le village de l'enfance de l'auteur a été touché par les phénomènes bien plus massifs. L'économie liée au tourisme, à la spéculation, au milieu du banditisme, a déplacé le centre de gravité de la société. C'est sur la côte que se concentrent les activités lucratives. À la fin de son texte sur le riacquistu, Marcu Biancarelli évoque les luttes culturelles pour que survive le corse :

"Cette langue parlée dans nos îles d'Aran à nous, quelque part loin des villes. ${ }^{31}$ "

On peut comprendre que la langue corse est perçue par cet écrivain comme une expression liée à des lieux « quelque part, loin des villes ", donc, dans les villages, donc dans les montagnes. Aussi, la place de la montagne dans Prisonnier n'est pas à chercher dans les seules rares occurrences du terme "montagne", ni dans les occurrences complémentaires de "village». Peut-être faut-il comprendre ici que la montagne est un univers symbolique qui, pour Marcu Biancarelli, se trouve aussi dans la totalité des mots de la langue utilisée pour écrire ces nouvelles.

\section{La représentation de la montagne dans Murtoriu $^{32}$}

\section{Murtoriu, propos général du roman}

19 Murtoriu est un roman signé par Marcu Biancarelli en 2009. L'histoire est écrite à la première personne. Le narrateur évolue dans la Corse contemporaine. Porteur du regard du moraliste, il vit son quotidien comme une résistance aux mutations de son île. Il a passé son enfance sur le continent. De profession, il est libraire, un avatar du professeur de Prisonnier. Il met un point d'honneur à fermer son commerce au moment où commence la saison touristique, retiré dans la maison de village dont il a hérité, il écrit. 


\section{L'apparition du terme « montagne » dans Murtoriu}

20 La relecture du texte m'a permis d'identifier 46 occurrences du terme au long des vingt chapitres qui le composent, soit une moyenne de plus de deux apparitions par chapitre. Ces occurrences peuvent être regroupées en trois grandes catégories. La première désigne la montagne comme le lieu qui permet au narrateur de vivre en ermite, en marge de la société («je suis reparti vers la montagne pour regagner ma tanière d'ours »). La deuxième évoque la montagne comme un terme qui permet de situer un lieu, de donner une direction vers laquelle se passe une action («je vivais à la montagne »). Enfin, la troisième fait correspondre la montagne à ce que l'on pourrait qualifier de fait de civilisation avec cette réflexion :

«J'ai repris mon existence montagnarde..$^{33}$ "

21 Dans Murtoriu, la montagne est le lieu de refuge du narrateur, de la culture corse. La ville, le littoral, avec ses boîtes de nuit et ses bars, sont envahis par l'activité touristique. La montagne est décomposée en deux parties, les confins de la moyenne montagne où se trouve le hameau originel de sa famille. Puis, plus haut, dans les alpages, se trouvent des façons de vivre qui remontent à la Préhistoire. Tandis que le temps d'une partie de chasse, ses contemporains se muent en "hommes de Filitosa", pour le narrateur, en fin de récit, «la plaine est perdue. D’ici peu, je crois que la montagne le sera aussi ${ }^{34}$ ». Aussi, ayant renoncé depuis longtemps à mener une lutte révolutionnaire paysanne ${ }^{35}$, énonçant que «le paradis terrestre n'existe malheureusement $\operatorname{pas}^{36} »$, le narrateur, comme dans Prighjuneru, comme le fit Marie Susini des décennies plus tôt, quitte la Corse.

Tandis qu'au début des années 2000 les autorités politiques de la collectivité territoriale de la Corse proposent un PADDUC (plan d'aménagement et de développement durable de la Corse) basé sur le « tourisme résidentiel », qui selon le Conseil économique, social et culturel de la Corse «tourne le dos à l'histoire », Marcu Biancarelli montre comment dans une commune ou, tout au plus, un canton donné, les deux dimensions existent. Cohabitent d'une part une plaine ou une plage, vendue aux appétits touristiques, et d'autre part, sans avoir à monter spécialement dans le Niolu, les hauteurs de chaque canton où résistent quelques Corses qui habitent des lieux où survivent des vestiges des temps néolithiques.

En 1981, Marie Susini témoignait des transformations vécues à un rythme accéléré depuis 1960. Elle n'évoque pas la guerre de 1914-1918 qui, pour des raisons attachées à l'historiographie corse, fascine les auteurs $\mathrm{du} \mathrm{xxI}^{\mathrm{e}}$ siècle, comme l'illustre Le petit soldat ${ }^{37}$. Malgré la francisation par l'école et la télévision, Marie Susini distinguait alors en Corse les villes côtières des villages montagnards. Elle observait comment la révolte naissait avec le cri «Francesi fora ». Trente ans plus tard, Marcu Biancarelli, décrit une génération qui a été désabusée par les meurtres commis au nom de la «lutte de libération nationale » dans les années 1990. Il constate comment la corruption et la mise en tourisme de l'espace gagnent sans cesse du territoire, partant du littoral, gagnant les villages et s'attaquant à la montagne. Puis il conclut comme Marie Susini qui, pour se libérer d'une aliénation qu'elle avait ressentie dans l'île, fait partir son narrateur hors de Corse, loin d'une montagne témoin d'assassinats et d'abandon. 


\section{La représentation de la montagne chez Jérôme Ferrari}

\section{La représentation de la montagne dans Variétés de la mort ${ }^{38}$ et Balbo atlantico ${ }^{39}$}

À quel genre littéraire correspond Variétés de la mort? Il s'agit apparemment d'un recueil de neuf nouvelles publié par Jérôme Ferrari en 2001. Les actions apparaissent dans un désordre chronologique et les intrigues s'entremêlent souvent. Aussi, sans trancher pour savoir si ce texte n'est pas en fait un roman décomposé en chapitres, c'est par convention qu'on appellera chacune de ses parties une nouvelle. Dans la plupart d'entre elles, les indications géographiques sont rares et pauvres. Les termes de «montagne» ou celui de "village» sont dans cet ensemble quasiment inexistants. Analyse a été faite au plus près de la manière dont sont distribuées les informations liées à la situation spatiale des personnages.

En lisant Variétés de la mort, on découvre une série de nouvelles qui montre comment les habitants d'un village du sud de la Corse évoluent dans une société qui tourne le dos à son passé montagnard. Sous l'emprise de cocaïne pour nombre d'entre eux, ils vivent sur la côte au rythme des saisons touristiques. Dans deux autres nouvelles, on a une description de deux aspects du monde de la montagne. Le premier apparaît par l'entremise d'un fantôme qui décrit ce que fut la Révolution corse au XvIII ${ }^{e}$ siècle. Une société montagnarde qui choisit de mettre sa capitale à Corte. Le second aspect est lié au riacquistu. Issue des combats culturels des universités d'été livrés à partir de 1973, l'Université de Corse, créée en 1765 et supprimée lors de la conquête, rouvre en 1981. Jérôme Ferrari nous montre comment, après un XVIII siècle où la raison d'État et le mensonge l'emportaient déjà sur la droiture, le nouveau professorat cortenais est marqué par l'imposture et les choix d'étudiants marqués par la fièvre d'une volonté de retour aux sources mythifiées dont l'auteur ne se prive pas de moquer les élucubrations qu'elle produit.

C'est à la fin d'Ethnologues que Jérôme Ferrari fait cohabiter plusieurs réalités dans une très longue phrase. Le premier élément à retenir est le suivant: " tandis que dans les villages de montagne les murs périssaient d'ennui », suivi par le second:

«Tandis que les discothèques porto-vecchiaises étaient assaillies par une meute

d'adolescents en rut avides de sons électroniques et d'extasy. ${ }^{40}$ "

Est clairement exprimée ici la place de la montagne dans la Corse de 2002 : c'est une part mineure. Mais tandis qu'elle est marquée par l'abandon et le déclin, la jeunesse issue d'une société agro-sylvo-pastorale, est pour sa part victime d'aliénation, à un degré allant, comme l'indique la nouvelle qui suit, jusqu'à l'overdose.

Le propos de Jérôme Ferrari est de montrer comment, de façon complexe, avec des personnages qui ont des trajectoires de vie diverses, la représentation de la montagne est centrale dans la société corse contemporaine. Elle est d'une part une racine oubliée par une partie de la société qui fonce en voiture de sport vers l'overdose, et d'autre part, sa connaissance, malgré les promesses liées à la réouverture de l'Université de Corse, n'est pas permise par les défauts structurels des parcours des enseignants et des étudiants. Celui qui peut montrer les différentes facettes de la montagne aujourd'hui est alors l'écrivain qui signe Variétés de la mort. 
29 En 2008 paraît Balco Atlantico. Jérôme Ferrari y décrit un monde en apparence plat, sans montagne. Ses personnages évoluent surtout en Corse, principalement dans un réseau de villes portuaires. Deux exceptions pourraient être le village et la ville montagnarde de Corte. Le village tourne le dos à la montagne. Toutes les activités décrites se font vers la côte, vers la station balnéaire proche ou la ville préfecture d'Ajaccio. Corte est décrite également comme une bourgade qui accueille une université de seconde zone, étouffée par des conflits idéologiques. Elle aussi tourne le dos à sa montagne. Ainsi, dans Balco Atlantico, la montagne est bien présente mais ses personnages ne la voient plus, font comme s'il s'agissait d'un ensemble de vieux outils mis à la cave et oubliés. Le contraste entre la réalité géographique et les représentations des personnages est le fond de décor consubstantiel des drames qui arrivent dans cet ouvrage sombre.

\section{La représentation de la montagne dans Le sermon sur la chute de Rome}

30 Considérons le livre le plus célèbre de Jérôme Ferrari, celui qui lui a permis d'être lauréat du prix Goncourt de l'année 2012, Le sermon sur la chute de Rome. Comme pour les ouvrages précédents, on va interroger les deux cents pages de ce récit avec un crible géographique. Où se passe cette histoire? La présentation la plus synthétique retient qu'il s'agit de la narration du retour au village de deux jeunes Corses des années 2000. Avec le récit décrivant leur reprise de l'unique bar de la localité, on s'attend à lire un ouvrage concentré sur le monde rural, tout en se demandant quel peut être le rapport avec le nom de la ville qui apparait en titre. Une lecture plus attentive permet de constater que trois histoires sont présentées en torsade. La première raconte comment la prise de Rome par les Wisigoths fut vécue par Saint Augustin. La deuxième présente la vie d'un Corse ayant traversé le $\mathrm{xx}^{\mathrm{e}}$ siècle. La troisième enfin est l'histoire qui retient le plus l'attention, celle des deux diplômés de philosophie qui décident de vivre à l'année au village. On relira donc l'œuvre en prêtant une attention particulière aux toponymes ainsi qu'à une série d'autres indices de situations topographiques.

31 Le $\mathrm{xx}^{\mathrm{e}}$ siècle est présenté par l'auteur avec pour fil rouge la vie de Marcel Antonetti. Ce personnage naît dans un village. La vie de Marcel tourne autour du même lieu appelé «le village» :

«Les lignes de fuite sont des cercles secrets dont la trajectoire se referme

inexorablement et le ramène vers le village détesté de son enfance. ${ }^{41}$ "

Père de Jacques qui passe sa carrière à Paris, Marcel est donc le grand-père de Matthieu qu'il soutient financièrement afin qu'il prenne en gérance le bar du village. Le présent se déroule dans la continuité de Balco Atlantico. On retrouve plusieurs personnages dans les deux livres. Avec leur inexpérience, leur insouciance et, de fait, leur inconscience, Matthieu et son ami Libero vont reprendre le bar qui avait connu une effervescence au moment de leur enfance.

33 Cette gérance se réalise dans un espace qui est distribué par cercles concentriques. Le bar unique du village en est le cœur. Il se trouve au centre d'un village. Aucun toponyme qui puisse faire "couleur locale ». On apprend peu à peu qu'il y a une église où se déroule la cérémonie du Jeudi saint, où ont lieu aussi les cérémonies religieuses liées aux obsèques des habitants. Le cimetière est le lieu où un homme comme Marcel Antonetti enterre les siens avec soin. Dans les confins du village, accessibles en 4X4 par 
des pistes de terre, est nichée la bergerie de Virgile Ordioni, sous une montagne couverte d'une forêt où se trouvent les ruines d'une chapelle.

Dans cet espace, la montagne apparaît à plusieurs reprises. Pour le jeune Marcel, né après la Première Guerre mondiale, malade chronique, la montagne, c'est une barrière :

«Les montagnes dissimulent le grand large et se dressent de toute leur masse

inerte contre Marcel et ses rêves inlassables..$^{42}$ "

Marcel a « la vue bouchée par la barricade de montagnes ». C'est la représentation d'un jeune qui rêve de partir comme son grand frère en Indochine. Arrive la guerre. Alors, du village, des jeunes, velléitaires, se demandent s'ils vont entrer en résistance. Le lecteur les voit aller à "un rendez-vous nocturne dans la montagne ${ }^{43}$ ". La peur physique les empêche de suivre ce chemin. Pendant ce temps, Ange-Marie Ordioni et sa femme qui mènent "une vie sauvage de chasseurs néolithiques", lui qui a une "stature d'homme des cavernes", considère "qu'en montagne, il faut de bonnes chaussures ", raison pour laquelle il élimine plusieurs soldats italiens !

Virgile Ordioni, né à la Libération, est l'éleveur que Matthieu et Libero fréquentent depuis toujours. On retrouve ici la figure du berger, «l'archétype du savoir-faire et savoir-être à préserver $\aleph^{44}$. La montagne apparaît pour la première fois dans la période récente en contraste complet avec Paris. «Il y avait deux mondes, peut être une infinité d'autres, mais pour lui, seulement deux ». En quelques paragraphes, l'auteur décrit les escapades de ces adolescents à la bergerie de Virgile. Ce qui a été tenté dans le bar, c'est de faire la synthèse de tous les lieux, de la côte à la montagne. Dans le même temps où l'expérience est en cours, un soir, Marcel Antonetti et Aurélie, sa petite-fille, «marchaient sur la route, en direction de la montagne ». C'est un passage de rare sérénité d'entente entre les générations, entre le village et les désirs futurs qui se feront ailleurs, sur la recherche d'un autre temps. La montagne pour Marcel, n'est plus là une barricade comme dans son enfance mais une direction à prendre pour atteindre l'harmonie.

37 La montagne offre plusieurs facettes. Elle peut être comprise comme une barrière qui enferme, sensation ressentie par le jeune Marcel. On retrouve là une facette de la montagne de Marie Susini. Mais dans Le sermon, la montagne est surtout le lieu des origines. S'y trouvent des vestiges immémoriaux où Moyen Âge et Résistance se confondent dans une chronologie dissoute qui est le propre du temps des légendes, celui que décrivait Marie Susini. Ses seuls habitants, les bergers, comme l'avait noté Dorothy Carrington dans sa description des bergers du Niolu, comme le personnage Mansuettu de Murtoriu, vivent comme aux temps préhistoriques. C'est parce qu'ils connaissent cette expérience de la montagne que Libero et Matthieu décident de rentrer au village. Mais tandis que leur bar fonctionne à plein régime... ils ne vont plus à la montagne. Les personnages d'Aurélie et Marcel n'oublient pas de l'observer comme un phare tandis qu'au milieu des chants le drame se prépare au village.

\section{La montagne, un lieu tragique?}

La visite de quelques œuvres majeures de la littérature représentant l'espace corse permet de saisir comment la montagne apparait dans l'imaginaire collectif contemporain. Chez un même auteur comme Marie Susini, avec A fiera ou La renfermée, le vocable peut être invisible ou être un élément essentiel du discours. On a vu comment le seul terme polysémique de "village » pouvait selon le contexte être 
compris comme "village de montagne ». S'il n'y a pas de règle de type comptable qui prévaut, dans les deux cas, la montagne est néanmoins là. Chez les auteurs étudiés, la montagne se définit systématiquement dans son rapport au littoral. Dans cette tension apparaît toujours un troisième élément. Il s'agit du continent français, de l'empire colonial, d'un pays d'origine pour des migrants dans la plupart des cas. Le même terme correspond à des réalités différentes entre 1981 et la période 2001-2011 où sont écrits les derniers ouvrages. On n'est donc pas dans une création mythique détachée du réel, bien au contraire. En 1981, malgré la diffusion de la télévision qu'observait attentivement Marie Susini, il y a encore une séparation visible entre littoral et montagne. Vingt ans plus tard, le centre de gravité de la société a bougé. Comme les tableaux statistiques de l'INSEE l'indiquent, dans les œuvres littéraires, la démographie et l'activité économique se situent, pour ne pas dire ont dévissé, sur le littoral. Les auteurs étudiés ici ne nient pas la réalité du processus démographique décrit par José Martinetti. Ils expriment la manière dont les territoires du quotidien ont changé de nature. Il faut aller en fait au-delà de ce que le personnage de Murtoriu peut analyser quand il distingue avec les outils de la géopolitique centre et périphérie. Grâce à la géographie culturelle et sociale, on peut constater que son espace vécu est en effet travaillé par d'autres phénomènes qui se combinent à ceux dont il arrive à avoir conscience, de son perchoir situé au-dessus de la plaine. Il semble que la montagne recouvre aujourd'hui les caractéristiques de ce qu'était un lieu, tandis que le littoral, marqué par la sur-modernité, soit devenu l'espace de déploiement des non-lieux. Là :

"C'est à la façon d'une immense parenthèse, que les non-lieux accueillent des

individus chaque jour plus nombreux. ${ }^{45}$ "

Les personnages décrits dans les fictions entretiennent des rapports différents à cette évolution. Dans Murtoriu ou Le sermon, on rencontre des individus qui incarnent l'être montagnard, l'archétype du berger. Comme les pasteurs que rencontrait Dorothy Carrington dans l'immédiat après-guerre, ils incarnent la transmission localisée de la culture corse depuis le Néolithique. C'est au contact de ces êtres que les personnages principaux entrent en contact avec ce qui les rattache le plus à lîle. Ils participent à un univers culturel que Marie Susini a décrit comme n'étant pas celui de l'histoire mais celui de la légende. Mais, dans ces deux livres, ces bergers au statut symbolique si important finissent tragiquement assassinés. C'est de cette violence silencieuse de notre temps que témoignent les auteurs.

Le personnage le plus près du narrateur se situe dans une zone intermédiaire entre le berger et les professionnels du tourisme: le professeur de corse, de philosophie, le libraire ou le jeune intellectuel qui tient un bar. Il a un rapport affectif fort avec la montagne, avec ses derniers habitants, les bergers. Mais ces personnages, proches du narrateur, constatent un même phénomène lié au processus de déclin général propre aux montagnes méditerranéennes d'aujourd'hui. Les contemporains, intégrés dans un monde sur-moderne (défini par Marc Augé par la surabondance évènementielle, spatiale, l'individualisation des références) tournent le dos à leur part d'identité montagnarde. Or, il semble qu'ils n'aient pas conscience qu'ils ne se réduisent pas à de simples attributs décoratifs, mais qu'il s'agissait de fait de leur "pass » de survie dans le monde violent qui est le leur. Aussi, sur la côte, où règnent les valeurs de la prostitution, certains meurent assassinés ou d'overdose. Étrangement, parfois dans la même scène, cohabitent des personnages qui ont oublié la montagne et d'autres, moins mis en lumière, qui continuent (rappelons que M. Augé précisait que non-lieu et lieu étaient plus des pôles que des lieux) à la regarder, à marcher vers elle, ou bien y aller. 
Cette montagne n'est pas seulement un lieu géographique. Il est clair qu'il ne s'agit pas que des plus hauts sommets de l'arête centrale de l'île accessible par une éventuelle variante du célèbre GR20. Les auteurs nous montrent comment elle est une portion, voire une dimension de chacun des territoires communaux de l'île. Comme Marie Susini qui écrivait sur la montagne corse en vivant à Paris, alors qu'on est passé de la modernité à la sur-modernité, les personnages de Marcu Biancarelli et de Jérôme Ferrari sont à un moment donné de leur parcours, parce qu'ils aiment leur montagne, et que ceux qui la gouvernent ne pensent qu'à un espace couvert par un carroyage en deux dimensions, confrontés au douloureux choix de l'exil. Celui-ci s'impose aussi à eux sans doute parce que c'est de loin qu'on peut comprendre qu'au quotidien on habite sur une montagne. En 2017, tandis que la Corse a acquis le statut officiel d'île-montagne, notons que le mot «montagne » n'apparaît dans aucune des professions de foi des onze candidats aux élections présidentielles. Il semble probable que l'imaginaire de la société corse continue un moment encore à être teinté de tragique.

\section{BIBLIOGRAPHIE}

AlBERA Dionigi, « Montagne », dans ALBERA Dionigi, CRIVELlo Maryline et TOZY Mohamed (dir.), Dictionnaire de la Méditerranée, Arles, Actes Sud, 2016, p. 975-988.

AUGÉ Marc, Non-lieux, Paris, Seuil, 1992.

BERTONCINI Pierre, Graffiti bombé et territoire corse (1973-2003), Thèse de doctorat en anthropologie, Corte, Université de Corse, 2005.

BERTONCINI Pierre, « La mémoire et le tourisme dans Le sermon sur la chute de Rome de Jérôme Ferrari », dans CHERUBINI Bernard (dir.), Patrimoine et identités locales, Paris, L'Harmattan, 2017, p. 165-187.

BIANCARELli Marcu, Prighjuneri, Ajaccio, Albiana, 2000.

BIANCARELLI Marc, Vae Victis et autres tirs collatéraux, Calvi, Materia scritta, 2010.

BIANCARELLI Marc, Murtoriu : ballade des innocents, Arles, Actes Sud, 2012.

CARRINGTON Dorothy, Corse. Ile de granit, Paris, Arthaud, Édition 1999.

DI MEO Guy, «Territoire vécu et contradictions sociales : le cas de la vallée d'Aspe », dans DI MEO Guy (dir.), Les territoires du quotidien, Paris, L'Harmattan, 1996, p. 51-86.

FERRARI Jérôme, Variétés de la mort, Ajaccio, Albiana, 2001.

FERRARI Jérôme, Balco Atlantico, Arles, Actes Sud, 2008.

FERRARI Jérôme, Le serment sur la chute de Rome, Arles, Actes Sud, 2012.

FRÉMONT Armand, La Région. Espace vécu, Paris, Flammarion, 1999.

FUSINA Jacques, Le petit soldat, Ajaccio, Albiana, 2014.

GROSJEAN Roger, La Corse avant l'histoire, Paris, Klincksieck, 1975. 
LENCLUD Gérard, Économie et société dans une commune de la montagne corse, Thèse de doctorat d'ethnologie, Paris-V, Paris, 1982.

MARTINETTI José, «Les représentations de la ville en Corse, le stéréotype d'un rejet », Encyclopedia corsicae, Bastia, Éditions Dumane, 2004, volume 3, p. 1244-1245.

PESTEIL Philippe, « Autour du Reacquistu », dans LE COADIC Ronan (dir.), Identités et société de Plougastel à Okinawa, Rennes, PUR, 2007, p. 161-183.

PONCIN Jacques, Paysages bâtis en Corse, Ajaccio, La Marge, 1992.

RATZEL Friedrich, «La Corse. Étude anthropogéographique », Annales de géographie, n 40, 1899, p. 304-329.

RAVIS-GIORDANI Georges, Bergers corses. Les communautés villageoises du Niolu, Aix-en-Provence, Edisud, 1982.

RECLUS Élisée, Histoire d'une montagne, Paris, Hachette, 1880.

SUSINI Marie, A fiera, Paris, Seuil, 1954.

SUSINI Marie, La Corse. La renfermée, Paris, Seuil, 1981.

WEISS Michel-Claude, LANFRANCHI François (de), «À la découverte des anciens vestiges », dans POMPONI Francis (dir.), Le Mémorial des Corses, Ajaccio, Le Mémorial des Corses, Marseille, 1981, tome I, p. 12-25.

\section{NOTES}

1. D. Albera, « Montagne », p. 975-988.

2. M. Augé, Non-lieux, p. 49.

3. F. Ratzel, « La Corse. Étude anthropogéographique », p. 304-329.

4. E. Reclus, Histoire d'une montagne.

5. A. Frémont, La Région. Espace vécu, p. 266-267.

6. G. Di Meo, «Territoire vécu et contradictions sociales : le cas de la vallée d'Aspe ", p. 56.

7. Ibid., p. 52.

8. P. Bertoncini, «La mémoire et le tourisme dans Le sermon sur la chute de Rome de Jérôme Ferrari ».

9. J. Ferrari, Le serment sur la chute de Rome.

10. J. Martinetti, «Les représentations de la ville en Corse, le stéréotype d'un rejet », p. 1244-1245.

11. M. Susini, La Corse. La renfermée.

12. Chris Marker (1921-2012), (Christian Bouche-Villeneuve) photographe, illustrateur, réalisateur et écrivain.

13. M. Susini, A fiera.

14. A. Frémont, La Région. Espace vécu, p. 254.

15. P. Bertoncini, Graffiti bombé et territoire corse (1973-2003). 
16. Dorothy Carrington (Frederica Lady Rose) (1910-2002), arrivée en Corse en 1948 avec son troisième mari, le peintre Francis Rose.

17. D. Carrington, Corse. Ile de granit.

18. M. Susini, La Corse. La renfermée, p. 33.

19. M.C. Weiss et F. de Lanfranchi, «À la découverte des anciens vestiges », p. 22.

20. D. Carrington, Corse. Ile de granit, p. 242.

21. G. Ravis-Giordani, Bergers corses : les communautés villageoises du Niolu.

22. G. Lenclud, Économie et société dans une commune de la montagne corse.

23. R. Grosjean, La Corse avant l'histoire, p. 91.

24. D. Carrington, Corse. Ile de granit, p. 258.

25. M. Biancarelli Marcu, Prighjuneri.

26. Le riacquistu est un mouvement culturel de réappropriation de la culture et de la langue corses qui se développe à partir des années 1970.

27. M. Biancarelli « Vae Victis et autres tirs collatéraux », p. 44.

28. P. Pesteil, « Autour du Reacquistu », p. 165.

29. M. Biancarelli, «Vae Victis et autres tirs collatéraux », p. 44.

30. J. Poncin, Paysages bâtis en Corse.

31. M. Biancarelli, « Vae Victis et autres tirs collatéraux », p. 50.

32. M. Biancarelli, Murtoriu : ballade des innocents.

33. Ibib., p. 80.

34. Ibid., p. 46.

35. Ibid., p. 65.

36. Ibid., p. 268.

37. J. Fusina, Le petit soldat.

38. J. Ferrari, Variétés de la mort.

39. J. Ferrari, Balco atlantico.

40. J. Ferrari, Variétés de la mort, p. 174.

41. J. Ferrari, Le sermon sur la chute de Rome, p. 148.

42. Ibid., p. 69.

43. Ibid., p. 79.

44. P. Pesteil, « Autour du Reacquistu », p. 165.

45. M. Augé, Non-lieux, p. 139.

\section{RÉSUMÉS}

La Corse vient d'obtenir le statut officiel d'« île Montagne ». Cela apparaît comme la traduction politique de la formule de Friedrich Ratzel qualifiant cet espace de «montagne dans la mer ». La 
société corse a néanmoins connu des transformations profondes entre la rédaction de ces deux textes. Afin de les mesurer, nous analyserons, avec des outils fournis par l'anthropologie et la géographie, la représentation de la montagne corse dans la littérature au xxi siècle. Au cours d'une étude sur Le Sermon sur la chute de Rome de Jérôme Ferrari est apparu comment l'écrivain utilisait un vocabulaire emprunté à la géographie. La ville située sur le littoral y est présentée comme l'opposé du village situé quant à lui dans la montagne. Cela reprend le stéréotype comprenant le village comme lieu de l'authenticité. On relira l'œuvre de J. Ferrari que l'on comparera à celles de Marcu Biancarelli et mettra en perspective avec celle, plus ancienne, de Marie Susini afin de montrer comment ces auteurs mettent en scène les mutations rencontrées par le fait montagnard.

\section{AUTEUR}

\section{PIERRE BERTONCINI}

Docteur en anthropologie, chercheur associé à l'UMR LISA de l'université de Corse - Pascal-Paoli, président de l'APARMA (Association patrimoine recherche de Méditerranée et d'ailleurs) 


\section{Un classique du roman de montagne : Joseph Peyré (1892-1968)}

\section{Pierre Peyré}

1 Des peurs ancestrales au tourisme de masse aujourd'hui, la montagne a toujours fasciné. Elle est un sujet de contemplation quand le poète dialogue avec elle, mais, plus prosaïquement, on la reconnaît comme un objet de recherche scientifique et de développement économique et social. Comme la mer, elle a inspiré les sciences, les lettres et les arts. Certes accueillante, aussi bien que contrainte, elle s'est ouverte aux activités humaines qui en fixent l'image et conduisent le destin. Entre nature et culture, la montagne, au-delà de sa dimension physique, c'est tout à la fois le monde de ceux qui $\mathrm{y}$ vivent, en vivent et la font vivre, que celui de ceux qui en rêvent ou ne font qu'y passer.

2 Je rejoins donc Bernard Debarbieux quand il note que «l'étude des montagnes constituait la voie d'accès privilégiée à la connaissance du monde ${ }^{1}$ ». Mais je ne suis ni historien, ni géographe. Mon côté du pont, c'est la psychologie, au carrefour de l'anthropologie culturelle et de la psycho-biographie sur fond de paradigme constructiviste de la complexité et de transdisciplinarité 2 . C'est pourquoi, du global au local, du général au particulier et de l'individuel au collectif, je vais vous parler d'un romancier, philosophe, juriste, journaliste et poète à la fois, inspiré par la montagne. Il est aujourd'hui au " purgatoire des écrivains». Ce romancier qui va me permettre de porter un regard transversal et convergent sur l'objet qui nous réunit, n'est autre que mon oncle (fig. 1), Joseph Peyré, bien connu des gens de montagne et autres amateurs de beaux textes, pour ses évocations pyrénéennes et sa trilogie montagnarde. 
Fig. 1. - Joseph Peyré à son bureau et vu par Ben, « La France littéraire ».

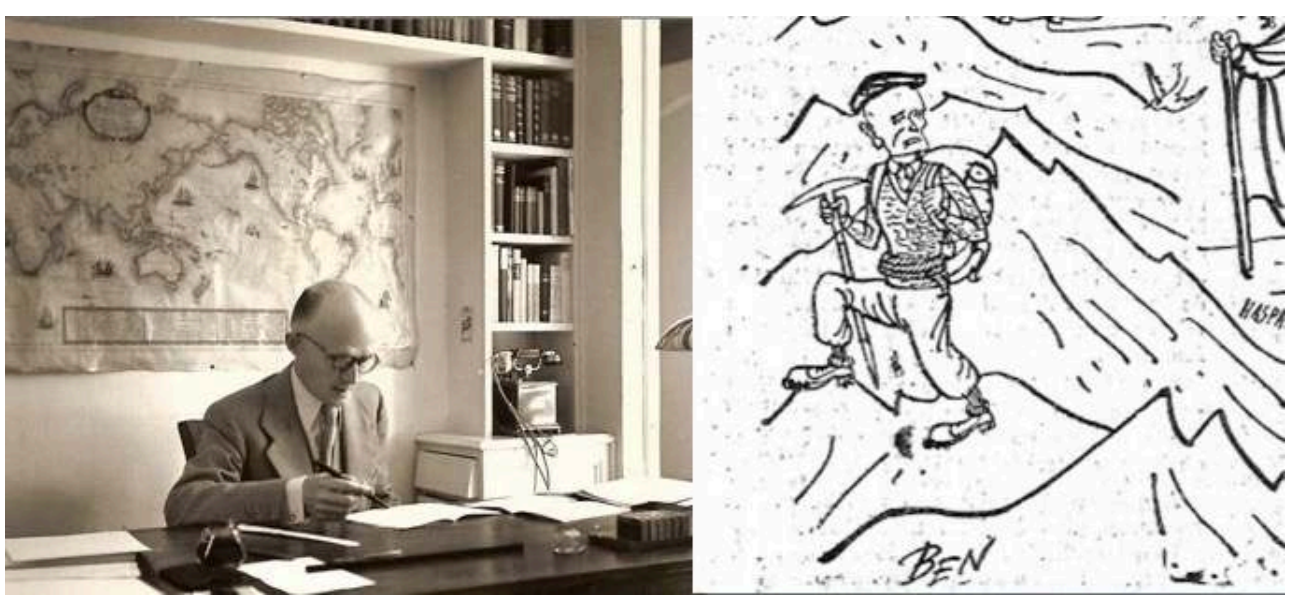

(c) Les Nouvelles Littéraires, juillet 1955.

\section{La fresque romanesque de Joseph Peyré}

Inspiré par le Pic du Midi d'Ossau, son "Cervin ${ }^{3}$ » pyrénéen, l'écrivain a parcouru en rêve et en réalité les Pyrénées, les Alpes et l'Himalaya. Il nous laisse Sur la Terrasse, De mon Béarn à la mer Basque, Matterhorn, Mont Everest et Mallory et son dieu.

\section{De la vue des Pyrénées}

Mort à Cannes en 1968, Joseph Peyré est né à Aydie en 1892, dans le nord-est du Béarn, le Vic-Bilh (le vieux pays en gascon béarnais) à une soixantaine de kilomètres de la chaîne des Pyrénées. Comme il le décrit dans De mon Béarn à la Mer Basque (1952) soustitré « essai de géographie personnelle », Aydie est pour lui un belvédère qui ouvre sur le monde et attise son goût inné de l'ailleurs :

« Les divers itinéraires que je devais suivre dans ma vie ou dans mes livres, j'en eus le pressentiment confus mais impérieux, dès mon enfance. Les routes qu'allait emprunter l'homme ou l'écrivain, je peux vraiment dire qu'elles sont parties de mon village béarnais natal. »

Entré à huit ans et demi au Lycée de Pau, l'enfant des coteaux du vieux pays se rapproche des sommets dont il connaît tous les noms. Au Sud, il devine l'Espagne voisine, et pressent l'appel de l'Afrique exotique. Interne, la promenade du jeudi sur le boulevard des Pyrénées n'est que récompense pour lui. C'est là son second belvédère après celui du haut d'Aydie d'où il contemplait déjà le Pic du Midi d'Ossau au Sud, et embrassait le panorama qui va du Pic du Midi de Bigorre à l'Est, aux Pics d'Anie et d'Arla à l'Ouest, en direction du Pic des Trois Couronnes au Pays Basque.

Le boulevard des Pyrénées est un haut lieu de la vie paloise (fig. 2). Les visiteurs l'apprécient: «Pau est la plus belle vue de terre comme Naples est la plus belle vue de mer $^{4}$ ", proclame Lamartine. Peyré en juge de même et rend hommage, à son tour, comme Barrès, Saint-John Perse, Zola, Colette, Stendhal et Victor Hugo, au célèbre boulevard, en composant en 1922 une plaquette intitulée Sur la Terrasse. Il est alors avocat stagiaire à Pau et c'est sa première publication: «Images exactes de paysages, images vaines de la Rêverie, il n'y a entre elles que nuances d'âme » écrit-il en exergue, 
comme pour s'autoriser à penser après Lamartine qu'« il n'est sans doute pas de ville en France qui est l'égale de cette perspective de montagne ».

Fig. 2. - La vue depuis la célèbre terrasse du Boulevard des Pyrénées, à Pau. Couvertures de Sur la terrasse, (édition originale de 1922, et édition de 1987).

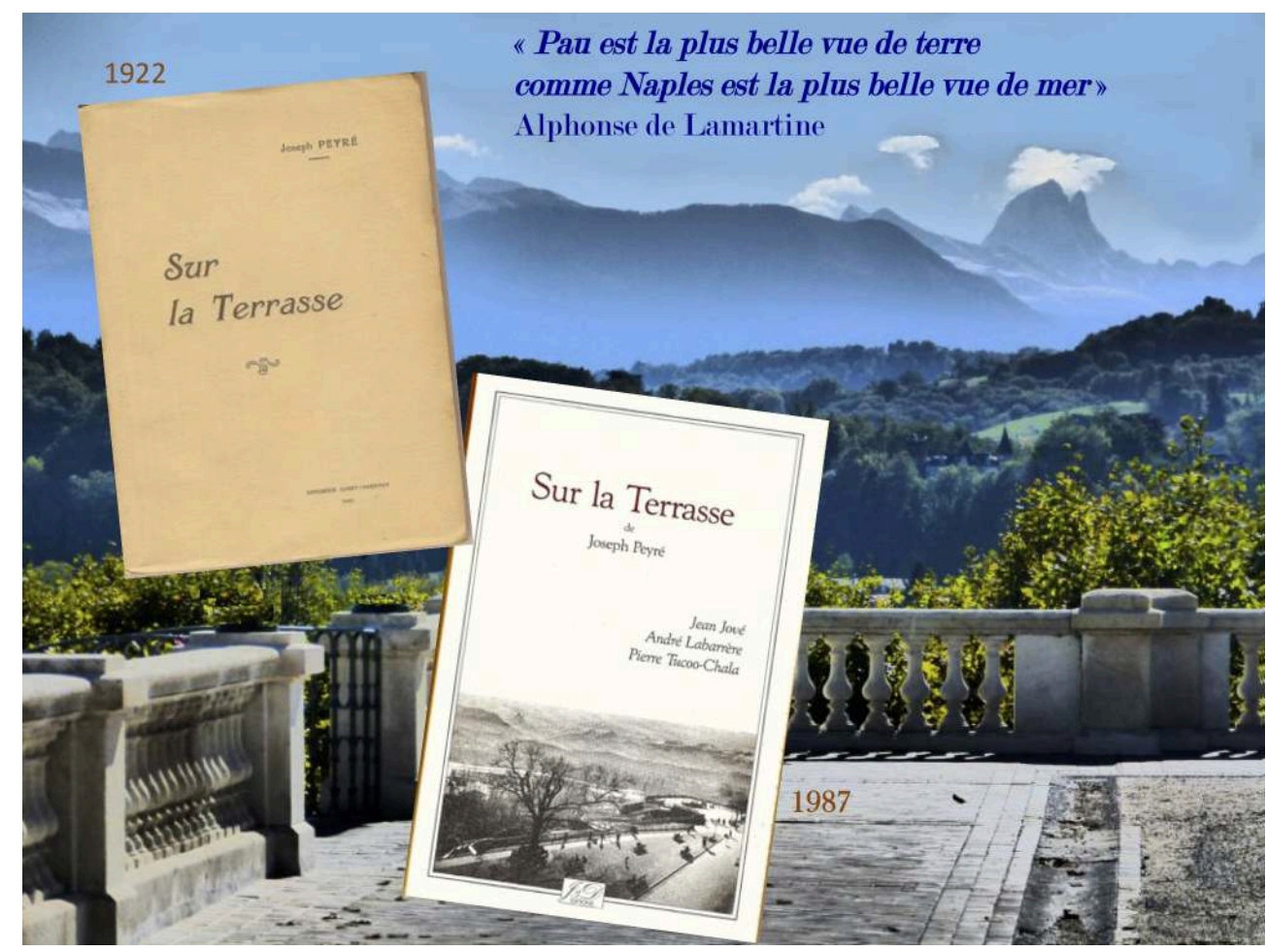

(C) Pierre Peyré

7 Hélas, de constitution fragile, Peyré, ne sera pas plus montagnard qu'il n'a été méhariste ou torero. Le fait est qu'en matière de montagne, le Béarnais, n'est guère alpiniste au sens où le terme est synonyme d'exploit sportif, indépendamment de toute appartenance aux Alpes. Pyrénéen, il est par contre pyrénéiste, si l'on considère (avec l'historien-géographe Henri Béraldi) que le pyrénéisme ne dissocie pas l'expérience physique de la montagne de l'émotion esthétique et culturelle qu'elle procure. Certes, l'écrivain béarnais n'a jamais connu que la voie normale du Pic de Ger où l'avait conduit, à 2613 mètres, le curé de son village, mais tout ému, il en racontait encore l'aventure, 45 ans après, dans son émission «Vent du Sud» à la radiotélévision française en 1958.

8 Ainsi, quasi paradoxalement, Joseph Peyré est-il devenu un expert de la grande épopée de l'alpinisme et du pyrénéisme.

\section{L'attraction mythique du Cervin}

Entre 1923 et 1935, Peyré est chef de cabinet du préfet de la Haute-Vienne, puis journaliste à Paris et à Madrid. En 1931, il obtient le Prix de la Renaissance avec L'Escadron Blanc. Pas plus qu'à la montagne, il n'est jamais allé au désert du Sahara, mais les récits de son frère, médecin méhariste, ont suffit à guider son imagination, au point que l'on a cru que le lauréat était un officier des compagnies sahariennes. En 1935, le Prix Goncourt le couronne pour Sang et Lumières. Et ce n'est qu'en 1939, après ses 
voyages imaginaires au Sahara, sa vraie vie en Espagne et ses séjours à Zermatt, qu'il publie son premier roman de montagne en 1939 : Matterhorn.

10 Avec ce roman édité chez Grasset au début de la guerre, l'écrivain inaugure une voie nouvelle dans laquelle il ne s'était jamais risqué : la haute montagne. C'est sous son nom suisse, le Matterhorn, que près de 100 ans après qu'il ait été vaincu par l'Anglais Edward Wimper, l'auteur raconte à sa façon poétique et réaliste à la fois l'histoire symbolique d'une ascension au Cervin.

11 Montagne-phare, sommet mythique, le Matterhorn culmine dans l'imaginaire des alpinistes. Il nourrit la mémoire des anciens, obsède les plus jeunes, et exaspère les guides qui se voient pris au piège, non pas des périls de la montagne elle-même, mais des succès du tourisme qui les conduit parfois à devoir choisir entre vivre au chômage, ou «traîner » au sommet des clients peu aguerris. Ce Cervin-là, chez Peyré, c'est la montagne qui se fâche, car elle veut rester inviolée :

«Le Matterhorn étend encore au monde son église. Ses néophytes et ses fidèles laissent suspendus à ses flancs des ex-voto secrets, à faire crouler les murs d'un autre temple.»

12 L'histoire du Cervin, qui bouleverse Zermatt et ses guides, a inspiré Peyré. Mais au-delà du mythe attracteur de foules et pourvoyeur de légendes, c'est l'esprit qui règne sur les faces vertigineuses et le sommet du Matterhorn qu'il a retenus.

\section{La mystique himalayenne}

13 Trois ans après Matterhorn et quelques détours en Espagne et au Maroc, voici Peyré reparti vers d'autres ascensions physiques et spirituelles, pour lesquelles la figure emblématique de l'Everest lui fournit le cadre des deux autres romans de sa trilogie : Mont Everest et Mallory et son dieu.

\section{Mont Everest (Grasset, 1942)}

14 L'expédition est conduite par Jewar Singh, un prince hindou qui affiche à son actif les plus grands sommets alpins. Il est accompagné par Jos-Marie Tannenwalder, le guide de Zermatt déjà mis en scène dans Matterhorn. Mais rien ne les a préparés à l'exténuante marche d'approche de l'Everest, qui met à vif la gorge et ronge les poumons.

15 Le vieil «Everester », autour duquel les difficultés se multiplient en progressant vers le sommet est pourtant servi par la chance, car il sera de la première cordée, celle qui redescend vaincue mais sauve de l'assaut final, alors que de la seconde paire d'assaillants, seul reviendra le sherpa Nima qui, à bout de forces, a laissé Jos-Mari continuer seul vers le sommet tout proche, dans un brouillard de glace. Jos-Mari a-t-il vaincu l'Everest? On ne le saura jamais.

16 Ici, comme dans Matterhorn où Kate prie au pied de la Croix du sommet, malgré l'épuisement et la menace de la tempête, l'ascension physique de Jos-Mari sur le « toit du monde" se double d'une mystique de l'ascension. Ici comme dans le précédent roman, un chemin spirituel double la voie qui mène au sommet de l'Everest. 
Mallory et son dieu (Grasset, 1947) préféré, l'écrivain croit à sa victoire. Cinq décennies plus tard, les faits lui donneront raison.

\section{Entre le réel et l'imaginaire, l'univers littéraire et montagnard de Peyré}

Poète qui éclaire de sa passion ses fresques exotiques, historien qui ne laisse rien aux dérives de l'imaginaire, géographe attentif qui dessine lui-même ses cartes (fig. 3), ce sont ces fonctions constantes que l'on retrouve dans l'univers montagnard de Joseph Peyré. Pour l'anecdote, il confesse avoir rêvé d'enseigner la géographie, qu'il préfère à l'histoire. Par respect pour l'histoire, explique-t-il, car il a conscience qu'« un excellent historien - comme l'a dit Fénelon - est peut-être encore plus rare qu'un grand poète ${ }^{5}$ ». 
Fig. 3. - Carte de Mont Everest et de Mallory et son Dieu dessinée par Joseph Peyré.

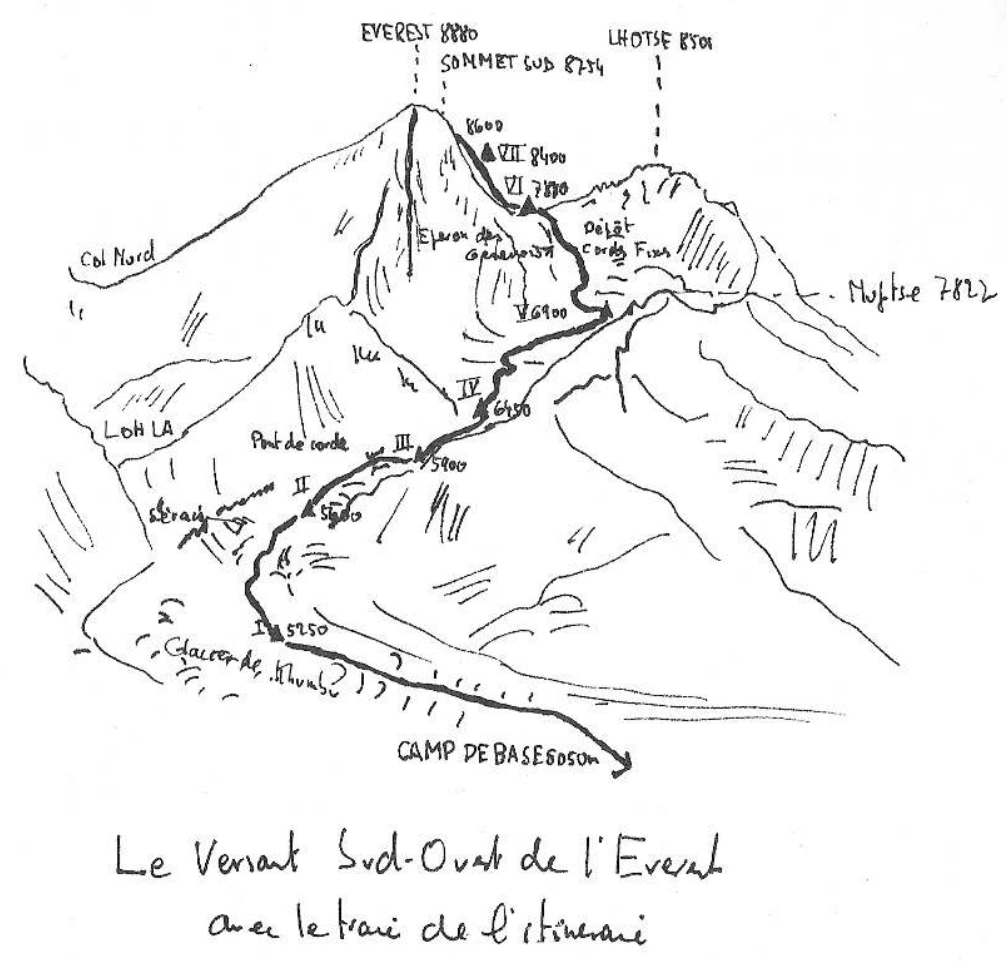

\section{Un écrivain voyageur}

Plus il se déplace du Nord de son Béarn natal vers le sud de l'Espagne et l'Afrique du Nord, et plus il parcourt d'Est en Ouest ses autres terres d'élection: Provence, Alpes, Himalaya, Andes, Nevada, plus le romancier ressent la force des liens qui l'attachent à "sa terre ", l'univers de son enfance. Aydie est son « centre du monde », et il l'exprime à sa façon, au gré de ses voyages exotiques. L'ancien ministre, député-maire de Pau, André Labarrère, l'a bien compris, qui relève le trait: pour lui, Joseph Peyré est un éternel voyageur, un migrateur "insatiable et insatisfait comme tout Béarnais qui connaît la nécessité de partir, de découvrir les ailleurs du monde afin de mieux revenir. Pour toujours dans sa petite patrie ${ }^{6} »$.

Auteur éclectique et fécond, il a tenu la scène littéraire pendant toute une moitié du $\mathrm{xx}^{\mathrm{e}}$ siècle. Témoin de son temps, ses personnages sont des gens simples, des "héros ordinaires" qui ont tous, comme chacun d'entre nous dans la vie, leur «montagne à gravir ».

Ses livres portent la marque d'une véritable philosophie humaniste, d'une conscience. Il aime les voyages pour s'enrichir des cultures dont il s'imprègne. Alors, il va à la rencontre de ses héros sans s'identifier à eux, mais en leur faisant partager son goût de l'ailleurs et sa quête d'absolu. Jusqu'à en être visionnaire.

Écrivain voyageur, J. Peyré s'est rapproché de la haute montagne avec les mêmes élans de cœur et de raison, qu'il s'est passionné pour d'autres horizons. Entre la terre et le ciel, la montagne est pour lui une frontière sacrée, une assomption. Et c'est ce qui fait de sa trilogie une œuvre qui n'a pas d'âge. 

écrit sur la montagne, c'est qu'il l'a aimée. C'est aussi parce qu'il a appris à la connaître et à la respecter. Il s'y est fait des amis qui l'ont guidé dans ses découvertes. Mais, magique et cruelle, la montagne reste un mystère pour lui, comme pour Michelet, d'ailleurs, qui concevait que tout ce que cache et révèle à la fois la montagne, « est pays de $\operatorname{roman}^{7}$ ».

Peyré s'est appliqué avec bonheur à l'exercice de ce roman. Si bien que, de la critique littéraire à l'authentification de son intuition visionnaire de la mort de Mallory, les témoignages abondent, qui nous renseignent sur cet univers qui a fait de Joseph Peyré un classique du roman de montagne.

\section{Du point de vue de la critique littéraire}

\section{À propos de Matterhorn :}

«Bien que n'étant pas alpiniste, Joseph Peyré a fort bien compris l'alpinisme et en a parfaitement assimilé la terminologie..$^{8}$ "

«On atteint là ce que devrait être tout roman de montagne : l'intime alliance d'un paysage et d'une action, la création, par cette alliance, d'un univers imaginé. Joseph Peyré, dans les derniers chapitres de Matterhorn, avait lui aussi [comme Roger Frison-Roche] réussi une telle création. ${ }^{9}$ "

À propos de Mallory et son dieu :

«La réédition de cet ouvrage, paru en 1947, apporte la preuve que l'aventure humaine lorsqu'elle passe par certains sommets, en l'occurrence l'Everest, ne prend pas une ride. ${ }^{10}$ »

"Quel plus bel hommage pour un écrivain que d'être réédité après sa mort... ${ }^{11}$ »

\section{Du point de vue des témoignages d'experts}

31 René Dittert, le chef de l'expédition suisse de 1952 à l'Everest, correspondait depuis le camp de base avec Joseph Peyré. Ses lettres ${ }^{12}$ attestent de ce don que possédait l'écrivain d'assimiler des mondes qu'il ne connaissait pas, au point de pouvoir dialoguer d'égal à égal avec les plus grands comme avec les plus humbles, sans jamais se prendre pour un pair.

- Lobeje, 21 avril 1952 (4 800 mètres) :

"Cher Monsieur, je vais au-devant des plus belles heures de ma carrière d'alpiniste [...] Je ne voudrais pas laisser passer ces jours sans vous dire aussi combien [...] je me sens petit et humble devant la grande montagne, je veux vous dire combien je revis votre Mont Everest et combien il respire avec exactitude la vie que nous menons. »

- Genève, 8 août 1952 :

«Tout au long de notre longue progression, le long des interminables pentes menant au Col Sud, j'ai souvent pensé à vous, à votre Mont Everest. »

Et dans une autre lettre écrite au retour de l'expédition :

«Mon cher ami, Je ne saurais trouver les mots pour vous dire combien j'ai été touché par vos lettres reçues sur le glacier de Khumbu... »

Plus saisissante encore, cette dédicace collective de Chevalley, Ditter, Lambert et Tensing de leurs Avant-premières à l'Everest (Arthaud, 1954) (fig. 4).

«Genève, mars 1954. À Joseph Peyré, avec toute notre sympathie et en témoignage de profonde admiration pour son livre Mont Everest, qui nous a préparés à notre expédition. » 
Fig. 4. - Dédicace des membres de l'expédition suisse : Ditter, Chevalley, Lambert et Tensing.

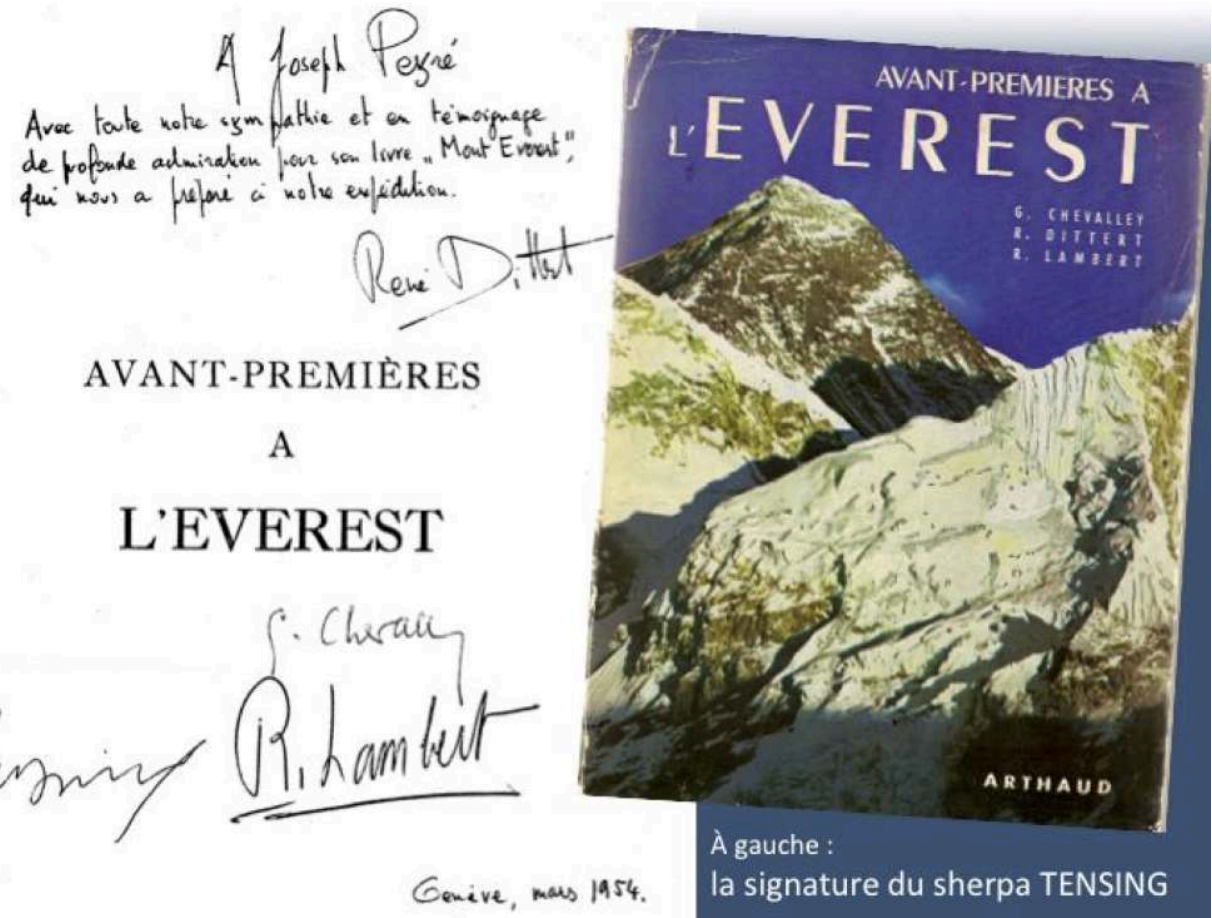

(C) Pierre Peyré

Empathique, celle de Jean-Jacques Languepin en tête de son Himalaya, passion cruelle (Flammarion, 1955) (fig. 5).

«À Monsieur Joseph Peyré qui sait si bien deviner les secrets des grandes montagnes et ceux des hommes qui en subissent l'envoûtement, en témoignage d'admiration et en hommage de l'auteur et de ses camarades " 
Fig. 5. - Dédicace de J.-J. Languepin, auteur de Himalaya, passion cruelle (Flammarion, 1955).

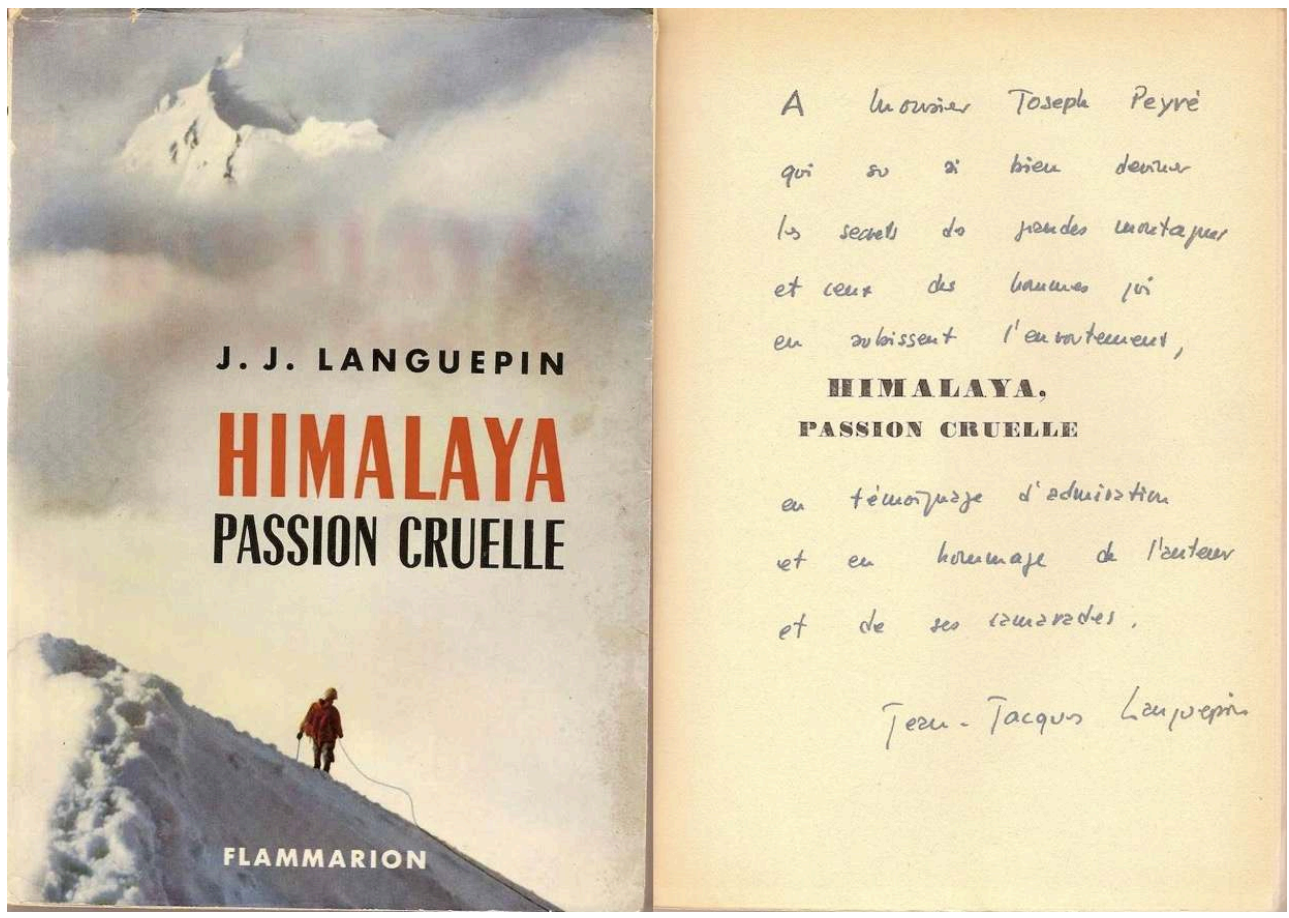

(c) Pierre Peyré

Révélatrices aussi, celles des livres d'Alain de Chatellus (fig. 6 et 7) : De l'Eiger à l'Hiharen (J. Susse, 1947) :

«À Monsieur Joseph Peyré, en témoignage de sympathie et d'admiration pour le premier romancier français qui ait su comprendre les véritables mobiles des alpinistes. "

Alpiniste est-ce toi ? (Arthaud, 1953) :

«À Monsieur Joseph Peyré qui connaît si bien la valeur des actions dangereuses, cette tentative d'explication. Hommage de l'auteur. » 
Fig. 6. - Dédicace de Alain de Chatellus, auteur de De l'Egeir à l'Iharen, (J. Susse, 1947).

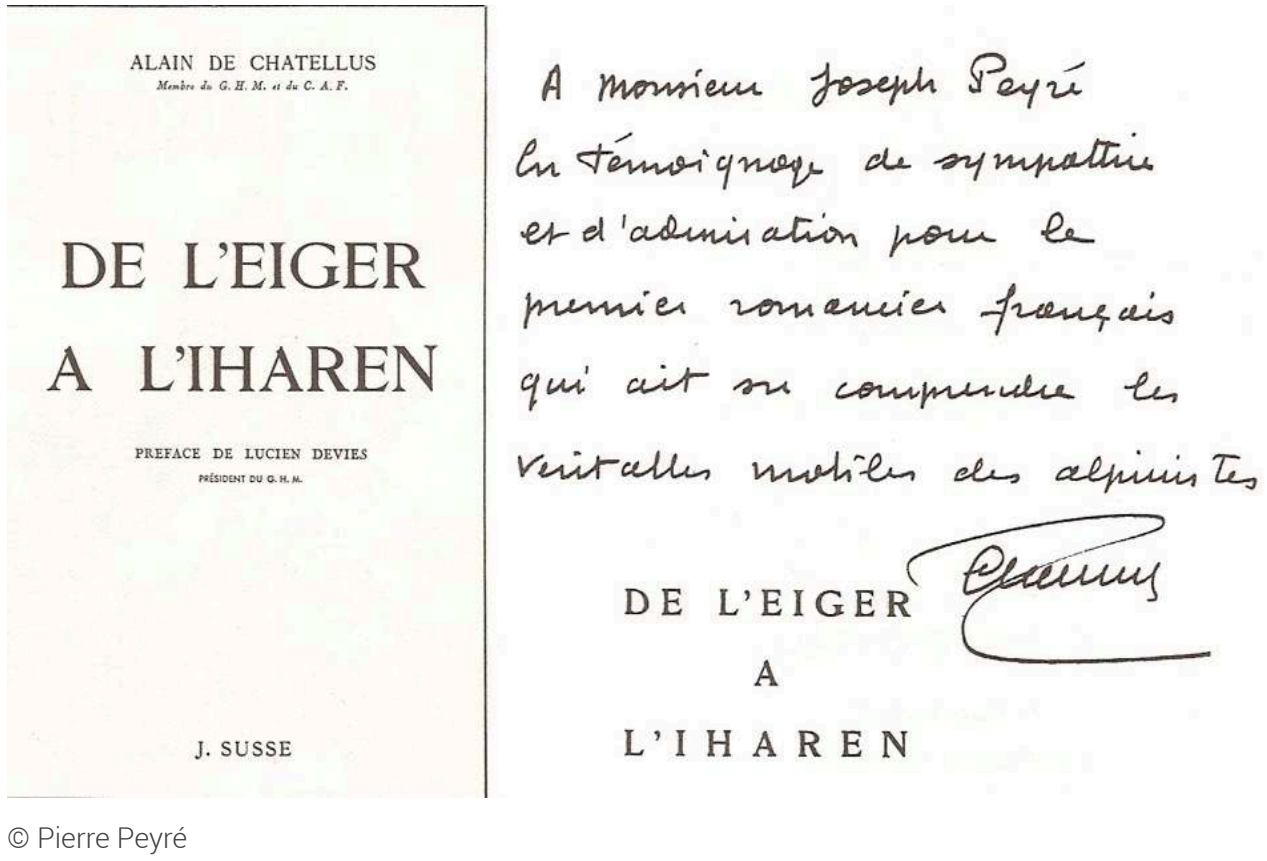

Fig. 7. - Dédicace de Alain de Chatellus, auteur de Alpiniste est-ce toi ? (Arthaud, 1953).

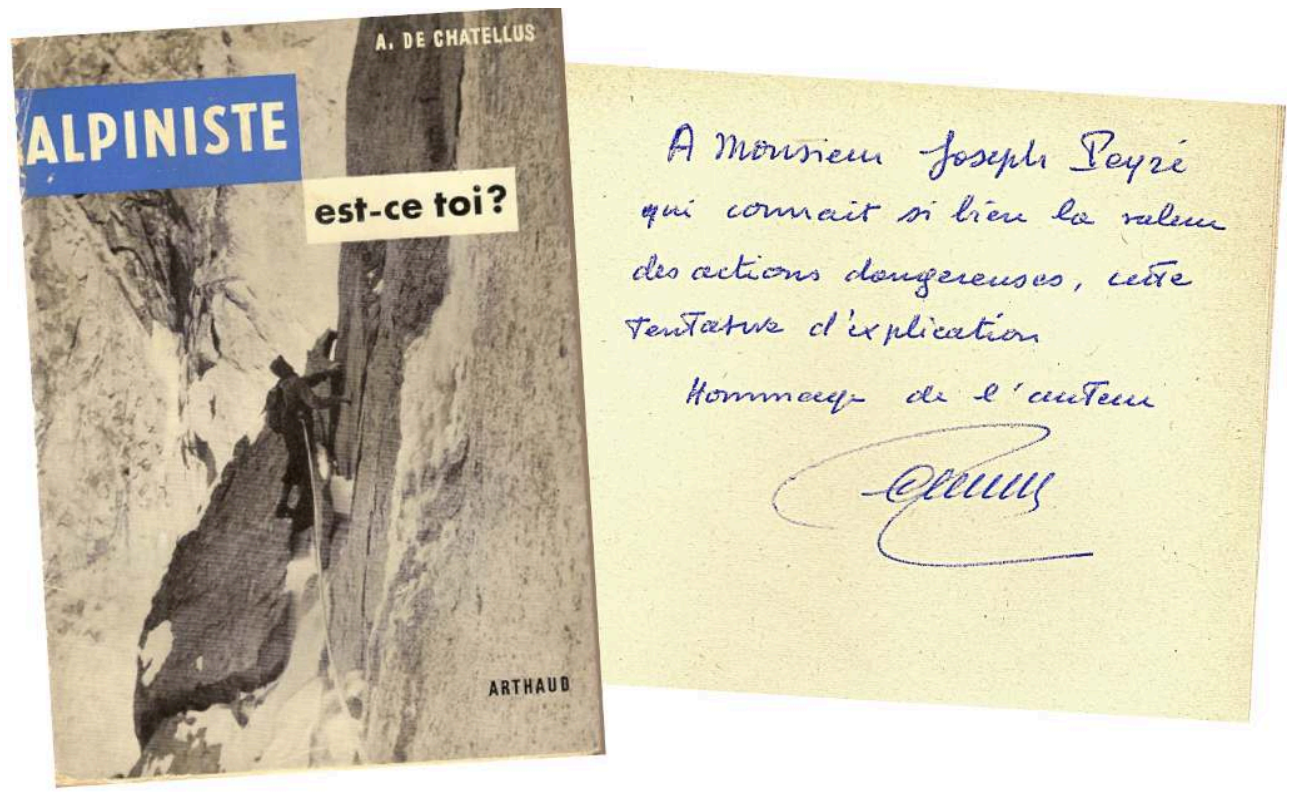

(c) Pierre Peyré

Fidèle en amitié, Roger Frison-Roche répond toujours présent en 1992 pour la célébration du Centenaire Joseph Peyré, à Pau :

«Sans hésitation possible, je dois à Joseph Peyré ma vocation saharienne [...]; son escadron blanc m'a poursuivi, harcelé, comblé mes désirs d'aventures. [...]. Un " grand merci » Joseph Peyré, pour m'avoir fortifié par ces récits prémonitoires de la montagne et des déserts, qui m'ont dicté ma propre aventure. » 
Émouvante d'humilité, cette dédicace de Robert Ollivier dont Le Pic d'Ossau (J. Susse, 1948) évoque l'attachement commun du guide de haute montagne et de l'écrivain à leur beau Cervin pyrénéen :

«À mon grand préfacier Joseph Peyré en le remerciant infiniment du grand honneur qu'il a fait à ce modeste livre. »

Fig. 8. - Dédicace de Robert Ollivier, Le Pic d'Ossau, (J. Susse, 1948).

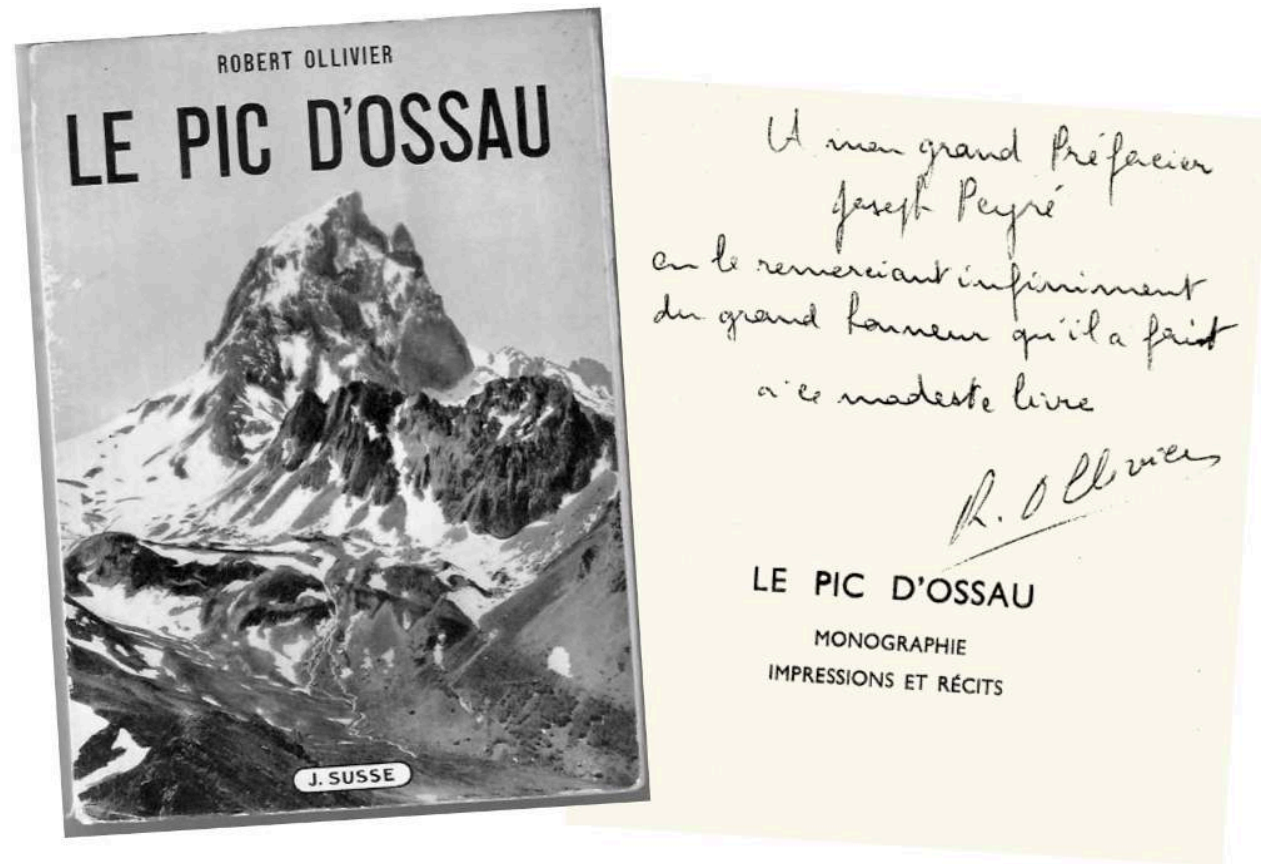

(c) Pierre Peyré

\section{La force d'une intuition}

Cinquante-deux ans après qu'il eût écrit Mallory et son Dieu, les faits sont venus corroborer la version du drame de l'Everest imaginée par le romancier (fig. 9). « Le héros de l'Everest retrouvé ", titre Le Figaro Magazine du 15 mai 1999. La montagne, en effet, a rendu soixante-quinze ans après sa disparition, le corps de Mallory, découvert par une expédition américaine le $1^{\text {er }}$ mai 1999 à 8300 mètres, le torse et les deux bras émergeant des glaces, à peu près là où le romancier l'a fait disparaître aux yeux de Nima, dans son roman de 1947. 


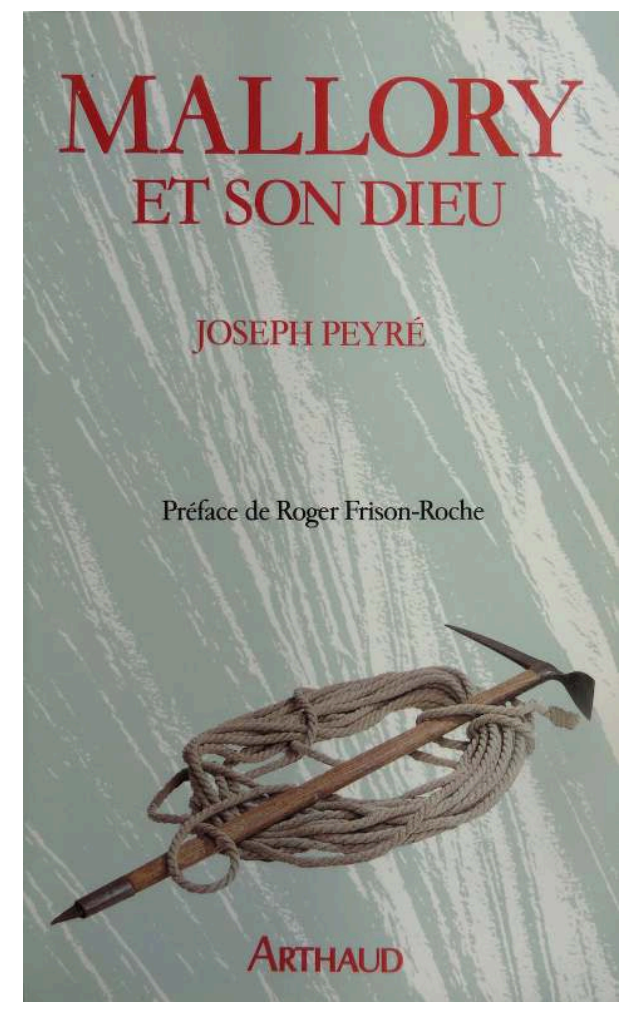

Une philosophie de la montagne

(c) Pierre Peyré

Toutefois l'énigme de la victoire reste entière : l'instituteur alpiniste anglais a-t-il été le premier à fouler le plus haut sommet de la planète, vingt-neuf ans avant l'apiculteur néo-zélandais Edmund Hillary et le sherpa népalais Tensing Norgay? Peyré a voulu le croire, mais il s'est tu. C'est la montagne qui a toujours le dernier mot. De fait quand il parle de montagne, à ces heures de guerre en Europe, Peyré - en quête du vrai bien emprunte les voies directes qu'il n'a jamais faites, car pour lui :

"Que l'Everest soit encore ou ne soit plus vierge, que Mallory ait fait ou non la plus haute montagne du monde, ce n'est pas ce qui importe. Ce qui compte à nos yeux, c'est l'aventure spirituelle du héros, son ascension. ${ }^{13}$ "

41 Hergé l'a bien compris dans Tintin au Tibet (1960), qui nous mène au Tibet mystique et magique, pour voir Tintin sauver Tchang d'une mort certaine, malgré l'incrédulité du capitaine Haddock. Assurément, l'Everest n'en finit pas de fasciner et de tuer les aventuriers de l'extrême: plus de 235 morts depuis presque un siècle, chiffrent les bilans officiels en 2017. Et la liste s'allonge chaque année. Sans compter les dizaines de tonnes d'ordures qui défigurent les glaciers!

Analysant la "poétique de la montagne dans l'œuvre de Joseph Peyré ", l'écrivain palois Pierre Minvielle, retient que, «à la différence de ce qui se passe dans les œuvres de Ramuz, de Giono, de Frison-Roche ou de Troyat, la montagne n'est jamais quelconque chez Peyré », elle est "un personnage animé ». Et la poétique est là, conclut-il « qui propulse la vérité géographique vers les hauteurs de la spiritualité ${ }^{14}$ ». 
Il y a là, en effet, une dimension universelle et intemporelle dans la trilogie de Peyré, qui, entre roman et poésie, littérature et écologie, à travers et au-delà de toutes les sciences et croyances, me fait penser (au sens de l'évolutionnisme et de la transdisciplinarité) à cette formule du biologiste généticien Théodore Dobzhansky :

« En changeant ce qu'il connaît du monde, l'homme change le monde qu'il connait ;

En changeant le monde dans lequel il vit, l'homme se change lui-même..$^{15}$ "

Que de frontières, de ponts et de cols à passer, que de sommets à vaincre; que de richesses et de drames, la montagne ne nous pousse-t-elle pas à connaître ? Que de cultures à découvrir ? Toutes ces perspectives, on voudrait les comprendre à la fois. Les embrasser. On voudrait les conjoindre, et atteindre avec Miguel Torga le graal de son aphorisme :

«L'universel, c'est le local moins les murs »!

Assurément, la montagne est une porte ouverte à la culture universelle. Une culture, écrit le médecin écrivain portugais, c'est "une lumière qui brille", et toutes les cultures sont riches de leurs spécificités. Mais, dit Torga :

«Elles doivent partager leurs expériences, ouvrir leurs portes, abattre les murs, être universelles sans jamais perdre pour autant leur identité. ${ }^{16}$ "

C'est de cette universalité qu'il s'agit chez Peyré en général, et dans sa fresque montagnarde écrite aux heures de guerre, en particulier. Et c'est de cette universalité là qu'il s'agit, encore, dans son article des Nouvelles Littéraires intitulé "À chacun sa montagne ", où il ouvre une porte sur lui-même en disant sa vérité : à savoir qu'au propre comme au figuré, nous avons tous jusque dans la moindre de nos journées, «notre montagne à gravir ». Le médecin alpiniste Isabelle Janin le sait bien, qui a fait de cette devise le symbole de son association au service des enfants malades:

«À chacun son Everest. »

Certes, l'écrivain béarnais, n'a jamais couru le risque des hautes altitudes, mais grâce au roman il s'est élevé avec elles. Michel Ballerini en atteste :

« Le cas d'Henri Troyat est comparable à celui de Joseph Peyré : l'un et l'autre n'ont jamais pratiqué l'alpinisme, mais l'un et l'autre ont écrit des œuvres qui comptent parmi les meilleures de la littérature alpine romanesque. ${ }^{17}$ »

\section{BIBLIOGRAPHIE}

BALLERINI Michel, Le Roman de montagne en France, Paris, Arthaud, 1973.

Collectif : Les voyages romanesques de Joseph Peyré, Sahara, Espagne, Himalaya. Préface d'André LABARRÈRE. Exposition du Centenaire Joseph Peyré, Bibliothèque municipale de Pau, Imp. Géronis, Pau, 1992, 69 p.

DEBARBIEUX Bernard, « La montagne, un objet géographique ? », dans VEYRET Yves (dir.), Les montagnes, discours et enjeux géographiques, Paris, Sedes, 2001, p. 1-21.

DELAY Pierre, Joseph Peyré (1892-1968), l'homme et l'œuvre, à la rencontre des héros. Préface de JeanLouis Curtis, Biarritz, J. \& D. Éditions, 1992, 250 p. 
DOBZHANSKY Theodosius, L'homme en évolution, 1961, traduit de l'américain par Georges et Simone PASTEUR, 1966, Paris, Flammarion, 432 p.

FÉNELON, Euvres complètes, tome III, Lettres sur les occupations, Lefevre (éditeur) 1835, p. 242.

FRISON-ROCHE Roger, " Je dois à Joseph Peyré ma vocation saharienne ", Les voyages romanesques de Joseph Peyré, Bibliothèque municipale de Pau, catalogue de l'Exposition du centenaire : 1892-1992, p. 33.

MICHELET Jules, La montagne, $1^{\text {ère }}$ édition 1868, $7^{e}$ éd. Hachette Livre, 2012.

MinVIELle Pierre, «Poétique de la montagne dans l'œuvre de Joseph Peyré », Revue Pyrénées, $\mathrm{n}^{\circ}$ 172, Pau, 1992, p. 457-466.

NICOLESCu Basarab, La transdisciplinarité, Manifeste, Monaco, Ed. du Rocher, 1996.

PEYRÉ Joseph, Sur la terrasse, Pau, Garet-Haristoy, 1921.

PEYRÉ Joseph, Sur la terrasse, Préface d'André LABARRÈRE, Postface de Pierre TUCOO-CHALA, Photos Jean JovÉ, Biarritz, J \& D Éditions, 1987.

PEYRÉ Joseph, L'Escadron blanc, Paris, Éditions des Portiques, 1930.

PEYRÉ Joseph, Sang et lumières, Grasset, 1935.

PEYRÉ Joseph, De mon Béarn à la mer basque, Paris, Flammarion, 1952.

PEYRÉ Joseph, Matterhorn, Paris, Grasset, 1939.

PEYRÉ Joseph, Mont Everest, Paris, Grasset, 1942.

PEYRÉ Joseph, Mallory est son dieu, Paris, Éditions du Milieu du Monde, 1947.

PEYRÉ Joseph, « À chacun sa montagne », Les Nouvelles littéraires, nº 1099, 23 septembre 1948.

PEYRÉ Joseph, «Vent du Sud », Série de 11 émissions de la Radiodiffusion-télévision Française, présentée par Marguerite Taos sur Antenne 2, du 5 janvier au 16 mars 1958.

TORGA Miguel, «O universal é o local sem muros », Diário XV, 1990.

UPPA (Collectif), « Joseph Peyré, L'homme de ses livres », actes du Colloque International, Université de Pau et des Pays de l'Adour (Laboratoire d'Identité régionale) Pau, 3 et 4 avril 1992. Préface de Pierre tucoo-chala, Avant-propos de Hélène CHARPENTIER, Biarritz, J. \& D. Éditions, 1994, 414 p.

\section{NOTES}

1. B. Debarbieux, «La montagne, un objet géographique?» Y. Veyret (dir.), Les montagnes, discours et enjeux géographiques, p. 1.

2. Pour Kant la connaissance [...] frontières entre les disciplines. Ce nouveau paradigme a poussé les constructivistes à établir une « Charte de la transdisciplinarité ». Celle-ci a été adoptée lors du Premier Congrès Mondial de la Transdisciplinarité, qui s'est tenu au Couvent d'Arrábida, au Portugal en 1994. Le physicien Basarab Nicolescu présente l'essentiel de ces travaux dans un ouvrage intitulé : La transdisciplinarité, Manifeste (Ed. du Rocher, 1996). 
3. Le Cervin, Matterhorn en allemand, est le $12^{\mathrm{e}}$ sommet le plus élevé d'Europe avec ses $4478 \mathrm{~m}$ d'altitude, situé entre dans le Valais à la frontière italo-suisse.

4. Phrase attribuée au poète selon le Guide littéraire de Pau de Louis Ducla et André Sarrail publié en 1962. Citation non retrouvée dans sa correspondance malgré sa venue à Pau en juillet 1840. Une première mention de cette attribution à Lamartine paraît en 1902 dans Le monde moderne et la femme d'aujourd'hui, p. 670.

5. Fénelon, Lettres sur les occupations, p. 242.

6. Les voyages romanesques de Joseph Peyré, Préface d'A. Labarrère, p. 4.

7. J. Michelet, La montagne, p. 84.

8. H. Hisselin, «À propos d'un livre récent ».

9. M. Ballerini, p. 136.

10. B. Vadon, Nice-Matin, 11 juillet 1987.

11. L. Laborde-Balen, Sud-Ouest, 23 avril 1987.

12. Voir le fonds Peyré à la bibliothèque municipale de Pau.

13. J. Peyré : Mallory et son dieu, p. 15.

14. P. Minvielle, p. 466.

15. T. Dobzhansky, p. 391.

16. M. Torga, Diario XV.

17. M. Ballerini, p. 136.

\section{RÉSUMÉS}

Éclectique, Joseph Peyré bien connu pour ses romans sur le Sahara (L'Escadron Blanc, Prix de la Renaissance 1931) et l'Espagne (Sang et Lumières, Prix Goncourt 1935) s'est aussi passionné pour les altitudes, théâtre où l'homme se magnifie et se grandit comme dans la solitude du désert ou sur le sable de l'arène. Écrivain voyageur, animé par le goût de l'ailleurs et la quête d'absolu, la conquête des sommets et l'esprit qui y règnent lui fournissent un sujet sur mesure. Inspiré par le Pic du Midi d'Ossau, son "Cervin pyrénéen ", l'écrivain se transporte dans les Alpes d'où il revient avec Matterhorn (1939), et s'évade en Himalaya pour étayer sa fresque montagnarde avec Mont Everest (1942) et Mallory et son dieu (1947). Ainsi Peyré est-il considéré comme un classique du roman de montagne. Pourtant l'écrivain, à la différence de Roger Frison-Roche par exemple, n'a jamais couru le risque des altitudes comme le souligne Michel Ballerini [« Le cas d'Henri Troyat est comparable à celui de Joseph Peyré : l'un et l'autre n'ont jamais pratiqué l'alpinisme, mais l'un et l'autre ont écrit des œuvres qui comptent parmi les meilleures de la littérature alpine romanesque». M. Ballerini, Le Roman de montagne en France, p. 95-110]. Philosophe de formation, humaniste, poète, journaliste, conférencier, l'écrivain s'est battu pour les espaces de neige et de roc. Entre la terre et le ciel, la montagne est pour lui une frontière sacrée, une assomption. Et c'est ce qui fait de sa trilogie montagnarde une œuvre qui n'a pas d'âge. 
AUTEUR

PIERRE PEYRÉ

Professeur émérite STAPS (Sciences et techniques des activités physiques et sportives, et santé), Université de Pau et des pays de l'Adour, ancien psychologue des hôpitaux, psychosociologue de la santé 


\title{
Joseph Peyré (1892-1968) : radiographe pyrénéen
}

\author{
Christian Manso
}

1 Le 29 septembre 1950, Joseph Peyré met un point final à son œuvre De mon Béarn à la mer basque qui fera l'objet d'une première publication en 1952. L'intérêt suscité par cet ouvrage est, assurément, accentué par son sous-titre, Essai de géographie personnelle, lequel éclaire l'usage du possessif du titre, mon, en renforce la portée et place immédiatement ce livre sous le sceau d'une subjectivité pleinement assumée, revendiquée même, en un domaine, la géographie, qui, a priori, en est totalement dénuée ou, à tout le moins, se définit intrinsèquement comme telle. Un paradoxe, donc, sur lequel repose une originalité qui ne peut manquer de piquer la curiosité du lecteur potentiel et ce d'autant plus que la démarche énoncée dans la Préface, tout en s'inscrivant résolument à rebours de l'Histoire, a pris le parti d'appréhender l'immuable, l'inaltérable :

«Aussi le témoignage que j'apporte ici à mon pays ne va-t-il pas aux vivants, ni aux morts. ${ }^{1} »$

2 Ce dernier porte sur «les choses qui demeurent ${ }^{2}$ ", à savoir sur "les terres et les paysages $^{3}$ ». Toutefois, l'approche de semblable réalité géographique ne saurait se concevoir chez Joseph Peyré sans méthode ni gradation :

« Mais un pays ne s'apprend pas, il n'est pas donné d'un seul coup [...] Il fait l'objet

d'une manière de découverte, d'une expérience personnelle qui change avec chaque homme, et forme les traits d'un visage particulier. ${ }^{4}$ "

3 À cet effet, il a cru bon de diviser son livre en deux parties pour rendre compte au mieux de sa quête, l'une délibérément tournée vers l'apprentissage qui le replonge chez l'enfant qu'il fut dans les rapports à son environnement, l'autre fondamentalement axée sur la synthèse qui se dégage de la réflexion de l'homme arrivé au soir de sa vie sur une semblable thématique. 


\section{Radiographie de son enfance}

4 Dans la première partie, Joseph Peyré tente, avec succès, de réinvestir l'univers mental du jeune Aydien qu'il fut, pour lequel la configuration géographique dont il a bénéficié a été, selon lui, déterminante dans la formation de sa propre personnalité. Ce village d'Aydie, qui se trouve aux confins du département des Basses-Pyrénées jouxtant celui des Hautes-Pyrénées ainsi que celui du Gers, a immanquablement contribué à générer en lui ce sentiment exacerbé de refuge, d'abri à l'écart du monde, voire de "sauvagerie ${ }^{5}$ » qu'il n'a eu de cesse de préserver jalousement. Deux éléments tutélaires en sont, à ses yeux, les garants : la " côte qui s'élevant vers l'ouest, nous protégeait de son épaule ${ }^{6}$ " et le Sagé, «le ruisseau frontière que la route ne franchissait pas $^{7}$ ». L'usage de la prosopopée qui se double d'anthropomorphisme appuyé n'a rien de feint en pareil cas, bien au contraire : Joseph Peyré capte au mieux les représentations émotionnelles que s'est forgées peu à peu en son intimité cet enfant qu'il fut, confronté à la prépotence des phénomènes naturels, à des réalités ultrasensibles relevant du magico-religieux, dont il était entouré. Ainsi en va-t-il du vent dont l'air vif au sommet de la côte du Haut-d'Aydie " annonçait l'océan ${ }^{8}$ ", qui l'amène à ce commentaire :

« Sans doute battait-il la crête pour rappeler au timide village [...] la présence des

espaces, et, ainsi, tenter ses esprits voyageurs. ${ }^{9}$ "

Ou encore de la profondeur de l'horizon qui « inspirait une espèce d'effroi ${ }^{10} »$. Dans de telles conditions, passer le limes d'un semblable territoire n'est pas sans risque ni dérangement. Encore que se déplacer jusqu'à Lembeye, à quelque quinze kilomètres, ne se puisse concevoir comme un total dépaysement. À cet égard, la présence de plusieurs côtes raides, d'est en ouest, est, à vrai dire, quelque peu rassurante, qui sont autant de remparts protecteurs : ne forcent-elles point les voyageurs à descendre de la jardinière où ils ont pris place et par conséquent à les enjamber non sans effort jusqu'à leur sommet pour regagner ensuite leur siège ? Cependant, deux signes sont annonciateurs d'une étrangeté quelque peu inquiétante. La route fait état de marques de l'ailleurs : composée de " boue argileuse " $^{11}$ ", elle est « rechargée à la saison de plaques de cailloux coupants - le gravier bleu de l'Adour ${ }^{12} "$, et puis, aux abords de cette ville, la voiture court sur des lieues de " poutge ${ }^{13}$ » plate. S'amorce, à l'évidence, une rupture qui n'est pas tant due au relief lui-même qu'à un paysage qui va prendre des aspects nettement dysphoriques. En effet, si entre Lembeye et Pau la route est en butte à l'opiniâtreté des côtes qui rappelle celle des précédentes, la mutation de la végétation ne peut être plus marquée :

«Plus de vignes pour égayer les pentes de l'est de leur chaude rougeur d'octobre

[...] Les couverts du touya - la lande béarnaise - étouffaient maisons et clochers. ${ }^{14}$ »

La tristesse l'emporte donc, par ses toits d'ardoise; de plus, s'entrouvre « un pays de terres froides, de châtaignes et de lait ${ }^{15} »$. Après six heures de route le séparant de sa terre aydienne et le franchissement du bourg de Morlaàs, le voyageur Peyré touche enfin à une ligne de démarcation sise exactement " au tournant de Serres-Morlaàs ${ }^{16}$ ». De ce belvédère qui départage Béarn des Côtes et Béarn des Gaves, il lui est loisible de percer à jour l'existence du Gave (de Pau) de par la vapeur qu'exhale la vallée qui «se déroule maintenant à (ses) pieds ${ }^{17}$ » ainsi que " l'oppressante proximité de la chaîne des Pyrénées $^{18}$ ». Cette longue et cahoteuse traversée d'Aydie à Pau, qui a arraché Joseph Peyré à son havre aydien, l'a plongé dans un ailleurs tout aussi déconcertant que riche d'enseignements. Un ailleurs qui ira croissant avec son approche de la haute montagne. 
7 C'est vers la fin de son «âge de lycéen ${ }^{19}$ » vécu au sein du lycée de Pau, que s'offre à lui l'opportunité de rencontrer "le prince du paysage ${ }^{20}$ ", c'est-à-dire le Pic du Midi d'Ossau. Cette vallée qui va lui en donner l'accès va provoquer en lui un choc émotionnel tel qu'il n'hésite nullement à la monter au pinacle :

« Ni la vallée de Zermatt sous l'apparition du Cervin, ni la vallée sainte de Rongbuk sous celle de l'Everest [...] n'ont eu sur moi pareil pouvoir. ${ }^{21}$ "

Un avant-goût de cette idiosyncrasie pyrénéenne lui est fourni en chemin par la présence du Gave (d'Ossau) dont il remonte le cours :

«Il gardait la fougue de ses sources. Il se déchirait à ses propres écueils, les couronnait de ses écumes, précipitait ses chutes scintillantes. ${ }^{22}$ "

Le grandiose et l'impétueux s'imposent d'emblée. Toutefois, en un tel cadre tout semble concourir pour le troubler. À commencer par la réalité minérale de la route qui n'est pas sans le frapper :

«La route blanche restait dure au pied, comme un marbre, le marbre qui en faisait le grain [...] et je m'émerveillais, moi qui étais né d'une terre dénuée de pierre. ${ }^{23}$ »

Après une halte nocturne chez le curé doyen de Bielle, se désaltérant à la fontaine du village, c'est l'eau qui le fait tressaillir d'aise :

«Jamais je ne devais goûter eau plus glacée, plus savoureuse [...] Elle avait un parfum de neige et de prairie en fleurs. ${ }^{24}$ "

Il va sans dire que pour cet aydien, cette eau " dont nous étions si durement sevrés ${ }^{25}$ ", lui fait «l'effet d'un miracle ${ }^{26}$ ». Enfin, s'écartant peu à peu de la route, il s'engage dans les alpages environnant les Eaux Bonnes et les lacets du Gourzy où, à nouveau, il est pris d'un saisissement qui l'ébranle tout autant que la minéralité de la route :

"Je glissais sur des prés de réglisse et de fleurs vives, dont aucune n'avait les violets, les jaunes ternes de celles de mes champs. ${ }^{27}$ "

Le constat qu'il dresse ne peut être plus péremptoire :

"Je passais vraiment dans un autre monde, où rien de mon expérience passée ne servait. ${ }^{28} »$

13 Lequel, cependant, suscite chez Joseph Peyré, à la faveur d'une subtile mise en abyme, l'idée d'une entité microcosmique protéiforme renvoyant du macrocosme l'intégral reflet :

« Notre patrie se suffisait, et suffisait à nous fournir toutes les images du monde..$^{29}$ »

L'approche du Pic du Midi d'Ossau, qui s'effectue depuis une sente conduisant au Pic de Ger, réunit pour l'occasion tous les ingrédients d'un rituel mystique d'accès au sancta sanctorum. Tout d'abord, un voile en cache la face sans que pour autant la puissance sacrée ne puisse manifester son omnipotence :

« De grands nuages blancs, gonflés comme des voiles de navire, dérivèrent [...] dans

la direction du col, où devait se produire l'apparition. Un frisson froid courut l'herbe rase..$^{30}$ "

Puis l'adolescent se trouve à son tour recouvert par ce voile, par le truchement duquel il est, dès lors, en mesure d'espérer obtenir satisfaction :

«Lorsqu'il m'enveloppa, je frissonnai et fermai les yeux, car je ne doutai pas qu'il

descendît sur nous pour nous préparer à l'apparition. ${ }^{31}{ }$ ”

16 Et c'est comme dans une sorte de transport que le narrateur peut assister à la transfiguration tant attendue : 
«Mon cœur [...] s'était mis à battre à coups tumultueux [...] et [...] lorsque l'Ossau surgit de la coupe du col de pierraille d'un seul élan, sur le ciel qui amassait autour de lui ses orages, je fus saisi d'une manière d'épouvante. ${ }^{32}$ "

\section{Réflexion au soir de la vie}

Reprenant, dans la deuxième partie de son ouvrage, ses considérations sur Aydie du haut de ses soixante ans, Joseph Peyré en complète sa vision. Il en souligne la joie qui y règne en raison de la culture de la vigne, laquelle «humanise la vallée ${ }^{33}$ ». À celle-ci s'ajoutent un «air neuf ${ }^{34}$ ", le «côté riant du paysage ${ }^{35}$ », son «renom de jardin ${ }^{36}$ ». C'est, pour sûr, un locus amcenus dans un hortus conclusus qui s'est peu à peu imposé dans son esprit et ce, grâce à l'amour que l'écrivain n'a cessé de lui prodiguer, un amour qui n'a rien d'un aveuglement, mais plutôt qui a été source de connaissance. Cependant, si pour Joseph Peyré, Aydie est un intangible enclos, du belvédère du Haut-d'Aydie se multiplient néanmoins en son esprit les hétérotopies, et par conséquent, les ouvertures :

«Il est ici ma jetée du Socoa, ma terrasse paloise, mon lieu de rêverie. ${ }^{37}$ »

Joseph Peyré en tire plutôt orgueil :

« Il me plaît d'embrasser ainsi, par-delà mon particularisme rebelle, l'harmonieux ensemble auquel j'appartiens et dont le site de mon village, site qui en est la clef, semble fait pour rendre visible le mouvement, l'articulation décisive [...] J'embrasse l'étendue, la profondeur de mon pays. Je sens, tout comme si j'y présidais moimême, le ruissellement, l'immense dérive, à partir de la vague bleue de la chaîne, des piémonts béarnais et basque, appelés par leur océan. ${ }^{38}$ »

De retour sur son promontoire aydien, après avoir parcouru cet immense et composite territoire pyrénéen, après l'avoir percé à jour dans ses composantes orographiques, minéralogiques et hydrologiques, Joseph Peyré a tout loisir d'en discerner « l'harmonie essentielle ${ }^{39}$ ", le sens. Aussi n'est-il point déraisonnable de concevoir, dans de telles conditions, Joseph Peyré comme un authentique radiographe pyrénéen, substantif faisant référence, bien évidemment, à Marcel Proust qui, le premier, a introduit le terme « radiographie » en littérature dans sa quête d'une réalité non donnée d'emblée et, à coup sûr, non exempte d'un prisme subjectif ${ }^{40}$. Pour une telle approche, la psyché seule est à même de dévoiler les essences entrant en jeu dans cette quête, laquelle recourt bien volontiers pour ce faire à la synesthésie si proustienne émanant d'une cénesthésie pleinement assumée: "Jamais les Pyrénées ne paraissaient aussi prochaines ni aussi hautes qu'à l'automne », fait remarquer Joseph Peyré.

«Leur ton mauve des soirs d'octobre reste pour moi lié à l'odeur des chais, des cèpes sur la lande, au roulement des chars de vendange, au cri des oies, auquel répondait, du haut des nuages voyageurs celui des oies sauvages surprises par la montée rapide de la nuit. ${ }^{41}$ " 


\section{BIBLIOGRAPHIE}

CAZALA Jean-Pierre, « Joseph Peyré, Aydie et le temps parfait », dans Joseph Peyré, L'homme de ses livres, Biarritz, J et D éditions, 1994, p. 83-91.

DELAY Pierre, Joseph Peyré (1892-1968), L'homme et l'œuvre : À la rencontre des héros, Biarritz, J et D éditions, 1992.

MANSo Christian, «L'hydrisme dans Guadalquivir », dans Joseph Peyré, L'homme de ses livres, Biarritz, J et D éditions, 1994, p. 287-300.

MANSo Christian, « España y Francia, Azorín y Joseph Peyré (1935), Una Academia Goncourt en España », dans Monóvar. Revista Cultural de la Asociación de Estudios Monoveros, $\mathrm{n}^{\circ}$ 32, Alicante, Instituto Alicantino de Cultura Juan Gil-Albert, sept. 2004, p. 12-13.

MANSo Christian, « Un Goncourt transpirenaico : Joseph Peyré, Le Pont des Sorts (1959) », dans Desde ambas laderas. Culturas entre la tradición y la modernidad, Barcelona, Universitat de Barcelona Edicions, 2015, p. 205-210.

MANSo Christian, «Tradición y revolución en la España del Frente Popular : La Tour de l'Or de Joseph Peyré ", dans Diálogos en la frontera. De la cultura popular a la cultura de masas en la era moderna, Zaragoza, Institución Fernando el Católico (CSIC), Excma Diputación de Zaragoza, 2016, p. 29-34.

PEYRÉ Joseph, De mon Béarn à la mer basque, 2e éd., Paris, Arthaud, 1987.

PEYRÉ Pierre, «Joseph Peyré, l'homme de toutes les énergies, Essai de psychobiographie », dans Joseph Peyré, L'homme de ses livres, Biarritz, J et D éditions, p. 15-31.

PROUST Marcel, À la recherche du temps perdu, Le temps retrouvé, Paris, Jean de Bonnot, 1989.

\section{NOTES}

1. J. Peyré, De mon Béarn à la mer basque, p. 9.

2. Ibid., p. 9.

3. Ibid.

4. Ibid.

5. Ibid., p. 18.

6. Ibid., p. 13.

7. Ibid.

8. Ibid., p. 16.

9. Ibid., p. 17.

10. Ibid.

11. Ibid., p. 21.

12. Ibid.

13. Ibid., p. 22.

14. Ibid., p. 29. 
15. Ibid.

16. Ibid., p. 30.

17. Ibid.

18. Ibid.

19. Ibid., p. 39.

20. Ibid., p. 38.

21. Ibid., p. 39-40.

22. Ibid., p. 41.

23. Ibid.

24. Ibid., p. 43.

25. Ibid.

26. Ibid.

27. Ibid., p. 45.

28. Ibid., p. 43.

29. Ibid., p. 46.

30. Ibid., p. 47.

31. Ibid.

32. Ibid., p. 48.

33. Ibid., p. 150.

34. Ibid.

35. Ibid.

36. Ibid., p. 157.

37. Ibid., p. 166.

38. Ibid., p. 173-174.

39. Ibid., p. 112.

40. M. Proust, À la recherche du temps perdu, tome sixième, p. 187.

41. J. Peyré, Ibid., p. 18.

\section{RÉSUMÉS}

Ancré en son village natal d'Aydie qui forme "l'angle extrême des Basses Pyrénées ", Joseph Peyré ne peut rêver de meilleur poste d'observation pour en sonder le site tout en en scrutant les alentours montagneux qu'il embrasse de la Bigorre au Pays basque. Ainsi en ressort-il notoirement dans De mon Béarn à la mer basque (Arthaud, Paris 1987). Ce faisant, il se livre à une systématique herméneutique paysagère, laquelle passée au crible de l'épistémologie, de l'esthésie et de l'esthétique, s'inscrit dans une véritable quête ontologique. 
AUTEUR

CHRISTIAN MANSO

Professeur Émérite en langue et littérature des civilisations espagnoles, Université de Pau et des Pays de l'Adour, ITEM, EA 3002 (Identités, territoires, expressions, mobilités) 


\title{
Une ré-Vision du Pyrénéisme pour le Xxie siècle
}

\author{
Manel Rocher Gonzalez et Claude Molinier
}

«Il faut continuer à enseigner selon les valeurs du Pyrénéisme ${ }^{1}$ »

1 Cet article constitue une réflexion sur le Pyrénéisme et, plus concrètement, sur l'importance et la transcendance qu'il a eu à son origine, au xix siècle, ainsi que sur la décadence dans laquelle il sombra au $\mathrm{xx}^{\mathrm{e}}$ siècle. Nous présentons une proposition pour sa renaissance.

2 Il est aujourd'hui indispensable de tirer la sonnette d'alarme et de faire une ré-Vision $\mathrm{du}$ Pyrénéisme pour affronter le $\mathrm{xxI}^{\mathrm{e}}$ siècle, si nous ne voulons pas qu'il tombe définitivement dans l'oubli. En effet, si nous regardons les résultats des recherches effectuées sur Google, nous nous rendons parfaitement compte de la situation actuelle (tabl. 1.).

Tabl. 1. - Recherche de mots clés.

\begin{tabular}{|l|l|}
\hline Terme & Résultat \\
\hline Pyrénéisme - fr- & 80700 \\
\hline Pirineisme - cat- & 4210 \\
\hline Pirineismo - esp- & 12200 \\
\hline Alpinisme - fr- & 11600000 \\
\hline Alpinisme - cat- & 7390000 \\
\hline Alpinismo - esp- & 8350000 \\
\hline
\end{tabular}

3 Il est évident que le terme "Alpinisme » a " écrasé » le terme « Pyrénéisme »; l'objet de cette étude n'est pas d'analyser les causes, mais bien de faire une proposition pour préserver la connaissance de ce mouvement, et, par conséquent, la vigueur de cette riche culture que constitue le Pyrénéisme (fig. 1 et 2). 
Fig. 1. - Refugi Rosta.

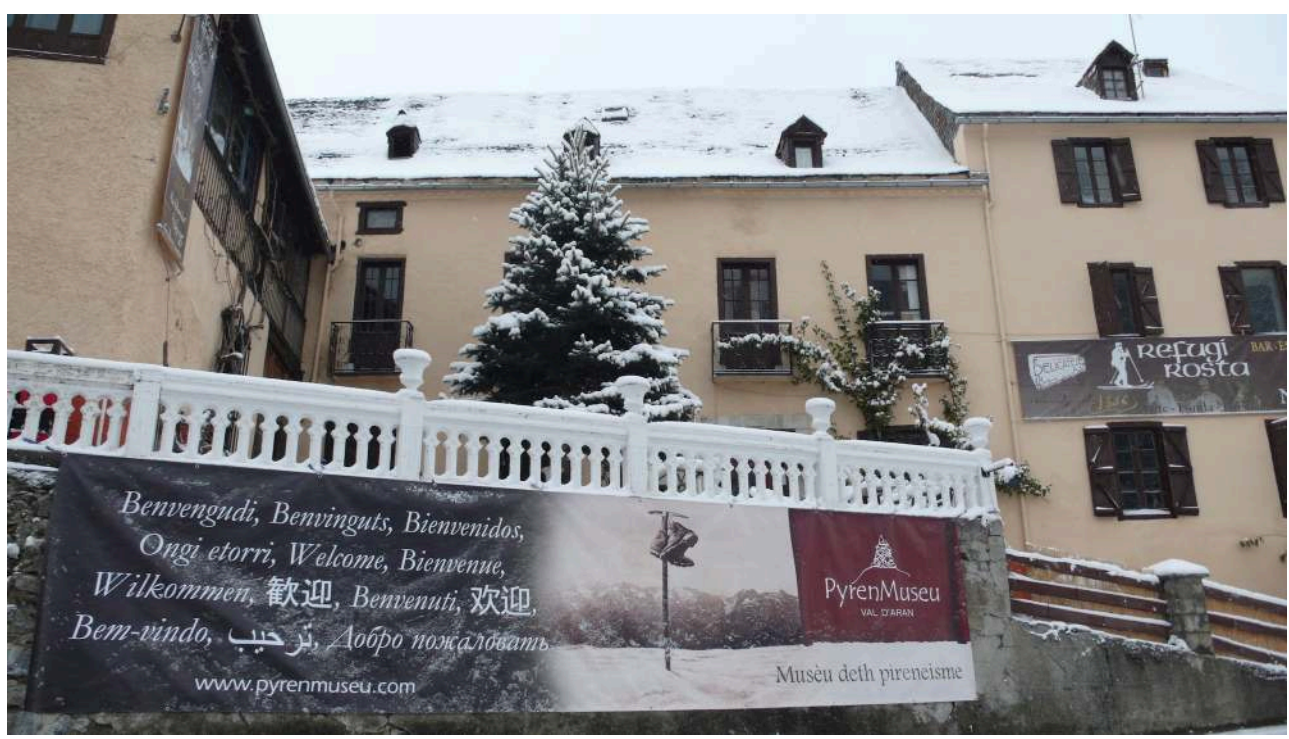

(c) Manel Rocher, Collection PyrenMuseu.

Fig. 2. - Salle Galeria, PyrenMuseu-Refugi Rosta.

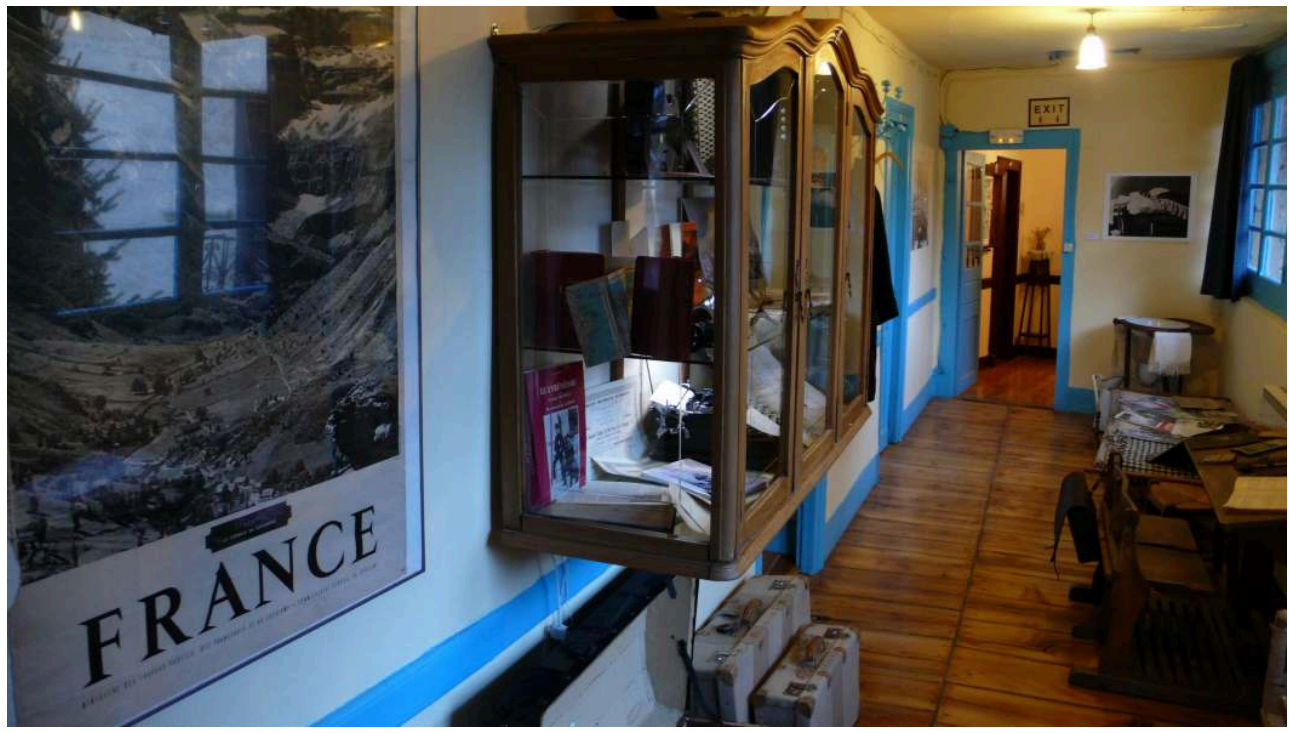

(c) Manel Rocher, Collection PyrenMuseu.

4 Le Pyrénéisme est un mouvement scientifique, naturaliste et culturel ${ }^{2}$. De fait, c'est un mélange, un recueil de grands concepts qui se rencontre en de très rares occasions : science, philosophie, recherche, arts, exploration, littérature, culture, aventure, sport... C'est « pratiquement » un courant civilisateur.

$5 \mathrm{Au} \mathrm{xx}^{\mathrm{e}}$ siècle, le Pyrénéisme désigne le courant qui rassemble des personnes très différentes qui partent à la découverte et à la conquête des montagnes et des vallées des Pyrénées. De nombreuses personnes sont venues d'horizons lointains pour participer à cette aventure, et profiter de la richesse et de la beauté que la culture et les paysages pyrénéens leur offraient. 
Il n'est pas possible de parler de tous, mais il est nécessaire d'en mentionner certains : Louis Ramond de Carbonnières (1755-1824), envoyé de Paris à Pau, grand poète et naturaliste, considéré comme le "père » du Pyrénéisme ; Henri Russel (1834-1909), aristocrate audacieux et pionnier, aux très nombreuses ascensions. Maurice Gourdon (1847-1941), grand découvreur de ma "demeure ", le Val d'Aran. Franz Schrader (1844-1924) géographe, personnification de la sensibilité et de la constance au travail : un génie. Juli Soler i Santalo (1865-1914), qui introduisit le Pyrénéisme dans la société catalane. Henri Beraldi (1849-1931), auteur de l'œuvre immense Cent ans aux Pyrénées, et à qui nous devons la merveilleuse maxime : "Marcher, sentir, écrire ", qui résonnera très longtemps, pour définir et différencier la culture montagnarde des Pyrénées de toutes les autres cultures montagnardes du monde (fig. 3).

Fig. 3. - Maurice Gourdon avec ses guides.

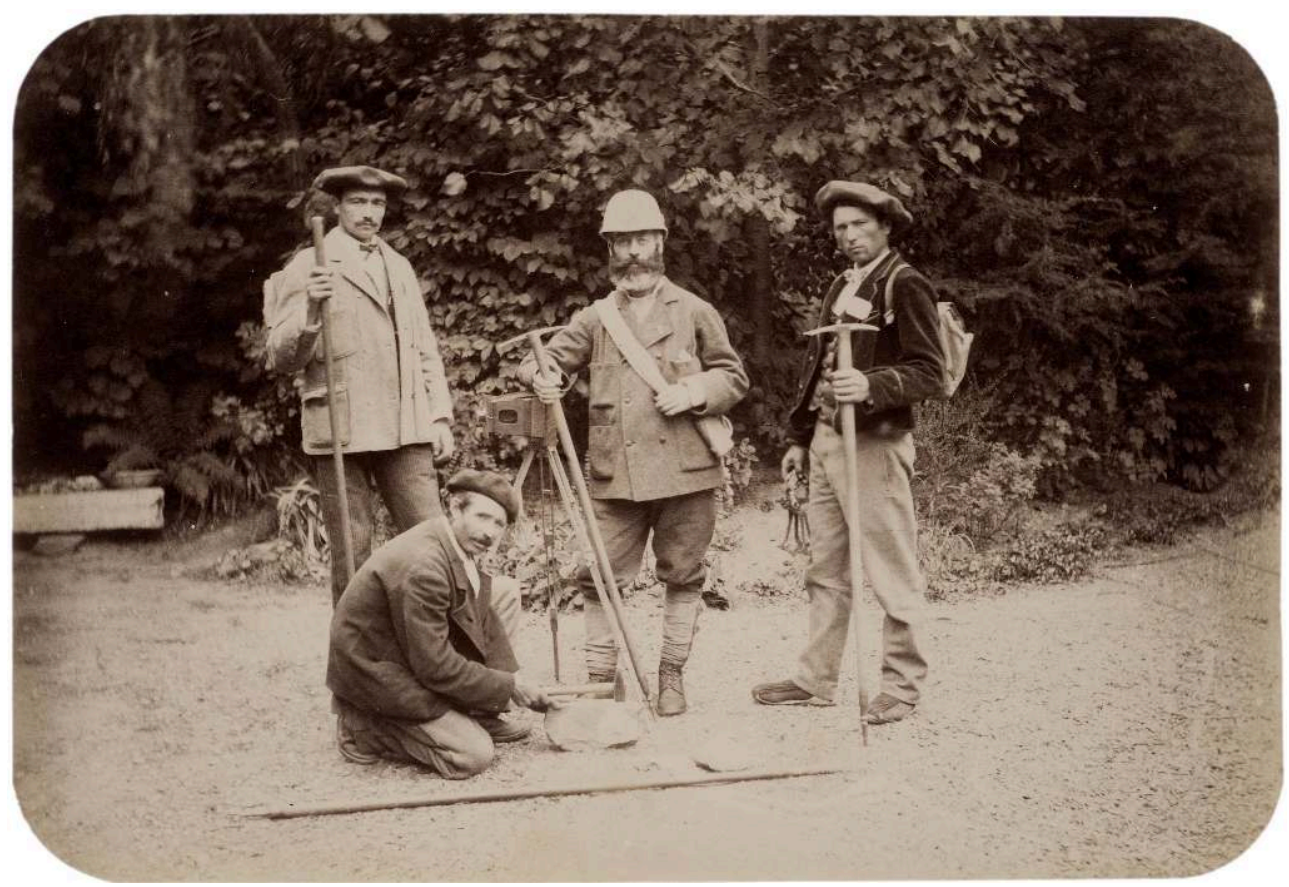

(c) Maurice Gourdon, Collection PyrenMuseu.

$7 \quad$ Tout au long du $\mathrm{xx}^{\mathrm{e}}$ siècle se sont développées diverses cultures montagnardes dans les Alpes, l'Himalaya et les Andes ; mais, il n'existe pas de mouvement similaire à celui qui s'est produit dans les Pyrénées au XIX ${ }^{\mathrm{e}}$ siècle.

8 Le Pyrénéisme a construit une vision scientifique et humaniste du territoire et de ses habitants. C'est un concept d'«exploration" totale. Le plus secret comme le plus commun est mis à nu jusqu'à ce qu'il soit compris et divulgué (fig. 4). Le défi que constitue l'ascension d'une montagne évolue en permanence. À la lumière de la révolution industrielle et par la fascination croissante de la conquête des montagnes apparemment inatteignables, ce défi a évolué jusqu'à créer un nouveau sport, une marque qui définit la pratique sportive en montagne qui a été baptisée d' " alpinisme ». C'est à ce moment qu'a commencé le déclin progressif du Pyrénéisme face au « monstre » qu'il a lui-même contribué à créer (fig. 5). 
Fig. 4. - Carte du Val d'Aran. Franz Schrader.

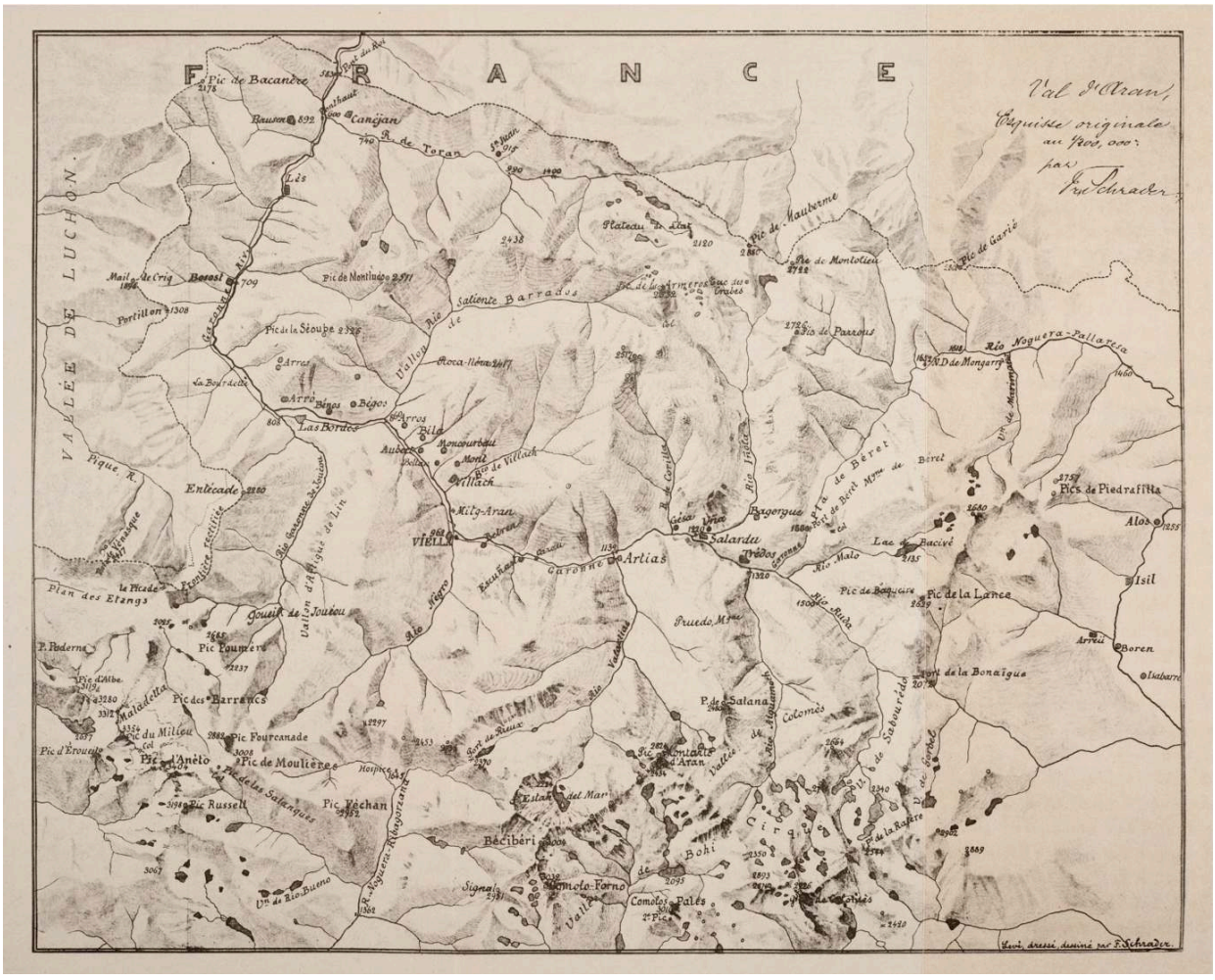

(c) Manel Rocher, Collection PyrenMuseu.

Fig. 5. - Pic de Posets.

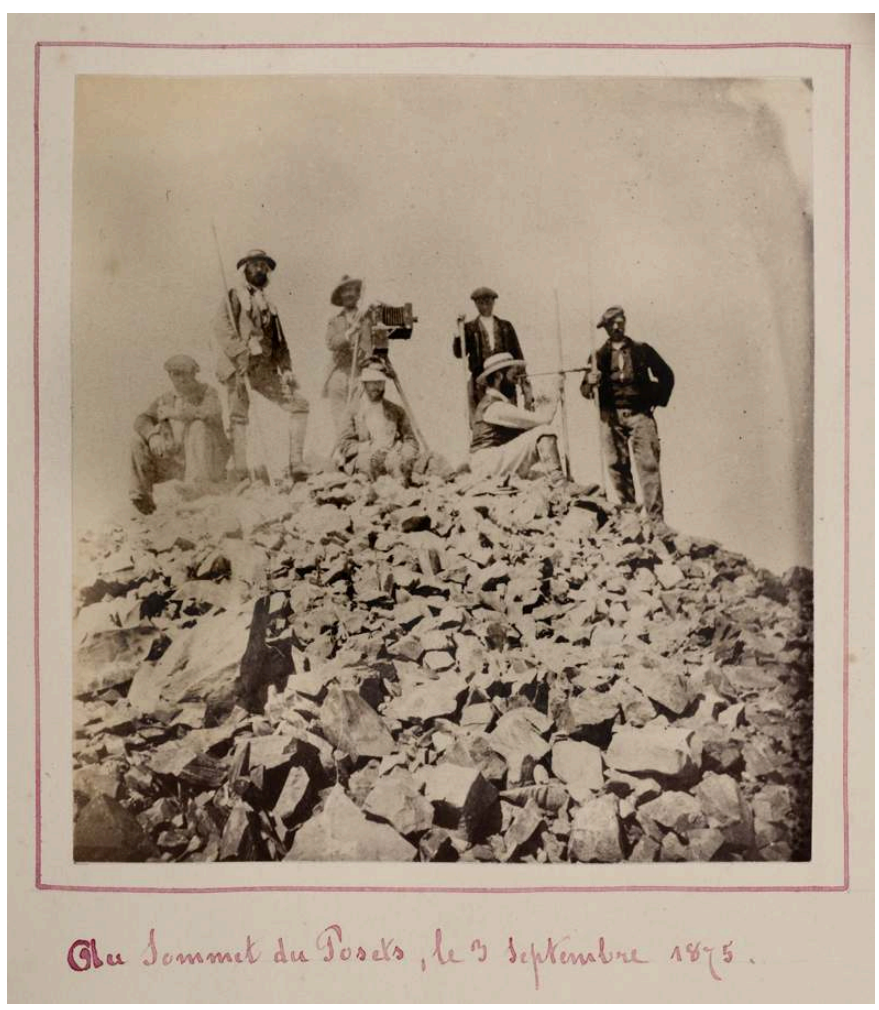

(c) Maurice Gourdon, Collection PyrenMuseu. 
Nous avons vu en introduction que la comparaison des résultats des recherches sur Google pour les termes "Pyrénéisme » et «Alpinisme » fait apparaître une relation inférieure à $0,01 \%$. D'autres éléments confirment le constat suivant : selon les enquêtes réalisées au PyrenMuseu de Salardu, seulement $1 \%$ des visiteurs ont entendu parler du Pyrénéisme.

10 Ainsi, au $\mathrm{xx}^{\mathrm{e}}$ siècle, le Pyrénéisme en tant que courant, après un siècle de brillantes réalisations, d'énormes découvertes et d'incroyables apports, au sens le plus large du terme, succombe devant la terminologie « Alpinisme». Et aujourd'hui, au début du Xxi ${ }^{e}$ siècle, il doit affronter sa disparition de l'Histoire... ou bien vivre une renaissance. Ce constat constitue la motivation de cette proposition de "ré-Vision », de cette réflexion urgente, si nous ne voulons pas que le Pyrénéisme soit réduit à un courant du passé. Le cadre du congrès Circulations montagnardes, circulations européennes, à l'université de Pau et des Pays de l'Adour, a constitué le lieu idéal pour envisager cette question.

\section{Le $\mathrm{XxI}^{\mathrm{e}}$ siècle}

11 Le $\mathrm{xxI}^{\mathrm{e}}$ siècle débute par d'importants progrès dans de nombreux domaines: communication, nouvelles technologies, mobilité et grands défis de tous types (politiques, environnementaux, sanitaires, économiques, sociaux) auxquels il faut répondre avec rapidité et sagesse. C'est dans ce contexte que les valeurs du Pyrénéisme peuvent être extrêmement utiles pour inspirer la société pyrénéenne dans cette vertigineuse évolution ou révolution! Ce qui nous attend, ce qui est en train d'arriver, ce qui est en train de se construire, nous ne le connaissons pas, nous ne pouvons l'imaginer, mais il est absolument nécessaire, que cela se développe sur une base humaniste (fig. 6 et 7).

Fig. 6. - Reproduction du panoramique de Maladetas.

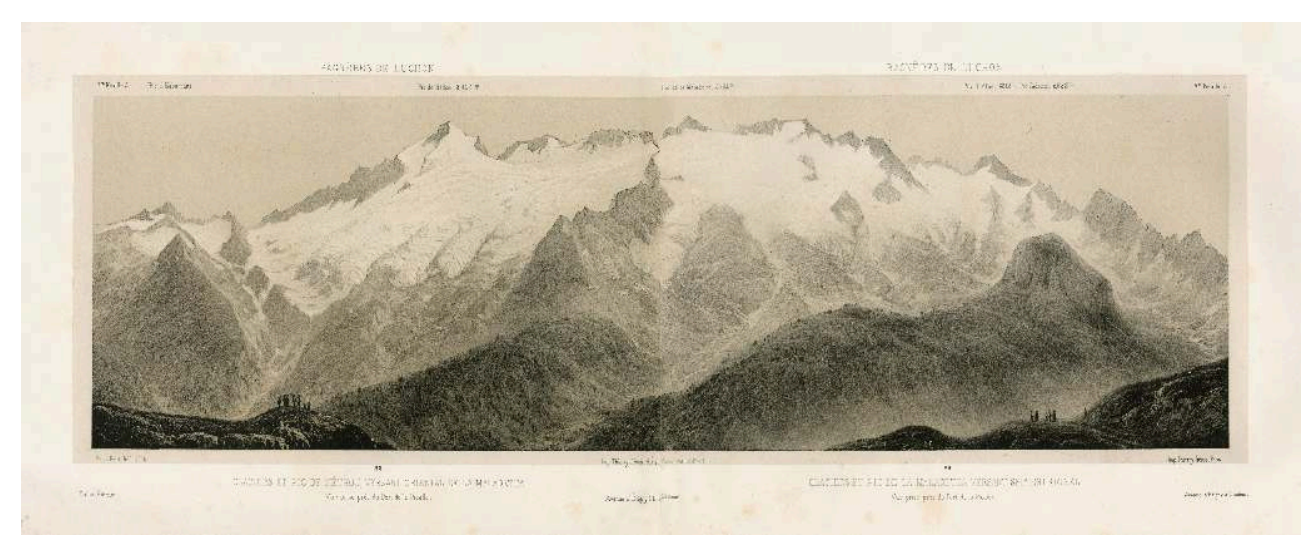

(c) Victor Petit, Collection PyrenMuseu. 
Fig. 7. - Manuscrit Maurice Gourdon.

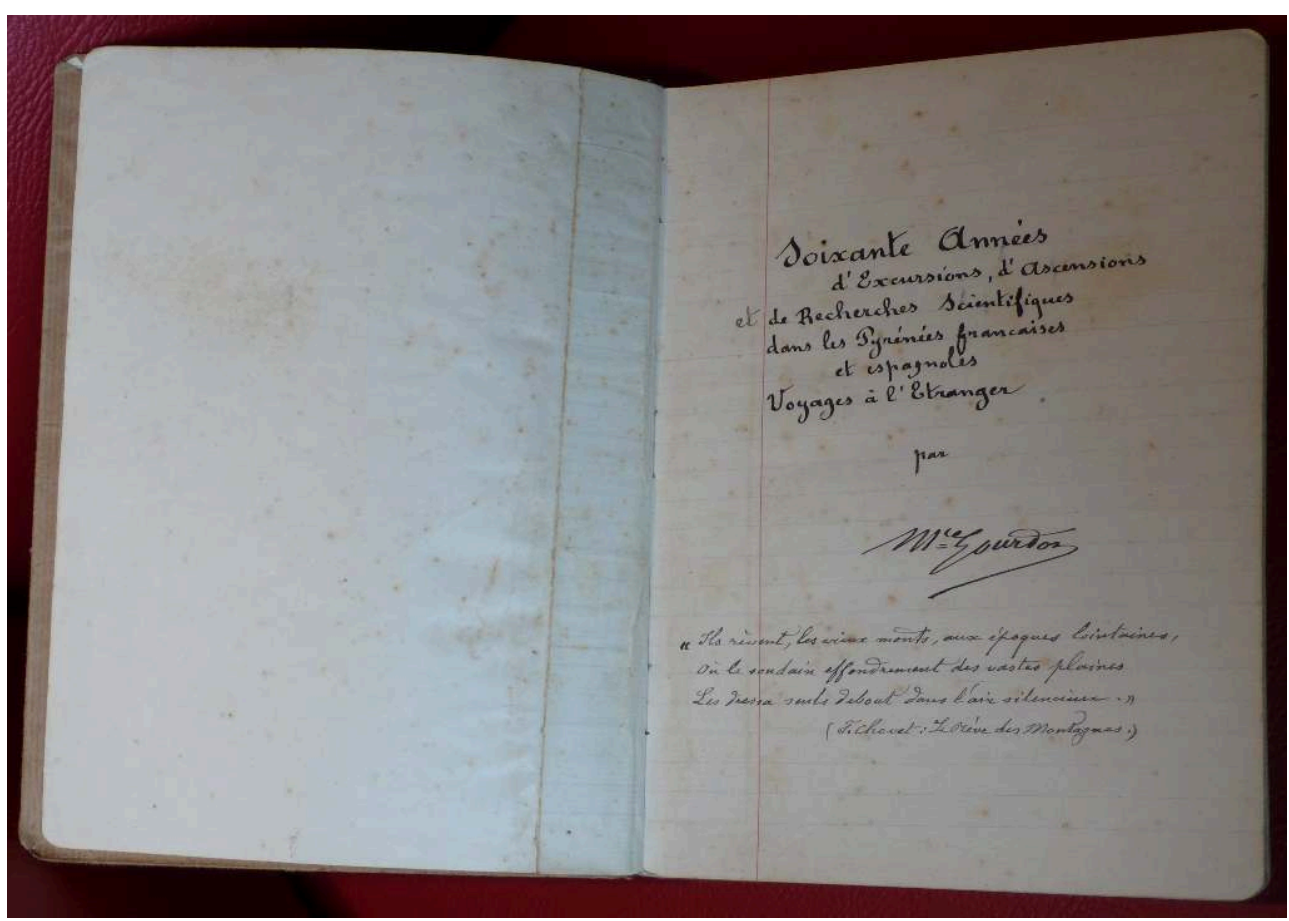

(c) Manel Rocher, Collection PyrenMuseu.

\section{La vision}

12 «Marcher, sentir, écrire ». Lorsque Henri Beraldi synthétise le Pyrénéisme dans cette phrase, il crée quelque chose de plus qu'une définition : il présente une vision. Les visions sont très puissantes, car elles sont indispensables pour générer l'ensemble des idées nécessaires et les diriger avec efficacité vers la réalisation d'un but, si nous ne voulons pas perdre les ressources et le temps disponibles. La valeur d'une vision est immense. Ce fut le cas en 1895, lorsque Beraldi l'écrivit dans le premier volume de son œuvre Cent ans aux Pyrénées, où il s'est attaché à rendre compte de la " découverte » des Pyrénées, de l'exploration de ses sommets, de ses vallées, de sa culture et de ses paysages. En lançant le siècle d'or du Pyrénéisme dans une œuvre de sept volumes, il a aussi voulu témoigner pour les siècles à venir (fig. 8). 
Fig. 8. - Reprogravure de l'Entecada.

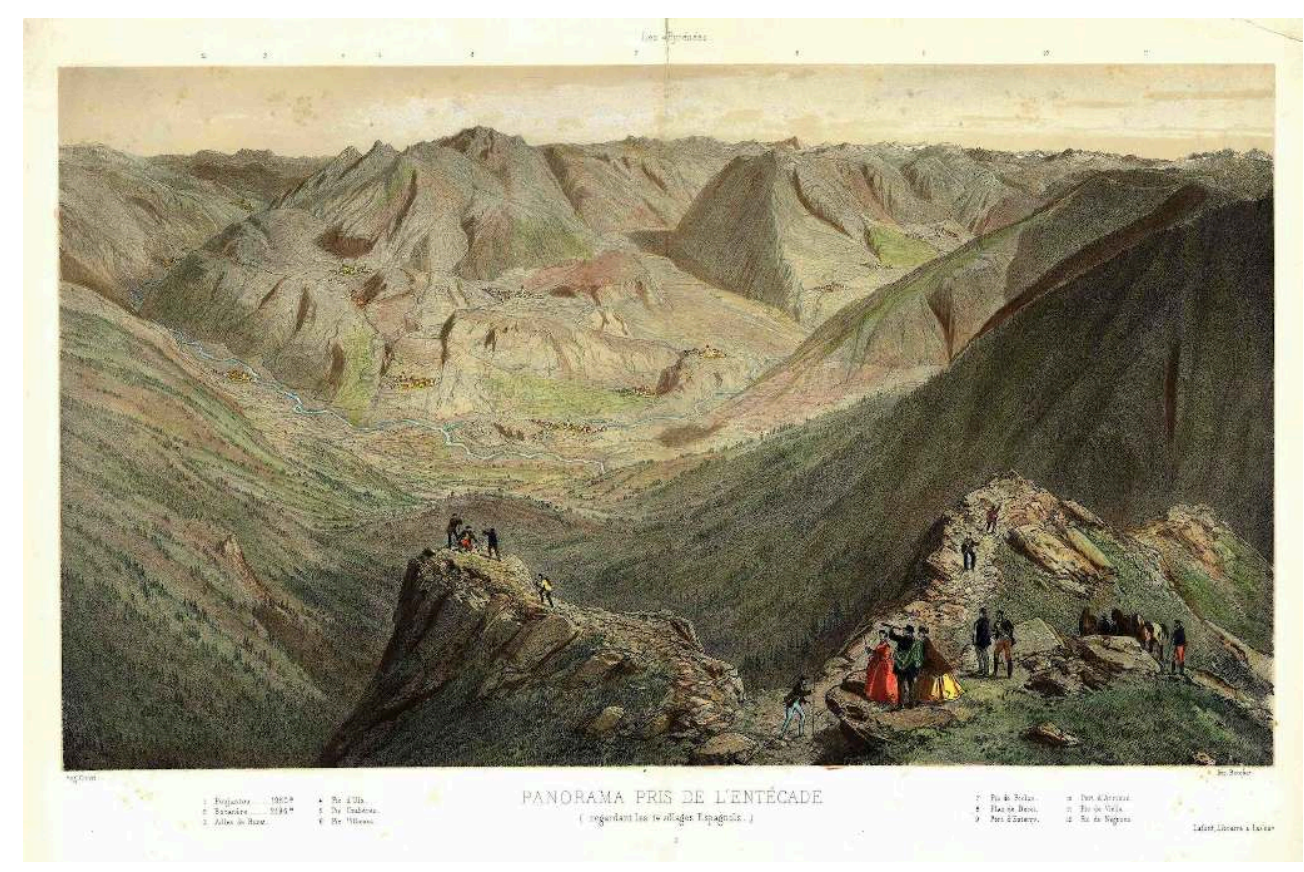

(c) Eugène Ciceri, Collection PyrenMuseu.

Les visions stratégiques doivent s'adapter aux changements qui se produisent avec le temps qui passe. Et ce moment, comme cette proposition, est venu pour le Pyrénéisme. C'est un mouvement trop important pour le laisser dans les oubliettes de l'Histoire. Il doit beaucoup apporter à notre société pyrénéenne. De fait, au-delà de la question, que faisons-nous ? il peut nous aider à répondre à la question, pourquoi faisons-nous cela ?

Une vision stratégique, c'est la capacité de prévoir le futur dans un projet, sur un territoire : c'est le fondement, la ligne à suivre. Et nous les pyrénéistes, nous l'avons : «Marcher, sentir, écrire» nous dit Henri Beraldi. Ne la perdons pas de vue! C'est l'essence du Pyrénéisme! Laissons-la inspirer nos pas. Utilisons la vision d'Henri Beraldi pour dissiper la confusion. Récupérons les valeurs du Pyrénéisme pour ce nouveau $\mathrm{XxI}^{\mathrm{e}}$ siècle. Avec toutes ses réalisations, ses farces et rêves brisés, le $\mathrm{xx}^{\mathrm{e}}$ siècle est déjà passé ; mais ce nouveau Xxi ${ }^{e}$ siècle sera celui dans lequel vivront nos enfants et nos petits-enfants. Nous pouvons travailler pour que les Pyrénées soient le lieu le plus merveilleux du monde (fig. 9). 
Fig. 9. - Les touristes sur le glacier de Paoule.

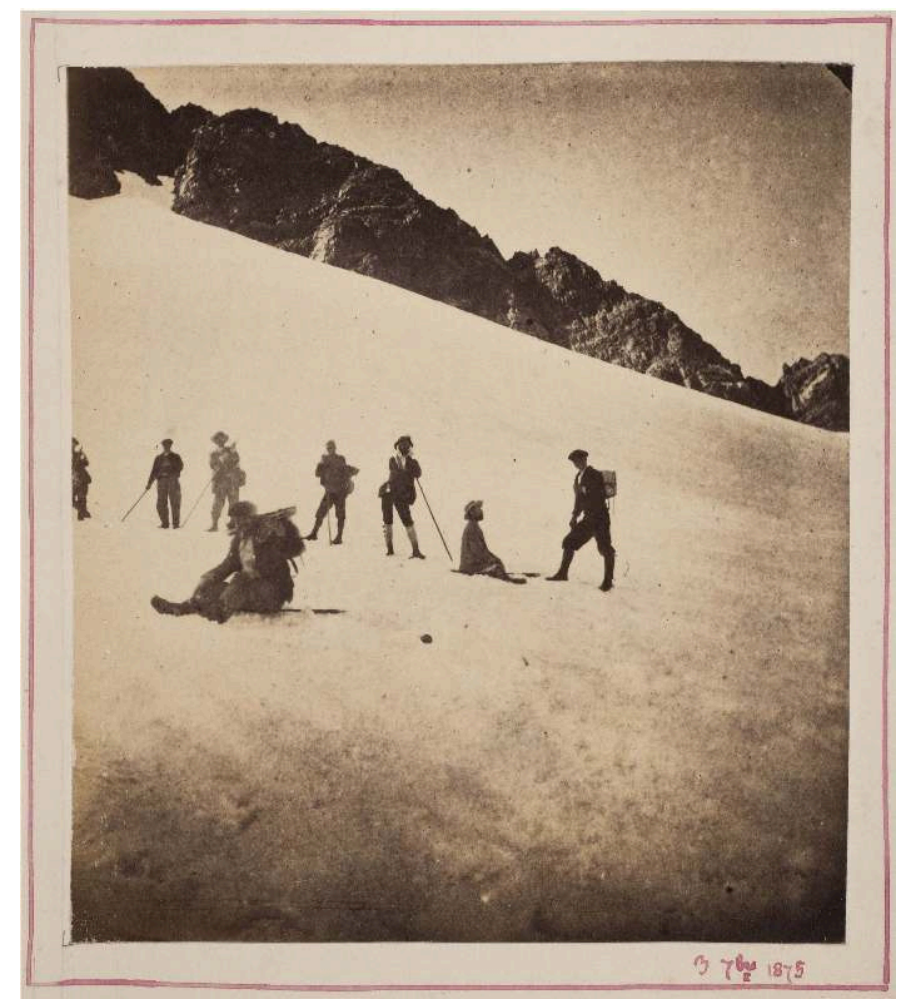

() Maurice Gourdon, Collection PyrenMuseu.

\section{«II faut poursuivre l'éducation avec les valeurs du Pyrénéisme »}

Cette phrase d'Eduardo Martinez de Pisón pourrait être revendiquée par tous les Pyrénéistes, par tous les amoureux des Pyrénées. Tous les connaisseurs de l'histoire du Pyrénéisme rêvent de perpétuer, grâce à l'éducation et à l'enseignement, les valeurs du Pyrénéisme. Et cela est possible. L'éducation et la formation, comme base de création du talent, peuvent être la voie pour freiner le dépeuplement des villages pyrénéens, en créant l'empathie nécessaire pour aller vers une meilleure qualité de vie. Si l'on pouvait obtenir que les vallées des Pyrénées soient territoires leader en créativité et en connaissances, on créerait l'attraction nécessaire pour stabiliser une population, et pour cela « Marcher, sentir, écrire » associé à « éduquer » est fondamental.

\section{Éducation et Pyrénéisme : la vision d'Henri Beraldi révisée}

En résumé, différentes questions nous assaillent: quels sont les instruments nécessaires à la continuité du Pyrénéisme, comment obtenir sa reconnaissance, et comment instaurer un cadre de discussion qui permettra d'inclure les valeurs du Pyrénéisme dans l'éducation?

La réponse existe. Nous proposons de présenter une candidature à l'Unesco afin d'inscrire le Pyrénéisme sur la liste du Patrimoine Culturel Immatériel nécessitant une 
sauvegarde urgente ${ }^{3}$. Ainsi le terme serait préservé et se ferait connaître dans le monde entier. Le Pyrénéisme remplit largement les prérequis nécessaires pour obtenir une telle inscription :

- Critère 1. L'élément est constitutif du patrimoine culturel immatériel tel que défini à l'article 2 de la Convention du 17 octobre 2003.

- Critère 2.

- L'élément nécessite une sauvegarde urgente parce que sa viabilité est en péril, en dépit des efforts déployés par la communauté, le groupe ou, le cas échéant, les individus et l(es) État(s) ou partie(s) concerné(s) ;

- L'élément se trouve confronté à une nécessité extrêmement urgente de sauvegarde parce qu'il fait l'objet de menaces sérieuses auxquelles il ne pourrait survivre sans intervention immédiate ;

- Critère 3. Des mesures de sauvegarde sont élaborées pour qu'elles puissent permettre à la communauté, au groupe ou, le cas échéant, aux individus concernés de poursuivre la pratique et la transmission de l'élément;

- Critère 4. L'élément a été soumis au terme de la participation la plus large possible de la communauté, du groupe ou, le cas échéant, des individus concernés et avec leur consentement libre, préalable et éclairé :

- Critère 5. L'élément figure dans un inventaire du patrimoine culturel immatériel présent sur le(s) territoire(s) de(s) (l')État(s) partie(s) soumissionnaire(s), tel que défini dans les articles 11 et ou 12 de dite Convention.

- Critère 6. Dans des cas d'extrême urgence, l(es) État(s) ou partie(s) concerné(s) a (ont) été dûment consulté(s) sur la question de l'inscription de l'élément conformément à l'article 17.3 de dite Convention.

18 Le Pyrénéisme remplit les prérequis nécessaires pour obtenir une telle inscription :

- Malgré sa fragilité, le patrimoine culturel immatériel est un facteur important dans le maintien de la diversité culturelle face à une globalisation croissante. La connaissance du patrimoine culturel immatériel de différentes communautés contribue au dialogue entre les cultures et promeut le respect envers les autres modes de vie.

- L'importance du patrimoine culturel immatériel ne réside pas dans la manifestation culturelle elle-même, mais dans les acquis des connaissances et des techniques qui se transmettent de génération en génération. La valeur sociale et économique de cette transmission de connaissances est pertinente pour les groupes sociaux aussi bien minoritaires que majoritaires dans un État, et revêt la même importance pour les pays en développement que pour les pays développés.

Ce travail doit être assumé par les agents territoriaux, les institutions et organisations sociales, et peut constituer un puissant élément de cohésion transpyrénéenne.

- Le patrimoine culturel immatériel ne s'évalue pas simplement comme un bien culturel, à titre de comparaison, pour son exclusivité ou sa valeur exceptionnelle. Il s'épanouit dans les communautés et dépend de ceux dont les connaissances des traditions, des techniques et coutumes se transmettent aux autres membres de la communauté, de génération en génération, ou à d'autres communautés.

- Basé sur la communauté, le patrimoine culturel immatériel ne l'est en tant que tel que s'il est reconnu par les communautés, groupes ou individus qui le créent, le maintiennent et le transmettent. Sans cette reconnaissance, personne ne peut décider pour un autre qu'une expression, ou un usage déterminé, fait partie de son patrimoine. 
Ce projet constitue une bonne voie à suivre. Avec passion et avec un travail commun, nous pouvons ouvrir un chemin de renaissance pour le Pyrénéisme.

\section{BIBLIOGRAPHIE}

BAQUÉS I SOLER Josep, Les muntanyes d'Aran i el Pirineisme. Una síntesi histórica, Tremp, Garsineu edicions, 2010.

BERALDI Henri, Cent ans aux Pyrénées, Paris, 1898-1904, (7 vol.).

GOURDON Maurice, À travers l'Aran. Itinéraires d'un touriste, rééd., Nîmes, éd. Lacour, 2005.

JOANNE Paul, Pyrénées, Paris, éd. Hachette et $C^{\text {ie }}, 1888$.

LÉVY André, Le dictionnaire des Pyrénées, Toulouse, Privat, 1999.

RAMOND de CARBONNIÈRES Louis, Lettres inédites, Bagnères-de-Bigorre, Société Ramond, 1927.

RITTER Jean, Le Pyrénéisme avec Henry Russell et Bertrand de Lassus, Louveciennes, 2001.

SAULE-SORBÉ Hélène, Pyrénées Voyages photographiques, Pau, Éditions du Pin à Crochets, 1998.

SCHRADER Franz, Les Pyrénées, Toulouse, Privat, 1932, (2 vol.).

SOLER I SANTALó Juli, Guia de la Val d'Aran, 2ª́d. Barcelona, CEC, 1933.

\section{NOTES}

1. Eduardo Martinez de Pisón. Conférence «Alabanza de los Pirineos" à Pyrenades Festival, 24/3/2018. [https ://www.youtube.com/watch? v=DTUlpKROcGc\&list=PLjoQCAj47jAz3pNy8rG1M4Ko71ijlWpoE\&index=13].

2. Pyrenades. Conferencias y debate: «Retos de futuro del Pirineismo» [http:// www.pyrenades.es/futur-pirineisme.html]

3. Unesco. Patrimoine Culturel Immatériel. Procédure d'inscription d'éléments sur les Listes et de sélection de bonnes pratiques de sauvegarde. [https://ich.unesco.org/fr/ procedure-d-inscriptions-00809] 


\section{RÉSUMÉS}

Le Pyrénéisme est un mouvement culturel et sportif. Dans les faits, c'est un recueil de grands concepts tels que la philosophie, la recherche, l'art, l'exploration, la littérature, la culture, l'aventure, le sport, la science, qui se combinent parfaitement pour définir le flux de personnes qui, au XIx siècle, part à la découverte et à la conquête des Pyrénées, afin de profiter de la richesse de la culture et des paysages qui leur sont offerts. Au cours du xx siècle, ce mouvement tombe en décadence: le mot alpinisme s'impose comme la définition du sport pratiqué en montagne, alors que la maxime d'Henri Beraldi, "marcher, sentir, écrire » était toujours présente à l'esprit des montagnards pyrénéens. Face aux défis du Xxi ${ }^{\mathrm{e}}$ siècle, il est nécessaire de proposer une "ré-Vision» du Pyrénéisme, comme mouvement lié à la société pyrénéenne. Le Pyrénéisme, au lieu d'être un mouvement de "visiteurs des Pyrénées», doit devenir un mouvement des « habitants des Pyrénées ». Le XxI ${ }^{\mathrm{e}}$ siècle, avec tous ses progrès, peut transformer notre chaîne de montagnes en une des sociétés les plus avancées au monde. Il est seulement nécessaire que ses habitants croient dans cette «ré-Vision». Pour cela, nous proposons de présenter la candidature du Pyrénéisme à l'inscription sur la liste du Patrimoine Culturel Immatériel de l'Humanité de l'Unesco.

El Pirineismo es un movimiento cultural y deportivo. De hecho es una miscelánea de grandes conceptos que combinan como en muy pocas ocasiones, la filosofía, la investigación, el arte, la exploración, la literatura, la cultura, la aventura, el deporte, la ciencia, para definir la corriente de personas que en el siglo XIX consigue el descubrimiento y la conquista de las montañas y los valles de los Pirineos. Muchos personajes vinieron de tierras lejanas para participar en esta aventura y disfrutar de la riqueza que las culturas y paisajes Pirenaicos les ofrecían. Durante el siglo XX este movimiento fue decayendo curiosa y principalmente por una cuestión semántica : la palabra alpinismo se impuso como definición del deporte en la montaña, si bien la máxima de Henri Beraldi, «caminar, sentir, escribir » seguía presente en el espíritu de los montañeros pirineistas. Ahora, ante los retos del nuevo siglo XXI, es necesario plantear una reVisión del Pirineismo como movimiento vinculado a la sociedad pirenaica. El artículo que presentamos se orienta en este sentido: el Pirineismo, en lugar de ser un movimiento de visitantes de los Pirineos ha a ser un movimiento de los habitantes de los Pirineos. El siglo XXI, con todos sus avances, puede convertir nuestra cordillera en una de las sociedades mas avanzadas del mundo. Para ello, solo es necesario que sus habitantes crean en esta reVisión : propongamos presentar a la Unesco la candidatura del Pirineismo a la denominación de Patrimonio Cultural Inmaterial de la Humanidad.

\section{AUTEURS}

\section{MANEL ROCHER GONZALEZ}

Directeur - Fondateur du PyrenMuseu - Musée du Pyrénéisme

\section{CLAUDE MOLINIER}

Guide de montagne, traducteur et pyrénéiste 